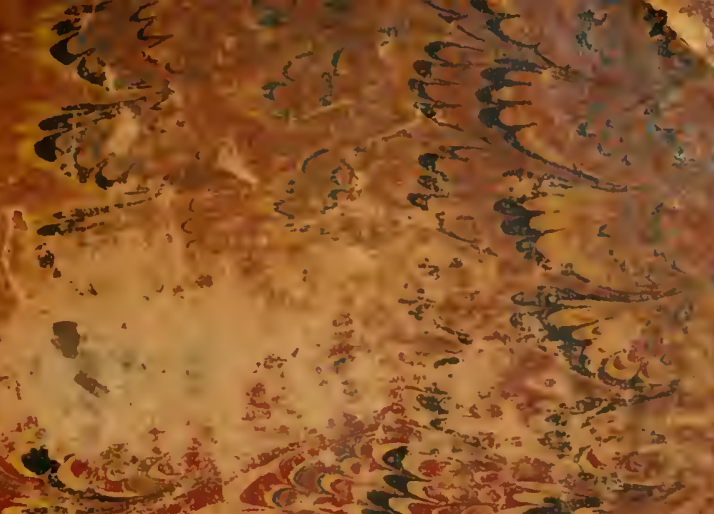

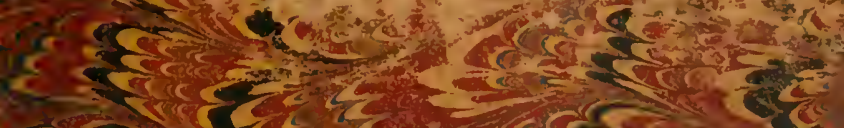

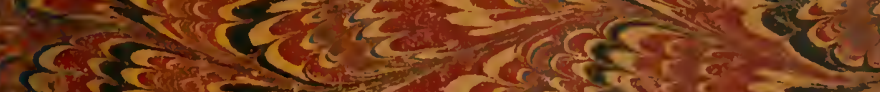

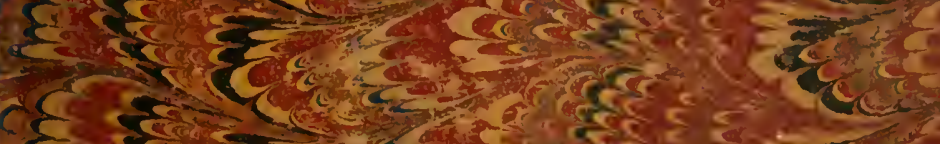

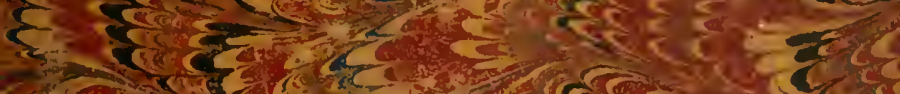

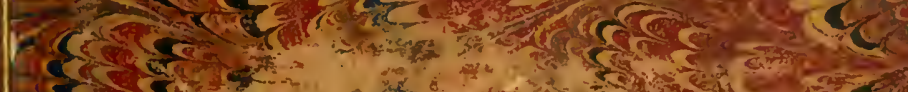

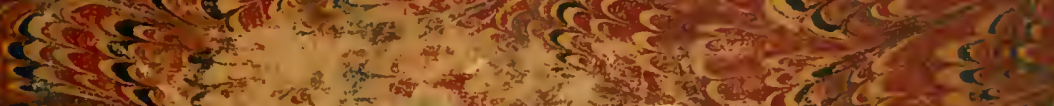

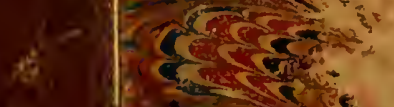

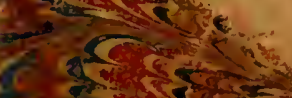

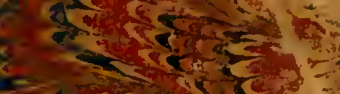

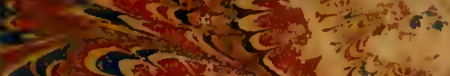

$\left.\left.16 c^{2}+2\right)^{2}\right)^{2}$

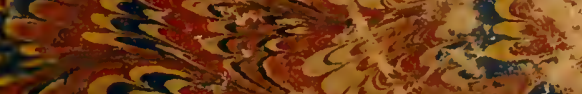

है।

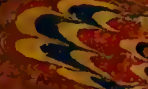

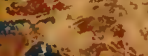

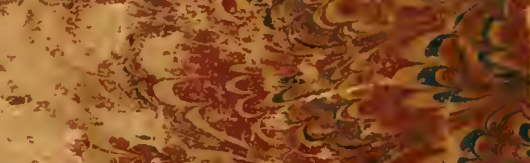

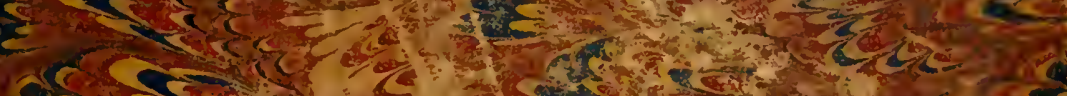

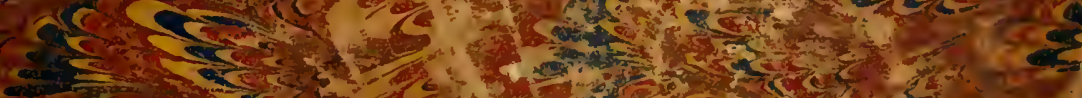

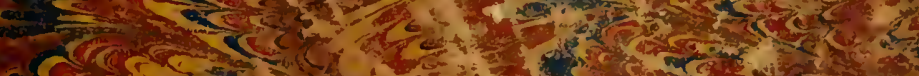

Ser

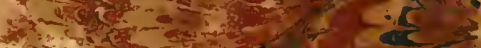
z. 4 in

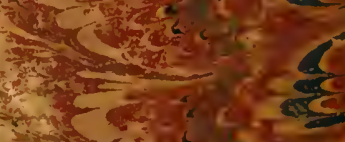

as

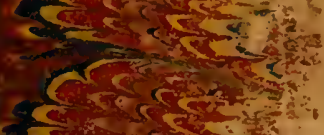

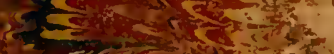

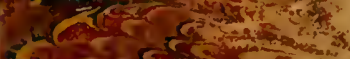

हैं

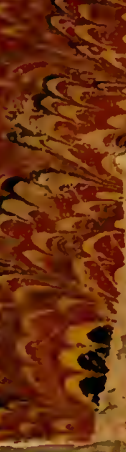

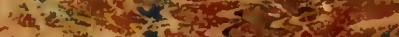

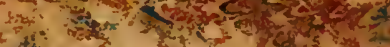

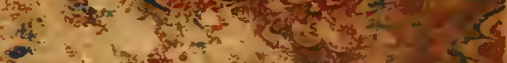

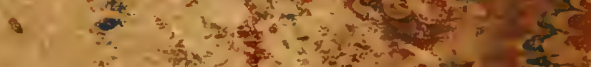

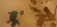

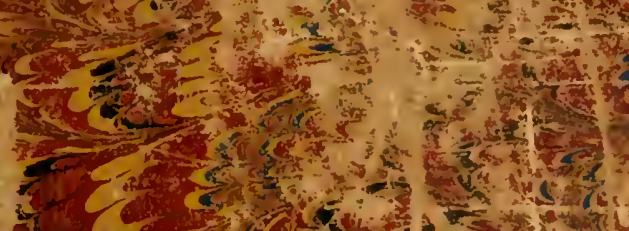

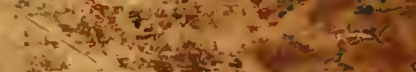

tite

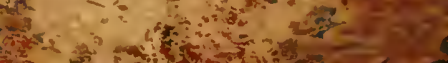
Q

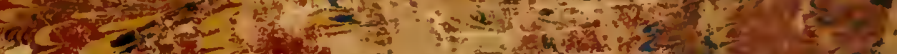
(1)

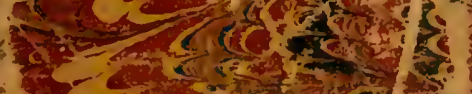

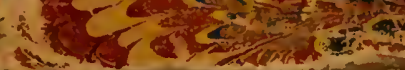

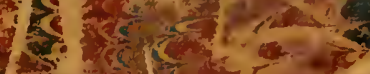



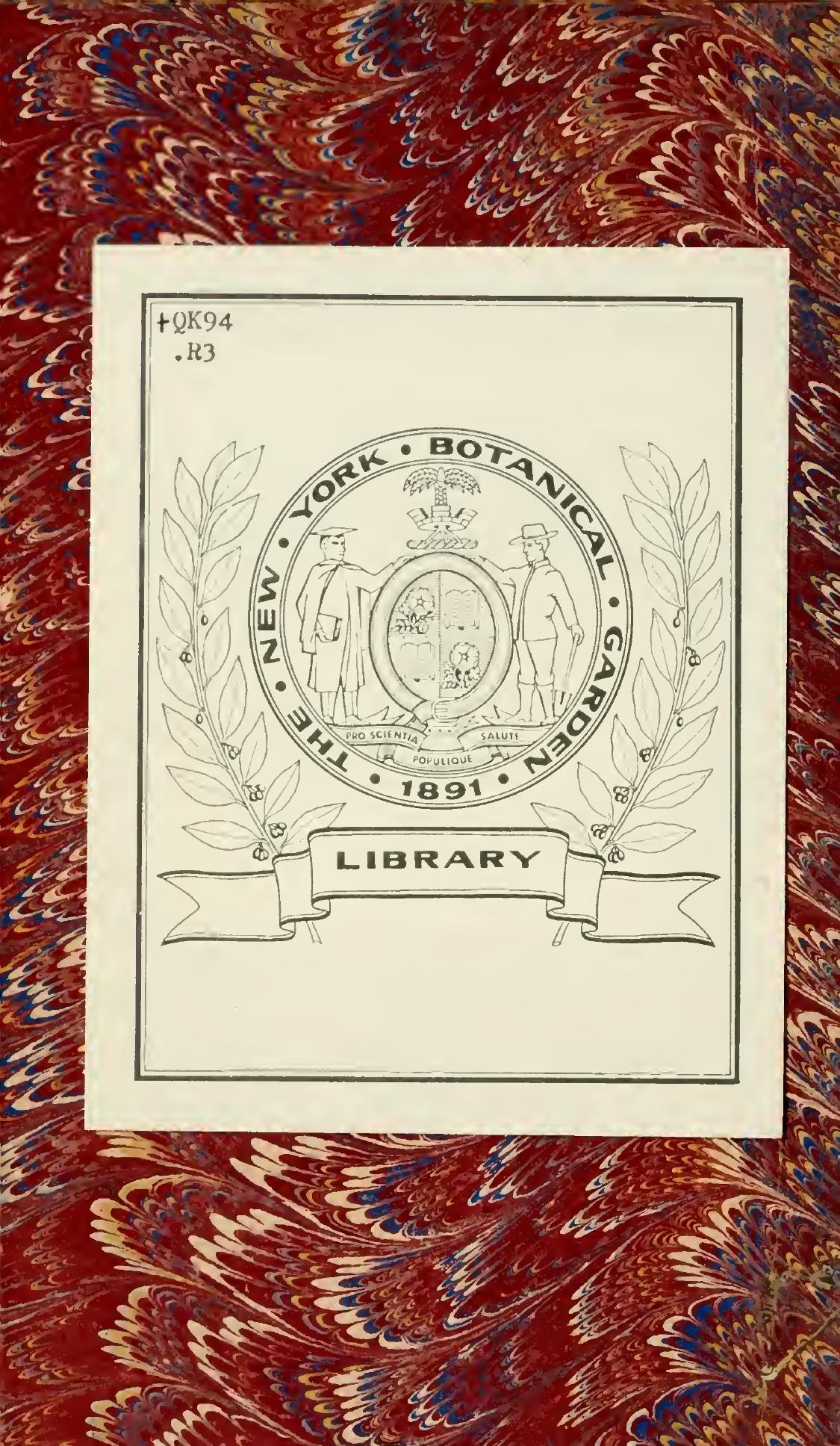
Nof

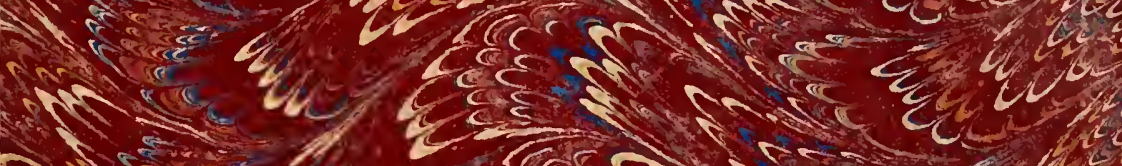
I.

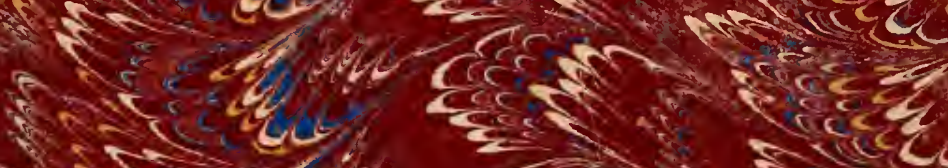

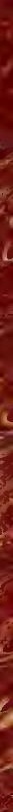

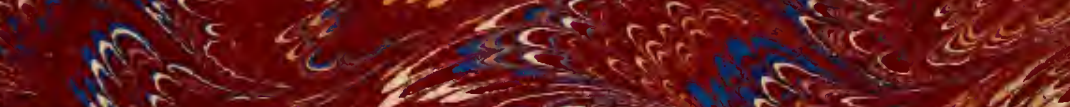
M है त ईहै है

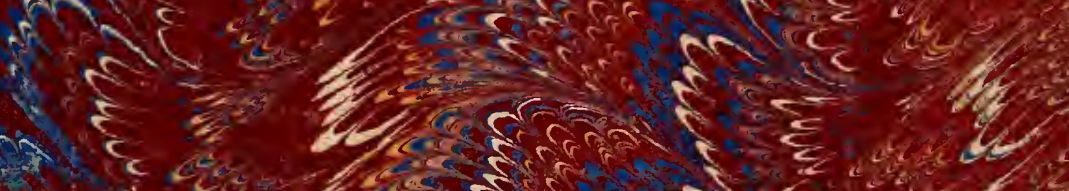

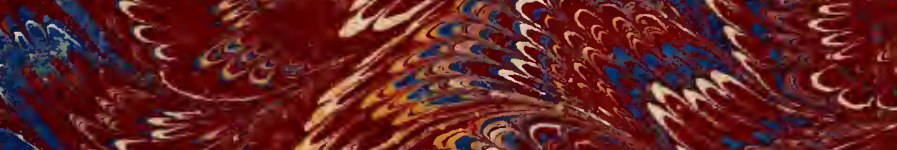
है

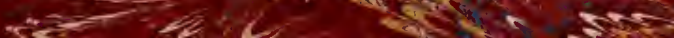





\section{THE DATES OF RAFINESQUE'S NEW FLORA AND FLORA TELLURIANA}

\section{By JOHN HeNDley BARNhaRT}

As far as I am aware, no question has ever been raised concerning the reliability of the dates given on the title-pages of any of the works of Rafinesque. His Autikon Botanikon, to be sure, is dated I 8 I 5-I840, while no portion of the text was published until I 840 ; but this text was intended to illustrate an herbarium which the author had accumulated during the years I 8 I 5-I 840 , so that the meaning of the date he gives is manifest.

About a year ago I noticed in the Flora Telluriana (4:27) a brief criticism of Gray's monograph of the Melanthaceae of North America, which was not published until November, I 837 ; and this, of course, showed that Raninesque's criticism could not have been published earlier than that date. A hurried examination revealed further internal evidence of the erroneous dating of the Flora Telluriana and its companion-work, the New Flora of North America, but the investigation of the subject was not carried very far at that time.

When the last number of the North American Flora was in press, it became necessary for Dr. Small to decide upon the relative priority of Mesynium Raf. ("I $83_{6} 6$ ") and Cathartolinum Reichenb. ( 1837 ), and this led to the study of which the results are here reported.

The New Flora of North America was undertaken by Rafinesque as a supplement to the works previously published by others upon the same topic; and as a result of his labors upon

* GRar, A. Melanthacearum Americae Septentrionalis Revisio. Ann. Lyc. Nat. Hist. N. Y. 4: 105-140. N 1837 . 
it, he was led to undertake the preparation of its "sequel," the Flora Telluriana, dealing with the plants of the rest of the world. The pages of these two works contain many descriptions of "new genera " of plants, so that the dates of their appearance are of considerable importance. As the books themselves are quite scarce, a brief preliminary account of them may not be out of place.

Each was planned to consist of six "parts" or volumes, but was completed in four. Each of the eight parts is separately paged, and has a separate title-page and subtitle of its own; and each is dated " $1 \&_{3} 6$."

\section{New Flora and Botany of North America}

First part. Introduction; Lexicon, Monographs. Ioo pages. 1836 .

Second part. Neophyton. 96 pages. 1836 .

Third part. New Sylva. 96 pages. I 836.

Fourth part. Neobotanon. I I 2 pages. 1836. (This contained also a general title-page for the entire work, dated 1 \$ 36 .)

\section{Flora Telluriana}

First part. Introduction and Classification. 103 pages. I $\$_{3} 6$. Second part. Centuria I, II, III, IV. I I 2 pages. I $8_{3} 6$. Third part. Centuries V, VI, VII, VIII. 100 pages. I 836 . Fourth part. Centuries IX, X, XI, XII. I 35 pages. I $8_{3} 6$.

(This contained also a general title-page for the entire work dated 1836 .)

Of these eight parts, the first part of the New Flora was the first to appear. It contained a dedication dated at Philadelphia, September, I $S_{3} 6$; and pages 73-80 are occupied by a monograph of the genus Lulluia, dated October, 1836 . These facts alone are sufficient to make one suspect that perhaps the eight parts were not all issued before the end of that year! There is not lacking other internal evidence on this subject, in addition to the citation of Gray's monograph (IFl. Tell. $4: 27$; also New Fil. 4: 103 , where the date of "Grey's " paper is distinctly stated as " 1837 "). Flora Telluriana, part 3 (which in turn is cited by 
New Fl. 3:4I, 5I), on page 57 refers to Bot. Reg. pl. 1906 (I $\mathrm{N} 1 \&_{3} 6$ !); and on page 37 to Bot. Mag. pl. 35.70 (I D i $\& 36$ !), which could not well have reached Philadelphia before the end of the year ${ } S_{3} 6$. Flora Telluriana, part 4 (which in turn is citcd by New Fl. $4: 56,57,63,98$ ), on page 124 cites Bot. Reg. pl. I95S ( I My I $S_{37}$ ). But, in spite of these references, I know of no intemal evidence that the two works were not completed before the end of the year i 837 .

From internal evidence, too, it is possible to arrange the parts serially, in the order in which they were printed. This may be done by means of the exact citations, by page, of one work by the other; chiefly of the Flora Telluriana by the New Flora. The result is as follows: New Fl. I ; Fl. Tell. I; Fl. Tell. II ; New Fl. II ; Fl. Tell. III ; New Fl. III; Fl. Tell. IV; New Fl. IV.

In order to approximate more closely than might otherwise be possible the exact dates of issue of each of these parts, the series of letters written to Torrey by Rafinesque during the years ${ }_{1} \&_{3} 6$ to $I S_{39}$, and preserved in the Torrey correspondence at the New York Botanical Garden, was searched, and the search was well rewarded, as is shown by the following quotations:

September 5, I 836.- “I having leisure have resolved to begin to print my New flora of North Amer. by alphabetical order. . . When this Work is printed, my botanical labors from I $\mathrm{SO}_{2}$ to $\mathrm{I}_{3} 6$, in America, will be better known."

December 21, I \$36. — "My flora proceeds very slowly \& was even suspended awhile for lack of a compositor that could print Botanical terms! . . I I have concluded to close the Lexicon of monographs very abruptly, and give instead selected monographs \& my N. Genera \& species."

This shows that only ten days before the close of the year I 836 even the printing of the first part of the New Flora was not completed.

April I 8 , I 837. - "I wanted to surprise you with a great Botanical Work - my Flora telluriana . . to which I was led by my New flora of $\mathrm{N}$. Amer, but I could only print 2 parts or volumes. I. Classes \& Orders. 2d. 400 N. Gen. my other engagts have compelled me to suspend for a while." 
By the middle of $A_{\text {pril, }} S_{37}$, then, had been printed one part of the New Flora and two of the Flora Telluriana.

October 24, I 837. - "I am still going on slowly with my New flora of N. America and Flora telluriana at once. . . . I have circulated but few copies of the numbers published, wishing to surprise you and all Botanists when the whole shall be out; but if you wish to see then earlier I may send you 5 numbers of 100 pages Svo each very soon, and more next March."

From this it appears likely that a second number of the New Flora had appeared when this letter was written, and that a third number of the Flora Telluriana was nearly ready; or else that the two parts were nearly ready to be issued together.

January IO, I $S_{3} 8$. - " My New flora or Mantissa begun to print in ${ } \&_{3} 6$ is still going on \& altho' interrupted by my flora Telluriana \& 2 works published this Spring (1. The Universe. 2. Safe Banking) is proceeding as fast as correct exam. can allow. I wished to issue the whole work together; but I shall be compelled to issue when half is ready 3 numbers of 100 pages as in Flora tellur. My $3 \mathrm{~d} \mathrm{~N}$. on the Trees and Shrubs or a New sylva is not quite ready."

At the end of 1837 , then, three numbers of the Flora Telluriana had been issued, and two of the New Flora, but on January IO $18_{3} 8$, the third part of the New Flora was " not quite ready."

Marcl 20, 1838 . - "I have long ago concluded 600 pages of my Supplemental Flora \& Flora Telluriana or 6 parts. If I had not undertaken these 2 works together, the first would have been completed ere now, but will be ere $1840 . "$

The third part of the New Flora had evidently been published since the date of the January letter. It appears that Rafinesque still intended each work to consist of six parts, and for this reason allowed himself until i 840 to complete them.

February I, I839. - "My 4th part or Volume of New flora was completed so as to give you time to go on with your flora. I also completed my Flora telluriana in 4 Vol. or 1225 articles. But immedy after begun \& have concluded last $\operatorname{Dec}^{r}$ my Synopsis of N. G. \& Sp. of Trees \& Shrubs of N. Amer."

From this it appears that prior to December, I 838 , both the 
Flora Telluriana and the New Flora had been completed. The last sentence refers to Rafinesque's Alsographia Americana, which was dated I 838 , and from his own statement above was probably issued in December of that year.

The extracts from Rafinesque's letters show that few, if indeed any, copies of either the Flora Telluriana or the New Flora had been actually distributed until three parts of each had been printed (in the spring of $18_{3} 8$ ); but, as he says in the letter of October 24,1837 , "I have çirculated but few copies of the numbers published," we must give him the benefit of the doubt, and assume that he had distributed a few copies.

Rafinesque's Bulletin of the Historical and Natural Sciences was an advertising sheet issued by him at irregular intervals from 1834 to 1839 . No. 7 , dated "Spring of 1838 ," is devoted chiefly to the two works here under discussion. He says in part : "I had long contemplated to give a New Flora of North America. ... I resolved ... to add the improvements on Natural classification. These last, however, increased so much under my revision, as to become a work by itself, and a companion rather than addition to our Flora. Both works were begun in 1836 , and our plants would all have been published by this time, if I had not thus been compelled to double these botanical labors. I once proposed to issue the whole at once when completed, but this delay and others arising from different pursuits and labors, have induced me to publish the parts as soon as printed, and now that 3 parts of each (being half a volume,) are published, I issue this Bulletin to acquaint the Botanists of Europe and America with" them. "Each work is to consist of 6 parts of IOO to 120 pages, thus forming a volume large octavo of 600 to 700 pages, which shall be completed in 1840 or sooner. . . The 6 parts now printed, 3 of each work, will be sold together for \$5."

The dates of the two works under discussion, as nearly as they can be determined from the evidence here submitted, may be summarized as follows :

New Flora $\quad$ Part I. I 836 (December).
II. 1837 (second half).
III. I 838 (first quarter).
IV. I 838 (late in year).


Flok. Telluki.is. Part I. I $\delta_{37}$ (first quarter).

II. 1837 (first quarter).

III. 1837 (November or December).

IV. 1838 (near middle of year).

Nail lork Botanical Garien. 




\section{FLORA}

\section{T E L L U R I A N A}

$\rightarrow \gg$ e0kr-

BY PROF. RAFINESQUE.

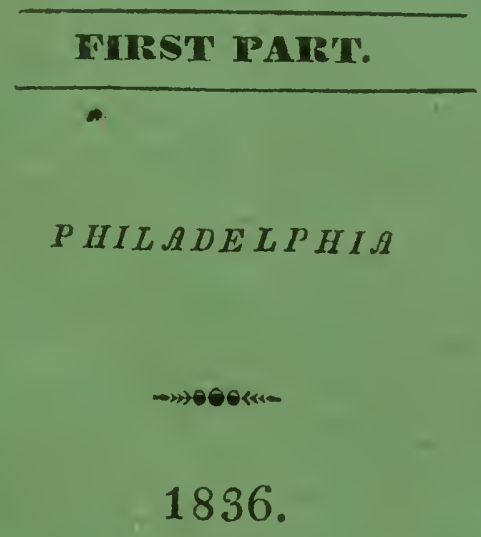




\title{
FLORA TLLLURIANA
}

PARS PRIMA.

\section{IN'TROD. ELASSHFIC.}

AD MANTISSA SYNOPTICA

\section{0}

NOZ GENERA PLANTARUM

\author{
VEL NOV. ORD. ET SPEC.
}

I N ORBIS TELLURIANUM

Determ.coll. inv. obs. et descr. ad

C. S. RAFINESQUE, Bot. Prof. \&c.

Anv. 1796 ad 1836.

$P H I L \Omega D E L P H I \Omega$

1836. 


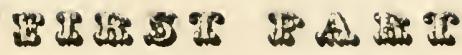

OF THE

\section{SYNOPTICAL FLORA TELLURIANA,}

\section{INTRODOCT:ON \& CLASSIFICAIIONT,}

With new Natural Classes, Orders and families: preamble of the 2000 New or revised Genera and Species of 'Trees, Palms, Shrubs, Vines, Plants, Lilies, Grasses, Ferns, Algas, Fungi \&c. from North and South America, Polynesia, Australia, Asia, Europe and Africa, omitted or mistaken by the authors, that were observed or ascertained, described or revised, collected or figured, between 1796 and 1836 ,

\section{BY, C. S. RAFINESQUE, A. M.}

Prof. of Botany, historical and natural sciences-member of many learned Societies in Paris, Vienna, Bruxelles, Bonn, Bordeaux, Zurich, Naples \&c. Philadelphio, New York, Cincinnati, Lexington, \&c.

To observe and compare, to correct or approve By good names and new facts thal convince and improve.

PHILADELPHIA :

PRINTED FOR THEIAUTHOR

BY H. PROBASCO, NO. 119, NORTH FOURTI ST.

\section{6.}




\section{Les noms font les choses.}

\section{Names realize Entities.}

Plus nos noms sont géneraux, plus non idées sont incompletes-Plus nous avons de noms, plus elles se completent. Lamark, Leach \&c. 


\section{DEDICA'TION.}

To Decandolee and Agardi worthy Improvers, and esteemed fellow laborers-and to all the Botanists aiming to improve the lovely Science of Botany by accuracy and discrimination, or who may wish to discard the Errors, Misnomers and Absurdities of former times or our own-These synoptical labors of accurate observations, proper distinctions, good denominations and correct analysis, the result of forty years of botanical researches and travelsARE INSCRIBED BY

THE AUTHOR. 


\section{NO'TICE.}

This whole work will comprize 6 similar parts, including 2000 new Genera and Species, with many new natural orders and families. 'This first part containing the Introduction and Classification.

Price of the whole work $\$ 5$, each part one Dollar.

This work is a sequel to the $N_{E W} F_{L O R A}$ of Norti America, and is the complement of the author's Botanical Works-The Genera of fossil plants and primitive types of our actual vegetation may be a subsequent sequel to this.

My Fauna Telundata or Synopsis of the new animals, living and fossil, Quadrupeds, Birds, Fishes, Reptiles, Crustacea, Shells, Polyps \&c. which I have observed or ascertained between 1796 and 1836 will form the complement of my discoveries and researches on organized beings. 
INTRODUCTION.

It is the duty of all the observers of natural productions to communicate their discoveries and researches. When a botanist has spent a long life in travelling over both hemispheres, collecting 100,000 botanical specimens, drawing 2000 plants, and discovering a multitude of new objects, as I have done: this duty becomes still more imperative. When to these exertions he may have added deep researches in the critical examination of many thousands specimens of plants from all parts of the Earth; and in consulting Books and Libraries, former authors and figures, Gardens and Herbals ... as I have also done, this duty assumes the aspect of necessity; particularly if what he has ventured to publish heretofore in unconnected works, has not been widely spread nor duly appreciated or quoted, owing to the difficulty of times, circumstances, shipwrecks, or scattered tracts in remote places.

Such having been my case; I felt the need of revising and combining all my botanical labors, both published and unpublished, while I was engaged in printing my New Flora of North America, a kind of Mantissa or Supplement to all the previous Floras of that continent by Linneus, Clayton, Michaux, Muhlenberg, Pursh, Robin, Nuttal, Torrey, Beck, Bosc, Lamark, Hooker, Elliot, Eaton, Riddell, Bigelow, \&c. Besides the numerous plants unnoticed by them, I found so many Species and Genera blended or in disorder, that it required a very extensive critical survey of those connected thereto elsewhere, to compare and ascertain the truth. 
'T'bus I was induced to begin a complete revisal and critical examination of all doubtful or involved Genera chiefly; of which the number is incredible, owing to the absurd usual mode of forming Genera by a single sp. or a few only, to which others are referred at random, by mere habit, external appearance, or in spite of peculiar generic features or characters.

In fact when Limneus began a Century ago to reform Botany, he was compelled to go on by gradual steps; any other mode would have been too abrupt. He had the merit to fix Generic names, and to invent Specific names, adding to these a short diagnosis in imitation of the former phaseologic names.

It has been very well observed that the specific diagnosis or essential character of plants can only become fixed, when all the Species of a Genus are known; which will never happen until the whole Earth is explored thoroughly. Thus the epitomic characters applied to $\mathbf{S p}$. by Linneus, have been found totally inadequate and inacurate, always involving many distinct species. Botanists were compelled to change and swell them gradually to a kind of epitomical description, until they have lately run into the opposite extreme, and Hooker has even some of $\mathbf{7 5}$ words! or as long as a common minute description. It is our duty to seek the most conspicuous, constant or discriminating, and to reduce them to the most essential terms in the least compass.

Generic diagnosis were also too much condensed by Linneus and his school; they have been improved by making them essential in their respective tribes, and adding some important features of the habit, inflorescence \&c. 'The 
generic descriptions of Linneus in his Genera plantarum are totaly useless, since they were made upon one or a few sp. alone, which are not even mentioned ....! and almost never apply to the whole Genus, when it has many species.

As to the sexual system, once so much insisted upon, I have lived to see it exploded, as such unnatural and indelicate system deserved. The beautiful natural method, the same pursued by Linneus for animals, has taken its place, in spite of sturdy opposition or delayed assent. But unfortunately often falling into reluctant hands the substitute has not yet reached its due perfection like Zoology. If my suggestions in $\mathbf{1 8 1 4}$

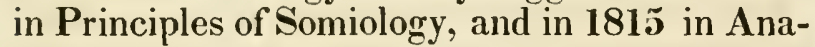
lysis of Nature had been attended to, it might have been otherwise; but the best Botanists persist to this day in making classes, orders, families or tribes without available and distinguishing essential characters, common to all the refered Genera.

While Sir James Smith the friend of Linneus, and possessor of his Herbarium, corrected so many of his glaring mistakes in Rees Cyclopedia: he expressed his deep regret at the impending fall of his beautiful botanical fabric: which implied a wish to retain his erroneous"system, Genera and Species. Such as the shameful and patched up Genera Sophora, Geranium, Cactus, Mimosa, Lichen, Conferva, Acrostichum, and 300 like them, that have been split and reformed even by the Linneists.

A number of less enlightened pupils or tenacious worshipers of the Linnean System, have insisted on preserving all its inconsistencies and blunders, bad Genera and Species. Others like 'Thunberg, Wildenow, Persoon, Smith, have 
more or less corrected them: which was called a inutilation by some worshipers of his Errors. Most of them have still insisted on the perverted axiom that the Genus gives the character! which for them meant that the Linnean bundle of plants, called a Genus, was to afford a common loose generic definition, whatever might be the essential features peculiar to each Sp. wrongly put in the bundle. I was compelled to transpose this axiom, by maintaining that the character makes the Genus, or that no proper Genus can exist without a character applying to all the species it contains. This principle fully applies also to Tribes or families, Orders and Classes; altho' quite neglected by the actual Botanists, who do for them what Linneus did for genera.

My own improvements in finding discriminating characters for all generic and other groups extend chiefly to frame none but positive and exclusive characters of a permanent nature in contrast-and besides to shorten long descriptions by avoiding repetitions, or merely stating how a Genus may differ from another, which always implies that they agree in every thing else.

Every Genus ought to find a place in the natural method, when properly known: none but those partialy described can be doubtful. Hence Jussieu was wrong in having so many Genera inserta sedis, which no one could find by his method, with so many $\mathbf{G}$. improperly added to families; while both were often types of new families since established. But Linneus, Adanson, Necker... did worse in forming many families of plants loosely connected by habit rather than the fructification. 
Since Gaertner anatomy of seeds, too much stress has been laid upon this internal structure; which is unavailable for practical purposes, and only useful in botanical physiology. If every one was compelled to dissect a seed or an egg, before he could ascertain the Genus or family of a Plant or Bird, the sciences of Botany and Zoology would become unattainable.

The axiom of Lamark that prolific Genera ought to be divided, holds true for most of them, as much for Carex, Euphorbia, Vaccinium, Solanum \&c. as for Scirpus, Geranium, Amaryllis, Lichen, and all the polymorphous $\mathbf{G}$. For instance, in Grasses and Lilies, the nuimber of Stamens and Stigmas is generic; so important as to divide Families: Jussieu has based thereon many of his families, and the sections of grasses.

A great advantage results from multiplying good Genera: since by it we lessen the constant repetitions of many common characters. But when Lamark said that small Genera might be conveniently united, he overlooked that $\mathrm{Na}$ ture does not limit them in that way; but admits of many distinct Genera of one or few species, either as late deviated types, or remains of nearly extinct types of generic forms. Besides, most of the Linnean Genera of one Sp. have been found to have several, when the earth has been better explored; this was the case with Kuhnia, Parnassia, Hydrastis, Fragaria, Dionea, Hippuris, Gaura, Samolus, Oryza, \&c. The type of a family may also be single at first, but soon becomes multiple, when we explore the Earth.

Meantime since the Linnean period, his own attempt at the enunciation of Natural Orders, 
vithout characters! perhaps based on his own views of the transmutation of characters, has been much modified, amplified and improved; and even the transmutation of Species and Genera insisted on by some : yet the more rational opinion of Necker that Species alone could (at least in the actual state of our Globe) be multiplied as breeds of their peculiar Genera, has been little attended to, probably owing to his deviation of terms, since he insisted on considering the natural Orders as Genera, these as mere Species, and our Species as Proles or Breeds. The subject of specific varieties was much neglected by Linneus, and left to the Horticulturists, and yet he admitted of Pelorian Genera, Hybrid Species and permanent varieties.

If 40 years of botanical observations, with many herborizations in similar spots of North America at a distance of 32 years, may entitle me to state my impressions on this abstruse subject, and add my testimony thereto, I must declare my conviction that $\mathbf{1}$. Vegetation produces only individuals! whose permanence is limited by their life. Our Species, Genera, Families, and Orders are well known to be mere abstract terms of successive groups, formed by a Synthetic operation of our mind, in order to study more conveniently such collective groups of Individuals. Their permanence in continual succession of forms can only be temporary : since their permutation of forms takes place spontaneously in their natal soils, as well as our gardens where it is increased by art; while new varieties and species were often met by me at long intervals in wild places well explored be- 
fore, grown from seeds of akin species. See my remarks and facts collected in my new Flora.

2. Plants vary gradualy, in features, aspeet, size, color \&c. by a natural spontaneous deviation from seedlings. This may happen quicker in annuals, less quick in perennials, slower still in trees, except when the tendency has already become active. These deviations may gradualy form distinct varieties, next Breeds, at last becoming separate Species, when they assume a striking difference, and peculiar specific characters of a more permanent nature. The disparities in the descriptions and figures of old and modern botanists amply verify this.

3. Even perennials may vary slightly in annual shoots from the same root, and trees in different branches or annual growth. When a tendency to deviation by monstruosity, hybridity or variety is taken by an individual, the seeds produced will unfold them when growing, particularly if removed from the native place into gardens and new soils.

4. Pelorian Genera, or Generic Deviations in flowers and seeds, happen slower or more seldom ; being often unnoticed, or the produced seed is not always fertile. When it is, the offspring may become the type of a New or distinct Genus. Many such perish before they reproduce the deviation by fertile seeds; but a few survive and are the types of akin Genera.

5. The periods of these deviations are doubtful, much fluctuating and various in length or existence. But we may assume as an average 30 to 100 years for the deviating or spliting range of specific deviation, and 500 to 1000 years for the Generic deviation; altho' their 
real permanence is much longer. Specific and generic Lives have not yet been calculated.

6. 'Therefore many of our actual or newly described Genera and Species, may be of recent origine, and all may have once sprung at the last rinovation or cataclysm of this Globe, from a lesser number of original types, perhaps found in the fossil plants of our Earth, which are far from being all knowu as yet, and whose seeds were preserved in mountains, earth, mud or water tiil the catastrophe was over,

7. It is even possible to ascertain the relative ages and affinities of actual species and Genera, sometimes their very parents or connections in the Genus or the tribe. Those we call hybrids are not always such, they may arise fiom other deviations; but artificial hybrids are evidently such. All these deviations are still less permanent.

8. As a general rule the real Genera (not the false ones of blending Botanists) of single or few species are the newest in order of time, and the most prolific the oldest in the Series. 'The same for tribes perhaps. False Genera like $\mathbf{E r i}$ ca, Carex, Aster, Allium, Lichen, Euphorbia, Mimosa, Geranium f.c. comprizing a crowd of generic distinctions, are as many collections of related Genera, springing from very early sources or types of forms. Extensive natural Genera prolific in Sp. like Rosa, Iris, Quercus, Salix, Oxalis. Malva, Vitis, Lactuca \&c. had also a very old or primitive source. Species prolific in individuals and varieties are always the oldest, and rare Species probably the newest of all, unless they are fragments of extinct groups.

Such exposition of my principles, and expla- 
nation of motives were perhaps needful, when I am going to increase the generic groups, perhaps beyond any thing ever done of the kind. Linneus had only $\mathbf{1 4 4 4}$ Genera, in his last cdition towards 1778; Persoon in 1807 had already 2300 phanegamous Genera. Jussieu in $\mathbf{1 7 8 9}$ had nearly 2000 ; but Necker in 1790 only 1842 . The 48 Cryptogamic Genera of Linneus have swollen to 400. Every year and every writer adds to the number. Ever since $1815 \mathrm{I}$ had ascertained and classified nearly 3000 , whereof 500 were my own. It is this labor, indicated in my analysis of Nature. that I now propose to enlarge, rectify and publish: whereby as many as Linneus ever had will be added or revised, and about 1000 will be totaly new, even now, as late as 1836 , or not yet generally adopted.

Altho' this attempt may astonish or perplex some timid Botanists, my labors will be duly appreciated ere loug, and my unceasing efforts to improve the science meet with a kind reception from the new improving school. The axiom that a multiplication of names enlarges our ideas, holds true in all cases and sciences, since they are based on facts or mental entities. Some Linneists have vainly tried to to throw discredit on generic reform, and called us Genera-mongers. We may in return call them Genera-Shuffers, who want to squeeze plants into improper Genera, and delay iuprovements by opposing the corrections of botanical blunders. It is to them that we owe the superfluity of synonyms: they often shuffle plants into 3 or 4 Genera, as Linneus did for Heliopsis, until it must at last form a Genus of itself. It is a fact that almost all plants of doubtful Genera, are 
types of peculiar ones; the chances of it increase, as they are shifted.

As to names, some botanists are very careless, and deem them of little consequence, forgetting the very rules of their Linneus, whose philosophia botanica they never read. I can boast at least of some accuracy and taste in my Nomenclature ; I frame none but good or meaning Names, none of mine are bad, unless preocupied unknown to me, as my Calistachya, Darwinia, Diplogon . . A All previous names, anterior in dates, ought to prevail, and dates must be given in doubtful cases. If I have made use sometimes of native names, I have only followed Linneus, who in spite of his strict injunction had adopted Coffea, Jasminum, Yucea, Pandamus, Piper, T'amarindus, Cocos, Canna, Cassia \&c. from Arabic, Celtic and foreign names. My Genera Zaga, Lolanara, Ramotha, Jupica, are as geod as these, and my Tilcusta, Kozola equal to Vanilla or as pretty.

I have often dedicated new Genera to Botanists, or to worthy men, philosophers and naturalists, eminent Horticulturists or promoters of knowledge \&c. My Genera Fenelonia, Empedoclia, Platonia, Thalesia, Adlumia . . . are as good as Aristotelia, Enpatorium, Euphorbia \&c.-If I have lost my G. Pythagorea, Bivonia, Savia, Torreya \&c. by preocupation; Lindley has lost his Clintonia, mine being the first dating of $1817,1819,1825$ ! So many Botanists, establish Genera at remote places that these clashing names must often occur : to prevent the loss of my names, I may sometimes give a double substitute in case of need, as I did for Darwinia or MIonoplectra. I am never 
at a loss for names, as Linneus was when he framed Quisqualis; I could readily supply 20000, all good: and Adanson table of Synonyms is an unfailing mine of old Classical names. As I have not yet heard of a Genus dedicated to me, I shall perhaps have to imitate Roxburg, and choose one for myself, as a Rafinesquia!

Altho' Linneus gave strict rules of nomenclature, he has broken them himself in 100 instances. He would have no generic names derived from each other, nor made up by adding or substracting a letter or a syllable: and yet he has Ambrosia, Ambrosinia-Pyrus, Pyrola -Zea, Zeus-Thea, Itea, Althea-Aster, Asterias-Apis, Apium, Sinapis-Capra, C'apraria-Linum, Talinum, Selinum-Pinus, Carpinus, Lupinus-Delphinus, Delphinium -Canna, Cannabis, Canarina, Canarium, Melia, Bumelia, Bromelia. foc.-The natural Botanists disregarding still more his tasteful principles, have added a crowd of similar bad names, Portulacu-ria, Oryz-opsis, Aquila-ria, Actinella \&c. Helianthemum identic with $\boldsymbol{H e}$ lianthus, which is my Anthelis-Calamagrostis! my Amagris, which have encumbered nomenclature. It appears that by the increase of names, Botanists begin to be at a loss for them, or cannot seek for good derivations.

Linneus objected also to names either too short or too long, under 2 or above 4 syllables; yet he has Bos, Mus, Sus, Boa, Poa, Thea, Zea, \&c. which I changed for him into Taurus, Musculus, Aper, Theaphyla, Mayzea-He had also Securidaca, Aeschynomene, Indigofe$r a$ \&c. of 5 , which may be tolerated; but $T a-$ bernomontana of 6 is intolerable, and must become Tabernaria-Bou and Pou are both ton 
alike and too short, why not say Ophisboa and Poagris? I have constantly insisted for the purity of tasteful nomenclature; but regret to see it oft neglected by the very best Botanists. Good names ought to be either classical or full of meaning; the best even to describe the main essential character.

Another source of mistakes arises from blunders in Orthography, or errors of the press, copied inadvertently. Thus it is now well ascertained that these gave rise to the Prunella read Brunella, Befaria read Bejaria, Amsonia read Ausonia, Galardia read Gaillardia, Gualteria read Gaultiera, Pentstemon read Pentostemon, Sarracenia read Sarazinia, Scilla read Skilla, Diclytra read Dielytra, Marsilea: read Marsiglia, \&c.

A new general Pinax of Names, like that of Bauhin of old, is very much wanted; but who shall undertake the herculeun task? It might be done however for Genera at least, and the admirable table of old generic names collected by Adanson, might serve for model. The generic synonymy of Decandole and Sprengel are but incomplete attempts. This surabondance of names arises from the timid or unskilful Botanists, who are not able to refer Plants to their proper Genus, nor able to make New Genera of those that disagree. It will never cease till skilful Botanists alone meddle with Names.

The compilers, translators, editors and commentators of the Linnean School have for $\mathbf{6 0}$ years past, often tried to keep Botany nearly at a stand, or impeded its progress. They have often neglected to avail themselves of the works, researches and discoveries of those who were not strict Limneists. 'They neglected for a long 
while Adanson, Necker, Richard, Lamark, and even Jussieu the fathers of natural Botany, whose labors are now superseding theirs. No wonder then they have also neglected mine of the same tendency.

Among the best Limnean writers must be reckoned Schreber, Richard, Murray, Smith, Salisbury, Vitman, Vahl, Wildenow, Persoon, Gmelin, Aiton, Romer, Shulze, Sprengel, Pallas, Fontenille, Lehman foc. whose works I have duly studied and used.

But I value above all the improving Botanists, such as Mench, Gaertner, Swartz, Desfontaines, Lamark, R. Brown, Decandole, Kunth, Esenbeck, Lindley, Agardh, Desvaux, \&c. that have enlarged or continue to improve the Science. It is among them that $I$ have aimed to deseive a place.

Some applying themselves to a single Class, Order or even Genus of plants have introduced admirable monographs, that become the bases of future stability. I may mention as models Persoon and Fries on Fungi, Acharius on Lichens, Agardh on Algas, Smith \& Swartz on Ferns, Palissot on Grasses, Cassini and Lessing on Composite, Richard and Lindley on Orchidea, Bentham on Labiate .. Besides the many families already illustrated by Decandole. To him we chiefly owe the practice of dividing large and incongruous Genera, into Sub-Genera, which will surely become gradualy as many Generic groups, unless not based on the fructification.

The greatest botanical discoveries have been made since Linneus, by travellers to distant regions, and authors of local Floras. Australia, Polynesia and both Americas have doubled the number of recorded plants. Linneus only had 
about 8000 sp. and only knew 3000 well, now we know about 5000 Genera and 100,000 species; yet we yearly increase their number. 'Those who have mainly enlarged our knowledge of Genera, were chiefly in North America, Michaux, Pursh, Nuttal, Elliot, Bosc, Hooker, Torrey, Beck, Kunth, Llave and Legarza.-In South America, Aublet, Mutis, Dombey, Ruiz, Humboldt and Bonpland, Poiteau, Swartz, Spix and Martens, Molina-In Oceania or Polynesia and Australia, Forster, Labillardiere, R. Brown, Commerson, Thouars, Cunningham, Thunberg -In Asia, Pallas, Clarke, Fischer, Ledebour, Hamilton, Walich, Roxburn, Forskahl, Loureiro-In Africa Desfontaines, Delille, Caillaud, Bruce, Schousboc, Palissot, 'Thunberg, Afzelius -and in Europe Waldstein, Jaquin, Sibthorp, Allioni, Viviani, 'Tenore, Brotero, Gilibert, Bivona, Gussone, \&c.-Ihese worthy laborers deserve our thankful gratitude: and it has been properly deemed that every word they have set down in their writings is of real value; such actual observers alone mainly increase the range of Science; Researches in Gardens, Herbals and Libraries only come next: I am at least one of them, if no more, and I belong to both hemispheres.

Another Class of Botanists by publishing collections of splendid botanical figures, have afforded many materials; but their costly works, whose figures and descriptions do not always agree, are often heyond common reach. Such are Jacquin, Ventenat, Delille, Labillardiere, Oeder, Sibthorp, Lheritier, Catesby, Redoute, Ruiz and Pavon, Curtis, Sims, Ker, Andrew, I indley, Hooker, Cavanilles, Tenore, Humboldt, Delessert, Roxburg, \&c. 
Others have issued annals or journals of Botany, else vast compilations or Encyclopedias of Botany, where are found many useful accumulated materials; such were Lamark and P'oiret, the dictionaries of Nat. history, Dumont-Courset, Miller and Martyn, Smith in Rees, Loudon Sc. I have read and consulted them all : with many more here omitted, and even some authors of rare works seldom quoted; such as Petagni, Vitman, Scopoli, Gouan, Bartram, Llave, Legarza, Dumont, Fontenille, Cupani, Chabreus, Gilbert, Thouars, Loureiro, Lunan, Russel, Clarke, Robin... In all there was something to glean.

Yet the result of all my researches upon these former writers, has been merely with the view to rectify their mistakes and generic blunders, or add to their knowledge. 'This work is not to be a compilation of their labors; but rather a supplement to all theirs, and the complement to my own. 'The fields wherein I was led to seek for original knowledge, were Italy and the South of France from 1796 to 1802. North America 1802 to 1804 . Italy and Sicily from 1805 to 181.5. Spain and the Azores in 1815. North America again from Canada and Boston, to the Mississipi and Apalachian mts. during 1616 to 1836 . My travels and researches may be seen in my Life of Travels published this year 1836.

I have chieffy studied and collected plants in their native wilds, from the Summit of Etna to the falls of Niagara: but $I$ have also visited many botanical and private Gardens in Marseilles, Genoa, Pisa, Leghorn, Palermo, Messina, Philadelphia, New York, Boston, Albany, Cincinnati, I exington, Washington, \&c. where 
I examined many exotic or rare Genera. My own library and Herbals of botanical specimens, with the public or private collections of books, figures and plants, have afforded me many materials. Every Genus which I venture to establish has usualy been examined alive or dried, or I have had a good figure or good description to depend upon, sometimes both, or even all those means combined.

A complete Catalogue of all my botanical works and tracts will be found in chronological order in my Herbarium Rafinesquianum 1833. Most of them are now collected in my Amenities of Nature. In 1808 by publishing my N. G. with 69 N. Sp. of North America-In $1810 \mathrm{my}$ N. animals and plants of Sicily, 21 N. G. 80 N. Sp. of plants-my career of discoveries was begun.

In 1814 my chief works were Compendium of Discoveries, with 14 N. G. 78 N. Sp. of plants-22 N. G. and $30 \mathrm{~N}$. Sp. of plants in my Cyclopedical Journal-besides the principles of Somiology wherein I gave the rules of natural classification for animals and plants : with principles of nomenclature. In 1815 my main work Analysis of Nature wherein I indicated $\mathbf{3 1 0}$ families of plants properly distributed into 66 Orders and 10 Classes. As Decandole had then only 150 families, 160 of mine were then new, altho' many have since been adopted without due credit for my previous sagacity and good names. Also my Chloris Etnensis published in Recupero history of Etna, classed naturaly.

My florula Ludoviciana of $\mathbf{1 8 1 7}$ had $30 \mathrm{~N}$. G. and over 160 N. Sp. admitted from Robin; I was blamed for having done for him what 
Gronovius did for Clayton, and Wildenow for Loureiro!

My florula Mandanensis and Missurica based upon the plants and specimens of Bradbury, Lewis, Miller and Beck, written between $\mathbf{1 8 1 7}$ and 1820, but never published. and a copy sent to England was lost. Other similar mpts. of mine, yet unpublished, I deem useless to enumerate.

Between 1816 and 1818 I rectified many errors of Pursh, Nuttal, Barton, Bigelow, Elliot \&c. in reviews, and published 40 new plants In 1819 my 50 N. G. of American plants were published in Journal de physique of Paris.

In 1820 my annals of nature had $25 \mathrm{~N}$. G. and $124 \mathrm{~N}$. Sp, of animals and plants, and my monographs of Rubiacea, Rosa, Houstonia, Lysimachia, Convolvulus \& c. were published in the Annales des Sciences physiques of Bruxels.

In 1825 I proposed 66 American N. G. in my Neogenyton. In my Medical flora of the United States 1828 to 1830 I gave many N. Sp: and figures, with monographs of Vitis, Gentiana, Heuchera, Trillium, Unisema, \&.c.

In 1830 and 1831 I sent to Decandole at his request several mpts on $\mathbf{1 6}$ New families, and 175 New Genera of Plants chiefly North American, among which $60 \mathrm{~N}$. G. of Composite besides 90 New Species of the same Order, and 45 N. Sp. of other orders, with 188 Specimens of rare, new or doubtful plants.

In my Atlantic Journal 1832 to 1833, I gave 150 N. G. or Sp. of Plants. In 1833 was begun my Herbar. Raf. with many new plants, Genera, and Catalogues of my discoveries, botanical collections \&c.

Altho' I am a draftsman, and can draw my 
New plants, I have seldom been able to publish my figures. My edition of Cupani, and the Amer. plants engraved before 1815 , were lost plates and all in my Shipwreck of 1815 with my herbarium, only few copies have survived. I only gave 100 wooden cuts in my medical flora, and about $80 \mathrm{in}$ my School of flora; 36 in American Florist, cheap popular works. 'Thus I resolved to publish my 500 ICONES rariorum in mpt. and also my Autikon Botanuion or Self figures by Specimens of 2500 new or rare plants, to be sold at the same rate as the actual usual printed figures.

At last in $1836 \mathrm{I}$ began to print my New Flora of North America, Supplemental to all the others, with $1000 \mathrm{~N}$. Sp. at least; which has led to the actual Synopsis or Mantissa, as a recapitulation of all my scattered works and observations, or their principal facts.

As to classical arrangement $I$ have aimed at none at first, because my own natural improved families, now amounting to $\mathbf{3 7 5}$ would have still more staggered the reluctant Botanists. I divide this work in Centuries with numbers, keeping often together akin Genera.-This is the actual plan of many books of botanical novelties, Hooker, Lindley \&c. The alphabetical order would have been useless where so many new Names occur, but Indexes shall be given, tables of Natural Orders at the outset, and a general classification at the end of the whole work. 'Those anthors who admit only what they see, or upon trust of particular friends, would not probably pay more attention to my researches, if given under any other garb. Those who seek for truth and new materials, will easily find both here, and mould them into their own shape or 
method. Whoever may wish for further proof in costly figures and specinens will find them in my Icoves and Aurikox (and possess them by buying them), else in the $\mathbf{N}$. Aner. plants of my Flora: meantime this work is perhaps the first ever published in America on General Classical Botany; and it will be a mine of botanical knowledge, to those willing to avail themselves of such help any where.

Such have been my labors and exertions in my favourite Science, the most amiable of all, and the earliest as it shall be the latest of my pursuits thro' life. Reader, kind or unkind . . ! do not disdain these results of long experience and criticism ; dismiss bad names and groups ; adopt or further improve my generic and tribal clusters. They are the natural evolution of spontaneous vegetable life exerted in wisdom thro' ages. Imitate my zeal, and be happy in the lovely study of flowers.

'To seek the truth in floral grifts concealiug,

Is pleasing task ; to lofty mindsrevealing Their secret beruties clad in bright arruy, That wislom teach and to the mind convey. 


\section{NATURA1, CLASSIFICA'TION.}

The fathers and improvers of the Natural method have not settled, which are to be the first and last Genera in the serial arrangement. Adanson in 1763 began with Tremella, ending with the Mosses, thus going in a circle. He was followed by Scopoli who began with Incompletes ending with Fungi, but Linneus from the Palms to Fungi. Jussieu in 1789 began with IMucor ending with Abies, from the most simple plant to the loftiest trees. Decandole in his flora gallica 1806 began with Nostoch ending with Actea. In his synopsis plantarum he has reversed this order in imitation of Zoology, beginning with Clematis. Necker in $\mathbf{1 7 9 0}$ began with luala in the Radiate, ending with the Confervas.

'Therefore I thought in 1815 that I could improve thereon by beginning with Rosa, the queen of flowers, as Homo is the king of animals! ending with Mucor, but now I end with Spunges. 'ilhe nost perfect flower ought to open the descending Series of organization, and not the Umbellifer of Lindley series. Agardh has again taken the ascending series and begun with Fungi in 1822.

'I'hus they all differ in this, and do not agree better in their clusters of Classes, Orders and families. From Cesalpini who in $\mathbf{1 5 8 3}$ made the first attempt at a natural method to our days, all the methods are variable; but begin to improve since Adanson or rather Jussieu, and have increased from his $\mathbf{5 8}$ families to nearly 100; which have been called Sub-Orders, Sub- 
families, Sections, and even Colsorts and Legions by some driller's.

I have contended with Limneus and Jissieu, that we ought to have only natural Classes and Orders, but admit families as main Sections of Orders, and many other Sections, in all to help the analysis. 'The natural method may become strictly analytical, as much so as in Zoology; whence I perceived that Botany could be analyzed in a paralel number of Classes, if not of Orders with Animals; each as distinct of each other as are Birds, Fishes, Worms, \&c.

Having read and studied whatever has been added or stated on natural Botany since 1815, I have not found needfin to change materialy my proposed improvements matured between 1800 and 1815 ; as to Orders, but some new Classes and families must be added, or find a place in my method, which has the advantages of facility, universality and exclusiveness, or general application, and easy analysis by exclusive characters of all the groups, like the analytical tables of Lamark.

Having published nearly 200 such new families in my analysis of Nature 1815, fixing them by mentioning their generic types, I deem proper to lay claim to the following, dating as early. They are all exclusive and different from the 100 of Jussieu.

\section{TABLE OF NEW NATURAY, FAMILIES}

And their TyPss in 181.).

I. Class, Eutrogines, Rosa the first Genus, type of real Rosaceous plants.

1. Senticosia, types Genera Rubus, Fragaria, ofc.

2. Poterinia, types Poterinm, Agrimonin. 
3. Govoligin, type Alchemilla, Sibbaldia.

4. Spireadia, type Spirer.

5. 'Thyaxia, type Zanthoxylum.

6. Acnenopsia, types Coriaria, Aylanthus.

7. 'Thalictin, types, 'Thalictrum, Tetracera

8. Peonidia, types, Peonia, Caltha.

9. Hetralonia, types Aconitum, Nigella.

10. Axarcodia, types Morus, Artocurpus.

11. Endophoria, " Ficus, Dorstenia.

12. Mesophoria, "Xanthium.

13. Ulmidia, " Ulmus, Celtis.

14. Amyridia, "Amyris.

15. Rivinidia, "Rivinia.

16. Phylicia, "Phylica, Ceanothus.

1\%. Peplidia, " Peplis, Ammania.

18. Dionidia, " Dionea, Monotropa?

19. Violidia, "Viola.

20. Iberidia, "Iberis.

21. Isatidia, "Isatis.

22. Alyssinia, "Alyssum.

23. Hesperinia, " Mesperis.

24. Sinapidia, " Sinapis. 'These 5 from 20 form the Order of Cruciferous.

25. Acteasia, type Actea.

26. Glinidia, "Glimes.

27. Tragidia, "Tragia.

28. Phalarsia, "Chytia.

29. Ricinidia, " Ricimus, Acalypha.

30. Droseria, " Drosera, Paruassia.

31. Empetridia," Empetrum.

32. Alsinia, "Arenaria, Cerastium.

33. Linidia, "Linum.

34. Tamarixia, "Tamarix.

35. Passiflorea, "Passiflorn.

36. Strigilidia, " Strigilia.

37. Gordonisia, "Gordonia.

38. Gossypidia." Gossypiuin. 
39. Malopidia, type Malope, Paluria.

40. Zeibania, type Zeiba.

41. Celosidia, "Celosia.

42. Loasinia, " Loosa.

43. Andirania," Andira.

44. Arthrocytia," Hedyssarum.

45. Diadelphia," Lathyrus, Trifolium.

46. Anthylidia, " Onomis, Anthylis.

47. Amorphinia," Amorphus.

48. Bauhinidia," Bauthinia.

49. Cassinia, "Cassia, Mimosa.

50. Prosopia, "Moringa.

51. Dalidia, "Dalea. The above from 43 to 51 are families or sections of the great Natural Order Crtenntuia or Leguminose

52. Rhodoracea, type Rhodora.

II. Class. Mesogines.

53. Sarcoditia, type Cotylaria.

54. Contortia, "Siapelia, Echites.

55. Nolanidia," "Nolana.

56. Echidia. "Echinm.

57. Monieridia, "Nomiera.

58. Dichondrania, "Dichondra.

59. Staticidia, "Statice.

60. Cuscutaria, "Cuscuta, Evolvulus.

61. Cressaria, "Cressa.

62. Ilexia, "Ilex, Cordia.

63. Chironidia, "Spigelia, Exacum.

64. Gratiolidia, "Gratiola.

65. Clythrelia, "Utricularia. [mus.

66. Verbascidia, "Verbascum, Hyoscin-

67. Hallerinia, types Halleria, Cyrtandra.

68. Sesamidia, "Sesamum.

69. Psychanthia, " Polygala.

70. Veronicia, "Veronica.

71. Justicillia, " Justicia, 
72. Petridia, type, Petrea, Lippia.

73. Pyrenaria, "Callicarpa.

74. Phrymaria, "Phryma.

75. Synarthia, "Gilobularia.

76. Stilbaria, " Stilbe.

77. Aegiphilia, " Ehretia,

78. Strychnidia," Strychnos, Capsicum.

79. Epacridia, "Phlox, Epacris.

80. Azalidia, "Azalea, Kalmia.

81. Styraxia, "Styrax.

82. Symplocia, " Hopea.

83. Micranthia," Micranthes.

84. Olaxia, " Olax, Bassiu.

85. Hilospermia, " Achras.

86. Inocarpia, "Ardisia.

III. Class. Endogynes.

87. Loranthia, type Viscum.

88. Mangidia, "Rhizophora.

89. Samolia, "Samolus.

90. Cinchonaria, "Cinchona.

91. Geniparia, "Gardenia.

92. Hamellidia, "Hamellia.

93. Linnedia, "Linnea.

94. Diervillaria, "Diervilla.

95. Sambucia, "Sambucus.

96. Viburnidia, "Viburnum.

97. Gitonanthia, "Valeriana.

98. Triostinia, "Triosteum.

99. Ixorinia, "Coffea.

100. Andromia, "Mathiola.

101. Morindia, "Morindu.

102. Echinopsia, "Echinops.

103. Gundelinia, "Gundelia.

104. Centaurinia, "Crupina.

105. Carduacea, "Cynara, Carlina.

106. Eupatorinia, "Conyza.

107. Spilanthia, "Grangea. 
108. Absynthia, type Cotula. 109. Ivaria, "Ita.

110. Parthenidia, "Parthenium.

111. Anthemidia, "Achillea, Bellis.

112. Helianthia, "Silphium.

113. Inulidia, "Solidago, Arnicu.

114. Lactucaria, "Lactuca, Seriolu.

115. Hieracidia, "Prenanthes, Hieracium.

116. Cichorinia, "Cichorium, Hyoseris.

11\%. Scolymia, "Scolymus, Lapsana.

All the above were blended in Rubiacea and Composite Orders. The tribes of Cassini in the latter were not known to me then.

IV. Class. Syuphogines

118. Jasionidia, "Jusione.

119. Lobelidia, "Lobelia.

120. Scevolidia, "Scevola.

121. Vaccinidia, "Vaccinium.

122. Sicidia, "Sicyos, Gronovia.

123. Scleranthia, "Scleranthus.

124. Homalidia, "Homalium.

125. Ribesidia, "Ribes, Cercodia.

126. Gastonidia, "Gastonia.

12\%. Saniculea, "Sanicula, Cussomia.

128. Scadianthia, "Anethum.

129. Periactia, "Seseli.

130. Diplactia, "Daucus, Tordylium.

The 4 above were families of Ombeliferous

Order.

131. Eryngidia, type Eryngium.

132. Begonidia, "Begonia.

133. Quercidia, "Quercus, Fugus.

134. Lecythidia, " Recythis.

135. Melaleucia, " Mrelaleuca.

136. Eugenidia, "Eugenia.

137. Stravadia, "Struradium.

138. Nyssidia, type Nyssa, Elcagmus. 
139. Osyridia, type Osyris, Santulum.

140. Thesidia, " Thesinm.

141. 'Trapacea. "Trupa, Mippuris.

112. Hederacea, " Hedera, Cormus.

13. Ophiracea, " Huchsia.

14. Melastomea," Melastoma.

\section{Class. Angians.}

145. Stratides, type Firatiodes.

146. Pistides, "Pistia.

147. Valisneridia," Valisneria.

148. Phyllacnia. "Phyllacne.

149. Diplantheria," Cypripodium.

150. Ananidia, "Ananas.

15̄. Gethylidia, "Gethylis, T'ımus.

152. Hydnoridia, " Hydnora s. Aphyteia.

153. Galaxidia, "Sisyrinchium.

154. Aplimia, "Burmania.

155. Amaryllides," Hypoxis.

156. Aechmidia, "Aechmea.

15\%. Ubidia, "Rajania. .

VI. Class. Grunians or Lilies.

158. Aloidea, type Aletris, Crinum.

159. Commelinea, " Tradescantia.

160. Aphylanthes, " Tillandsia.

161. Xuridia, "Xuris.

162. Helonidia, " Helonias.

163. Smilaxia, "Smilax.

161. Trillidia, " "Trillium.

16.5. Unisemia, "Unisemr.

166. Alismaria, "Alismu.

167. Potamidia, "Potrmogeton.

VII. Class. Pinanerians.

168. Coryphinia, type Corypha, Lomlans.

169. Arecaria, "6 Arecu.

170. Phenixia, " Phenix, Cocos.

171. Calamia. "Calamus, "Sugus.

17:. Cycadia, "Cyces, Zamia. 
These last $\mathbf{5}$ form the Palm tribe. 173. Julacia, type Saururus.

174. Dracontidia, type Dracontium.

175. Orontidia, "Orontium, Acorus.

176. Carexidia, "Carex, Scuria, Raf.

177. Pharidia, " Pharus, Nastus.

178. Olyracea, " Olyra, Nardus.

179. Agrostaria, "Agrostis, Phleum.

180. Frumentaria," Triticum, Arundo.

181. 'Iripleia, " Oryza, Luziola.

182. Trimeia, "Anthoxanthum, Cinna

VIII. Class. Cryptiaxs.

183. T'mesipteria, type Pilularia.

184. Stachyopteria, "Ophioglossum.

185. Poropteria, "Marattia.

186. Schizopteria, "Schizea.

187. Rhizospermia, "Isoetes. $\dagger$

These are akin to Filixia, the Ferns.

188. Diplostomia, type Hypurm.

189. Aplostomia, "Dicranum.

190. Apogonia, "Phascum.

191. Carpodia, " Marchantia.

192. Phylomalia, "Riccia, Blasia.

And these form the Mosses.

IX. Class. A lginss.

Of this Class and the next, I give all my families to show the whole connection, altho' some were not new.

193. Hypoxilia, type Nyloma, Spheria.†

191. Opegraphia, " Ifysterium.

195. Lepraridia, "Variolaria.

196. Beomydia, "Beomyces.

197. Squamarinia, "Psoroma.

198. Lobarinia, "Lobaria.

199. Cladonaria, "Cladonie.

:00. Usnearia, "Usnea. End of Lichens

201. Fucaria, " lucus, Virsoides. 
20\%. Deloxia, type Dictyota, Phytelis. 20:3. Ulvaria, " Ulva, Caulerpa.

204. Rivulinia, "Rivularia. 205. Physudria, " Physudrium. 206. Corallinia, " Corallina. 207. Spongidia, "Spongia. 208. Ectospermia, "Vaucheria. 209. Ceramia, "Ceramium. 210. Arthrinia, "Conferva. 211. Endonemia, " Mesasperma.

\section{Class. Fungians}

212. Byssidia, types Byssus, Hymantia.

213. Conoplidia," Conoplea.

214. Monilidia, "Monilia.

215. Clavaridia, "Clavaria.

216. Tremellaria, " Helvella.

217. Pezizaria, "Peziza, Teleobolus.

218. Lithecia, " Clathrus, Phallus.

219. Agaricia, " Amanita, Merulius.

220. Boletidia, " Boletus, Phorima Raf.

221. Hydnidia, " Hydnum, Merisma.

222. Cyathidia, " Stictis, Nidularia.

223. 'Tuberidia, " Isclerotium, Gramularia

224. Trichidia, " Diderma.

22:5. Dermosporia, " Geastrum, Batarea.

226. Gymnosporia," Uredo, Mucor.

$\dagger$ These few were adopted from Decandole flora gallica 1806. Some others were published by Brown in 1810, but I did not know his labor in $\mathbf{1 8 1 5}$.

Many of these have been admitted and published by other Botanists between 1815 and 1835, without quoting my labors. As usage and equity requires in Botany that all previous labors and names should prevail or be acknowledged, I hope that future Botanists of a liberal onind or correct principles, will in future duly 
refer to them in their works, as they do to other improvers in their synonymy.

'I'hey never can be at a loss to know what familes or groups I meant: as far as Hetizalonia I gave the proper exclusive characters, with Sub-families and all the Genera of each, as a generil method. For the others I quoted from 2 to 10 Genera of each family: altho' I now still reduce the quotations to one or two genera as main primitive types, any sagacious Botanist may know at once my original families of 1815 by these types. When the quoted Cienus belongs to any newer family, that family was established by me in 1815.

Why should I then admit or follow later labors and arrangements not so perfect nor complete as mine of I815? Decandole's method is not yet complete, and is still obscure. Agardh's is rather better; but follows the ascending series: while we all know now, that the real Serial Order of organization is neither ascending nor descending, not even circular, but Reticulate, or Geographical, as in a Net, or rather a Map: where Classes represent Islands, Orders and Families, their regions and districts; while Genera and Species are the hills and mountains of this botanical geography.

The most proper Natural Series must then follow a geographical plan, wherein the mutual affinities are expressed by vicinity and drawn lines of Seas, Rivers \& c. expressing or defining common characters: which can only be accuratly expressed in tables and maps; while in Serial books we must attempt to follow the plan as nearly as possible, as it is done in books of Geography.

My own peculiar improvements in natural 
Classification, consist, therefore, 1 . in finding the first Genus of the most perfect organization, ROSA, to begin the Serial Order, and Spunges to end it. 2d. Fixing the natural Classes and Orders on nearly a paralel plan with those of Animals. 3d. Giving them proper good names singular and plural so as to express as in Geography, Europe, Europeans. 4th. In increasing largely or triplicating the families of Jussieu, giving them similar good names. 5th. Fixing the characters of all these groups by good and essential characters, whereof some must always be exclusive. 6th. Applying the process of analysis in their formation, sub-divisions, and to acquire their knowledge. 7th. Improving the botanical maps by grouping according to mutual affinities, and separating my mutual disparities.

'To evince how preferable and improved was my method in 1815 even above the clever general method of Agardh in 1822. I shall give here his families called Orders of his $2 \mathrm{~d}$ Class called Series, while he called Classes our reai Orders, thus transposing all the terms.

iII. Series. Cryptocotyles.

I. Class. Macropodes.

27. Order, Nayades, Juss.

28. "Podostomea, Rich.

29. "Alismacea, DC.

30. " Hydrocharides, Rich.

31. " Nymphacea, Juss.

II. Class. Spadicinae.

32. Order, Pistiacea, Raf. I815,

33. " Aroides, Jus.

34. " Acoroides, my Orontides 1815.

35. " Pandanea. Ag.

36. " Cycarlea, Raf. 1815. 


\section{Order, Palma, J.}

III. Class. Glumiflorae.

38. Order, 'Typhina, J.

39. " Cyperacea, J.

40. " Graminea, J.

41. "Juncacea, J.

42. " Xyridia, Raf. 1815.

IV. Class. Liliflora.

43. Order, Asparagoides, J.

44. " Asphodela, J.

45. " Coronaria, J.

46. "Veratrea, my Helonides 1815.

47. " Commelinea, Raf. 1815.

48. " Pontederea, Ag.

49. " Dioscorides. Ag.

50. " Hemodorea, Ag.

51. " Iridea, $\mathbf{J}$.

52. " Narcissea, J.

53. " Bromelinea, my Ananidia 1815

V. Class. Gynandres.

54. Order, Musacea, J.

55. "Cannacea, Ag.

56. " Scitaminea, J.

5\%. " Orchidea, J.

These 31 families answer to my V, VI, VIII, Classes, wherein I had 48 new families, 7 years before Agardh; whereof he has several under same or akin names, without quoting me; as he had not seen my analysis of Nature. He had however the sagacity to perceive some of them, and the good sense to find exclusive characters for all, which Lindley could not do, nor imitate much later, prefering to return to the obscurity of Adanson: whereby he has impeded the general adoption of the natural System, as a general method.

It may be regretted that Botanists do not 
even quite agree as yet on the terms to be given to Natural groups, and mix or transpose the terms of Series, Classes, Orders, Tribes, Families, Legions, Cohorts \&c. as Necker did Genera, Species and Proles. I gave the rules for this in 1814 and I now give a table of the proper terms in Latin and English.

The vegetable or botanical World or Empire or Kingdom, may be gradualy divided by complete analytical process into 6 main or essential successive Sections of the whole, or into 12 lesser Sections, as follow.

- First Series or Primary Classes, or Clusters of Classes-Series vel Classes Primordiales.

I. 2. Chasses, the regular common Natural Classes-Classes Natur.

II. 3. Primary Orders or Sub-Classes-Ordines primaris vel Sub-Classis.

II. 4. Natural Orders-Ordines Naturalis.

5 T'ribes or Sub-Orders; 'Tribu vel Sub-Ord. III. 6. Natural FAurues-Familia Naturalis.

7. Sub-families-Sub-familia s. Genera primordialis.

IV. 8. Geners, or Generic groups and types.

9. Sub-Genera, their Sections not based on fructification.

V, 10. Species, Specific types of Individuals.

11. Breeds or Proles, Specific deviations. VI. 12. Varieties of Individuals.

Individuars alone have a separate physical existence, all the other clusters are useful botanical groups of ideal abstractions based on physical characters, by successive proportions of affinities; as political institutions collect men in successive clusters of families, clans, ranks or castes, communities, tribes and States.

Therefore, Individuals are the main object 
and first aim of Botanical knowledge ; the study of their clusters becomes the aim of systematic Botany: nomenclature and classification, which may be compared to a kind of Statistical Science, under a philosophical method, based on accurate principles.

SPEcies are the collections of individuals perfectly alike in all their parts. Varieties are slight casual deviations. Breeds or Proles are permanent Varieties. Therefore Species are natural altho' variable.

Genera are the collective groups of Species, that agree in the characters of the fructifieation. No Species belongs to a Genus unless it agrees with all the others therein included. Sub-Gencra are lesser groups or sections with some slight deviations chiefly in the habit, seldom in the floral organs. 'Therefore proper Genera are also natural.

Natural Families are groups of Genera having some striking characters in common, chiefly floral and organic.

Natural Orders are groups of families united by one or several important characters, chiefly floral and organic.

Natulal Classes are groups of Orders, possessing some very peculiar floral characters, and common organization.

By attending to these successive groups, and never forcing into them any stranger by organic characters, we may hope to rectify them, improve and fix invariably.

If the natural Classes of Plants were as striking as those of Animals, and known at first sight like Birds, Snakes, Insects \&c. we should not have had so many difficulties in seeking them. But even Reptiles, Insects \&c. offer 
many forms and difficulties, whence Lizards, Frogs, Snakes, Crabs, Spiders \&c. are now becoming peculiar distinct Classes. 'This happens likewise in Botany, and the two organized series of Beings may be deemed almost parallel.

In the valuable but oft neglected work of Adanson on natural families, we find almost a Cyclopedia of botanical knowledge, history, classification, authors, names and genera until his time 1763. It begins now to be appreciated, and I refer to it for all ancient Botany, botanists, Classes and names. It may be often consulted with advantage by improvers.

We learn from him that botanical classifications are numberless, and have been based on all kinds of consideration of forms, organs and uses. To show the absurdity of contriving such artificial systems, he had himself contrived $\mathbf{6 5}$, and calculated their value; till at last the result was the combination of all into the Natural system.

Whatever has been done by Botanists since Zoroaster and Moses (deemed the first by Adanson) till Linneus, is now of little account, and belongs to historical Botany: wherefore I have began my researches at Linneus and Adanson. Yet many eminent Botanists flourished since the revival of letters, among which Cesalpini who in 1583 first contrived 15 natural Classes, and Zaluzianski in 1592 had 22 such, but only few very really natural-Magnol who was the first in 1689 to attempt 68 natural Orders. Tournefort who in 1694 was the first to fix definite Genera, and reduced 698 of them to 22 artificial Classes.

These Genera were increased to $\mathbf{1 1 7 4}$ before Linneus and by him: Adanson increased them 
to 1615 , all deserving attention: this number was sivelled to 1832 by Necker in 1790 , and has been swelling ever since; until Genera are now nearly as numerous as known Species were a Century ago.

Adanson ventured to prophesy that botanists would at last be compelled to attend to Genera only, and neglect the Species, both for their number and natural spliting. He was like Linneus, Necker and myself (in fact like all acute observers) a strenuous supporter of the doctrine that Species were unlimited, and increasing by the natural process of semination, deviation, variation, hybridation \&c. Whence he concluded that we could hardly ascertain the primitive types of species, that many known to ancient Botanists were lost or no longer found, while new ones were evolved in mountains, groves, fields and gardens.

The practice of uniting incongruous and unlike plants in the same Genus, has long prevailed and is yet followed by Hooker, Torrey and many eminent botanists, who do not perceive the fallacy of this plan: whereby their species are in fact often real types of overlooked Genera, and their Genera are artificial like the first made by 'Tournefort and Linneus.

Botany will never reach perfection till this arbitrary mode of naming and refering plants is discarded: and until all the species of a Genus offer similar characters; as in fact they naturally ought to do. 'The many polymorphous Genera are mere artificial and heterogenous combinations of unskilful or wavering Botanists, and not real gencra!

In some instances these cautious botanists appear to be positin cly blind to dieparitise, and 
unite in the same Genus, species with a capsule, a berry or one seed!--else with equal or unequal calix, petals, pistils, stamens \&c.!else with Ovary inferior and superior!-'They might as well unite a Grass with a Rose, Men with Monkeys, and Bats with Birds.-They deplore the increase of Synonyms and overwhelm us with useless names; since all theirs must be changed, and will be.

'The only plea ever given for artificial systems was their utility in the facility of finding plants by analysis; but this use utterly fails when the admitted aberrations are numberless. In the sexual system they abound, and I have often amused myself by defying a botanical Student to find out some plants by it; Cleome dodecundra for instance, my Polanisia graveolens. But by the natural method uniting the analytical process as I do, there is no difficulty to find out Genera: while for Species, all being reduced to their proper Genera, there is the same facility. Not so by the distorted Genera of many Botanists, one third of their Species not possessing the generic characters ascribed, can never be found out by beginners, while experienced botanists are directed by mere habit, aspect, affinities, or something which cannot be expressed, and is neither definite nor real nor natural nor true.

Nature in the spontaneous evolution of vegetation, baffles all our petty incongruities by making new Species out of varieties, and new Genera out of floral deviations! the process is not always so quick as to be pereeived in a few years; but "is very obvious to botanical observers who happen to study plants during $\mathbf{4 0}$ or $\mathbf{5 0}$ years. This fuct is then a truth, whoever 
doubts it is a mere tyro or beginner in the study. On this truth must be based our Genera and Species, instead of admiting improper clusters of individuals. We shall then be better able to ascertain the formations, deviations and filiations of plants, with all their connections, relations and affinities to each other.

Some botanists deem that in nomenclature and classification, the majority must rule: this may be true for artificial systems; but not in the natural method. There Nature alone must rule, and her close observers who notice the botanical laws, phenomena, exceptions and forms. All other Botanists may be wrong, and are often so, when they wish to make these bend to their own petty views and absurd classes or Genera.

Names are also quite essential, because they fix and convey the knowledge thus acquired. Bad names can only be tolerated for awhile. 'Those of Aublet were changed by Necker and Schreber and we had 3 for one of his. Rosa, Quercus and Labiates for instance are good invariable names. If the philological absurdities of vulgar languages as to Grasses, Lilies \&c. are admitted into the Scientific language of Botany, we should fall into confusion of ideas and applications. Names are not arbitrary : they impart ideas, and ought to be proper, clear and distinct, in order to suggest or convey such ideas to the mind, fix them in the memory, and be generaly applicable and practical.

Botanists have like other men their whims, preferences, systems, theories and hypotheses; but all must give way before observations, facts and realities: and thus by truth shall the Science progress. 
In result 1 . It is better to distinguish and insulate by good names, than to blend and conceal by wrong references and bad names. 2. All bad Genera must be reformed, revised and corrected, till they become unobjectionable and invariable. 3. All bad names must be changed for good Names. 4 The same for Natural Classes, Orders and families. 5 And also for Species or the Generic types.

It is this I propose partly to do in this work, as far as my observations avail and my researches extend. 'To my fellow Botanists I say-Do likewise or better still; but never neglect a botanical reform, based on nature, and proper discriminations.

\section{NATURAL CLASSES AND ORDERS}

\section{of C. S. Rafinesque, 1815.}

The study of mutual affinities and disparaties, is the base and true path of methodical and natural Botany. Cesalpini in $\mathbf{1 5 8 3}$ began modern Classification on a natural plan by 15 natural groups; most of the Botanists prefered, since artificial systems, until Magnol in $\mathbf{1 6 8 9}$ and Linneus who in $\mathbf{1 7 5 1}$ produced 58 supposed natural Orders along with his artificial sexual Classes.

Adanson had also 58 families in $\mathbf{1 7 6 3}$, and $\mathbf{4 5}$ were Natural, they were reduced to 36 by Scopoli in 1783, and to 54 by Necker in $\mathbf{1 7 9 0 .}$ But Jussieu improving thereon had in 1789 as many as 100 natural families in 15 artificial Classes, which have been gradualy increased or improved by Lamark, Ventenat. Decandole, 
Richard, Mirbel, Agardh, R. Brown, Lindley and others.

Meantime as early as 1802 I began to perceive the necessity of rectifying the presumed Orders of Jussieu, and after many observations in both hemispheres, I published my Natural Classes in 1814, and my 66 Natural Orders in 1815. 'I'wenty years of additional researches have convinced me that they need but little additions, unless we change their Sections into Orders ; but that altho' I had increased their families to 310 , they may now become about 400 .

I have only published my Chloris Etnensis 1815, Florula Ludoviciana supl. 181\%, Annals of Nature 1820, according to this arrangement. But it is susceptible of general application any where: and every Genus finds its place in it, because it is both natural and analytical. Every one of the $2000 \mathrm{New}$ genera or plants of this work, will easily be refered to my Orders, it not to my families, by any one acquainted with analytical Botany

Therefore I shall proceed to give tabular views of my Classes and Orders, with their essential analytical Characters, comparative and distinctive of each; the examples and types are both in my New families, and some quotations of Jussieu's.

\section{Table of Natural Classes.}

I. Primary Class. Endogenia or Dicotylia or Mesotyula. 'The Endogenes or Dicotyles or Mesotyles-Trees, Shrubs, Vines or Plants; stems and roots vascular fibrose, vessels and fibres in concentric layers, around a central pith or cellalar hollow. Outward bark or epidermis, often woody beneath it. Leaves often articulated or oppesite, nerres commonly reti- 
culate, flowers conspicuous with perigone stamens and pistils. Germination commonly dicotyle or polycotyle, and central, growth by outward increment. They correspond with the Vertebrate or Bony Animals, and the binary or quinary numbers prevail, $2,4,8$, or $5,10,20$.

1st Section. Eltranthia, the Eltranthes. Flowers with one or more Pistils, quite free not coalescent with the perigone (superior Lin.) Flowers commonly free and separate from each other. Fruits free.

I. Class ELTROGIA, The Eltrogins. (meaning free pistils) Stamens free or only connected together, not coalescent with a corolla or inner perigone into a tube, unless the fruit may be a pod. Equivalent of the Mammalia.

II. Class MESOGIA, the Mesogins (mg, middle pistils). Stamens connected with a corolla or inner perigone, or inserted on it, and forming together a tube around the pistil. Fruit never a pod. Equivalent of the Birds.

2d. Section. Synanthia, the Synanthes. Flowers often united into a compound flower with only one pistil, united or coalescent with the base of the perigone (inferior L). Fruit always connected with it and often crowned by it.

III. Class GYNENDIA, the Gynendes (mg. inside pistils) Stamens always as in the 2d Class, more or less connected with a Corolla, or often connected together also, and both inserted on the pistil. Equivalent of Reptiles,

IV. Class SYNOGIA, the Synogins (mg. united pistils) Stamens free unconected with the corolla when it exists, and commonly inserted on the perigone, Equivalent of Fishes.

IId. Primary Class. Exogenia or PleuroTrua. The Exogenes or Pleurotyles-Palms, 
Lilies, Grasses, Ferns and Mosses with stems and roots vascular fibrose, ressels and fibres fasciculated and intermixt, without a central pith, the pith scattered or lacking. No proper bark, nor wood, the epidermis only of closer texture. Leaves seldom articulated or opposite or whorled, nerves commonly parallel ; flowers more or less conspicuous or anomalous, with or without perigone and stamens, but always a pistil or the equivalent, Germination lateral, commonly monocotyle or heterocotyle or cryptocotyle; growth by inward increment. T'hey correspond to the Anostians or unbony Animals, and the ternary numbers prevail, $1,3,6,9.12$.

Ist Section. Isanthia, the Isanthes. Flowers always regular and conspicuous with a perigone, stamens and pistils, never glumaceous, nor spadiceous.

V. Class ANGINIA or STEGINIA, the Angines or Steginians. (Mg covered pistil) Pistil single inferior, coalescent with the base of a perigone, Stamens on either, fruit covered or crowned. Equivalent of the Crustacea.

VI. Class GYMNOSIA or LIRIDIA, the Gymnoses or Lirides, (mg uncovered or Lilylike) Pistils one or many free and central, with a perigone and stamens around. Equivalent of the Insects.

2d. Section. IIeteranthia, the Heteranthes Flowers anomalous or inconspicuous, seldom with a perigone, commonly glumaceous, Spadiceous or without Stamens.

VII. Class PHANERIA, the Phaneres (mg conspicuous) Flowers conspicuous with spatha spadix or glumaceous bracts, or a perigone epispadix, stamens and pistils conspicuous. Equivalent of the Worms or Annelides. 
VIII. Class CRYPTOSIA, the Cryptoses (mg hidden) Flowers anomalous without perigone, inconspicuous or concealed, conimonly no stamens and hardly apistil, assuming various uncommon forms. Equivalent the Millusca.

IIId. Primary Class. Lannagenia or AcotyuAd, the Cellulars or Acotyles-Lichens, Algas and Fungi, or plants without stem nor roots nor leaves, nor flowers; neither vessels nor fibres; formed of cellular tissue variously expanded, Frủctification concealed and granular or gemmular, germination acotyle growth by mère expansion. They correspond to the Zopsians, or Animals without blood, nor nervous system, and there is no prevailing numbers.

IX. Class ALGOSIA, the Algas. Commonly a frond or tallus, imitating leaves or threads or stems, fructification often evident producing gongyles or gemmules. Color often greenish and station aquatic. Equivalent of the Polyps or Zoophytes:

X. Class. MYCOSIA, the Fungi-Neithe? frond nor thalus, expansion variable often glo bular, fructification in spores or a powder ofter invisible. Color seldom green, station never aquatic, either terrestrial or parasite. Answering to the lowest animal class of Porostomes or Animalcula.

\section{Trable of the Natural Orders. \\ I. Class, ELTROGINES.}

Ist Section, Polygynia. Pistils multiple, or petals anomalous, fruit not a pod.

1. Order, Rhodantiua, the Rhodanthes. (Roseflower) stamens peristomic, antliers not adnate.-Types Rosa, Spirea. 
2, Order, Perimesia, the Perimeses (around middle) stamens hypogyne or perigyne basilar, anthers not adnate-Type Sedum.

3, Order, Annantieria, the Adnanthers, stamens hypogyne, commonly many, anthers adnate.-Types Anemone, Magnolia.

2d, Section, Eltrandria, Pistil single, Stamens commonly free, petals never anomalous, nor united in a peripetalic form, fruit never a pod.

4, Axantina, the Axanthes (fl. on axis) Flowers symphoric axanthic or amentaceous, apetalous, diclinous, often a lepigone instead of perigone, fruit often monosperm -Types Pinus, Populus, Ficus.

5, Movosperma, the Monospermous-Flowers neither symphoric nor axanthic often apetalous, fruit monosperm, stamens isarine not opposite-'Types, Urtica, Rumex, Ulmus, Laurus, Protea.

6. Plymontia, the Plyrontes (opposite), stamens opposed to petals or alternate to calix, isarine often several stigmas-Types, Rhamnus, Berberis, Vitis.

\%, Isandria, the Isandrous (eq. st.) Stamens alternate to petals or opposed to calix, stigma simple, fruit often polysperm-Types Lythrum, Ruta, Viola.

8, Struridia, the Cruciferous-Stamens heterines commonly tetradynamic, fruit siliquose.'Types, Iberis, Sinapis.

9, Monostrima, the Monostimes (single stig,) Stamens many or not $\mathbf{4}$ dynamic, fruit se!dom Siliquose, one stigma.-Types I' $\bullet$ paver, Cistus, Citrus.

10, Porymesia, the Polymeses (many midclle) Stamens heterines or many, commonly 
epimesial, several stigmas, and seeds'Pypes Sapindus. Portulaca, Hypericum, Luphorbia.

11, Isostuma, the Isostimes (eq. stig.) Stamens isarine or regular, never epimesial, many stigmas and seeds-Types Drosera, Saxifraga, Dianthus.

3d. Section, Symphandria, stamens commonly united, or fruit a pod. or a peripetal corolla (monopet.)

12, Adecrumid, the Adelphides, Stamens is arine, regular, united, corolla regular,many stiginas and seeds, fiuit not a pod-Linum, Tamarix, Geranium. Passiflora.

13, Omoplitia, the Omoplites (reg. union) Stamens heterine or many, well united, many stigmas and seeds, fruit not a pod-Types Adansonia, Hibiscus, Malva.

14, Perinonia, the Perimones (around single) Stamens isarine united, petals not papilionaceous nor united, one stigma, fruit not a pod-Types Melia, Celosia, Fumaria.

15, Cytenntira, the Leguminose-Petals papilionaceous or various, stigma single, fruit a pod.-C'Yyes Amorpha, Colutea, Buuhinia.

16, Peritalia, the Peritales (around petal) corolla peripetalic, but not staminiferous, fruit never a pod.- Types Ledum, Clethra, Plumbago.

II. Class, the MESOGINES.

1. Section, Polydia, several pistils or a lobed ovary, one or several styles and stigmas, several fruits or secds.

17, Porygia, the Polyges. Several pistils and fruits-Types Echites, Asclepias.

18, Lobogynia, the Lobogynes-Ovary lobed, 
one style, several seeds-'Types Nolana, Borrago, Echium, Salvia, IMoniera.

19, Polvis, the Polymes. One ovary, several styles, fiuit simple-'Types Statice, Cuscuta, Cressa, Cordia, Carica.

20, Epicina, the Epicles (on valv) fl. regular one ovary, one or several styles, fruit simple, valves seminiferous-'Types Gentiana, Orobanche.

2d. Section Heterolia. One Ovary and style, fruit simple, valves not seminif. Corolla irregular.

21, Chasmantuia, the Personate. Fruit a capsule unilocular or bilocular with paralel septum, or multilocular partitions alternate to valves-Types Gratiola, Gerardia.

22, Plasyrata, the Plasyrges. Fruit a capsule polysperm, with 2 or many cells, partitions opposed to valves-Types Polygala, Veronica, Justicia, Sesamum, Mimulus.

23, Olisperma, the Olispermes. Fruit monosperm, or with monosperm cells, often a drupe or berry-Types Vitex, Phryma, Scabiosa.

3d. Section Isorolia. One ovary and style,fruit simple, valves not seminif. Corolla regular.

24, Arcytima, the Arcythes. Stamens alternate, fruit monosperm or drupe or berryTypes Ehretia, Solamum, Jusmimum.

2.5, Darynia, the Darynes, Stamens alternate, fruit a polysperm capsule-Types Convolvulus, Phlox, Kalmia.

26, Stemisia, the Stemises. Stamens opposed to the sepals of the corolla-'Types Primula, Plantago, Olax, Achras, Ardisia. III. Class, the GYNENDIANS.

1. Section, Eltranthia, Flowers separate, or 
without a common periauthe, anthers free.

27, Nantiandria, the Nantiandres, Stamens opposed to the sepals of the corolla, or to staminiferous petals-Types Viscum, Rhizophora, Sumolus.

28, Polyspia, the Polyspes. Stamens alternatè. fruit bilocular or multilocular polysperm -Types Cinchona, Gardenia, Hamellia, Linnea, Diervilla.

29, Spiranichia, the Sphanicles. Stamens alternate, fiuit monosperm or monolocular, or with monosperm cells.-Types Sambucus, Viburnum, Valeriana, Rubia, Coffea, Morisuda.

2d. Section Symphanthia, Flowers agregate on a common phoranthe. surrounded by a perianthe,fruit monosperme, anthers united.

30, Frosculia, the Floseular. Corollas uniform tubular or irregular, but none ligular'Iypes Echinops, Centaurea, Carduus, Conyza.

31, Actinantina, the Radiate. Corollas of the radiusligular and radiating-Types Bellis, Helianthus, Arnica.

32, Glossantiri, the Glossanthes, all the corollas ligular-Types Taraxacum, Ci chorium.

1V. Class the SYNOGIANS.

1. Section, Sigollia, Internal perigone or penpital corolla not stamindfewus.

33, Conntrina, the Codanthes (bell flow.) Fruit commonly capsular, never a berry, flowers sometimes agregated and irregular -'Types Jasione, Lobelia, Scaevola, Campanala.

34, Iniogrnia, the Idiogynes (separ. fem) Fruit a herry, flowers neither agregated 
nor irregular-Types Vacinium, Cucumis. 2d. Section. Polystylia. Corolla not peripetal or wanting, styles and stigmas multiple.

35, Ascadia, the Ascadia (not umbel) Stamens isarine, fruit never 2 seeds -'Types Scleranthus, Adoxa, Ribes, Arcilia.

36, Onbelimfrera, the Ombelliferous. Stamens isarine, fruit 2 seeds or capsule 2 seeded flowers commonly umbellate-Types $\mathbf{S} a$ nicula, Buplevrum, Eryngium ofc.

37, Polvandria, the Polyandrians. Stamens heterine or many.--'Types Fagus, Juglans, Begonia, Pyrus.

3d. Section, Monostylia, Corolla not peripetal nor wanting, a single style.

38. Dapsilitia, the Dapsilitians, stamens indefinite-Types Myrtus, Eugenia, Cactus.

39, Acascotia, the Acascotians. Stamens determinate, fruit monosperm-'Types Nyssa, Santalum, Thesium, Trapa.

40, Convphitia, the Coriphians. Stamens determinate, fruit polysperm-'Types Cormus, Escullonia? MLelastoma, Ludwigia, Asarum.

V. Class the STEGINIANS.

1 Section. Gymandria. Stamens epigyne inserted on the Ovary or style.

41. Cratodia, the Cratodians. Frnit multilocular-'Types Musa, Hydrocharis, Amomum.

42. Orcindin, the Orchides. Fruit unilocular. -Types Pistia, Vallisneria, Phyllacne, Cypripedium, Arethusa.

2d. Section, Gynosynia. Stamens perigynes inserted upon the perigone.

43. Acrnitia, the Acinitians. Fruit a berry, 
stamens free-'Types Ananas, Gethylis, 'Tumus.

44. Synema, stamens monadelplous or united 'Types Hydnora, Sisyrinchium.

45. Iridisin, stamens free, less than 6 , a capsule-Types Crocus, Gladiolus, Burmannia.

46. Yynodia, six free stamens, a capsuleTypes Amaryllis, Narcissus, Pitcairnia.

VI. Class the LIRIDIANS.

1. Section, Carpiclia fruit simple, capsular and commonly polysperm.

47. Liriantuia,capsule trilocular, partitions opposed to valves-Types Lilium, Asphodelus, Crinum.

48. Gonotidia, capsule unilocular, or trilocular with partitions formed by valves-'Types Tradescantia, Eriocaulon, Juncus, Helomias.

2d. Section, Achenacia, fruit simple or multiple, berry or achena often monosperm.

49. Acanopsia, Fruit simple, commonly a berry-Types Smilax, Trillium, Asparagus, Unisema.

50. Polachena, fruit multiple. commonly of several monosperm achenas-Types Aliona, Potamogeton.

VII. Class. PHANERIANS.

1. Section, Spadicea, flowers on a spadix and often a spatha, without glumes.

51. Pocilma, the Palins. Spadix often ramose with spatha and perigone, stem cauliform, leaves polytome-Types Phoenix, Calamus, Zamia, Pandanus.

52. Emprotia, the Aroides. Spadix simple, often no spatha nor perigone, stem commonly annual and leaves entire-Types 
Equisetum, Saurums, Zostera, Pothos, Acorus, Typha.

2d. Section Gilumacea. No spadix, nor spatha flowers glumaceous, fruit achena.

53. Cortodia, the Culmiferous. Style single, stem commonly without articulations'Types Carex, Cyperus, Phams, Olyra, Nardus.

54. Acmirosia, the Grasses, Styles 2 or 3 , stem articulated; 'Types Agrostis, Avena, Oryza, Cinna.

VIII. Class. the CRYP'IIANS.

Ist. Section. Pteridia, fructification sessile capsuliform, not calyptrate.

55. Diclipteria, the Diclipters. fructification bivalve or multivalve, commonly in spike or axillary-'Types Plananthus, Pilularia, Ophioglossum.

59. Dorsiferia, vel Filices, 'The Ferns or Dorsifers. fiuctification globular opening by pores or splits, commonly behind a frond or on a root-Types Marattia, Schizca, Asplenium, Salvinia.

2d Section. Phryganisia, Fructif. pedunculate calyptrate, uniform or tubuliform.

57, Etheogania, the Mosses, Fructif. calyptrate, stem leafy-Types Hypmum, Dicranum, Phascum.

58. Hepatidia, the Hepatides. Fructif. uncovered not calyptrate, stem or expansion fron. dose-Types Marchantia. Riccia.

IX. Class, the ALGIDIANS.

1st. Section, Lichenia, the terrestrial algas or Lichens.

59. Tremaria, the Tremians. Fructif. opening by a pore or slit-'Types Hypoxylon, Xyloma, Verrucavia. 
60. Pinsmaria, the Phymians. Fructif. solid, tubercular, not opening; 'Types Lepraria, Squamaria, Lobaria, Cladonia, Usuea.

2a. Section, IIydrophytia, the aquatic AIgas.

61. Fucidia, the Fucidians. Form variable, but never filamentosc-Types Fucus, Ulva, Rivularia, Physidrum, Spongia.

62. Hydronema, the Confervians. Form filamentose, commonly tubular and with partitions; 'Types Vaucheria, Ceramium, Characias (chara L.) Oscillaria, Stereonema.

$X$. Class, the FUNGIDIANS.

1st. Section, Exosporia, fructification external, in spores or sporanges, form seldom filamentose

63. Adelisteb, the Adelistians. Hysterium or receptacle hardly conspicuous; 'Types $\boldsymbol{B}$ y $\boldsymbol{s}$ sus, Conoplea, Momilia, Clavaria, Helvella, Peziza, Phallas.

64. Pinaneristia, the Phaneristians. Hysterium very conspicuous, in rays, veins, pores, tubes or tubercles-Types Aguricus, Boletus, Hydmum.

2d. Section, Endosporia, fructif. internal in spores or sporanges, form never filamentose.

65. Sarcosporia, the Sarcosporians, fructif. fleshy or gelatinose, not pulverulent; 'Types Cyathella, Stictis, Gramularia.

66. Coniosponia,the Coniosporians,fructif. pulverulent-Types Diderma, Lycoperdon, Fuligo, Mucor.

In these Orders every family and Genus admitted was to possess the essential character applied to all, except in very few instances; 
when by natural anomalies (to be added and expressed) they might somewhat deviate: as happens in some Genera, Trifolium and Saponaria \&c. But the great anomalies allowed by Jussieu in the Amentacea, Saxifragea, Rhamnoides, Bicornes \&c. are by no means deemed to be such ; since they arise from blending together different families, united by trivial characters of lesser importance. Such orders must therefore be divided by all means.

But besides such regular Orders based on the fructification, an essential but temporary part of organization, the vegetable world offers another series of permanent forms, which have been deemed essential and very easily distinguished at first sight. In fact they are the only ones available iu the study of fossil Botany, since few fossil flowers and fruits are found; and the three great Series of plants have been hased thereon.

1. Exogenes or Concentric Vascular, types Trees,

2. Endogenes or fascicular Vascular, types Palms.

3. Larnagenes or Cellular, types Lichens.

Altho' these Series are subdivided by the organs of reproduction, their permanent organs of support and nutrition offer very important bodily forms susceptible of indicating natural Orders; and in fact we now always add them to the description of tribes and even Genera, since Adanson and Jussieu gave the exumple. See my new views on that score at the end of my rectified Classes. 


\section{'HHE NA'TURAI, CLASSES, Rectified 1835.}

During 20 years from 1815 to 1835 , I have continued to rectify my Natural Classes. 'The later improvers differ yet widely on their number and serial Order. Many appear prone to acknowledge only 3, Exogenes, Endogenes and Acotyles. 'This is as preposterous as if we made only 3 classes of animals, Bony, Unbony and Anemates, uniting Mammalia, Birds, Reptiles and Fishes into the first; altho' others incline to separate even the Mammalia into 3 , Primates, Quadrupeds and Cetacians, and also the Reptiles into 3, Saurians or Lizards and 'Turtles, Ophidians or Snakes, and Batracians or Frogs and Salamanders,

However Agardh in his later works wishes to amplify too much the Natural Classes of Plants, having made 25 of them, and increased the Orders or rather the families to about 200 .

The rapid increase of botanical knowledge, and the immense materials yearly added, appear to require an addition of Classes which altho' necessarily taken off from others, become better distinguished by insulation or separation. My first Natural Class was so numerous in Orders, and with so many natural anomalies that it may very conveniently be divided. It answered nearly to the 'Thalamiflores of Lamark and Decandole, a bad name being mongrel half Greek and half Latin. The Leguminose are so anomalous and numerous as to deserve probably to become a peculiar Class, instead of an Order, or be united to Symphandria. 'The first Orders distinguished by a plurality of pistils would be a natural Class if the Genera Consol- 
ida, and others with single pistils are removed, as I have already Acter and Botrophis, or this anomaly introduced as exceptions.

As early as 1820 I proposed to form a Natural Class, Naxtrandria (in Bory's annals) from all the scattered Orders that have, Stamens opposed to petals, or to sepals of a corolla, or alternate to a calix. 'This I conceive will be a very natural and definite Class; this reversed insertion and position being very essential and constant, as the Rhamnides with a different interposition form quite separate families. Most of the Gentianides belong to it.

The plants with a peripetalic corolla (monopet) surrounding the stamens, but not bearing them, as Erica, Vaccinium, Campanula appear to demand also the formation of a peculiar class, this mutual freedom of essential Organs, heing quite striking. Thus 4 Classes might be taken from my Eltrogines chiefly.

In the next, Heteroma or all the Orders with irregular corolla or stamens, including the Labiate and Echides, might form a very natural Class. The same is true of the Synanthes or Compound flowers. Thus we have 6 additional Classes of Exogenes.

Among the Endogenes, the Gynandrous or Orchidea and akin Orders, are a very natural group, deserving now to become a Class by the vast number of new Genera lately ascertained. The Grasses and Ferns are both so distinct and peculiar as todeserve also that name. This gives 3 other natural Classes; another or tenth in the whole may be found in the Lichens or terrestrial Algas: whereby a linnean Genus became gradualy a family, next an Order, and now may be a Class! 'The labors of Acharius 
and Agardh on the two divisions of $\boldsymbol{A}$ lgas have led to this. Agardh in his excellent Systema Algarum 1824 has in fact divided the aquatic Algas into ( 0 orders and 101 Genera, altho' he has omitted ny Physidria family, besides the Spunges that are real plants.

I'lius we may double my 10 Classes into 20 equaly Natiral, and this is the extent of Classification we may venture to admit. If we were to have more, or 40 and 50 , they would be in fact mere Orders by a different name. If the Cactoides were more numerous they might perhaps form a Class also; but they are very akin to Myrtus and Ribes. Having thus revised and rectified my own Classes, I shall present their tabular analytical View in Latin.

Series I. EXOGENIA. Rosa prim. G.

Classis, 1. POLYGIA, Pistilis plurimis liberis, vel petalis staminisque anomalis, Stam plurima S. pancis alternantis, \& libera. Ord. 1, 2, 3 ut supra. Rosacea, Sedoides, Ranunculacea, \&c. auctoris.

2. EL'TROGIA, Pistil, liberum unicum, petalis regularis vel nullis, perigono vel lepigono, stam. plurima s. paucis alternantis liberis, fructo vario non legumen \&c.-Ord. 4, 5, 7, 8, 9, 10, 11, Crucifera, Caryophylea and alia Ord. auctoris.

3. SYNANDRIA, Pistil. plurinis s. unic. liberum. Stamina plerumque coalitis, vel fructo legumen, Corolla varia regularis s. papilionacea -Ord. 12, 13, 14 15, ut supra. Leguminosa dividenda inter Ord. Papilionaria, Lomentaria, Mimosaria? Genera Fumoria, Melia, Malva, Geranium \&.c. ad fructo solo distinguenda.

4. HE'TEROLIA, Stam. paucis ad corolla peripetala insertae adnatis, alternantis $s$. hete- 
rolis. vel inequualis. Corolla inequalis s. Iabiata. Pistilum liber unicum vel lobatum plerumque stylo unico-Ord. 18, 21, 22, 2:3. Labiata, Per sonata, Verbenacea, Polygalea \&c. auctoris. An Orobunche addenda? ut Convolvulacea et Verbascides

5. MESOGIA, diff. Heterolia, Corolla et stamina equalis, plerumque isarinis, alternansOrd. 17, 19, 20, 24, 25. Borragines Asclepides Cuscutea, Solanea, Jasminea \&c. auctoris.

6. ElTRAN'THIA, Pistil. unic. coalitum vel infero. Stam. ad corolla peripetala adnata inserta, alternantia, paucis, antheris liberis, fructo vario, flores non agregata in periantho-iOrd. 28, 29. Rubiacea, Valerianea \&c. auctoris.

7. SYNAN'THIA, vel Composita,diff.Eltranthia, antheris plerumque coalitis, fructo achenaceo monosp. flores agregata in perianthoOrd. 30, 31, 32, Corymbifera, Cichoracea \&c. auct.

8. ENDOGIA, Pistil. unic. coal. interno. Stam. libera, plerumque periginis, plurima vel definita alternantia. Corolla polypetala vel nulla-Ord. 35, 36, 37, 38, 39, 40, includens Ombellif. Cactoides, Myrtides, Epilobia \&c. auct.

9. NANTIANDRIA, Stam. determinata, isarina apposita, ad petalis s. lobis corolla opposita, vel ad calix sepalis alternantia (in omnia alia opp.) pistillum plerumque unicum-Ord. 6, 26, 27, Rliamnoides. Berberides, Sarmentosa, Menisperma, Primulacea, Plantaginea, Hilospermia, Loranthea, Samolides $\mathcal{S} \cdot c$. auctoris, et plurima Gentianea, an omnis?

10. ANEPERIA (not upon around) Perigono duplex, interno corolla peripetala non staminifera (in omnis alia cor. peripest staminif.) Stam. centralis s. periginis. Pistil. unic. liberos. 
coalito-Ord. 16, 33, 34 sed Bicornes, Nyctagynea, Jasionidia, Campanulea, Lobelides, Vaccinides, Cucurbitea \&c. auctoris.

\section{SERIES II. ENDOGENIA.}

11. GYNANDRIA, Pistil. unicum coalitum, Stam epiginis s. epistylis-Ord. 41, 42. Scitaminea, Orchidea \&c. auctoris,

12. S'TEGINIA, Pistil. unic. coal. Stam. periginis supra perigono inserta-Ord. 43, 44, 45, 46, Irides, Narcissides \&c. auctoris.

13. LIRIDIA, Pistil. liber. unic. plurimis, st am. periginis vel. hypoginis, spadix et glumis nullis, perigono sepe corollato-Ord. 47, 48, 49, 50, Liliacea, Asphodelides, Juncides, Smilacina, Alismides \&c Auctoris.

14. SPADIXIA, Diff. Liridia, spadix flor. ferens, minime corollatis seu apetalis non glumosis-Ord. 51, 52. Palmae, Cycades, Aroides, Acorides, Equisetides, Saururides, Typhides \& $\mathrm{c}$ Auctoris.

15. GLUMOSIA. vel. Graminea, flores apetalis, bracteis glumosis vel. squamosis vestitis, stam. hypoginis, firuct. achenaceo-Ord. 53, 54, Carexides, Cyperides, Agrostides, Graminea, \&c. auctoris.

16. P'TERIDIA, flores anomalis, apetalis, stam. pistilisque cryptis vel. nullis, fructibus capsuliformis dorsiferis vel spicatis vel acalyptris -Ord. 55, 56, Lycopodia, Stachiopteris, Poropteria, Filices, Rhizosperma \& c auctoris.

17. PHRYGANISIA, difl: Pteridia, fructib. urniformis calyptratis vel tubulatis vel explanatis, non capsuliformis.-Ord. 57, 58, Musci, Hepatica \&o auctoris.

Series III. LARNAGENIA, Cellular.

18 LICHENIA, 'Terrestris vel parasitis sepe viridis, Frons vel thalus, squamosis, crus- 
taceis vel varians, fructif. evidens, scutellis, tuberculis vel. gemmulis dehiscens-Ord. 59,60 , Hypoxilia, Lichenes, Thaliferis of auctoris.

19. MYCOSIA, Terrestris vel parasiíis, coloratis vix virides. Eftusis, carnosis stuposis, fungosis, lignosis, gelatinosis. Form a varians. Fructif. sepe crypta vel inclusa, hysterium sporis ferens-Ord. 63, 64, 65, 66, omnia Fungi Auctoris.

20, AlgOSIA, Aquatiles sepe virides intedum terrestris vel parasitis. Effusis, frondosis, thalosis, filamentosis, herbaceis, vesiculosis. Fructif. crypta, gelatinosa, vel gongyles-Ord. 61, 6:, Chara,Conferva, Ulvacea, Fucides, Spongides \&c auctoris.

I admit in this Chara and Nostoc with Agardle, but with doubt. Perhaps all terrestrial algas are to be removed to the preceding classes, but all the Spunges belong here; since they are yet deemed animals by some Naturalists, they properly form the last link of vegetation, as Roses the first link.

'The needful increase of natural families is evident as we proceed in discoveries ; but the increase of orders much less needed. To change families into orders is not requisite to better their knowledge. My $\mathbf{3 7 5}$ families may yet be increased to about 400 ; but my 66 Natural Orders require very little improvement, and only admit of Sub-Orders. If such are to be deemed peculiar distinct Orders I venture to indicate those I ascertained and established since 1815.

ADDITIONAL ORDERS.

1. Senticosia, differing from Rhodanthia by pistils definite, types Agrimonia, Sibbaldia, Spirea, \&c. 
$\because$ Isotonu, diff. from Perimesia, by Stam. indefinite, types Annona, Magnolia, Aconitum.

3. Gropolis, diff. fiom Adnantheria by pistils and fruits indefinite on a gynophore, types Clematis, Anemone, Magnolia.

4. Axepia, diff. from Axanthia by flowers on a phoranthe or flat or hollow receptacle, types Ficus, Gnetum, Ambrosia.

5. Sarcodia, diff. from Monospermia by a fleshy fruit, berry or drupe, types Amyris, Rivinia, Laurus, Daphne.

6. Calycantiuia, diff. from Isandria by stam. perigine summigere, types Lythrum, Rhexia.

\%. Polarxia, diff. from Monostimia by fruit multilocular, types Cistus, Citrus.

8. Epinesia, diff. from Polymesia by flowers dicline stamens inserted in the middle, fruit cocular, types Euphorbia, Ricinus, Begonia.

9. Lonentalzia, diff. Cyteanthia by corolla not papilionaceous, and stamens often free; all the regular leguminose plants.-All these are from my first class.

10. Carytuin, the true Labiate plants with 2 or 4 unequal stamens.

11. Monornuia, diff. Chasmanthia by capsule unilocular, types Gratiola, Utricularia.

12. Puropsanthia, diff. Plasyrgia by stam. not didynamic, types Polygala, Veronica, Justicia.

13. Distemia, the regular monopetalous with 2 stamens, types Olea, Lilas, Nyetanthes.

14. Anisandria, the regular monopetalous with 5 or 10 unequal stamens. types Convolvulus, Vesbascum, Celsia.

15. Praxarpia, diff. Stemisia by a capsule, types Primula, Plantago. 
16. Osaritra, diff. Sphanidia or Rubiacea by fruit unilocular or monosperm, types Sambucus, Viburnum, Valeriana.

17. Cynaridia, diff. Flosculia by style articulated, flowers often irregular, types Cynarä, Echinops, Gundelia.

18. Sy nodra, diff. Codanthia by flowers aggregated with a phoranthe and perianthe, types Jasione, Opercularia.

19. Cactidia, The Cactus family and akin genera Mesembryanthus, Tetragonia, hardly distinguished except by fleshy substance, many petals, ovary adherent, fruit polysperm. But Ribesides and Myrtides, Begonia and Escalonia, are very near with a different habit.

20. VACCINIDIA, this small family of mine may become an order also, being very distinct from the next orders Campanulea and Cucurbitacea : by far more akin to Ribesides and Cactides only differing by the single style and corolla peripetal not staminiferous.

\section{EXOGENIA.}

21. Calicinia, diff. from Emprotia by a true perigone, types Dracontium, Orontium, Typha. LARNAGENEA.

22. 'Thalaria, diff. from Lichenia, by having a real stem called thallus, types Cladonia, Stereocaulon, Usnea.

23. Byssinia, the filamentase Fungides, types Byssus, Conoplea, Monilia.

24. Ceramidia, diff. from Confervides, fructification external, types Coramium, Ectospermia, Chara.

25. Stereopsia, diff. from Fucidia and the Ulvinia division by having nothing like stem nor frond, types Rivularia, Physidrum, Spongia.

These 2.) new orders added to my 66 of 1815 
would make about 90, and perhaps the whole may be stretched again to 100 very soon by insulating as orders rather, than families, the Spunges, Oaks, Laurines, Menispermes, Proteides, Hypericines, Coniferes, Gentianea, ofc. which will render the Natural Orders equal to those of Jussieu, but by far better disposed in a series, well distinguished and fixed by comparative characters.

I conclude by a complete tabular view of my serial Classes and Natural Orders.

\section{EXOGENIA.}

1. Polygia, \{1. Rhodanthia, type Rosa.

2. Senticosia, Spirea.

3. Perimesia, Sedum.

4. Isoteria, Annona.

$\{$ 5. Adnantheria, Peonia.

6. Gynopolia, Ranunculus.

$\{$ 7. Axanthia, Populus,

$$
\text { (Pinus? }
$$

8. Axepia, Ficus.

9. Monospermia, Rumex, (Protea?

10 Sarcodia, Amyris (Laurus.

11. Isandria, Ruta.

12. Calicandria, Lythrum.

13. Styridia, Draba.

14. Monostimia, Papaver.

15. Polarxia, Citrus,

\{16. Polymesia, Portulaca,

Reseda, Hypericum?

17. Epimesia, Euphorbia, Be-

\section{gonia.}

18. Isostimia, Dianthus.

2. Synandria, 19. Adelphidia, Geranium.

20. Omoplitia, Malva.

21. Perimonia, Melia,

(Tamarix? 
\{22. Cyteanthia, Pisum.

233. Lomentaria, Cassir.

3 Heterolda, $\{$ 24. Chasmanthia, Gevardia.

25. Monorimia, Gratiola.

\{26. Plasyrgia, Rhinanthus.

27. Pliopsanthia, Veronica.

28. Olispermia, Verbenu.

29. Carythia, Mentha.

30. Anisandria, Convolvulus.

31. Asclepidia, Asclepias.

5. Mesogia, 32. Lobogynia, Borrago.

33. Polymia, Cuscute,

\section{Carica?}

34. Darynia, Polemonium.

\{35. Arcythia, Solanum.

36. Distemia, Olea.

6 Eutrantilia $\{37$. Osaritia, Valeriana.

38. Sphanidia, Rubia.

39. Polyspia, C̈̈nchona.

7. Synantilia, 40. Cynaridia, Cynara.

41. Flosculia, Cacalia.

42. Actinanthia, Heliunthus.

43. Glossanthia, Lactuca.

8. Endogra, 44. Ascadia, Aralia.

45. Ombellifera, Daucus.

46. Polyandria, Pyrus,

(Quercus?

47. Dapsilitia, Myrtus.

48. Cactides, Cactus.

49. Acascotia, Nyssa.

50. Coryphitia, Asarum.

9. Nantiandria,51.Plyrontia, Vitis, Berberis?

Cocculus?

52. Viscoidia, Viscum.

53. Plaxarpia, Primula,

(Gentiana?

54. Stemisia, Achras. 
10. Aneperia, 55. Peritalia, Erica. 56. Vaccinium, Vaccinium.

55. Synodia, Jasione.

58. Codanthia, Campanula, 59. Idiogynia, Cucurbita.

II. ENDOGENIA.

11. Gynandria, 60. Cratodia, Musa.

61. Orchidia, Orchis, (Pistia?

12. Stegrnia, 62. Acinitia, Gethylis.

63. Synemia, Sisyrinchium.

64. Iridisia, Iridis.

65. Ymnodia, Narcissus.

13. Lirmia, 66, Liriantha, Lilium. 57. Gonotidia, Helonias. 68. Acinopsia, Trillium. 69. Polachenia, Alisma.

14. Spadrxia. 70. Pocilmia, Phonix (Pandanus?

\{7. Calicinia, Acorus.

72. Emprotia, Caladium.

I5. Guunosia. 73. Cortodia, Scirpus (Carex? 74. Achirosia, Panicum.

I6. Pteridia, 75. Diclipteria, Ophioglossum. 76. Dorsiferia, Adianthum

17 Phryganisia, 77. Aethogamia, Hypnum. 78. Hepatidia, Riccia.

III. LARNAGENIA,

I8. Lichenia, 79. Tremaria, Xyloma. 80. Phymaria, Lepraria. 81. Thallaria, Usnea.

19. Mrcosia, 82. Byssidia, Byssus. 83. Adelistia, Clavaria. 84. Phaneristia, Boletus. 85. Sarcosporia, Stictis. 86. Coniosporia, Mucor.

20. Algosia, 87. Hydronemia, Conferva. 


\section{Ceramidia. Ceramium, Chara. \\ 89. Fucidia, Ulva. \\ 90. Stereopsia, Spongia.}

Such then are now the Natural Orders to the best of our actual knowledge ; but each includes from I to $\mathbf{1 0}$ families or secondary groups quite as natural; each family from 1 to 50 genera ; each Genus from 1 to 50 species, seldom more : those of 100 or 200 species are small natural families. Such are Carex, Erica, Euphorbia, Vaccinium, Salix, Aster, Polygonum \&.c. long ago properly divided by myself or others: like Conferva, Lichen, Agaricus, Cactus \&.c. now become families, orders or even classes!

But this Serial Order interrupts or conceals many mutual affinities, since they are not akin to the two Orders only, before and after in the series; but also to many removed in the series. 'Thus the following are closely allied altho' far removed, as Nations may have colonies or be allied altho' distant on the Earth.

The Gynopolia $\mathbf{6}$ and Polachenia 70.

Cactides 48 with Idiogyna $\mathbf{5 9 .}$

Gynaridia 40 with Synodia 57.

Dapsilitia 47 with Vaccinidia 56.

Darinia 34 with Peritalia or Bicornes 55.

Isandria 11 with Adelphidia 19.

Axanthia 7 with Emprotia 72 \&c.-And whenever a natural table or map or the whole will be given, they must be approximated or connected by some means.

Various modes may be devised to elucidate these affinities.

1. A botanical inap on a graphical plan would best represent them by contiguity; a second mode would be by a kind of net work, $3 \mathrm{~d}$ by a 
kind of genealogical tree, 4 th by a triple concentric cercle, divided in rays, the inner circle being the cellular plants, the outer or largest the Exogenous plants and trees, 5th by a triple series or 3 columns one for each great series, with lines accross to connect affinities.

Few of these excellent modes of elucidation have been employed as yet, the best in use are the Analytical and Synthetical tables; but all the serial modes whether circular or linear or triple, are all inadequate and imperfect. This explanation was needful as an apology for having used this vulgar mode even here; but I had made a complete Botanical map as early as 1815. Such botanical illustrations may exercise the ingenuity of Botanists well skilled in botanical affinities; which are the highest results of phytographical knowledge, as stated by Jussieu - Their relative value, importance and permanency, must equaly be ascertained; and thereby we invariably fix the botanical groups, whatever be the names we may apply to them, or place we may allow them.

'The general aspect and organs of plants must also be studied and attended to, more than usual at least; and experienced botanists can often detect or conjecture the natural Classes and Orders of many plants without flowers by such means, called the habit or general form. But as this indication is liable to many exceptions and anomalies, it must always give way to the floral certitude.

Meantime I venture to propose the following arrangement, which $I$ have devised, and found very useful in practice; as it will be indispensable in the study of fossil Botany, or the primitive forms of vegetable organization. 


\section{Series-EXOGENES.}

I. Class. Driopses or Trees. Woody concentric leafy and branched, roots similar. Wood hard. Shrubs are only small trees, and branches of trees are shrubs. 3 Vines are climbing trees or shrubs. 4 Bushes are the smallest or weak shrubs commonly cespitose or with many stems 'The orders may be.

1. Polones, (many frame) Branches, leaves and often flowers assuming a compound form either vertical or horizontal.- Synophyles, leaves compound with articulated folioles. Polytomes leaves united with folioles not articulated. These are again divided in bipinnate, pinnate, decompound \&c.

2. Trocnomes, (wheeled) Branches, leaves and often flowers verticillate around an axis, or terminal-Rotules around stem or axis. Umbellites terminal wheel or umbel.

3. Diplones, (doubled) Br. leaves and fl, opposite to each other often jointed-Disticates, in two rows, Decussates in four rows crosswise.

4. Spiroues, (Spiraly) Br. leaves and fi. in a spiral position around the stem or axis-Disticales, spiral by 3. Alternutes, spiral by 4, Pentales spiral by 5 . Densales spiral by more than $\mathbf{5}$ and usually crowded imbricate. These are seldom jointed.

II. Class. Malaxyles or Anomalous Trees. Wood hardly concentric, with few branches, often articulate or no leaves: wood soft and cellular ; roots similar-Very singular arboreal form hardly noticed as yet offered by Carica, Ephedra, Ficus, Piper, and other tropical trees, 5. Sicomes, Trees, with leaves and branches, 
sometimes simple stem, wood often spnngy, Ficus, Carica, Adansonia? Pavia?

6. Piperones, Leafy vines, or jointed stem, leaves alternate, Piper.

7. Viscones, Stem articulated, opposite leaves, Viscum.

8. Ephenrones, Stem articulate, no leaves, Ephedra, Casuarina.

III. Class. Sarcomes. Perennial fleshy plants not woody, but thick or juicy, seldom hardened to soft wood (like Opuntia) but permanent anomalous forms, seldom a stem and leaves, vessels hardly concentric. Roots fleshy, dissimilar scanty.

9. Phorbones, Massive unblanched not articulated, Euphorbia.

10. Stapelomes. Massive branchëd articulate Stapelia, Salicornia.

11. Cастомes, Jointed, branching or with fleshy leaves, Cactoides.

IV. Cass. Perennials or true plants. Root and Stem dissimilar, Root perennial woody or tuberose or fleshy concentric. Stems annual, concentric or hollow, with leaves or scales. In fact here the roots are subterranean small trees or sarcomes, and the stems mere annual shoots or branches; thus they may be subdivided like Trees into

12 Polomes with compound foliage, Lathyrus.

13 Trochomes with verticillate foliage, often joints, Rubia, Frasera.

14 Diplomes with opposite foliage, often joints, Silene, Salvia.

15 Spiromes with spiral foliage, Borrago, Hesperis.

V. Class. Scapigeres or Scapose perennials, 
They differ from true stemy or caulescent plants by the roots being crowned by radical leaves and sending forth a leafless stem called scape, which is merely a peduncle bearing the flowers. They blend often with them, and with annuals.

16. Cladomes, with branched or umbellate Scape, Primula, Statice.

17. Scapomes, with a simple scape, Plantago, Dionea.

VI. Class, Botanes or Herbs, Annuals. Root dissimilar, but slender, fibrose, annual or biennial ; sending forth only once a stem, bearing flowers, with or without leaves-In fict Plants and Herbs are hardly distinguished by usage, yet easily known by their small roots: they blend by biennial roots, and have sometimes scapose stems. Hence the Orders must be different, since here ends the woody or hard stems or roots.

18. Dinomes, commonly biennial root, radical leaves, and stem leaves different, Verbascum.

19. Stemilomes, Stem leafy, Papaver.

20. Rhizilomes, Root leafy or crowned, scape leafless, Erophila.

21. Aphilomes, No leaves, Cirscuta, \&c. 2d Series, ENDOGENES.

VII. Stelmians or crowned 'T'rees. Stem cylindrical seldom branched, with spungy wood, crowned with leaves on the top ; roots dissimilar.

22. Palms, Leaves flabellate or pinnate, flowers central.

23. Aplostelmians, Crown of simple leaves, flowers central, Yuca, Drucena. The Lily trees.

21. Pterostelmians, the Fern trees, Crown of leaves bearing the fructification. 
25. Spathelmians, Crown of leaves, and corols or spathas bearing the fructification, Aroid-trees, P'andamus.

VIII. Class Rinzonians, Stem concealed under ground, assuming the shape of a root often horizontal, sending forth annual shoots, leaves and flowers; roots fibrose dissimilar.

26. Aloidians, Roots crowned with leaves, stem often leafless, Aloes,-Iridians with few lateral leaves.

27. Paridians. Roots not crowned, a leafy stem crowned or whecled with leaves. Paris.

IX. Class, Bubbosins, the Bulbose plants and Lilies, with a thick perennial bulbose stem under ground, formed of concentric scales or coats, increasing from within, sending annual shoots, leaves and flowers from the centre. Roots fibrose dissimilar.

28. Liviuns, a leafy stem. Lilium.

29. Criniaus, radical crown, scape leafless, Crinem, Skilla.

$X$. Class Orcumbins, Fleshy roots or stems under ground or above ground, simple or divided, perennial; sending yearly lateral shoots, stems or scapes sometimes articulated.

30. Satyrians, roots double or tuberose, under ground.

31. Cladorians, roots branched thick under ground.

32. Georlizians, roots simple or agregate above ground.

XI. Class, Culminins, roots or stems jointed hollow, either perennial or annual, with leaves or scales, roots slender.

33. Equisetians, stems jointed, with verticillate leaves or scales. Equisetum.

34. Graminiams, stems jointed with alter- 
nate sheathing, leaves or seales. 'The true Grasses.

35. Alismians, stems jointed, leafless, or leaves radical. Alismu.

XII. Class, Plaroniaxs, stems not jointed, solid or thick, but of various forms, roots perennial dissinilar.

36. Smilacians, stems hard perennial, leafy by expansions, often bearing flowers, Ruscus, Similax.

37. Heloniuns, stems soft annual, roots perennial, thick or tuberose ; leaves expansive, Dioscorea, Helonias, Asphodelus.

38. Hydnorians, Leafless, stemless only roots and flowers. Aphyteia, Rafflesia.

XIII. Class, Filixiavs, stem rhizomian, under or above ground, leaves crowning this radical stem bearing fiuits. The Ferns.

39. Dorsiferians, true Ferns, leaves bearing fruits on the back.

10. Radiciferians, roots bearing fruits between the leaves, or on pedundes.

XIV. Class, Muscidins, the Mosses, roots small creeping, stem bearing leaves or scales persistent, or membranose frondose.

41. Phylosians, leaves or scales, Mosses, Lycopodians.

42. Frondulians, no real leaves, a winged or expanded membranose stem. Hepaticans.

III. Series LARNAGENES.

XV. Class, Licmens. Expanded crust with scutelas or tubercles.

43. Scutellites-Scutellas.

44. Phymites-Tubercles.

XVI. Class, Neurtes, Filamentose bodies.

45. Usnites, Scutellas, no joints, solid. 
16. Cercmites, joints, and grains outside, as in Chara.

47. Confervites, joints, and grains inside, tubular.

48. Byssites, no joints, nor scutellas, solid.

XVII. Class, Fungrtes, form variable, fungiform, fleshy or fungose or suberose, colored, with or without a stipes or support.

49. Monilites, strings of grains, or granular clusters.

50. Endosporites, grains inside or in the substance.

51. Clavites, no grains nor extraneous appendages.

52. Hydnites, with points, bristles or warts.

53. Boletides, with pores or tubes.

54. Agarites, with gills, rays or veins.

XVIII. Class, Augites. Green bodies, frondose or tubular, simple or ramose, with or without a stem.

55. Pilites, with a stem and head or bulb.

56. Stemites, with a stem and leaves.

57. Fucites, Leafless, various, solid or tubular.

58. Ulvites, membranose or frondose, complanate.

59. Physidrites, Vesicles full of water, closed.

60. Spungites, spungy celular, or open vesicles.

These Orders might be divided into Genera by the inflorescence, and be greatly increased if more minute forms are taken into account. They coincide very nearly with the Natural Orders in the lower series, and afford in the upper series a very striking additional mean of knowing Orders and Genera; by far more useful in practice than the hidden modes of germination, and embryonic forıns. 
But I am fully aware that any methodical division of the Habit of Plants is liable at present to many objections. Some Botanists may perhaps deem foliage more important than stems or roots. Inflorescence is no doubt more important than either; but belongs to floral Classification, or may afford the generic groups in fossil botany. The study of Roots is just beginning, they had long been overlooked, and have not yet found a Gaertner; but it is now admitted that many presumed roots are real stems.

Some of my views and suggestions on the subject are nearly new; my Classes of Sarcomes, Rhizomians and Nemites are quite so, with many of my Orders. My Malaxyles and Piaromians deserve attention and to be better studied. The soft texture of many trees, and the expansive floriferous leaves of Ruscus, Phyllanthus \&c. appear to connect with the Cactoides, and to indicate another great organic Series of Vegetation, different from the $\mathbf{3}$ admitted ; to be called perhaps SARCOGENES, wherein vessels and fibres are drownded in a fleshy tissue. 'These are susceptible of becoming lignose by induration or dessication, as it happens in Opuntia and thus to form trees.

'The joints and knots of stems indicate also a peculiar organization or process of evolution; all the opposite leaves appear to indicate it even when not conspicuous. Many leaves and flowers are evidently articulated to their support, as are all the buds of our northern trees. It is so in the Coniferes, Polygones, Caryophyles, Ombelliferes, Leguminoses, Sedoides, for stems or leaves, and all the florets of composite flowers. While all the spiral leaves, or drying on the stem, appear to be mere continuous appendages 
of it, grown by expansion and not super-addiion.

'I'his view of physiological Botany might suggest again another arrangement based thereon; and is perhaps susceptible of being improved and made available. At present I merely throw these hints, and invite the attention of Botanists to the study of these forms of growth. To fix them still better, I will give a short table of such supposed modifications of growth, in $\mathbf{4}$ Organic Series.

EXOGENES-1. 'Trees or plants with joints or articulations, growing by addition of parts. 'Tomogenes, foliose, aphyle.

2 , Trees or plants of a simple continuous form, growing by expansion of parts. Aplogenes foliose, aphyle.

SARCOGENES-Fleshy trees or plants. 3 Tonogenes, foliose, aphyle.

4. Aplogenes. foliose, aphyle.

ENDOGENES-5 'Touogenes, Equisetians Orchidians, Culmidians.

6. Stelmogenes, Stelmians, Rhizomians, Bulbosians, Filixians. All Aplogenes, growth foliose, terminal crowning.

7. Synogenes, Very simple aplogenes, growth by shoots with false leaves or frondose expansions quite persistent. Muscidians, Frondulians.

LARNAGENES.-Sipiogenes, growth by tubular partitions, or agregated grains, vesicles \&c. Confervites, Ceramites, Monilites, Physidrites.

9. Condrogenes, with fronde or membrane or crust expanded and lobed, Lichens, Ulvites, Fucites, Usnites, Podospermes?

10. Mrcogenes, with pileus or head distinct 
often with rays or pores or papillas. Pilites, Agarites, Boletides, Hydnites.

11. Unigenes, simple solid bodies, Byssites, Clavites.

12. Corlogenes, simple hollow bodies, with cavities, Endosporites, Spungites.

'The difficulties or exceptions to this general arrangement are very few, chiefly arising from some plants not yet well observed or quite anomalous: Carica, for instance which has the wood and habit of Palms, with the floral organs nearly like Solamem! and which is probably a Scarcogene tree. Stated by Hooker to bear fertile seeds without fecundation, as does Morus, and Vitis sometimes, and to afford Fibrine like Animal Flesh, and some Mushrooms. Also Podostemon with the structure and habit of Fucus, but floral organs like Hippuris and Ruppia, is it also a Scarcogene plant? 'These and the Cactides, Euphorbias, Stapelias \&c. evidently indicate one or even two New Series of vegetable evolutions, distinct from those admitted since the discovery of Desfontaine. I claim therefore the merit of having first noticed and indicated those new vegetable forms which I believe had been overlooked by all Botanists as an organic Series, altho' long noticed as anomalies.

I invite Botanists to watch their germination and evolution, so as to ascertain their morke of increment. My own observations lead me to believe that it is expansive in Podostemon, Carica, \&c. but partly peripheric in Euphorbia aud some Cactides, while in Opuntia and Stapelia it is evidently articulated and gemmular as in Epleedru and budding trees. But their wood is totally unlike that of Oaks or concen- 
tric trees, nearer to the fascicular wood of Palms, and yet different. In Carica it has been called fungose by Jussieu; it is rather suberose like cork, and cellular like a spunge, without rings. In Opuntia the fleshy articulations change gradualy in a woody mass of fasciculate fibres as in Palms, but softer still, more cellular and never hard nor concentric.

All the trees with soft wood, often as light as cork, and unfit to burn! deserve also to be examined again, such as Ficus, Aesculus, Puvia, Adansonia, Rhizophora, \&c. in order to trace their analogies of evolution and increment in a soft state. 'Their anatomical structure requires a new examination. 'They are deemed Dicotyle, as well as Carica and Cactus; but this seminal character is fallacious, and not so important as stated by Jussieu; since Cycas and Juncus are said to be dicotyle also, and the Coniferes are polycotyle: while Nelumbium is anisocotyle or nearly monocotyle, altho' united with Nympheacea or Ranunculacea. Perhaps it is, as well as Nymphea, of the Rhizomian Class; since their roots are evidently rhizomes, and the structure is hardly concentric. 'The aquatic ferns and some other aquatic Genera appear to have for seeds mere Embryos or radicles called Somorhizes by Nuttal in Ruppia. 'They are evidently acotyle therefore. Some plants deemed Vascular, hardly show any vessels but cells, Monotropa and Orobanche have such annual fleshy stems. These and other anomalous plants will offer a fine scope for observations, dissections, and careful investigations. 


\section{THE FIFTY RULES OF GENERIC}

\section{NOMENCLA'TURE,}

\section{By Linneus and Rafinesque, 1814.}

These rules were chiefly established by Linneus in his philosophia botanica. In 18141 gave their principles with additions and remarks, applying them also to Animals, and to Classes, Orders and Species. I shall now add here their main features and applications, recomending them to the attention of the accurate botanists : the Linneists ought at least to deem them peremptory, yet they often violate them.

1. All the species united by some essential definite characters must form a Genus. Lin. phil. bot. 210.

2. Each Genus must have a name, Lin. 218. Anonymous Genera like those of Walter are deficient and inadmissible.

3. None but skilful Botanists ought to form and name Genera.

4. All the plants possessing similar characters must form a Genus, and bear the same name. Lin. 215, 216.

5. All the Species with generic disparities must form different Genera and bear different names. Lin. 214.

6. Therefore when one or several species differ essentialy from their typical Genus, they must be separated and bear other names. This applies to the Genera Valeriana, Justicia, Convolvulus, Polygala, \&oc. and similar anomalous groups.

7. If a Genus has been made upon erroneous characters, it must be annulled, and united to the Genus that bears the real character. Ex- 
amples Sheffielda which is a Samolus, Hecatonia is Adonis, of c.

8. As soon as a good name is given to a Genus, it must never be changed. Lin. 219.

9. Not even for a better one, or another more convenient. Lin. 245.

10. Names must not be transfered from a Genus to another on any account of convenience, becoming a double change. Lin. 245 .

11, If similar names are given to two or several Genera; this name must be left to the first in date, and the later must be changed. Lin. 21\%. Apply this to Aubletia, Persoonia, Heritiera, \&c.

12. If several good names are given to a Genus, the first in date must prevail, and the others be dismissed. Example. Artocarpus of Forster which has prevailed over Sitodium, Polyphema and Rademachera.

13. But when of two names, the first is improper, the second in date if good must prevail. Example Pyrularia Mx. dismissed for Hamiltonia W.

14. If two names are given the same year, the best must prevail, even if later by some months. Ex. Polypogon Desf. prefered to Santia of Savi.

15. Generic names must have a meaning or derivation, even if obscure. L. 220. But names must not be changed on that mere score, and thus Linneus kept Bryonia, Acalypha, \&c.

16. Generic names contrasting or at variance with some Species of the Genus, must be avoided. Lin. 232. Ex. Gratiola, Cyanella.

17. Synonymic names in greek and latin must be avoided, but not changed. Ex. Stellaria and Asterias; but Aster is worse. 
18. If a new Genus to be established has a convenient specific Synonyme, it must be adopted for the Genus.

19. If a Genus is to be divided, the old name must be left to the majority or best known species.

20. Generic names must always be substantive and never adjective. Lin. 221. Thus Gloriosa and Mirabilis were changed by Jussieu to Methonica and Nyctago.

21. Generic names must always be simple, never double nor triple. Lin. 222.

22. 'They must be singular, never plural, but may be masculine, feminine or neutral.

23. A specific name changed to generic name becomes substantive. Ex. Agrimonia eupatori$u m$ and Eupatorium.

24. The name of a Class, Order or family must never become Generic. unless properly modified. Lin. 212, 233. Pteris which means Fern must be changed to Peripteris or Pterilis.

25. The generic names of animals must not be applied to Plants, nor viceversa. Lin. 230. Taxus animal must be IIelesius. Lencosia plant Leucipus, being posterior to Leucosia animal.

26. Names of minerals must be avoided in Botany, but a few adopted may be tolerated. Ex. Hyacinthus, Plumbago, Heliotropium.

27. Names derived from arts and sciences, or religious ideas, can only be admitted when properly modified. Ex. Jacobea, Angelica, Vulneraria, Ternatea, \&c. are tolerable names; but Cotyledon, Baca, Baltimora, ought to be changed in Cotylaria, Cleterus, Baltimorea.

28. The best names are derived from the greek and from striking habit and characters or 
generic peculiarities. Lin. 222. Ex. Cepkalanthus, Rhizophora. Eriophorum, foc.

29. Those derived from the latin in the same way are next; but the mongrel names derived from the two mixt languages are inadmissable. Lin. 223. Cornucopia, Cimicifuga, Baccaurea are wholy latin; but Vinceioxicum, Scyphofilix are mongrel, and must be changed to Gonolobus, Scyphopteris.

30. Generic names may be made by abreviations, contractions, elongations or blending one, two or three words; but never from a single word without alteration. Therefore Chelone $\mathrm{L}$ meaning turtle must be changed in Chlonanthus, and Chelone Latr. in Chelonias. Chlora L in Chlorita, and Chloris W. in Chlorostis \&c. but Crassulu, Salsola, Clypeola are good.

31. Biended names must not be formed from two other Genera united L. 224. Malvaviscus and Calamarrostis are absurd, and must be changed to Acharia and Amagris.

32. Doubtful or equivocal names must be excluded, particularly when made by adding one or two syllables before or behind another name. Lin. 225. Homalo-cenchrus, Hippo-crepis, Calam-agrostis, foc. are bad, Leersia, Hippocris, Amagris have been substituted.

33. The same for names made from others by substracting one or more syllables before or behind. Linneus fell in this error sometimes, Ex. Bromelia, Bumelia, Melia, BromusSinapis, Apis. Of these the shortest are the worst.

34. But names casualy with one or more added letters in the middle, may be tolerated.

35. And also when the termination changes as 
well as the first letters, Ex. Chimaris, Marica, Narica, Mariscus.

36. Generic names made by a mere modification of terminations, are inadmissible, and all those made by adding oides, ella, ola, ilus, i $\iota$, ium, aria, ea, ata, ita, astrum, ago, formis, opsis, emum, \&c. must be changed. Lin. 226, 22\%. This useful linnean rule has been often neglected, producing a crowd of equivocal names to be rejected: such as Polygonella, Centanrella, Cicutaria, Portulacaria, Linaria, Helianthemum, Oryzopsis, \&c.

37. Equivocal names pronounced nearly alike must be avoided; but need not be changed, unless the difference is only in termination. Lin. 228. Ex. Mitella, Mitchella, Michelia, are bad names on that account, but the following still worse. 1 Apis L, 2 Apus Cuvier, 3 Apios, Pursh, 4 Apium 5 Apion, Herbst, might be changed to 1 Apicula, 2 Apodium, 3 Gronancylis, 4 Apium, 5 Apionus.-Zea same as Zeus I make Mrayzea. - Scolopendra,Scolopendrium this last must become Glossopteris or Phyllitis -Delphinus and Delphinium or rather Plothirium \&.c.

38. We must avoid to give barbarous names to Genera, Lin. 229, but we may sometimes admit Arabic,Celtic, Indian, African or American names, when not too harsh, and easily latinized. Linneus did so for Yucca, Datura, Ribes \&c. -Pacurina, Palovea are much better than Messerschmidia, Schwenlifelda.

39. Foreign names must be latinized, softened or receive a proper termination. Lin. 248. Thus we must write Calesia, Areca, Cupuya, instead of Calesjam, Arec, Coupoui \&c.

40. Names too long must be abreviated, and 
when too short, must be lengtliened, even when personal. Lin. 249. The best names ought to have 2 to 5 syllables, those of one or six are objectionable. 'Ihus Krascheninikofia and Mesembryanthemum, must be changed to Kranikovia and Mesenbryanthus.-While Lea, Neea, Zea must become Leania, Neeania, Mayzea. Linneus changed Anapodophyllum into Podophylum.

41. Harsh and uncouth names must be softened and modified, upon the same plan. Tournefort changed Gundelsheimera into Gundelia. Tabernamontana and Lightfootia must become Tabernaria and Lifutia.

42. Some of the best botanical names are patronymic, or dedicated to botanists. But instead of being the reward of merit, this practice has been too much extended, by dedicating them to mere Amateurs and Catalogue makers.

43. These patronymic names must not be formed of two names; Gomortega and Carludovica are become Adenostemum, Ludovia.

44. We may dedicate Genera also to eminent Naturalists or Philosophers, great Travellers, Horticulturalists and Protectors of Botany; but never to mere friends or by flattery. Ex. Furcroya, Virgilia, Comptonia, Solomonia, Cliffortia \&.c.

45. Names may also be borrowed from my thology or ancient poetry, dedicated to Nymphs or Legislators, Heros \&c. Ex. Arethusa, Chironia, Artemisia, Euphorbia,Calypso \&c.

46. Ancient names of plants may be applied to New Genera, nearly alike or related. Ex. Datisca, Adike, Selepsion, Verbena \&oc.

47. Genera must not bear the names of their organs; but flowers and fruits may bear the 
same name. Bacca, Cotyledon were bad; Rosa, Tulipa, Prunus are good, Necker erred in changing them to Rhodophora, Prunophora.

48. 'The pronunciation of Genera must be as in latin, the Greek or Foreign names must be pronounced as in latin or italian. 'This applies to the vowels $\mathbf{A}, \mathbf{E}, \mathbf{I}, \mathbf{O}, \mathbf{U}$, which are distorted by english botanists. $P h$ is meant for $\mathbf{F}$, and CH commonly for K. Acacia which is pronounced Acasia like Cassia must be spelt Acakiu as in Greek.

49. Names must be changed if they disagree with one of these rules, unless it be of little Consequence; but when they disagree with several they must be altered by all means.

50. As soon as a Generic name is changed or modified according to these essential rules, so as to be correct ; this correction must be adopted, and no longer liable to changes.

Such are the correct principles and rules of generic nomenclature, not yet generaly known nor attended to by all the Botanists. Those who neglect them evince a deficiency of taste, learning and acuracy. They will be the test of correct and accurate Botanists. Those who follow them will thereby evince that they know end admit the necessity of fixed principles in Names as well as Genera.

It is to be regreted that Decandole himself, in the hurry of his great labors on Species, has given sanction to admit some very improper names; but no doubt either he or his future continuators will see the propriety to dismiss them.

At present the greatest source of generic perplexity arises from the great number of improvers, laboring and writing unknown to each other, 
and in remote places; where they happen occasionally to clash by applying similar names to distinct Genera, either discovered or reformed : While they may also give different names to a single Genus.

Else we may have double and triple names for similar Genera, as was the case with those of Aublet, changed by Necker and Schreber. It is not yet perfectly settled which are to prevail, many of Aublet were barbarous; but others quite good and easily latinized. This difficulty will always occur, unless we stand by the rule of always admiting the previous name, if good or even tolerable.

The names of some Botanists happen sometimes to be given to several Genera, because many wish to reward their labors: while others are left unrequitted for years. I was very prone to dedicate such Genera; but now always add a second name as a substitute in case of previous application.

There have been for instance, 4 Genera called Scopolia, the first in time of Jaquin was found to be a Hyosciamus. The second of Linneus jun. has since been united to Daphne, altho' it has capitate flowers with a perianthe or involucre, and must at least form a subgenus. Forster made the $3 \mathrm{~d}$, but deeming since the $2 \mathrm{~d}$ good, he changed his to Griselinia. The 4th was made by Smith, by changing the good name Todalia of Jussieu; a very improper change, yet imitated by Schreber who made it Crantzia, but there was a previous Crantzia of Swartz! Todalia is better than Coffea of Linneus and must be preserved as previous. Thus Scopolia has been multiplied and made doubtful, unless we restore it to the $2 \mathrm{~d}$ as a genus or subgenus. 
This is an instance of the difficulties occurring in generic nomenclature : similar cases abound, and become complicated by the different views taken of the matter by the various botanists. Thus Centerurella a bad name of Michaux, made worse still by Persoon Centaurium, both derived from Centaurea, was changed to Bartonia by Wildennw; but some botanists dissented and made another Bartonia, while Andrewsia was proposed for the first Bartonia; but there was another Andrewsia? then Nuttalia was soon after proposed; which is not deemed a genus, but a mere subgenus of Sida, yet it has been called Calirhoe also, which is a name already applied to a genus of animals, and the 2d Bartonia was made a Torrcya! while there are two other Torreyas! What a heap of ambiguities! yet they could be avoided by attending to the generic rules confirming the first Bartonia of Wild. and my Nuttallia of 1817 for the second Bartonia.

Therefore it is obvious that correct Nomenclature is an art based in scientific principles, and that all dubious or deffective names arise from neglect of such rules of this branch of Botany. Thence we may distinguish 3 series of generic names-

1. Goon Naves, including excellent names, Defining Names, Appropriate-Dedicated-Accurate-Early greek-Ancient-Compounded-Easy Names \&c.

2. Indifferent Naures including unmeaning Names, Anomalous-Contradictory-Transfered-Equivocal-Latinized----Synonymic--Mineralogical-Anagramic-Religious-Geographical-Mythological Names \&c.

3. BAD Names including Erroneous Names, 
Anonymous-Barbarous-Harsh-Sesquipedal Syllabic-Double-Multiple-Adjective-Zoo. loģical -Organic-Blended-Doubtful-preoc. cupied-Modified-Relative names \&c.

If the 2nd series may be tolerated, these bad Names cannot; they must be abolished and changed.

Many of these erroneuus Genera and Names, are not only deffective, but absurd; yet to these some incorrect Botanists appear to cling with tenacity.

They will form a test of accuracy : whoever admits them, declares himself a friend of Erroneous Botany and Nomenclature.

As to names neither good nor bad of the second Series, some latitude and disparity of opinions may exist. Most of them when once established may be tolerated; but ought never to be imitated.

\section{CONCLUSION \& NA'TURAL GENERA.}

The object of these preliminary remarks was not to enter any further into generic, anatomical or physiological researches. My main object is to reform and improve Genera : in the course of my hints and elucidations of all the natural clusters, I have been led to convey the result of my observations, both on their floral and organic structure. To enter into explanations and details wou'd swell these remarks bey ond my proposed limits; but I hope to have thrown the seeds of some future discoveries, and further improvements.

I chiefly insist upon the practical rectification of Genera. I blush for the science and my fellow botanists, when I see yet a crowd of arbitrary and perplexing Genera, quite as loose and 
incorrect as those of Tournefort or Lichen of Linneus, not only in use, but insisted upon as correct, by Botanists neither blind nor unskilful; but who shut their eyes and ears, so as to discard the use of their senses-For instance in Euphorbia, Veronica, Saxifraga, Vaccinium, Andromeda, and 200 snch limnean Genera, which are strange medleys of blunders, ambiguities, and absurdities.

If they admit the mistaken and absurd rule that a wrong Genus must give the character, they ought at least to take it from all the strange plants they mix together, and the character of Saxifrian should be Calix inferior or Superior, equal or unequal, with 5 to 10 parts, Petals 5 equal or unequal, with glands or no glands, with claws and nerves or none. Stamens 5 or 10 or 20, equal or unequal, flat or filiform, fertile or sterile, Ovary inferior or superior with glands or no glands, 2 Styles or 3 to 6 or no Styles, Capsule bifid or bicorne or 2, 3, 4, 5, 6, Capsules, naked or crownerl or covered ....! At which rate and with many .. OR .. OR - we can make a single Genus of Roses and Cabbages! and without any OR, a single Genus of all the Cruciferes.

The fact is they are ashamed to give the real characters of such Genera, and discarding the linnean rule, give us instead a paltry unmeaning character that does not apply to all the Sp.

Linneus did the same, and that is their apology. And so did the old Botanists before Linneus. He himself has broken occasionaly every one of his own rules, either by oversight or by the difficulty of discarding all errors at once. Even his golden rule of two names only for each plant; since he had some with three-Aspleni- 
um ruta muraria and Pedicularis Sceptrum carolinum, for instance!- $\mathrm{All}$ kinds of barbarous names Coffeca, Piper, Patetta, Yucca \&cMany derived names as Passerina from Passer -Galium and Allium-Arum, Asarum and Comarum \&c. altho' he had abolished all such.

1 do not mean by this to imply that Coffea and Yucea are bad names. 'They are quite as good as some harsh greek names. And many greek and latin names came from the Celtic and Oriental Languages. Kroscheniliofia, Lightfoutia, Mesembryanthenum are certainly much worse, and must be softened or latinized into Krasnikovia, Lifutia, Mesembrirenthus.

But I mean to say that his oversights are not to mislead us, and that mistaken Genera, whether in fact or names, either of Linneus, Jussieu or even Decandole, (since he has some also) or of Hooker, Lindley ofc are not to be adopted; but must be corrected; even now, if so long overlooked by neglect.

All such arbitrary and absurd Genera must then be revised and reformed-Such is my aim - Any one who may disapprove of this plan, must be blinded by prejudice or inveterate love of errors.

Decandole has done much, but only reached one fourth of Vegetation, and overlooked many forms, or slightly noticed them, owing to the immense labor he undertook on all the specir .

A fair example of blunders by a Linneist, - may be seen in Loureiro flora Cochinchinensis, where he has referred a crowd of new plants and new genera, to linnean objects, quite different; yet he was commended and reprinted by Wildenow, and has been quoted by Smith, Lamark foc, without detecting his blunders. 
Another instance, more recent still, is met in the labors of Hooker, a clever botanist, once a Linneist, but now trying to adopt the natural method; wherein he brings the defects and absurdities of Iinneus. I have detected a crowd of generic and specific mistakes in lis Flora Boreali Americana, and in the new series of the Botanical Magazine written by him, he falls into the most palpable errors as to genera and Species. In his Sth volume alone there are $\mathbf{3 0}$ plants out of 90 reduced to wrong genera.

If such is yet the actual course of eminent Botanists, the copists and compiler's follow the same tract ; even my old friend 'Torrey in America, deems that to follow or creep on their steps is quite needful, whatever be the glaring blunders they may commit; the fear of innovation, and of improving the Science too quickly is the prevailing fecling.

For my part I entertain no such fear, and should wish to see Botany reaching a greater perfection in my days; but if it is a hopeless wish, I will at least do something towards it.

Whoever will imitate my zeal, must attend with care to the intrinsic value of organic characters, and their relative importance, in Orders, Genera and Species. This is the test of a real follower of natural affinities, bases of the Natural system of Botany. 'T'heir neglect is the sure evidence of arbitrary Botany.

This knowledge teaches us that the importance of floral organs stands in the following. order, in Phenogamous plants.

1. Pistil and fruit-whether free or coalescent, simple or multiple, definite or indefinite, with one or several stigmas, with or without style, \&c. 
2. Stamens-where inserted, if free or coalescent with a corolla, a calyx, alterne or opposite to them, or united together, with filaments or none, with anthers with $1,2,3$ or 4 cellsas to number, if definite or indefinite, isarine or equal to perigone, heterine with less or more, equal or unequal between themselves, \&c.

3. Perigone or floral covering-If simple or double or multiple, free or coalescent with the pistil, if equal or umequal, with or without corolla or inner perigone, whether simple and lateral, or with many pericentric petals in one or two rows; or if with a peripetalic corolla, its shape, lobes, persistence, $\mathcal{E}$.

4. 'The fruits and seeds-Their nature, form, number and structure, cells, partitions, placentas, seeds, arillas, embryos.

5. Accessory parts--Glands, nectaries, scales, appendages, crowns, spurs, and other auxiliary additions to the flowers; lastly bracts, in all their forms and names of Involucre, spatha, perianthe, pericline, glume, palea, lepigone, anthophyle, \&c. besides the various receptacles or supports, phoranthe, gynophore, spadix, column, \&c.

It is very important neither to invert this Order of values, nor to ascribe more power to any than realy can be ascertained. If Botanists would attend to this, they should never join together in the same Genus or family, the plants that offer a difference in the most essential characters. Such as Pistils single or many, free or coalescent, superior and inferior of Linneus-Stamens free or united, equal or unequal, few or many, \&c.

They should know that Bracts stand at the very end of the floral series, and altho' useful to 
distinguish Genera when assuming the shapes of Perianthe or common calix, phoranthe or common receptacle, involucres of Ombellifera, Glumes of Grasses, \&c. they never ought to be employed alone to fix families and Orders.

'They ought to know that when there are no other essential Organs than Pistils and Stamens as in Grasses, except the mere bracts called Glumes, these must give way to the first; and their number becomes then of paramount importauce, in defect of additional organs. 'Therefore $\mathbf{J}$ ussieu was very right to divide the grasses in first instance by the numbers of Styles and Stamens: while the modern Botanists who unite in the same Genus Grasses, with $1,2,3$ or many stamens, are utterly in the wrong. 'They even sin against Linneus who divided Cinna, Anthoxanthum, Oryza, \&.c. 'To unite in the single Genus, Carex, plants with 2 or 3 stigmas or styles is still worse; and not to perceive that such a Genus of 300 Species is a fine Nat. family with many Genera distinguished by this and the seminal covering, proves that the absurd linnean principles evinced in the Genus Lichen now a Class! prevail as yet among us. Whoever preserves Carex entire ought to keep $\mathbf{L}$ chen and Agaricus entire, and make a single Genus of Ombellifera.

I conclude by asserting that GENERA ARE NA'TURAL, and that every actual Genus that is unnatural, arbitrary or polymorphous, IS NOT A GENUS, but an incorrect and artificial aggregation of aliens! Wherefore all Genera must be reformed till we reach these natural clusters of species quite alike in floral organs.

When in the course of time this truth shall be acknowledged, and it shall be wondered how 
we could have put together in a single Genus all the Lichens, all the Bats, all the Monkeys, all the Heaths, $\mathbb{S}$. let it be remembered that I had duly rectified 500 such preposterous or artificial Genera between 1800 and 1815 , and now write this synopsis to evince the fact, and propose or complete the needful reform of all such perplexing and obsolete Genera. 'They are for instarce:

$\begin{array}{lll}\text { Euphorlia, } & \text { Erigcron, } & \text { Saxifiaga, } \\ \text { Veronica, } & \text { Inula, } & \text { Orobanche, } \\ \text { Salvia, } & \text { Iythrum, } & \text { Aristolochia, } \\ \text { Gentiana, } & \text { Passiflora, } & \text { Erica, } \\ \text { Vaccinium, Allium, } & \text { Conferva, } \\ \text { Pontederia, Scilla, } & \text { Solidago, } \\ \text { Polygonum, } & \text { Prunus, } & \text { Habenaria, } \\ \text { Tradescantia, Andromeda, Carex, } \\ \text { Commelina, Helianthus, }\end{array}$


ters are ample, conclusive, essential, exclusive and general, applying to all the species: this makes them natural and proper. Therefore all Natural Genera ought to be like them, and all artificial Genera divided or rectified till they reach this perfection or rather correct form, by revising and amplifying their single or confined generic character : while anomalous and polymorphous Genera must of course be reduced to their natural limits.

The rectification of Genera is important above all in Botany, as in Zoology ; since they afford the generic names, and a wrong Genus multiplies the wrong names of Species, overloading them with useless artificial synonymy. Improper names will therefore be never exploded until bad Genera are forgotten, as were gradualy the unmeaning and indefinite Genera of old.

Names and Genera are thus intimately connected; they stand and fall together. T'he necessity of both being proper and definite, natural and perspicuous, is therefore quite evident. A bad name given to a good Genus annuls it in the eyes of many, until rectified or changed.

In this sense Natural Genera shall really afford the natural character and name: while actual Genera have often a false character and improper name, or else they appear nearly as bad as the vulgar names in modern languages: quite loose and incorrect in import or application.

Species altho' less important since they are variable, must however be attended to in the same way; they ought to bear a good name that applies, if not exclusively, at least properly. We ought to be guided for them by a due knowledge of their fixed forms, and those that may 
vary to produce breeds or proles, until these assume the specific rank by important features, united to permanency, multiplicity of individuals or insulation in distinct climes.

Many of our admitted Species are in fact such new breeds or productions of a genus, that had perhaps once a single type on the Earthas it has happened to our knowledge with MANKIND or the Genus HOMO, once a single TYPE, that has produced during many ages, so many natural varieties and breeds, gradualy become permanent, divided and multiplied, that they now form 3 or $\mathbf{4}$ great Races or Breeds, deemed Species by some philosophers: and even others admit 10 or 15 Species of MEN. 'Thus the natural spliting of MANKIND has realy produced many divisions of the type. If however we acknowledge that they all come from a SINGLE GENERIC TYPE, it is immaterial whether we call them Species or Races, Breeds, or Proles, Varieties . . , But if deemed real natural new Species as in Plants, Oaks, Vines, Roses \&c. they obtain a name and better Entity.

MEN form both a Genus, a family the HUMAN tribe, and an Order the BIMANES: which are both very striking and obvious groups ... Roses do the same, and the botanists clo not agrce on their species and varieties; altho' so numerous now, they probably came like MEN from a single gencric Type? Let us then study species in that point of view; both as distinct, and connected by the common Generic tie.

GENERA therefore are mainly essential; they give form, existence, characters and names to Species. T'he generic types are either in- 
variable or slightly and slowly transforming; but specific types may and do eventualy vary in all their frame and parts, except the essential floral organs of the Genus ... as MEN have varied in color, size, features, hair, Sc. but preserved the great generic characters of limbs and teeth, and are BLMANES or with two hands, forming an Order distinct from the Quadrumanes or four handed Monkeys.

Rosa and Rubus were once united in the same Order and family, but they are as unlike as Men and Monkeys, Rosa has a calix berry like enclosing the germs or pistils, while Rubus bears them on a central gynophore or fructal receptacle. 'These are character's perfectly essential and exclusive, like 2 hands or 4 hands. All good characters ought to be such; when they vary they lose their importance; but when we merely suppose they do, because we unite alien plants, the mistake is ours, not a natural consequence nor real fact.

This view of natural Botany opens a wide field to us : the aggregation or segregation of individuals in various successive real Cluster, ruled and led by several physical laws of opposite tendencies, may gradualy unrol before us the mysteries of Vegetable organization and frames, with their mutual contending aims.

These great laws that rule living bodies and vegetation, are, SYMETRY that gives the bodily forms to Genera, casting the moulds of typical frames-PERPETUITY that by reproduction, perpetuates these original primitive forms-DIVERSITY that bids and compels all living bodies to assume gradualy, a variety of slight changes when reproduced, and never evolves individuals perfectly alike, nor two leaves 
quite similar in all points on the very same tree.

Lastly INSTABILI'IY that does not allow any forms nor frames to be perpetual nor ever the same, giving to plants as to animals birth, growth, decuy and death! in succession, within a term of a few hours, a day, a month, a year, or 1000 years.

By these contending laws, always balancing each other, Vegetation is regulated like the Worlds and Skies by Gravitation and Repulsion.

Symetry and Diversity are ever moulding or changing the Vegetable forms : while Perpetuity and Instability are ever controling their existence by successive reproductions, or vital evolutions.

Let us study these laws, let us ascertain their effects, let us contemplate and admire the innumerable forms they evolve, sustain and improve upon our EAR'TH-This is the study of natural Botany, of floral beauties, and of GOD working wonders there as elsewhere.

HE rules the skies and in his hands upholds The solar Worlds: while from his breath di[vine

Spring living souls, that men and beings move. By him alone the trees and shrubs are set, And with the lesser plants, the spark of life Receive, imbibing solar heat and light.

Then to the Sun their leafy limbs eqpand, And nuptial buds with dazzling beauties bloom of thousand shapes and hues, or sweet per[fumes ;

The Earth adorning with a verdant dress, Sprinkled with floral gems like lucid stars, 
Sparkling throughout the skies, adorned all By gilding light, with colors of the prism : Thus they delight the human senses, showing The deeds of GOD in floral wonders growing. 



\section{TABLE OF CONTENTS.}

Introduction, - - - page 7

Natural Classification, - $\quad-\quad-26$

New Natural families, - $\quad-\quad-27$

Natural Classes and Orders, - - 44

Nat. Classes, 1814, - - - - $\quad 45$

Nat. Orders, 1815, - $\quad-\quad$ - 48

Rectified floral Classes, - $\quad$ - $\quad$ - 58

Additional Orders, - $\quad-\quad-63$

Classes by Habit, - - - $\quad$ - 71

Rules of generic nomenclature, - $\quad 81$

Conclusion and natural genera, - 90 



\section{NOTICES.}

Late works published by Prof. Rafinesq

History of the American Nations, before after Columbus - 2 volumes published-\$ 6 volumes to subscribers.

Life, travels and researches of Prof. $R$ esque, in both Hemispheres-one vol. 12 75 Cents.

The philosophy of Instability-one vol 8 vo. $\$ 1,50$.

New Flora of North America-First vol 8vo. - \$5.

Herbarium Rafinesquianum-\$1.

Atlantic Journal, with 200 tracts on Scien one vol. 8vo. complete- $\$ 2$.

A few copies of former works for salelysis of nature-Principles of Somiologylines of discoveries-New Genera of Ani and Plants of Sicily-Ditto of North Ameri Fishes and Shells of the River Ohio-Med flora of the United States, \&.c.

Unique Copy of Autikon Botanikon or ? Self figures of new and rare plants, folio \$

Icones plant. rariorum N. Amer. folio, figures-\$300. 
FLORA TELLURIANA CENTUR. I.--XII.

\section{MANTISSA SYNOPTICA.}

\section{0}

N. Ord.-N. GeN.-N. SP.

\section{PLANTARUM}

IN ORBIS TELLURIANUM 。

Determ. coll. inv. obs. et. descr.

Ann. 1796-1836.

Auctore C. S. RAFINESQUE, Bot. Prof,

PHILADELPHIA.

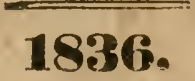


Les noms font les choses.

Names realize Entities.

Plus nos noms sont generaux, plus non idees sont incompletes.-Plus nous avons de noms, plus elles se completent. Lamark, Leach, \&c. 


\section{FLORA TELLURIANA}

PARS IV ET ULT.

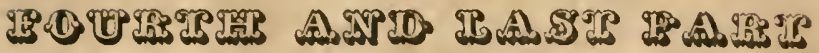

OF THE

SYNOPTICAL FLORA TELLURIANA,

CENTURIES IX, X, XI, XII.

With new Natural Classes, Orders and Families : containing the 2000 new or revised Genera and Species of 'Trees, Palms, Shrubs, Vines, Plants, Lilies, Grasses. Ferns, Algas, Fungi, \&c, from North and South America, Polynesia, Australia, Asia, Europe and Africa, omitted or mistaken by the Authors, that were observed or ascertained, described or revised, collected or figured, between 1796 and 1836.

BY C. S. RAFINESQUE, A. M.

Prof. of Botany, historical and natural sciences-member of many learned Socicties in Paris, Vienna, Bruxelles, Bonn, Bordeaux, Zurich, Naples, \&.c. Philadelphia, New York, Cincinnati, Lexington, \&c.

To observe and compare, to correct or approve

By good namcs and new facts that convince and improve.

\section{PHILADELPHIA.}

PIRINTED FOR TILE AUTHOR

By H. Probasco, No. 119, N. Fourlh St. 1838. 


\section{NO'TICE.}

This fourth part of the Flora Telluriana will conclude the work. It had been intended to divide it into 6 parts; but even these could not contain all what $I$ have to add or correct on the Orders, Genera and Species of the whole world and all the classes of Vegetation : therefore it is better to limit this Work to Plants and Lilies chiefly; while I mean to publish separate $W$ orks additional to this on the Trees and Shrubs or a Sycva Telluriana, on the Ferns and Grasses, Fungi and Algas, with perhaps Monographs of some peculiar Families of great interest, and probably a complete account of my New Class of the Nantiandres, having stamens alternate to calix or opposite to corollas, contrary to the usual order.

The price of this work will still be $\$ \mathbf{5}$ or $\$ \mathbf{4 0}$ for 10 copies, only 160 copies were printed, which makes it high, as but few copies can be sold in America, where Botanists cannot duly appreciate it, and they must be sent to Europe, to be often exchanged instead of sold. 


\section{P R E A M B L E .}

\section{TO 'THE FOUR'TH AND LAST PART.}

In the process of this work I have met with many interruptions and disappointments. It is neither easy nor agreeable to stem the current of botanical errors and blunders, and whoever swims against the streams of scientific prejudice may reckon on difficulties. I have met such in all my attempts to increase and correct knowledge ; but I persevere nevertheless, and write for posterity rather than the actual Schools. I feel that ny weary labors are not now appreciated except by a few, but am confident that in 50 years hence they will be more valued. Of this $I$ have received already some assurances, when young and skilful Botanists have partly approved and adopted my views.

Meantime I must again repeat that whatever I now state or correct, had been mostly done by me between 1806 and 1815 chiefly, when I had matnred my botanical reforms as stated in my Analysis of nature 1815. I then possessed the ample materials of all the works of Wildenow, the great dictionary and illustrations of Lamark and Poiret, Martyn's large edition of Millers in 4 volumes Folio, and the New Dictionary of Natural History in 24 vol. 8vo . . . besides many rare and valuable works... which were lost with my Mpts. in my Shipwreck of 1815. Having thereby been thrown back upon the world and entered new paths of life, I could only gradualy collect again my materials, and restore my labors imperfectly. Yet $I$ have since then consulted and studied many other 
modern labors similar to mine, without finding the same accuracy and perspicuity of Generic reform. While it is with the utmost difficulty I can obtain even for money some late works of similar tendency, although 1 try to correspond direct with the Authors.

Therefore I distinctly state here again, that my feelings being of the most liberal and friendly kind for all Botanists and Naturalists, all over the Earth, if my labors ever interfere with theirs it must be accidentaly and unknowingly, either because my Genera were those established by me between 1806 and 1815 , or because I have not obtained yet their works, although willing to buy them or exchange them with mine, unless they be too costly like Audubon or Jacquin-I hereby call publicly upon all synoptical and improving Botanists (and even Zoologists and Oryctologists) such as Agardh, Decandole, Endlicker, Schreber, Sprengel, Fries, R. Brown, Lindley, Hooker, Don, Sweet, Arnott, Bentham, Nees, Fischer, Link, Tenore, Ledebour, Blume, Martius, St. Hilaire, Bory, \&c... with others unknowu to me by name as yet, to send me their works in exchange of mine and also to exchange specimens. I have been permanently established in Philadelphia again since 1826 , and shall probably ever continue here, although I may become connected with various literary institutions, particularly the Central University of Illinois of which I am one of the founders. Books sent me for the University will be free of duties, and equaly received in exchange.

When I have accumulated all the latest Materials or Genera, and the great work of Decandole is completed, I may then revise the 
whole, connect naturaly the scattered Genera, and add the needful corrections. I have been called already a Veteran in Natural Sciences even by my foes, I hope to become the Nestor of Botany like Adanson was, and if my zeal does not abate, to publish in 1850 , a real Mantissa of all Botanical Genera till then.

Meantime I proceed with my New Flora and New Sylva of North America, and am now preparing a peculiar work on tho Ferns of North America and other parts. I am going to reprint all my early essays on Botany and Zoology, as my Amenities of Nature. I have begun my Mantissa of Zoology, or new or revised Genera of all classes, many also framed previous to 1815 .

My Historical and Philosophical Works are also proceeding, I shall publish my Celestial Philosophy, and my Genius or Spirit of the Helrew Bible, preparing afterwards my good Book of Knowledge and Wisdom to be probably issued periodicaly, and including the restoration or increase of much knowledge on all Sciences whatever. These vast labors, besides those of private life, in order to obtain the means to produce my works, at my own expense chiefly (since they are too good and too learned for the vitiated scale of our publishers) have partly induced me to curtail the actual work, and conclude it here; but to continue it in another form or reproduce the additions as a Sylva Telluriana and otherwise.

Althongh the articles are now only 1200, yet they include with the species and subgenera, orders \&c, 2000 or more added or revised objects and groups. 


\section{FLORA TELLURIANA.}

\section{CENTURIA IX MONOCOT.}

Number 801. Oтоsмa Raf. (ear scented) diff. from 679, spatha lato cuculata basis convoluta, spadix clavato, apice nudo, pistillis inf. subrot. stylosis, antheris truncatis sup. baccis 1 loc. 6-12 sp. sem. teret. oculatis. Scaposa, fol indiv. spathis amplis albis odoratis. - I continue here the Aroides, Lilies, and other monocotyles. This Genus blended with Colla of $\boldsymbol{L}$. is totaly unlike, see my Provenzalia 679, and New Flora N.A. 481 to 485.-Type $\boldsymbol{O}$. ethiopica R. Calla do. L. auct. fol. cord. sagit. cuspidatis, lobis obt. well known plant of Africa, often cult. seen alive since 1806.

802. Spirospatira R. Spatha spiralis clausa, spadix obl. pistillis mixtis trilobis, stigma sessile concavo trifido, antheris ad bas, mixt. ad ap. solit. baccis 3lobis 3locul-polysp.-Still more unlike Calla with flat spatha, no style, berries yellow uniloc, Type Sp. occulta R. Calla do Loar. Sm. fol. ovat. cord. petiolis canaliculatis. -From Anam. Perhaps the Arum Spirale of Retz Vitm. Sm. is a $2 \mathrm{~d}$ sp. with lanceol leaves and sessile flowers.

803. Pleurospa R. diff. fiom Caladium and Colocasia by the flowers unilateral on one side of the spadix, and stem frutescent.-The singular structure of spadix requires attention, perhaps several Genera and Species blended as Arum arborescens L. Types 1. Pl. reticulata R. fol. sagittatis, spadix reticul the linnean sp. of South America, stem 6pedal, leaves pedal, flowers white inside green outside, base dark 


\section{FLORA}

\section{T E L L U R I A N A}

$\Rightarrow \gg 000 \ll-$

BY PROF. RAFINESQUE.

SECOND PART.

$P H I L A D E L P H I \Omega$

$\rightarrow \gg 000 \ll$

1836. 



\title{
FLORA TELLURIANA
}

\section{PARS STCUNDA.}

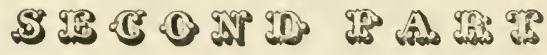 \\ OF THE
}

SYNOPTICAL FLORA TELLURIANA,

CENTURIA I, II, III, IV.

With new Natural Classes, Orders and families: preamble of the $2000 \mathrm{New}$ or revised Genera and Species of Trees, Palms, Shrubs, Vines, Plants, Lilies, Grasses, Ferns, Algas, Fungi, \&c. from North and South America, Polynesia, Australia, Asia, Europe and Africa, omitted or mistaken by the authors, that were observed or ascertained, described or revised, collected or figured, between 1796 and 1836.

\section{BY C. S. RAFINESQUE, A. M.}

Prof. of Botany, historical and natural seiences-member of many learned Societies in Paris, Vienna, Bruxelles, Bonn, Bordeaux, Zurich, Naples, \&c. Philadelphia, New York, Cincinnati, Lexington, \&c.

To observe and compare, to correct or approve By good names and new facls that convince and improve.

PhiladelpiIIA :

PRINTFD FOR THE AUTEOR

BY H. PROBASCO, NO. 119 NORTH FOURTI ST. 
Les noms font les choses. Names realize Entities.

Plus nos noms sont géneraux, plus non idées sont incompletes.-Plus nous avons de noms, plus elles se completent. Lamark, Leach, $\mathcal{f} \cdot \mathbf{c}$. 


\section{EXPLANATIONS}

\section{OF SOME BOTANICAL 'TERMS.}

WE are indebted to Limneus for a beautiful glossology or language, whereby we are able to express by words every form of vegetable organization, and to paint by words as it were, every plant, fixing in the mind the Genera and Species.

'These botanical terms ought to be well known to Botanists. 'The language of Botany is to be learned at the outset by every student, and there are many grammars of it. Several gradual additions and improvements have been made since Limneus .. . chiefly by Richard, Necker. Jussien, Mirbel, Agardh, Persoon, Decandole, \&c.-I have ventured to add but few, following or adopting mainly those of Richard and Decandole.

It is unfortunate that all the Botanists do not quite agree even on this: and use sometimes various terms for the same organs.-For instance, the Common Calix of Limneus, an improper double word, had been very properly changed to Perinntue by Richard, a good single word derived from around the flowers. But other Botanists have proposed the synonyms of Anthodium, Periclinium, Involucre, Perigynande, \&c., which are both later and worse. It is true that Perianthe had been applied by Limneus to the floral coverings, but the name was wrong. since these coverings, the calix and corolla, form the flowers and are not around it; they have since been collectively named Perigone (around the sexes) by Jussieu. and this name has been adopted by all the Natural Botanists.

I therefore adopt and use the terms Perianthe and Perigone; also, Phoranthe (bearing flowers) of Richard, instead of common receptacle of Linneus, or Clinanthe of later Botanists. 
It would be useless to discuss in this way the merits of the different terms: it will be sufficient to designate those which I will employ throughout this Work, wherein I shall venture upon very few innovations.

Sepalis-Sepals, the folioles of the Calix or perigone.

Petalis, Petals, those of the Corolla.

Receptacle.-Only the centre of a flower bean. Placenta.-The receptacles of the seeds in a fruit. Gynophoro.-'The receptacle of the pistils or sceds, commonly swelled or protruded.

Androphoro.--The disk or pillar bearing the stamens.

Columna.-The Androphore of the Orchideous flowers united to the pistil.

Phorantho.-The receptacle of compound flowers.

Diclinis.-Dicline, Separated beds or Stamens and pistils.

Pistillis.-Pistils, the whole female organs, Ovary, style and stigma.

Ovarium.-Ovary, the germen of Linneus, this name is now restricted to the real germen of the seeds.

Ovarium adherens vel liberum.-Ovary adherent to the perigone or free, as called by Jussieu, \&c., answering to Germ inferior and superior of Linnens.

Peristomic, around the mouth of the perigone, Peripetalic.-Corolla monopetal of Linneus, which is not of one petal, but a circular petal around the stainens or pistils.

Lepigono.-Lepigone or bract bearing the sexes. Isarinis.-Isarine, stamens of equal number to the perigonal parts, or Diplarine when double. 
Heterinis.-Heterine or in unequal number compared to perigone.

Epimesis.-Epimesical, stamens standing in the centre of the flowers, where the pistils generally are.

Sporulis.-Sporules, the minute seeds of Fungi and Lichens.

Sporangis.-Their Capsules.

Gongyles.-The seeds of Algas and Aquatic acotyle plants.

Heterolis.-Heterolic Corollas, irregular, and not equal in number of parts with stamens. Achena.-Achene, a dry firuit that does not open, commonly with only one seed.

Glumis.-'T'he scaly valves or bracts of Grasses and Cyperacea, wrongly called calix and corolla by the Linneists.

Corolla.-The inner colored floral covering, when there are two, when only one colored, it is called perigone.

Involucris.-T'he bracts surrounding umbels. 'The minor ones or secondary called $\mathbf{I n}$ volucelis.

Galea.-Helmet like appendages or parts of Corollas.

I have given the descriptions or essential characters of the Genera and Species in the usual Botanical Latin Language; but the roots of every word are similar to the proper English Botanical Glossology, and may be understood by any English Botanist. who knows the usual terms of the Science; besides being available to all other Botanists. The remarks, explanations, rescarches, \&c., will be given in English: this double mode of expression is now often employed, and has many advantages; while the use of abbreviations is well understood. 
SOME ABBREVIATIONS USEU IN 'THIS WORK.

G. for Genus.

Sp. . . Species.

do. . . Ditto.

Cal. . . Calix.

Cor. . . Corolla.

Stam. . . Stamina.

Pist. . . Pistilis.

Per. . . Perianthus.

Perig. . . Perigone.

Pet. . . Petalis.

Sep. - . Sepalis.

Phor. . . Phorantho.

Col. . . Columna Sexualis.

Gynoph. . Gynophoro.

Nect. . . Nectarium.

Fil. . . . Filamentis.

Anth. . . Antheris.

Styl. - . Stylis.

Stig. - . Stigma.

Gland. . Glandulis.

Ov. Ovar. . Ovarium.

Caps. . . Capsula.

Sem. . . Semina.

Recept. . Receptacle.

Sq. . . . Squamis, osis.

Bract. - . Bracteis.

Fl. flor. . Floribus.

Spic. - . Spicis, atis.

Panic. . . Paniculis, atis.

Corymb. . . Corymbis, osis.

Umb. . . Umbellis, atis.

Sess. . . Sessilis.

Petiol. . Petiolatis.

Ped. . . Pedunculis.

Lob. . . Lobis.

Segm. . . Segmentis.

Fol. . . Foliis, leaf. 
Lab. . for Labio, labelum. lip.

Int. . . Internis.

Ext. . . Externis.

Nerv. . . Nervis, osis.

Lin. . . Linearis.

Rad. . . Radiis, atis.

Flos. . . Flosculis.

Eq. . . Equalis.

Ineq. . . . Inequalis.

Adn. . . Adnatus.

4loc. . . quadrilocularis.

4valv. . . quadrivalvis.

Obt. . . Obtusus.

Ac. . . . Acutus.

Obl. . . Oblongus.

Ov. ovat. . . Ovatus.

acum. . . acuminatis.

Ic. . . . Icones.

filif. . . filiformis.

polysp. . . polysperma.

multil. . . multilocularis.

tubul. . . tubulatis.

infund. . . infundibuliformis.

hypocr. . hypocrateriformis.

unifl. . . uniflora.

valv. . . valvis.

cord. . . cordatis.

dent. . . . dentatus.

monad. . monadelphis.

diad. . . diadelphis.

didyn. . . . didynamis.

diff. . . . different.

fid. . . . . fidus, cleft.

opp. . . . oppositis.

alt. . . . . alternis.

vertic. . . verticilatis.

char. . . characteris. 


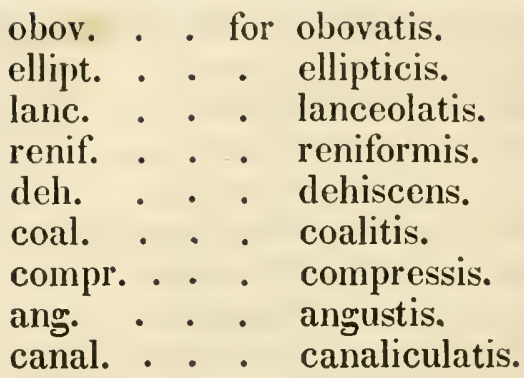

ABBREVIATIONS OF SOME AUTHORS.

$\begin{array}{llll}\text { L. Lin.-for Linncus. } & \text { N. Nek. for Necker. } \\ \text { Ad. } & \text { Adanson. } & \text { Mx. } & \text { Michaux. } \\ \text { J. Jus. } & \text { Jnssieu. } & \text { Nut. } & \text { Nuttall. } \\ \text { Dec. DC. } & \text { Decandole. } & \text { Hook. } & \text { Hooker. } \\ \text { W. Wild, } & \text { Wildenow. } & \text { Lind. } & \text { Lindley. } \\ \text { R. Raf. } & \text { Rafinesque. } & \text { Tor. } & \text { Torrey. } \\ \text { Sm. } & \text { Sir James Smith. } & \text { Cat. } & \text { Catesby. } \\ \text { Sw. } & \text { Swartz. } & \text { Big. } & \text { Rigelow. } \\ \text { Ag. } & \text { Agardh. } & \text { Roxb. } & \text { Roxburg. } \\ \text { P. Pers. } & \text { Persoon. } & \text { Humb. } & \text { Humboldt. } \\ \text { R. P. } & \text { Ruiz and Pavon. El. } & \text { Elliot. } \\ \text { Br. } & \text { Robert Brown. } & \text { Leg. } & \text { Legarza and Lavo } \\ \text { b. m. } & \text { botanical maga- Th. } & \text { Thunberg. } \\ & \text { zine. } & \text { Lour. } & \text { Loureiro. } \\ \text { b. reg. } & \text { botanical register. Forsk. } & \text { Forskahl. } \\ \text { Jaq. } & \text { Jacquin. } & \text { Forst. } & \text { Forster. } \\ \text { Lab. } & \text { Labillardiere. } & \text { Vitm. } & \text { Vitman. } \\ \text { Cav. } & \text { Cavanilles. } & \text { Scop. } & \text { Scopoli. } \\ \text { L'her. } & \text { L'heritier. } & \text { Del. } & \text { Delille. } \\ \text { Ach. } & \text { Acharius. } & \text { Spr. } & \text { Sprengel, \&c } \\ \text { Lod. } & \text { Lodiges. } & & \end{array}$




\section{FLORA TELLURIANA,}

CENTURIA PRIMA.

Synopsis Prantarum select-This Synopsis will contain nearly 2000 new or revised Genera, with some new or corrected Families and species of Plants, from North and South America, Oceania, $\Lambda$ sia, Africa and Europe; which having been neglected or mistaken by the Botanical writers, were by myself ascertained, and even several published, between 1805 and 1835 . It forms the complement to my New Flora of North America, Autikon Botanikon (of 2500 New or rare Gen. and Sp.) and all my Botanical works since 1805 .

Number 1. Nretosma Raf. (sweet smellingo by might) G. Orchid. ad. Eypdendmu distincto. Sepalis 5 eq. angust. elongatis, labellum columna adn. ineq. 3partito, 2 auriculif. 1 calcarifore basi biglandul. columna 3 appendices dentatis, anthera 4 loc. 4loba, alata, intus clausa. N. nocturna. Lid. do. L. \& omnis anctoris, Lind. Hooker bot. $m$ 3298. fol distichis ellipt. retusis. caule compresso unifl-fos magno citrino, Antillis, an Florida?

2. Kadzi, R. (Nomenindicum) 1815. Catcarmia R: 1830. Pentederia Sp. Sepalis 6 sessilib, carinatis ineq. 3 ext. lanc. ac. patens, $;$ int obov. obt. supero erecto. Stan. 6 inec. subul. sep. insertis, 5 fertiles adscendens antheris lin. l,isulc. apice dehiscens, 1 stam. major erccta appendicul. Ovar. 3gonum superum, stylo. filif. incurvis, stigna obt. Caps. or. acum. 1lec. 3 valvis, treta basi sepalis marcescens contortis. Semina 
plurima, recept. centrale affixa. Habitus Ponted. spatha monophyla multifl. fol. oppos-Plurima Sp. Ponted. G. maxim. mal. ordin. 'Typus C. acuminate Raf. P. dilatata Buch. Sym. Ava, Ic . . Andr. rep. 490, Sm. Caule unifolio fol. cord. acumin. petiolata fl. corymbosis ceruleis ad petiolo erumpens-Ava. certainly as distinct a G. as my Unisema. the capsule is uniloc. by Buchanan figure, but triloc. in his descr. to make it agree with $\boldsymbol{P}$. as our Botanists do with Unisema (P' corlata) that has only one seed. $\boldsymbol{P}$. hastata and $P$. vaginalis perhaps belong here as Sub Gen. The G. is in utter confusion and shall be partly revised here.

3. Carigola R. (nom. ind) hastata. Ponted. hastata L. Auct. Cor. regularis sepalis 6 eq. ceteris ut preced? fol. hastatis, florib. umbellatis.

4. Gompinna R. (club stigma) vaginalis, Ponted. do. L. auct. Stigma clavato, florib. racemosis, fol. cordatis.

5. Pontederia vere ch. Cor. 6 fida bilabiata, stam. 6 iner. caps. carnosa 3 locul. fl. spathaceis spadiceis capit. s. spicatis-Typis P.rotundifolia, P. ovita (S. G. Narukila florib. cap.) $\boldsymbol{P}$. "zurer? \&e.

6. Uniseita (one seed) Raf. 1808, med. Al. 1830. Cor. Gida bilabiata, Stam, 6 ineq. ovar. obl. stylo filif. stigma simplex. Fructus Semen nudum ut in Granineis! unicum, corolla marcescens 6costata obsito. Perisperm. farinos. Embryo centrali tereto. Spadix spicato, erumpens, caulib. umifoliatis-This fine N. G. of mine has been doubted by many, yet Nuttall and Torrey have verified the singular fruit, but deemed it the proper one of Pontederia! and our Botanists continue to admit the blunder. We have 9 sp. or more in N. Amer. well indi- 
cated by me in my Med. flora, and U. deltifolia figured tab. 93, yet all blended in 2 or $3 \mathrm{Sp}$. by them. How slow are Botanists to redmit improvements and even accurate obserations! This G. is the type of a new family UNISEMEA, nearer to Aroides, Piperacea, \&c. than Pontederacea, indicated in my Analysis Nat. 1815, but put then near Asparagicies in order Acinopsia.

7. Lunavia R. $1830 \mathrm{~m}$. fl. (Luman bot.) cor. tubul. Gfida ineq. Stam. 3 in tubo, stylo 1, stigma 3-6. Caps. 3 loc. 3 valv polysp-L. uniflora, fol. ovat. cordatis, scapis uniflor is ; Antillis, Mexico. Pontederia limosa Św. \& plur. auct. Another distinct Genus forming with Schollera, Leptanthus, Heterandra . . a subfamily of Pontederacea, with 3 Stamens instead of 6. Mnasium, Pollia and others of same family have 6 . The inequality of Corolla or Stamens or both, forms the essential character of the whole natural family, with the regular fruit.

8. Apenox R (Solan. Diosk) Datura auct. Cal. tubul. tercto apice fisso integro ut spatha latere dehiscens. cor. tubo angulato, limbo plano plicato 10 dent. 5 alt. minor. Stam. exserta subeq. anth. 4 gon. 4 loc. stigma obt. Caps. 4locul. levis-A. crassicule. Caule crasso dichotomo, foliis oblongis sinuato-repandis, subtus glaucis tomentosis, fructo globoso pendulo- $\mathrm{Cu}$ ba Antillis, fl. magnis albo-ceruleis, estivatio spiralis-D. ceratocaula Ortega \& omnia auctoris! D. macrocaulis Roth, plurima ic. bot. reg. 1031. bot. mag. 3352. How could Hooker and Lindley leave this in Datura? which has cal. ang. 5 dent. cor. 5 dent .... It is nearer Solandra by calix, but has not a berry. v. v.

9. Askolame R (Asphodel.nentiq.) Milla auct. 
nomen inclusum in Camomila! ch. ref. Cor.infund. Gfida. sep. alt. major. anth. 6 eq. ad fauces sep. Orar ped. stylo fil. stigm. 3 Caps. 3 loc.polysp. A. bifizora $R$. scapo bifloro, fol. lin. laxa, $\mathrm{fl}$ albis marnis-Mexico. Milla bijorn Cav.t. 196 and omn. auct.

10. Imuson R (Asphodel. anit.) Irilla unifora Hookci bot.m. 3327. cor. infund. 6 fida, sepalis eq. Stam. 6 in tubo, stam alt. brevior, ovar. sessile, stylo fil. stigma 1 capit. caps. clavata 3 loc. polysp-I. uniflorum R. Scapo unifl. compr. medio vaginato spatha bifida, apice unifl. sepalis oratis acum. fol. lin. obt. scapo eq-B. Ayres, A. albis. Itilla sp. auctoris G. duplex, ad stam. ovar. stigma conspic. diversis: ambi ad Asphodelides pertinent. v. v.

11. Śtomadeva R. (glandular mouth) Ipomea aitoni Lindl. b. reg. 179.1. Aff. Convolv. cal. ineq. cor. tubo brevi crasso, camp. intus ad insertio stam. multiglandul. Stam. ad fauce $\mathbf{5}$ ineq. 2 major, stigma bilobo, caps 2 loc. 4sp.St. violacen volub. villos. fol. cord. 3lob. acutis brevis, pedic, multift. bract. lanc. acum-Florida, fl. viol. med. v. v. The Convolvulacea are yet in utter confusion, Choisy has made the matter worse by conversion of characters, the real Ipomea has caps. 3loc. but 20 fine $\mathbf{N}$. $\mathbf{G}$. are blended in these 2 , and the ineq. of calix and stam. is not attended to, as the next evinces.

12. Cormaneva R (hollow gland) Ipomen horsfalia Hook. 6. m. 3315. Cal. sepalis $5 \mathrm{cq}$. imbric. Cor infund 5loba, lobis emarg. Stam 5 equalis filam. basi glandulis. 5 cavis insertis, ad disco hypogyno ferens. stigma bilobo. capsula 2locul-C. Iryenaris volub. glabra, fol. quinatis lanc. acum. undul. pedunc. multifl. cymosis, cal. sepalis obt-Africa? fl. purpur. hyemalis. This 
G. is not even a Conrolmulacen, lut by the equal stam, rather a Polemoniacer, I have shown since 1820 that this is the true essential distinction; but by the insertion of stamens on disk instead of corolla, it may be akin to $N_{y j c}$ tagymea. Perhaps other plants belong to this N. G. compare Ipomea 5phyllin Jaq. with hairy leaves; but Ip. 5phylla Cav. is a different sp. Ip. puniculata Br. or Convolv. do $\mathrm{L}$. is akin, lut has palmate leaves, and forms my G. Morier:

13. Srmma L. mispelt Scilla. char vere. Petalis 6 sessilis planis, patulis caducis, Stan. 6 oppos. filiformis. stylo filif. stigma simplex. caps. 3 loc. polysp-Type \$\%. mavitima and all the sp. with filiform filaments as stated by $\mathbf{L}$. but nany sp. united that lack this good character.

14. Oncostema R. (swelled stamens) Scilla. auct. Diff. Pet. (6 apice villosis, Stam. (6 basi petalis adnatis, medio inflatis cum fovea nectarifera, stylo conico 6 sulcato, stigma obt. villoso. caps 3loc. polysp. extus Gsulcata intus dissep. duplex, receptac. centrale-Typ. O. villosa, fol. cuneatis villosis acutis, scapo glabro, fl. corymb. bract. lanceol. petalis ovatobl. olt. alboceruleis-Barbaria, Sicilia, v, v.-Scilla villosa Def. t. 85. bot. mag. 3211, omn. auct. Probably other sp. belong here.

15. IItexontx R- (6 claws) Scilla L diff. Petalis unguiculatis et alia char. aliena- $\boldsymbol{H}$. $j a-$ ponica Raf. Scilla do Thunb, L. flores umbell. ut in Allium cum spatha.

16. Eprufrimion R (nom. antiq) Scilla I. diff. Petalis (6 campanulatis basi coalitis. $f l$. racemosis ut in Slitla. 1 E. hayarinthoides 
Raf. Scilla. do Jar. Sc. companul I. - 2 E. undulatum Raf. Scilla do Desf. Persoon \&c.

17. XURIDIA Raf. A. N. 1815. The Xurines. Stamina terna libera. Ovarium liberum. capsula polysp. Perigonum 3-6 part. 'This tribe of monocotyle plants is certainly distinct from the Restidia, type G. Restio, which has single seeds, nuts or fruit, like UNISEMIa. It differs from Irides chiefly by fiee Pistil, from GadaxIDEs (the monadelphous Irides) besides by free stamens. Xuris is the typical G. Eriocculon, Xiphidium, Mayaca, Wachendorfia, \&c. belong to it, as well as the following: 19 to 21 .

18. XURis mispelt Xyris by L. too near Iris. vere char. cal. persistens squamis 3 ineq. 2 minor carinatis internis. cor. evanescens petalis 3 unguicul. Stam 3 anth. subsess. ad ung. adn. anther obt. ovarium obov. 3 lobo. stylo 3 fido, 3 stigma, caps. 3 valvis uniloc. seu ad basis sub 3locul. valvis septiferis set placentiferis polyspScaposis, fol. gracilis, fl. capitatis, bract. squamosis imbricatis-Linneus had only one sp. $\boldsymbol{X}$. indica, R. Brown alone added 15, and now we know 35 species! but some have peculiar characters, and form the 3 next Genera. The following $25 \mathrm{sp}$. belong here; but several are yet united by mere habit, the fructif.. not being properly known. $\boldsymbol{X}$. pubescens Desf. platycaulis Poiret, capensis Thunb. macrocephala Vahl, anceps Lam. t. 132, pusilla, denticulata, paludosı, scabra, levis, bractcosa, juneea, gracilis, filifolia, flexifolia, brevifolia, lacera, vivipara, lanata, \&c. of Brown and others; besides 6 N. Amer. sp. brevifolia Mx, caroliniana.W. fimbriata Eiliot, fistulosa Raf. (juncea Baldw, not Br.) obovata Raf. retusa Raf. see my monogr. in New flora N. Amer. 
19. Synomga $R$ (united few) Diff: 18, Capsula unilocul. valvis non placentiferis, receptac, centrale. Important characters perhaps united to others omitted, habit also different, a stem with distichal leaves, head with 2 or 3 flowers only: the $\mathbf{X}$. subulata of Ruiz, X. paciflora W. and perhaps others.

20. Ramotha' $\mathbf{R}$ (nom.ind) Diff: 18. Nectaris seu appendices 3 fimbriatis- interjectis inter petalis. Stylo simplex, stigma capitatis plumosus. 'This includes several sp. blended as $\boldsymbol{X}$. indica by authors, thus the original G. of Lin. also $X$. operculata and others. The following sp. were

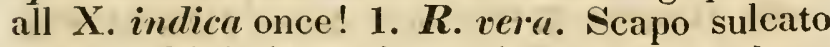
spiralis, fol. latis, capit. ovatis, squamis subrot. glabris. Asia-2 R. Africana. Scapo sulcato basi spiralis, fol. angustis striatis, capit, globos, squamis subrot. fuscis ad medio pilosis. Africa$3 \cdot \boldsymbol{R}$. pubcscens, Scapo tereto, vagina foliosa pubescens, fol. latis, basi dilatatis, capit. squamisque ovatis. obtusis; Antillis. Xyris pubesc Desf. anct-4 R. floridama. Scapo contorto compresso, apice 4gono, fol. tenuis, striatis contortis, capit. globosis, sq. subrot. emarginatis, Florida. X. iudica Pursh, an Xuris? aff. $\boldsymbol{X}$. relusa Raf.

21. Jupica R (nom. amer.) Diff: 18. Stam. filamentis villosis, antheris 4 gonis, stylo trifido, stigma 9-The type or perhaps only $\mathrm{sp}$. is $\mathbf{J}$. cerulea Raf. Xyris americana Aubl. auct. with blue flowers, all others are yellow.

22. 'Tradescantia $L$. vere ch. Cal. 3part eq. pers. Cor. 3 petala plana, tenuiss. evanescens equalis. Stam 6 subeq. filam. hirsutis. stylus filif. stig. simplex caps. 3 loc. oligosp. fl. umb ? spathis. 'To this Gr. belong virginica, rosea, subaspera, hirsuta, discolor, ma?abarica? ge- 
miculata, crassifoliu, umlutu, and many more, with my 8 N. sp. from N. America.

2:3. Sarcopers R. Zamnonia Cramer, Persoon. non aliis Diff. Corolla crassa persistens baccata. S. bibracterita Raf. Commelina and Tradesc. Zanomia auct. is very different.

21. 'Fonningia Necker. Diff..22 Cor. 3partita, 3fidisque, basi tubulosa, coalita, ovar. lanat. fl. axill-I'. axillaris, 'T. speciosa \& aliae. Tradesc auct.

25. Smprosmua R (tubular stigma) Diff. 22. Stylus clavatus, stigma tubulosum crenatum. S. cristata Raf. Trudesc. do Jaq. auct.

26. Gibasis R (gibbose base) Diff. 22. Cal. triang. basi trigibbosus, stigma bilobo, caps. 2loc. 2 sp.Sem obl-G. pulchella Raf. Caule procumbens, fol. ovat. acut. glabr. serrulatisTrad. do Kunth mexico, compare Callisia L.

27. Frimosantires R. 1825 (Evanescent flowers) Diff. 22. Cal. ineq. cor. 3 pet.ineq. 1 pet. major concavo, Stam 6 ineq. 3 major incurvis. Il. racemosis-E. cianta R. Neog 42. Caule erecto dichot. ol. ovat. acum. undıl. ciliatis, racemis secundis-Louisianna, 'lexas 3pedal, fl. blue, lasting only one hour. Is Eothinanthes better?

28. Tripoganiora $R(3$ bearded st) Diff. 22 . Stam 6, 3 brevis glabris, 3 longis apice barbatis, A. cymosis-Tr. melliflore R. procumbens, fol. ovatis basi ciliatis, cymis pedunculatis; Caracas. Tradesc. muligl. Jaq. ic 355. procumbens Wild. Auct.

29. Phyonina R. (growing by twin) Dift. 22. Stam 3, fil. antheris binis divaricatis gerens, stylo basi barbato; Ph. gracilis Sm. Auct. Thor: do. near to Callisia L. IIapalanthus Jaq. which has same Stamens, but capsule bilocular. 
30. Leiandiza R: (smooth st.) Diff. 22. Stain. levis non barbatis ped. multifl. 1 L. cordifolia, repens, filif. fol. cord. ped. term. multifl. 'Trad. do Swartz. 2 L. divaricata. Dichotoma, fol ovlanc. glabris, vag. vill. pedunculis paniculatis. Commelina hexundra Aublet. Tradesc. auct. amb. amer. sp.

31. Hemnema R (half filam) Diff. 22 Stam. 3 glabris, ut in Commelina sed Cor. ut in 22H. multiflora Raf. Tradesc. do Sw. and auctoris, fol. cord. ciliat. ped. axill. multifl-Antillis.

32. Artomen $\mathbf{R}$ (single smooth) Diff. 22. Stam. unica levis.-A. diffusa, fol. ov. cord. glabr. ped. axill. multifl.-Hayti. 'Trad. monandra Sw. auct. - These 3 last Genera lack the very essential character of bearded Stam. that once was the only distinction of Tradescantia from Commelina, but these 2 Genera are in utter confusion, as the above proves. Compare also my genera of Commelina. It is deplorable to see Botanists forcing sp. into genera, in spite of characters. There is not a single generic character common to all the above G. 22 to 32 ! my reform and revision were indispensable, and begun in 1815 . The Tr. malabarica is probably also a peculiar genus, to be called TAmpuris, petalis cuspidatis, ped. longis unifloris. But the Toadescantia! nervosa Lin. is an Orchideous N. G. called Thelypogon angustifol. by Kunth, what a blunder! this whole Genus is a mass of linnean errors.

33. Agltwieis R. va ${ }^{n}$. nom. anticus, Allium L. et auct. pessimum aff: Galium, Homalium, Allionia \&c. Sir James Smith says of this G. the wholerequires to be reformed as to name: 
characters and species alike. Allimn has no meaning and clashes with many genera. Several ancient Gencra, Onion, Leek and Garlick, were blended by L. without just cause; they must be kept apart, having all good essential cliaracters. 'The sp. are in utter confusion, altho' very easy to distinguish; I shall here chiefly attempt a generic reform-The real $\mathbf{G}$. alciun or rather agritueis has, Petalis 6 subequalis dorso carinatis s. nervosis, sessilis patulis. Stam. 6 sub equalis ad basis, filamentis planis subulatis simpl. Ovar. sessile, Styl. simplex, stigma acutum. Caps, 3loc. 3valvis oligos perma, sem. angulata. Flores umbell. spatha 1-2valvis gerens-Io this $\mathbf{G}$. belong all those not enumerated in the following, but not the $A$. sativum ! 'There are yet $40 \mathrm{sp}$. in it; the types being A tatarica, carinata, ursina, \&c. I have added many $\mathbf{N}$. Sp. from N. Amer. triflora, 5flora, stenia, geminata, petiolata, latifolia, triphyla \&c. see Monograph, Herb. Raf. and New flora.

34. Gynomon R (pistil toothed) Diff. 33. Petalis connivens. Ovar. 3dent. coronans, stigma obtus. Caps. globosa dentata 3 sperma-many 3 seeded sp-belong here, A.tricocum? mutabile, cernum, Elioti, mpestre Raf. The types are these 3 last sp. 1. G. cermumm. (A. Roth, Curtis 1824. Sm. W.) Caule 2ang. fol. lin. canal. umb. cernua, petalis ovatis, stam. inclusis, caps. tricornis. Mts. Cancasus. 2. G. Ellioti (A. cernuum mg. E.) scapo compresso 2ang. fol. lin. planis striatis, umb. nutans petalis Janc. Stam. exertis, caps. glob. 3dentata. imts. Alleghany: 3. G. rupestre Raf. Scapo tereto 2ang. fol. lin. brevis obtusis, levis, umb. nutans, laxa pancifl. petalis 
lanc. Stam. eq. Caps.olotuse 3dentatis. ad rupes Kentucky, v. v.

35. Steluesus R (Crown middle) Diff. 33. Petalis ineq. 3 ext. minor carinatis, Ovar. 3 gono apice 6dent. Caps. turbinata 3gona, Gdentata, 6sp. loculis 2spermis-Type St. stellatus Raf. Allium do Sims b. mag. 1579. Scapo 3gon. fol. canal. carin. umbella multifl. N. America.

36. Geboscon R (nom. grec.) Dift. 33. Petalis erectis concavis, alterni latiora. Filamentis filiformis lóngissimis. Caps. globosa bisp.-Grobliquum. Allium Gm. Sib-t-9. Lin. et Auctoris.

37. Kalabotis R (Cepa gr.) Diff. 33. Cor. campanulata, petalis 6 erectis concavis obov. 3 ext. major, stylo conico, brevis Caps. polysperma? many sp. Allium pallens, clusiumum, flavum, nigrum, molly, canadensis, paniculatum auctons, et alsa sp.

- 38. Maligra R (nom. lat.) Diff. 33. Cor. campanul. basi tubulosa 6fida, petalis hasi unguic. connivens, cum. Stam 6 basi monadelpha. Ovarium depressum 3 gonum. Caps 6sp._-3 sp. blended as $\boldsymbol{A}$. angulosum \&c. 1 M. laxa Raf. Caule anceps 2angul apice recurvo, fol. lin. carinatis, umbella laxa. North America. A. angul. Pursh 2 var. fl. incarnatis, fl. albis-M. fastigiata. Scapo 2. 4angul. compresso striato recto, fol. lin canalic. vix carinatis, umbella fastigiata globosa. Europ-Sibiria, fl. purpureis-3 M. gracilis. Scapo tereto, fol. lin. canal. Jamaica. Al. gracile Ait. auct. striatum Redonte, Curtis. All these are fragrant, and form a very natural genus. 'The $\boldsymbol{A}$. striatum Jaq. is a true $\boldsymbol{A g l i -}$ theis not Maligia, from Africa, but $A$ striatum. of Pursh and N. Amorica is A. Ormithogaloides of Walter that has received 7 names.

39. Kres Tourn. Ad. Necker \&c. Diff. 33. 
Petalis crassis persistens, filam. crassis conicis planis basi dilatatis-'The true G. Onion, Kepa esculenta (al. cepa L.) and $\boldsymbol{K}$. fistulosa are the types; but other sp. may yet be blended with Aglitheis.

40. Porrun Tournef 1700, Adans. 1763. optim. G. Diff. 33. 6 Stam. alt. 3cuspidatis, filam. dilatatis, petaliformis ad apice 3 fidis, anthera ad dente medio-500 Genera are based on less important characters. 'This includes all our Leeks, the Garlick itself, Allium sativum, porrum, ampeloprasum, lineare, rotundum, Scorodoprasum, arenarinm, spluerocephalon, mutans, escrlonicum \&c.

41. Getuonis R (nom. gr.) Diff. 33. Stam. 3. alternis subulatis, 3 alt. bifurcatis cuneatis petaloideis, antheris medialis.-Perhaps only a Sub. Gr. of the last. This includes Al. vineale, and perhaps others.

42. Penxistena $\mathrm{R}$ (segmentnarrow) Diff. 33. Petalis nervosis, obovatis concavis, Stam. 3 alt. linearis planis angustis trifidis, segm. lat. divaric. medialis brevior antherifero. Ovar. turbin. Caps. Ggona, 6sperma, umbella cum Involucro polyphilo-Pl. margaritea (A. do Sm. fl. greca) Caule tereto, fol. canalicul. Grecia, pulchra sp. f. albopurp. nervis viridis.

43. Stremonoxis R (Stam. dent.apex) Diff. 33. Stam. 3 alt. subul. 3 ait. cuneatis antheris medialis. Ovar. 3sulc. Caps. oblonga 3sulcata, 3sperma-St. juncea Raf. (A. do Sm. f. gr.) Scapo tereto, fol. 2. fistulosis, umb. globosa, spathis 2 ovatis, petalis ellipticis acutis purpureis. Grecia.

43. Panstenua $\mathbf{R}$ (all narrow) Difí 33. Petalis linearib, angustis, planis enervis reflexis. Stam. filiformis erectis. equalis, Caps. 3sp? - 
P. monspessulamum. Al. do Gouan t. 16, Vitm. Scapo tereto, fol. lanceolatis, umbella globosa.

45. Exnotis $\mathbf{R}$ (inside eurs) Dift. 33. Petalis internis basi biauriculatis, alia char. examinanda-E. mprenaica Caulescens, fol. ang. lin. subearin. A. appendiculatum Ram. Pers.

46. Kйомох $\mathbf{R}$ (nom gr.) Diff. 33. Petalis ineq. planis, 3 ext. latior brevior, 3 internis lanc. duplo longior, Stam. Stylogue longissimis filiformis, $-K r$. parviflorum. Al. do $\mathbf{L}$. an aff. Geboscon?

47. Loncostemox $\mathbf{R}$ (lance stam) Diff. 33. Petalis eq. scariosis vix patulis, Stam. eq. exserta, filam. lanceolatis s. medio latior, apice filif. Caps. 3cocca, 3sperma ?-type L. victoriale, Allium do. auct, and akin sp.-Thus the single G. Allizm of authors, offers $\mathbf{1 5}$ generic groups; altho' some may be deemed mere sub. genera, it will be hard to refer them properly. By better and closer rescarches in the view of correct distinctions, some of these will surely be further improved. At present the Limean G. is only kept together by pure mistake, and mere habit of flowers in unbels, yet some sp. have only 2 , 3,4 , or 5 flowers.

48. Mesonpris R. 1815, (black linavel) Mieleromphate Renealm. Ornithogalum arabicum L. et. auctoris. Petalis $\mathbf{6}$ equalis concavis deciduis. Stam. 6 equalis hypogynis, basi vix coalitis, late subulatis planis. Disco hypogyno 3gono, Ovar. globos. 6sulc. stylo 3gono, stigma 3lobo. Caps. globosa 3loc. 3valv. polysperma, semina obl. induplice series Scapis teretis, fl. corymbosis, suuxcolens, alb, bract. amplectens, Disco, Ovar. et Caps. nigris vernicatis-A very natural and beautiful Genus, merely indi- 
cated long ago by Renealm, overlooked by Linneus, and containing 3 sp. blended in one !

49. Melomphis arabica Raf. Scapo multifloro, fol. canalic. corol. camp. petalis externis obsolete 3dentatis-Arabia, Egypt, Madeira, Cape. often figured and the usual sp. of nearly all authors.

50. Melomphis sicula Raf. Scapo paucifloro 3-6, fol. subcarin. pedicellis abreviatis bracteis subequante cor. subglobosis, petalis obovatis retusis integris.-Sicily near Segesta, where 1 found it in 180\%, in Corsica says DC. never figured, it is $\boldsymbol{O}$. arabicum of DC. fl. gallica: 15 inches high, flowers large; shaped like those of Yuca. Estival.

51. Melomphis peruviana Raf. Scapo multifloro, fol. planis marg. involutis, pedunculis elonglatis ad bract. longior, corollis subpatentib. petalis ellipt. obt. subintegris, stigma pubescens. In Chili, Peru. Scape 2 or 3 feet with 11-15 flowers. Vernal. It is the O. corymbosum $\mathbf{R}$. P. fl. per. t. 300, Lindley bot. reg. 806 . Hooker bot. mag. 3179. who calls it also O. umbellatum! and is at a loss to distinguish it from $O$. arabicum!

52. Synconium R (union bell) Petalis 6 patulis ad basi coalitis cum Stam. 6 monadelphis campanul. (ut in Narcissus) 6fidis, 3 fil. longior emarg. bicornis antheris erectis. Stylo, stig. simpl. Caps. 3loc.- V Very distinct Genus, overlooked by all; Ornithogalum mutans of L. and all authors! Type S. nutans. Scaposo fol. lin. carin. obt. fl. 5-20 racemosis secundis pendulls, bracteis fucis-In Europe, flowers greenish white, often figured.

53. Ornitiogalon Diosk. I. omn. auct. Ver. Char. Perig. Gpart. corolliformis persistens, ba- 
si connivens, superne patens, equalis. Stam. 3 lib. filam. subeq. subul. 3 alt. vix dilatatis. petalis ext. insertis. styl. stig. simpl. Caps. 3loc. 3valv. sem. plurima. Scapis; fl. racem. scu. corymb, brocteatis.-All the botanists had been puzzled to distinguish this G. from Skilla, with filiform Stamens, because the sp. had been blended, and thrown into such a inedley, that at last the blue color of some Shillas became their only distinction! The type of this ancient G. is O. umbellatum, and contains many corymbose sp. with lacteum, nanum, Sm. revolutum, conicum, and all those with equal subulate stamens. Besides many Scilla of authors, unifolia L. cuthericoides Desf. \&c. and my next N. sp.

54. Ornithogalon ceruleum Raf. Car. 204. Fol. lanceol. scapo fistuloso, fl. corymbosis, bract. lin. lanc. pedicellis elongatis-Discovered in Sicily in 1808, published 1810, not a Skilla, filaments subulate equal. Fig. Cup. t. 201.

55. Gagea Salisb. Diff. 53. Petalis 6 deciduis, filam. equalis angust. subul. ad basi petal. adn. liberis. Styl. clavatus, sem. parva subrot. Plıntae graminea facies Hypoxis, fl. paucis s. corymb. bractcatis, luteis extus viridis.-Salisbury mentions $7 \mathrm{sp}$. in his monogr. once all deemed Ornithog. 1 fasciculanis S. luteum sm. 2 bracteolaris S. luteum L. 3 stellaris S. miniuin L. 4 spathacea, 5 pygmea, 6 bulbifera, 7 reticularis $\mathrm{S}$. circinnat. $\mathrm{L}$.

56. Fenelonia R (Fenelon. phil) Diff. 53. Petalis ext. 3 trinervis latior, Stam. 6 eq. filam. lin. subul. angustis. Ovar. obl. sub. 3gon. stylo, clavato 3gono, stigm. capitato 3lobo. Scrpis bracteatis uniforis.-F. bracteata Raf. atl. 
Journ. pag. 145. Ornithog. do. 'Torrey. Oregon mts. see my Flora. 'This G. is nearer Gagca.

57. Lonconelos $\mathbf{R}$ (lanc. membr) diff. 53. Petalis uninervis, 3 internis brevior latior. Stam. subeq. membranaceis lanceolatis liberi Stylus. brevis. fl.racemosis. Nearly all the racemosesp. of Ornithog. belong here, pyrcnaicum, narbonense, japonicum, latifolium, secundum, pyramidale, suaveolens, \&c.; but many of the $\Lambda$ frican sp. require to be revised. I can already detect the 3 next Genera.

58. Eurokanmos R (Nom. grec.) Diff. 52, 53, 57. Stam. ineq. 3 fil. alt. major cuneatis emarg. s. furcatis, liberis. $l$. corymb s. racemosis chiefly different from Syncodium by free Stamens, 4 sp. all Ornithog of authors. E. thygrsoides, aureum, coarctatum, caudatum, and probably other blended sp.

59. 'T'rmelopter R (3.memb. wings) Diff. 53. Stam. ineq. 3 filam. alt. major lanceolatis, 3 minor subulatis, Ovar. Capsul. trigona, trialata. Fl. racemosis-Typ. Tr. fuscatum. Orn. do. Jaq. W.

60. Lonconilis $\mathbf{R}$ (lanc. bell) Diff. 57. Corolle campanulata, pet. basi connivens, filam, 6 lanceolatis in tubo adnata. Fl. racemosisTyp. L. scapigera Raf. Ornithog. paradoxum Jaq. W. P.

61. Eriospermum Wild. Diff. 53. Petalis 6 persistens in corolla campanul. connivens. Filam. 6 basi dilatatis (monadelphis in cylindro L.) Semina lana involuta-Wildenow has $3 \mathrm{sp}$. latifolium, lancifolium, parvifolium. The first was Ornithog. capense of Lin. who assert it to be monadelphous as Syncodizm.

62. Lagoconss $\mathbf{R}$ (Hare bell) Cor. camp. petalis 6 basi connivens (ut Hyacinthus) Stan. 
6 equalis filiformis glabra ad petalis adnatis. Styl. simpl. Caps 3loc. 3valv. sem. plura rotunda. Fl. racemosis-Scilla and Hyacinthus L. but a striking peculiar G. the English Harebell. Several sp. the type was put by Lin. in both Genera! and made $3 \mathrm{sp}$ ! It is 1 Lag. mutans Raf. S. do Sm. DC. S. eermua L. Hyacinthus cermuus et II. nonscriptus L. 2. L. cernua, S. do Sm. 3 L. hyacinthoides. S. do Jaq. 65. 4. L. patula. S. do DC. fl. gal. \&c.

63. Kozola Raf. (nom. Jap.) Petalis 6 unguiculatis patulis. Stam.6. filiform ? Stylo 3gono, Stigma capitat. 3lobo, fructus ut Skilla ?-Scilla japonica 'Th. L. auct. my Kozola japonica, flor. umbellat. fastigiatis. Petals as in Melantiium purple white.

64. Quamasia 1827 (nom. am.) Petalis 6 sessilis persistens equalis, Stam. 6 libera, glabra, filiformis equalis. Ov. 3gon. Stylo filif. trifidus, stigma 3 remotis acutis. Capsula 3loc. 3valv. Sem. panca. Scapo, fl.racemosis, ceru?. s.albis. I shall conclude this long series of revised correct Grenera akin to Shilla and Ornithog. by the S. esculenta of $\mathbf{N}$. America and two akin sp. each the type of another Genus. Since the best Botanists have admitted such a mass of blunders on those $2 \mathrm{G}$. which they could not distinguish and reform, our Amer. botanists are partly excusable for similar mistakes on my 4 N. Amer. G. Fenelonia, Quamasia, Oxytrian. and Amblostima. The type of Quamasia is Phalangium quamash Pursh, P.esculentum of others, Scilla escul. bot. mag. 1596, spread from Kentucky to Oregon, perfectly distinguished from Shilla and Phalangium by the trifiri style. If any Phal. have that character thes they belong here. If Qunmasia is deemed a 
name too barbarous, I ofler two substitutes, Lemotris and Bulbedulis! I call it $Q$. esculento.

65. Oxy tria R (sharp 3) Diff. 64. Stam. subulatis, stylo conicus trifid. stigma 3 comnivens. fl. racemose yellow-O crocea Raf. Ph. alangium croceum Nut. (not Mx.) fol. gramineis, racemo paucifl. bract. obtusis, petalis ovatoblongis. N. amer. fl. croceis.

66. Amblostima $R$ (obtuse stig) Diff. 64. Stam. subulatis planis, Stylo conico simplex, stigma obtuso unico. Sem. globosis nigris. $f$. racem. albis s.luteis-1 A. albiflora Raf. (Ornithogalum croceum Elliot) fol: planis nervosis, scapo tereto, racemo laxo, bract. ovatis, brevis, petalis ovatis obtusis. In Georgia, florida, $\mathbf{f}$. white-2. A. crocea (Phalangium croceum. Mx. P.) fol. gramineis, scapo brevis, racemo pyramidal. bract. obtus. brevis. In Georgia, ff. safron color. Elliot has best described the flowers, Michaux the seeds. All the above Genera akin to Allium, Scilla, \&c. belong to the natural tribe of Asprodelia.

67. Anthericum L. another absurd linnean G. whose essential character was merely Cor. 6petala potens Caps. ovata! which might include Allium, Scilla and 20 other genera. Some botanists ashamed of this blunder, confined it to the Sp. with bearded stamens, taking out of it Phalangium, Narthecium, Tofielda, Abama; \&c. But even all these require correction, including many distinct Genera. The true $\boldsymbol{A} n$ thericum has Petalis 6 sessilis patens deciduis, filam. 6 lanatis filiformis equalis, anther. versatiles, stylus filif. rectus, stig. integr. papillosus. Caps. glob. 3loc. 3val. Sp. having different characters are to be separated. Types $A$. frutescens and akin African sp. 
68. Nemopogon R (fil. barb) Diff. (67. Stam. declinatis ineq. filam. filif. ad medio barbatis. Ovar. 3lobo. Stylo declinato flexuoso, stigma acutum, Rad. fibrosis-'Type $N$. glaucum Raf. glauc. fol. canalic. scapo teretis, fl. racemosis, pedic. adpressis, petalis ovat. obtusis. 'Tasmania Anthericum semibarbatum R. Br. Lod. bot. cab. 330, Hooker bot. mag. 3129.

69. Fusirilum R. (spindle fil) Diff. 67. Stam. glabris fusiformis ad medio dilatatis. $3 \mathrm{sp}$. Phalangium physodes, pusillum Jaq. W. coarctatum RP. Pers, \&c.

70. Onsitila R (drest fil) Diff. 67. Stam. non barbatis sed vestitis muricatis, s. pubescens. Perhaps several G. or at least Sub. G.-1. PubiLARIa bicolor, filam. pubesc. Phalangium bicolor DC. 2. Trachnena vespertina, filam. muricatis. Phal. do anct. 3. Tr. hispida Raf. ditto. Antheric. hisp. L. W. auct.

71. Lepicaulon R. (sq. caule) Diff. 67. Corolla infundibul. campanulata 6partita. Stam. glabris-L. Squameum. Anther. do W. \&c.

72. Hesperoscondu Lindley. Petalis 6 carinatis Stam. 6 filam. dilat. subcoronatis membr. Ovar. ad apice 3glanduloso (ut Hyacinthus) stig. simplex, valvis septif, sem. angulatis; Genus akin to Syncodium, Hyacinthns \&c. according to mere habit, it ought to be Allium! H. lactcum bot. reg. 1639. Scapo teres, fol. canalic. umbella multiflora, bracteis linearib. sepalis ovatis acutis, 3 int. emarg. ad California.

73. Hemerium $R$ (half wooly) Diff. 67. Filamentis ad basis lanatis, apice glabris, $\boldsymbol{R} a d$. crassa, caule ramoso, $f$. purp. albisq.-Typ. H. planifolium Raf. 2. grecum, et alia. sp. Antheric auct.

74. Endogona $R$ (inside angular) Vere 
Phalangium 'T. Juss. P. nomen G. arachnides similis! Diff: Stam. glabris filiformis. 'This G. hardly differs from Skilla, except by the angular seeds: the type is End.ramosa-Antheric. Phalang. of authors, and the akin sp. E. adenanthera (Forster) with the anthers on a gland must form a Sub. Genus at least.

75. Buldine L. Shult. Liliago Lob. T. Diff. 67. Stam. glabris filiformis ineq. Stylo declinato, fl. racem. albis-Type B. or Antheric. liliago L. Phal. do W. DC.

76. Pleisolin ron R (near Lilly) Liliastrum 'Г. Diff. 67. Stam. glabris filiformis declinatis, Stylo recto, Cor. campanul. petalis connivens. Scapo fl. racem. secund. albis, liliformis'Typ. P. liliastrum. auct. A. Ph. do.

77. Collonox $R$ (hollow tip) Diff. 67. Petalis vix patulis apice fornicatis, dorso carinatis; 'Type C. albucoides Raf. Phal. do Pers. Albuca Ait.

78. Cronyxium R(colored claw) Diff. 6\%. Petalis unguiculatis, stam. glabris, Subcaulescens, unifl. flos magn. albo variegato-Cr. serotinum Raf. auct. sub. A. Phal. enum.

79. 'Tofielda Hudson. Abama Ad. Rydbeckia Neck. Heriticra Sternb. Iridrogalvia RP. Antheric. calycul. L . . . Diff. 67. Calix trifid. subt. corolla, stam. glabris. Stylis 3-what a linnean blunder! just like those daily made by his worshipers. many akin sp. now united require a further division, see my New fl. N. amer. True Tofielda. Petalis equalis, filam. subul. caps 3cocca, intus dehiscens, sem. paucis angulatis; 4 Species.

80. Abama Raf. Diff. 79. Petalis alt. longior, Stam. filif. Caps. 3 gonis, loculis apice bivalvis 2sperm-Type $A$. scabra $R$. Tofielda pubens $\mathrm{E}$. 
3 other sp. each with some peculiar characters, the Northecium of Mx. his N. pubens, Sub. G. 'T'vianthe, has petalis angust. caps. globosis, loculis polysp. sem. teretis caudatis.

81. Nartincium Sm. Aniheric. ostifragum L. Difl. 67. Petalis persistens, filam. hirsutis, Caps 3gona, sem. appendiculatis. Thus no cal. like many of the above Gienera. Botanists must be blind to blend stich disparities. When all these $\mathbf{G}$. will be properly noticed and studied, the stigma and seeds will offer other additional features. From 67 to 81 the Genera belong to ASI'HODELIA.

82. Melantmum Gron. L. auct. another G. requiring a radical reform. The very name meaning black flowcr does not apply, and the authors have united thereto many plants quite unlike, Helonias, Tulipa, Wurmbea! The following G. 83 to 100, are chiefly reformed out of this heterogenous mass, all belonging to HELONIDIA.

83. Eronvxis R (well claved) Melanthium Gron. Petalis 6 equalis persistens, rotatis, basis unguic. elongatis staminiferis, s. pustulatis, 2 glandulosis. stam. eq. filif. antheris. globosis stylis 3. caps. 3 basi coalit. intus deh. Sem. plura alata. Caulescens, ramosis, fl. albescens panicul-Fine natural G. many Sp. in N. Amer. not yet well distinguished. E. virginica, glauca, monoica, hybrida, all Melanthium of authors, with several N. Sp. see my New Flora. I add one.

84. Evonyxis (Melanthium) undulata Raf. Caule elatior, fol. glabris striatis, panicula multifl. puberula, pedic. elong. petalis reniformib. undulatis-Alabama, fl. large, white with 2 red spots above the claws. 
85. Ziganenus Mx. Diff. 83. petalis sessilis erectis glandulis binis ferens. Ov. 3gon. Stylis 3 obt. contiguis (in fig. Mx. Stylus trifido acuto) Caps 3gona 3loc. Sem. angulatis - I give this $\mathbf{G}$. to contrast it with the next. Several sp. in my new Flora.

86. Gomphostrums R. n. fl. (club styles) petalis 6 patulis persistens, sessilib, subcoalitis, biglandulis. Stam. 6 basi dilatatis ovar. adpressis, apice deflexa filif. Styli 3 clavatis, Caps. ut in 85. Caulescens, fl. subspicatis, bracteis obsitis.

87. Gonpu. bructeata Raf. Caule flexnoso, fol. lin. lanc. acum. brevis, spicis oblongis, $\mathrm{fl}$. subsessilis, bracteis petalisque ovatis acum. involutis. Virginia. Helonias bracteata Brereton mpt. Is the Veratrum Virginicum Ait. bot. mag. 865 a second sp. of this Genus? or a Zigadenus? 2. Gomphostylis? paniculata R. racemis paniculatis, pedicelis bracteis longior, petalis bigland. ut. in Genus. and another sp. is perhaps the Veratrum Virginicum of Sm. who says it is the real Melanth. Virgin. of Lin! and also Helonias Virginica Curtis bot. mag. 983! 3 Gomph? or $Z$. fuscatus Raf. fol. nervosis plicatis, fl. panicul. petalis ellipticis sessilib. extus pubescens, intus viridis, basi pustulis 2 fuscatis.

88. Veratrum L. this differs from the last by having no glands. on the sessile petals. Petalis 6 sessilis egland. Stam. subhypogynis. Caps. 3 polyspermis. Caulescens, fol. latis, fl. panic. $V$.nigrum, $V$. album, $V$. viride of $N$. amer. $V$. luteum is Abalon albiflorum Raf. fl. white. V. sabadilla is probably a peculiar $\mathbf{G}$. as the habit is different; Sabad. offic, fol. lin. lanc. nervosis, fl. racem.. secundis, pet. ovatis atropurp. Mexico. akin to next Genus. 
89. Anepsa R (Verutr.grec) Cor. campanul. 6 part. eglandula, sepalis eq. angustis. Stam. 6 breviss. ad bas. sepalis. insert. filam filif. antheris subrot. Ov. 3fid. stylis $\mathbf{3}$, caps. 3 coalitis oligosp. Caulescens, fol. angustis, fl. panicul. sepe diclinis. 4 Sp. at least. v. v.

90. Anepsa spicata Raf. fol. longiss. filif. canalic. spica basi ramosa, fl. subsess. bract. subul. brevissim. sepalis lanc. acutis. mits. A palach pedal, fl. purplish dioical.

91. Anepsa graminifolia Raf. Caule angulato, fol. gramineis planis, striatis, panicula racemosa, bract. setaceis fl. longior, pedic. 1-3 floris, sepalis lanc. acum-West Kentucky, pedal, fl. incarnate.

92. Anepsa carinata $\mathbf{R}$ (V. angustif. $M x$. auct) fol. longis lin. carinatis panic racemosa, sepalis linearib-mts. Apalach. fl. dioicis viridoluteis.

93. Anepsa latifolia R (V. parvifl. Mx. auct) fol. ov. lanc. planis, panic. racem. gracilis, pedic. brevis, sepalis lanc. acutis-mts. Apalach. fl. polyg. viridis.

94. Epionix R. (on claws) Petalis 6 unguicul. eglandulis, lanceol. Stam 6. adnatis ad ung. apex. Ovar. tereto 3sulcato, Stylis 3 recurvis. Caps. 3locul. Cauleseens, pancifl. grandifFine African Genus made $2 \mathrm{G}$. by Linneus! Tulipa breyniana, Melanthium ethopieum! Sir J. Smith who corrected this, calls it $M$. . $l a-$ vum, but there are $\mathbf{2}$ sp. M. unifl. Jaq. W. is the 2d. others copy these blunders as usual1. Epionix rubra R. Caule 3-4 floro, fol. lin. lanc. fl. rubris. 2. E. flava, Caule, unifl. fol. lin. lanc. fl. flavo.

95. Aphoma R (no pustule) Petalis 6 sessiis. pustulis nullis. Ovar. ad basis Gglandul. 
Caps. 3gona 3locul. Cetera ut Zigadenus. Rad. bulb. C'aulesc. paucifl-Typ. Melanthium indicum L. auct. plurima sp. confusa. 1. A. angustifiora R. fol. lin. petalis linearib. acutis. 2. cumerta, fol. lin. lanc. petalis cuneatis obtusis. Both in India.

96. Onixotis R (claw eared) Diff. 83. unguiculis pet. sepe canalic. basi vel apice bidentatis s. biauriculatis, eglandulosis. Caps. 3loc? fl. spic.s. racem-Several sp. from Africa, called Melanthium by authors. 1. O. ciliata, $O$. secunda, \&c. have auricles at base of claw. 2 a Sub. Genus 'Zigotila, auric. ad apice ung. fl. racemosis. viridis Raf. fol. ovatobl. scapo 2-3fl. fl. viridis. Mel. racemoides Jaq. 450. Curtis 641 . Sm. \&c.

97. Ornithogloson Salisb. Diff. 83. Petalis sessilis reflexis basi nectariferis, Stam. 6 hypogynes. 'Type O. s. Melanth. viride $\mathrm{L}$.

98. Sкizima R (split stig.) Diff. 83. Petalis 6 sessilis cuculatis egland. stylis nullis, stigma 3 bifidis, Caps-3loc. 6 Sp. Scapis paucifl-S. pumila R. Scapo brevis 3-4fl. fol. cespit. lanç. rigid. canal. basi barbatis-Fuegoland, fl. allis. Melanth. pum. Forst. W. P.

99. Plexiniun $R$ (segm. union) Diff. 83, Corolla Gpartita, s. 6fida, sepalis basi coalitis, sessilib. ad basis poriferis staminiferis. Stam. glabris? Type Pl.punctatum which is Melanth.capense L. and M. monopetaluen! L. 2 sp. are only one. MIel. sibiricum probably belongs here also, having united sepals.

100. Crosperia Raf. 1825 neog. (colored seed) Melanthium, Helonins, auct. Corolla persistens, rotata, 6part. sepalis sessil. glandulis o. Stam. 6 filif. Stylis 3. Capsulis 3 vix coalitis monosp. sem. arillatis, arillis coloratis, Caules- 
cens fol. ang. ft. racemosis-This G. includes several sp. that have been united to 5 or $6 \mathrm{Ge}$ nera! now conmonly blended in Helonias but with different fruit. Chiefly from N. Amer. and in great confusion. See my New flora. The following are the types, 1. Crosperma loeta Raf. M. loetum, erythrosp. Helonias do auct. 2. Cr. phalangioides R. Mel. do W. P. antheric. subtrigynum Jaq. \&c. 3. Cr. angustif. R. not $\boldsymbol{M}$. do Mx. and several new species.

\section{FLORA PELLURIANA}

\section{CENTUNIA SECUNDA.}

101. ZaGa R (nom. ind.) Cal. persistens 5part. eq. Petalis 5 subeq. Stam. 10 libera. Stylo curvo. Legum. ovato compr. crasso duro apex uncinato, intus monoloc. '2-8 sperm. Sem. angul. rubris. Arbor. fol. imp. pinnatis, fl. panicul1. Z. latifolia. Foliolis 7 oppos. petiol. ovato lanc. leg. rectis uncinatis dispermis-2 Z . parrifolia foliolis alt. sessilib. ellipt. leg. falcatis 7-8spermis. Both trees called Zaga by Rumphius, grow in Molucas. The G. is near Cesalpina, and also $X_{y}$ locarpa by the fruit.

102. Alagopiryla R. diff. Gesneria, Cor. tubulosa, limbo 5lobo equalis, glandulis 2 hypog. posticis ad ovar-A. dasyanthes Raf. Gesn. alagophyla, Martens, Lindley bot. reg. 1767. Villosa, fol. obl. obt. crenatis, fl. spicatis luteis, villosis, limbo flavo. In Brazil. The true Gesneria has the Cor. camp. bilabiate! another Gesneria with unilabiate! corolla, is my Megrapleilis bnlbosa 204.

103. Calacinun R (Cal. bacca) Diff. Polygomm, Fagopyrum, Hclxine, Cal. spart. eq. 
conc. persistens baccans Stam 8. Ovar ovat. Stylis 3 dilatatis, foliaceis, stig. acutis. Sem. ovato incluso in bacca globosa calycina. Frutex volubil. fl. racemosis polygamis $\mathrm{G}$. intermed. betw. Polygon. and Cocolaba, another added to the 10 good Genera blended with the first by authors-1. C.adpressum Raf. (Pol. do Lab. 127. Hook. bot. m. 3145) ramis teretis, fol. cord. sag. marg. scabris. Australia edulis. 2. C. australis $\mathbf{R}$ (Cocol. do Forst) fol. cord. ovatis. N. Zeland.

104. Cocoloba L. differs from last by, Cal. basi carnoso, ovar. immerso. Stam. basi coalitis. in annulo. stylis 3 simpl. stig. obt.-Тур. C. uvifera L. fol. cord. baccis nutans pyriformis, spicatis. 'Tree, Florida Antilles \&c. often figured. many Sp. united thereto must be examined again.

105. Naucorepies $R$ (nut covered) Cocoloba auct. diff. styli dilatatis cuneatis dentatis, Ovar. lobat. Nux. 3-6loba, 1-6sperma in cal. baccans inclusa-Typ. 1. C. pubescens L. 2. puretata auct. et alia sp.

106. Lolanara $\mathbf{R}$ (nom ind) Cal. bisquamosus, Petalis 6, duplice series, 3 int. major. Stam. plurima hypogyna. Drupo ovato, nucleo bivalvis intus pulposo polysp?-L. odorata, fol. ovatis sparsis scabris. Oceanic tree, Lolanwara of Rumphius. Family Hesperidia.

107. Turipa L. Cor. camp. petalis 6 decid: glabris subeq. nectaris nullis, Stam. 6. subeq. filam. glabris, antheris erectis. Stigma. sessile magno 3 lobo trigono. Florib. erectis-Genus very near Fritillaria, Erythromium \&c. many sp. not well described. T. gesneriana, clusiana, suaveolens, oculus-solis, and several N. Sp. all seen alive. 'Type of tribe Tulfrides. 
108. Tulipa unguiculata R. unift. fol. ov. tanc. corolla ovata, petalis flavis apice unguiculatis. Caucasus.

109. Tulipa laciniata R. unifl. fol. ov. lanc. undulatis, corolla dilatata camp. petalis obovatis undulatis laciniatis versicolor. In Hortis.

110. T'ulipa bicolor Raf. atl. J. 1833. Caule flex. unifl. fol. ov. lanc. planis acum. petalis acuminatis, 3 int. obov. albis, 3 ext. ovat. incarnatis. Arkanzas.

111. Tulipa aurea Raf. A. J. 18:33. unifl. fol. ang. canal. apice falcatis, petalis acum. luteis, 3 ext. lanc. 3 int. ovatis. In Gardens from Texas.

112. Tulipa montana R. A. J. 1833. Caule unifol. unifl. fol. rad. angustis planis, petalis lanceol. acutis croceis. Alleghany mountains.

113. Liniopogon Raf. (Lily bearded) Tulipa auct. Diff. Petalis apice barbatis, Stam. barbatis ad basis vel. apice-Types 1. celsiamum, sylvestre, biflornm \&c. all Tulipa of authors; but the first was made Melanthium miflorum by Curtis b. m. 717!

114. Amblimion Raf. 1817. Diff. 10\%. Filamentis toto hirsutis, Stylo clavato piloso, stigma 3gono. floribus nutans ut in Fritillaria-Typ. A. grecum Raf. Tulipa sibthorpiana $\mathrm{Sm}$. unifl. fol. 2 ovatis, petalis obtusis-The doubtful Lilium pudicum of Pursh, since united to Fritillaria, Tulipa, appears to belong here by habit, and a clavate style; but it is perhaps smooth. A mbl. pndicum Raf. 1816 unifl. fol. lin. lanc. petalis obovatis retusis flavis. Oregon.

115. Varroxia L. Cal. tubul. persist. 5dent. Cor. tubul. limbo parvo patulo 5fido, equalis. Stam. 5 eq. in tubo, Stylo 4 fida, Stigma 4. 
Drupa nux Alocul. sepe 4sperma.-Typ. V. linentu, bullata, globosa, alba, geniculata, alia sp. Order ARCYTHIA, tribe AGIPHILIA Raf. 1815.

116. Catovia Raf. (Cato agr.) Diff. Cor. campan. subintegra crenata. stylo dichot. Drupa monosp-Typ. C. lantanoides Raf. Varronia monosperma auct. spicis globosis, fol, ov. lanc. Carracas.

117. Habevaria W. Br. auct. Whoever deems the numibers of Stamina unimportant in Grasses, Lilies \&c. must regret that this G. and many other Orcmines are based on their double number, altho' a most essential character : 1 and 2,3 and 6,5 and 10 stamina, atways distinguish very distinct Genera, and whoever unites Azalea to Rhododendron sins against Linneus and Nature! meantime notwithstanding the learned labors of Swartz, Brown, Richard, Lindley ... on Orchines, they have left Hutienaria, Orchis, Epidendron and other G. in utter perplexity. The last char. of Habenaria is merely a Cor. ringens, labellum calcaratum, antheris nudis binis-while other Genera have elaborate definite characters of 50 or 60 words. Habenaria thus includes many blended $G$. and requires complete reform. I shall now begin it, and give a new essential character of Habenaria Raf. Cor. ringens, lab. ad basi calcarato, glandulis nullis, col. simplex, antheris 2 divisis nudis anticis vertical. Caulescens. fl. racemosis-Týpes the Amer. Sp. having those char. H. fimbriata, O. habenaria, and akin Sp.

118. Platantirera Richard. Diff. Cor. patula, columna dilatata, antheris 2 terminalis lateralis nudis. remotis. Scaposis, fol. binis 
rad. amplis.-'Type the various sp. blended as Orchis or Maben. bifolia, now 7 or 8 Sp. many new in the Alleghany mits. I do not know the sub-genus Mecose of lindley, is it my next Genus?

119. 'Tuotis R. (wart ear) Difi. 117. Label: ad basis supra uniglanduloso, ad latere 2 auricul. s. bidentato. Col. brevis dilatata biloba, antheris ut Platanthera, habitus ut IIabenariu, floribus fragrans. Types 4 Sp. described 1832 in my Herb. Raf. H. herbiola, fuscescens \&c. of authors. See my Flora N. Ainer.

120. Digonphotis R. ( 2 club cars) Diff. $11 \%$. Sepalis connivens subeq. Label. cuculato, basi calcar saccato. colum. ad latere appendiculata, auriculis claratis, (an antheris ster?)-Types the 2 following sp. and others.

121. Digomphotis cordata Raf. Foliis binis alt. cordatis acum. 5nervis, fl. viridis bracteis equante, lab. trilobo, calcar breviss. obt.-Portugal, Madera. Habenarin cordata Br. Hook. misc. t. 55. b. mag. 3164. "Satyrium diphylum Link.

122. Digomphotis undulata Raf. Fol. pluris ellipticis obtusis undulatis, spica densa, bract. fl. longior, sepalıs 3 ovat. obt. ext. viridis, petalis int. albis. obov. lab. integro ovato, calcar globoso-India, Nepal. Haben. goodyeroides (n. barb) Don. Spreng. Hook. b. m. 3397.

123. Pecterlis R. (comb. lip) Diff. 117. Corolla patula ineq. sepalis 2 int. minimis, label. amplissimo 3 part. med. minor integro, lobis later. amplis flabellatis. pectinatis. Col. elongata, antice bidentata. antheris lateralis, apice annexis. basi auriculatis. Florib. amplis, paucis, fragrans. a beautiful $\mathbf{G}$. near Bartholinia of Lindley 
'Jypes 3 sp. which roots are tuberose, Ovary terete, Spur long, and fiagrant flowers.

124. Pecteilis gigantea R. fol. ovatis, florib. 4-5, bract. lanc. ovar. tereto subeq. petalis albis, 3 ext. obov. obt. 2 int. lin. falcatis-India, Nepal. Stem 4 feet, flowers 5 inches. Habenaria gigantea Sm. Ex. bot. 100. Hook. b. m. 3374 .

125. Pecteilis susunna R. Fol. ensif. canalic. florib. sub 3 petalis albis, 3 ext. subrot. lab. lobis ciliatis-Amboyna, Stem pedal, fl. 2 inches. Orchis susumna L. \& auctoris.

126. Pectuilis radiuta Raf. Diff. 125. Petalis 2 ext. ovatis acuminatis.-Japan. Orchis radiata Pers. O. susanna. 'Thunberg.

127. Blepinariglotis Raf. (ciliate glotis) Ovar. desinens in appendice tereto subtus fl. Petalis 3 ext. in galea connivens, label. ligulato fimbriato; Col. dilatata, utrinque latere glandula magna, antheris lateralis remotis clausis inter cella bialata bivalvis - striking $\mathbf{G}$. of North Amer. Types the 5 next Sp. Similar habit, stemleafy angular spike short, $f$. handsome inodorous, leaves few carinate lanc. pollen caudate, only one in each cell or anther. All seen alive.

128. Bleplear. albiflora Raf. Elatior, fol. acutis spica oblonga, petalis albis, 2 internis obl. apice laceris, calcar elongato, label. convexo margine reflexo, apice acuto panlo ciliato- $\mathbf{N}$. Amer. paludis, Orchis et Haben. blephariglotis auctoris, O. ciliaris alba Mx. oft. figured.

129. Blephar. longicornis Raf. Elatior, fol. obtusiusc. nervosis, spica oblonga, petalis albis, 2 internis apice ciliatis, calcar longissimo divaricato, labello lineari, basi ciliis brevis, apice lacero fimbriato-Alabama, Louisiana. fig. antikon bot. as all the sp. 
130. Blephar. bicolor Raf. Caule biped. fol. venosis obtusiusculis, spica ovata, petalis fulvescens 2 int. subintegris calcar elongato albo, label. albo plano pinnato fimbriato, apice truncato lacero-Nova Cesarea, in aquosis: apparently a hybrid of 128 and 131, yet Orchides cannot produce hybrids, the fecundation being so intimate: it is rather a deviated N. Sp. beautiful, the yellow is pale, nearly buff color.

131. Bleplear. flaviflora R. Caule 1-2ped. fol. subnervosis obtusiusc. Spica ovato, petalis flavis croceis, 2 int. subintegris, calcar elongato label. plano pinnato. fimbriato, apice truncato longe fimbriato-N. Amer. ad pratis. 'The most common sp. in mts, and plains. Orchis s. Hraben. ciliaris auct. fl. smaller than in the others.

132. Blephar cristata Raf. Humilior, fol. acutis subnervosis, spica oblonga, petalis croceis, 2 internis subrotundis cristatis, calcar brevis ad ovar. dimidio, labello obl. pennatim lacero-Virg. ad Florida. Flowers smaller still. Orchis cristata Mx. et auctoris.

133. Mesicera R. neog. 1825 (middle horns) Diff. Orchis. Petalis internis bipart. Lab. tripart, lobis lateralis setaccis. anthera unica 2 pollen, ad basis bicornuta. capsula trigona-Types 2 Sp. 1 Mes. Michauxi R. O. 5seta Mx. and 2 Mes. repens R. Habenaria do Nut. El. see Herb. Raf. and new flora.

134. Galearis.R. h. 1833 (helmet like) Diff. Orchis. Petalis 3 supernis coalitis in unico galeaformis concavo fornicato, labello indiviso anthera bipollen. Radix ramosa, scapo paucifolio paucifloro-Striking peculiar habit, types 2 sp. Gr. spectabilis and Gr. biflora Raf. herb. Raf. both blended as Orchis spectabilis by authors. fig. autikon bot. 
135. Prectrumus Raf. 1819, neeg. 1825 (spu: tail) 'Tipularia discolor Nut. nom. absurd. ad Tipielteria, et nonnulis auct. Orchis et $\mathbf{L i}$ modorum alia auct.-Difr. Orchis Petalis patulis spatulatis, lab. integ. Col. aptera porrecta libera, anthera opercul. articul. bivalvis 4 pollen. Rad. monilif: fol. rad. unica, scapis, $f$. racem. nutans ebract-Habit quite different firom Orchis, the spur and anther only akin. nearest to O. abortiva, another N. G. Epipactis.

136. Orcnis L. Br. auct. Corolla ringens, lab. basi calcaratum, diviso, sine glandulis, anthera unica 1-2glandulosa, cuculata. Caulescensfoliosis, fl. spicatis - Such are the characters of the true Orchis. But many Sp. offer yet disparities that deserve to form sub. genera at least. 1. Tephrosanthos $R$. petalis connivens, calcar incurvo. 2. Pomoplis-R. petalis connivens, calcar recto. 3. Rhizarina R. petalis patulis s. reflexis: which may offer other subdivisions.

137. Larnandra R. neog. 1825 (cell st) Diff. Epidesdron ovar. filif. Lab. lobato adnato supra biglanduloso. Col. elongata cuculata fornicata anthera unica ; inclusa operculata 4 locul. globosa. Rad. vermicularis, Caulib. paucifol, paucifloris-Type L. conopsea Raf. Epid. do Br. et auct. Hook. bot. m. 345\%. 'This and the next G. are no more real Epidendr. than my Nyctosma. Many G. are yet blended under the absurd characters of, lab. libero vel adnat. calloso, costato, vel tuberculato nunc. calcarato! 'The only common char. being petalis patens, col. erecta, anthera carnosa 2-4loc!

138. Caularturon $\mathbf{R}$ (Stem jointed) Diff. Epideudron. Label. libero, ad lasi alato s. 
glanduloso. Col. libera bialata dentata, anthera terminalis 4 pollen. Cuul. urticulato, vaginato, bifolio, paucifloro-Habit very singular. Types ¿Sp. 1. Canil. bicormutum. Epid. do. Hook. b. m. 3332. Bulbo caulifómis, fol. rad. ligul. retusis, scapo paucifl. label. trilobo, medio lanc. ac. basi bicorne, petalis ellipt. acutis albis. Trinidad-2 Cuul. umbellatum R. Epid. stenopetalum Hook. b. m. 3410. Bulbo ramoso, caule scaposo, fol. terminalis lin. obl. obt. ft. umbellatis purpureis, labello integro obovato obt. basi glandula maǵna quadrata, petalis ovatis acum, 3 ext. angustior, col. alis dentatis. Jamaica.

139 Telesia R (Telesio phil) Periantho oligophylo imbric. ext. major. Phorantho paleis carinatis, fl. radiatis, radiis paucis fem. 3 dent. achenis trigonis tridentatis, in disco triaristatis. Fol. oppos. habitus Helianth.-Telesia aurea Raf. Wedelia? aurea Don, Hook. b. m. 3384. Scabra ramosa, fol opp. sess. ov. lanc. serratis acutis, ramis trifloris, per. sepalis ovatis acutis. Mexico. very near Helianthus, merely diff: by few leaved perianthe, and trigone seeds. Wedelia with rays or no rays! seeds urccolate or uniaristate, is far more remote, and a confused Genus.

140. Solmago L. auct. This G. with Erigeron, Inula and Aster were in utter confusion, the determination of Sp. hardly attainable, altho' offering many excellent permanent charar:ters for N. Genera. I began in 1807 to reform them; since then my labor has been partly anticipated by Cassini, Esenbeck, Nuttal and Lessing, but they have left the others nearly untouched. I now mean to revise them, leaving most of the Asters for further enquiries. For the N. Sp. see my Flora. 
141. Sommaco Raf. Car. ref.-Perianthus obl. imbricat. clausus. Radiis 3-10 ineq. integris, flosculis paucis, phoranthus nudus, Semina obl. pubesc. pappus caliculatis, levis pilosus. Caul. erectis, fol. sparsis fl. vacemosis parvis bractcolatis, plcrumque flavis-many sub-genera. 1. 'Triactis. Rad. 3-4 brevis cuneatis, flosculis 3-5. 'Typ. 1. S. retrorsa Mx. 2 tortifolia E2 Brachyactis, Rad. 5 cuneatis brevissimis in periantho inclusis. 'Typ. 3. S. juncea L. 4 canadensis L. 5 procera Ait. 6 aspera Ait. et. alia. sp.-3. Albigula, Rad.5-10 albis elongatis cuneatis deinde reflexis. Typ. 7. S, bicolor L. et. Sp. affinis-Plemactius, Rad. 5-10 lanceolatis brevissimis, plurima Sp. S. flexicaulis, villosa, ulmifolia, cinerascens, \&c.-5. StenacTILA, rad. 5-10 linearib. elongatis, plur. sp. S. sempervirens, augustifolia, pubescens, elliptica, Virgaurea? \&c. Doria Adanson, Virgaurea Tourn. are the same Genus as Solidago.

142. Leioligo $R$ (smooth few) Diff. 141. Radiis 5-10, Semina glabra, pappus non caliculatus, fiorib. nonmelis corymbosis-3 sub. G.-1. Brevigula, Rad. 5. brevissimis cuneatis. Type. L. cesia L. 2 pyramidata P- 2 Lininque, Rad. 5 linearib mediocris. 4 L. speciosa Nut. 5 salicina E. \&c.-3. Doria, Rad. 6-10 elongatis lin.lanceol. plurima sp. L. petiolaris Ait. erecta P.corymbosa E. pulverulenta. N. rigida L. elata P. lithospermif. \&c. all Solidago of authors: and several N. Sp.

143. Aplactis R (simple ray) Diff. 141, Radio unico, flosculis 5, Sem. glabra, 17 . paniculatis-Type Apluctis paniculata Raf. Solid. pauciftosculosa Mx. et auct. near to Sub. G. Triactis. 
144. Lepiactis R (scaly rays) Difl: 141, Perianth. ovato, rad. 5-7 ineq. squama ad tubis radiis, flosculis 7-8, Sem. striatis villosis, pappus calicul. piloso, scabro. Racemis virgutis-Type Lepiactis virgatu R. Solid do Mx. et auct.

145. Dasiorma R (hairy pits) Difl: 141, Perianth. globoso, phorantho alveolato, marg. ciliatis. Rad. 7-10 elongatis, disco multifloro, Sem. pubescens. Racemis paniculatis-Types several sp. blended as Solid. mexicamı anct. 1. D. mexicana Raf. Caule corymboso, fol. lanc. sessilis, pedunculis nudis uniflorus. Probably the original Sol. mexicunu L. Dod. t. 219. Loud. 12099-2 D. limonifolia (Sol. do Pers) Glabra paniculata, fol. lanceol. infimis ellipt. pedicellis 1-3fl. squamosis. N. Am.-3. D. ellioti Raf. Sol. lim. E) Glabra panicul. fol. angusto lanceol. marg. scabro, imis amplex. pedicellis sq. 1-3floris. Carolina.

146. Eutuama Nuttal diff. 141, Periantho tereto, phorantho setoso, rad. brevissimis, $\boldsymbol{f l}$. corymbosis-Chrysocoma of Amer. authors.

147. Actipsis Raf. (Rays false) Diff. 141, Periantho turgido campanul-sepalis laxis $\mathbf{s}$. reflexis, Rad. 10 brevissim. obov. disco multifl. Sem. glabris, pappus scabro.-Types $\boldsymbol{A}$. glomerata Raf. (Solid. do Mix.) fl. glom. per. lax. 2. A. squamosa anct. fl. racemosis periantho reflexo. 'This quite akin to Asters.

148. Dectis R. (nom. antic) Diff. 141, Periantho sepalis carinatis, ext. laxis internis connivens. Rad. 7-10 ovatis. Sem. glabra. Fruticosis, fl. coryml. s. thyprsoideis-Types Dectis (Solid. anct.) arborescens, leucud, rugosa (spuria)-decurrens Lour. Rad, 7-8, fl. thyrsoideis. 'These are Asiatic or African shlrubs. 
149. Ongactis Raf. 1804! (few rays) Aster auct. Comyza asteroides L. et Sp. affinis. Perianth. tereto imbricato, sepalis scariosis coloratis, phorantho punctato, Rad. sub 5 ineq. bidentatis. Sem. angulata pubescens pappus pilosus scaber. Fl. corymb. albis-G. as near the Solidlago group as to $\boldsymbol{A}$ sters, Types $\boldsymbol{O}$. linifolia, conyzoides, tortifoliu, and several N. Sp. in my Flora.

150. Mesoliaus $\mathbf{R}$ (middle few) Diff. 149. Per. sepalis adpressis subul. nonscariosis, rad. 2.̈-30 brevissimis, ineq. 3dent. disco paucifloro 6-10f. Sem. glabris teretis striatis fl. panic. rad. purpareis-'Type MI. subulatus Raf. Aster do Mx. auct. et alia sp. G. near Erigeron, Cenotus, \&.c.

151. Galatella Cassini auct. Diff. 149. Rad. integris abortivis, phorantho alveolaris, marg. lacero. A. purp. 'Types Aster acris, hyssopifol. punctita \&c. auct. mod.

152. Avactis $\mathbf{R}$ (no rays) Aster discoideus Elliot. Periantho hemisph. imbricat. sepalis subul. squarros. laxis, Phorantho parvo nudo. Rad. nullis, flosculis 12-15, omnis herm. Sem. oblongis glabris, pappus simplex scabro $f l$. panicul. purpureis.-1. A. pilosa, Caule erecto villoso, fol. spathul. oratis pilosis. Carolina-2. A. glabra. Glabra, fol. lanceol. spatulatis. Alabama. Near Eupatorium and discoidal Erigerons.

153. Аgсотомa $R$ (uncut lig) Diff. 149. Per. sepalis squarrosis ciliatis non scariosis, Rad. paucis 3-8 integris oblongis. $f l$ panic, ceruleis; Type. Aster multiflorus M. alia sp.

154. Asteriscus Tourn. Aster L. auct. Pinardia Necker Aplopapus Cassini. This vast G. is now greatly reduced and confined to the 
sp. with Per. hemispher. ineq. ext. laxus imbricatus, Radiis 10-20 simpl. series ang. vix. dentatis, sem. ang. pubescens, pappus scabro pilis caliculatis-The name Aster root of Asterius and others must be lengthened as did T. I refer to Esenbeck \&e. for Sub. G. and akin Genera; but some of his are falacions, Symphotrichum for instance says Hooker, in fact many Asters have annular pappus at base. Meantime I add the indication of my own Genera settled between 1815 and 1825 .

155. Leincinenis Raf. (smooth ach) Diff. 154, Sem. levis, pappus simplex non caliculato; Several Sub. G. 1. Psukelis. Per. foliaceus, radiis 15-25. A. paludosus L.-2. Merisis. Per. laxis, Rad. paucis 10-12. A. cordifolius, paniculatus \&e. - 3. Dodecalis Per. sep. adpressis, rad. 10-12. A. corymbosus, dumosus Ac.-4. Finibramis. Per. sep. adpr. fimbriatis, rad. 15-25, A. foliolosus, sparsiflorus \&c. auctoris. The shrubby Asters are akin to this Genus. A. sericeus of America has 10 rays, lanc. acute, entire, $\boldsymbol{A}$. argopluylus of Australia has only $\mathbf{3}$ rays, $\boldsymbol{A}$. angustif. of Africa has many oblong rays nearly biserial. They probably form as many Sub-Genera. My Venatris is another perhaps, see 163 .

156. Drplactis R (double rays.) The Asters with crowded long rays in double or triple series, narrow linear subentire, pappus simplex. Such are A. alpinus, caucasicus, novanglia, cyaneus, blandus \&c. Akin to Leiachenis, seeds commonly smooth.

15\%. Stenactis Cass. auct. Differs from Diplactis by Per. subequal. phoranth verrucose, pappus duplex, ex. brevis paleaceis-Aster chinensis type and many Sp. to he distinguished 
from the last. 'I'he name means narrow rays, and not to sigh as stated by Lindley.

158. Kalimares R. (nom. diosk) Diff. 155. Periantho sep. ext. reflexis, internis erectis scariosis coloratis. Rad. duplice series-Type A. amellus L. Genus Amellus 'T. Ad. but Linneus has given that name to another Genus.

159. 'Tripolion $\mathbf{R}$ (nom. diosk) Diff. 155. Per. sep. subequalis duplice series adpressis membranaceis. Radiis unica series-Types Tripolion maritimum Raf. Aster tripolium L. auct. obs. v. 1801-2. A. tenellus L. 3 A. tataricus $\mathrm{L}$.

160. Fimbristina R Diff. 154. Pel. laxis. Rad. 16-20 lin. lanc, apice 3dentatis, stigmata fimbriatis, Sem. et pap. ut 154-Aster squamosusI. sparsijl. P. flexuosus N. maritimus Raf. et alia.

161. Diplopapus Cassini. Diplostephion Kunth. Chrysopsis Nut. Diplogon Raf. 1817 not R. Brown, name preocupied. How many names! this shows that many did perceive the striking characters of the double pappus. Meantime this G. must now be restricted to those nearest Asteriscus 154. differing by, Ovarium tereto hirsuto, pappus, biserialis conformis scabris, ext. minor,-It may be divided in many Sub. genera and has some blended G. yet-1. Chrysopsis fl. yellow terminal. 2. Leucalis fl. white corymbose. 3. Cerulinis ft. blue terminal. Aster aurantins and pinnatns of Mexico with pinnate leaves and yellow flowers are probably a peculiar G. Diplostephion?

162. Vingulus R (small rod) Diff. 161. Periantho sep. adpressis. Rad. paucis integris, Sem. obovata villosis, pappus duplex. ext. brevissimo villoso ut caliculo, interno aristis color- 
atis ineq. levis, florib. racemosis purp.-'Гype Aster concolor L. and akin sp. habit of Miatris.

163. Vranatzes R (3 veins) Per. campan. sepalis laxis imbric. subeq. Phorantho punctato. Rad. 10-1:2 linear. integris trivenis. Sem. teretis tenuis glabris. Pappus simplex brevis coloratis equalis levis-Types A. pauciflorus N. linifolius, temifolius, sulicifolius? Ec. and the next N. Sp. best type of the $G$.

16.1. Venatris falcata Raf. glabra, canle subangul. 3-5floro, fol. erectis sessilib. falcatis lin. lanceol. acutis uninervis, sulintegris, pedunc. pubescens squamosis, sepalis oblongis, $f$. purpureis-East Kentucky, falls of Cumberland, pedal.

165. Crontron R (col. feather) Diff. 154. et. 61. Pet. Rad. paucis integris, Sem. obl. teretis hispidis, pappus coloratus scabr. simplex ad pilis caliculatis, $f l$. paniculatis flavis.-This G. would be near Imula of L. but now this is restricted to I. helenium and the bisetose sp. 'The G. Pulicuria, Lioydia Necker, Limbarda $\Lambda d$. are badly distinguished; but having the perianthe variable are different from thisType Cropt. divaricatum R. Chrysopsis do N. E. fol. lin. lanc. hispidis serratis ciliat. panicula divaricata. Florida, anthers mutic.

166. Stelmanis $\mathbf{R}$ (cor, diff.) Perianth. tereto 100 phylus imbricatus phorantho favoso, Rad. 20 lanc. nervosis. anther muticis Sem. hispidis ad radi cupula integra membr. coronans, sem. disci pappo duplex, externo membr. polyph. interno piloso scabro. fl. panic. flavis-a very distinct G. Chrysopsis scabra Elliot, who sugested Culycium for a name, but it is bad. Stelm. scabre fol. scabris punct. rad. petiol. ovatis, caulinis cord. sessilibus. Carolina-I add the 
varions Genera of Imula 167 to !7\% to show the contrast.

16\%. Invla L. anct. Per. squamosus foliosus globosus, rad. plurimis, antheris bisetosis, pappus simplex scabro \&c. 'Type I. helevium, $\boldsymbol{I}$. squarrosa, hirta, salicina, hirsuta! \&c. fl. yellow in all.

168. Liovdia Necker, diff. 167, Per. subeq. sepalis 10-15. 'I'ypes doubtful.

169. Pulicaria Raf. diff. 167, Per. imbric. adpresso, rad. paucis brevissimis 3dent. pappus caliculatis \&c. Types P.vulgaris (Inulu pulic) 2. I. indica. 3. I. dysenterica, 1. fétida etc. 170. Limbarda Ad. diff. 167. Per. tereto s. ovato adpresso \&c. Types Imula crithmifolia, arabica, japonica, pinifolia ofc.

171. Astrop'terus Vaill. Ad. diff. 167. Phorantho reticulat. foveolaris. Pappus duplex, extern. membrana ciliata. Frutic. fl. cervuleis'Typ. Inula cerulea L.

172. Orestion R. (n. gr. Inula) Olearia Mench, nom. mal. ad Olea!-Diff. ad omnis Aster, Inula, Pappus plumosus, basi comatus. Frutex-'Type O. dentata of Australia. Aster do Andr. t. 61. tomentosus W. 6.

173. Pappocirroua R. Periantho tereto imbricato. Rad. 20 cuneatis 5dentatis, stigmata 2 clavata, Sem. ang. Pappus simplex coloratusP. uniflora Raf. Erigeron pappochroma Lab. nearer Asters.

174. Erigeron L. auct. Panios Ad. In Linneus only differs from Aster by having many narrow rays as Stenactis and Diplactis! and containing a crowd of Generas or deviations: I restrict the G. as follow and separate the Genera $\mathbf{1 7 5}$ to 182-Erigeron Raf. Per. hemispher. imbric. sepalis lin. subeq. deinàe reflexis. 
Rad. multiplis sepe duplice series, linearis integris elongatis. Phorantho nud. punct. antheris muticis. Sem. obl. hispidis, pappus duplex, ext. brevi, interno 15-25 aristis. fl. scpe corymb. albis-'Types E. philadelphicum, asperum, pumilum, strigosum, gramineum, \&c.

175. Paniopsis Raf. (Erig. grec) diff. 174, Per. obl. laxis seu squarrosis ineq. Rad. brevis unica series sepe 2-3dent. antheris bisetosis ut in Inula Pappus subsimplex. fi. sepe panicul. rad.luteis -Near to Inula, only difference short rays, per. hemisph. 2. Sub. G.-1. Graveolaria types E. graveolens, glutinosum fetidum, \&c. 2. Squar elaria, E. viscosum, \&c.

176. Deinosuos $R$ (Conyza diosk) diff. 174. Per. obl. sepalis ext. laxis elongatis, Radiis nullis, flosculis. ad rad. femineis, antheris bisetosis pappus simplex. fl. axill. luteis-Type D. siculus Raf. Erigeron do L. W. Inula, Solidago Chrysocoma! of other authors, thus shuffled iuto 4 genera, till it will rest in its own! fol. lin. lanc.'scabris marg. revol. subintegro. Sicilia.v.v.

17\%. Enemias R (Conyza gr.) diff. 174. Per. globosis. Radiis minutis inclusis brevissimis vagineformis, flosculis paucis, pappus simplex. fl. corymbos. albis-Types Conyza egyptiaca and gouani L.

178. Brepiocton R (Conyza gr.) Diff. 174. Per.lenticularis, florib. discoideis, rad. nullis flosculis ad ambitu plurimis femineis apetalis, stylis nudis. flosc. centralis paucis. Frutic. fl. axillTyp. Br. cuneatum Raf. Erig. do. L. fol. cuneatis trilobis. Mexico. Near to Baccharis, Gynmostylis and Leptogyna Elliot. The 3 Genera 176 to 178 are nearer Conyza than Erigeron; but the female floscules are outside 
and unmixed. 'The Erig. camphoratum is a Leptogynn:

179. Fragmosa $R$ (Conyza gr.) Diff. 174. Rad. simpl. series 20-30. Sem. glabris. Pappus duplex, ambi subeq. aristis brevis 10-12, vel. simplex duplice series definita. A. unicus $s$. pancis term. albis-Types Erigeron alpinum, uniflorum, pumilum, asperum. nudicaule, glabellum? \&c.

180. Musteron $\boldsymbol{R}$ (Conyza gr.) Diff. 174. Per, vere hemisph. equalis, sepalis dupl. series, Rad. longis unica series lin. bident. Phorantho convexo punct. Stigm. clavat. integ. Sem. glabra, compr. marg. Zalato. Pappus simplex aristis unica series 25 -40 scabris. fl. paucis term, ceruleis-Type Musteron bellidifolium Raf. Erig. do anct. v. v. nearer Asters, habit of Daisies and 179. Several blended Sp. M. pulchelum, uniflorem, glabrum Raf. see Flora.

181. Camotus Nut. doc. diff. 174. Per. tereto obl. s. ovato, radiis brevissimis minutis vix exsertis, flosculis sepe $\mathbf{4}$ fidis, stigma clavato integ. Sem, obl. compr. biangul. levis. Pappus simplex, aristis paucis 10-15. fl. sepe paniculatis flavulis-Types Erig. canadense, pusillum \& c. longifolium auct. with my N. Sp. C. altissimnes, mavitimas, uniflorus, diffusus ofc. see my Flora.

182. Diplenum $R$ (double half) diff. 174. Phorantho convexo. Rad. dupl, series multiplex 100-200 apice 2-3dent. Pappus radii simplex, pappus disci duplex. fl. corymbosis albidisTypes E. Erig. carolinionum L. and quercifolium Lam.-2d Sub-G. Leptinum, diff. pappus radii brevis squamatus, pappus disci ext. ut radiis. fl. panic-Types 3 Erig. nervosum P. not W. 4. Erige. strigosum E. not W. Diplem. 
s. Lept. paniculat. Rat. Does Erig. Buhumense scop. t. 7. Vitm. belong here? a Shrub with per. round, many rays 3 dent. Perhaps a peculiar G. Tanaxion tomentosum Raf.' fol. pet. lanc. toment. fl. corymb.

183. Orgasis $\mathbf{R}$ (nympha) Orchidea diff. Oncidium et Cymbidium, Sepalis 5 ut Cypripedium, 2 ext. calicinis oppos. inferum bidentatum, subtus lab. planum. Columna basi cum glandula et labello confluens apice latere bialata, anthera unica antica in forea, pollen bilobo pedicellato. Scaposa, f. spicatis-Olgasis $t \cdot i$ quetra Raf. fol. triquetris. scapo subnudo, spica multif. laxa, petalis ovatis, labello subtrilobo. Jamaica, pretty flowers variegated white and red. It was Cymbidium do Sw. Orecidium do Br. Hook. b. m. 3393: but very distinct from both Genera, nearer Plerostylis $\mathrm{Br}$.

184. Deppia R (bot) Maxillavia deppi Lod. b. c. 1612 Hook. b. m. 3395. diff. Maxil. Sepalis 6. ext. 3. calicinis nervosis patens, binis subcalcaratis ad basi coalescens. Labello cucul. basi glanduloso. Col. pet. adnata, anthera term. subrot. pollen bilobo. Bulbis terrestris, fol. et Scapis mifl. ferens-Deppir mexicana Rat. fol. ellipt. undul. Scapis brevis squamosis, sepalis 3 ellipt. viridis, petalis albis obovatis, labello bigulato undulato. Mexico large flowers. Totaly different from MLaillaria.

185. Eltroplectris $R$ (fiee spur) difi. Neottia et Sienorynchus, petalis 3 sup. ringens, 2 patulis acum. lab. basi cucul. calcar elongato libero basi coalit. cum ovar. Columna bilabiata, anthera lanc. ad lab. sup. Scap. vorgin. paucifl. fol. inferis-E. rcuminata Raf. fol. petiol. ellipt. acum. undul. Spica 5-61t. calcar incurvo ovar eq. petalis lanc. acum. lab. conforme den- 
ticulato. Antillis. fl. viridescens. Neottia calcarata Sw. Hooker b. m. 3403. Sten. calcaratum Rich. Sprengel. \&c.

186. Endeisa R. (Nympha) diff. Dendrobium. Petalis patulis, 2 inf. basi connatis saccatis, lab. lobato basi cucul. apice reflexo, Col. quadrata decurrens, anth. term 2loc. 4poll. globosa. Caule artic. fl. spicatis-Type Endeisa flava Raf. Caule sulcato, fol. term. lanceol. spica lateralis laxa multifl. petalis ovatis acutis flavis, labello croceo, trilobo serrulato, lobis subrotundis. Nepal. Dendrob. densiflorum! Wallich, Lindley, Hooker b. m. 3418, totaly unlike the Amer. sp. of G. Dendrobium which is yet a very loose incorrect Genus.

18\%. Vindicta $R$ (Epim. lat.) Cal. 4 ph. eq. color. Petalis 4 planis oppositis. Stam 4 oppositis subsess. anth. linearis biloc. Ovar obl. Stylo lateralis, tereto, stigma truncat. Caps. uniloc. polysp. sem. unica series dorso insertis. Caulescens, fol.paucis obliquis, fl. racemosis-Vindicta begonifolia Raf. filiformis foliis $2-4$ foliolis petiol. ellipt. obtus. integris, basi oblique cordatis, racemis secundis paucifl. fl. cernuis albis, petalis obovatis cal. obl. superans. Japan. Epimedium diphylum Lod. 1858. Hooker b. m. 3448. Quite a distinct Genus like the next.

188. Sculeria $\mathbf{R}$ (Scooler bot) Cal. sepalis 8 ineq. reflexis. Petalis 6 cuculatis reflexis. Stam 6 opposita. Stigma capit. Caps. polysp. Scaposis, fol.comp. ff. racemosis-Sculeria geminata, Raf. foliis. bi-triternatis, foliolis petiol. cord. 3-5lobis. fl. racemos. luteis, geminatis. Ad. Oregon-Epimedium hexandrum Hook. fl. bor. Am. I add the real G. Epimedium to show the striking contrast. 
189. Eprmenium L. auct. Cal. sepalis 8 ineq. 4 opp. internis. Petalis 4. cyathiformis. Stam 4. Capsula acum. monoloc. bivalv. polysp. Caulesc. fol. tritern. fl. panicul-Ep. alpinum. foliolis cordatis dentatis, fl. panic. purpure-flavis, sepalis emarg. Alpis. Thus these 3 G. altho' all belonging to BERBERIDEs, agree in nothing but the fruit; the opposite stamens with rolling valves of the anthers are common to the whole tribe.

190. Gypsopinla L. auct. Lanaria Ad. Cal. camp. 5dent. 5ang. nudum, petalis $\mathbf{5}$ obovatis subsessilis non unguicul. Stam. 10 subeq. hypogynis. Ovar. sessile ovato, Styli 2. Caps. monoloc. semi 4 valvis, polysperma. Sem. centralis non alatis-This is the strict essential character, which applies to many Sp. in 3 sub-G. VACCARIA petalis emarg. G. vaccaria, repens, cretica graminea \&c. 2. Gypsaria petalis crenatis reticul. G. muralis \&c.-3 Aploma petalis integris. G. struthium, fastigiata, perfoliata \&c. Auctoris. Sir J. Smith has united to this G. Saponaria vaccaria, cretica, illyrica, wondering how L. could put them elsewhere, their calix is 5angular, but they must be examined again as to stamens, capsule, seeds, \&c.

191. Arrostia (Arrosto bot) Raf. car. G. 53 in 1810. Diff. Cal. camp. prof. 5fidus, non angulatus. Stam. 10 eq. Caps. globosa bivalvis oligosp. Sem. obl. alatis-I justly separated this G. long ago: the Gypsoph paniculata and altissima may belong to it; but the type was my next N.Sp.

192. Arrostia dichotoma Raf. car. 189. Caule erecto dichot panic. fol. lanceol. glabris univervis acutis crassiusculis, fl. pedic. ad dichot-Mts. of Sicily, Nebrodes, 3 to 4 feet, 
much branched, fl. white not dioical petals obovate entire. 'To show how the 2 akin $\mathbf{S p}$. difler, I add them here.

193. Arrostia? paniculata. Caule humifuso panicul. basi villoso, fol. lin. lanceol. uninervis margine scabris, fi. panic. dioicis, petalis revolutis-Austria, Sibiria. Gyps. panicul. L. Jaq. Aust. t. 1. et auctorib.

194. Arrostia? altissima. Caule giganteo panicul. erectis, fol. caul. obl. obtusis glabris trinervis subtus glaucis, ad ramulis longo lanceol. acutis. fl., corymbosis exiguis-Sibiria. Gyps. altiss. L. auct. Gm. 4. t. 60. The figure of Bocconi t. 5. f. 3 refered here belongs rather to 192 ; but no doubt these 3 plants have often been mistaken for each other by authors.

195. Tunica Raf. (old name) Diff. 190. Cal. basi caliculatis ut Dianthus, petalis subunguic. Stam. ineq. 5 alternis ad unguis ut Dianthus. Caps. subsessilis obl. teretis 4 dentata-Types Gypsoph. rigida. 2. G. saxifraga once his Dianthus do, both have notched petals: 3. G. dianthoides Sm. fl. gr, Fl. capit. bract. pet. integris, fol. lin. has the habit of Dianthus armeria. This G. is nearel Dianthus than Gypsophila. Is the calix angular in all? It is the old G. Tunice of Dalechamp, but that of Dillen and Adanson was the real Dianthus of.L. The G. Asophila of Necker, dicline with 5 sterile stamens, is unknown to me: did he mean Gyps. paniculata? or saxifiuga stated to have 8 or less stamens sometimes?

196. Endopogon $\mathbf{R}$ (inside beard) Cal. super. 4 dent. Cor. tubulosa campanul. 4 fida, intus barbata, antheris 4 sessilis, capsula 4 dentata ' 2 sperma. Fol.opp. imbric.jl. axill-G. of Rubiacea, near to Hedyotis, Spermacoce, Diodia, Hous- 
tonia, differing firom all by the bearded corolla inside, concealing the anthers and stigmaType $\boldsymbol{E}$. pumilum. Caule pumilo erecto basi nudo, fol. imis imbricatis, linearib. acutis marg. revolutis, capsulis obov. pubescens-Mts. Apalaches, in IVasioto hills of Last Kientucky. annual, biuncial, fl. axill. solitary, sessile, rather large; whitish, capsule large. I can find no synonym to this plant, and it is probably quite new, discovered in 1823. Autik. bot. and Ic. rar

197. Comarum L. Pancovia Heist. Ad. Some late botanists have rejected this linnean $\mathbf{G}$. and made it a mere section of Potentilla; yet it is as distinct as Tormentilla and Fragaria; it comes nearest this last; but has a larger foliaceous colored cadix 10-14 parted, petais shorter. 5 to 7 and red; receptacle and fruit ovate similar thick, but fungose instead of fleshy, besides the pinnate leaves. I can increase it to 3 Species; all in my Autikon. Only one was known. If Arum is retained Com-arum is a bad compound name, Pancovice is better.

198. Comarum (or Pancovia or Potentilla) palustre L. fol. pinnatis, petiolis vaginatis nudis, foliolis 5-7 ellipt. grosse serratis subtus pallidis, fl. corymbosis petalis ovatis acuminatis-In Europe and Sibiria ; it has even 2 varieties or deviations. 1 stipulatum stipulis parvis lanceol. petalis ovatis ligulatis obtusis-2 villosum, foliis villosis. Pluk. t.. 212. Pers. Probably several Sp. blended yet, as the two next were.

199. Comarum (Panc. Pot.) digitatum Raf. Caule hirsuto, fol. glabris, digitatis 3-5nis, stipulis ovatis, foliolis :3-5) ellipt. hasi integris, apice argute serratis, subtus glaucis; fl. corymbosis, petalis ovito lanceol. acuminatis-North America, Canada \&c.: this is C. palustre of all the 
American botanists! flowers much larger than in last, purplish as in all the Sp. Folioles from nearly the same point not properly pinnate.

200. Comarum (Panc. Pot.) angustifolium Raf. Caule glabro, fol. pinnatis, petiolis vaginatis nudis, foliolis 5 cuneatis angustis, pectinato serratis, subtus pallidis; florib. term. 2-3 parvis, petalis ovatis acuminatis-Oregon or N.W. Amer. Semipedal, flowers half size-The above 3 species now distinct, appear to have a common origine as deviations of a type; by calling them varieties we should overlook their characters and this formation of Species. They are a fair example of this phenomenon: or of local deviations in remote clines.

Whenever plants have long been removed in different continents or botanical regions widely apart, they assume in general a different shape and virtually becomes new species. Therefore Decandole has properly stated that all plants deemed alike in remote regions must be examined again. None of the North American trees and plants are exactly alike to their Asiatic or European types, except among Grasses and Cryptogams, a fact as yet unexplained for these. The boreal plants of both continents have however mostly identic Genera, and many identic species, but South of Canada, the Species and Genera gradually become different or unlike. 


\section{FLORA TELLURIANA.}

\section{CENTURIA TER'TIA.}

201. Euscynetra R (well unit. 4) Cal. 5fid. folios. dent. subeq. Cor. tubul. recta bilabiata, lab. sup. trifido, med. major bif. lab. inf. integro. Stam. 4 didyn. antheris coalitis in singula quadrata. Glandula biloba supra basi Ovar. stylo declinato, stig. obt. Capsula baccata uniloc. bivalv. polysp placenta '2. Frutex fol. opp. fl. axill-Very distinct G. totaly unlike Columnea and Achimenes by lips, \&c., not Family Gesnerides but Gratiolides by fruit onecelled.

202. Eusynetra bicolor Raf. Columnea hirsuta Sw. Auct. Hooker b. m. 3081. Achimenes major Br.t. 30-Scandens fol. pet. ovatis acutis vix serratis, superne hirtis, fl. solit. s. geminatis, Cal. lanc. Cor. villosis coccineis intus flavisJamaica, on rocks and mts.

203. Cupulissa Raf (cup split) Diff. from Bignonia, cal. cupularis integro latere fisso, Cor. tubulosa campanul. limbo undulato subeq. 5 lobo, lob. inf. fisso. filam. basi glandul. hirsutis, didyn. quinto ster. ovar. supra disco glanduloso, stigma bilamel. Scandens, fol.comjug. fl. racemosis-Type $\boldsymbol{C}$. grandifolia, foliolis 2 ovatis undul. cirrhosis verrucosis, racemis multifl, pedic. bibract. flexuosis.-Carracas, large yellow flowers. Bignonia grandif. Jaq. hort. 287, Bot. reg. 418, Bot. mag. 3011, anctoris, Probably several Bignonias belong here, the real have cal. dent. cor. bilabiate $\mathbb{S}$ c.

204. Megapleilis, R. (big single lip) Cál. 5 fidus ineq. Cor. tubulosa, unilab. basi 5gibbosa, apex dilat. labio superne amplo emarg. lab. inf. subnullus. Stam. cxserta, antheris 
didyn. coalitis. Stylus clavat. Caps. Zloc. ¿valv. placentis parietalis. Tuberos. fol. opp. fl. term. racem.-M. tuberosa. Raf. fol. cord. ovatis serratis villosis, subpetiol. bract. sessil. cord, integris, raccmo paucifl. fl. oppositis coccineis. In Brazil, large singular flowers, base yellow with 5 inflate lobes. Gesneria bulbosa. Ker. bot. reg. 343. Hook. bot. m. 3041. How could they unite to Gesneria, such conspicuous N. Genus? not even of same family::

205. Endocona R. (ins. hairy) Perig.6 partit. ineq. patens pers. 3 internis minor fimbriato comosis. Stam. 6 eq. subul. glabris ad basi sepalis, Ovav. 3 gon. stylo brevi, stig. 3, Caps. 3loc. 3v. polysp. sem. subr. Rad. tub. fascic. Caulesc, fol.ung. /l. term. paucis.-Fine N. G. near Quamasia and no Anthericum! two sp. both called Autheric. plumosum, the first by Ruiz t. 309, the second by Hooker bot. m. 3084 .

206. Endocoma peruvianc Raf. (Ruiz) fol. linear. Caule subnudo 3-5fl. albis.-Peru, large white flowers.

207. Endocoma parviflora Raf. (Hooker) fol. linear canal. Caule bifolio bifloro.-Chili, small green flowers, margin white, larger plant. Is not this a deviated species?

208. Caldiprora Lindl. Cor. subcamp. Gpart eq. stam. 6 fertilia, 3 breviora, filam, petaloideis bifidis, antheris sessilis intra lobis. Ovar, stipit. stig. 3 lobo, Caps. triptera. Scap. fl. $u m b$. bipathis, luteis.-G. of tribe Asprodenidia like the last, near to Porrum, Getuonis and Brodiea.-Type. C. lutea bot. reg. 1590. Scapo teres, fol. canalic. longissimis, umbella multifl. From California.

209. 'Tracteua R. (spindle fil.) Cor. patula rotata eq. 6 partita, sepalis subunguic, uninervis, 
Stam. 6 eq. filam. glabris basi dilatatis sub fusiformis. Ovar. trilobum, stylo sulcato, stigm. obtuso. Bulbosa, unifolia, scapo racemoso. Altbo' I had nearly exhausted the reform of Skilla, and akin Genera, this which was a Slilla, proves that many distinct $\mathbf{G}$. are yet blended. It is as near Fusifilum 69 ; but the habit is peculiar. It is at least a distinct SubGenus.

210. Tractema pumila Raf. Skilla pumila Broterofl. lusit. Hook bot.m. 3023. Folia lanceolata undulata involuta, acum. carin. Scapo subeq, paucifloro, fl. cermuis, bracteis breviss. sepalis ellipt. obt.-In Portugal, 3 to 4 inches, fl. blue or lilac or white about 5 , the fig. and descr. of Hooker do not agree.

211. Blepilantmera R. (cil. antlı) Cor.patula equal. 6partita, Stam. vel stylo declinatis, Stam. subeq. filam. subulatis ciliatis villosis, antheris ciliatis. Ovar. globos. glabrum, stylo flexuoso declinato filif. stigma acut. Bulbosa, fol.fascicul. canal. scapo racemoso.-Another fine G. blended with Authericum african G. nearer Nemopogon 68, australian like it, but the bulb and stamens separate them: the English authors have even blended two species of it into one as Anthericum bulbosum.

212. Blephanthera depressa Raf. Antheric. bulb. Brown, Bulbine Australis Sprengel. Bulbo depresso, filamentis declinatis. Australia, not a Bulbine having smooth unequal filaments.

213. Blephanthera hookeri Raf. Bulbo ovato, filam. non declinatis, Antheric. bulb. Hooker bot. m. 301\%, who himself suspected it was a new sp. Yet gave no new name to it. Australia. Leaves long narrow thick semiterete canal. Raceme long multifl, yellow. 
214. Dotumus Raf. (papilar lip) Orchidea. Sepalis ringens ineq. 3 internis undulatis papillosis, labello subeq, basi cuculato, lamellis 6 longit. obsito. Ovar. clavat. Columna semiteres, erecta basi dilat. bident. stigma bilabiato, anthera dorsalis ad apex, ovata biloc. 4 pollen. 'Ierresiris. caule folioso, fl. term. amplis.Beautiful N. G. quite peculiar, not a Neottia, as Hooker even presumed, and has since called it Ulanthia; but our geueric characters are different.

215. Dothilis grandiflora Raf. Neottia? grand, ft. Hook. bot. m. 2956. not 2730! another plant of same name! Fol. imbric. glaucis oblongis acutis planis nervosis, florib. paucis, magnis undulatis, albo viridis, striatis, labello obovato crispo.-Large flowers 3 inches. Native place not stated, probably S. America.

216. Anisantuera Raf. (uneq. anth.) Leguminosa. Calix 5part ineq. Vexillum reflexo cuculato, alis obov. Carina eq. Stam. monadel'phis, vagina tota fissa, apice filam. pectinatis reflexis. Antheris ineq. alternis 5 linearis, 5 alt. subrot. Ovar. sessile. obl, villosum, Stylo clavato glabro. reflexo, stigma obt. Legumen turgidum villos. polysp, Anmua. fol. simplices stipulatis, fl. racemosis. - Very distinct G. blended with Crotalaria by all: yet nearer Lupinus, anthers quite peculiar.

21\%. Anisanthera Versicolor Raf. Caule flexuoso 4 gono. fol. glabris ovatis obovatisque, obtusis, stipulis lunulatis, racemis terminalis.Beautiful flowers variegated of white, blue and purple. Said to grow in the tropics of both hemispheres. Often figured. It is the Crotulariu verrucosa of W. and D. C. but there is nothing warty about it. Andr rep. 308, bot. reg. 
11:37, bot. mag. 3034. Crot. cerulea Jaq. Ic rair. 144 an Sp. diversa? Crot. angulosa Lam. Cav. 321. Probably several sp. blended. the Asiatic and Antillian plants must be again compared, and perhaps other Crotal. belong here, like the next.

218. Anisanthera hastata Raf. diff. fol. hastato lanceol. acutiss, deemed a Var. of last by Lam. Persoon. \&c, Certainly distinct. From Mauritius and Bourbon Ids.

219. Isorium R. (Equal lobes) diff. from Echium.-Cor. longo tubulosa, limbo 5lobo equalis, Cal. 5part. ineq. Stam. 5 ineq. stigma capit. Nucibus 4 stylo adnatis.-Very distinct G. of nat. family Ecmidis, that differs from Borraginea by unequal cal. cor. or stam. 'Type Isorium formosum Raf. Ech. do Persoon. Ech. grandiflorum Andr.t. 20. African shrub, leaves lanceol. hispid, fine large red flowers.

220. Nemurantues R. (fil. caud. fl.) Orchidea. Ad Habenaria et Blephariglotis diff. Cor. bilabiata, pet. ext. supero concavo trinervo cuculato, 2 ext. reflexis, petalis 2 internis bipartitis, labello tripartito segm. linearis, medio brevior, Calcar longissimo filiformis (pedalis.) Ovar. pedic. clavato sulcato, columna trifida brevis, antice glandulis 2 appendiculatis. Anthera Unica! terminalis 2 loc. antice cornuta. Canle folioso racemoso non spicato!-Wonderful Genus, no Habenaria since unic anther! floral structnre quite peculiar, really racemose. Types the real Orchis hubenaria $\boldsymbol{L}$. or Habenaria macrocera of $\mathrm{W}$. and the following fine species.

221. Nemuranthes longicauda Raf. fol. lanceol. acutis striatis, bract. lanc ad ovar. brevior, f. racemosis pedic. laxis, petalis ext. ovatis acum. pet. int. et label. segmentis linearibus.- 
In Demerara, fl. greenish white very large, ovary and pedicel 5 or 6 inches, spur 10 to 12 inches! long. Ilubenaria longicauda Hook. bot. mag. 295\%.-The Hab. macrocera Hook. 1. $\mathbf{2 9 4 7}$ belongs here also and merely differs by leaves obt. bracts longer, spur shorter, fl. subspicate, it is my $\boldsymbol{N}$. Habenaria.

222. Uloma R. (crisp border) diff. Bignonia, cal. camp. 5dent nervosus persistens, cor. campanul. lobis $\mathbf{5}$ subeq. margine plicatis sulcatis crenatis, filam. incurvis. Bacca teres carnosa edulis uniloc. bipartibilis, dissep. spongioso, sem. circumalatis. Arbor. fol. opp. imp. pinnatis, panic trichotoma.-How different from 203! yet both made Bignonias! altho' both types of New Genera. This has a true berry and not a dry siliqua.

223. Uloma telfairia Raf. foliolis 13-19 ovatobl. integris. oppos. petiol. panicula laxa.Elegant and useful tree of Madagascar, beautiful large pink flowers, fruit excellent flavor, called Vuakidzi, cultivated.-Bignonia telfairia Bojer in bot. mag. 2976, dedicated to a lady botanist.

224. Xeilyatuum (lip fiddle) diff. from Oncidium. Petalis 5 rotatis eq. undulatis liberis, labello plano pandurato emarginato basi papilloso cristato, columna brevis antice biloba, apice bident, anthera supera bipollen. Parasita, bulbo terrestris costato basi involucrato, apice unifolio, Scapo lateral. ramoso paniculato.One of the finest new Orchidean G. shuffled into 3 Genera, habit striking, nearer Renanthera which $I$ add for contrast.

225. Xeilyathum Altissimum Raf. Folia et invol. carnosis carinatis elong. acutis. panic. flexuosa divaric. petalis obl. undulatis-Antillis, 
leaf 2 or 3 feet, Scape 4 to 6 feet, ft. rather large, lip bicolor base fulvous and yellow. Oncidium Altiss. Sw. W. auct. Hook. b. m. '2990, Epidendrum Altiss. Jaq. am. t. 141. Swart\% put it in 3 Genera by turns those 2 and $\mathrm{Cymbidium}$ besides!

226. Renanthera coccinea Lour. bot. reg. 1131, bot. mag. 2997. This only diff. from Xeilyathum by-Petalis 2 inf. major, magis undul. labello integro basi saccato, col. abov. anthera operculata. Caule folioso, Panicula lateralis extra axill.-fol. ligulatis obt. distichis, petalis cuneatis coccineis, labello ovato acuto.

227. Exopirya R. (outer growth) Oirchidea, cor. rotata pet $\mathbf{5}$ subeq labello 3 lobo, col. semiteres bident. Anth. term. semispher. 3 loc. 4 pollen. Bulboterrestris sulcuto, canle later, ad basis bifolia, $f$ racemosis.-Very near also to 224. chief difference the anther; yet Hooker would force it into his new G. Encyclia by spoiling its character, bot. mag. 2831 of petals conmivent \&c.

228. Exophya fuscata Raf. Encyclia patens Hook. b. m. 3013. Bulbo ovato, fol. lin. lanceol. planis acutis, racemo paucifl. petalis obl. 2 int. spatul. acutis, labello lobo medio ovato acutoFrom Brazil, small, but fl. large dusky and fragrant.

229. Kuritis R. (Verbena gr.) diff. Selago, Cal. ineq. tripartitus, Cor tubulosa, limbo ineq. 5fidus. Stam. 4 didyn. fil. clavatis, antheris unilocul. Stylo filif. Stigma acut. Caps. biloc. bipartibilis dispermis, Arbusc. fol. sparsis. integris, fl.bracteatis term.-The G. Selago includes many Genera, with cor. 4 or 5 lobed, cal. camp. 5dent.. The family Selaginea of 
Choisy, hardly differs from Verbenacea. 'This G. is peculiar by calix, and includes $2 \mathrm{Sp}$.

230. Kuritis gilii Raf. Selago do H. b. m. 3028. Caule teres pub. fol sparsis lin. obl. glabris fl. glomeratis-South Africa, small shrub, flowers lilac, lobes of corolla obl. obtuse.

231. Kuritis ciliata Raf. Selago do Choisy. Fol. ovatis ciliatis imbricatis, fl. spicatis elongatis, bracteis ciliatis-South Africa, The Selago ciliata of Thunberg with ovate spikes, is a real Selago, and different from this.

232. Saxifraga, L. auct. This pretty group of plants has, been made a single Genus by nearly all the Linneists, being however united by no common character, as I have shown in the first part of this work. 'They had nothing in common, not even habit! It was a shameful artificial Genus; since the united plants belong even to 3 different Natural Orders and Families, of two different Natural Classes.

Order ASCADIA, nat. fam. Diceracea, all those with coalescent calix and ovary.

Order ISOSTIMIA nat. fam. SAxifragen, all those with free calix and ovary, only one Capsule.

Order PERIMESIA nat. fam. SArcopiyuLIA, near Sedum, all those with free pistils and several capsules, now the Genera Eriogyna and Leptarhena 261, 263.

Many Botanists have published monographs of this Genus, but overlooking this capital blunder. Smith has only illustrated $50 \mathrm{Sp}$. Hooker has 42 from North Amer. alone, others have 80 Sp. divided in Subgenera, Tausch monograph I never saw, but has no generic reform. I shall, propose 12 new Genera quite as good as Eriogyna and Leptarkena of Hooker, and present 
here a complete reform of the whole Genus, from 233 to 263 .

233. Saximica Raf. Calix libero camp. 5 fido persistens, staminif. petalis $\mathbf{5}$ sessilis, equalis, Stam. 10 eq. Ovar. liber, in Stylis 2 desinens. Caps. libera, monoloc. bifida, inter fissura dehiscens, polysperma, bivalvis, valvis seminiferis. Habitus Varius, fol. sepe crassis.-The free pistil Sp. are the types of the G. and family Saximragea, along with Mitella, Timella Sc. the Sub-Gen. Hirculus, Hydatica, Arabidia, Micranthes Tausch, chiefly belong here, and only apply to the habit. Necker called Hydutica all these true Saxifragas; but I shall confine it to the next Genus. 'The Sp. belonging here are Sax. aspera, bryoides, media, cermua, sibirica rotundifolia, spicata. arguta, nelsoniana, mudicaulis, nivalis, vernalis, virginiensis, integrif, dahurica, flagellaris \&.c. but many must be examined again. Sux. orientalis Jaq. Vitm. or Sax mollis b. m. only differs by petals trinerve, perhaps type of a subgenus IVIulagea Raf. it is the Geum orientule 'Toum it. 2 ic $\mathbf{1 4 8 .}$ Caule procumb, stolonif. fol. lobatis, pet. obov.

234. Hydatica Raf. diff: 233, Cal. 5 partito reflexo, petalis unguiculatis, biglandulosis, glandulis pustuliformis.- Types Saxifr. umbrosa, hirsuta, cuneifolia, Stellaris, foliolosa, Geum,

235. Apiononix Raf. diff. 233, Cal. semi adherens, concavo,quinque fido, petalis unguicul. subrot. enervis glandulis vel pustulis nullisType Saxifraga hederacea, but others may perhaps belong here also. This G. must go into nat. fam. Diceracea, with the next.

236. Ponista Raf. (ad Pona) Cal. adherens 5fidus, Petalis 5 trinervis eq. Stam. 10 alt. 5 
brevior, fil. subul. antheris bilobis. Ovar. adl. globos. supra planiusc. Stylis 2 brevis, Stigma obt. Caule ramoso, fol. divisis, fl. term.-Annuals; several of the Saxifragas with trincrve petals belong here probably, such as Sax. hypnoides, moschata, platipetula, cotyledon, tridactylis, aquatica, elongela, \&c. but the real Types are-

237. Ponista petrea Raf. Saxifr. do L. Jaq. 181. anct. fol. pet. palnatis tripartitis, lac. trifidis, caule decumb. ramosisino, pedunc longiss. unifl. petalis emarg.-Alps of Europe, Sux. rupestris, adscendens and pona said to be the same must be compared again.

238. Ponista oregonensis Raf. Saxifr. petrea Hooker fl. and bot. mag. 3026. Glanduloso pilosa, caule erecto, fol. trilobis, lobis integris trifidisque, radic. petiol, caulinis sessil. cuneatis pedic. brevis eal. obl. obt. petalis cuneatis retusis -Mts. Oregon of North America, certainly a widely deviated species, not a mere variety, sometimes abortive flowers.

239. Hexaphoma Raf. (6 pustules) Cal. libero 5partit. reflexo, petalis 5 ineq. 3 unguic. major bipustulatis, 2 minor sessilis nonpustulatis. Stam. 10. alterna 5 brevissima, filam. filif. Ovar. liberum bifidum. Subacaulis seapo paniculato - How can a sensible botanist deem this of same Genus as Ponista for instance? 'The types are the Saxifraga leucunthemifolia of North Anerica, $\approx \mathbb{S}_{p}$. blended in one, and a third is the pyrenaic Sp. even hardly of this Genus! this is an instance of utter confusion of ideas, principles, characters and species, by not attending to the original descriptions.

240. Hexaphoma ferruginea, Raf. Saxifr. do. Graham. Sax. leucanth. Hooker flora. bot. 
mag. 2959 ! fol. sessil. obor. grosse serratis acutis glabris. scapis ferrug. glandul. panic. laxa dichot. petalis 3 ovatis, 2 oblongis minor.-Mts. Oregon of North Aner. fl. white,with 6 yellow pustules, Hooker Synonymy is quite wrong since he blends the $3 \mathbf{~ S p . i n t o ~ o n e ! ~ T h i s ~ h a s ~ s e v e r a l ~}$ scapes uncial.

241. Hexaphoma petiolaris Raf. Saxifr. leucanth. Mx. Elliot Pers. and all the Amer. bot. not Hooker nor Europ. botanists! fol petiolatis spatulatis elongatis ovatis grosse serratis scapo hirsuto, panic diffusa,-Apalachian Mts. a single bipedal scape, leaves 3 or 4 inclies long. fi. small variegated with yellow and pink, probably by the pustules and red pistils.

242. Hexaphoma? pyrenaica Raf. Sax. leucanth. Lap. t. 25. et europ. auct. Sax. clusi? Gouan non Clusius, Bauhin ic 708 monente Smith-Fol. cuneatis petiolo brevi alato hirsutis lato dentatis, scapo bracteisq levis. vestito panicul. petalis pustulatis?-Mts. Pyrences and Alps of Emope, flowers large white stated without spots by some, perhaps 2 Sp. blended as in America. Smith distinguished this plant from that of Michaux, refering that to Rupifraga, did he mean that in this the petals are equal? then this would be a real Myplulica like Saxifr. Stelluris. The $\mathbb{S}$. clusi of Goun and Vitman has a foliose viseid Stem, leaves spatulate cuncate viseid, racemes axillary dichotome, petals white. It must be a peculiar Sp. Hexaphoma viscida Raf.

243. Rupifraga L. Mpt. Adenogyna Raf. Cal. basi coalito, 5 firus subineq. Petalis 5 inequalis, 3 superis brevis, 2 inferis elongatis, stam. 10 subeq. Ovario sub lilerum, glandula magna carnosa ad dorso supera, stylis 2 , capsula bivalvis. 
Stolomifere, fol. radice peliol. fl. pamiculatis. - Certainly a beautiful distinct Genus, with habit of Ilexuphoma, which Simith tells us Linneus meant to establish: 1 did the same before kuowing of this. 'The type is Rupifraga. Sarmentosa L. herb. of China and Japan, well known in our gardens with round lobate leaves. It is saxifraga Sarmentosa L. et Auct. Curtis b. m. 92. S. stolonifera Jaq. Meers t. 23. \&c. It is as different from Saxifraga as Iberis is from Alyssum.

244. Pramopirya, R. (thick leaf) diff. Saxifrogr, Cal. campanul. 5-8fidus. petalis obovatis $5-8$ campanularis, Stam. 10-16 ineq. alternis latior, ovario libero, stylis 2-3, Caps. 2-3 valvis. Fol. rad. binis. crassis amplis, scapo nudo, florib. cymosis.-Habit quite peculiar indicating a Genus, 3 types blended as Sraxifruga cressifolice of authors.

215. Piarophyla sibirica Raf. Fol. petiol. ovato subrotundis retusis serratis, fl. densis purpureis-Mts. of Sibiria. Gmel. t. 66. Curtis bot. mag. t. 196. Sim. W. DC.

246. Piarophyia cordifolia, Raf. Fol. petiol. cordatis ovatis serratis, fl. glomeratis. Sibiria. Saxifr. cordifolia, Haworth, Aiton. Var. of crassifolia Śmith.

247. Piarophylu elliptica Raf. Fol. subsessilib. ellipticis obtusis subintegris vel vix serratis, cyma glomerata pedata, florib. nutans-In Sibiria and N. W. America. This is the real Sax crussifolia of Lin. dec. t. 14. Buchoz dec. t. 1 and my American specimen hardly differs. 248. Stemanrsia, R. (sterile uneq.) Cal. 5 partitus, petalis 5 equalis, sessiles, Stam. 10 planis subeq. nonnulis sterilis ineq. petaloideis vel clavatis-Very distinct anomalous Genus 
with Stamens mostly sterile. Types Saxifi. punctata, veflexa, heteruntha of c.

24!). Steiramisia punctutu, Raf. (Sax. do L.) Fol. subrotundis dentatis longe petiolatis, scapo nudo, petalis punctatis. Sibiria.

250. Steirunisia reflexu Raf. (Sax. do. Hook. fl. bor. t,85) (ano pubcscens, fi. corymbosis, petalis bimaculatis, staminibus lanceolatis. In Oregoll.

258, Steiramisia heterantha Raf. (Sax, do. Hook, fi. bor. t. 78) Staminib. sterilis plurimis varians clavatis et petaloideis, fertilis filiformis, Boreal America. Is it another G. Heterisia? having more than 10 Stam,

252. 'Telesonix, R. (perfect claws) Cal. 5 partitus petalis 5 equalis orbiculatis longe unguiculatis, Stam. 10 eq. filam. brevis. Ovar. liberis stylis 2, caps. - ut Saxifraga. Caulescens pauciflora. Type. Telesomix jumesi! Raf. (Sax. do 'Torrey, Hooker, Eaton) Fol. renif, crenato dentatis, papillosis, caule brevi paucifi. petalis integris purpureis-Mts. Oregon. near to G. Aphomonix.

253. 'Tulorina, R. (wart pits) diff. Saxifr. and Hydatica by CaI. reflexo 5partito, petalis unguicul. ad ung. fossulis binis ferens verrucis centralis anmulatis.--Type 'T'ul. cymbalaria Raf. Sax. do Anct. Caule procumb. fol. cordatis trilobis vel integris, petalis ovatis flavis. Oriental plant.

254. Hriculus, Raf. Cal. 5part. reflexo, petalis sessilib, nervosis ad basis bituberculatis vel verrucis 2 sine fossula, ovario ovato libero, Caulescens 1-2 fl, fol. simpl.-'Туре Hirculus punctatus Raf. (Saxifr. hirculus I. ) Fol. lanceol. petalis ellipt, flavis, nigro punctatis,---Alps, 
Sibiria, and boreal Amer. Perhaps several blended species.

255. Hemeva, Raf. (half well) Cal. adherens patens 5 fido, petalis $\mathbf{5}$ eq. Stam. $\mathbf{5}$ petalis alternis, Ovar. coalito infero, supradisco annularis cincto petalis et stam. ferens. Capsula cal. coronata, apice bivalvis. Caulescens, fol. lobatis, fl. Spicis corymbosis.-Fine G. mixt with Saxifiraga by IIooker. 5 Stamens and the disk are very essential characters, nearer to Tellima and Heuchera. 2 types.

256. Hemieva ranunculifolia Ráf. (Sax. do Hook. fl. t. 83) Pubera, fol. renif. 3part. infimis longe petiol. segmentis latocuneatis inciso lobatis, fl. corymbosis, petalis obovatis ad cal. duplex.-Falls of the R. Oregon or Columbia.

257. Hemieva richardsoni Raf. (Sax. do. Hooker fl.) Fol. orbic. cordatis crenato lobatis, spicis corymbosis, bract. lanceol-Arctic Sea of boreal America.

258. Evaiezon, R. (well everlasting) Aizoon et Aizoum of old botanists, Saxifraga Tourn L. auct-Diff. Saxifraga 233. Cal. adherens 5fido, pet. 5 eq. sessilis, Stam. 10 eq. Ovar. adherens infero vel semi inf. Stylis 2, Capsula coronata, semi-bivalvis.-This comprizes all the Saxifragas with coalescent Ovary and calix, that do not belong to Ponista, Aphomonix, Rupifraga, ILmieva \&c. They are numerous and of dissimilar habit indicating several Subgenera, such as Antiplnyla Haworth with opposite leaves, Aizonia, Porphyrion, Dactyloides $\& . c$ of authors. Here belong the Saxifraga oppositifolia, retusa, biflora, aizoides, punctato, gramulala, bulbifera, cuneata, palmata, cespitosa, hirta, cesia, ajugifolia, ladanifera, trifurcata, pedatifida, geranoides, ri- 
vularis, callosu, mutata, pensylo, erosu, androsacea, hieracifolia, diapensoides, burseriana, esholtzi, serpylifolia, cenosa, exarata, silenif. lanrentiuna, exilis, mutans, \&c.; but they are yet in great confusion of synonymy, and many Sp. are blended, of which I give two instances.

259. Evaiezon trichodes Raf. Cespitosa, caulescens fol. lanceol. acutis glaucis, pedunc. unifl, petalis cal. brevior.-In the Alps. Nax. trichodes Scopoli fl. carn. t. 15. Vitm. S. alpina Seg. ver. t. 9. S. muscoides Allioni t. (il?

260. Evaiezon pungens Raf. Caule viscido paucifloro, fol. crassis pungens subul. imbricatis ciliatis, fl. 5-7 fastig. petalis undulatis.-In the Alps and Pyrenees. Sux. burseriana Lap. t. 17. Sin. Var. Spinosa Pers.-Both these are different from Sax. burseriana with which they have often been blended.

261. Eiziogrna Hooker, difi. Suxifraga 233 by, Stam. 20 basi coalitis, cal. turbinato 5fido, pistilis et capsulis 4-6 liberis lanatis bivalvis, stylis 5. Caulescens, fol. divisis, fl. racemosis. It is a wonder Hooker ventured in this innovation, while he left Hemiera. Steiramisia, \&c. in Saxifraga; but the blunder of Pursh, copied by Nuttal, Eaton and all our blunderers on Genera, was too glaring : even Smith had declared it was no Saxifraga; I had done the same in 181\%. It does not belong even to the same natural order where Hooker leaves it; but near Sedum, Sempervivum and Penthorum in PERIMESIA, and natural family Sarcophylia.

262. Eriogyna pectinata Hook. f. t. 88. Saxifraga do. Pursh, Nut. Eat., \&·c. Cespitosa, fol. trifidis pectinatis linearibus.-Oregon Mts. 263. Lep'tanena Br. Hook. diff. Saxifraga. 
Cal. camp. 5fid. petalis 5 linearib. Stam. 10, pistilis 2 liberis, capsulis 2 intus dehiscens.Acaulis, Scapo paniculato.-Another distinct genus separated by Brown, but of same family as Scdum, altho' near Spiren by the dehiscence of Capsules, the definite Stamens unite it to Sedum. Several Sp. called Saxifrugas by Don, pyrolif. amplexif. micrantha, \&c. Type $L$, pyrolifolia IIooker ft. t. 89. Fol. ellipt. serratis, racemis paniculatis. Boreal America.

:64. Pectianta R. (comb oppos) Drumondia D. C. Cal. coalito turbinato, Segm. 5 refl. Petalis 5 pectinatis subepigynis staminiferis persistens. - Stam. 5 ad pet. oppos. et basi insertis, antheris bilobis subsessilis. Ovario coalito, apice plano, stigma 2 sessilis bilobis. Capsula cal. et pet. connata, uniloc. apex fissus subbivalv. polysp. placenta 2 parietalis. Acaulis, fl. spicatis.-Another striking new Genus overlooked by Hooker, and mixt with Mitella; all the akin Genera Tiarella, Heuchera, are in equal confusion. 'This is not even of the same Natural Class, since the opposite stamens remove it to NAN'TIANDRIA and near Pluglica, Ceanothus, \&c.; but forming even a new family as will be seen below-Meantime the type is

265. Pectiantiu mitelloides Raf. Drumondia do. Dec. Mitella pentandra Hook. b. mag. 2933. fl. bor. there was a previous G. of Mosses Drumondia.-Pubera scabra, fol. petiol. cord. lobat. crenatis, fl. parvis spicatis, petalis rigidis lutescens.-Oregon Mts.

266. EVANTEPIA Raf. New Natural Order of Class Nantiandria. The Evantepians, meaning well opposite over._Differing from PLYRONTIA by Cal. and ovary coalescent. 
Petals various, one or several stigmas.-This will contain like Plyrontia several Natural families, such as-

26\%. PEC'I'AN'TIDIA, the Pectantides Raf. Petals persistent, several stigmas, capsule uniloc. seeds parietal. 'Iype Pectuntia, ahove.

268. GUANIDIA, the Gouanides Raf. Petals squamiforn, one style, several stigmas, capsule multiloc, cells monosperm,. 'Type Gouania.

269. LORANTHIA, the Loranthians Raf. 1815, types Loranthus, Viscum, \&c. Berry monosperm. one stigma.

270. MANGIDIA, the Mangidians Raf, 1815, types Rhizophora, Bruguiera, Mangium Raf. Aegiceras. Capsule monosperm, several stigmas.

271. Ozoneus Raf. Cal. campanul. basi coalescens $\mathbf{5}$ fido, petalis $\mathbf{5}$ trifidis, vel nullis, Stam. $\mathbf{5}$ ad petalis alternis. Ovario semi infero stigma capitatis (cetera ut Mitella) Acaulis, $f$. spicatis polygamis. Very distinct G. also blended with Mitella by Hooker. 'I'ype Ozom. varians Raf. Mitella trifida Hooker fl. bor. t. 82. Fol. cord. obtusis lobatis crenatis, fl. spicatis, nonnulis apetalis vel masculis.-From Oregon. It belongs to Diceracea, but Mitella to Saxifragca.

272. Pleurendotria R. (side inside 3) Lithofragma Nuttal. (bad mongrel name) Cal. cyathiformis 5dentatus liberus. Petalis 5 unguicul. trifidis. Stam. 10. antheris sessilis, Orario libero, basi vix coalito, Stylis $\mathbf{3}$, caps. unilocul. placentas 3, valvis 3. Caulescens fol. divisis, fl. racemosis.-Another distinct Genus, which Nuttal would lately unite with the last, 2 types.

273. Pleurendotria parviflora Raf. (Tellima do Hook. f. t. 77) Hirsuta Scabra, fol. ternis inciso pinnatif. racemo brevis.-Oregon. 
274. Pleurendotria reniformis Raf. Lithofragma do Nuttall. excl. Syn. Hooker. Annua, pubescens, fol. renif. trilobis incisis, racemo cynıso-Oregon Mts. flowers pale rosate, Nuttal blends it with the last, but says it has sometimes 8 stamens only, and 2 styles. Both very different from Mitella grandiflora type of G. Tellima, which has 5 Stamens.

275. Petalosteira R. (pet. sterile) diff. Tiarella by no petals, but 12 to 20 stamens whereof 3 to 10 are sterile and petaloid, all in a row.--Types 1. P. unifolia Raf. 'Tiarella do Retz, Vitm. Hooker fl. bor. t. 81.-2 P. laciniata Raf.'Tiar. do Hook. t. 76. both from boreal America.

276. 'Tiarema L. auct. This G. properly differs from Mitella by Capsule of two unequal valves and petals entire, it is therefore less natural than many of the above. The type is Tiarella cordifolia, with calix 5parted; all the other sp. are doubtful, each appearing the type of a subgenus at least, while ' $\boldsymbol{T}$. Bracteata and biternata lack the 10 Stamens or unequal valves, and inust form the two next Genera.

277. Oreotrys (Mt. cluster.) Raf. atl. journ. p. 145. in 1832. Diff. Tiarella, calix campanul. $\mathbf{5}$ fido, petalis $\mathbf{5}$ linearis parvis, Stam. 5, petalis, alt. subul. Stylis 2, capsula semi-infera birostrata, valvis equalis staminiferis. Acaulis fl. racemosis bractcatis.-Nearer Heuchera to which Dec. unites it than to Tiarella. One type.

278. Oreotrys bracteata Raf. Fol. rad. cespitosis subrot. cordatis, inciso serratis glabris petiolis pubescens: racemo simplex, bracteis lanceolatis.-Mts. Oregon. Tiarella bracteata, Torrey, Eaton, Heuchera do Dec. Peronnial, calix obovate segments. 
279. Blondra (Necker) diff:'Tiarella, Cal. conc. campan. 5fido, Petalis 5 linearis elongatis, Stam. 10 elongatis, ovario birostro, Stylis 2. Stig. obtusis. Capsula uniloc. birostra equalis, inter stylis dehiscens. Cumlescens, fol. compositis alt. fl. paniculatis.-'The description is from Elliot, who properly says it is nearer Saxifraga than 'Tiarella; but habit quite peculiar, similar to Aruncus, where Michaux once united it.

280. Blondia biternata Raf. 'Tiarella do Vent. Pursh. Nat. Elliot, Eaton, Spirea aruncus Var. Mx. Fol. bitern. foliolis ovato cord. obliquis, inciso lobatis dentatis, panicula racemi flora divaricata pubescens bracteis linearibus.Saluda Mts. of Carolina, perennial, styles inflexed, segments of calix deep and ovate.

281. Blondia trifoliata Raf. Tiarella do L. et auct. Fol. trifoliatis, foliolis rhombeis angulatis serratis pilosis, panicula corymbis racemosis stylis rectis.-In E. Sibiria and Oregon. Cal. realy campan. 'This was the type of Necker's Bloudia, who ascribes to it a double capsule, as Leptarhena, which comes still nearer to Amucus. In Bl. bitcruata the 2 capsules are coalescent and the calix deeper more open: is it a Subgenus Saluda Raf? the definite Stamens remove them however from Spirea and Aruneus, while the habit is also near Alchemilla.Yet the G. probably belongs to Saxifragea.

28:. Yayama R. (nom. Amer.) diff. Heuchera, cal. turbinato ineq. 5lobo, petalis nullis, Stam. 5 inclusis. Acaulis, fl. racemo Simplex.-Type, Yumala cyliudricu Raf. Heuchera do Dec. Hook. fl. fol. cordatis truncatis lobato crenatis, racemo tereto spicato. In Oregon.

283. Leptaxis Raf. diff. Heuchera et Tiarella, Cal. infundih. sfido, peialis ã linear. Stam, 
5 ineq. 3 exsertis longior, stylis 2, capsula onnino libera, Acaulis, rucemo simplex.-Type Lepiaxis menziesi Raf. Heuchera do Hook. fl. t. 80. Tiarella do of some authors; but not of Pursh, his being a real 'Tiarella with 10 stamens, caulescent and spicate.

284. Dianonon R. (Solanum diosk) diff. Solanum. Cal. ineq. bilabiato, 4partito, lab. inf. emarginato. Corolla rotata, subineq 5 loba, Stam. 5 eq. libera, filam. brevis, anth. magnis ellipt. apice biporosis. Frutex fl. term. solit.-Type. Diamonon coriaceum Raf. Solanum do. Hook. b. m. 2708. Glabrum, inerme, fol. petiol. oblongis coriaceis nitidis integris subvenosis, pedunculis subunifl. corollis plicatis undulatis mucronatis cerulescens. - A pretty Sp. from Mexico with large bluish flowers.

285. Evotrochis R, (well wheeled) diff. Primula, Cal. campan. 5fido, lac. serratis, Cor. hypocrat. tubo angulato, limbo 5lobo; antheris 5 sessilis, Stylo filif. stigma concavo, Caps. globosa uniloc. polysp. sem. ad placenta glob. magno centrale inserta. Caulescens fol. et fl. verticillatis.-It is astonishing how the botanists could unite this fine distinct Genus with Primula : probably several $\mathbf{S p}$. blended as $\mathbf{P r}$. verticillata.

286. Evotrochis involucrata Raf. fol. radic. spatulatis inciso serratis, acutis nervosis bullatis, subtus farinosis flavo maculatis, fol. caulinis sess. ovatis trinervis 5 -\%rotatis, fl. axill. rotatis 5-12. corollis crenatis. - In Eigypt, fl. pale yellow hardly odorous. This is $\mathrm{Pr}$. vertic. of some authors Grah. Hook. b. m. 2842, not of Forskal.

287. Evotrochis odorata Raf. fol. ovato-lanc. serratis subtus farinosis, radicalis petiolatis, pe- 
tiolis alatis basi dilatatis, florib. verticillatis, corollis lobis integris.-In Arabia, Mts. of Kuman, flowers sweet scented like a primrose.-This is the real Prim. vertic. of Forsk, Vahl t. 5. and all the authors.

288. Coniphus R. (bell tubular) Ovario infero adherens elongato 4 gono, cal. 5partito, corolla tubulosa 5fida, Stam. 5, antheris coalitis Stylo filif. Stigma bifidum, Caps, 4ang. 4valvis siliquosa, 2locul. sem. plurimis biserialis ad dissepimento libero inserta, Frutex, fol. sess. $f$. axill sess.-Blended with Campanula and Prismatocarpa, different by corolla, anthers, capsule; same natural family, yet near Lobelia by the anthers comnected.

289. Codiphus nitidus Raf. Prismatoc. nitidus L'her. t. 3. Campanula prismatoc. Ait. W. auct. Honk. b. m. 2733. Glaber assurgens, fol. lanceol. rigidis laxe serratis spinulosis, bractea unica linearis.-South Africa, small white flowers. The Campan. plicata? and tomentosa? probably belong here also ; but the Genera Prismocarpus, Apenula or Legouzia, \&c. of which Polemonium ruelloides and Campan. Speculum are the types, appear quite distinct. Camp. biflora and amplexic, are also types of peculiar Genera, see my Flora.

290. Benaurea, Raf. (well gilt) diff. Campannla, Cal. campan. 5fido 5angulato. Cor. camp. stellata 5 fida, ad basi cal. adnata, Stam. 5 ad basi corolla adnatis vel insertis, stylo 1 , stigma 5 linearis undul. Caps. 5 gona 5locularis. Frutex carnosus, fol. alt fl. paniculatis.This G. and the 3 next differ from the real Campanula, Prismocarpus, Legouzia having a tribocular capsule, by capsules with $\mathbf{5}$ cells and often angular: but this $\mathbf{G}$. deriates besides 
from the essential character of free stamens not inserted on corolla, and probably belongs rather to the family of Drervillaria ; but it has leaves alteruating?

291. Benaurea sempervirens Raf. Campanula aurea L. auct. Dumont. Foliis ovato lanceol, glabris serratis, fl. panic. cal. coloratis. -Madera, flowers golden, shrub with fleshy stem-Dumont has well described the flowers. 292. Coxcilium, R. (Campanula lat.) diff. from Campanula, by-Capsula cylindrica, 5locularis, stigmas 5, corolla subrotata. Frutex ericoideus.-This G. like the 2 last has a shrubby habit, the type is, Concilium peduncularis Raf. the Camp. fruticosa L. auct. of South Africa.-Fol. lineari subulatis, fl. longissime pedunculatis.

293. Decaprisma, R. (10 ang.) diff. Campanula. Stigma 5. Caps. decagona 5locularis, valvis angularis.-Type Decaprisma cespitosa Raf. Camp. do. Scop. fl. carn. t. 4 et auct. Caulib. cespit. angulat. fol. sess. imbricatis lanceol. ovatisque, serratis glabris, subtus punctatis, bracteis lin. integris, fl. nutantes-Alps of Carniolia, flowers blue.

294. Mindrum Ad. non Jus, diff. Campanula. Cal. 10-12 partitus alt. reflexis Cor. camp. 5-6fida, Stam. 5-6, basi valvatis distans, apex patens, antheris pendulis, Stylo clavato, stigma 5-6fido. Capsula 5-6gona, 5-6 locularis. Rad. tuberosa, caule nodoso, rames et fol. ternis, fl. ad dichotomia.-Habit totaly unlike Campanula, similar to Rubiacea, perhaps of family Diervillaria like 290 if stamens inserted on corolla. 'The G. Forgesia and Ceratostema of Jussieu appear to be akin, and of same family ; 
but Chupalon is nearer Vuccinium and Rubiacea.

295. Mindium canariense? Raf. Fol. petiolatis hastatis dentatis, levis, caulinis ternis, rameis oppositis, fl. solit. cernuis.-Fine plant of the Canaries, root fusiform, stem erect tripedal, flowers large rufous, branches dichotome. Of this plant Linneus made $2 \mathrm{Sp}$. and 2 Genera! Campanula canariensis and Canarina campamula! And was imitated by his disciples: the generic name Canarina is inadmissible being formed from Camna and Canarium! 'The Mindium of Jussieu was a different G. now called Michauxia.

296. Petalostrua, Raf. diff. Campanula, Corolla infundib. tubo brevis intus villoso, limbo 5 fido. Stigma 5 petaloideis obovatis subtus hispidis, capsula 5locul. 5valvis, sub 10 gonus. Fol. oppositis, pedunc, axill. ramosis.-Another ont of the 20 Genera blended in Campanula. Habit peculiar, and strange form of stigmas.

297. Petalostima capensis Raf. Camp. do L. auct. Commel. t. 35, Curtis, b. in. 785. Caulescens, fol. lanc. dent. hispidis mucronatis, pedunc, longissimis, cal. et caps. hirsutis.-South Africa, flowers blue.

298. Hecale, R. (Nympha) diff. Campanula, Cal. 3-5 fidus, Cor. infundib, 3-5fida, Stam, 3-5, Stigma bilobo, Capsula oblonga bilocul. Canlescens, fol. alt. fl. panicul. secundis.-This G. is nearer Codiphus by capsule, but it is not angular, nor corolla tubular. Type, Hecale lobelioides Raf. Campan. do. L. auct. Glabra, ramosiss, fol. lanc. serrat. sessilibus-In Madera, many small flowers, whitish purple in terminal secund panicles. 
299. Prloren Raf. (head hill) diff. Campanula, Stigma bifidum, Capsula bilocul. flores copitutis.-Near the last, but Corolla campanulate 5fid, perhaps a sub genus of it. 'I'ype Pilorea graminif: Raf. Campan. do L. auct. Cespitosa, fol. lin. subul. basi ciliatis, caulinis subamplex, bract. ovatis acuminatis.-Mts. of Italy and Sicily, flowers violet blue in terminal heads on short stems. Seen alive.

300. Pentropis Raf. (5 keels) diff. Campanula, Calix 10fidus, 5 alt. reflexis, Corolla 5 fida campan. stigma 5fidum, Capsula globosa, 5locul. 5carinata.-Fol. alt. Al, relt.-Another G. of this fine tribe with 5 cells, Type Pentropis Saxatilis Raf. Camp. do L. auct. Barr. 813. Boc. 64. Fol. obov. crenatis, fl. nutantibus.-In Creta, large blue flowers.

I shall resume the New Genera of Campanula with those of Vaccinium. Enough has been stated to show that the $\mathbf{G}$. Campanula was absurd and unnatural, since it had Cal. with 3 to 15 parts, Corollas of all shapes with 3 to 6 parts, 3 to 6 Stamens, free or united, on calix or corolla, 3 to 6 styles or stigmas. 5 to 6 cells to Capsule, opening by valves or pores, \& $\mathrm{c}$. 'The true Campanulas will be confined to those with Cal. 5fid, Cor. camp. 5fid, 5 Stamens; 3 stigmas, 3 cells and no angles to the eapsule opening by pores. Those with angles and valves from the G. Prismocarpa, Apenula, Legouzia, Stephalea, Blepheuria, Pleurima, Palaeno, \&c. 


\title{
FLORA TELLURIANA.
}

\author{
CENTURIA QUARTA.
}

301. Pinaopus R. (thick fect) Pontederia azurea et crassipes auct. When I refered these plants with doubt to my reformed Pontederia 5, I had not before me Hooker's figure, which proves that they hardly belong there, and confirms my opinion that the whole wants reform, and to be examined again. These form the type of a beautiful N.G., probably with many overlooked, sp in S. America.-Corolla tubo incurvo, limbo amplo ineq. nonbilabiato, 6partito. segm. obovatis, uno supero major. Stam. 6 ineq. 3 intubo, 3 longior exsertis, omnis curvis. Ovar. ovato, Stylo longo flexuoso, Stigm. capit. papilloso. Capsula 3locular polysperm, sem. central. Plant. natans, radic. fibris fimbriatis, fol. radic. petiolis inflatis fusiformis celulosis, fl. amplis spicatis, rachis triangularis non spadix. What a striking G. by habit and characters! 3 Types at least.

302. Piaropus tricolor Raf. Fol. cordatis ovatisque, acutis, corollis purpurascens, petalo major tricolor, stam. piloso. glandulosis, pistilis viridis. From Guyana to Guayaquil, probably several sp. that of Swartz and Antilles has azure flowers, this has them pale purple, the upper petal blue in the middle with a yellow spot, fl. 3 inches.-Pont. azurea Hook, b. m. 2932 Synon. dub. ad Kunth, Hoenk, confer.

303. Piaropus mesomelas Raf. Ponted. crassipes Mart. braz. ic. Fol. rhombeis, corollis 
azureis, stam. glabris, pistillis nigris._Brazil and B, Ayres.

204. Piaropus azureus Raf. Ponted. Azurea Sw. Wild Pers. fol. subrot. ellipticis, corollis azureis.-Antillis. Hooker in order to obscure the subject has blended these 3 into one-it is thus that Botany is made to retrograde, and distinctions are overlooked. 'These plants are as different from my Unisemas G. 6, as may be Roses and Geraniums.

305. Kunda R. (nom. Sanscr) Aroid. Spatha coriacea campanulata dimidiata dilatata undulata plicata, Spadix fungiformis basi columnaris tereto florifero, apex capitato dilatato undulato. Pistilis inferis, globosis stylosis, stigma trilobo. (bacca 3sp.) Stam. superis 4 locul. poris 4 apice dehiscens. Acaule, rud. tuberosa fibris comata flos subsess., fol. radic. divisa.-Most extraordinary Indian Genus, near Arum by habit; but quite distinct by spatha, spadix, styles and anthers, The flower and leaf appear at different times. The huge root is esculent and cultivated for food. I have given to it the old sanscrit name. Perhaps several blended species, but the type is

306. Kunda verrucosa Raf. Tuber depresso zonato gemmulato fibrillato: folia decomp. trifida, deinde bifida pinnatifida aspera foliolis obl. acutis ; flos. verrucosus intus leve luteo, margine rubro, spadix capitulo equante magno atropurpureo mesenterico.-India, Ceylon, Molucas. Root brown 4 to $8 \mathrm{lb}$. weight crowned by white fibres, flower 1 or 2 feet wide, on a short thick warty peduncle with some uneq. spreading sheaths. It is Arum campanulatum Roxb. cor. t. 272, Hook. b. m. 2812, Arum zeylanicum 
Com. t. 53. Tacea phalifera Rumph t. 112. Schena Rheed 11 t. 19. Not in Linneus.

307. Spatulima Raf. Legum. diff. Orobus, Cal. urceolatus, 5nervis 5dent. eq. Vexillum elong. emarg. Stylo reflexo Spatulato marginato plano subtus pubescens, stigma margine obtuso. Foliolis binis ternatisque sessilib. pedicellis elongatis multifloris. The style or stigma of this G. is quite peculiar. Probably several sp. blended as Orobus sessilifolins, ensifolius, digitatus, pyrenaicus, \&c.,-all refered here by Hooker.-Type.

308. Spatulima angustifolia Raf. Caule flex. striato, foliolis 2-3 mucronatis lin. angustis acutis, stipulis subul. semisagitt. pedic. 3-4floris, fl. pendulis. Crimea, Greece, Sicily. fl. purplish, vexillum darker. Orobus sessilif. Sm. DC. Hook. b. m. 2796. Or. digitatus? Bieb. Spreng. 309. Petaloxis Raf. (pet. sharp) Commelinea, Cal. 3phyl. Cor. petalis 3 acutis persistens, eq. Stam. 6. subsess. subeq. glabris 3 ext. 3 int. Antheris lin. 2loc. apice 2porosis. Stylo filif. stigma obt. Caps. globosa corolla baccata, 3locul. polysp. Fol. vaginans, fl, racemosis.Wrongly united to Dichorisandra of Mikan by Hooker, which has 5 Stamens only, in 2 unequal phalanges, anthers not porose, petals not acute.-It is near my G. Eothinanthes, having same habit.

310. Petaloxis purpurea Raf. Dichorisandra oxypetala Hook. b. m. 2721. Caule tereto striato basi squamoso apice folioso, fol. ellipt. acum. striatis, undul. racemo flexuoso, pedicellis biftoris, petalis ovatis acutis purpureis.-South America, stem simple or forked, raceme terminal, pretty purple flowers.

311. Dimanisa, (Twin uneq.) Raf. Cal. 
5part. bibract. Cor. tubul. bilab. lab. sup. erecto emarg. lab. inf. eq. 3fido. Stam. didyn. Antheris biloc. loculis ineq. Capsula ut Justicia. Frutex artic. nodosis, fol. opp. fl. axill.-How could Hooker unite to Justicia a plant with 4 Stamens! 2 types if Link has made same blunder for another.

312. Dimanisa latifolia, Raf. Justicia nodosa Hook. b. m. 2914. Fol. ovatis acutis subintegris, bracteis linearib. cal. longior-Beautiful shrub with scarlet flowers, locality unknown.

313. Dimanisa? oblongata, Raf. Justicia do Link H. ber. t. 9. fol. oblongis lanceolatis, bracteis foliosis lanceol. cal. duplo longior. If this is a brazilian plant, the former may be such also, has this really 4 stamens like the last?

314. Faulia, R. (Olea. gr'.) Cal. 4fidus, caliculatus bracteis 4. Cor. rotata plana 4fida, Stam. 2 opp. divaric. longis. Ovar. ovat. stig. globoso subsess. Bacca 2loc. 4sp. Fol. opp. fl. Spicatis panic. Very distinct from Ligustrum by cal. cor. \&c. nearer Olea. The Lig. japonicum, Sinense, lucidum of Asia may belong to it; but the types are the two next Sp.

315. Faulia verrucosa, Raf. Ramis teretis verrucosis, fol. petiol. lato oblongis acum. glabris undul. panicula trichotoma glabra. cal. scabris. In Nepal. fl. white inodorous? Ligustrum nepalense var. glabrum Wallich, Hook. b. m. 2921 .

316. Faulia Odorata, Raf. fol. ovatobl. acum. subtus villosis, panicula villosa densa, racemulis spicatis, cal. villosis-In Nepal, flowers white sweet smelling, berries oval blue. $\mathbf{L i}$ gustrum Spicatum Don. fl. nep. or Nepalense 
Wallich.-The authors appear to have blended 2 species, and thus not well marked the mutual distinctions.

31\%. Egena, R. (nympha) Cal. glob. 5dent. Cor. tubulosa incurva, limbo 5lobo eq. Stam. 4 didyn. exserta, stylo filif. stig. acuto. Bacca in calice 4 sperma. Frutex fol. opp. fl. corymbosis-Pretty G. blended with Clerodendron with Cal. 5fid. drupo 4pyreno. 'Type E. erminensis fol. opp. ternisque ovatis petiol. integris vel apice serratis, corymbis term. Clerodendron do. Hook. b. m. 2925. fl. yellowish or incarnate white Madagascar.

318. Colax, Sprengel. Orchideous G. properly distinguished from Maxillaria altho' blended by Hooker and Lindley. Petalis non resup. basi coalitis, 2 infimis in cuculo calcariforme adnatis. labello basi cucul. Col. apice glandula bifida ferens, Pollen 4 supra glandula ineq. 2 minor. Scapo artic, 1-2 fl. bulbis terrestris unifol.-Colax harisoni Spr. Dendrobium do Hook. ex. fl. 120. Maxillaria do Lindl. b. reg. 897. Hook. b. m. 2927. Grandiflora would have been a better name; large yellow fl. 4 inches broad odorous, lip red, petals oval, leaf lanceol. plicate.

319. Tritelandra, R. ( 3 perf. St.) Orchidea with 3 perfect stamens, quite distinct from Epidendrum with one! instead of an anomaly it is deemed now the real type of the tribe! and yet was left in Epidendrum! Petalis 5 patulis subeq. label. major basi adnato ad columna, tereta, apice antice antheris ternis subeq. liberis in triangulo positis, supera major 4 pollen, 2 infimis latere valvatis 2pollen. Rad. vermicul. fol. distichis, fl. capitatis-Distinct by habit, lip and anthers, as near Octomeris as Epiden- 
drum. Probably several sp. mixt. compare Epid. fuscatum, anceps, secundum \&c. the main type is

320. Tritelandra fuscata, Raf. Fol. obl. acutis, caule basi et apice subnudo, petalis obovatis, label. ftabellato lobulato-Antilles, flowers small greenish brown. His Epid. fuscatum Sw. W. often figured by Sm. Andr. Lod. 472. Bot. reg. 67, Bot.mag. 2844. Is the E. anceps Jaq. Lod. 887, the same? and E. secnndum Sw? as Hooker says; but he is so much in the habit of confusing Sp. that these must be verified again.

321. Sacolla, R. (bag hollow) Orchidea diff. Neottia, Petalis - connivens teretis, 3 infernis basi coalitis saccatis unicalcaratis, labello indiviso, columna teres truncata aptera, stylo conico, anthera liaearis 2pollen postice posita. Caule squamoso, vaginato fl. spicatis-Very distinct by habit, united petals and style. Type Sacoila lurida Raf. (Neottia aphyla Hook. b. m. 2797) Tota rubescens fuscata, squamis acutis vaginatis, spica paucifl. fl. nutans, labello ligulato obtuso integro. Antilles, whole color lurid redish brown, flowers 5-8 large shaped as in Spiranthes, but calcarate not spiral: habit of Orobanche.

322. Neotria, of authors. They have mixt in this Genus a crowd of heterogenous plants, and so many have been removed that now we hardly know the real types of it. The characters of all the authors are quite different. Many Sp. of Ophrys, Orchis, Serapias, Satyrium, Limodorum, $\mathcal{S} \cdot c$. have been united thereto, and the Aristotelia of Loureiro. While the Genera Spiranthes and Goodyera have lately been distinguished. All the Sp. must be examined 
again, I have removed several of them: see my G. 214 Dothilis, and N. calcarata or my Eltroplectris 185. As many as 12 Genera may be made out of this; and the real reformed Neottia will merely comprize $N$. Speciosa, elata Orchioides, plantaginea of Hooker, not mine of 1817 which is a Spiranthes.

323. Neottia, Raf. Corolla ringens, petalis connivens, labello concreto subeq. basi saccato Col. tereta, stylosa, anthera stylo paralela. Fol. radic fl. spicatis.-Even this requires revision; it appears in this the lip is saccate while in Sacoila it is the external lower base of petals.

324. Annula, Raf. diff. label, et 2 sepalis tricalcaratis decurrens calcarib. ad ovario adnatis. 'Type Adnula petiolaris Raf. (N. adnaria auct.) fol. longe petiol, oblongis, spica simplex, labello deflexo bilobo. Antilles.

325. Narica, R. (Nympha) diff. Neottia, labello concavo bilobo, petalis liberis, fol. rad. Rorib.radic. sessilibus--Type Narica moschata Raf. fol. sessilib. ovatis undulatis, labello oblongo obtuso pubero dilatato undulato-ld Trinidad, fine large flowers smelling like musk, mixt of white and green. It is the Neottia acaulis of Smith.

326. Monustes Raf. (Nympha) diff. Neottia, labello non saccato basi appendicis binis ligulatis columna alata, lobis elongatis. Fol.radic. $f$. Spicatis Spiralis.-This G. is near Pterostylis with the habit of Spiranthes. Type Monustes australis, Raf. fol. lanceol. labello oblongo undulato. Neottia anstralis Brown, Smith.

327. Goodyera, Br. 1812? Thessaca Raf. 1814. for this G. see my new flora of N. America. Habit peculiar by dense spikes.

328. Spir.nvtues, Richard. Girostachis Per- 
soon 1807 proposed earlier but not established, and objectionable formed from Stachys. Genus now generally adopted aud easily known by the habit of spiral flowers. The lip is unguic. bicallose paralel, anther peduncled $\mathscr{f} \cdot c$. It is very prolific in species, not yet well distinguished and often blended. Many new species of N. Amer. are described in my Herbar. New flora, Autikon. \&c. but I add here some exotic species.

329. Spiranthes tortilis et Satyrium spirale Sw. Fol. longissimis linearibus, Spica tortilis, fl. secundis laxis, labello trilobo crenulato. In Jamaica, the plants of $\mathbf{N}$. Amer. and China, refered to this are all different.

330. Spiranthes laxiflora Raf. Fol. linearib. spica laxa, floribus glabris nutans, labello reflexo trilobo.-In China, fl. white large. It is Neottin tortilis Smith \&c. but not the $N$. sinensis of Wild.

331. Spiranthes flexuosa Raf. Caule basi folioso, fol. oblongis patulis, florib. villosis, label. ovat. undul.-In Nepal, fl. whitish very spiral. Neottia flexuosa Smith. Roots vermicular.

332. Spiranthes Sibirica, Raf. fol. lin. lan- ceol. erectis, spica gracilis, labello obovato crenato. In Sibiria, blended with our Sp. estivalis and many other peculiar Sp.

233. Spiranthes glauca, Raf. Foliis ovatis petiolatis glaucis, fl. albis adoratis, labello ovato crispo.-In South Europe, blended with Sp. autummalis, or Neottia spiralis by many authors, which has oblong green leaves subsessile. 'There are two other odorous Sp. in North Amer. and others in Sibiria.

334. Spiranthes parviflora, Raf. (Neottia do $\mathrm{Sm}$.) same characters as $\mathbf{S}$. flexuosa except flowers hardly spiral unilateral, lip red 
crenulate.-In Nepal also, Sp. bicolor perhaps a better name.

335. Spiranthes diuretica, Raf. (Neottia do Auct),fol. radic. lin. fl. unilat. vix spiralis, labello obl. obtuso crenato reflexo.-In Chili, Nil Feuil. t. 17.

336. Spiranthes quadridentata, Raf. (Neottia do Auct) Caule basi folioso, fol. obl. obt. spica secunda vix spiralis, labello obovato Adentato-Guyana \& Antilles.

337. Nerissa, R. (Nympha) diff. Neottiot, petalis sub patulis obovatis campanul. labello cordato concavo. Floribus racemosis, pedunculatis-Type Nerissa glandulosı Raf. Neottia do Sims. bot. mag. 842, Smith \&c. Fol. radic. ellipt. obt. recurvis, fl. racemosis, labello acuto -Antilles, fl. white with green stripes. Compare with G. Corallorhiza.

338. 'Tonotris R. (cut cluster) diff. Neottia, labello basi inflato, apice plano, calcarib. nullis, petalis vix concretis. C'aule ramoso articul. geniculato, folioso, racemis compositis.-Very near Neottia and Sacoila, but habit quite peculiar indicating a different Genus. 'Types $\mathbf{T} \boldsymbol{o}$ motris polystachia and flava, Neottia do of authors. Once Serapias fluta.

339. Strateuna Raf. Stenorynchus? Auct. Diff. Neottia culcar elongato ut in. Eltroplectris, angusto libero, labello bilobo. Rad.fascicul. caule folioso, spica foliosa.-Type Strateuma zeylanica Raf. Orchis strateumatica! Lin. Fol. lineari lanceol. infimis brevioribus, calcar ad ovario equante. Id Ceylon.

340. Synoplectris $\boldsymbol{R}$. (united spur) Petalis connivens, bilabiatis 3 supernis coalitis galeatis-labio inf. bifido petalis 2 basi coalitis et decurrens in tubo calcarif. ad ovar. toto concreto 
adnato, labello elongato spatulato integro basi gibboso, decurrens in tubo. Columna erecta, tereta, basi mellifera. apex glandula, anthera infera ad glandula dependens, loculis 2 lanceol. pollen 2 utrinque. Fol. magnis radicalis, scrtpo bracteato, fl. spicatis flavis.-This G. is very peculiar and distinct from Admula by the position of the bilabiate petals, anther and habit, 2 types.

341. Symoplectris viridis Raf Neottia viridis and grandiffora. Hook. 6. m. 2730. (not the same as 2956) Spiranthes grandiflora, Lindl. b. reg. 1043 , not at all like a Spiranthes!-Fol. sessilib. carnosis ovato lanceol. undulatis nervosis, scapo sulcato bract. ov. lanc. fl. bract. lin. lanc. puberis, petalis connivens, labello basi involuto, apice undulato.-Brazil, large leaves and flowers occupying most of the scape and greenish yellow.

342. Synoplectris picta. Raf. Neottia vel Spiranthes do Auct. Fol. petiolatis ovato-lanc. maculatis pictis, petalis subpatulis labello revoluto.South America, flowers whitish yellow smaller.

343. Diplectraden (double spur gland) Raf. Orchidea. Petalis 3 ext. concavis, superior galeato, lateralis obliquis, petalis 2 internis semi sagittatis, labello lin. basi trifido, calcarato. Anthera unica ovata sulcata, biloc. bipollen pedic. basi bicalcarata, biglandulosa. Cuulescens, spicuta.-Neither Neottia nor Habenaria as supposed: Anther peculiar, like Orchis.

344. Diplectruden leptoceras Raf. Neottia orchioides. Auct. Habenaria leptoceras Hook. b. m. 2726. Caule angul. fol. lanceol. carinatis, spica laxa, bract. ovato lanc. calcar filif. compresso ovario duplo longior.-South America, flowers green many, lip yellow acute, ovary striated twisted. 
345. Exporna Raf. (in pits) diff. Conyza, Periantho imbric. tereto scarioso, phorantho favosum, alveolis magnis ineq. laciniatis, flosculis omnis fertilis tubulosis 5dent. antheris bisetosis. Sem. obl. verrucosis in alveolis immersis, pappus filis clavatis plumosis basi coalitis interdum furcatis. Habitus Comyza, fl. pedunc.-Very striking distinct Genus by phoranthe, seeds and pappus, nearer Conyza than Astelma and Guaphalium.

346. Endorima modesta Raf. Astelma do Sieber, Gnaphal. do. Hook. b. m. 2710. Fruticosum ramosum, fol. lin. fil. canalic. cano tomentosis, pedunc. term. unifl. elongatis, periantho sq. cuspidatis roseis tomentosis, flosculis flavis.South Africa, pretty plant, discovered by Sieber; but the G. Astelma of Brown has a smooth mutic phoranthe.

347. 'Trilonisa Raf. (3 edges eq.) diff. Begonia by, fl. masc. pet. 4 ineq. 2 patulis, 2 internis minor erectis, fl. fem. petalis 5 subequalis, stigm. 3 bilobis convolutis angulatis, Capsula alis 3 equalis, loculıs 3 equalis. Frutex artic. $f l$. subsess.fl. panic.-Type 'Trilomisa undulata Raf. Begonia do Auct. H. b. m. 2723. Caule albo macul. fol. brevi pet. obliquis undulatis ellipticis glabris, panic. axill. dichot. ft. fem. pendulis.-Brazilian shrub with white flowers.

348. Strepsiphus Raf. (twisted tube) Cal. 5phyl. subeq. Cor. tubo elongato tortilis, limbo bifido bilab. lab. sup. obl. 3dent. inf. integro s. bif. minor. Stam. 2 Antheris loculis 2 superpositis obliquis. Stylo filif. stig. emarg. Caps. ut Justicia.-Frutex, fol. opp. pedunc. axil. involucr. 3floris.-Very distinct G. near Dianthera, corolla and stamens quite peculiar.

349. Strepsiphus speciosus Raf. Justicia 
speciosa Roxb, Hook. bot. m. 272\%. Fol. opp. petiol. ovatis acutis crenulatis glabris, infimis subcord. pedunc. axil. et term. involucris duplicis, utrinque Aphylis ineq. spatulatis trifloris ciliatis.-Bengal, fine shrub with many large violaceous flowers.

350. Delonix R. (evident claw) Legum. diff. Poinciana, Cal. eq. non fornic. Pet. 5. subeq. unguis longissimis, limbo. crenatis flabellatis. Stam. 10 ineq. glabris, declinatis.-D. regia Raf. Poinciana regia Hook. b. m. 2884. inermis, fol. bipin. ovatobl. muticis. A beautiful tree of Madagascar, with large scarlet flowers. The G. Poinciana DC. separated from Caesalpinia is American with uneq. cal. hooded, petals unequal, stamens hairy. \&c.

351. Oronora R. (Papaver diosk) Eschscholzia Auct. barbarous russian corrupt name, similar besides to the prior Elshozia of Wild. Auct. being dedicated to the same family of botanists! wrongly united to Loasacea by DC. and by Hooker to Papaveracea; I have ascertained on the living plant, that it belongs to my nat. family Glinidia of 1815 near to Glinus and Portulaca, all the Papaveracea with several styles belong there also; but it is the type of a sub family Omonoidea by calix and capsule. Such diversity happens in Hypericea and Portulacea, both very near families of same order POLY: MESIA. My family Resedacea differs by unequal calix, petals and stamens.-Cal. calyptratus, pet. 4. Stam. plura hypog. epipetalis. Ovar. conico, Styli 4 ineq. lin. siliqua bivalvis angul. uniloc, polysp. valvis seminif. fol. decomp. $f l$. term. solit. amplis, flavis.-Omonoia californica Raf. foliolis linear. apice multifidis. Eschscholzia do. Chamis, DC, bot. reg. 1168, bot. mag. 2887. Sweet t. 265, \&c. 
352. Streptua R. (twisted st.) Cal. tubul. 5ang. 5 dent. Pet. 5. unguic. eq. Stam. 6. ineq. 3 major hypogynis. Stylo filif. apice trifido contorto flexuoso. Caps. uniloc. 3valvis, valvis seminiferis polysp.-Frutex fol. opp. vertic. vel fascic.fl.axill.sess, vel.ad dichot.-Str. pauciflora Raf. Fronkenia pancifl. DC. Hook. b. m. 2896. fol. lin. obt. marg. revol. canescens, petalis cuneatis apice crenatis roseis.-Another plant evidently united to Frankenia by mere habit, altho' akin; both belong to POLYMESlA also, and are akin to Omonoia, Reseda and Portulaca, differing by Stam. definite ; but they are neither equal nor isarine as in Alsinides and Phorandres, the Caryophyles of authors: they belong therefore to Hypericea near Sarothra and Triadenum with Menetho, Nothria and some others, having few stamens. The Frank. revoluta of Forskal with ovate revolute leaves, dichotome stem, belongs here perhaps, it is an Egyptian shrub. Str. pauciflora altho' supposed to be Australian is perliaps African also. Ifooker doubts if his own plant is really that of Decandole, because scabrous. All the shrubby Sp. of Frankenia are also African, such as corymbosa and thymifolia Desf. except $F$. microphyla of Patagonia. But all the sp. must be examined again, since Adanson gives a different character to some species, and the next real Australian Genus is very distinct from both. 353. Menetio R. (nom mythol) Cal. 4fidus, petalis 4 unguic. integr. Stam, 6 ineq. stigma 2, Caps. uniloc. bivalv.-Type M. sedifolius Raf. frutex prostratus, fol. subul. crassis calcaratis, fl. term. sessilib. In Australia, the Frankeria quadripetala of Labil. t. 114.

354. Frankenia, I. Franca Mich. Cal. teres 
5dentatus 10 gonus persistens, Petalis 5 integris ung. squana ad tunguis, stig. 3 vel. 6 (Lin.); Caps. umiloc. 3valv. sem. centralis? This includes many herbaceous $\mathbf{S p}$, of the mediterranean region, Fr. levis, hirsuta, \&c. which I have seen alive, and found with 3 stigmas and central seeds.

355. Notimia, Berg. Franca Ad. I take the name from the first, but characters from Ad. Cal. tubul. 5dent. Pet. 5 crenatis, Stam. 5-10 (an 6-9 ?) stylo, stig. 3 caps 3 valv. unil. Jussieu says Adanson meant 3locular. The type of this is the Frankenia pulverulenta of several authors, found from Sibiria to Senegal ; but perhaps several Sp. have been blended. The real Nothria repens of Berg and South Africa has acute petals, and must be examined again. In fact the authors have neglected to notice the inequality of stamens and their proportions, which are quite essential ; altho' heterines for the Corolla, they are isogyne or proportionate to stigmas in all.

356. Lacanthis, R. (much spinos.) Euphorbia splendens Hook. b. mag. 2902. of all the beautiful and strange plants blended in Euphorbia, this is one of the most singular and distinct Genus.-Periantho cupularis 10lobo, lobis alt. minor glanduliformis, Phorantho villoso. Androphoris bifidis, stam. 2 ferens, antheris subrot. uniloc. Gynophoro nullo, ovar. sessile obl. stylo 3 fido, stig. 3dent. Frutex toto aculeis vestito, fol. parcis sparsis, umbella dichot. bracteis binis coalitis coloratis unifl.-How can a botanist of sense unite this with even the other fruticose Euphorbias?

35\%. Lacanthis splendens, Raf. Aculeis basi dilat. confluens, fol. cuneatis cuspidatis. bracteis 
orbic. mucron. coccineis-Disc. in Madagascar by Bojer, the bracts are scarlet as in Pleuradena, while the fl. are of a dull yellow. The bifid stamens and $\mathbf{1 0}$ lobed fl. are quite striking: deemed generic in Salvia! why not here? I would have dedicated this fine G. to Bojer, if I had been sure there is no other Bojeria.

358. 'Tropilis, R. (keel lip) diff'. Dendrobium, Petalis 5 linearib. vix patulis basi coalitis, labello parvo cucul. basi cum appendicis ad columna adn. apice reflexo, dorso 3carinato. Rad. fibrosis, bulbis terrestris costatis pancifol. Scupo laterali paucifl.-'Type Tr, emulum Raf. Dendrob. do. Br. Hook. b. m. 2906. fol. ovatobl. scapo brevi 3-5fl. labello tricarinato, apice acum. latere unidentato-Australia.

359. Eusteralis, R, (mentha diosk) diff. Mentha. Cal. ovato inflato 4 fido, eq. Cor. tubul. 4dentata subequalis, Stam. 4 subeq. filam. ad media barbatis, antheris uniloc. transv. dehiscens. Anmu, fol. vertic. fl. spicatis-Fine Genus habit and characters quite peculiar. Type Eusteralis pumila Raf. caule vesiculoso, adscendens, fol. 4-5nnis lin. lanc. sub serratis, spica term. densa, bract. ovatis lanc. ciliatisIn Nepal, corolla rosate. It is Mentha pumila Grah. Mentha verticillata Roxb. Don. Hook. b. m. 2907.

360. Strrosinia, Raf. (cross union) Cal. 5 part. ineq. libero, Cor. tubulosa clavata basi supra bigibbosa staminifera, limbo 5lobo. Stam. 4 didyn. antheris $\mathbf{4}$ coalitis planis cruciatis $\mathbf{4 l o b i s .}$ Ovario libero ovato, basi gland. 4 ineq. supera major, stylo filif. stig. obt. Fol. petiol. oppos. axillis multifl.-W -Wongly united to Gesneria, not same family since ovary free and stamens on 
corolla, rather near Scrophularia; but do not the united anthers indicate a peculiar tribe?

?61. Styrosinia coccinea Raf. Gesneria agregata Bot. reg. 329. Hook. b. m. 2\%25, G. pendulina B. reg. 1032! Villosa, fol. ovatobl. rugosis crenatis acutis, axillis 2-4 $\mathrm{fl}$. pedunc. ineq-Brazil, pretty flowers scarlet, one inch long.

262. Rafinesquia vel Flundula, Raf. Leguminosa, Cal. tubul. basi carnoso, apex 4dent. dent. sup. bifido. Petalis longe unguic. undulatis, subeq. Vexillum major reflexo cuculato. Stam. 9 monadelphis, filam. apex liber. parvo, 4 alternantis fil. et antheris minor, filam. decimo toto libero sterilo. Ovar. lineare, stylo filif. incurvo, stigma capit. glabro. legum. lin. acum: compr. subarticul. polysp. sem. ellipticis. Fol. imp. pinnatis, fl. umbellatis-Another G, wrongly refered by Hooker to Lotus, by mere inflorescence: lacking even the trifoliate habit, It is a beautiful distinct Genus by habit, calix, corolla, stamens, stigma and fruit... It is one of those I propose to dedicate to myself, as Linneus did for the Linnea, but I propose a second substitute, in case there is another previous Rafinesquia. 'This is so distinct that I rather fear it may have already a third name unknown to me.

363. Rafinesquia (vel Flundula) comosa Raf. Lotus pinnatus! Hook. b. m. 2913. Glabra, ramis teretis striatis ftexuosis, stipulis ovatis, foliolis 7-9 obovatis (vel oblongis) pedunc. elongatis, 5-7floris umb. sessilibus-In Oregon, on Columbia R. \&c. Hooker says the wild specimen liad obovate leaves; but in the gardens they changed to oblong! as in his figure. Perhaps two Sp. collected by Douglas, $\boldsymbol{R}$. comosa, 
$\boldsymbol{R}$. obovata, or else the species has undergone an evident transmutation by seeds in Scotland. 364. 'T'riconlendus, R. (3 inside hollows) Legum. ad Lofus toto celo diversus. Cal. camp. ineq. 5 fidus pers. Vexillum dilatatus alae obliquis equante, Carina non rostrata latere tubercul. Stam diadelph. basi submonadelphis, ineq. Ov. lin. pub. Stylo glabro persitens, stigma capitato glanduloso. Leg. bivalv. tereto oblongo, trilocular. $3 \mathrm{sp}$. loculis serialis, sem. glob. punctatis. Facies ut Lotus.-Very distinct N. G. by the pod, stigma, petals, calix, refered to Lotus by Hooker by the mere habit or aspect of the type.

365. Tricoilendus microphylus, Raf. Lotus do H. b. m. 2808. Decumbens filif. puberus, fol. petiol. foliolis 3 parvis obl. acutis carinatis, stipulis subul. pedic axill. elongatis, fl.5-6 capitatis roseis.-Bouth Africa. Delicate annual with minute leaves and flowers.

366. Liniactis, Raf. (lily star) Petalis 6 equalis patulis stellatis, 3 ext. apex subbident. Stam. 6 brevis eq. filam, subul. glabris, antheris linearis, ovar. sess. trigono, stigma sessile trigono supra plano. Caulescens, 1-2/l-Fine new G. united to Tulipa by Hooker; but really distinct by petals, stigma foc.

367. Liviactis albiflora, Raf. 'Tulipa stellata Hook. b. m. 2762. Fol. gramineis convolutis, infimis falcatis, superis tortilis, fl. amplis, albis, petalis ellipticis obtusis.-From Kumana in East Indies, two feet high, brown bulb. large white flowers 4 inches broad spreading in the day as Ornithogalum, closing at night.

368. Pleurostima, Raf. (lateral Stigma) Corolla infund. basi adh. tubo angulis 6 verrucosis, limbo 6fido, lac. lanceol. 3 ext. angustior. 
Stam. 6 petaloideis bifidis, antheris linearib. ad basis intus arlnatis. Ovar. adherens obl. scabro Ggono tuberc. stylo conico trigono acuto, stigm. 3 glandulos. adnatis ad medio stylo. caps. 3loc. polysp. Caudex foliosis, scapis lateralis radicalis mifloris.- Fine (r. of family Narcissides, near Herucritiun, not Hemodorea as stated by Hooker. The fruit is very peculiar with 3 large stellate partitions, bearing in their angles 3 placentas and many seeds. Bavbaceniu differs by anthers in the fork of stamens and different style.

369. Plenostima purpurca, Raf. Barbacenia do Hook. b. m. 27\% . Fol. lin. acum. carinatis spinuloso serratis. scapo fol. longiore, scapo trigono scabro unifloro, capsulis scabris angulis 6 tuberculatis.-In Brazil, flower large violet purple, base green.

370. Tulisma, Raf. (warty cleft) diff. Gesneria by Cal. adher. 5dent. Cor. tubulosa, basi 5 gibbosa, apice 5loba, subeq. verrucis ad sinubus, stam. didyn. epicorollis, antheris coalitis, orario apice libero, dorso bigland. stylo filif. stig, obt. Ilerba fol. verticil. fl. term.-Really distinct from Gesneria, and not of same family, unless it has staminiferous corollas as this.

371. Tulisma verticillata, Raf. Gesneria do Hook. b. m. 2\%6. Pubescens, fol. quaternis petiolatis ovatis subcord. serratis, pedunc. 2-4 term, unifl. recurvis, florib. pendulis-Brazil, fine plant one foot high, stem terete. petiols colored, flowers large, 3 inches long, red with darker spots.

372. Mrenanena, Raf. (moon gland) Orchidea diff. Truxillarin Petalis connivens non resupinatis, labello basi cuculato cum basi Col. adnato, ad medio costato. Col. semiteres, stigma Lang. 
Anthera operculata term. pollen ineq. :minor ad glandula lunulata inserta. Baivo rugeso terrestris unifolio, scupis lateralis squmulis unifl.-Very distinet G. by habit, flower and anther.

373. Menatena partieri, Raf. Maxil. do Hook. bot. m. 2729. Bulbo ellipt. rugoso, folia lanceolata pedalis, scapis brevis, squamis imbric. ovatis coloratis, petalis 3 ext. ellipt. 2 int. lin. lanc. labello ligulato trilobo, undulato.-Demerara, flowers fulvous, 2 imner petals white with purple spots, lip yellow with purple border. 374. Matura, Raf. (Nympha) Orchidea. Petalis patens 5 sub-equalis, 2inf. coalitis, labello sinplex basi ztuberc. zalato. Col. semiteres, anthera operc. pedicello pollen 2 ferens, Ovario clavato, Bulbis terrestris lasi et apicem foliosis, scapis radicalis, h. spicatis, bracieis persistens.-Beautiful G. totally unlike Pleurothalis in habit, flowers, anthers, \&c. 375. Muturna suaveolens, Raf. Pleurothalis foliosa Hook. bot. m. 2746. Bulbo oblongo, vix striato, fol. inferis vaginans, fol. sup. binis larceol. carin. scapis multifl. bract. lanceol. $\mathrm{n}$. luteis odoratis, petalis linearibus. labello ovato acuto.-Brazil, fine spike of yellow flowers very fragrant, like Primrose.

376. Mencus, Raf. (honey lip) Orchidea, Petalis ineq. difformis, distortis 3 ext. involutis obliquis, 2 int. erectis undul. Labello heteromorpho, pedunculato saccato mellifuo, operculo magno galeato ferens. Columna elongata tereta, basi bidentata apice incrassata biloba, anthera pollen 2 sess. ceracea. Pulbis terestris striatis bifoliatis, Scapis basillaris biflo ris.-A most extraordinary N. G. which Ioolier is at a loss to describe and wrongly refers to 
Gougora. 'The Lip distils honey that fills the hollow bag.

37\%. Melichis speciosa, Raf. Gongora do Hook. b. m. 2755. Bulbis oblongis, fol. lin. lanceol. longissimis, scapo compresso artic. bifl. galea tridentata.-Brazil, very large yellow flowers 4 inches broad, with a strong smell.

378. Peralantuera, Raf. Cal. 5part ineq. Cor, tubul incurva bilab. lab. nervosis, sup.integro sulcato, infero apice 3lobato. Stam. 2, Antheris obliquis petaloideis ineq. bilobis, uno subrot, alio acum. ambi loculo ferens. Ovario supra glandula magna. stylo filif. stig. acut. Frutesc, fol. oppos. fl. capit. bracteatis.-Another fine G. distinct fiom Justicia and Dianthera by the singular peculiar Anthers with unequal lobes.Near G. Echolium and Dianthera.

379. Petalanthera punctata, Raf. Frutic. fol. petiol. ovatis obovatisque integris glabris. Capitulis obl. term. bracteis imbric. subrot. fl. subternis sessilib. punctatis. - China, fine sp. fl. white with purple dots. It is Justicia Ventricosa Wallich and Hooker bot. m. 2766, but there is nothing ventricose about it.

380. Cruchundula, Raf. Crucifera diff. Hutchinsia. Cal. eq. concav. margine scariosis. Petalis 4 eq. oblongis obt. undulatis, stylo longo persistens. Siliqua oblonga cuneata emarg. Valvis carinatis basi dehiscens, loculis 5 spermis. Pretty G. wrongly united to Hutchinsia, Iberis and 'Thlaspi !

381. Cruciundula minima, Raf. Thlaspi do Ard. t. 15. Iberis Stylosa Tenore fl. nap. 37. Hutchinsia Stylosa. Dec. Spr. Hook. b. m. 2772. Minima, multicaulis, fol. radic. cespitosis obovatis petiolatis subcarnosis, subintegris, caulinis obl. sessilib. stylo siliqua subeq.-Mts, of South 
Italy, pretty little sp. with rose flowers, (Iooker) white (Dec.) quite peculiar by undulate petals. Biennial 2 or 3 inches.

382. 'Tolumina, Raf. (Nympha) Orchidea, Petalis 4 patens, subeq. 3 sup. 1 infero bidentato, labello magno 4 lobum, ad basi 3cristato, Columna semiteres bialata, anthero ovata acuta, pollen 2 supra pedicello clavato. Parasitica, Rad. vermicul. ramosis, fol. radic, carnosis scupó mudo, fl. racemosis.-Another beautiful N. G. of Orchidea refered to Oncidium, altho' it has only 4 petals or 5 with the lip, and different anther, habit, \&c.

383. Tolumnia pulchella, Raf. Oncidium do Hook. bot. m. 2773. fol. carinatis triquetris acutis basi striatis, florib. racemosis secundis, petalis obovatis, labello subquadratum 4lobo, lobis equalibus.-Demerara, lovely sp. with large white flowers in a cluster, with pink and yellow shades.

384. Anipe, Raf. (Nympha) Orchidea, diff. Maxillaria, petalis connivens subeq. 2 externis coalitis in calcar conico, labello unguicul. ad. col. adnato, ad medio tuberculato, sub cuculato. Columna porrecta inter calcar, anthera opercul. pollen 2. Bulbis terrestris 4 gonis, unifoliatis, scapis lateralis. fl, racemosis.-Very distinct G. by habit, conic spur, 2 pollens instead of 4, \&c.

385. Adipe racemosa, (s. fulva) Raf. Maxil. do Hook. bot. mag. 2789. Bulbo compresso 4gono squamis ad basis, folia lanceol. trinervia undulata, racemo laxo multifl. petalis ovatis acum. labello obovato, margine involuto, obtuso integro.-Brazil. flowers fulvous. Hooker says the Dendrobium or Xylobium Squalens may be akin. Is it of same Genus? Why not attend 
to the generic distinctions! Hooker can hardly make out the genera of his new Orchidea, and Lindley bases his genera on inconspicuous characters, instead of plain, striking distinctions.

386. Zenonops, Raf. (Zelon, Datepalm) Palma, diff: Phenix Stain. 6, Stylis 3, Ovar. 3sperm. Drupa monosp.- The Date palm to which it has been wrongly united has $\mathbf{3}$ Stamens and 1 style!-Type Zelonops pusilla, Raf, Fol. pinnatis inermis, foliolis linearib. Caule pusillo pedale vel bipedale. - Small dwarf palm of India and Anam. It is Phenix pusilla Lour. Gaertn. t. 24. Ph. farinifera Roxb. Cor. t. 74. Smith et Auctoris.

387. Prarruula, Raf. (thick stigma) Cal. bipart. Cor. tubulosa ineq. 4 loba, Stam. 4 ineq. Stigma incrassato, Semen unicum. Fol.oppos. fl. capitulis pedunc. cum periantho.-T'ype the Phyla chinensis of Loureiro, which has the habit of Verbena nodiffora: the name Phyla is objectionable meaning leafy like Phyllis of Lin.-Piarimula chinensis, Raf. Fol. ovato lanceol. glabris, apex serratis, fl. violaceis longe pedunculatis, perianthis spatulatis.-In China. 388. Prlopus, Raf. vel Bertolonia, 1812.Types all the several blended species miscalled Verbena and Zapania or Lippia and Blaeria nodiflora, and akin, which are in utter confusion. I wrote in 1812 their monograph and sent it to Sir James Smith, calling the G. Bertolonia, which name has since been employed for another Genus; I therefore substitute now $\boldsymbol{P i}$ lopus, meaning peduncled head, if needful. See my monograph of $\mathbf{N}$. American Sp. in my new flora. The Genus differs from all those blended in Verbena by creeping habit and calix bilabiate 
not 5dentate, and only 2 seeds as in Zapumiu. I add these for illustration of diversities.

389. 'Zapania, Auct. Cal. 5dent. Cor. ineq. 5loba, Stam 4, Stigma. capit. fructus utricularis :sperm. Frutex Fol. opp. fl. copilutis.The type is Zap. odtoruta. Persoon. Z. lantanoides Lam. Verbena globifera Lher. W. \&c. Fol. lanceol. crenat. scabris rugosis.-A South American Shrub.

390. Panope. Raf. (Nympha) diff. Zapania Stam. 2. Type Ptechadifolia Raf. Verbena and Zapania do Auct. Fol. lanceol. serratis plicatis, spicis ovatis. $\mathbf{A}$ shrub from Florida and Jamaica, probably 2 blended Species.

391. Tarpueta, Raf. Stachytarpheta Vahl. Pers. \&c. Cal. tubul. Adent. Stam. 2 fertilis, 2 sterilis, Ovar. 2lob. stigma peltatum. Sem. 2. This chiefly differs from Zapania by the calix, and has many Sp. formerly called Verbena. The G. Lippia differs chiefly by Corolla 4 lobed and a drupe. The G. Larituna is also very near both.

392. Cymburus, Salisb, Diff. 'Tarpheta by, Corolla ringens, bilabiata, lab. sup. cmarg. inf. 3lob. Frutex, fol. alt.--Type,C. squamosus Raf. Stach. do Vahl. \&c. Canle fruticoso, fol, alternis ellipt. lanceol. serrulatis, pedunc. squamis ciliatis obtectis, apice subramosis.-Verlena Squamosa Jaq. ic. South American Shrub vith very different habit. Cymburus was aplied by Salishury to all the Tarphetas, I retrict it to this type and akin if any. 'Ihe Lipvia umbellate and cymosa may be akin, and 'mith asks to compare Elyiravia.

393. Vermena, L. Auet. 'To this G. are left ill the species with 4 stamens and 4 seeds; Cal. 5dent. Cor limbo 5lobo iner. \&c. But many. 
require revision as yet, some have Calix and Cor. subequal, others only 2 fertile stamens, or a peculiar style or fruit like the next $\mathbf{G}$.

394. Styueurodon, Raf. Cal. tubul. 5dent. ineq. Cor. tubulosa, limbo subbilab. lab. sup. lato enrarg. inf. 3lobo. Antheris 4 sessilis. Stylo latere unidentato, stigma capitatum. Nux indehiscens dura, Aloc. 4sp._Blended with Verbena and Phryma by all our authors, habit similar, but fruit peculiar, the name means lateral toothed style.

395̃. Styleurodon carolinianum, Raf. Verbena do L. Phryma do Walt. Scabro erecto, fol. cuneatis oblongis ineq. serratis, spicis filiformis.-From Carolina to Florida and Alabama, seen alive. Very near Verb. ringens in habit. See my New flora.

396. Prexipus, Raf. (Nom. mythol.) Cal. tubul. 5angul. 5dent. Cor. tubul. limbo 5lobo equalis. Stam. 4 didyn. Ovar. oblong. Stylo brevis, stig. simplex, Nux 4 gona 2loc. 2sperma, sem. clavatis dehiscens. Hubitus ut Verbence Buchnera, nearer the last Genus.

397. Plexipus cuneifolius, Raf. Buchnera cuneifolia T'hnnb. L. W. P. auct. Phryma dehiscens Lin. supl. Wild. Fol. cuneatis glabris apice dentatis. - South Africa, put in two Genera by Linneus, $W$. \&c. very near Buchnera and above all $B$. Cermur ; but the true Sp. of Buchneras have a real Capsule, with emarginate lobes to the Corolla. By this $G$. Verbena is linked to them, as it is to Lippia by Rapania.

398. Micalia, Raf. (Nympha) diff. Buchnera by, Cal. infundibulif. 5dent. Cor. tubo longissimo, limbo plano 5̆lobo, lobis rotundatis.Fol, opp.fl. axill. pedunc. bibract.-Habit pe- 
culiar, very distinct G. many other Buchneras must be examined again.

399. Micalia grandiflora, Raf. Buchnera do L. \&c. Scabra, fol. obl. integris, pedunc. unifl. bibracteatis.-A beantiful plant of South America, with large flowers 4 inches long.

400. Aloysia, Ortega, Vitm. Pers. Beautiful Genus which the Linneists have persisted to unite to Verbena, altho' perfectly distinct by shrubby habit, whorled odorous leaves, whorled spikes and flowers, \&c. It has besides Cal. 4lobus, Cor. 4loba, stigma emarg. Stam. 4, Sem. 2. Whoever unites it to Verbena must be blind. There are $2 \mathrm{Sp}$. of this fragrant G.-1. $A l$. citriodora, fol. ternis lin. lanceol. 2. Al. virgata, fol, ternis ovatis crenatis. Both are from Peru, Chili, \&c., and now common in our gardens. Seen alive.

There are other new Genera connected with these, blended in Priva, Buchnera, Verbena, Lippia, Lantana, $\mathcal{f}^{\circ}$., that deserve to be studied, and I shall perhaps resume the Verbenacea tribe. 


\section{INDEX OF WIIE GENERA, Ac.}

oF

CENTURIES 1, 2, 3, 4 .

New familics are in Capitals, Synonyms

in italic.

Abama, 80 . Abalon, 88, Achimenes, 201. Actipsis, 147. Adipe, 384, 5. Adnula, 324. Aizoon, 258. Albigula, 141. Aloysia, 400. Aglitheis, 33, Aglotoma, 153. Alagophyla, 102. Allium, 33 to 47. Anthericum, 67 to 81 , 205, 211.

Amblostima, 66.

Anactis, 152. Anepsa, 89 to 93. Apemon, 8. Aploleia, 32. Aplopapus, 154. Aploma, 190.

Anisanthera, 216 to 218.

Aphoma, 95.

Aphomonix, 235. Aplactis, 143. Askolame, 9. Ascadia, 232. Astelma, 346. Amblirion, 114. Amellus, 158. Arrostia, 191 to 194. Arum, 306. Asteriscus, 154. Asophila, 195.

Barbacenia, 388. Begonia, 347. Benaurea, 290, 91. Bertolonia, 388. Bignonia, 203, 222. Blephanthera, 211 to 213. 
Blephariglotis, 127 tol Dasiorima, 145. 132.

Brachyactis, 142.

Brephocton, 178.

Brevigula, 14\%.

Buchnera, 396 to 399.

Bulbedulis, 64.

Bulbine, 75.

Caenotus, 181.

Calacinum. 103.

Calycium, 166.

Calliprora, 208.

Dature, 8.

Dectis, 148.

Deinosmos, $\mathbf{1 7 6}$.

Delonix, 350.

Dendrobium, 186, 318, 358.

Deppia, 184.

Dianthus, 195.

Diamonon, 284 .

Dichorisandra, 309,10.

Digomphotis, 120 to 122.

Canarina, 295.

Carigola, 3.

Dimanisa, 311, to 313 .

Diplectraden, 342, 4,

Campamula, 288 to 300.

Catonia, 116.

Diplemium, 282.

Diplactis, 156.

Caularthron, 138.

Clerodendron, $31 \%$.

Chrysopsis,161,165,166.

Cocolaba, 104, 105.

Codiphus, 288, 9.

Coiladena, 12.

Columnea, 201.

Coilonox, 77.

(Diplopapus, 161.

$\{$ Diplogon, 161.

Diplostephion, 161.

Dodecalis, 155.

Doria, 142.

Dothilis, 214, 215.

Eliokarmos, 58.

Comarum, 197 to 200.

Commelina, 30, 31.

Concilium, 292.

Cymburus, 392.

Conyza, $\mathbf{1 7 6}$ to $\mathbf{1 7 \%}$.

Colax, 318.

Crotalaria, 216, 218.

Cronyxium, 78.

Crosperma. 100.

Cruciundula, 380.

Croptilon, 165.

Cupulissa, 203.

Endocoma. 205.

Endogona, 74.

Endotis, 45.

Endopogon, 196.

Edemias, 17\%.

Epidendrum, 1, 137,

$138,225,318$, to 320 .

Epimenidion, 16.

Epionix, 94.

Erigeron, 174 to 182.

Eriospermum, 61. 
Echium, 219.

Egena, 317.

Etheosanthes vel.

Eothinanthes, 27.

Eltroplectris, 185.

Endorima, 346, 7 .

Eriogyna, 261, 2.

Evonyxis, 83.

Evotrochis, 285, 7.

Euthamia, 146.

Eusteralis, 359.

Endeisa, 186.

Epimedium, 187 to 189.

Eusynetra, 201.

Euphorbia, 356.

Exophya, 227.

Evaiezoa, 258 to 260.

Evantepia, 266.

Fenelonia, 56.

Faulia, 314 to 316 .

Flundula, 362.

Fimbramis, 155.

Fimbristima, 160.

Frankenia, 352 to 355 .

Fragmosa, 179.

Fusifilum, 69.

Gagea, 55.

Galearis, 134.

Geboscon, 36.

Gesneria. 102, $361,371$.

Getuonis, 41.

Gibasis, 26.

Glinidia, 351.

Goodyera, 327 .
Gomphima, 4.

Gongora, 377 .

Gomphostylis, 86. 87.

Guanidia, 268.

Gynodon, 34.

Gypsaria, 190.

Gypsophila, 190.

Habenaria, 117 to 135 ,

$220,221,344$.

Hecale, 298.

Helonias, 100.

Helonidia, 82.

Heritiera, 79.

Hemierium, 73.

Hemieva, 255, 7 .

Heminema, 31 .

Heuchera, 277 to 283.

Hesperocordum, 72.

Hexonix, 15.

Hexophoma, 239 to 242.

Heterisia. 251.

Hirculus, 254.

Hyacinthus, 62.

Hydatica, 234.

Inula, 167.

Inula, 165 to 171.

Iridrogalvia, 79.

Isorium, 219.

Isostimia, 232.

204, Ipheion, 10.

Ipomea, 11, 12.

Jupica, 21.

Justicia, 311 to 313 , 379. 
Kadakia, 2.

Kalabotis, 37 .

Kalimares, 158.

Kера, (Сера) 39.

Kozola, 6:3.

Kunda, 305, 6.

Kromon. 46.

Kuritis, 229 to 231 .

Lagocodes, 62.

Lanaria, 190.

Larnandra, 137.

Lacanthis, 356 .

Leiandra, 30.

Leiacherus, 156.

Lemotris, 64.

Leucalis, 161.

Lepicaulon, 71 .

Lepiactis, 144.

Liliago, 75.

Liliastrum, 76.

Loncodilis, 60.

Leptilium, 182.

Loncomelos, 57.

Loncostemon, 47.

Lolanara, 106.

Lotus, 263. 364.

Liriopogon, 113.

Liriactis, 366, 7 .

Lininque, 142.

Limbarda, 170.

Lunania, 7.

Lithofragma, 274 .

Lioydia, 168.

Leptarhena, 263.

Lorantilia, 269.

Leptaxis, 283.
Ligustrum, 314 to 316.

Malagea, 233.

Maligia, 38 .

Manghida, $2 \% 0$.

Maturna, $\mathbf{3 7 4}$.

Maxillaria, 184, 372, 385.

Megapleilis, 204.

Melanthium, 82 to $\mathbf{1 0 0}$, 113.

Meliclis, 376 .

Melomphis, 48 to 51 .

Merisis, 155.

Mesicera, 133.

Mesoligus, 150.

Menadena, 37\%.

Milla, 9, 10.

Mindium, 294, 5.

Mitclla, 264, $2 \% 1$.

Menctho, 35:3.

IHentha, 359.

Micalia, 398, 99.

Modeca, 12.

Monustes, 326 .

Musteron, 180.

Narthecium, 81.

Narica, 325.

Naucorephes, 105.

Nemopogon, 68.

Neottia, 185, 215, 321

to $\mathbf{3 4 4}$.

Nemuranthes, 220, 221 .

Nerissa, :3:37.

Nothria, 355.

Nyctosmi, $\mathbf{l}$. 
Obsitila. 80.

Ononoia, 351.

Olgasis, 183.

Oligactis, 149.

Orcotrys, $277,8$.

Oncidium, 225, 382.

Onixotis, 96.

Orobus, 307, 8.

Orchis, 136.

Orchis, 117 to 136,339 .

Orestion, 172.

Oncostema, 14.

Ozomelis, 271.

Omithogalum, 48 to 61 .

Oxytria, 65.

Ornithoglosson, 97.

Pancovia, 197.

Panios, 174.

Paniopsis, 175.

Panstenum, 41.

Pappochroma, 173.

Pectantidia, $26 \%$.

Pectianthia, 264, 5 .

Pecteilis, 123 to 126.

Perimegia, 232.

Petalosteira, 275.

Pinardia, $15 \mathbf{4}$.

Piarimula, 387.

Piarophyla, 244 to 247.

Phalanginm, 64 to 69 , 79.

Piaropus, 301 to :304.

Petalanthera, 378, 9 .

Phyodina, 29.

Pliryma, 394 to 397. Phonix, 386.
Plexistena, 4\%.

Plyyla, :38\%

Plexinium, 99.

Pilopus, 388.

Plexipus, $396,7$.

Pleisolirion, 76.

Pontederia, 2 to $\%, 301$ to 304 .

Polygonum, 103.

Platanthera, 118.

Plectrurus, 135.

Peurothalis, 375.

Pleiactila, 141.

Pleurostima, 368.

Pleurendotria, 272, 4.

Porrum, 40.

Ponista, 236, 238.

Potentilla, 197.

Ponoplis, 136.

Petalostima, 296, 7.

Pentropis, 300.

Pilorea, 299.

Petaloxis, 308, 9.

Pornciana, 350.

Psukelis, 155.

Polymesia, 351, 35̃2.

Pulicaria, 169.

Quamasia, 64.

, Rafinesquia, 362, 3 .

Ramotha, 20.

Renanthera, 226.

Resedacea, 351.

Rhizarina, 136.

Rupifiaga, 243.

Rydbeckia, 79. 
Sacoila, 321 .

Saluda, 281 .

Sarcoperis, 23.

Sutyrium, 121, 329.

Saxifraga, 233.

Suxifiruga, 232

Sculeria, 188.

Selago, :29.

Skilla, (Scilla) 13 to 16 ,

62 to $64,209$.

Skizima, 98.

Siphostima, 25.

Solanum, 284.

Solidago, 140 to 148.

Solidago, 141.

Stelmanis, 166.

Stelmesus, 35.

Stenactis, $\mathbf{1 5 7}$.

Stemodoxis, 43.

Stenactila, 141.

Stomadena, 11.

Steiranisia, 248 to $\mathbf{2 5 1}$

Spirea, 280.

Spiranthes, 328 to $\mathbf{3 3 6}$,

$$
\text { 340--2. }
$$

Strateuma, 339.

Strepsiphus, 348, 9.

Syncodium, 52.

Synoliga, 19.

Synoplectris, $\mathbf{3 4 0}$ to 342 .

Streptima, 352.

Styrosinia, 360, 61.

Styleurodon, 394, 5.

Talipulia, 32.

Tanaxion, 182.

Tarpheta, 391.
T'Telesia, 139.

Telesonix, 252.

Tipularia, 35.

Tellima, 27:3.

Tiarella, 275 to 283.

'I'oficlda, 79.

'Tolumnia, 382, 3.

'Tonningia, 24.

'Tomotris, 338.

'Tephrosanthos, 136.

Tractema, 209.

Tradescantia, 2: to 32 .

Tricoilendus, 364, 5.

Trimelopter, 59.

Tripogandra, 28.

Triactis. 141.

Tritelandra, 319, 320.

Trilomisa, $\mathbf{3 4 7}$.

Tropilis, 358.

Tulipa, 94, 113, 114.

Tulipa, 107 to 112.

Tulisma, 370.

Tulotis, 119.

'I'ulorima, 253.

Tunica, 195.

Uloma, 222.

Unisema, 6.

UNISEMEA, 6.

Vaccaria, 190.

Varronia, 115, 116.

Venatris, 163, 164.

Veratrum. 88.

Verbena,388 to 395.400 .

Vindicta, 187.

Virgulus, 162. 
Wedelia, 139.

Xeilyathum, 224.

Xuridia, $1 \%$.

Zaga, 101.

Zannonia, 23.

Zapania, 389.

Xuris, (Xyris) 18 to 21. Zigadenus, 85.

Yamala, 282.

Zigotila, 96. 



\section{NOTICES.}

Late works published by Prof. Rafine

History of the American Nations, befo after Columbus-2 volumes published6 volumes to subscribers.

Life, travels and researches of Prof. esque, in both Hemispheres-one vol. 75 Cents.

The philosophy of Instability-one 8 vo. \$1,50.

New Flora of North America-First 8vo. - \$5.

Herbarium Rafinesquianum-\$1.

Atlantic Journal, with 200 tracts on Sc one vol. 8vo. complete-\$2.

A few copies of former works for salelysis of nature-Principles of Somiology lines of discoveries-New Genera of A and Plants of Sicily-Ditto of North A m Fishes and Shells of the River Ohio-1 flora of the United States, S.c.

Unique Copy of Autikon Botanikon c Self figures of new and rare plants, folio

Icones plant. rariorum N. Amer. fol figures-\$300. 


\section{FLORA}

\section{T ELLUR I A N A}

-mwocenk-

BY PROF. RAFINESQUE.'

THIRD PART-

PHILADELPHIA

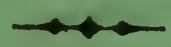

1836. 



\section{FLORA TELLURIANA}

PARS TERTIA.

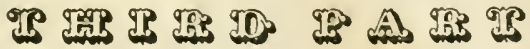

OF THE

SYNOPTICAL FLORA TELLURIANA,

CENTURIES V, VI, VII, VIII.

With new Natural Classes, Orders and families: containing the $2000 \mathrm{New}$ or revised Genera and Species of 'Trees, Palıns, Shrubs, Vines, Plants, Lilies, Grasses, Ferns, Algas, Fungi, \&c. from North and South America, Polynesia, Australia, Asia, Europe and Atrica, omitted or mistaken by the authors, that were observed or ascertained, described or revised, collected or figured, between 1796 and 1836 .

BY C. S. RAFINESQUE, A. M.

Prof. of Botany, historical and natural sciences-member of many learned Societies in Paris, Vienna, Bruxelles, Bonn, Bordeaux, Zurich, Naples, \&c. Philadelphia, New York, Cincinnati, Lexington, \&c.

To observe and compare, to correct or approve

By good names and new facts that convince and improve.

\section{PHILADELPHIA.}

PRINTED FOR TEIF AUTHOR

BY H. PROBASCO, NO. 119, NORTH FOURTH ST, 1836. 


\section{Les noms font les choses.}

Names realize Entities.

Plus nos noms sont generaux, plus non idees sont incompletes.-Plus nous avons de noms, plus elles se completent. Lamark, Leach, \&c. 


\section{PREAMBIE \\ TO THE THIRD PART.}

This part has been somewhat delayed by some occupations foreign to my favorite sciences of Botany ; but every delay is attended with ultimate good results and greater accuracy.

I have been much amused lately by looking over all the singular contradictions of Lindley in the botanical Register, and particularly his remarks in the $22 \mathrm{~d}$ volume on the labors of Spach on QEnothera of Fuchsia.-Lindley is one of the best English Botanists, having fully adopted the natural method, and trying to improve it; yet he has his own blemishes, and appears to be jealous of Spach, because he has rectified these Genera by divisions, endeavouring to ridicule the minute characters he has employed for it ..... This must appear singular from him, who has ventured to establish the Genus Lowea for the Rosa berberifolia, merely upon having single leaves and no stipules! contending that mere habit may form good Genera! in which case all the species of $\mathbf{G}$. Oxalis, Lupinus, Jasminum, Fraxinus, \&c. with single leaves ought to be Genera also! compound leaves and various inflorescence will also become generic accordingly, and we should return to the absurdities of old genera before Linneus, as indeed Lindley advises in his overzeal to explode the Linnean sexual system, Necker was more rational when he made Genera of all stemless species!

Lindley is a great Genera monger as we are called, or framer of New Genera, and thus a real improver; but all his Genera are not good, and he shows too much propensity to seek for 
inconspicuous microscopical characters into the seeds and pollen; which belong to Anatomical Botany, rather than descriptive Botany, being quite useless in practice. 'This absurdity is too prevaiiing at present, it is as wrong as if we were to make the Genera of Birds upon their Eggs! instead of their bills and feet. Some modern Ornithologists led astray by the same anxious wish to find distinguishing marks, have made Generic distinctions upon the tails and feathers of birds, which answer exactly to the blunder of Lindley about his Lowea.

They appear to have forgotten that the different habit, leaves and inflorescerce of plants, when they are outward indications of Genera, have corresponding essential characters in the flowers to be sought for and on which the Genus is to be based, as Jussieu did explain long ago. Thus in Lowea there are some partial characters in the flowers somewhat different from Rosa! which Lindley neglected to seek, and I shall describe; but they merely indicate a Sub Genus, not a Genus; else all the Sub Genera of Decandole are Genera.

Meantime I shall probably adopt all the Genera of Spach, or any other, when properly known to me, as based on permanent characters of the fructification. Lindley will only admit of Godetia because it has fringed seeds (always the seeds for him;) but I know already that most of the other Genera out of Oenothera are good, Boisduvalia is very distinot by unequal stamens, even Brebissonia is good. The Oenotheras with capitate or 4 partite stigma, winged capsules, bifid calix, must certainly form peculiar Genera, and my 3 Genera Onosuris, Pleurandria and Chamerium established in 
1817 in my florula Ludoviciana, on such distinctions were adopted by Decandole.

Lindley seldom quotes me, and only by ricochet as the French say, not lnowing or not caring for my works or labors, I shall show his blunder about the Skilla Cupaniana, to which he adds two of my plants, both distinet, with bluc and white flowers, while his has purple flowers.-When he shall receive this Work and be astonished by my 2000 rectified Genera, he may if he likes probably treat me even worse than Spach; but although he may deny my New Genera, he cannot say that I make them on leaves, nor stipules, nor sperm-pollen, nor inside of the seeds or eggs of plants. Thus they cannot involve any one in perplexity, and are all conspicuons. Nor shall he find me adopting any barbarous or compound exploded name, such as Cotone-Aster (my Cotonea, nor Amelanchier (my Amelancus); nor Mus Cari the Cari Mouse, else Musca Ri the Ri fly; for my Genus Eubotrys \&oc.

I hope he may deen my Genera of the Linnean G. Allium, as good as his own Hesperoscordum and Nectaroscordum, rather long uncouth names, which however need not be changed." I hope he may admit that my previous Genera Clintonia (1817) Oxyurus (1810) \&c, ought to prevail over his of same names, changed by me into Gynampsis and Steiractis.

Whatever he or others may think or say of my labors, they cannot find me inconsistent, nor in contradiction. Within 40 years of botanical investigations I have always been consistent in deeming that Genera and Species ought to be reformed till they become perfect, and un- 
excepionable. Nor can I be accused of forming Genera on minute or invisible characters, or to give any bad names to my new or reformed Genera. As to my own errors and oversights (to which all Botanists are liable) I have always corrected them myself, as soon as perceived, those that may occur in this work will be corrected at the end. I wish all my fellow Botanists would do the same; but some are very tenacious of their conceits and mimomers.

I must now for instance mention that $I$ was right when I said that the Genus Flundula or Rafinesquia 362 of this Flora had perhaps a third name since it is the Hosackia bicolor of Bentham and Lindley. I wrote that article upon the egregious blunder of Hooker who called it Lotus pinnatus, but he lias since rectified it himself in his flora Americana. Yet as the 5 Species reduced to Hosackia may probably form two Sub Genera, the name of Flundula may yet be applied: as to a Rafinesquia, I have provided half a dozen, out of which I hope some one will suit the fancy of botanists and be adopted-although I may be blamed for this conceit, $\mathbf{I}$ blame instead for it those maker's of New Genera that dedicate them to obscure individuals that have not added one page to the Science, and have not thought of me for 40 years, although I have added 1000 pages to it, and 3000 New Genera or Species. Now by the absurd contending whims of Botanists it is become often needful to provide two names for a New Genus, for fear one should be already employed-or if you wish to secure a good name, it must be repeated till adopted, for fear that some jealous exploding Botanists may 
annul it by not deeming the first application given to a proper Generic group. When correct principles shall always guide Botanists, this will be avoided and rectified. I find that my G. Eusteralis 359 is a Dysophyla of Blume, but yet a Sub Genus at least, differing as Euhemus does from Lycopus.

While Lindley was so severe on Spach, he has admitted the whole labors of Bentham on the Labiate, an admirable rectification of 108 Genera to be sure; but exactly similar to that of Spach: The Genera of Bentham are often based on very minute characters likewise, and yet he has left many Genera, Salvia, Teucrium, Phlomis, Sideritis, Marrubium, \&c, hardly rectified, as I will easily prove in this Work. He has omitted my Empedoclia of 1810, also Cephaloma and Bonamia of Neeker: my G. Vleckia of 1808 unknown to Bentham or Lindley, although republished by Desvaux, is exactly their Lophanthus, a name of Limneus and Adanson, different from that of Forster. (Thus the Microstylis of Nuttal 1818 is my Acroanthes of 1808, published by Mitchell and since by Desvaux in their works) while the divisions of the family are actually absurd, being based on the direction of the Stamens! and many Genera of Adanson, Mench, \&c. are omited.

When Lindley shall take the trouble to consult my Works, as does Decandole, he will I hope rectify all such oversights.

I might proceed to state how loose and inaccusate are the characters of many natural families of Lindley. I have already stated that they are often like those of Adanson \& Necker without definite evident common chavacters 
.... not so with mine. I will give a single instance now, Lindley so intermixes the Convolvulides and Polemonides, that it is impossible to know what he means by them. But I have long ago shown that they are perfectly distinguished by the Stamens unequal in Convolvulides; but equal in the Polemonides-This is the plain evident character that any one can sce, and by which many of the Genera of Lindley must be removed, and properly placed in each family : the position of valves is less evident.

As to Species Lindley often blends many into one, and mistakes others; but these errors will be rectified hereafter I suppose, or at least are of lesser importance, if all species are variable. Of all variable characters, the colors of flowers are well known to be the least permanent; yet I was much surprised to see Lindley confess (in article Slillla plumbea bot, reg. 1355) that the Genera Skilla and Ornithogalum were merely distinguished by the colors of their flowers since Linneus ... ! what a confession, and what a blunder! for a correct botanist ... ! blue or purple flowers make a \$7illa! white or yellow an Ornithogalum! what a mistake! since the Skilla maritima type of Skilla has white flowers! I have proved 30 years ago that the filiform stamens form the real Slcilla. I hope my reform of akin Genera, will be deemed by him equal to that of the Amaryllis by Sims.

If $I$ have chiefly noticed Lindley in this introduction, it is because I value him as a colleague in improving Botany; but I might have made similar remarks on many other European Botanists, that fall into the same mistakes, or 
appear to neglect my improvements in Genera and fumilies. They may apply to themselves every one of the deffects and contradictions that I have thus exposed, and then endeavour to avoid then.

I shall conclude by the remarks of Lindley on the Genus Aster, which he states in the 18th vol. of Bot. Reg. to have been the disgrace of Bolanists by imperfect descriptions, unscientific arrangement, false species, confused synonymy, and multiplied names, till the second rectified monograph of Nees. Those remarks apply equally well to 100 other Linnean Genera, and this work is a perpetual comment on such disgraceful general blunders of many Botanists to this very day.

\section{FLORA TELLURIANA.}

\section{Centuria V.}

Number 401. Polygonum L. auct. Nearly all the botanists acknowledge that this Linnean Genus is absurd ; even Lindley says it ought to be divided: Adanson and Necker partly did so, but their useful labors were neglected. I now mean to revise the whole, and rectify or establish 20 Genera out of it ; all perfectly plain and distinct : the linneists may vince and evade, but this must be done at last. My labor dates of 1815, my true Polygonum Raf. will have, Calix 5partito equal, Stam. 8, internis 3 fertiles, ()var. 3 gonum, Stylis 3. Semina trigona, cal. vestita.-This includes $\boldsymbol{P}$. avicularis, maritimum, setosum, beliardi, glaucum, erectum, serratum, temee, and all similar sp. with axilla- 
ry flowers chiefly, of which many new ones irs N. Amer. see my flora. I have not yet seen the late monograph of this Genus, by Meisner, but it appears he has kept it entire as yet, only using sub genera as Persoon and Jussieu.

'The Species with colored flowers in panicled racemes, like arliculatum, fimbriatum, divaricatum, alpinum, undulatum, sericcum, \&c, must probably be innited to Stopinace but offer but few essential characters in the flowers. The frutescent $\boldsymbol{P}$. grandiflorum with large axillary flowers is perhaps another $\mathbf{G}$. or sub $\mathbf{G}$.

402. Fagopyron 'Tourn diff. 401. Cál. coloratus, Stam 8, externis 5 antheris bilobis remotis, internis 3 brevis sepe sterilis, alternis cum glandula 3 hypogyna. Sent. ut P. flores sepe corymbosis vel glomeratis.-This G. called Buckwheat in English, is perfectly distinct, and known at first sight, the leaves are commonly broad and cordate; the types are Fag. cereale (P. fagop.) tutaricum, chinense, perfoliatum, cressifolium, \&c. If any one may object to the name being rather mongrel, beech (lat) wheat ( $\mathrm{gr}$ ) they may use instead Trachopyron of Gerard.

403. Hnixine L. frst ed. since wrongly rejected, may be applied to all the species with unequal calix, Dift 401. Cal. colorat ineq. lac. 3 major sepe carinatis s. alatis. Sem. in Cal. 3alato sepe. Caule sepe scundens, fl. racemosis-Types, T1. Sccudens, dumetorum, convolvulus, multifornam, cilinode, sngitiatum, foc, and akin with mequal calix. Those with carinated rather than winged fruit, form the $G$. Fallopia of Admson, who ascribe to it 9 Stamens, if so it is a good Genus; lut I only saw 8 stamens in 4 . Secturess, as in the others. 
P. Sagittatum forms another Sub G. Belotrachis by fl. polygamous glomerate Cal. colored .3 parted with a calicule of 2 small green segments, style trifid \&c.

404. 'T'ermis Raf. (non Ad) Asicaria Nerk. Pedalizm Ad. non L. Atraphaxoides Sub G. auct. Diff. 403. Cal. ineq. lac. 2 ext. reflexis parvis, 3 internis erectis planis persistens reticulatis Fruir, ll. racemosis. This differs fiom 403 chiefly by calix, perhaps only a subgenus. Type rephis rutescens and zorvitolia Raf. 'l'ephis was Adanson name for Atraphaxis.

405. Sropruaca Raf. (nom. lat.) Polygonella Mx. difl. 401. Cal. subineq. coloratus, Stam. 7-8, stigma 3 clavatis. sem. 3gona, sepalis 3 ampliatis vestita. Aruticul.fl. racemosis dioicis S. polygamis. Type St. parvifolia Raf. P. do. Mx. P. polygamum auct. This appears entitled also to be a Genus, with $\boldsymbol{P}$. gracile of Nuttal,

406. Karkinetron Raf. (nom gr) Cal. 5fido, basi turbinato subcarnoso, limbo lac. eq. Stam. 8 monadelphis in unica serie coalitis in disco. ovar. subrot. stylis 2-3. sem. globosa in Cal. basi baccato immersa. Frut. fi. racemosisGenus quite peculiar near to Coccoloba like Calacinum 103. Types 2 Sp.

407. Karkinetron undulatum Raf. Polygonum injucundum Lindley b. reg. 1250. Frutic. fol. petiol. ovatis deltoideis acutis undulatis, ochreis truncatis, racemis axill. paucifl. foliosis, stylis 2. Andes of chili, fl. green.

408. Karkinetron hastatum Raf. Coccoloba sagittefolia Ortega, Dec. 60. Polyg. acetose fol. Persoon. Scandens fol. hastatis lanceol.crassiusculis, stylis 3. Brazil. an S. G. ad stylis, vere bacca? 
409. Kunohale Raf. (rom. dio:k) near $F a-$ gopyron diff. Stam. 10 ineq. 5 interna brevior, glandulis 10 hypogyis, Sem. truncato emarg. 3alate-Type K. curneum Raf. Folyg. emarg. W. Don, Lindley b. reg. 1065. fol. cord. sagitatis, superis sessilib. racemis corymbosis. Cult. in Nipal and China as Buckwheat, perfectly distinct by 10 Stam. flowers incarnate: the Polyg. chinense with ovate leaves is perhaps a second species. The Genus is rearer to Brunnichia than Polygonum!

410. Bistorta 'Tourn, Ad. diff. $401 \mathrm{Cal}$ 6partitus, Stam. 9 (ex Ad) Radix tuberos. florib. coloratis spicatis.-Types B. officinalis R. 2. B. americana R. 3. B. vivipara, \&c.

411. Antenonon Raf. fl. lud. 1817. Calix 4fidus, Stam. 4, stylis 2, persistens, sem. ovata striata stylis coronata fl. racemosis.-Type A. racemosum Raf. hirsutum, fol. petiol. obl. acutis, racemo longissimo. fl. remotis albis, bracteis scariosis vaginatis. Louisiana.

412. Tovara Ad. cal. 4fidus. clausus ineq. 2 minor. alt. Stam. 4 ineq. Stylis 2. Sem. lenticularis levis. fl. spicatis.-Type Tovara virginiana, the Polyg. do. L. totaly unlike the other species, a Genus as distinct as any, but near the last. Adanson and others ascribed 5 Stam. to it by mistake, I never saw but 4.

413. Pleunostena Raf. (narrow side) cal. rhombeus 5fidus ineq. Stam. 5, Stig. 3 sessilib. Sem. conicus cal. longior, ineq. triqueter, lato uno angusto. $\boldsymbol{l}$. axill. polygamis.-'Type my St. serotinus Raf. Polyg. do Raf. an. nat. 1820. Suffruticosus, ramis plurimis virgatis angul, fol ovatobl. acutis ochreis fissis laceris, fl. axill, fascicul, pedunc. In Kentucky, it flowers in October. 
414. Cnopos Raf. (nom. gr.) Cal. 5 part. ecp. Stam 3, stylis 3, Sem. trigona. fl. subvericilatis - Type Cuopos romosissimum Raf. Polyg. do lix. Ifere the Stamens are reduced to the minimum number and are isostyle. 'Thus we see in the 10 momm of I limneus, blended Genera with $3,1,5,6,7,8,9$, and 10 Stamens! thus belonging to eight sexual classes, what a delightfol absurdity to swallow!

415. 'Tracanlon Rinf. (romgh stem) Cal. colorato 4partito, lac. '2 ext. ninoribus, 2 int. major sepe emarg. stam. \& fertilia, 4 sterilia brevis alternis. ovar. compr. stylis 2 , stig. capitatis. sem. ovato biangulato. Habitus Helxine sed fl. glomeratis-The type wil be Tr. arifolium, a well marked Sp. very distinct from Helxine sagittata, although the stem is equally rough. Flowers more like Tozara, but habit quite unlike. Michaux saw 6 stamens, but the mistake arose from the sterile filaments first noticed by Elliot. The 3 Genera Tovara Tracaulon and Antenoron will form a group by the Calix and stamens. Trucaulon is also near Kunoliale, the stamens being diplarine instead of isarine (equal to perigone) but here 4 are sterile.

416. Spermaulaxin Raf. (secd canaliculate) monoical. fl. masc campan. 4-5́fidis. lac. subeq. obtusis coloratis, Stam. 4-5 brevi-simis. Flor. fem. similis majoi magis ineq. lac. erectis obovatis, stigma 2-3subsess. Semen conicum cal. duplo longior, basi accretum, tiiqueter, uno latere excavato sulcato. Fratex, $\boldsymbol{\pi}$. spice tis.-Another very distinct Genus, near to Pleurostena, but different habit; it has also some affinities with Polyg. grasidillorum, 
which perhaps belong to it, as well as Polyg. Ochroutum; in this last are blended 2 species, the Siberian of Gmelin, and the Jamaican of Slome; but the type of my genus is the following N. sp. of Florida.

417. Spermaulaxen dichotomus Raf. caule fruticoso dichot. sulcato, fol. petiolatis oblong is lanceol. acutis levis margine scabris, cchreis subnullis, spicis filif. fl. remotis nonnulis pedunculatis.-In Florida, fl. small greenish purple, male and fenale on the same spike. I can find no synonym for this: it differs from the usual character by lacking the tubular stipules, a small subulate deciduous stipule appears instead in some leaves and flowers; described on a dry specimen.

418. Chulusium Raf. (nom. anticus) Cal. 5fidus inequalis, lac. 2 major, Stam. 5, ovario compresso, stylis 2, Sem. lenticularis. fl. spicatis coloratis,-Types Polys. amphibium, natans, filiforme, and several North Amer, Sp. blended in punctatum, such as the following, also $\boldsymbol{P}$. fluitans of Eaton.

419. Chulusium acaminatum Raf. Pol. punctatum Raf. annals nat. 9\%. T'ol. lato lanceol. acum. peticlatis, subtus punctatis, ochreis nervosis muticis, spicis densis, fl. geminatis, bracteis ovatis-In West Kentucky, fl. white.

420. Peutalis Raf. (nom. gr.) Persicaria Tourn. Ad. Necker, ad Persica and Crrya malum. Cal. 5part. subeg. Stam 6, una interjecta ad latere ovar. compr. stylo 1 bifido, Sem. lenticularis. flores spicatis coloratis.- Types $\mathbf{P}$. persicaria, nodosa, hylropiper, minus, incana, and several N. sp. from North America, see my fiora, also P. incarnata Eiliot. 
421. Discolenta Raf. diî. 420. stylis 2, Sem. discoformis utrinque latere concavo.Type, D. lapathifolia and my scabra.

422. Heptarina Raf. (7 masc) dif. 420. CaI. corollato equalis, Stam 7 , bina interna latere ovario, stylis 2. flores racemosis.-Type $\mathbf{I 1}$. orientalis.

423. Droctis Raf. 1817 ( 2 and 8 ) diff. 420. Stam. 8, internis 3 minor, stylis 2.-'Types $\boldsymbol{l}$ ). peusyloarica, and $3 \mathrm{Sp}$. of my fl. Ludov. bicorne, maculatum, vernum, besides equisetifolia of Egypt.

424. Mitesia Raf. (mildness) diff. 120 , stylo 1 bifido, ineq. Sen. ovato subtrigono, Stam 8. - Types M. albiflora, hirsuta, punctata Elliot and my M. divergens, M. montana \&o $c$.

425. Pogalis Raf. (beard diff.) diff. 420, stam 6, Stylis 3, Sem. vix. lenticularis-Types P. barbata, tinctoria, tomentosa, \&c. 'This conchudes the reform of Polygonum, with my Calacinum 103, in all 22 Genera. If all those with lenticular seeds and 5fid calix have been commonly deemed Persicaria, the difference that $I$ have shown, prove that at least Sub Genera are required. I am not yet sure to have exhausted this Genus, and may return to it if needful. I have in my herbal many new species of it as yet, some of which I add.

426. Polygonum festigintum Raf. caule erecto ramoso fastigiato fuscato, fol. lin. lanceol. adpressis, ochreis laceris, axillis uniftoris -annual, Alleghany mts.

427. Polygonum crassicaule Raf. caule crasso striato diffuso ramoso, ramulis brevis divaricatis, fol. parvis subpetiolatis oblongis, obtusis, axillis 1-3floris-Perennial, Alleghany mt.s. stem pedal rigid, near P. erectum. 
128. Poiggonm imbricatum Raf. multican'e, caulib. brevis flexuosis vix ramosis, fol. imbricatis linearibus. ochreis laceris scariosis, axillis mifloris-annual, Kentucky, dwarf only 3 to 4 inches.

429. Polygonum angustifolium Raf. caule erecto flexuoso ramoso, fol. longis. linearib. angustis, ochreis integris, axillis unifloris- $O_{n}$ the Sea shore and islands of Jersey and Virginia, perlal different from P. tenue.

430. Discolenta scabra Raf. Polyg. lapathifol. of Amer. bot, non alis. Caule erecto subramoso, fol. longe lanceolatis acuminatis scabris, infimis petiolatis, ochreis laceris, spicis paniculatis gracilis carneis-North America, 2 or 3 feet, large leaves, 6 to 8 inclies long.

431. Mitesia divergens Raf. caule nodoso dichotomo ramis divergens vel retroflexis debilis, fol. sessilis longis lineari lanceol. glabris, ochreis ciliatis, spicis filiformis carneis-annual in Kentucky, singular sp. with widely spread branches.

432. Peutalis linearis Raf. caule simplex nodoso, foli is linearib. elongatis, margine glabris, ochreis ciliatis, spicis plurimis filiformis rubris-Carol. and Florida, one or two feet, Howers very small sessile and pedicellate.

433. Gononeus Raf. (knces swelled) I have to add here a very singular Genus, that unites many anomaiies on the same spikes. Polygam. cal vix coloratus, ineq. 4-5fidus 2-3ext. major, stam. 6-8, ovar. et Sem. trigonis vel lenticula. ris, stylis brevis $2-3$, stig. capitatis. Flores $\mathbf{S p i -}$ cais, masculis et supernis sepe 4fidis. 'Therefore this appears to unite the characters of Toura, Peutulis and Mitesia, yet differs from all by the calix loss divided, hardly color- 
ed, except in the male flowers. Is not this a hybrid Genus? and hybrid new Species?

434. Gononcus undulatus Raf. Glabrum, diffusum, ramosum, fol. lanceol. undulatis acuminatis obtusiusculis, geniculis inflatis oblongis, ochreis tubulosis ciliatis; spicis terminalis nutans gracilis imbricatis, basi interrupte foliosis. -In the swamps of Delaware, New Jersey and near Philadelphia, but rare: leaves acrid pungent, unspotted, nervose beneath; flowers green somewhat incarnate inside, male often white, the lower ones commonly with trigone seeds, the upper ones with lenticular seeds. Has it sprung from Nitesia albiflora? but this has narrow flat leaves, slender naked white spikes.

435. Gentiana. One of the finest and yet most obsure linnean Genus, Jussieu said of it, an G. dividendum? Sir James Smith said that Linneus knew not this Genus! many of his Sp. were doubtful, ard he has put in it even Sp. belonging to other families. G. aphyla, filiformis and heteroclita, have since been united to Exacum, but the last is a peculiar Genus of Acanthacea!' The whole G. was often divided, but the blundering linneists would not admit the propriety. Tournefort had 140 years ogo 2 Genera, Gentiana with campanulate flowers, and Centaurium infundibuliform. Renealm, Morison, Adanson, Necker, Richard \&c, have tried to improve it, but the Erythrea of Necker adopted by Richard has only been generally admitted. 'The whole requires a radical reform ; this G. and itc family has always been a peculiar favourite of mine, and I will be able to rectify the whole, adding many new Sp. also. It is as bad as Saxifraga was, having 4 to 9 Stamens, free or united, calix and corollas of all 
shapes $\mathcal{E} c$, thus belonging to 7 limnean classes, and being merely united by the fruit, as if the fruit alone was to form Genera, whereby all the Cruciferes should be one Genus! all the Gentians have a bivalve unilocular capsule, and so have $\mathbf{1 0 0}$ other Genera. Meantime I have detected in the whole group 4 important characters overlooked by all the botanists. 1st. All the Stamens are opposed to the segments of the Corolla as in the Primulacea! this will remove the Gentianides in the serial order: any one with alternate stamens must be removed from the family. 2d. The segments of the calix are more or less unequal in length or breadth in nearly all, this is a generic character. $3 \mathrm{~d}$. The stamens are equal, when they are unequal as in Lisianthus \&c, those Genera belong to Zisianthides, a subfamily of Convolvulides, 4 th. All the leaves are entire sessile, opposite or verticilate with reticulated veins besides the nerves, which are easily perceived in all their leaves. I shall now proceed with this fine group, dividing it into many good Genera with the akin Chironia, and adding some new species; but many more are in my flora of North America.

436. Gentiana Ad. non Necker, cal. camp. anomalo spathaceo seu truncato, 1-6lobato. Cor. camp. 6-9loba equalis, stam. 6-9liberis. Rad. crassis amara, Fol. et fl. oppos. seu vertic.This must remain as the typical Genus, and the typical Sp. is $G$. lutea or officinalis, to which that name was given by the greeks. But the Genus is thus reduced to but few species, and even requires to form three subgenera. 1 . Picriza (bitter root) Cal. 3-6lobato, Cor. camp. rotata. This includes G. lutea, pannonice, 
campanulata sfc.-2. Pleuroglossa (lateral tongue) Cal. truncatus, latere sepalo unico lingua similis, cor. camp. subtubulosa. Types $\boldsymbol{G}$. punctatu, G. purpurca, \&c.-3. T'ulbela, Cal. camp. 5-7fid. ineq. Cor. camp. 10-14fid. lac. alternis minor ciliatis, stam. 5-7. 'Type $G$. rossica Ref. (7 fida Pallas, Frol. Persoon.) These subgenera might even become Genera if more Species are found belonging thereto.

437. Dasistepina Renealm. Ad. Cal. tubul. 5dentato, Cor. tubulosa, campan.. plicata 5dentata. Stam. 5, antheris coalitis-Types $D$. arclepiadea, and D. cespitosa R. Gentiana auct.

438. Diplona Raf. Cal. camp. 5fidus, Cor. infundib. inequalis 10fida, Stam. 5, antheris coalitis. Stylo et Stigma bilamel.-Types $D$. altaica, ungustifolia, pyrenaica Raf. \&c. see 484, 485.

439. Cinuralts Morison, Mench, Thylacitis Renealm. Cal. camp. 5 fido, sinubus obt. Cor. camp. 5dentata, sinubus plicatis integris, Stam. 5 liberis? stigma capitato 4 lobo undulato.Type C. grandiflora, angustif, alpina \&॰c which were Gent. aphyla and 2 var. of Authors, Grenus very distinct from Pnenmonanthe by the stigma; the Ciminalis of Adanson was the Xolemia blended with Picriza.

440. Pneunonantie Tournef. Neck. Cal. tub. camp. subtrunc. 5fidus ineq. Cor, tubul. camp. 5 fida, sinubus integris. Antheris 5 coalitis vel connivens, stylo elongato, stig. 2lam.This is a very extensive Genus, containing many of the European and American Sp. of Gentians, the types being the various Sp. blended by L. under G. Pneumonanthe and the akin Sp. such as the following and 487, 488, 441. Pnenmonanthe media Raf. Gent. 
pneum. N. Am. botanists, Gr. pseudopneum, Romer Sch. Caule erecto 1.3floro, glabro fuscato tereto latere sulcato, fol. lin. lanceol. obtusis, internodis eq. flor. pedunc. Cal. trunc. sepalis ineq. linear. obt. Cor. tubul. subventric. cal. duplo longior, apice obt. 5fida, sinubus unidentatis.-In New England and Canada, flowers blue, stem about pedal. This is even hardly a Pneumonanthe, the sinusses not being enture, and rather a Xolemia S. G. Cutlera.

42. Amarella Raf. Hippion Schmidt. cal. camp. sepalis $\mathbf{5}$ ineq. Cor. tubulosa, apice camp. 4-5fida eq. squamis 4-5 barbatis alternis intus cor. Stam. 4-5filif. antheris liberis. Ovar. linear. stigma bilamel. Plant. annua, caule angul. glabro. 2 Sub Genera, 1. Amarella the Gent. do L. and akin Sp. with $\mathbf{5}$ divisions. 2 Hippion, with 4 divisions, type the Exacum viscosum or Gent. viscosa of Authors, perhaps distinct Genus if cal. 4phyle, in Amarella it has a campan. tube. The Gent. germanica with 4 or 5 Stamens connects them. I add here the blended Sp. that I possess in my Herbarium.

443. Amarella vulgaris Raf. Gent. amar: auct. Glabra, caule 4gono multifloro, fol. 3-5 nervis internodis subeq. inferis obovatis obtusis, medis ovatis acutis, summis ovatobl. acutis. fl. pedunc. ax. et term. Cal. sep. lanceol. tubo longior, Corolla ad cal. duplo longior.---Europe fl. blue, many varieties that are perhaps forming Sp. 1. A. Simplex. caule simplex semipedale, fol. omnibus obl. internodis brevior, cor. lac. latis acutis.---2. A. brevifolia, caule pumilo simplex sub 3floro, fol. omnib. deltoideis brevis, cor. gracilis, lac. angustis acuminatis--3. A. ramosa, caule fuscato ramosis, fol. in. 
feris amplis obovatis, cal. sepe deltoideis revolutis, corollis acutis---4. A. fastiginta crule rubello ramoso fastigiato.

446. Amarella bievicaulis Raf. Glabra, caule 4 gono multifl. brevis ad fl. eq. fol. imbricatis ovatobl. acutis 3nervis, fl. pedunc. magnis. cal. sepalis linearib. revolutis, cor. ad. cal. triplo longior---Oregon, only 3 inches high including the flowers nearly half of that length, blue, peduncle and sepals equal to tube of calix, simuses of cal. and cor. obtuse.

447. Amarella gracilis Raf. caule gracile 4 gono multifl. fol. remotis lanceol. acutis 5nervis, $\mathrm{fl}$. axill. pedunc. ad medio bracteis binis linearib. cor. gracilis parvis cal. duplo longior. capsulis cor. superans---Sibiria semipedal, leaves and fl. uncial, upper leaves nearly linear, fl. very slender, teeth acute narrow erect. 448. Amarella acuta R. Gent. do Mx. El. Caule 4gono, fol. ampl. ovatobl. acutissimis, fl. fascic. cor. lac. lin. lanc.---Mts of Carolina and Canada, fl. small greenish yellow?

449. Amarella rugosa Raf. caule 4gono, pumilo simplex, fol. amplectens oblong lanc. ob. tusis rugosis undulatis vix trinervis, fl. subsess. ax. et term. cal. lac. lin. obt. rugosis revolutis, corollis brevis calix vix superans.---Oregon, triuncial.

450. Alortis Raf. differ ad Amarella. Cor. tubulosa clavata, 5dent. dentib. mucronatis, squamis nullis, Stam. 5 liberis, Ovar. stipitatum obl. stylo brevis, stig. 2lamel. Annuis, fl. fasciculatis.---Type Gent. 5flora L. and akin species, such as

451. Aloitis parviflora Raf. Gent. amarella Elliot non Auct. Caule ramoso brachiato 4alato flavescens, fol, remotis ovatobl. acutis 5-7ner- 
vis, fl. term. fascic. 3-10floris, ped. brevis, $f$. ineq. Cal. sep. linearib. Cor. brevis cal. duplo longior, lac. acum.-In the Mts of Carolina, Tennessee and Fast Kentucky, 2-3feet, flowers bluish half size of $\boldsymbol{A}$. 5flora.

452. Aloitis quinqueflora $R$. Gent. do. auct. Differs from the last chiefly by stem pedal, simple or branches erect, $\mathrm{fl}$. in fascicles of 3 to 5, large uncial slender, 4 times the length of calix, leaves broader deltoid. Fig. bot. mag. 3496.

453. Aloitis anceps Raf. Gent. amarelloides Mx. caule simplex tereto biangulato, fol. intern. eq. ovatobl. 5nervis, pedunculis 1 floris 4gonis, ad apice subumbellatis, Cal. sepalis obl. acum. Cor. cal. duplo longior, lac. acuminatis.-Kentucky, fl. large above one inch, blue but becoming yellowish in drying.

454. Xocemia Raf. (shut half) Cutlera Raf. 1807. Cal. tubul. camp. 5fido, sepalis foliaceis, ineq. Cor. ventricosa seu fusiformis apice coarctata multident. 5 major dilatatis, alternis in sinubus dentatis. Stam. 5. filam. subulatis, antheris subcoalitis. Perennis, fl. fascic, seu capitatis bracteatis.-Types the various $\mathbf{S p}$. blended or akin to Gent.saponaria and ochroleuca, such as my $\boldsymbol{X}$. clausa, acuminata, latifolia, palustris, trachiloma, catesbei, striata, rotundifolia, heterophyla, serpentaria, shortiana, Elliotea, axillaris, Collinsiana, obovata, enervis, fistulosa, longiflora, albiflora, ternifolia, rubella \&c, described in my Monographs med. flora 1828, and New Flora 1836. Every American botanist has blundered about these plants, since Linneus whose G. Saponaria was the catesbei and ochroleuca blended ... The G. Xolemia chiefly differs from 
Pneumonanthe by the superadded alternate segments of Corolla. The true Xolemias have the corolla ventricose nearly shut, the $\mathbf{S}$. G. Cutlera has it more open and tubular, but the change is very gradual.

455. Ricoris Renealm. cal. tubul. 5fido, cor. hypocraterif. 15fida, 10lac. interjectis minimis binis in sinubus. Stam. libera, stylo elong. stigma capitato orbic. scutellato concavo.Types R. or G. verna, pumila, bavarica \&ec. Here as in Ciminalis the Stigma is very different from the usual kind.

456. Chiophina Raf. (snow friend) differ. ad Ricoila, cor. tubo longo, limbo dentib. 5patulis eq. in sinubus nullis. stigma..?-Type $C h$. nivalis.

457. Govrris Raf. (angles under) Chirvnia et Gentiana L. Centaurion Ad. Erythrea, Neck. Rich. Pers. Cal. 5gonus 5dent, tubulosus, cor. infund. basi tubul. apex 5fida, stam. libera. stylo unico, stigma capitato bilobo-Genus easily known by the pentagonal calix : the names already given are objectionable being similar to Centaurea and Erythrina! the type is G. centaurium and akin sp. Persoon had 11 sp. to which 1 can add 8 other sp. of my herbarium, 4 of each continent, all are annual.

458. Gonipia pancifolia Raf. Caule humile 4gono, 1-3floro, fol. paucis remotis parvis subrot. obtusis, imis obl. cal. elongato, cor. eq.Sicily, 3 to 4 inches, 3 or 4 pairs of minute leaves, limb of corolla small, one fourth of calix.

459. Gonipia sicula Raf. caule 4gono pedale gracile, apice alterne ramoso, fol. cuneatis et lanceol. trinervis acutiusc. adpressis internodis dimidio brevior, fl. Iaxis subternis, me- 
dia sessilis, bract. lin. cal. elongato cor. eq.-In Sicily over a foot high, leaves uncial, fl. rose as in nearly all.

460. Gonipia linearis Raf. caule humile ramoso vix 1 gono, fol. linearib. uninervis, fl. fascicul. bract. lanceol. cal. 5fido dentib. elongatis lin. cor. eq.-North of Europe, blended with G. centaurium by many botanists, 3 to 6 inches.

461. Gomipia rotundifolia R. Pumila, caule subtereto, fol. plerumque orbiculatis obtusis, $\mathrm{fl}$. agregatis parvis, cal. elongato 5 dent. cor. subeq-Nortl of Europe, also blended like the last: the real G. centrurium has stem Agone, leaves ovate obl. acute, calix very short.

462. Gonipia pulchella Raf. Chironia do L. caule ramoso subtereto, sulcato, ramis 1 -2floris, fol. oblong. obtusis internodis longior, cal. 5fidis linear. tubo cor. equante---Florida, 3 to 6 inches, corollas large acute. Not the Exacum pulchelum of Pursh which is a Sabutia.

463. Gonipia pumila Raf. caule pumilo 4gono paucifl. ramis unifl. fol. paucis ovatis vel oblongis acutis internodis eq. cal. profundi 5 fidis sepalis subulatis, subeq. ad tubo cor. gracilis, lac. lanc. acutis---Florida, minute plant of 1 or 2 inches.

464. Gonipia bicolor Raf. caule sub dichotomo. sub 4 gono multifl. fol. remotis ellipticis obtusis trinervis, fl. fascic. term. pedunc. 2-3fl. cal. 5dent. ad cor. tubo dimidio brevior, cor. obtusis, bract. lanceol.---On Lake Ontario; a beautiful sp. stem semipedal, fl. with yellow tube and rosate limb, leaves uncial, style exerted, stigma capitate as in most.

465. Gonipia tenuiflora Raf. caule dichot. 1 gono gracile, fol. internod. eq. linearib. obt. $\mathrm{f}$. 
corymbosis, ramulis 1 fl. pedune fl. eq. cal. tenuis subul. tubo cor. brevior, cor. filif. lac. subulatis, stam. et stylo exsertis-Louisiana and Texas, stem 4 to 6 inches.

166. Psalina Raf. diff. Gonipia Cal. inflatus utriculosus, angulatus bilent. cor. hypocraterif. 5fida.-Types Gent. utriculosa, exacoides.

467. 'I'my racitis (nom. gr.) diff. Gonipia calix sub. 5part. subang. tubo camp. stylis 2, stigma 2.- Type Geniuma or Erythea maritima of Authors, blending 3 species.

468. Thylacitis marilima $R$. caule dichot. tereto corymboso, fol. obl. lanc. fl. pedunc. luteis-Sea shores of Europe.

469. Thylacitis compressa R. (var. Sch. Pers) caule dich. compresso, fol. lanceol. trinervis-In Marocco.

4\%0. Thylacitis leptina R. Gent. do Raf. precis 126. caule pumilo uncialis tereto sub unifl. fol. ovato lanc. acutis, cal. subul.-Sea. shore of 'Tuscany and Sicily, disc. 1800 descr. 1814, fl. white, tube yellow.

471. Antropogon Necker 1790. Eubleptuis Raf. 1814, Crossopeialon Beck 1833. cal 4 partit. ineq. cor. camp. 4 fida vel 4 loba, lobis ciliatis, stam 4 libera, stig. bilobo-'The finest Genus of Gentians, perfectly distinet, types Gent. ciliata, crinita, barbata, detonsa, and the following N. Sp.

472. Anthopogon virgatum Raf. caule gracile sub 4gono, virgato apice nudo unifl. fol. remotis adpressis longo linearib. infimis cuneatis obtusis, cal. sepalis lanc. cor. fimbriataCanada and Alleghany nts. very rare, stem 1 or 2 feet, flower blue very large two inches long.

473. Tretorniza Renealm. cal. 4fid. ineq. 
2 alt. minor, cor. hypocr. tubo longo, limbo plano 4 fido, dentib. 4 alt. ad lacinis in sinubus. Stam. 4 liberis.-Type Gent. cruciata and akin sp.

474. Cicendia Ad. Cal. tubulos. Afidus, cor. 4 fida hypocrat. barbata Stam. 4 libera, stigma bilamelatis.-Type Gent. campestris and akin sp. tenella, glacialis, $f^{\circ} c$.

475. Pogoblepuis Raf. Cal. 4part. sep. 2 alt. duplo longior, cor. tubulosa camp. 4fida, faux barbata ad squamis 5setis, stam. 4 libera-Genus near the two last. 'Type the following sp.

476. Pogoblephis missurica Raf. Gent. acuta Nuttal non alis, caule 4gono ramoso, fol. ampl. ovatis acutis trinervis, fl. axill. solit. longe ped. cor. lobis ovatis acutis-Upper Missouri, pedal, fl. greenish purple.

47\%. Narketis R. (nom. gr.) Gentiana! Necker, non alis. cal. 5 part. cor. rotata 5part. stam 5brevis subul. liberis, faux squamosisTypes the Gent. or Sweriia rotata of Author's G. carinthiaca, sulcata of $c$, besides the next sp. 478. Nar\%etis rotata R. caule pumilo 4 gono fol. remotis ovatis obt. cor. albis oblongis--Altaic mts of Sibiria, in my herb. blended with the next by Frolich, and the Suertia rotata of Thunberg is also a peculiar sp. Narketis japonica.

479. Narketis hyperborea R. caule gracile ramoso 4 gono, fol. remotis obl. obt. fl. pedunc. cor. ceruleis ovatobl. acutis-N. W. America, at Kotzebue Sound, seen dry, stem 6 inches.

480. Lepinema R. (scaly thread) cal. et cor. ut Gonipia 45\%. diff. stam. squama nectarif. ad basis filam. stylus, stig. capit. capsula semibiloc. fl.verticilatis-Types the Gent. verticillata and 
exaltata L. both of Antilles, with octoflora of India?

481. Lepinema verticilata R. Gent. do L. Exacum do Vahl, Wild Pers. caule simpl. fol. lanc. fl. sess. vertic. acutis luteis-Antillis et Florida. Many sp. of Exacmm are yet obscure althongh the G. Mirrocale has been removed from it. Ex. filiforme is a Microcale rather than Cicendir.

482. IIETeroclita Raf.cal. incurvus 4dent. subul. cor. hypocrat. limbo '2part. lac. bifidis, lobis 2 erectis, 2 deflexis, stam. 4 inequalis! minor inclusa, stigma bilabiat.-A very distinct G. wrongly united to Gentiana and $\boldsymbol{E} x$ acum, not even of same family, nearer to Cutubea and probably both of family Acanthides, or Orobanchicles, unless types of a new family. 483. Heteroclita dichotoma R. Gent. heter. L. Exacum heter. W. P. caule dichot. 4 gono, fol. ovatis, summis linearib. fl. purp. solit. ad dichotomia-Malabar. Before concluding the Linnean Gentians, and beginning his Chironias, I shall give some interesting sp. of the above Genera.

484. Diploma hudsomica Raf. Gent. pneumoncentíe Mx. non alis. fol. lin. lanc. fl. majusc. term. et axil. lacinis $\mathbf{5}$ rotundatis, $\mathbf{5}$ alt. plicatis unidentatis-Hudson bay and mts of Canada, very different from the others, not even a Pneumonanthe, having 10 teeth or parts to corolla.

485. Diploma tenuifolia Raf. caule. fllif. flexuoso unifl. fol. remotis angusto linear : flos magnus albus, cal. sinub. truncat. sepalis linear. cor. lac. alt. laceris.-Florida, beautiful sp. seen in the herh. of Torrey, very distinct firom D. angustifolia, with many blue flowers. Only semipedal. 
486. Dasistephu cespitosa Raf. Gent. glauca? Pallas. Parvula glabra, fol. radic. cespitosis, caulinis binis, ovato subrot. obtusis, fl. fascicul. cal. urceolaris 5dent. obt. sinub. obt.N. W. America, Kotzebue sound, plant only 3 inches, and flowers one inch, tubular, blue.

487. Pneumonanthe vulgaris Raf. Gent. Pneum. of L. and many European bot. not of Am. bot-caule tereto paucifl. fol. internodis longior cuneatis seu obl. lingulatis obt. summis sublanceol, fl. subped. cor. cal. triplice longior, cal. sepalis lin. obt.-Europe, stem. pedal, fl. biuncial.

488. Pneumonanthe minor Raf. caule subangul. 1-2fl. fol. lin. obl. obt. rugosis revolutis, internodis eq. fl. ped. cal. sep. obl. obt. cor. triplo longior-Europe, deemed a var. probably of last; but very distinct, stem 4 to 6 inches, usualy one flower over one inch long, a var. is biflore-None of these two are found in $\mathbf{N}$. America unless perhaps north of Canada, all the American sp. akin are different, such as $\boldsymbol{P}$. media 441, P. rigida, gracilis, torreyana of my monograph of 1828 .

489. Xolemia trachiloma Raf, caule scabriusc. rubescens, fol obt. et sublanc. subt. glaucis, margine et nervo scabris, fl. term. 2-5, cal. sepalis ovatobl. cor. subclausa ad cal. duplo longior, sinubus ineq. trifidis---New Jersèy, near waters, autumnal like most of the American sp. of this G. fl. pretty blue. Some varieties, 1. biflora, minor $2 \mathrm{fl}$. fol. obl. lin. cal. sepalis subovatis. 2 major. fl. 2-3 purpuro cerul. cal. sepalis lanceol, N. Carolina, called Gent. loomesi in Herbal of 'Torrey.

490. Cinronia L. This Linnean G. was nearly as loosely framed as Gentiana, and chi e- 
fly differed from it by the declinte style, and twisted anthers, all the other characters being often common to Gentiena ; but it had from 4 to 12 stamens and parts to the corolla: when above 5, this character blended with Chlora. Adanson ascribed 4 stigmas to Chlora and only one to his Sabbatia formed by the Chl. dodecandra of $\mathbf{L}$. which is not true. Pursh transfered the Sabbatic to the American Chironias, without giving any definite peculiar character to it, and Sir J. Smith would not admit therefore of this Genus. 'The whole is yet in utter confusion, there is a crowd of fine $\mathbf{N}$. sp. from N. America, forming two distinct Genera at least. The African Chironias offer also several anomalies and may form various Genera : even Chlora must be divided, and I shall try to fix their true essential characters. The fruit is as in Gentiana except in Roeslinia.

491. Chironia Raf. cal. camp. 5part. equalis? cor. hypocrat. limbo 5fido, stam. 5, antheris spiralis, stylo declinato, stig. capitato bilobo. caps. uniloc. bivalvis.---Type the South African sp. but they all require to be verified, as the 4 next Genera prove.

492. Crondropis Raf. (membr. keel.) diff. 491 cal. ineq. sepalis membranaceis carinatis, stylo erecto \&c.---Type Ch. trinervis R. Chironia do L, Pers. Exacum do alis auct. Fol. lanc. acum. 3nerv. fl. ped. opp. coruleis.---In Ceylon and South Arica, perhaps 2 sp. blended. Ch. Agona is another sp. of this Genus.

493. Eupodia Raf. (well pedunc) diff. 491. cal. tubulosus 5fidus 5nervis, stylo apex flexo--Type E. purpurea Raf. Ch. peduncularis bot. 
reg. 1803. frutic. fol. ovato lanc. acum. 3-5venis, ped. $1 \mathrm{fl}$. longissimis, cal. tubo cor. brevior. ---S. Africa? large purple flowers, corolla stellate, segments ovate acuminate.

494. Onefera Raf. (Rapunt. nom. antic) Rupuntium Breyn. diff. 491. cal. camp. subintegro semi-bilobus-.-'Type $O$. coccinea Raf. Chironia linoides L. fol, lin. crassis, fl. ped. coccineis---S. Africa.

495. Roeslinia Mench. diff. 491. corolla subrotata, stigma peltatum, bacca unilocul, vel caps. carnosa---very good Genus 2 types 1 . $\boldsymbol{R}$. 4 gona M. Chironia boccifera L. 2 R. frutescens.

496. Sabbatia Raf. cal. ineq. 5part. tubo camp. corolla 5 fida rotata, antheris involutis, style declin. stigma 2 linearis contortis, caps. 2 valv. uniloc.--'Types the American sp. which a re very numerous, see Elliot and my N. Sp. in my monograph, S. obtusif. lanceol. cymosa, lingulata, petiolata, amena, umbellata, pumila, nivea, stricta, tenuifolia, diffisa, heterophyla, anceps, \&c.

497. Plenenta Raf. (more added) Sabbatia Ad. non. alis. diff. 496, cal. 7-12part. stam. 7-12 stylo sepe recto \&c.---Although this G. chiefly depends on extra numbers, it is a very natural one, prolific also of Amer. sp. It chiefly differs from Chlora by the unequal calix. 'Type the Chl. dodecandra L. which included 12 blended sp. see my monograph and N. Sp. Pl. leucantha, rigida, flexuosa, fasciculata, capitata, \&c I add here this last.

498. Pleienta capitata Raf. caule rigido tereto bisulcato, fol. remotis obl. lanceol. acutis uninervis, inferis ellipt. fl. term. capit. involucr. sub 8 andris, cal. lanceol. cor. brevior, lac. cor. 
spatul. obt.-Unaka and Cherokis mts. very distinct sp. next to $\boldsymbol{P l}$. gentianoides, leaves uncial, fl. white or incarnate in sessile heads of 3 to 5 , involucre of 4 leaves.

499. Plurimaria Raf. Chlora L. anct. cal. equalis rotatus 8-10part. cor. rotata 8-10part. stam. 8-10 non spiralis, stylo unico, stigm. 2 bifidis.-'Types the Chl. perfoliata, mascariensis and quadrifolia? but Chl. sessilis W. ог Gent. do L. is a sp. of my G. Narletis having a rotate 4 fid corolla, or a N. G. if it has 8 stamens at the same time. All have yellow flowers.

500. Disinsty lis Raf. (2 un. st.) diff. 499, cal. camp. Gfidis, cor. rotata 6 fida, stam. 6 . stylis 2 coalitis, stigma 2 obtusis-Type $\boldsymbol{D}$. italica Raf. Chlora imperfol. L. fol. sess. ovais oppositis. Very distinct Genus. 'Thus all theChloras were of different Genera! I changed the name because too similar to Chloris and Chloranthus.

After such an evidence of indispensable reform in 3 Genera of old in this Centuria alone, it will appear that those Genera Polygonum, Gentianc and Chironia were without any real peculiar characters, the species merely held together by no one linows what, except a kind of fascies or appearance. The Gentians now very numerous, amounting perhaps to 150 species, may be all reduced to the reformed genera, by a little care, and I may even do it hereafter. But there are several anomalous species types of subgenera. 'The G. pratensis probably a Narlictis has a very unequal calix. The G.auriculata has also a similar calix with 2 sepals cordate; it has 4 or 5 parts to the campanulate corolla, and 4 or 5 stamens. Is it a Genus? Dicndiotis Raf. near Pogoblephis. 


\section{CENIURIA VI.}

501. LINIDIA Raf, 1815. Cal. persistens 35 partitus, Petalis 3-5isarinis, stam. hypog. definita basi sepe coalitis. stylis 1-5, stigma 3-5, capsula multicoca multiloc. multiv. loculis valvatis monospermis-These characters of my new nat. family of 1815 ought to have been the characters of the G. Linum of L. instead of the false ones usually given. This family is intermediate between Alsinidia and Tamarixia. It contains the G.Linum, Radiola, Scheflera, Cometes, Glochidion, and my Numisaureum, Meiapinon, Mesynium.

502. Numisaureum (gold coin) cal. 5part. petalis 5, stam. 5. stylis 3-4longis, stig. capit. capsula depressa 6-8loc. nonpartibilis evalvis. Frutic. fol. alt, fl. auratis-Indian Genus, 3 types $N$. repens, petiolatum, acuminatum.

503. Numisaureum petiolatum Raf. Linum trigynum Sm. ex, bot. t. 17 Lod. 1193, Curtis 1100, Ramis teretis erectis, fol, petiol. ellipt. acutis serratis. fl. corymb. petalis flabellatis, stylis 3.-A fine shrub of the mts of Ceylon and India, called Gul ashafi or the flower of golden coin, whence my generic name.

504. Numisaureum acuminatum Raf. Lin um Agynum Colebrook, Fol. ellipt. obl. acum. serrat. brevi petiol. fl. capit. corymb, sepalis ovat. acum. stylis 4-Shrub of Nipal.

505. Meiapinon (least flax) Raf. Cal. 3part. 
petalis 3 , stam. 3 liberis, stylis 3 , caps. 3loc. 3sp. 3valv. Herba. fol. opp.-Very near to Mollugo, but really a Linidia by monosperm cells, and nearer Radiola.

506. Meiapinon saginoides Raf. Glabra, erecta, ramosa, fol. lin. obl. acutis, fl. axill. ped. -In Florida, small plant one or two inches high, flowers white.

507. Messmium R. (middle un.) diff. ad Linum, stylo unico, stigna 5 capitatis, vel stylis 5 plus minusve coalitis, in stylo 5fido, caps. mucronatis 5 valvis 5locularis 5sperma.Types III. africamum or Limum monogymum, M. mexicanum, texense and chilense (L. macrei Lindl.) Lindley indicated this G. although he says that in some sp. with yellow fl. the styles are slightly united at the base, such are $\boldsymbol{L}$. ethiopicum, virginicum, vigidum, mysorense : but they have capsule 10loc as Linum,

508. Mesynium texense Raf. Linum berenderi Hook. b. mag. 3486. Herbac. multicaule angul. fol. alt. lin. rigidis glabris mucronatis, $\mathrm{fl}$. racem. flavis, sepalis lanc. acum. serrul. petalis basi villosis-Texas, quite monostyle, caps. globose acute.

509. Mesynium mexicanum Raf. Linum do. Kunth, DC. bot. reg. 1826. Herb. paniculat. glabr. fol. inferis oppos. ovatis, sepalis ovatis.Mexico, style 5fid at top. capsule mucronate.

510. MOLLUGIDIA a new small family of mine, only differing from Linidia by cells or capsule polysperm, valves septifer, seeds central, and petals often lacking, Types Rotale, Cherleria, Bergia, MIoltugo, Nemallosis, Lampetia, Hermannia, MI Thernia, Pharnace$u m$ they had been referred by me formerly to $\mathrm{Al}$ sinidia, but this branch of the old Caryophyles 
have all unilocular capsules. Sagina belongs to Alsinidia as Smith proved that the caps. is uniloc. and not Alocular. Leaves opposite or verticillate as in Tinidia and Alsinidı. Hermannia belongs here, but has united stamens, like Linum. Mahernia also and united styles like Mesynium, are they a N. Fam?

511. Nemallosis Raf. (fil. variable) cal. 5 part. petalis 5 linearis emarg. stam. 10, fertilia 5 , sterilia 5 alt. stylis 3 , caps. 3loc. polysperma. Canle artic. fl. verlic. 19. nonnulis 3 andris apetalis.-Types the two following plants with habit of Mollugo verticillata.

512. Nemallosis prostrata Raf. Pharnaceum mollugo L. auct. Burm. t. 5. caule depresso tereto, fol. 4-5ineq. ellipt. fl. vertic. pedunc.India, fl. whitish.

513. Nemallosis erecta Raf. Pharn. mollugo L. Alsine Burm. zoyl. t. \%. \&c. Caule erecto, fol. 5 lanceol. $A$. vertic-Ceylon, larger white flower's. See Sir J, Smith for remarks on these plants, which he states had been called Mollugo spergula once by L. when showing only $\mathbf{3}$ stamens.

514. Lampetia Raf. (Nympha) cal. 4fidus, petalis nullis, stam. 4, stylis 4 , caps 4 loc. polysp. acaulis, fl. ponic-Very near to Phasnaccum, habit peculiar, but many sp. of that Genus have various forms and must be examined again. Habit of Dionea and Drosera.

515. Isampetiu nudicaulis R. Inollugo do Smith, Burm. t. 8. fol. radic. obovatis. scapis dichotomis paniculatis-Ceylon and Africa, perhaps two sp. blended also.

516. ALSINIDIA. This family of Adanson was Caryophyles of Jussieu, who united thereto the Spergulides Ad. and many heterogenous 
plants, without common characters. They can be distinguished easily thus.-Arsinines. capsule unilocular polysperm, seeds central, calix parted. Such as Alsine, Siellaria, Arenaria, Sagina, Mrenchia, Buffonia, Holosteum, Polycarpon. Cerastizm, Spergula, Iresine, Velezia, Frantionia, Ielephium, Cosmia Isgarum.

51\%. DIAN'THIDIA or Phorandivia 1815 Raf. The Dianthides or Caryophyles (bad name meaning clove?) cal. tubulos. petals 5 unguic. 10 stam. 5 ad ung. pet. inserta, caps. 1-5loc. sem. centralis. fol. opp. fl. ped.-These are the real akin to Dianthus, Silene, Lychnis, Agrostema, Cucubalus \&c which all require generic reforms as yet.

518. DIONIDIA Raf. 1815. This new family differs chiefly from Alsinidia by a single style and stigma: the habit is often peculiar.Types Dionea, Ortegia, Loeflingia, Adolicton Hagea, Lahayer. \&c.

519. AMAR AN'IHIDIA. This family must be confined to the $\mathbf{G}$. with free stamens, several stigmas, and yet with capsule monosperm, as Amaranthus, Dimeianthus, Quevia, Anychia, Digera, Xerandra, Paromychia, Herniaria, Drypis, Corigiola of ${ }^{2}$, these 3 last have petals and form sub families.

520. ACHYRANTHIDIA. This small group differs by a single stigma and sced, stamens united. 'Types Achyponthes, Illecebrum Gymnocarpon, Lophanthus Forst. All these nat. families are gradually connected in flowers and habit. All the Genera with monadelphous stamens, ought to be of same order: yet we see this tendency in Linidia likewise and Mollugidia. See 534. 
521. SCLERANTIIIDIA Raf. another small family near to Linidia but diff. by stamens fiee and double diplarine, ratlıer perigyne, fruit commonly dicocus-Types Scleranihus, Galenia, Floerleca, Cabomba or Nectris which is not monocotyle, as stated by a mistake of Jussieu, since the leaves are opposite as in Galeuia, but Floerlia has alt, divided leaves. Galenia has affinities with Hamamelis, Florkea with Limnanthes.

522. DROSERIDI- 1815. Fine family near Alsinidia, only difference valves seminiferous, Habit peculiar but variable, often like Dionea, scapigerous.-Type Drosera and the divided Genera of it, Aldrovanda, Parnassia. \&c.-Roridula differs from it as Dionea from Alsinides by a single style and stigma: it is probably the type of another family RORIDIA. Turneva differs only by the perigyne petals and stamens.

523. Rorella Raf. 1815 ad Drosera diff. stam. 10, stylis 5.---Type R. Insitanica Raf. Drosera do L. Arenaria do nonnulis auct. Fol. radic. subul. scapo flor. umbellatis.

524. Dismopiyla Raf. (binate leaf) ad Drosera diff. cal. 4-5part. pet. 4-5. stam. 4-5, ovar glab. 4-5lobo, stylis 4-5multifidis fol. rad. divisis, fl. corymb.--'Type the next sp. but probably Dr. pedata and others of Australia belong here.

525. Dismophyla binata Raf. Drosera do DC. Br. Labil. 105 bot. mag. 3082. fol. longe petiol. bipartitis linearib, glandulosis, scapo glabro, fl. paucis ochroleucis. Australia.

526. Drosera L. auct. This G. requires total revision offering many forms, which I now indicate as mere Sub Genera; but are perhaps 
Genera 1. Rossolis cal. 5p. eq. pet. 5. eq. stam. 5 eq. stylis 5. caps. 5ralv. Type Di. acamis, rolundifolia, of $c$.

527. Adenopu Raf. (ped. gland.) cal. 5p. ineq. petalis 5 subeq. mareescens, stam. 5. ineq. filam. planis subul. membranaceis, antheris obl. biloc. stylis 3-4bifidis, caps. oblongis, valvis $\mathbf{3 - 1}$ -'Types Dr. anglica and nearly all the $\mathbf{N}$. Amer. species, see new flora.

528. Filicirna Raf. (thread rolled)cal. 5p. ineq. 1. obov. major, petalis 5 eq. venosis, stam. 5 eq. fil. filif. anth. bilobis, stylis $3-4$ basi coalitis bipartitis caps. obl. 3valvis, jol. sine lamina, fl. racem. bract. secundis, roseis. Three types disc. by myself in New Jersey in 1802, leaves reduced to mere petiols circinated or rolled when young, with stipules, cotyledons 2 elliptic obtuse. All annual vernal.

529. Filicirna, s. Droser filiformis Petiolis filif. supra piliferis, stipulis laceris, bracteis subul. pedic. longior, petalis obov. stylis :3.This is my original sp. Pine barrens of $\mathbf{N}$. Jersey, scapes 8-15 inches.

530. Filicirna s. Drosera iemifolia. W. Rom. Big. bot. mag. 3340 as filiformis. Petiolis filif. supra canaliculatis piliferis, hasi lanatis, stipulis subul. deltoideis glahris integr is. bract. brevis, petalis obov. concavis, stylis $\mathbf{A}-\mathbf{N}$. Jers. Long Id. and N. England.

531. Filicirna s. Drosera leionema Raf. Petiolis filif. undique glabris, apice vix piliferis stipulis laceris, racemis pacicilloris, bract. subul. ped. eq. Petalis cuneatis stylis 3-South New Jersey, fl. rose white, scape 6-9inches. Probably all sprung fiom each other.

532. 'TAMARIXIA 1815. This small family chiefly differs from Droseridia by stamens 
more or less united at the base, and rather perigyne---Peculiar habit like Cistus-'Type Tramarix, Eutiplex, Rolejeka and perhaps Turnera.

533. Eviplex Raf. ad Tamarix diff. cal. pet. 4-5, stam, 8-10-Type Tam. germanica, caspica, songrarensis, the real Tamarix is isarine with 5 stamens and petals.

534. GOMPHIRENIDIA. Another small family that differs from Amaranthides by stamens monadelphous-Types Gomphrena, Bragantia, Alternanthera, Belutta, Aerua, Waltheria \&c, with many new G. The whole tribe of Amaranthides and akin are known to be in utter confusion, the sp. having been refered by mere habit, the whole requires revision. The Bragantia of Vandelli was the Gomplur. arborescens. The Caraxeron of Vaillant is probably a good G. it was both Gomphrence and Illecebrum Vermicularis L.-The Alternanthera Forsk. was the Gomphr. or Illec, sessile $\boldsymbol{L}$. the Coluppa of Adanson. The family $\boldsymbol{A}$ chyranthidia 520 is only a subfamily of this, the united stamens being more important than the stigmas, when there is only one seed.

535. Caraxeron Vaillant. Philoxerus $\mathbf{R}$. Br. Sm. Cal. 5part. conc, stam. 5 basi coalitis, stig. 2. capsula evalve-Type C. vermieularis and brasiliense (Gomphrena and Illeccebrum, $\mathbf{L}$ conicus, diffisus (Philoxerus R. Br.) these two australian sp. form perhaps a subgenus. The real Gomphrenc have only one capitate stigma says Smith; but the Genus appears to have been formed on the capitate flowers and mere habit! all those with a single style are Illecebrum of L. but the I. verticillatum forms the Genus Paronychia 'T. Juss. Ad. with free 
stamens and a bifid style, of fam. Amaranthidia.

536. Belutta R. (nom. ind) cal. 5part. calicul. sq. 1-3. stam. 5 ad basi tubo monadelpho, stylo 1, stig. 2. caps. circumsc. monosperma, sem. lenticularis. Fol. alt. capitulis axillaris. 'Two types united in Celosia nodiflora by $\mathbf{L}$. and Author's, but of a diflerent family by one seed only, therefore of Goumplurenides.

537. Belutta sessilis Raf. Lelosia nodifl. L. auct. Fol. subrot. olov. mucronatis, capitulis sessilibus.---Malabar and Ceylon, Burm. zeyl. t. 5. f. 2 .

538. Belutta peduncularis Raf. Cel. nodifl. var. auct. Fol. oblongis vel cuneatis undulatis acutiusculis, capitulis peduncularis---In Sumatra \&c.

539. Cadelaria Ad. Achyranthes L. cal. 5 part. reflexis, calieulus 2 , stam. 5 liberis ciliatis stylo 1, stigma bilobo, utriculus monosp. Frut. fol. oppositis, fl. spicatis. 'The types are the 3 following sp all blended as Achyranthes aspera by L. all the other Achyranthes require revision; of family Amaranthides.

540. Cadelaria indica Raf. Fol. cuneatis acum. subt. toment. argenteis---In India, often figured by Burman, Rumphius \& c, perhaps two sp. blended as yet.

511. Cadelaria sicula Raf. Fol. lanceol. acutis scabris subtus sericeis, .spicis adpressis--In Sicily and Barbary, figured by Bocc. Sic. t. 9, but the Sp. from Jamaica appears difierent.

542. Cadelaria punetata Raf. Fol. obovatis acuminatis glabris subtus punctatis glabris--Arabia. The Achyr. panienlata of Forskal with free stamens broad at base, is probably also of this Genus. It will be hard to say 
which are the true types of the real Achyranthes! see the next Genera, A. dicholoma and corymbosa are Anychior. Ac. celtissima with scandent stem must be a peculiar Genus.

543. Codivalia laaf. Pupal Ad. cal. 5part. 2ciliatis hamatis seu echinatis, caliculus 2, stam. 5 basi coalitis, stylo, stig. sem. unicum Fruct. Fol. oppos. fl. spicatis, glomeratis---Types Achipr. lappricea, patula, L. \&c.

514. Uretis Raf. Ouret Ad. cal. 5fidus, caliculus 1 , stam. 10 basi coal. 5 sterilis, 1 styl. 2 stigma, sem. 1. Ilerba, fol. calt. fl. capitatis. ---Type Achyranthes alternifolia L. and probably several others.

545. Kokera Ad. cal. Gpart. concavis, stam. 5 liberis styl. 1 . stig. 2 , caps. circums. 1 sperma. Fol. relt. glomerulis panic.---I am unable to indicate the type of this $G$. of Adanson, as he quotes no figure, it is probably found among the Achyranthes, although the firuit is like Amaranthus. Family Amaranthides.

546. Eczotoripa Raf. (Nom. Egypt. Amaranth) cal. squarrosus, 5part. ineq. 3 internis petaloideis major patens, stam. coalita in corpus calloso 3dent. antheris 3 internis, stigma ... sem. unicum. Fol. alt. fl. spicatis---another peculiar Genus blended in Achyranthes muricala of Tgypt, at least 2 species.

547. Eclotoripa fruticosa Raf. caule frutic. fol. petiol. ovatis, spicis pedunc. axill. elongatis, ped. 5gonis.---Egypt \&.c.

548. Eclotoripa anmua Raf. caule annuo ramoso patulo, fol. petiol. ciliatis, subcordatis, ped. ut supra---Arabia and Mollucas.

549. Steinemis Raf. (sterile half) Cal. duplex, ext. 3part. internus 5part. ineq. stam. 10 
monadelphis 5 alt. sterilis, stylo brevis, stig. 1 . obtus. glanduloso, utriculo monosp. sem. Ientic. Fol. opp. fl. capit-New American Genus near Uretia and Digera, of real subfam. Achyranthidia by unic stigma. 'Three types,

550. Steiremis repens Raf. Achyr, do Elliot. Gomphrena et Illecebr. polygonoides L. auct. Achyr. Lam. non Retz nec Vitm. quid et $\boldsymbol{D} i$ gera arvensis Forsk. Repens hirta, fol. pet. lanc. capitulis sessilibus ovatis-Carolina, Georgia and Antilles, the Antillian plant is perhaps difierent by stem dichotome, broader leaves and globular heads, St. globosa Raf.

551. Steiremis ficoidea Raf. Achyr. do EIliot, Illecebrum do L. auct. Repens, glabra, fol. petiol. lato lanceol. capit. globosis sessilis pubescens-Carol. Florida, Antillis ofc.

552. Steiremis sessilifolia Raf. Repens radicans glabra, fol, sessilib. obov. et subrot. acutis, capit. glabosis sessilis-In Spain and Africa, blended with the last by all Authors, probably several other sp. near these in both continents.

553. Phyllepidum Raf. 1814 sp. sc. cum. ic. cal. duplex, utrinque 5part. internis emarg. stam. 5 liberis filif. stylis 2. filif. utriculus monosp. Fol. alt, sess. Al. spic. bract-Of family Amaranthidia New G. of mine disc. 1804, the habit is peculiar, leaves scaly like, the internal calix has emarginate divisions, both are persistent as usual in the whole tube.

551. Phyllepidum squamosum Raf. ut supra, Desvaux $\boldsymbol{\mathcal { S }} \cdot \mathrm{c}$, caule erecto ramoso, fol squamul. semiamplex. oratis acuminatis, spica densa oblonga, bract. subul-In Maryland, sandy Pine woods, rare, flowers uncolored, stem 6 to 8 inches.

555. Dinemantuus Raf, (2 less in fl.) Bliton 
Ad. Differ. ad Amaranthus cal. 3part. stam. 3 - Types all. the triandrous Amaranthus that ought never to have been united with the pentandrous, ha!f the Genus belongs here. It is said that $\boldsymbol{A}$. oleraceus appears to unite both, having $\mathbf{3}$ or $\mathbf{5}$ stamens, if so it is like Gononcus a dimorphous sp. that ought to be a peculiar Genus, Pentrius Raf.

556. Euxouus Raf. (well shut) diff. ad $\boldsymbol{D i}$ meianthus, fructus utriculus ovatus indehiscens sem. ovata non lenticulata cal. longior-Type E. deflexus, or Amar. do L.

55\%. Amblogyna Raf. (obt. fem. ff.) diff. ad Dimeianthns fl femineis infurdibulif. 3fidis, lac. obl.-Type Ambl. or Amar. polygonoides L.

558. Amarantuus L. Bajan Ad. as A. sanguineus appears the type of this Genus, it must with the pentandrous sp. retain this name. Adanson's Amaranthus was Celosia L.

559. CELOSIDIA Raf. 1814. This family differs from Alsinidia just like the Gomphrenides from the Amaranthides by having united stamens. It differs from Gomphrenides by several central seeds in the capsule. The habit is nearer Amaranthides, leaves commonly alternate-Types Celosia, Lophoxera, Snkana, Hyparete, but Cedrela does not belong to it, nor Coilosperma, nor Belutta.

560. Lophoxera Raf. (crest dry) ad Celosia diff. stam. vix. coalitis, stylo trifido stig. 3, caps. 3sperma et 6sp.-Types Loph. comosa, paniculata, caudata, polygonoides, and racemosa (Cel. 3gyna L.) all Celosias. The true Celosias have one style, 2 stigmas, 2 ar 4 seeds. The Cel. lanuta is now Aerua tomentosa.

561. Sukana Ad. Diff. Celosia, stam, 5 sine 
filam. sterilis interjectis, cal. vix caliculatis'Type the Amarantlius of Barr. t. 193 says Adanson, Celosia has properly 10 stam. 5 alt. sterile, and cal. with 2 or 3 calicules.

562. Xerandra Raf. neog. 1825, ad Iresine diff. cal. duplex ext. 3part. int. 5 part. stam. 5 glandulis globosis, alternis. fl. fem. extus comosa, stylis 2, , sem. glabrum unicum in utriculo.'Type $\boldsymbol{X}$. celosioides, and elatior, both Iresine do auct. but this G. belongs to Amaranthides while Iresine bolongs to Alsinides by polysperm capsule.

563. Iresine L. auct. Dioica cal. 5part. bicalicul. stam. 5, squamulis alt. stig. 2 sess. utriculo polysp. sem. tomentosa-the stamens are slightly united at the base. this G. might be reduced to Celosides.

564. Collosperma Raf. (hollow seed) cal. du plex, ext. 2 part. int. 5 part. concav. stam. 5 liberis basi dilatatis, stylis $\mathbf{3}$, bacca 3sperma, sem. concavis, lucidis-A very distinct Genus from Celosia by free stamens, berry and seeds, whereby it belongs even to a different family Eirpetridia, see 633, but the habit is somewhat like Celosides. The type is C. corduta Raf. the Celosia baccata! Retz. W. P. fol. cord. acum. racemis laxis. India.

565. Deeringia Br. cal. duplex ext. 3part. int, 5 part. stam. 5 basi coalitis, stylus trifidus, bacca triloba polysperma uniloc. sem. centralis - This $G$. was wrongly deemed the Celosici baccata by Brown; it is not even of same family, being a real Celosidia. Type D. celosioides Hooker b. m. 2717. fol. petiol. ovatis acum. spicis gracilis, fl. remotis viridlis-Australia.

566. Hyparete Raf. (Nympha) cal. urceolatus 5dentatus stam. 5 basi coal. stylis 3, caps. 
3valvis monoloc. polysperma. Fol. alt. $-\mathbf{A}$ very distinct G. blended with Celosia, that has a circums. caps. or pyxidium, yet of same family. Type H. glauca Raf. Celosia do. Wendland, Persoon, fol. lin. lanceol. carnosis canis_-South Africa.

567. Litiopinila Sw. Sm, cal. duplex, ext. 3 part. int. 5part. ineq. 3 petaloideis, 2 squamosis, stam; 2 lib. stylo 1, stig. capit. emarg. capsula 2locul ? 2sperma? Fol. oppositis-Of family Scleranthidia? altho' near Ortegia and Loeflingia fruit not well known, on which will depend the main affinities.

568. Lithophila muscoides Sw. Sm. pumila, fol. lin. obt. canaliculatis, fl. congestis. minute plant of I. Navaza in Antilles.

569. ATRIPLEXIA. This natural family will be much reduced by separating the anomalous G. with berries or more than one seed: being thus reduced to those with a single seed akene or utricule, altho' with several stigmas, the Genera therein placed hardly differ from Polygonides, and might as well be united, the habit in those last of articulate stem and vaginate leaves being the chief difference! while Urticides differs by single stigma or hypogyne stamens. Uniting therefore Atriplexia and Polygonidia we may divide them into 6 more evident subfamilies, distinguished by the fruit and proportions of stamens. Salisbury detected the mistake of Jussieu who deemed them perigyne, while they are properly hypogyne.

570. Polycnemides. utriculus monospermus, -G. Polycnemum, Camphorosma, Petiveria, Chenolea, Kochia.

571. Baselundes. Calix baccatus-G. Basella 
Anabasis,Coccoloba, Calacinum, Naucorephes? see 105. Calostima? 589.

57\%. Salsolides. Akena, cal. isarine-G. Salsola, Chenopodium, Spinacia, Beta, Atriplex. Crucita,Acenida, Dondia, Isgarum, Axyris, Sovara, Tracaulon, Rumex, Oxyria, Triplaris, Koenigia, and all the pentandrous G. of Polygonum.

573. Polygonides. Akena, cal. meiarino; more stamens than parts--G. Polygonum and reformed Genera, Atraphaxis, Rheum, Calligonum, Pallasia, Anredera, Vibones, Menophyla, \&c.

574. Blitides. Akena, cal. pleiarino; less stamens than parts---Blitum, Ceratocarpus, Salicornia, Cnopos, Sclerosperma, Ofaiston \&c.

575. Corispermides. Akena nuda, not covered by the calix as in $572,573,574 .---C o r i s-$ permum and akin.

576. Menopixyla Raf. (moon. leaf) monoica, cal. duplex, ext. 3fidus, int. minor petaloid. stam. 12 antheris bifidis. fem. stylis 3 reflexis stig. plumosis, cal. in fructo ampliato, Frutex, f. panic--'Types $M$. lunaria. Rumex do $\mathbf{I}$. auct. 2. $\boldsymbol{R}$. polygamus Cav. t. 22. has granular calix and 6 sterile stamens.

577, Vibones Raf. (Rumex lat.) diff. Rumex, Dioicus, fl. masc. 3part. stam. 9-12 antheris bifidis---Type $\boldsymbol{V}$. auratus Raf. caule angul. fol. ovato lanceol. subcrenatis, nervis pilosis, cal. lin. canaliculatis. Alps of Switzerland.

578. Niro Mench diff. Rumex, Dioicus, fl. masc cal. 6part. equalis, 3 internis, fl. fem. cal. cupularis 3fidus spinosus reflexus punciatusType Rumex spinosus L.

579. Rilonoptera Raf. (rosate wing) diff. Rumex, cal. ineq. internus valvis membrana- 
ceis, una major aleformis.-Tye $R$, roseus $\mathrm{L}$.

580. Enex Necker, diff. Rumex, stam. 12, fructus uncinatum.- Type Rumex acutus L.

581. Analiton Raf. diff. Rumex, Dioicus, cal. 3part. eq. subrot. internis nullis. fl. fem. cal. 3part. cordatis sine granulis. fol. bipinnatis-Type R. bipinnatus of Marocco, habit very different from Rumex. stam. 6, not 9 as in Vibones.

582. Rumex L. auct. 'This G. was like Polygonum in great disorder, with many anomalies. The old G. Lapathum and Acetosa of 'Tourn. deserve to be subg. at least. $\boldsymbol{R}$. digynus is become Oxyria reniformis quite lately. I think the 6 foregoing Genera are quite distinct, and the following subgenera ought to be admitted besides. 1. Patientia hermaphr. cal. granulato-2. Rumex herm, cal. non granul.-3. Tomaris herm. cal. laciniato-4 Atecosa dioicis, cal. granul.---5 Acetosella dioicis, cal. non granul. 6 Eutralia Raf. dioic. cal. very unequal. ext. subulato, int. large orbicular, type $\boldsymbol{R}$. luxurians, this might even be a Genus perhaps.

583. Isgaru m Raf. (Salsola) cal. 5part. stam. 5 stylo bifido, stig. 2 capsula biloba uniloc. disperma. Fol. oppositis---Type I. didymum Salsola do L our. Sm. caule decumb. fol. obl. crassis, fl. axill. sessiles. East Africa. By the two seeded capsule and habit, this $\mathbf{G}$. is not of Atriplicides but rather of $\boldsymbol{\Lambda}$ lsinides family, some Kochias belong here.

584. Ofaiston Raf. (Salsola) diff. Salsola, stam. 1, while all salsolas have 5... ! Type $O$. paucifolium Raf. Salsola monandra Auct. fol. paucis alt. teretib. carnosis. Caspian region. neer Corispermum. 
585. Sarsola L. this G. ought to be distinguished by the spiral seed, and 2stignas : therefore $\mathbf{S}$. trigyma with 3 st ylis is probably a peculiar G. 'Tinitis (Raf.) triflora. 'I'he (G. Kochia, Chenolea, Sueda, Donslia are all good. Even in Kochia those with two soeds are probably sp. of Isgarum, Dondia of $\Lambda$ danson has one style with $\mathbf{3}$ to $\mathbf{5}$ stigmas and lenticular seed.

586. Salicornia L. One stam. 2 stig.-S. herbacea, fruticosa, polystachia, cruciata, arbuscula \&c.- -stem. artic. as in Polygonides.

587. Sarcatimia Raf. (fleshy artic) two Stamens, 2 stigmas-Here belong. S. procumbens, radicans, strobilacea, virginica, indica, ambigua \&c, all Salicornias of $\boldsymbol{L}$. and Authors. This $G$. was chiefly made by habit of artic. stems; it may offer yet other anomalies by calix entire or 4dentate f $^{\circ} \mathrm{c}$.

588. Untica L. Another G. full of anomalies, many of the $100 \mathrm{sp}$. united thereto were merely held by habit or aspect, Linneus had only 28. 'The Genera Boehmeria has been properly separated, I also separated Adike and Selepsion long ago; but must now add two others which are not even of same family! four parts to the male calix, but fem. cal. 2parted: the seed is thus as Atriplex, therefore very near that G. and its family, altho' Urtica is made the type of another, the main difference is in the single stigma, with hypogyne stamens. Parietaria differs from Urtica, by same means as Chenopodium from Atriplex.

589. Calostima Raf. (fine stig) diff. Urtica, Dioica, stigma radiato pilis stellatis, cal. fructif. inflato pulposo cordato.- This certainly a peculiar G. having a beautiful tuft of radiated 
hairs for stigma, thus many stigmas! and the fruit as in Baselides, therefore it belongs to that subfamily of A Treiplexia.

590. Calostima aculeata Raf. Uctica baccata L. plum. ic, 260 Andr. rep. 454 \&c. Arboresc. acul. fol, alt. cord. dent. acum. racemis axill. retroflexis-South America, habit of Nettles, but a tree, perhaps several blended species.

591. Lithocnine' Raf. (stone nettle) diff. Urtica fl. fem. 3part. stigma 3 , semina 3gona'This also belongs to Atriplexia by the stigmas multiple. type L. lappulacca Raf. Urtica, do Sw. W. P. caule repens, fol. alt. ovatis ciliatis, fl. axill. Antilles on stones, leaves not stinging.

592. Pentocnine R. (5 nettle) diff. Urtica, cal. 5part. stam. 5-Perhaps only a subgenus, near to Chenopodium, Type P. glomerata. suffiut. fol. alt. ovatis, f. axil. sessilis. Antilles. any other pentandrous sp. must be added.

593. Anıке Raf. 1803. diff. Urtica, fl. tripartitis, triandris, fl. fem. similis. caule carnoso, fol. glabris-Many American Sp. blended in Urtica pumila, see my New flora, and I add here another from Antilles.

594. Adike allophyla Raf. Urtica trianthemoides Sw. W. P. Sin. caule erecto ramoso carnoso, fol. oppos. ineq. oblongis et obovat. obt. integris-Hayti, near streams.

595. Selepsion Raf. 1814. diff. Urtica, fl. fem. 4part. ineq. 2alt. minor, stylo, fl. dioicisThis G. was indicated by Leers long ago for U. dioica and urens, it is a subgenus at least. Many Sp. belong thereto, see my New flora for S. vernum and montanum. Selepsion was an Egyptian name for nettles, and Adike a Greek name according to Adanson. 
596. Orbixilis Raf. 1818. diff. Urtica, Cal. masc. 5part. 5andris, fl. fem. 2phyl. ineq. persistens, stylo lateralis reflexo, stig. acuto, sem. orbic. obliqua plana acum.-'Types. U.divaricata, camadensis and my new species, see my new Flora. These plants have a singular structure of sced, with a very evident style and unequal small valves to it. Sir James Smith hoped that some American botanists would illustrate them. It is probable other sp. belong here, compare $\boldsymbol{U}$. membranacea.

597. Moxosteria Raf. (one ster) cal. 4part. corolla 4 fida infund. stam. 2 , una sterilia, stig. capit. caps uniloc. bivalvis, polysperma.-This must be added to the Gentiana? but is very near Heteroclita 482, and Pladera. It is the Hopea, of Vahl. W. Sm. but now L. which has wrongly been united to Symplocos.

598. Monosteria dichotoma Raf. Hopea do V. W. Sm. Exacum pusillum Roxb. caule dichot. 4 gono pumilo, fol. ovatis et lanceol. superis subul. fl. ad dichot. ramis $3 \mathrm{ft-Coromandel,}$ fl. yellow.

599. Pladera Roxb. Canscora Lam. cal.tubulosus 4alatus 4dentatus, cor. infundib. irregularis 4 loba, lobis 2 major. stam. 5 ineq. una minor, stigma stylosus bilobus. Capsula ut Gentianea-another G. blended with Exacum by $\mathbf{L}$. yet evidently not even of same family, but of Orobancimmes with Monosteria and Heteroclita, that family differing from Gentianides by irregular corolla or stamens not isarine nor opposite. Hooker says it has $\mathbf{5} \mathrm{sp}$. all ammuls, and that Canscora badly described by Lamark belongs here; but the type will be.

600. Pladera elccussata Roxb. b. mag. 3066. cate 4alato, fol. ovato lanc. trinervis, florib. 
paniculatis trichotomis albis.-East Indies. The G. Exacum is thus nearly as loose as Gentiana, I shall return to it with Sebaea of Solander.

In this Centuria I have chiefly attended to settle or reform the natural families of which Linum, Amaranthus, Atriplex, Polygonum, Urtica, Drosera \&c were the main types, with several akin Genera and families. By this labor it will be seen how utterly inconsistent was even Jussieu in framing his families or refering Genera to them, whereby it was almost impossible to separate them by any definite character. The later writers had done no better; but now I hope that it will be easy to know each family and refer genera to them by something definite and tangible. It is in this manner that all natural families ought to be revised, ascertained and ultimately settled.

\section{CENTURIA VII.}

I shall here resume again the beautiful tribe of Asphodelides and akin, begun in the first Centuria, and shall quote the very words of Lindley on that score- "In tribes of plants so simple in structure as Asphodelea, like Umbellifera and Crucifera, it is indispensable that Genera should be confined within most exact limits, and it is far better that this should be effected by the creation of many new Genera, than that it should not be done at all. Lindley bot. reg. 1486.

601. Lemotrys Raf. (meadow raceme) this 
name is to be substituted for my Quamasia 64. It now appears that this $G_{\text {. }}$ is based on the Skilla esculenta found from Kentucky to Missouri. my Lem. hyacinthina; while the same plant found in Oregon is even another Genus to which Lindley applied also the name of $\mathrm{Ca}$ massia in 1832, as follows-

602. Qunmasia Raf. 1827, Camassia Lindl. 1832. Petalis 6 inequalis, 5 adscendens, 1 inferu declinato unguicul. stam. 6 filif. hypog. equalis omnis adscendens, stylo declinato, stigma tridentatum-Habit of Lemotrys very distinct by irregular petals, stamens and style.

603. Quamasia esculcilta Raf. Lindl. b. reg. 1486 excl. Skilla syn. Phalangium quamash Pursh. fol. longissimis carinatis non glaucis, petalis lanceolatis purp. ceruleis-In Oregon, flowers double the size of Lemotrys hyacinthina that has glaucous leaves and pale blue or white flowers, but this has also a white variety or perhaps sp. figured by Hooker b. m. 27\%4. Sprengel united all these plants to $\boldsymbol{A} \boldsymbol{n}$ thericum, but totally different by stigma and smooth stamens.

604. Barnardia Lind. diff. ad Skilla, stam basi dilatatis in squamis valvatis ut Asphodelus, caps, 3loc. 3sperma-'Type B. scilloides b. reg. 1029. Bulbus ovatus, fol. rad. lin. canal. elongatis acutis, scapo racemoso, fl. carneis, bract. subnulis. China. 'This is a Gr. taken from Skilla on characters like my own Genera. 'The Skilla plumbea of Lind. b. reg. 1355 is a real Skilla by filiform stamens.

605. Uropetalon Echv. Zuccagnia Bodin non alis, Dipcadis Uster diff. ad Skilla et Lachenalia, cor. tubulosa 6ficla, lac. 3 externis, stam. adnatis, stigma 3-another G. near Le- 
motrys, by the 3 stigmas, Types 4 species. 1 Lachenalia viridis, 2 Scilla serotina Curtis 859 , 1185 ! and 2 N. Sp. 3. U. crispum, 4 U. glaucum, bot. reg. 156.

606. Borioya Raf. (greek name) corolla campan. semi 6 fida, stam. 6, membranaceis 3 dentatis, dente medio antherifero, filam. dilatatis coalitis ad cor. adnatis. stig. trilobo, caps. subrot. 3loc. polysp. sem. centralis. Fol. radic. fl. spicatis-A striking N. G. blended with Hyacinth, near to Lagocodes 6\%, but stamens not filiform! the real G. Myacinthus is now confined to $\boldsymbol{H}$. orientalis and amethystinus.

607. Borboya spicata Raf. Hyacinthus spicatus Sm. fl. gr. Lind. b. reg. 1869. Fol. lin. canal. elongatis, scapo brevis, fl. spicatis confertis $\mathbf{8 - 9}$, bracteis adnatis appendiculatis, sepalis obl. obtusis-Id. Zante. flower's cerulescent, modern greek name Borboya.

608. Nemaulax Raf. (fil. can.) diff. ad Albuca. petalis 6 , internis 3 apex fornicatis, stam. 6 ineq. 3 brevior alt. filam. canaliculatis, stylo 3gono, stig. trilobo-The G. Albuca is very near Skilla, in the hexandrous sp. but the real Albuca has 3 sterile stamens : all the other sp. united thereto are aliens like this. Type.

609. Nemaulax fastigiata Raf. Albuca do. Th. W. P. bot. reg. 27\%. Fol. lanc. et lin. planis, scapo multifl. corymboso fastig.--South Africa, fl. white green.

610. Albuca L. auct. diff. ad Skilla et Ornithog, cor, 6pet. 3 ext. patens, 3 int. alt. erectis, . stam. 6, alt. 3 sterilis, stylo 3 gono, sem. plana -Types A. altisima, major, minor, flaccida, viridifl. coarctata, \&c. all those with 6 fertile stamens are Nemaulax or of other Genera.

611. Tenicroa Raf. (colored ribbon) cor. 
petalis 6 rotatis, carinatis fornicatis, stam. (6 equalis subdeclinatis glabris, stylo declinato incurvo 3 gono, stigma concavo trifldo-Very distinct $\mathbf{G}$. wrongly united to Anthericum and Albuca, akin to Quamasia, but stamens not filiform.

612. Tenicroa fragrans Raf. Anthericum do, Jaq. W. Albuca fugax Edw. b. reg. 311. Fol. trigonis, filiformis. scapo racemoso multifl. elongato - South $\boldsymbol{\Lambda}$ frica, fl, white red.

613. Prlasia Rad (head villose) very near 'Tenicroa and Quamasia diff. Petalis nervosis non fornicatis, stam. filif. eq. glabris ovario 3 gono, stylo adscendens, stigma capitato villoso -Several sp. of Anthericum belong here or to the Genera Tenicroa, Bulbine, Quamasia ofc. by declinate style or stamens, such as $A$. exuviata bot. mag. 871, A. vittata. 1046, A. physodes 1329, but their real Genus and place depends on their stigmas, and not having now the figures before me I cannot ascertain the fact, but will do it hereafter. Meantime the type of this $\mathbf{G}$. is

614. Pilasia filifolia Raf. Anthericum do Jaq. W. Albuca do Edw. bot. reg. 557. Fol. filif. flexuosis longis, scapo brevis, racemo paucifl. albo carneo. South Africa.

615. Laothoe R. (Nymph) diff. 613. stigma bidentatum, caps. 2locularis, caulescens, paniculato-Habit and fiuit very peculiar. Type L. pomeridiana, Raf. Skilla do DC. pl. gr. Anthericum do Edw. b. reg. 564. Caule ramoso panic. fol. lanceol. carinatis glaucis scabrisAfrica, fl. white.

616. Ozrroe R. (Nymph) diff Ornithog. cor. 6pet. eq. integris, stam. 6 eq. filam. ovato lanc. acum. stigma trifidum. caps. polysp. sem. alatis -Very distinct G. near Lemotrys and Askolame by stigma. Type 
617. Oziroe leuchlore Raf. Ornithogalum chloroleucum Lind. b. reg. 1853. Fol. lanceol. canal. strictis, scapo equante racemoso, pedic. elongatis, bract. brevis lanceol. petalis obl. obtusis-Chili, fl. greenish white.

618. 'Tomoxis Raf. (cut end) diff. Eliokarmos, 58. Pet. 6 eq. stam. ineq. 3 alt. dilatatis apex. emarg. vel. bidentatis, antheris inter dentis, 3 alt. subul. ad pet ext. opposita, ovar. 3 gonum, stylo. trigono, stig. simplex-The stamens are here nearly as in Getuonis 41 , but the habit is like the racenose Loncomelos 57. Three types, for real Eliokarmos see 643.

619. Tomoxis odorata Raf. Ornithogal do. Jaq. ic 432. Andr. t. 260. W. P. Fol. lin. lanceol. brevis prostratis, racemo elongato, bract. ad ped. brevior.-S, Africa, ft. large greenish. fragrant.

620. Tomoxis virens Raf. Ornithog. do Edw. b. reg. 814. Fol. lin. lanc. elongatis apex teres acum. bracteis sphacelatis fl. longior-S. Africa, flowers smaller, hardly odorous virescent.

621. Tomoxis coarctata Raf. Ornith. do Jaq. ic. 436. W. P. \&॰c, Fol. linearib. canalicul. racemo coarctato, stam. alt. emarg.-Wouth Africa.

622. Nicipe R. (Nymph) diff. ad Ornithog. Petalis 6 lanceol. 3 ext. carinatis, stam. ineq. 3 alt. lanceol. major, 3 alt. subul. ovar. 3 gon. stylo 3gono, stig. 3gono, fl. racemosis.

623. Nicipe nivea Raf. Ornithog. niveum. Ait. W. P. Edw. bot. reg. 235. Bulbo globoso, fol. filif. canal. racemo brevi paucifl.-S. Africa, flowers perfectly white. I shall resume the akin Genera at 640.

624. Raxamaris Raf. (berry bitter) cal. 2 
part. petalis $\mathbf{5}$ concavis, stam. $\mathbf{5}$ subulatis, ovar. obov. bifidum, stig. 2 sessilib. Bacca biloc. 2 sperma, obcordata coriacca, sem, obl. planaVery peculiar Gr. of my family Sarcocinis, and also akin to Ścleranthides, blended with Ophioxilon by Sm. not L. Habit of Phytolaca.

625. Raxamaris parviflora Raf. Rex. amaroris Rumph 2. t. 41. Frutic. fol. alt. ellipt. petiol. racemis axillaris-A shrub of the Moluca Ids. intensely bitter, valuable specific for cholera, pleurisy and fevers.

626. SARCOCIDIA. A small family of mine very near to Linidia, differing by berry instead of capsule, and stamens perigyne : the berry is multilocular or polycoca, cells monosperm, leaves alternate, fl. racemose-The types are Phytolaca, Sarcoca, Schefferia, Raxamaris, Ec, see 624. I had reduced them to Empetriclia in 1814, but there the berry is unilocular. polysperm. Very distinct from Rivinidia.

62\%. Pirytoraca L. cal. 5part. stam. isostylis, liberis, 6-10, stylis 6-10, bacca 6-10loc. 6-10 sp-C'Types Ph. decandra, octandra, stricta,

628. Sarcoca Raf. (fleshy cells) diff. 561. stam. diplostylis 10-20, stylis 5-10, bacca 5-10. loc 5-10sp.--'Types Phyt. abyssinica,icosandra dioica, \&c.

629. SCHINIDIA, another small family very near the last, perhaps a subfam. of it, difference, petals present, 1 style several stigmas, leaves compound. 'Types Schinus, Spathelia.

630. RIVINIDIA 1815 Raf. ảiff. from Sarcocides, Empetriclia, Atriplices $\& \cdot c$, by a $m o-$ nosperm berry, although several styles, or stigmas-Types Rivina, Piercea, Mancoa, Salvadora, Rhagodia, Einadia, Bosea, 'Trophis, f.c. 'The G. Dobera is akin but has united 
stamens, R/uus or Sumacus is also very near, but has petals, and rather a drupe than berry. 'The Prperides differ chiefly by calix lepigone, stamens hypogyne.

631. Piercen Miller, ad Rivina diff. stam. 4, all the tetrandrous Rivinas belong here, the type of Rivina is $\boldsymbol{R}$. americana (octandra I. 12andra Jaq. with 8 to 12 stamens.

632. Mancon Raf. diff. Rivina stam. 4, cal. 4 part ineq. bilabiatus.-Type Mr. secunda (Rivina do fl. per. t. 100) sglabra, fol. ovat. acum. subdentic. Peru.

633. EMPETRIDIA 1815 Raf. This family will be reduced to Empetrum, Euleucum, Grubbia, Ceratiola, Batis, Coilosperma, \&c, with berry unilocular polysperm, stamens free, several styles or stigmas. The habit is often heath-like, dicline and no petals. The G. Shimmia, Nandina, Melicytus are akin, but have petals and only one stigma, they probably form another family Nandrnidis.

634. Euleucum Raf. (well white) ad Empetrum diff. Dioicus, stig. 3, bacca 3sp-Type $\boldsymbol{E}$. album. The others have 9 stigmas and seeds. But the whole akin Genera and sp. require revision.

635. Terogia Raf. (ad Ortegia) diff. Ortegia, stigmas 3. type $T$. dichotoma-The true Ortegia has a single capit. stigma, which makes it of a different family! Terogia is a true Alsinidia, and not Dionidia.

636. Endophectris R. (inside spurs) another Epimedium, difiering from my 3 G. 187, 188, 189-cal. duplex, ext. 4sepalis lin. int. sepalis 4 ovatis. petalis 4 calcaratis, stylo, stigma concavo. caps. obl. sessile. Fol. triternatis fl. racem.-Type the following sp. but it is said there 
are $z$ other sp. in Japan, Ep. violaceum and mnschionum. This G. quite peculiar by the long spurs of petals.

637. Eudoplectris tricolor Raf. Epimediun macranthum of Meren, Lind. bot. reg. 1906. Fol. 3tern. foliolis ovatis obliq. serratis, fl. racemosis-Japan, a beautiful sp. with large flowers of $\mathbf{3}$ colors, ext calix red, int. calix lilac, petals white.

638. Vaxessa Raf. (Nymph) cal. 4part. dentes 4 alt. internis, brevis, cor. tubulosa clavata 4dent. stam. 4, stylo filif. longissimo, stig. 2lamel. caps. 2loc. 4valvis Scandens, fl. axillRubiacea near Manettiu, that has cal. 4-5dent. thus differing as Bouvardia from Houstonia.

639. Vanessa cordifolia R. Manetta do Dec. Hook. b. m. 3202, fol. ovatis cordatis acutis petiol. fl. ax. longissime pedunculatis. Vine of Uraguay with scarlet flowers.

640. Ledebourea Roth. cor. 6pet. patens persistens, stam. 6 , erectis ad basis pet. ovar: stipitatum 3part. stylo simplex, stig. acum. utriculis 3 connexis 1 sp-A very distinct $\mathbf{G}$. of Asphodelides, altho' near Veratrides by fruitType L. hyacinthina b. m. 3226. Anthericum do W. Erythronium indicum Rottler, Melanthium do Heyne! fol. lanc. macul. scapo racemoso, fil. cernuis viridis. India.

611. Licrivia Raf. (Nymph) cor. 6part. extus hispidis medio nerrosis, stam. 6 eq. filif. acutis glabriusculis, stylo recto. stigma simplexChiefly different from Anthericum and Endogona by the smooth stamens and having petals. Type L. canaliculata R. Antheric. do Ait. W. (Phalangium do Pers.) bot. reg. 87\%. Var. rufa. Pilosa, fol. ensiformis, canalic. triquetris, scapo tereto raccmoso-S. Africa, several var. 
with whitish, greenish and rufous flowers. Habit of Aloes. 'The G. Trichopetalon of Lindley differs from this by the petals plumose or hairy inside not outside. It has 2 types $\mathbf{T r}$. gracile and stellatum, once Anthericum plumosuin.

642. Loncoxis Raf. (lance sharp) diff, ad Fusifilum 69, filamentis basi dilat. lanceolatis planis acum. caps. trigona oligosperma.-Another $\mathrm{G}$. or subg. distinguished by the flat stamens, very near to Loncomelos $5 \%$ but petals equal. Type $\boldsymbol{L}$. sulfurea Raf. Anthericum sulf. Waldst. W. P. Spr. b. mag. 2623. fol. lin. canal. obtusis, scapo tereto, racemo teres, petalis obl. obtusis pallide sulfureis. In Hungary. All the sp. of Anthericum or Phalangium, my Endogona, must be revised; as stated they would all be Skillas without bulbs! A.greminif. with 3 undulate petals must be a subgenus at least. 643. Eurokarmos Raf. see 58, my characters then were rather those of Tomoxis 618: as I meant Orn. thyrsoides and akin to be the type of this Genus I must rectify it-Petalis 6 patens ovatis subeq. stam. 6 ineq. lanceol. 3 alt. magis dilat. ad basis, apex bifurcatis. Ovar. glob. coloratum, stylo brevissimo crasso tereto, stigma capitato trilobo; fl. racemosis vel thyrsoideis-'Therefore this $\mathrm{G}$. is distinct.very near to Melomphis chiefly distinct by stigma nearly sessile and split unequal stamens. The types are the various sp. blended as Orn. thyrsoides, aureum, of $\mathrm{c}$, which are in utter confusion, and I can only indicate some of them here.

614. Eliokarmos thyrsoides Raf. Ornith. do. Ait. Jaq. W. Curtis 1164, Edw. b. reg. 305. Red. lil. 333-Fol. lanceol. racemo thyrsoides pallide luteis, bract. ovatis acum. petalis obtusis. 
In S. Africa, the ovary and capsule are ash blue, different thus from petals as in Melomphis.

645. Eliokarmos maculatus Raf. Ornithog. arabicum Red. lil. 63 non L. thyrsoides var. alba bot. reg. 316. Jaq. hort. 28. Miller ic. 129 -fol. longis lanceol. planis, racemo pyramidalis, bract. elongatis, petalis ovatis acutis albis basi macula fuscata. S. Africa, certainly a peculiar sp. not variety.

646. Eliokarmos aureus Raf. Ornith. do Wild Pers. fol. lanc. subdenticulatis, racemo coarctato corymboso. South Africa, several varieties of colors, perhaps blended sp. Ornith. miniatum, flavescens and flavissimum of Jaquin.

647. Lomaresis Raf. (edge turned) Petalis 6 ineq. patentissimiss ovatis, margine revolutis, 3 alt. latior, stam. 6 ineq. subulatis, 3 alt. basi dilatatis. ovar. 3sulcat. stylo brevi crasso, stigma capit. globoso vix Blobo-Near the last G. but simple stamens and stigma. Type $\boldsymbol{L}$. albre Raf. Ornithog. revolutum Jacq. sch. t. 89. Curtis b. m. 658. Edw. b. reg. 315 . fol. brevi lanceol. scapo racemoso flexuoso, bract. lanc. acum. petalis obtusis albis. South Africa.

648. Melomphis patens Raf. Ornithog. corymbosum Lind. bot. reg. 906. Fol. lato linearib. canalic, acutis, scapo tereto, corymbo paucifloro, bracteis scariosis albis ovatis amplex. petalis patens, 3 alt. retusis subtridentatis-A fourth sp. of this G. from Chili, wrongly blended with my M. peruviana 51. the syn. of Lindley must be erased, O. Narbonense has also 3 petals tridentate, but the stamens are equal, fl. racemose, is it a subg. of Loncomelos? to be called Tritriela Raf. 
649. Etnesin Raf. (Nymph) dift: ad Loncomelos, filamentis equalis basi ovatis dilatatis, stylo elongato striato, stigma capitat. pubescens. -Type E. prasina Raf. Ornithog. prasinum Edw. b. reg. 158. fol. glaucis canalicul. apice tortilis, scapo racemoso, fl. viridescens. South Africa.

950. Strrepsiphya Raf. (twisted leaf) corolla campan. Gfida, limbo obliquo, lac. ineq. reflexis. stam. connivens in cono, 6 ineq. 3 brevior alt. antheris lanceol. stylo conico, stigma 3gono. caps. Bloc. polysp. duplice series in loculo.- Very distinct G. near Drimia, Lachenalia, Hyacinthus \&c. but separated by the unequal segments. Type

651. Strepsiphyla villosa Raf. Drimia do Lind. b. reg. 1346. fol. lanceol. undul. tortilis acum. villosis erectis, scapo racemoso, bracteis brevis ovatis-South Africa, flowers greenish, but stamens incarnate, the Drimia undulata is probably a second sp. with smooth narrower leaves, Str. undulata Raf.

652. Triallosia Raf. (3 uneq.) cor. tubulosa camp. 6fida ineq. lac. 3 ext, lobis brevis ineq. lac. 3 internis longior ineq. stam. 6 ineq. cetera ut Lachenalia-Near the last G. such Genera with unequal corolla ought to form a family. Type.

653. Triallosia palliala Paf. Lachenalia do Ait. 'Th. W. bot. reg. 1350. fol. lanceol. carinatis acum. fl. racemosis nutans. South Africa, fl. pale blue or incarnate.

654. Cummingra Don. This Gr. differs from Conanthera, as Hyacinthus from Skilla, by cor. campanulate, the 6 stamens are connivent in cone also, and the ovary half inferior : therefore belonging to my order Yunodia probably fam- 
ily of Hypoxides-'Type C. andica Raf. Conanthera bifolia Sims b. m. 2196. C. campanula b. reg. 1193. fol. 3-4 lin. acutis, scapo ramoso, fl. panic. cernuis ceruleis. Andes of Chili!

655. Aurota Raf. (Nymph) cor. supera 6 part. patens, stam. in disco epigyno insertis 6 eq. stigma capit. caps. basi 3locul. apice monoloc. polysp. sem. globosis. Al. radicatis sessilis.-Very diflerent firom Curculigo except by habit. Type A. latifolia Raf. Curculigo do Ait. b. m. 2034, b. reg. 754. fol. petiol. lato lanceol. plicatis. Polynesia, fl. yellow sessile on the thick root, akin to Hypoxis and Hypoxides.

956. Curculugo Roxb. W. P. this G. of which C. orchioides was the type, is now increased to many sp. but all those that have not 3 stigmas and a monoloc. capsule with few seeds like this type, ought to be excluded. Is the Ovary realy free or inferior in all?

657. Leuconyne Lindl. diff. ad Brodiea, cor. hypocraterif. stam. 3 sess. in tubo, 3 infauce sterilis-Plants of Chili, 3 sp. L. odorata, irioides, alliacea. Near family Xuridia.

658. Triteleia Hooker near last, cor, Gfida basi tubulosa, stam. 6 fertilis, ovar. stipit. stigma 3partito-'Two sp. from Chili, T. umiflore, bivalvis, and perhaps a third from California Tr. peduncularis; but two others form my G. Tulophos 700.

659. Skilla fistulosa Raf. precis 1814. Fol. erectis linearib. obtusis glabris canalicul. scapus fistulosus tereto, racemo elongato, bracteis brevis, petalis ovatis obtusis albis-On Mount Etma on rocks, estival. 'This is a real Slitlla with filiform stamens and white flowers, which Lindley has blundered so far as to unite with my Ornithogalum ceruleum 54, with bluc flow- 
ers with flat subulate stamens, and also with the next plant of Gussone, which is not a Skilla neither, and another plant by purple flowers.

660. Loncomelos purpurcus Raf. Skilla cupaniana (Gussone? R. S.) Lind. b. reg. 1878. fol. lanceol. denticul. acutis, fl. racemosis. racemo oblongo inultifl. 20-30fl. bract. linearib. perl, dimidio, petalis ovatobl. obt. purpureisSicily : this description is irom Lindley figure, where the petals are purple, ovary blue, stigma 3lobe, filaments equal lanceolate, seeds central few : the descr. of Gussone appears to recede and apply to my Orn. ceruleum 54, by pauciflore corymb of blue flowers, but differ yet by ciliate leaves and rostrate capsule. There must be several akin sp. in Sicily, of different Genera; meantime I will state the great main distinctions of 3 species of 3 Genera ! Loncomelos purpureus, raceme of purplish fl. stamens lanceolate. Ornithogalum ceruleum, corymb of blue fi. stamens subul. flat. Skilla fistulosa, raceme of white flowers, stamens filiform.

661. Arisarum Tourn, Ad. Arum L. auct. (name included in Asarum, Comarum \&c.) 'This Genus is the type of the Aroides family, a fine singular tribe : in utter confusion as yet, because the Genus was based on the mere spatha, instead of the stamens. I mean to revise it partly, but all the species must be examined again, as well as those of Colocasia and Caladium.-My real Arisarum has, spatha cuculata uniloba, spadix brevior ad basis pistilifero, in medio staminifero, filam, sterilis medialis apice nudo clavato,antheris rhomboideis,sessiles stigma sessile. baccis monolocul. oligospermis. Rad tuberosn, scaposis s. acaules, fol. simpli- 
ces, sepe basi divisis-This will include A maculatum, italicum, pictum, serpenlinum ( $\Lambda$. arisarum L.) ovatum? \&oc with akin species. The 3 Genera of Necker were chiefly distinguished by habit, and thus improper, they were Colocasia caulescent, Alocasia stemless with compound leaves, Arum stemless, simple leares Many other essential distinctions are offered by the spatha, spadix, pistils and anthers. The singular $G$. Kunda 305 deviates greatly, also Seguinum $97 \%$.

662. Homand Ad. diff. 661. spatha angusta, spadix longior apex nudo tereto vermiculato, ad basis squamosus, et antheris mixtis cum squamis, baccis monospermis, Acaules, fol. angustis integris-Types the several sp. blended as A. temifolinm L. and gramineum Lam. The A. gromineum of Russel with lanceolate spathe and clavate spadix is diffent and perhaps a true Arisarum not Homaida; but A. probosciden is a Ilomaida.

663. Desnesia Raf. (separ. middle) diff. 661. spatha basi tubulosa seu globosa integra, pistilis et antheris divisis ab annulo nudo, filam. sterilis superis plumosis. Acaules, fol. divisis. -Several sp. probably, types the two following.

664. Desmesia orixensis Raf. Arum do Andr. rep. 356. Edw. reg. 450. fol. hastato tripartitis, scapo brevissimo basi globosa, labio ovato, spadix clavato-Orixa in India, inside of spatha and club red.

665. Desmesia venosa Raf. Arum venosum Ait. W. P. Edw. reg. 1017. Fol. pedatis, foliolis 5 ovatis acum. basi confluens, scapo brevi, spatha basi tubulosa, apex lanceol. longissima spadix tereto.-Brazil, spatha green, veined and marbled with purple inside, 2 large scales spa- 
thiform at base of spatha, by this character, lack of plumose sterile filaments, terete spadix and tubular base, it may be a snbgenus, or real type of $\mathrm{G}$. while D. orixensis might form subg. Steiroptilas.

666. Megotigea Raf. (big ear of ground) diff. 661. spatha basi tubulosa inflata recurva, apex coarctato, labio amplissimo auriculato cucullato emarg. intus crinito. spadix tuberc. crinito. ad basi squamis involucrato, pistilis cuneatis apex Ggonis, antheris confluens carnosis 2loc. biporosis, baccis 4-6spermis. fl. subraalic. fol. divisis radic-Very peculiar Genus.

667. Megoligencriaita Raf. Arum. do. Ait. W. Edw. reg. 8ã1. A. muscivorum L. fol. pedatis furcatis glaucis, foliolis lanceolatis, scapo brevissimo-Minorca and Spain, spatha green spoted of brown, inside dark red, spadix greenish black, this flower is like a huge ear or a flower of Aristolochia, and is fetid like many others of the tribe.

668. Amocasia Raf. name of Necker but only part of his Genus. Diff. ad 661, spathis triclinis, masc. fem. et herm. basi tubulosa, spadix clavato, basi et apice nudo, stigma sessile punctiforme, antheris subpedicellatis 2-4locul. In fl. herm. confluens, baccis oligospermis. subcaulescens, fol. 3-5natis-Distinct $\mathrm{G}$. by the division of sexes either monoical, dioical or trioical. 'Types the several sp. blended inA. triphyhum, ternatum. vingens, atrorubens and pentaphylum, see my new flora for these species. The 5 leaved sp. most form the subgenus Rhomphalis name of Zanoni.-A. triphyla includes $3 \mathrm{sp}$. A. lobata, pedata, Virginica Raf.

669. Dracunculus Tourn. Ad. diff, 661,spa- 
tha involuta angusta, spadix tenuis non clavato, antheris et pistillis confluens et nonnulis mixtis, Acaules, fol. sepe divisis-Several sp. belong here, 1 Dr. polyphylus (Arum dracunculus L) 2 spadiceus Raf. (A dracontium,) with spadix elongato. 3 divaricatus, 4 trilobatus: 5 cannefolius of c, all Arums of $\mathrm{L}$. with several others probably and N. Sp. Dr. tenellus, and crassicaulis. This G. is very near Homaida, but has no scales and several seeds. 'The sp. with entire leaves form the subgenus Neienshena.

670. Peitandra Raf. 1819. Lecontea Torrey 1824. Renselaria Beck 1833. spatha angusta involuta tubulosa, apice fissa, spadix incluso toto genifero, pistillis inferis, stigma sess. capitato, antheris confluens (ad apice sterilis) peltatis crenatis multilocul. Baccis 1-5spermis ineq. Acaules, fol. simpl. 'The types are the various sp. blended under Arum Virginicum L. descr. in my monograph of new flora $P$. undulata, hastata. Walteri, angustifolia, sagittata, latifolia, heterophyla, these 3 last have only one seed in ripe berries, and form subg. Renselaria. I noticed ${ }^{\bar{t}}$ this G. since 1804, I published it 1819 in my 50 N. G. Journ. phys. yet two other names were given to it since.

671. Colocasia Necker, diff. 661, antheris divisis cirrhis sterilis mixtis, baccis multilocul. polysp. Acaules, fol. sepe peltatis.-Types C. vulgaris (arum colocasia), C. esculenta, $C$. macrorhiza, peregrina, cuculata ofc, and similar useful sp. called Edoes in English.

67\%. Caladium Vent. W. P. diff. 661. spatha cucul. apice dilatata, spadix medio glandu lifero, apice antherifero, antheris peltatis multiloc. stig. umbilic. bacca monol. polysp.-Chiefly different from Peltandra by the glands or 
sterile stamens medial, and the spatha as in Arisarum, Persoon had $\mathbf{1 6}$ sp. some of which frutescent or twining: they must all be examined again, and compared with Pellandra, Arosma, Seguinum \&c. The C. glaucum of Elliot appears of this genus. C. bicolor has tho anthers unequal rhomboidal.

673. Arosma Raf. (Ar. odor) diff. 661, spatha cuculata basi inflata, spadix toto genifero, antheris 6gonis 6loc, planis, inferis sterilis, pistillis 6costatis, stigma 6lobo, baccis 6spermis, Caulescens radicans-Two types from South America, both very fragrant, perhaps the fragrant Arum cordatum belongs here also.

674. Arosma obtusifolia Raf. Caladium fragrans Hooker b. m. 3314. fol. cordatis oblongis obtusis, petiolis semiteretis marginatis, spadix tereto-Guyana, spatha white, base red, suaveolent.

675. Arosma acutifolia Raf. Calad. grandifol. Sims b. m. 26+3 non Jaq. Caladium simsi, Hooker. fol. cordato sagittatis lucidis acutis, petiolis teretib. spadix clavato obtuso-Also from Guyana, less fragrant than the last.

676. Trumpous Raf. (spotted feet) near Caladium 672, diff. spadix ad spatha coalito decurrens, antheris turbinatis angulatis 12locul. supra planis, pistillis lateralis obovatis, stigma plano umbilic, integro, baccis monosp? Caulescens, radicans-Type $T$. grandifolius $\mathbf{R}$. Calad. do Jaq. h. sch. 189. W. P. Hooker b.'m. 3345. fol. cord. sagitt. acutis opacis, petiolis, teretibus maculatis, spadix clavato obtusoGuyana, large leaves two feet long, flower inodore greenish white.

677. Seguinum Raf. spadix cuculato lanceol. brevis, spadix tereto longior unilatere toto gen- 
ifero sed ad medio nudo verrucoso, antheris rhomboidalis umbilic. squamiformis. Pistillis caliculatis! calix 3-4part. clavatis, stigma capitatis 3-4lobis, baccis 3-4spermis. Caulescens, fol. integris-Very peculiar Genus by a calix to fem. fl. thus approximating to the family of Potinines.

678. Seguinum maculatum Raf. Arum seguinum L. Calad. do. W. P. fol. ovatis lanceol. acum. albo maculatis. Jamaica, called Dumb Cane, spatha green, anthers brown.

679. Provenzalia Ad. Calla L. auct. bad name too near Calea, Caladium, Calamus! very near Caladium difference only male and fem. fl. mixt, a style and acute stigma. There are 3sp. of this G. in N. America, see my new flora. One from Oregon P. bispatha Raf. is very peculiar by double spathas and obtuse leaves.

680. Siderasis Raf. (rusty fur). cal. 3part. ineq. ferrug. petalis 8 ineq. basi connatis, stam. 4-6 ineq. antheris oblongis et difformis, stigma simplex, caps. 2-3loc, 2-3valvis, 4-6sperma. Acaulis, fl. radicalis.- I begin to add now same other new genera blended with Tradescantia and Commelina, see my former G. 22 to 32: they all belong to the family of Commelinides. This is akin to Etheosanthes, Callisia $\mathcal{S} \cdot c$, yet was united to Tradescantia. Type S. acaules Raf. Tradesc. fuscata Lod. Edw. b. m. 582. acaulis ferrug. hirsuta, fol. ellipt. acutis, pedunc. radic. 1-3fl.-Brazil, flowers pale blue.

681. Pogonesin Raf. (beard middle) diff. ad Tradesc. Petalis basi barbatis, stam. 3 superis brevior, 3 inferis longior. declinatis, stylo declinato incurvo-Type P. undata Raf. Tra- 
desc. do W. Kunth, Lind. b. reg. 1403. fol. amplex. ovatis undul. umbella term. 5fida ramosa corymb. bracteis ovatis, cal. pilosis. Cumana and Mexico, petals purple, bracts ivolucrating and on peduncles.

682. Tropitria Raf. (3 keels) a subgenus only of Tradescantia? difference, cal. carinatis, filam. basi villosis, stylo glabro, seminis biserialis in loculis-Type Tr. crassula Link. Grah. Hook. b. m. 2935. glabra ramosa, fol. obl. lanceol. ciliatis nervosis, umbella termin. petalis cal. brevior albis. South America.

683. Ovides Raf. Spatha ut Commelina, cal. 2phylo ineq. infero major bilobo. cor. petalis 3 ineq. 2 subunguic. rotundatis. stam. 5 glabris 2 sterilis, stylo declinato, stig. 3lobo, caps. 3locul. 3gona-Very distinct from Commelina by $\mathbf{5}$ stamens \&c. perhaps several sp. but the type is.

684. Ovidia gracilis Raf. Commelina do fl. per. t. 72, Hooker b. m. 3047. C. formosa Grah. caule assurgens articulato retropiloso, fol. lanceol. glabris acutis 7nervis, spatha plicata compr. cordata multiflora. Peru.

685. Eudipetala Raf. diff. Commelina, polygama, cal. 3fol. eq. pellucidis membr. petalis 2 superis eq. infero minimo seu nullo, stam. 3 fertilis, antheris ineq. 2ovatis, 1 lobata major, stam 3 sterilis lobatis-Type Eud. deficiens Raf. Commelina do Hook. b. m. 2644. erecta, ramosa, fol. ovatis lanceol. undulatis basi ciliatis. spatha cordata acum. nonnulis $\mathrm{ft}$. femineis -Brazil, perennial, petals blue, the third when present is white.

686. Connelina L. auct. a crowd of heterogenous sp. was blended here; it is difficult to say which ought to remain the real type. I 
venture the following characters-Cal. 3part. equalis coloratis, cor. petalis 3 eq. stam. 3 fertilia, 3 sterilia. nectariforme, stylo 4 , stig. simplex, caps. 3loc. 3valvis 3sperma, valvis oppositis. Spatha plicata multiflora. 'Types the tropical species C. cuculuta, fasciculata, nervosa, bracteolata, spirata \&c, but all must be examined again.

687. Stickmannia Necker. diff. 686. cal. 3 part. eq. cor. petalis 3 ineq. uno minor, stam. 6ineq. omnis fertilia, caps. 3loc. oligospermaTypes C. mollis, longicollis, africana? guyanensis or $\mathrm{C}$, communis of guyana, $\mathcal{d} \cdot \mathrm{c}$.

688. Ditelesia Raf. (2 perfect) diff. 686, stam. 2 fertilia, 4 sterilia nectarif. spatha mulla-Type D. mudiflora Raf.

689. Dirtea Raf. (Nymph) diff. 686. Polygama, cal. et petalis ineq. stam. 2 fertilia, 4 sterilia, stig. capitato, capsula loculis ineq. polyspermis-Types $\boldsymbol{D}$. japonica $\mathbf{R}$. and several sp. of $\mathbf{N}$. America blended as Com. erecta, communis, hirtella \&c, see my new flora.

690. Ananthopus Raf. fl. lud. 1817 diff. 686. cal. 3sepalis scariosis, petalis ineq. 2 major. stam 6 ineq. difformis, una lanceol. stylo coalita, stylo reflexo declinato. Spatha gelatinosa, multifl. pedunc. uno obortivo-For an ample account see my fl. ludov. page 21, 22. Several types, but chiefly A. clandestinus.

691. Ananthopus clandestinus R. fl. lud. 58. fol. lanceol, retrorsum scabris subtus glaucis, spatha saccata mucron. demum plana reniforme-Louisiana, petals blue, the spatha changes in form.

692. Ananthopus cordatus R. fl. lud. 59. caulib. virgatis, fol. lanceol. supra basi scabris, spatha cordata-Louisiana, an Dirtea? 
693. Ananthopus undulatus Raf. caule ramoso, fol. amplis ovatis acum. glaucis scabriusculis, margine aspero undulato, vaginis amplis tubuloso campanulatis, spatha cordata plicata acuminata-Alabama, in my herbal, stem 1 or 2 feet, leaves 5 to 8 inches long.

694. Allotria Raf. diff. 686, cal. 3sepalis ineq. Petalis 3 subeq. unguic. stam 3 fertilia antheris sagittatis, 3 sterilia amorphis lobatis cum glandulis pedunc,-Type A. scabra Raf. Commel. virginica L. Elliot $\mathcal{S} \cdot c$. caule erecto, fol. lanc. subpetiol. serrulatis supra cabris, spatho renif. scabra uniflora cum ped. ananthoVirginia and Carolina, 3 feet high, not C. virg. of all botanists.

695. Nephraldes Raf. (kidney diff.) cal. 3 part. ineq, concavis, supero minor. Petalis 3 ineq. infero minor sessile reniforme, 2 major unguiculis canalicul. lamina renif. obliqua. stam. 6 ineq. omnis fertilis glabris, filam. filif. erectis, antheris ovatis. Ovar. obl. stylo filif. erecto, stig. obtuso. caps. membranacea 3loc. 3sp. sem. obl. spatha plicata multifl.-A very distinct Genus akin to Stickmania, which I have described at length on the living plant in full bloom ; the flowers of all this tribe are so evanescent that unless they are caught at their blooming hour, no correct idea can be had of them.

696. Nephralles parviflora Raf. glabra, decumbens ramosa, ramis geniculatis divaricatis, vaginis tubulosis membran. fol. ovatis acutis spatha cordata pauciflora.-Kentucky and Tennessee, fl. small pale blue. estival, in bloom at noon.

697. Larnalles Raf. (cells uneq.) cal 3 sepalis ineq. color. supero minor, petalis 2 subrot. 
emarg. unguic. uno infero sessile vel carens ut in Eudipetala. Stam. 6 ineq. fertilis, 3 superis. brevior antheris cruciatis. 3 inf. longior, antheris ovatis, stylus filif. stig. simplex caps. 3loc. loculis ineq. polyspermis ut Dirtea.-Several North American species seen alive.

698. Larnalles dichotoma Raf. caule dichot. geniculato, fol. lanceol glabris, raginis fissis, fl. panicul. spathis cordatis brevis plicatis pedunculatis-Apalachian mts. stem slender 1 or 2 feet high, petals blue.

699. Lamalles glauca Raf. caule simplex, fol. glaucis lanceol. basi vaginatis, spatha term. subsess. corlata plicata-Kentucky, Illinois, pedal, flowers large pale blue.

700. Tulopuos Raf. (wart crest) near to Triteleia 658, diff. cor. tubulosa eq. 6fida, stam. 6induplice series ad tubo inserta, filam. 3 decurrens ad basis callosis vel. cristatis, Ovar. brevi stipit, stigma trilobo. Scaposa, fl. umbellatis ceruleis-Habit of the Hyacinth and mistaken for such by some or united to Triteleia. Two types 1. T. grandiflora Raf. Triteleia do Lindl. b. reg. 1293. fol. lin. glaucis, scapo brevior, spatha triphyla, umbella paucifl. bract, ped. eq. filam. callosis. Oregon. $-2 T$. laxa Raf. 'I'rit. do. b. reg. 1685. fol. lin. glaucis scapo longior, umbella 6flora, ped. laxis bract. longior, filamentis cristatis. California. 


\section{CENTURIA VIII.}

\%01. RESEDINIA, this little family of mine belongs to the Order Polymesia and is very near the Grininia see 351, differing by unequal calix, petals and stamens; the capsule is unilocular end polysperm, seeds parietal, as in some Hypericines, but the habit is very different. and also from Droseridia ; altho' the flowers are akin, but stamens not isarine. It is also akin the Euphorbiües; how Jussieu and others put them near the Capparides with a single style, is rather strange. The G. Reseda of L. is the type of it, and as it had a crowd of anomalies may be easily divided into several good genera, only united by the fiuit, which even differs in Astrocarpos.

702. REsEda Raf. cal. 6part. ineq. petalis 6-8ineq. nonnulis integ. et nonnulis divisis, stam. 12-14 hypog. ineq. disco glanduloso, stylis 3-4 caps. angul. verrucosa, apice $3-4$ dent, dehiscens, uniloc. polysp. placentis 3-4parietalis. fol. alt. sepe dissectis, fl. racemosis-Reseda odorata must be the type of this old Genus, with $\boldsymbol{R}$. suffinticosa, luter, gallica, mediterranea, tetragyna? \&.c.

703. 'Tereinanies Raf. (cut fl.) difl. 702, cal. 5 part. petalis 5 subeq. omnis 3 seu 5 fidis, stam. 11-15 ineq. stylis 3-5.-'Types $T$. undata Raf. Reseda do L. $2 T$. fruticosa. $3 T$. glauca, 4 T. alba: all Resedas.

704. Pectanisia Raf. (comb uneq) diff. 702. cal. 6part. reflexo magno, petalis 4 ineq. pecti- 
natis, stylis 3, caps. angulata 3dentata-Typc. P. phyteuma Raf.

705. Arkopoda Raf. (nom. gr.) diff. 702, cal. 4 fido, petalis 3,2 integris vel trifidis, uno major supero multifido, stam. 12-15, stylis 3, fol. integris-Type A, luteola Raf. Reseda do. L.

706. Hexastylis Raf. diff. 703. Ovario stipitato, stylis 6 , caps. 6dentata, fol. integrisIn this $\mathbf{G}$. the number of petals is anisostyle. Types H. canescens, 2 H. arabica Raf. Reseda hexastylis Forsk.

707. Dipetalia Raf. diff. 702, cal. parvo marg. membr. petalis 2 integris cuneatis, stylis 4. fol. integris-Type D.crpensis Raf. Reseda do Burm. R. dipetala Ait. W. P. fol. linearib. integris.

708. Astrocarius Necker, Sesamoides T. diff. Tereianthus 703, petalis 5 multifidis. stylis 4-5, capsula 4-5loba, demum 4-5valvis stellata. fol. integris---Type A. purpurascens, and Sesamoides, Resedas of Authors.

709. Nicotiana L. the plants akin to the real Tobacco form several Genera, and more must be added. 'They all properly belong to the family of Verbascides by the unequal stamens, the capsule bilocular with many central seeds, separates them chiefly from Solanides and Convolvulides. I will give the main distinctions of the old Gencra, so as to contrast them with my new Genera.

Nicotiana cal. tubulosus tereto 5dent. eq. cor. infund. plicata, 5 dent. stam. 5 ineq. filif. stigma capit. bilobo \&c.

Petunia Jus. diff. cal. profunde 5fidis, cor. hypocr. 5loba.

Lamarkea Rich. diff, cal. tub. 5gonus 5fidus, cor. hypocr. 5loba. 
Ninzembergia R. P. diff: cal. tubul. 5fido, cor. hypocr. tubo longissino, limbo subineq. stam. 5े exertis, filam basi connatis. Many sp. now known ; but some are anomalous and must form New Genera.

710. Sipinumax Raf. cal. tubul. 5dent. cor. tubo clavato curvo basi 5 gonus, apex 5sulcato, limbo parro cupularis 5́dent. stam. 5ineq. Ovario immerso in disco carnoso. stylo filif. stig. bifido. caps. 2loc. recept. centrale magno carnoso. Frut. fl. panic-This ample diagnosis evince its distinctions from Nicotiana, the type is S. glabra Raf. Nicot. do Gr. b. mag. 2837. Suffrutic. glabra, fol. ineq. oratis repandis acutis longe petiolatis, panic term. Buenos Ayres.

711. Langsoorfia Raf. vel Perieteris Raf. cal. camp. ineq. 5fidus cor. hypocr. tubo clavato, limbo eq. sepe integro rotato, stam. ineq. 1 minor-Thus nearer Petunia. main diff. the unequal calix. Types $L$. P. viridiflora Raf. Nicot. langsdorfi R. S. b. mag 2555. fol. sess. obl. acutis villosis, superis decurrens, fl. panic. parvis viridis. Brazil-2 P. tristis $\mathbf{R}$. Nicot. do Com. Sm. fol. undul. rad. obov. caulinis lanceol. fl. racemosis. B. Ayres, fl. purple.

712. Eucapnia Raf. (good smoke) cal. obl. 5 gonus 5 dentis ineq. cor. hypocr. tubo gracilis limbo stellato 5partito-Thus between Lamarkia and Nicotiana, diff. unequal teeth of calix. Type E. repranda Raf. Nicot. do Lehm. Hook. b. m. 2484. fol. amplis cordatis repandis undul. fl. axill. et subspic. sessilis, lac. limbo ovatis acutis uninervis.- This is the mild tobacco of $\mathbf{C u}$ ba, flowers small incarnate.

713. Caprorea Raf. (smoke of mts.) cal. camp. 5 fidus ineq. cor. hypocr. tubo clavato, limbo plano 5 fido, stam. 5 subeq. pilosis, disco 
annularis, Acanles, fol. et fl. radic-Very near 711, but different habit, limb divided and pilose stamens. Type $C$. nana Raf. Nicot. do Edw. b. reg. \$33. fol. pilosis lanceol. et cuneatis, fl. subsess. brevis. lobis limbo obtusis. Oregon mts. cult. by Indian tribes as a mild tobacco, leaves 2 or 3 inches only, flowers small white.

714. Ampinpleis Raf. (all more) cal. ovatus costatus 6-8fidus, cor. hypocr. tubo basi infl. limbo plano 6-8fido, stam. (6-8ineq. caps $4-81$ loc. 4-8valvis. stigm. capit. integrum. $f$. rxil.This G. deviates widely from the tobacco tribe, and is still nearer the Convolvulides. Two types, A. 4valvis R. Nicot. do. 2. A. fetida R. Nicot. multivalvis Lindl. b. reg. 105\%. Viscido pilosa, fol. lanceol. inf. petiolatis, fl. axill. subsess. cal. acuto, cor. obt. cult. on the Columbia R. hircose smell.

715. Conuba Raf. (Haytian) diff. Nicotiana cal. hirtus camp. cor. campanulata 5 dent. capsulis bicornis. Frutic. fl. racemosis-Type C. urens Raf. Nicot. do L. auct. Arboreus hispidus, fol. cord. crenatis, racemis compositis secundis. South Amcr. fl. white.

716. Buenocoes Raf. (Renealm) diff. Nicotiana et Petunia cal. magis ineq. lacinia una foliosa duplo major, cor. obliqua subringens, fl. racem.-Type Bl. glutinosa R. Nicot. do L. auct. fol. petiol. cordatis, glutinosis, racemis secundis-Peru, fl. rosate.

717. Siphonema Raf. (tube fil) cal. camp. limbo amplo 5part. ineq. foliaceis. cor. hypocr. tubo filiformis, limbo 5 lobo subeq. stam. 5 ineq. inclusis, filam. et antheris connivens vel coalitis. stig. oblongo transverso integro-Very pcculiar G. near Cohiba and Nierembergia, two types. 
718. Siphonema longiflora Raf. Nierembergia calicina Hooker b. mag. 3371. glandulosa, fol. opp. et alt. petiol. obovatis, pedic. extraxill. cal. obovatis-Paraguay.

719. Siphonema filicanlis R. Nieremb. do Lind. b. reg. 1649, Hook. b. m. 3370. Glabra erecta, ramis filif. fol. alt. linear. cal. linearibBuenos Ayres.

720. Struomphis Raf. (stig. umbil) cal. campan. 5fidus ineq. foliosus, cor. campanulata 5loba subequalis, stam. 5 ineq. 2 major, stylo filif. stig. turbinato umbilicato, $\boldsymbol{F l}$. axillaris-Another striking G. blended with Nierembergia and Salpiglossis; this last is very distinct by only 4 fertile didyn. stamens, and is of therefore another family. Type St, linearis Raf. Salpiglossis do Hook. b. m. 3256. Nieremb. intermedia Grah. fol. sess. lin. obl. caule ramoso, fl. ax. pedic. Buenos Ayres, fine purple and yellow flowers.

721. Stimoryne Raf. (stig. club) cal. 5partito ineq. cor. campan. 5loba subeq. stam. 5 fertilis ineq. stigma clavato. fl. axill.-Very near the last, chief diff. calix and stigma. Type. St. purpurea Raf. Nieremb. phenicea Gr. Salpigl. integrif. Hook. b. m. 3113. fol. spatulatis obl. integris, fl. solit. pedic. Uraguay, fl. purple.

722. Strimenes Raf. (stig. lunul) cal. camp. 5 fidus equalis, cor. hypocr. limbo plano ineq. 5 lobo, stam. liberis subeq. 2 longior, antheris renif. stigma lunulato magno transverso, $f$. terminalis-Near to $\mathbf{7 2 0}$ diff. equal cal. and stigma. Type. St. gracilis Raf. Nierembergia do Hook. b. m. 3108 caule erecto gracile, fol. sparsis linearib. fl. term. Uraguay, flowers white streaked of blue.

723. Ninanga Raf. (nom ind) cal. duplex 
utrinque 3part. persist. erectis coloratis, internis bifidis, tubo corolliformis mecolatus 5dent. antheris 5 intus ferens. stylis 2. utriculus 1spermus. Jol. oppos. Al. verticil-'This G. must be added to the Gomphrenides, but is very distinct from Gomphrena, the type of which must be the G. globosa and akin sp. that have cal. ext. 3p. int. 5part. one style, 2 stigmas, as I have verified: it is Oplothece that has only one capitate stigma. 'The types of Ninanga are $N$. bicolor Raf. Gomphrena perennis L. auct. b. m. 2614. and perhaps $2 N$. intermpta Raf. Gomphr. do L. Celosia procumbens Jaq. -Gomphr. arborescens is probably type of another G. Wadspes Raf. but I lack the distinction.

724. Megasea Haworth. aff, 'Telesonix 252. Petalis unguicul stam. 10 ineq. 5 alt. brevior. scaposa fl. cymosis-Another $G$. to be added to the Saxifragides, Ovary free and capsule as in them, but unequal stamens. 'Type M. ciliata Haw. Saxifraga ligulata Wallich. Hook. ex. fl. 49, b. mag. 3406. fol. radic. petiol. obov. erosis retusis ciliatis, scapo panic. cymoso-Nepal.

725. Evaltie Raf. (Nymph) cal. tubul. 5 gonus 5fidus eq. cor. hypocr. limbo 5fido rotato, stam. 5equalis, antheris linearib. 4 gonis 4 dentatis, stylo sulcato erecto, stig. capit. obtuso -Another fine G. of Gentianides neal Chironia 491. It shows well the family character of opposited stamens. Type E. jasminoides Raf. Chironia do L. auct. b. reg. 197. Caule 4gono, fol. cuneatis lanc. acutis, ramis unifloris. South Africa, fl. rosate.

726. Exacum L. auct. cal. 4 fido eq. cor. 4 fida basi globosa, stam. 4, antheris poro dehis- 
cens, stylo declinato, stig. unicum, caps. 2loc. 2 valvis-This is a corrected account of this $\mathbf{G}$. and includes chiefly E. sessile, peduncul, punctutum of $\mathrm{c}$, many $G$. have or must be taken from the old Linnean genus, like the next.

727. Seraen Solander, Br. Sm. cal. 5part. carinatis, cor. infund. Afida, tubo inflato, stam. 4, antheris elongatis stylo erecto, stig. 2, caps. compr. 2loc. 2valvis-This includes the $\boldsymbol{E} \boldsymbol{x}$. albens, aurea. ovata, \& $\mathrm{c}$, but not the cordata see next. The G. Cutubea Aublet chiefly differs by the 4 scales, caliq 4 fid and capsule nearly unilocular.

728. Parrasia Raf. (Nymph) diff. 727, corolla 5fida, stam. 5 calix dilatatus 5 part. membr.-_'Type $\boldsymbol{P}$. cordata Raf. Exacum do L. and Gentiana exacoides L. thus put into double Genera by him, caule dichot. angul. fol. cord. amplex. fl. luteis. S. Africa.

729. Episiphis Raf. (upon tube) cal. 5 part. eq. extus bracteis 2 magnis involvens, cor. campanul. petalis 5 connivens in tubo, basi intus squama lanceol. posita ut in Ranunculus, stam. indef. pauca in 3 phalangis basi tubulosis, glandulis 3 hypogynis ad basi ovar. stigma 3 subsessilis. caps. 3loba, 3loc. polysp. Frutex fol. opp. parvis, ramis 1 fl. $-\mathrm{A}$ very distinct $\mathrm{G}$. blended with Hypericum and Elodea by all Authors. 'Type E. parvifolia Raf. Hypericum egyptium L. auct. bot. reg. 196. caule suffrut. compresso, fol. ovatis acutis glaucis squamiformis, ramulis unifl. petalis spatulatis. Egypt, small yellow flowers.

730. 'Triadenum Raf. 1808 (3 glands) cal. 5part. equalis, Petalis 5 eq. stam. 9 in 3 phalanges planis trifidis triantheris, glandulis $\mathbf{3}$ magnis carnosis, alt. cum phalanges. Ovario 
3gono. stylis 3, caps 3ralvis subuniloc. axil. et term-I established this fine $\mathbf{G}$. long ago for the Hyper. Virginicum, to which must be added several blended or new species of North America, improperly united to Elodea of $\mathbf{A}$ danson. See in my new flora Tr.virginicum.

731. Elones Ad. Raf. diff. 730 Petalis 5 basi squamulis ut 729, stam. 9-12-15 basi coalitis, apice in 3-5phalanges divisis, glandulis interpositis nulli - $\mathbf{C}$ 'his $\mathbf{G}$. is thus between the two last, the types are $\boldsymbol{E}$. lubulosa, petiolata, \&c ; the Elodea of the actual botanists, includes all these 3 genera.

732. Sarotira Raf. 1808 non L. ad Hypericum vel Tridesma diff. stam. paucis 5-12 liberis rel vix coalitis, stylis 3, capsula 3valvis unilocularis, valvis seminiferis-The Linnean Sarothra was united to Hypericum ; but many sp. having few stamens, uniloc. capsules, and commonly small flowers and leaves, required to form a G. including many sp. S. fastiginta, pancifiora, trinervia, canadensis, parviflora, \& c, Choisy in his Monograph of this family in 1821, as well as Decandole since, have yet united this to Hypericum.

733. Hypericun Raf. I confine this G. to the first section of $\mathbf{L}$. et Dec. with $\mathbf{5}$ styles, $\mathbf{5}$ phalanges, caps. 5locular. many sp. I shall return on the fine family of Hypericines, but will now give the main distinctions of my Genera of 1815 before Choisy, to which I shall add 4 others licreafter.

734. Konana $\Lambda$ d. diff. stylis 5 coalitis seu stylo unico, stig. 5. caps. lloc. 5valvis. Centera subgenus with 1 style 5 fid.

735. Tridesua Dec. subg. cal. ineq. pet. 
eq. phalanges 3 , stylis 3, caps. 3loc. 3ralvis.Most of the species.

736. Streptaron Raf. cal. 5p. ineq. petalis 5 obliquis contortis, stan. pluris liberis, stylis $\mathbf{3}$, capsula uniloc. 3valvis- Type Str. dolabriforme, efoc.

737. Petalunisia Raf. cal. ineq. pet. ineq. stylis 3 , caps. 3locularis.

738. Pleurenonon Raf. cal. campanul. 5fidus, pet. 5eq. latere unidentatis, stylis 3 , caps. 3loc?

739. KnifFA Ad. cal. eq. 5fido camp. pet. eq. stylis 2, caps. 2loc. $\boldsymbol{H}$. brevistylis Choisy tab. 7 appears to belong here.

These $G$. are all distinct from the others of same family, such as Ascyrum, Androsemum, Arungana Jus. Palava R. P. Vismia W. or Caspia Necker, Eucryphia Cav. Carpodontes, Brathys Mutis \&c, but Triplaris with one seed is of Amaranthidia.

740. Anisn n'mera Raf. diff.ad Borrago,cor. hypocraterif. non rotata, tubo cal. longior, antheris magnis longis inequalibus-Type A. ciliata Raf. Borrago macranthera Russel fl. alep. fol. lanceolatis ciliatis, calicib. ciliatis. Syria.

741. Eunesurs Raf. (well fasc) diff. ad Hypoxis, cor. tubo longo filif. limbo 6 part eq. stam. 6ineq. 3 brevior alt. stylo filif. stig.obtuso, Spatha rad. fol. et fl. involvens latere fissa-Very distinct $G$. by habit, tube and stamens. Type, E. fascicularis Raf. Hypoxis do. L. Russel, fl. alep. fig. 9. Allium Rus. 1. ed. fig. 2. Bulbo subrot. fol. linearib. canalic. cum florib. fasciculatis. Syria, fl. white.

742. Cuetropis IRaf. (bristle keel) diff. ad Alsine, cal. carinatis aristatis, petalis 5 integris brevissimis---Type Ch. setrcea Raf. Alsine 
mucronata Gouan ill. 22. A. aristata Rus. alep. p 249 fol. setaceis, cal. glabris mucronatis aristatis, petalis linearibus. Syria and Italy. Alsine differs from Stellaria by $\check{5}$ stamens, but has bifid petals like'it. This is perhaps a subg. of the next.

743. Melargyra Raf. (membr. silvery) cal. 5part ineq. margine membr. Petalis 5 integris cal. eq. (rubris) stam. 5-8, stylis 3 , caps uniloc. 3valvis, fol. fascic. cum. stipulis membr. argenteis-Very near to the last $\mathbf{G}$. chief diff. calix and stipules. This $G$, includes all the pentandrous Arenarias like $A$, rubra, media, purpurea, canadensis ofc with the next $\mathbf{N}$. Sp.

744. Melargyra rosea Raf. Caulib. prostratis, apice pubescens, fol. subul. internodis eq. fl. axill. racemosis, cal. obt. pubesc. petalis ovatis roseis cal. vix brevior, caps. ovatis cal. eq. -dry hills of Pennsylvania, pretty flowers autumnal.

745. Disynoma Raf. (2 un. law) Crucifera, sepalis et petalis subineq. obov. obl. obt. stam. 4 minora 2 libera, 2 major binis coalitis, filam. $2 \mathrm{obl}$. planis bifurcatis 2antheriferis. Ovar. orbic. compr. stylo filif. stig. acuto. siliculis orbic. obcord-Singular G. near Thlaspi, but belonging to the tribe of Anomandria or cruciferous anomalous in the stamens. Type D. carnea Raf. 'Thlaspi carneum Russ. fl. alep. tab. 11. f. 2. caule superne ramoso, fol. cord. amplex. glabris integris, petalis cal. eq. carneis. Syria and Natolia, found by Tournefort and Russel. Not in Wildenow nor Persoon, who both appear to have overlooked all the $7 \gamma$ new plants of Russel in his floras of Aleppo and Libanus, although published in 1794 ! 
746. Andaca Raf. (nom. gr.) Legum. cal. camp. bilab. lab. 2-3fidis, vexil. unguic. subrot. emarg. carina brevis. stam. 9 monadelphis, 4 alt. brevis, antheris subrot. sterilis, 5alt. longior fertilis, antheris oblongis, apice poro dehiscens, stam. decima libera sterilis, anth. subrot. Ovar. genicul. lineare. stylo brevissimo, stig. capitato, Leg. tereto polysp. fol. ternatis, pedunc. axil. 1-3fl.-A fine G. that has escaped all the botanists altho well described by Russel, and quite distinct from Lotus by the singular stamens. Type

747. Andaca arabica Raf. Lotus arabicus L. et omnis auct. Russel fl. alep. tab. 14. Jaq. h. t. 155. Prostrata, fol. pet. foliolis 3 petiol obov. obt. subincanis glabriusc. stipulis fol. similis, pedunc. erectis 1-3fl. unibract. fl. nutansArabia and Syria, flowers fulvous with red veins.

748. Loreia Raf. Campanulacea, cal. 5part. ineq. serratis, cor. rotata 5part. ovar. villoso sulcato, stam. et stylis ut Camp. caps. 3loc. 3 poris dehiscens.-A pretty $\mathbf{G}$. to add to this family, it is near the true G. Campanula, but differs by the calix, rotate corolla and ovary. Type

749. Loreia baldensis Raf. Camp. do. Balbi, C, lorei R. S. b. mag. 2581. caule diffuso 5 gono, fol. pet. ellipt. obl. lanceol. serratis, pedunc. elongatis unifl, cal. ad cor. eq.-Mt Baldo and Alps, flowers dark blue.

750. Rafinesquia vel Dioneilis R. Labiata. cal. tubulatus rectus striatus subbilab. lab. sup. bident. inf. 3dent. faux intus villosa clausa cor. recta bilab. lab. sup. concavo emarg. lab. inf. subeq. 3lobo, stam 4 remotis, antheris cordatis bilobis sem. levis, Frutic. $f$. axill-Here 
is another Rafinesquia for a fine shrub mistaken by all the Authors, and forced into $\mathbf{3}$ Genera! Cunila, Melissa, Gardoquia! if not approved, I add a 5th name! abridged from Diodontocheilis! I add next the Gardoquia to compare them. The type of this is in my herbarium with several other shrubby Labiate of Florida.

751. Rafinesquia (vel. Diodeilis) coccinea Raf. Cunila! do Hooker, Melissa! do Spr. Gardoquia! hookeri; Benth, Don. Lind. bot. reg. 1747 ... Fruticosa, glaberrima, fol. obov. sess. acutis integr. pedunc. axil. 1-3fl. pretty Shrub from Florida, flowers scarlet or miniate, seen dry. Bentham doubted the $\mathbf{G}$. of this plant, he says it has stamens nearly like Origanum. I have another N. G. from Florida different by campan. calix \&c. see Diseldia.

752. Gardoquia R. P. fl. per. cal. tubul. incurvus 5dentib. subeq. faux nuda s. barbata, cor. tubulosa incurva. lab. sup. plano einarg. lab. inf. 3lobo, lobo medio latior. Antheris renif. sem. levis. Frut. $f$. axil-Thus every thing is in contrast with the last except the habit. Type G. chilensis bot. reg. 1812 fol. linearib. cuneatis obtusis, Chili---I shall begin to add some good new Genera to those of Bentham on the Labiate: I have already stated that his valuable labors are incomplete in many genera, and that his tribes of Labiate are artificial. 'The old division of Unilabiate, Diandres, and Didynames by Jussieu was more natural, the stigma lateral or terminal, the stamens bilocular or unilocular, with or without appendages, would form much better natural tribes. Therefore I offer the following tribes or families to be hereafter rectified and enlarged. 
753. Order LOBOGYNIA, suborder ARCYT'HIA Family LABIATA - -1 subfamily or tribe DIANDRIDIA, the Diandrides with two fertile stamens, filaments simple. Types Monarda, Lycopus, Cunila, \&c.

754. SALVIDIA, the Salvides or Sages, stam. 2 fert. filam. append. antheris uniloc. types Salvia, Sclarea, and Hemistegia, Calosphace \&c.

755. UNILABIATA, the Unilabiates, stam 4,corollis unilab---Teucrium,Ajuga, Scorodonia, Monipsis, Chamedrys, Melosmon. \&c.

756. BILABIATA, the true Bilabiate and Galeate, stam. 4. cor. bilab. divided into several smaller tribes.

1. Prasides, with baccate seeds. Prasium L. or Levina Ad.

2. Heterostrues, with terminal or unusual stigma, Sideritis, Lavandula, Cleonia, Perilla.

3. Pleiodontes, calix with more than 5 teeth Leonotis. Hemisodon, Marrubium, \&c.

4. Synandrines, stamens united or connivent Synandra, Coleus $\mathcal{S} \cdot c$.

5. Thymides, stamens divergent far apart. Types 'Thymus, Satureja, Hyssopus, Origanum, \&.c.

6. Ocrnides, stamens declinate, 7 th order of Bentham, very unnatural.

7. Brunellides with furcate stamens, Brunella, \&.c.

8. Nepetines. All the Genera excluded by the aboue characters ; and they are yet very numerous. I shall increase the 108 Genera of Bentham to about 160 ; but most of the N. Gr. beong to Unilabiates or Salvides or Phlomis \& $c$.

75\%. Teucrium Raf. non $\mathbf{L}$. et auct. I confine this $\mathbf{G}$. to the Species with cal. urceolatus, 
5 dent. eq. cor. lab. sup. vix nullo, infero lobato concavo, fl. racemosis. Types T. flavum, fruticosum, canadense, virginicum, and several sp. blended with these, see my new flora.

758. Scorodonia Ad. diff. 757. cal. tubul. incurvus ineq. sub labiato, dentis 5ineq.-Types Sc. trivialis, massiliense, sicula, arduini \&.c, and other blended sp.

759. Chamedrys Tourn. diff. 757, cal. tubul. lanato subintegro, cor. lab. supero brevis sed evidens dilatato, fl. axill.et glomeratis. Types Ch. marum, polium, capitatum, creticum, quadrulum, betonicif. nissolianum, latif: montanum, pumilum \&c. 3. subg. 1. Polium fl. cap. 3 Marum fl. racem. 3 Chamedrys fl. vertic axill.

760. Trixago Raf. (n. antic.) Iva Dillen non L. diff. 75\%. cal. gibboso, inflato tubul. 5 dent. connivens, $\boldsymbol{f}$. axill. Types $\boldsymbol{T}$. botrys scordium, \&c.

761. Melosmon Raf. (n. diosk) diff. 75\%. cal. amplo campanulato 5 fido eq. 10striato, dentis carinatis, fl. axill. Types $M$. bicolor, rotundifol. campanul. orientale, \&.c.

762. Scorbion Raf. (n. diosk) diff. 757. cal. curvus striatus, apex camp. 5 dent. ineq. 1 sup. major, 4 inf. spiuosis, cor. basi globosa. $f l$. axill - Type Scr. spinosum; but several sp. are blended under 'T'eucrium Spinosum.

763. Monipsis Raf. (single form) diff. $75 \%$ cal. camp. 5 fido subeq. ut in $\mathbf{7 6 1}$, cor. tota unilabiata, lab. sup. nullo, lab. inf. labelliforme 5lobo, lob. ultimo major, stam. et stylis superis adscendens. $f$. axill--.'Type $\boldsymbol{M}$. orchidea Raf. Teucr. do Lind. b, reg. 1255. frutex, fol, obl. obtus. sessil. glaucis subint, fl, axill. solit, sess. carneis. Chili. 
Thus I have tried to revise Teucrium into 7 Genera; but all the sp. must be examined again. Limneus had $35 \mathrm{sp}$. Schreber in monogr. unilab. and Vitman 26 more, Persoon 69 in all : we have nearly 100, of which but few are completely described. According to Smith several belong to Ajuga, such as T. iva, laxmani, chamepytis, and salicifolium, but this last has 4 fid calix ! if so it is a Subgenus. Vimenerba Raf.

764. Origanum Raf. non $L$. one of the absurd linnean Genera formed upon mere habit and the strobilaceous inflorescence, and therefore including many distinct $G$. I confine the name to the sp, with calix tubulose equal 5 dentate, such as $O$.vulgare, humile, glandulosum, americanum, \&c, and akin. The Authors having neglected to describe the Corollas of the sp. many genera are yet hidden therein perhaps, such as

Zatarendia R. type O. egyptiacum L.

Oroga Lobel. type O. hereaclontica.

765. Amaracus Mench. cal. tubul. 5dentes ineq. supera major labiatiformis, cor. basi saccata, faux compressa-Type Am. dictamnus and sipyleus.

766. Majorana Raf. cal. urceolatus bilabiato, lab. sup. magno integro, lab. inf. parva dentata-Type $\boldsymbol{M}$. fragrans Orig. majorana L. 2 M. suffruticosa. Orig. majoranoides W. 3. M. tenuifolia, 4 Syriaca? \&c.

76\%. Onites Raf. cal. bilabiato, labis subeq. subintegris-Type Onites tomentosa Raf. Orig. onites L. \&c.

768. ВеLтоком Raf. (n. gr.) differs from 764 by Corolla calcarata ut in Plectranthus!Type B. Tourneforti Raf. Origanum do auce. 
-Many other genera will probably be required, the Audibertia of Bentham would have been an Origanum for the linneists, although diandrous!

769. Prilonis Raf, non L. of all the absurd linnean G. of Labiate this was the worst (except Salvia) including a crowd of Genera united by nothing except rerticillate flowers. It was so bad that Brown took away Leucas and Leonotis, I confine my Phlomis by cal. tubulosus 5 gonus 5 dent. equalis, cor. galea compressa emarginata, such as Phl. fruticosa, italica, nissoli, lychnitis, purpurea, crinita, samia, pungens, lunarifolia \&c. and I establish the following 16 genera for the others till 785.

770. Trambis Raf. (n. gr.) cal. tubul. 5 gono extus glabro intus hirsuto, 5fido lac. patulis. Galea ovata dentata intus lanata-Types $\boldsymbol{T} \boldsymbol{r}$. tuberosa and alpina, both Phlomis of $\mathbf{L}$. and perhaps my Phlomis grandifolia, which is a subgenus Blephiloma see am. flor. and appendix.

771. Clueria Raf. (n. lat) cal. 5dentato eq. lanato, galea ovata plana fimbriata fol. pinnatis-Type $\mathbf{C l}$. laciniata, Raf.

772. Anemisis Raf. (wind plant) cal. dentib. 5 aristatis pungens subeq. Galea bifidaType A. rigida Raf. Phlomis herbaventi L.

773. Belonkon Raf. (n. gr.) diff. 772 Galea non bifida, villosa, apice crenata, labio infero latere dentato. lobo lato emarginato-Types B. luteum Raf. Phl. herbaventi Russel fl. alep. t. 16, non L. fol.' subsess, ovato lanc. acutis serratis, bracteis lanceol. ciliatis. mts of Syria, fl. yellow; while white and purple in Anemitis rigida, 2. B. tomentosum Raf. Phl. lunarif. Hook. b. mag. 2542. Phl. russeliana Lagasc. not 
Russel plant,fol. ovatis serratis ruogosis,'subtus tomentosis, bract. lin. lanceol. Spain.

774. Hersilia Raf. (nymph) cal. camp. profunde 5fido, galea profunde bipartita.-'I'ype H. biloba Raf. Phl. do Desf. alt. t. 127.

775. Leonotis Pers. Br. cal. tub. angulato ineq. 8dentato spinoso, dens supero major, Galea elongata concava villosa integra, lab. inf. parvo deflexo trilobo subeq.-Types $\boldsymbol{L}$. nepetif. $2 \boldsymbol{L}$. capensis. (Phl. leonitis L.) 3 caribea? cal. incurvus, subg. Eupalus.

776. Hexisodon Raf. (half. eq. teeth) diff. 7\%. cal. tubulosus regularis 10 dent. 5 dent. alt. minor-Type H. leonurus Raf. Phl. do L. Leonotis do. Br. b. mag. 478.

77\%. Buandina Raf. (Nymph) cal. campan. subinfundib. 10 dent. 5 alt. minor. cor. an idem 775 ?-Types Bl. biflora and chinensis. Phlomis do W. Stachys Forst. Leucas of Brown.

778. Isodeca Raf. cal. obl. 10striatus, 10 dentatus equalis. galea integra-Near to $\mathrm{He}$ misodon and Marrubium, but this last has bifid Galea. Type I. flaccida Raf. Leucas do Br. fol. ovatis membranaceis, verticillis multifloris. India.

779. Leucasia Raf. diff. 778. cal. obliquo 10 dentis subineq.-Type $\boldsymbol{L}$. zeylanica Brown, Leonurus indicus $\mathbf{L}$. Leucas was too short a name, root of Leucadendron and Leucanthes.

780. Hetrepta Raf. diff. 778, cal. obl. striato 7 dentes inequalis- $\boldsymbol{H}$. lavandulifolia $\mathrm{Raf}$. Leucas do Br. Sm.

781. Eneodon Raf. diff. 778 cal. membranaceo truncato obliquo. striato, 9 dentes ineq.Type $\boldsymbol{E}$. urticif. Phlomis do W. P.

782. Elbunis Raf. (n. gr.) diff. 778. cal. campanul, faux obliqua 5dent. subeq. cor. lobo 
medio ad lab. inf. obcordato major-Type $\boldsymbol{E}$. alba Raf. Phlomis do W. \&c Leucas Br.

783. Doriclea Raf. (nymph) diff. 778. cal. obliquato unilabiato 3dentato-D.indica Raf. Phlomis do L. \&c.

784. Heptrilis Raf. (7-3lip) cal. campan. bilabiato, lab. sup. 7dentato, lab. inf. 3dent. aristatis, cor. lobo medio ad lab. inf. majus obcordato-H. glabrata Raf. Phl. do W. Leucas Br.

785. Drctilis Raf. (net lip) cal. campan. bilabiato, labis integris, supero ovato acuto, infero dilatato membranaceo reticulato undulato non dentato-D. molucoides Raf. Phl. do Vahl. W. P. Leucas Br. Clinopodium fruticosum Forsk. fol. ovatis, vertic. multifl, bracteis setaceis. Arabia.

786. Vheckia Raf. 1808 (bot. Van Vleck) Lophanthus Benth. 1828! non Lophanthus Forst. 1780. This G. was ascertained by me in 1802, Bentham long after not knowing of my name, gave it that of Lophanthus already employed by Forster, whose genus had wrongly been united to Waltheria by Wild. Many types, Vlechia chinensis, multiflora (Nepeta.) nepetoides, scrofularif. anisata, urticifolia, all Loph. of B. besides my N. sp. Vl. cordata, alba, parvifolia see my new flora: united to $\mathrm{Ne}$ peta and Hyssopus by L. distinct by lab. infero dilat. crenato, stam. divergens, antheris loculis paralelis.

787. Hemistegra R. (half over) Labiate, Salvian-cal. urceol bilab. sup. integro. infero bifido. Cor. tubul bilab. galea recta integra, labello. patulo apice pandurato 4lobo, lobis subeq. stam. 2 elongata, appendice plano. stylo apice barbat. stig. lateralis acuto. Ovarium su- 
pra semitecto lobo ovato intus septifero ad medio, sem. 4 semitectis-Type $\boldsymbol{H}$. mexicana Raf. Salvia do L. auct. on which I have ascertained this singular structure of Ovary upon the living plant. Many other sp. probable possess the same new Organ, which is similar to a septiferous valve of half a capsule, being a passage to the capsular structure. Something similar was found by Bentham in his N. G. Physostegia but reduced to a clavate gland. All the sp. of Salvia must be now examined to ascertain if thoy possess it, or what kind of disk.

788. SAlvia Raf. non L. The S. officinalis and sp. agreeing with it must form this reduced G. 200 sp. of Sages have been unitcd to it, that have nothing in common except the appendages to the stamens, which form a good character for a family, not for a Genus, which must agree in all parts of fructification! 'The G. Sclarea, Jungia, Glutinaria, Schraderia of Heister must all be restored and properly fixed. I had formed 17 Genera out of Salvia, as early as 1810, I shall now give some striking types out of them like my Hemistegia and the next G. till 800 .

789. Codanthera Raf. cal. tubul. bilab. 3 fido. cor. longe tubulosa galea integra, labello brevis 3lobo: stam. 2 elong. appendices clavatis, antheris campanulatis i frutic. fl. term.striking $G$. by the singular anthers. Types 1 . C. glabra Raf. Salvia strictiflora Hook. b. m. 3135. suffrut glabra, fol. ovatis cordatis obt. serratis, vertic. paucift. 'Tucuman. ' 2 C. biflora Raf. Salvia do R. P. tubiflora Sm. frutic. villosa, fol. villosis cord. obt. serratis, vertic. bifloris. Peru.

790. Belospis Raf. (arrow ap.) cal. colorato 
tubul. bilab. sup. major integro, inf. vix bident. cor. fusiformis, galea concava integra, label. subeq. 3lobo. filam. dilatatis planis, appendices sagittatis, stylo pubescens. fl. racemosis bracteatis-'Type B.levigata Raf. Salvia do Kunth t. 147. spr. Salvia involucrata Cav. W. b. m. 2872 , b. reg. 1203 fol. ovat. acum. serr. bracteis coloratis deciduis,vertic. 6fl. Mexico, scarlet fl. and bracts.

791. Praradena Raf. (thick gland) diff. 787. cor. ventricosa, labello trilobo, appendicis spatulatis, disco antice glandula magna munito, stylo villoso---Here we have a glandular disk instead of the valved one of Hemistegia. Type P. fulgens Cav. W. b. reg. 135. s. Cardinalis Kunth t. 152 seen alive.

792. Lasemia Raf. (hairy half) cal. tubul, 2 lab. 1 et 2 dent. cor. brevis, tubo inflato, basi intus bidentatus, galea fornicata, integra, lab. 3lobo, lobo medio magno flabellato emarg. appendices parvis, stylus subtus villosus, glandulis nullis---Type $L$. coccinea $R$ af. Salvia grahami, Benth. bot. reg. 1370. frutex, fol. pet. ovat, obt. crenatis, racemis verticil. Mexico, fl. scarlet. This G. is well marked by style, lip and tube: seen alive.

793. Artopsis Raf. (n. gr.) cal. camp. bilab. 1 et 2 lobo. cor. brevis. galea erecta integra, labello 4lobo pandurato obcordato ut 787. stam. erectis flexuosis, appendices clavatis, stylo glabro, sem. triquetris levis---near 787 by Corolla, but calix, tube, app. style and seeds different. Type $\boldsymbol{A}$. foliosa Raf. Salvia do Benth b. reg. 1429. fol. petiol. ovato serrat. pubesc. racemo vertic. Mexico, fl. azure.

794. Calospirace Raf. (subg. Benth) near last diff. cal. tubul. 'striatus, galea ooncava. 
This includes the many narrow leaved sp. blended and confused as $\mathbf{S}$. angustif. virgata. reptans, azurea, acuminata \&c, of which I possess many, see my new flora.

795. Kiosmins Raf. (n. gr.) cal. tubul. 3 dent. eq. cor. brevissima, galea acuta villosa, labello vix 3lobo---Type Salv. hispanica and akin sp.

796. Meluigo Raf. (n. lat) cal. tubul. 3fido vix bilab. galea emarg. labio trilobo. filam. basi connatis! appendices glanduliformis-Type Salvia amena and akin sp.

797. Larnastyra Raf. (cells cross) cal, camp. angul. bilab. sup. 3dentato. inf. 2fido; cor. tubulosa, galea ovata emarg. labio trilobo, medio emarg. filam. cruciferis, antheris loculis 2 , valde separatis, uno interdum sterile, appendiculis nullis, sem. uniangulata-Types $\boldsymbol{L}$. $l y$ rata, claytoni, verbenacea, urticifolia,indica? and other akin species of Salvia.

798. Flitpanta Raf. [nymph] cal. tubul. 10 gonus, brevi 5 dent. subeq. spinulosis : cor. galea compressa villosa, labio trilobo, medio concavo emarg. sem. 2-4---Type $F \boldsymbol{~}$. ovata Raf. Salvia spielmani Scop. del. t. 15. Vitm. caule rigido ramoso piloso, fol. ovatis crenatis rugosis, ft. vertic. nudis. Africa, flowers violet.

799. Enipea Raf. [nymph] cal. urceol. trilobo subeq. cor. galea recta compressa barbata, labio dependens villoso trilobo, medio vix major emarg.---Type $\boldsymbol{E}$. formosa $\mathbf{R}$. Salvia do Lher. t. 21, Wild, Curtis b. m. 376. Salv. nodosa fl. peruv. \&c, suffiutic. fol. subcord. fl. axill. ot vertic. Peru, fragrant shrub.

800. Elelis Raf. [Salv. grec.] cal. bilab. 5 dent. 3fido et 2fido, galea compressa integra, labio cuculato subrot. emarg. lobis lat. falcatis, 
stam. 2 exerta divaricata, appendices calcariformis marginatis, disco carnoso---Type $\boldsymbol{E}$. austriaca Raf. Salvia do L. auct. often figured with akin sp. that may offer the same striking characters in corolla and stamens : the thick disk approximates to the glandular disk of Piaradena.

\section{APPENDIX.}

Thesc axamples are sufficient to evince how many fine Genera are involved in Salvia, nearly as many as in all the other diandrous Labiate. To regulate the whole tribe will be a task for Benthan or Decandole, since the flowers of nearly all must be examined alive to detect the disk and forms of appendages. Although I have 70 sp. in my Herbarium, 1 find that it is not easy to determine the Genera in the dry state : yet I will indicate for further study some of the main essential distinctions of other new Genera, or subgenera of mine,

1. Oboskon cal. bipartitis, stylis 2! Salvia cretica.

2. Hematodes. Galea compressa falcata, labio lobo medio saccato emarg. Salvia hematodes, indica \&c.

3. Glutinaria. cal. trilobus, galea falcata, labio lobo medio crenato. Salvia glutinosa et alia sp.

4. Crolocos cal. camp. membranaceo 3lobo. Salvia aurea, colorata, pomifera, calicina.

5. Megyathus cal. camp. patens 5dent. ineq. galea bifida, labio trilobo. S. acetabulum \&c.

6. Ormiastis cal. ovato inflato subintegro, cor. brevis, fol. pinnatis. S. pinnata.

7. Ruopormis cal. amp. ringens, cor, la- 
bio undulato, fol. pinnatis. \$. rosefolia.

8. Sobrso cal. bilab. 5dent. cor. ringens, fol. pinnatis. Salvia japonica 'Th.

9. Scrarea. cal. camp. 5dent. ineq. spinosis, galea brevis compressa emarg. labio lobis lat. deflexis, medio bilobo crenato Salvia spinosa, sclarea, tingitana, triloba \&.c.

10. Terepis. Galea falcata bifida, labio 4 lobo pandurato, subscaposi . Type $\mathbf{S}$. forskalei, \&oc.

11. OrMrLis. cal. reflecto in fructo! ... S. horminum, viridis.

12. Pleudia, Galea brevissima emarg. labio concavo ut Nepeta, stam. plerumque 4 fertilis! S. egyptiaca \&c.

13. Euriples. cal. camp, stam. appendices foliosis. Type E. rugosa Raf. Salvia sibthorpi fl. gr. t. 22. caule ramoso subaphylo, fol. radic. cord. lobatis crenatis rugosis. Zante.

14. Epiadena. cal. camp. bilab, 3dent. et bifido, galea falcata compressa, labio trilobo saccato emarg. reflexis, filam. arcuatis supra glandulis insertis, et basi appendices malleatis, disco glanduloso et semitecto. Type. $\boldsymbol{E}$. $b i$ color Raf. Salvia do Desf. t. 2. Parad. lond. t. 93. fol. cord. obl. repandis dentatis hirsutis rugosis, fl. racemosis vertic. 6fl. Atlas, beautiful flowers blue and white.

15. Addition to 415-Polygonum arifolium of Japan is totaly different firom the N. Amer. being a subgenus of Helxine, only difference stam. \%. I call it Tasoba from the Japanese name, and the $\boldsymbol{P}$. sagittatum of 'Thunberg, not Linneus, belong to the same-1 Helxine (Tasoba) arifolia Raf. Ramis heterogonis aculeatis. fol. hastatis acum. villosis strigosis, stipulis ciliatis truncatis, fl. glomeratis alternis. $2 \mathrm{Hel}$ - 
xine (Tasoba) sagittata Raf. ramis 4 gonis scabris, fol. sagittatis subtus pallidis, stipulis bifidis inflatis, fl. capitatis globosis, bracteis ovatis acutis. Both in Japan, see 'Thunberg fl. Jap.

16. Addition to 418-Polygonum filiforme of Japan is not a Chulusium; but another N. G. according to 'Thunberg's description. I will call it Sunania Frliformis, dedicated to Sunan a Japanese Botanist. cal. 4part. ineq (ut in Tovara) stam. 5 ineq. stylis 2 , sem. compressa subtriquetra. Habit of 'Tovara,stam. and seeds different. Fol. ovatis, stipulis ciliatis inflatis, spicis filiformis.

17. Addition to 770-Blephroma Raf. (cil. edge) cal. tubulosus membran. glaber, non angul. subinfl. subincurvus, apex obliquatus 5 dent. ineq. subul. margine et dentis ciliatis. cor. incurva, galea concava emarg. villosa, margine dense fimbriato, labello brevis 3lobo medio emarg. stam. glabris. Herbaceus, fol. cord. fl. vertic.-This may well be a Genus of itself likewise rather than subg. The type is a new N. Amer. plant, while no other Phlomis has been found in this continent. Bl. amplifolia Raf. fol, inf. amplis petiolatis cordatis deltoideis crenatis obtusis glabris, fol. floralis sessilib. parvis ovatis dentatis acutis, verticillis multifl. bracteis cal. eq. linear. ciliatis. In Texas and Arkanzas, 3 to 4 feet high,lower leaves 8 to 13 inches long, 6 to 10 broad, corolla white incarnate: seen alive in gardens. 
96

DNDEX OF IN CENTURIES 5, 6, 7, 8,

Families are in Capitals, Synonyins in ltalics. Achyrantuidia, 520. Astrocarpus 708. Achyranthes 539, 552. ATIRIPLEXIA 569. Adenopa 527. Adike 593. $\Lambda$ itopsis 793. Albuca 608-14. Alocasia 668. Aloitis 450, 3. Alsine 742. Alsinidia 516. Allotria 694. Amaraca 765. Amaranthus 555, 58. Amaranthidia 519. Aurota 65 .

Barnardia 604. Basellides 571. Beloakon 773. Belospis 790. Beltokon 768. Belutta 536, 8. Brlabiata 750.

Bistorta 411. Blandina $77 \%$. Blenocoes 716. Amblogyna 557. Amarella 440, 19. Amphipleis 714. Analiton 561. Ananthopus 690. Andaca 746. Anemitis 772. Anisanthera 740. Antenoron 411. Anthericum 613, 615, 640, 642. Anthopogon 471, 2. Arenaria 743. Arkopoda 705. Arisarum 661. Arum 661 to 678. Arosma 673, 5. Asicaria 404.

Blephiloma 7\%0, ap. 17. Blitides 574. Blondia 279 (omited) Borboya 606. Borrago 740. Brunellides 756.

Cadelaria 539 to $\mathbf{5 4 2 .}$ Caladium 672.

Calla 679.

Calosphace 794. Calostima 590. Campanula 748. Canscora 599. Capnorea 513 Caraxeron 535. Celosia 536, 560, 66. Celosidia 559. 
Chamedrys 759.

Clictropis 742.

Chiopliila $\mathbf{1 5 6 .}$

Chironia 490-88,725.

Chlora 499, 500.

Chondropsis 492.

Chulusium 418.

Cicendia 174.

Ciminalis 439.

Clinopodium 785.

Clucria 751.

Cnopos 411.

Coccolaba 108.

Codantliera 789.

Codivalia 543.

Cohiba 715.

Coilosperma 564.

Colocasia 671.

Commelina 683 to 699

Corispermides 575.

Crolocos, ap. 4.

Cummingia 654.

Cunila 7500.

Curculigo 655, 6 .

Cutleres 141, 454.

Cutubea $72 \%$.

Dasistepha $13 \%, 486$.

Decringia 565 .

Desmesia 662, 4.

Diandrida $85: 3$.

Drantmidia $51 \%$.

Dictilis 785.

Dioctis 123.

Biodeilis 650 .

Dioninia 518.

Dimeianthus 55.
Dipetalia $70 \%$.

Diploma 438, 484, 5 .

Dirtea 589.

Discolenta $421,430$.

Dismophyla 524.

Disynoma 745.

Disynstylis 500 .

Ditclesia 688.

Doriclea 783.

Dracunculus 669.

Drimia 650 .

Drosera 523 to 531.

Droseridua 522.

Eclotoripa 546-8.

Elbunis 782.

Elelis 800.

Eliokarmos 643-6.

Elodes 730-1.

Emex 580.

Empetridia 633.

Endoplectris 636.

Eneodon 781.

Enipea 799.

Epiadena ap. 14.

Epimedium 636.

Episiphis 729.

Erythrea $45 \%$.

Eudesmis 741.

Eudiplex 533.

Eucapnia 712.

Eudipetala 685.

Euleucum 634.

Eupalus 75.

Eupodia 193.

Euriples, ap. 13.

Eutralia 582. 
Euxolus 556.

Evalthe 725.

Exacum 483, 492, 726to 728 .

Fagopyron 402.

Filicirna 528-31.

Flipanta 798.

Gardoquia 750 to 52.

Gentiana 435 to 489 .

Glutinaria, ap. 3.

Gomphrena 723.

GompHrenidia 534.

Gonipia 457 to 465 ,

Gononcus 433.

Helxine 403.

Hematodes, ap. 2.

Hemisodon 776.

Hemistegia 787.

Heptarina 422.

Heptrilis 484.

Hersilia $\mathbf{7 9 4}$.

Heteroclita 483.

Heterostima $\mathbf{7 5 6 .}$

Hetrepa 780.

Hexastylis 706.

Hipion 140.

Homaida 662.

Hopea 598,

Hyacinthus 606, 700 .

Hyparete 566.

Isgarum 583.

Jsodeca 778.

Karkinetron 406-8.

Kiosmina 795.

Kokira 545.

Komana 7:34.

Kniffa 739.

Kunokale 409.

LaBiata 753.

Lachenalia 653.

Lamarkia 709.

Lampetia 514.

Langsdorfia 711.

Laothoe 615.

Larnalles 597-9.

Larnastyra 797.

Lasemia 792.

Ledebourea 640.

Lemotrys 601.

Leonotis $7 \mathbf{7 5}$.

Leonurus 779.

Lepinema 480.

Leucas $\mathbf{7 7 5}$ to 785 .

Leucasia 779.

Leucoryne $65 \%$.

Licinia 641.

Linum 502 to 509.

Linidia 501.

Lithocnide 591.

Lithophila $56 \%$.

LoBOGYNIA 753.

Lomaresis 647.

Hypericum 729 to 739. Loncomelos 660.

Hypoxis 741.

Loncoxis 642.

Lophanthus 786.

Iresine 563 .

Lophoxera 560. 
Loreia 748.

Lotus 746.

Mancoa 631.

Manetta 638.

Megasea 724.

Megotigea $\mathbf{7 2 6}$.

Megyathus, ap. 5.

Meiapinon 505.

Melargyra 743.

Melanthium 640.

Melissa 750.

Melligo 796.

Melomphis 648,

Melosma 761.

Menophyla 576.

Mesynium 507-9.

Mitesia 424, 131.

Mollugo 515.

Mollugidia 510.

Monipsis 763.

Monosteria 597.

Narketis 477-9.

Nemallosis 511-13.

Nepetides 752.

Nephralles 695.

Nibo 578.

Nicipe 622.

Nicotiana $\mathbf{7 0 9}$ to $\mathbf{7 2 2}$.

Nierembergia 709.

Ninanga 723.

Numisaurum 502-4.

Oblixilis 596.

Oboskon, ap. 1.

OCYMIDES 756.
Ofaiston 584.

Onefera 194.

Onites 767.

Oplotheca 723.

Origanum 760-64.

Ormiastis, ap. 6.

Ormilis, ap. 11.

Ornithogalum 616 to 623,643 to 649 .

Oroga 764,

Ovidia 683.

Oziroe 616.

Parrasia 725.

Patientia 582.

Pectanisia 704.

Pedalium 404.

Peltandra 6\% 6 .

Pentocnide 592.

Pentrius 555.

Perieteris $\mathbf{7 1 1}$

Persicaria 420.

Petalanisia 737.

Petunia 709.

Peutalis 420.

Parnaceum 512.

Philoxerus 535.

Phlomis 769 to 785.

Phylepida 553.

Phytolaca 627.

Piaradena 791.

Pioriza 436.

Piercea 631.

Pilasia 613.

Pladera 599.

Plcienta 497 . 
100

Pleiolontes 736 .

Pleudia, ap. 12.

Pleurenodon 738.

Pleuroglossa 436.

Pleurostena 4.13.

Plurimaoia 499.

Pucumondnthe 44.0, 487-8, ap.

Pogalis 425.

Pogoblephis 4.76 .

Pogomesia 681.

Polygonum 401 to 434 .

Polygonella 405.

Polygonides 573 .

Polycnemides 570.

Prasides 756.

Provenzalia 679.

Psalina 466.

Quamasia 602.

Rafinesquia 750.

Raxamaris 624.

Reseda 702 to 708 .

Reseuinia 701.

Rhodoptera 579.

Rhomphalis 668 .

Rhodormis ap. 7 .

Ricoila 455 .

Rivina 631-2.

Rivinidin 630.

Rocslinia 495.

Rorella 523.

Rossolis 526.

Rumex 576 to 582 .

Sabbatia 496.

Salicorria 586.

Salsola 5S4 to 587 .

SALSOLIDES 572.

Salvia 787 to 800 , app.

Sarothra 732.

Sareathria 587.

Sareoca 628.

Saruocidia 626.

Saxifraga 724 .

SoIIINidia 729.

Sclarea, ap. 9.

Scleranthidia 521

Scorbion 762 .

Scorodonia 758.

Sebaca 727.

Segninum 677 .

Selepsion 595.
INDEX.

Siderasis 680.

Siphaulax 710 .

Siphonema $71 \%$.

Skilla 659.

Sobiso, ap. 8.

Spermaulaxen 416.

Stciremis 549 to 552 .

Steiroptilus 665 .

Stimenes 622.

Stimomphis 720

Stimoryne 721 .

Stopinaca 405.

Strepsiphyla 650 .

Streptalon 736.

Sunania, app. 16.

SyNANDRIÑES 756 .

Tamarixia 532.

Tasoba, ap. 15.

Telipodus 676.

Tenicroa 611.

Tephis 494.

Tereianthus 703.

Terepes, ap. 10.

Terogia 635 .

Teucrium 757 to 763 .

Thlaspi 745.

Thymines 756.

Thylacitis 439,467 to 470 .

Tomaris 782.

Tomoxis 618-21.

Tovara 412.

Tracaulon 415.

Tradescantia 680-2.

Trambis 770 .

Tretorhiza 475.

Triadenum 730 .

Triallosia 652 .

Trichopetalon 641 .

Tridesma 735 .

Trikalls 587.

Triteleia 658, 700 .

Trixago 760.

Tropitria 682.

Tulbella 436.

T'ulophos 700.

UNILABIATA 752 .

Uropetalun 605 .

Urticn 588 to 596 .

Vanessa 638 (Vanasta)

Vibones 577.

Vimenerba 763.

Vleckia 786.

Wadapuo 723.

Xerandra 562.

Xole mia 454, 489. 




\section{FLORA TELLURIANA}

CENTUR. I.--XII.

\section{MANTISSA SYNOPTICA.}

\section{0}

N. Ord.-N. Gen.-N. SP.

\section{PLantareum}

I N ORBIS TELLURIA N U .

Determ. coll. inv. obs. et. descr.

Ann. 1796-1836.

Auctore C. S. R $\Lambda$ TINESQUE, Bot. Prof.

PHILADELPHIA.

1836.

[1838] 


\section{Les noms font les choses. Names realize Entities.}

Plus nos noms sont generaux, plus non idees sont incompletes.-Plus nous avons de noms, plus elles se completent. Lamark, Leach, \&c. 


\section{FLORA TELIURIANA}

PARS IV ET UIT.

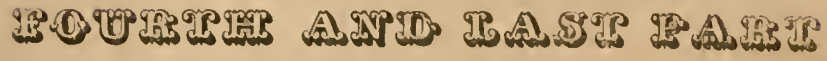

OF THE

SYNOPTICAL FLORA TELLURIANA,

\section{CENTURIES IX, X, XI, XII.}

With new Natural Classes, Orders and Families : containing the 2000 new or revised Genera and Species of Trees, Palms, Shrubs, Vines, Plants, Lilies, Grasses. Ferns, Algas, Fungi, \&c, from North and South America, Polynesia, Australia, Asia, Europe and Africa, omitted or mistaken by the Authors, that were observed or ascertained, described or revised, collected or figured, between 1796 and 1836 .

BY C. S. RAFINESQUE, A. M.

Prof. of Botany, historical and natural sciences-member of many learned Societies in Paris, Vienna, Brixelles, Bonn, Bordean, Zurich, Naples, foc. Philadelphia, New York, Cincinnati, Lexington, \&c.

To observe and compare, to correct or approve

By good names und new facts that convince and improve.

\section{PIILADELPIITA.}

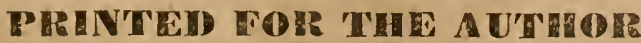

fiy H. Probasco, No. 119, N. Fourll, St. 


\section{NO'IICE.}

This fourth part of the Flora Telluriana will conclude the work. It had been intended to divide it into 6 parts; but even these could not contain all what $I$ have to add or correct on the Orders, Grenera and Species of the whole world and all the classes of Vegetation: therefore it is better to limit this Work to Plants and Lilies chiefly; while $\mathbb{I}$ mean to publish separate Works additional to this on the Trees and Shrubs or a Sylua Telduriana, on the Ferns and Grasses, Fungi and Algas, with perhaps Monographs of some peculiar Families of great interest, and probably a complete account of my New Class of the Nantiandres, having stamens alternate to calix or opposite to corollas, contrary to the usual order.

The price of this work will still be $\$ 5$ or $\$ 40$ for 10 copies, only 160 copies were printed, which makes it high, as but ferv copies can be sold in America, where Botanists cannot duly appreciate it, and they must he sent to Europe, to be often exchanged instead of sold. 


\section{PRE A M B L E .}

\section{"TO 'THE FOUR'TH AND LAST PART.}

In the process of this work I have met with many interruptions and disappointments. It is neither easy nor agreeable to stem the current of botanical errors and blunders, and whoever swims against the streams of scientific prejudice may reckon on difficulties. I have met such in all iny attempts to increase and correct knowledge; but I persevere nevertheless, and write for posterity rather than the actual Scliools. I feel that my weary labors are not now appreciated except by a few, but am confident that in 50 years hence they will be more valued. Of this I have received alrealy some assurances, when young and skilful Botanists have partly approved and adopted my views. is

Meantime I must again repeat that whatever I now state or correct, had been mostly done by me between 1806 and 1815 chiefly, when I had matnred my botanical reforms as stated in my Analysis of nature 1815. I then possessed the ample materials of all the works of Wildenow, the great dictionary and illustrations of Lamark and Poiret, Martyr's large edition of Millers in 4 volumes Folio, and the New Dictionary of Natural History in 24 vol. 8vo . . . besides many rare and valuable works... which were lost with my Mpts. in my Shipwreck of 1815. Having thereby been thrown back upon the world and entered new paths of life, I conld only gradualy collect again my materials, and restore my labors imperfectly. Yet $\mathbf{I}$ have since then consulted and studied many other 
modern labors similar to mine, without finding the same accuracy and perspicuity of Generic reform. While it is with the utmost difficulty I can obtain even for money some late works of similar tendency, although 1 try to correspond direct with the Authors.

Therefore I distinctly state here again, that my feelings being of the most liberal and friendly kind for all Botanists and Naturalists, all over the Earth, if my labors ever interfere witl theirs it must be accidentaly and unknowingly, either because my Genera were those established by me between 1806 and 1815 , or because I have not obtained yet their works, although willing to buy them or exchange them with mine, unless they be too costly like Audubon or Jacquin-I hereby call publicly upon all synoptical and improving Botanists (and even Zoologists and Oryctologists) such as Agardh, Decandole, Endlicker, Schreber, Sprengel, Fries, R. Brown, Lindley, Hooker, Don, Sweet, Arnott, Bentham, Nees, Fischer, Link, Tenore, Ledebour, Blume, Martius, St. Hilaire, Bory, \&c... with others unknowu to me by name as yet, to send me their works in exchange of mine and also to exchange specimens. I have been permanently established in Philadelphia again since 1826 , and shall probably ever continue here, although I may become connected with various literary institutions, particularly the Central University of Illinois of which $\mathbf{I}$ am one of the founders. Books sent me for the University will be free of duties, and equaly received in exchange.

When I have accumnlated all the latest Materials or Gencri, and the great work of Deeandole is completed, I may then revise the 
swhole, connect naturaly the scattered Genera, and add the needful corrections. I have been called already a Veteran in Natural Sciences even by my foes, I hope to become the Nestor of Botany like Adanson was, and if my zeal does not abate, to publish in 1850, a real Mantissa of all Botanical Genera till then.

Meantime I proceed with my New Flora and New Sylva of North America, and am now preparing a peculiar work on the Ferns of North America and other parts. I am going to reprint all my early essays on Botany and 'Zoology, as my Amenities of Nature. I have begun my Mantissa of Zoology, or new or revised Genera of all classes, many also framed previous to $\mathbf{1 8 1 5}$.

My Historical and Philosophical Works are also proceeding, I shall publish my Celestial Philosophy, and my Genius or Spirit of the Hebrew Bible, preparing afterwards my good Book of Knovledge and Wisdom to be probably issued periodicaly, and including the restoration or increase of much knowledge on all Sciences whatever. These vast labors, besides those of private life, in order to obtain the means to produce my works, at my own expense chiefly (since they are too good and too learned for the vitiated scale of our publishers) have partly induced me to curtail the actual work, and conclude it here; but to continue it in another form or reproduce the additions as a Sylva Telluriana and otherwise.

Althongh the articles are now only 1200 , yet they include with the species and subgenera, orders \&c, 2000 or more added or revised objects and groups. 


\section{FLORA TELLURIANA.}

\section{CENTURIA IX MONOCOT.}

Number 801. Otosma Raf. (ear scented) diff. from 679, spatha lato cuculata basis convoluta, spadix clavato, apice nudo, pistillis inf. subrot. stylosis, antheris truncatis sup. baccis $\ell$ loc. 6-12 sp. sem. teret. oculatis. Scaposa, fol indiv. spathis amplis albis odoratis. - I continue here the Aroides, Lilies, and other monocotyles. 'This Genus blended with Colla of $\boldsymbol{L}$. is totaly unlike, see my Provenzalia 679, and New Flora N. A. 481 to 485.-Type $\boldsymbol{O}$. ethiopica R. Calla do. I. auct. fol. cord. sagit. cuspidatis, lobis obt. well known plant of Africa, often cult. seen alive since 1806 .

802. Spirospatina R. Spatha spiralis clausa, spadix obl. pistillis mixtis trilobis, stigma sessile concavo trifido, antheris ad bas, mixt. ad ap. solit. baccis 3lobis 3locul-polysp.--Still more unlike Calla with flat spatha, no style, berries yellow uniloc, Type $\$$ p. occulta R. Calla do Loar. Sm. fol. ovat. cord. petiolis canaliculatis. -From Anam. Perhaps the Arum Spirale of Retz Vitm. Sm. is a $2 \mathrm{~d} \mathrm{sp}$. with lanceol leaves and sessile flowers.

803. Prevrospa R. diff. from Caladium and Colocrsia by the flowers unilateral on one side - of the spadix, and stem frutescent.-The singular structure of spadix requires attention, perhaps several Gronera and Species blended as Arum arborescens L. Types 1 . Pl. reticulata I. fol. sagittatis, sparlix reticul. the linnean sp. of South America, stem 6pedal, leaves pedal, flowers white inside green outside, base dark 
purple.-2 $\mathrm{Pl}$. cordifolia Raf. fol. cordatis, spadix non reticul. Madagascar, flowers fragrant.

804. Doxosua R. (glorious smell) diff. Epidendrum, anthera 4 loc. pollinis 4 unident. columna teres margine auricul. stigma trilobo. Terrestris, Acaulis fl. racem. fragrantissimis.-Probably several sp. Type D. gracilis R. Ep. do bot. reg. $1 \% 65$. fol. lanc. et ensif. racemo longissimo, sepalis cuneatis, labello trilobo, lobo medio obov. dentato costato-Beautiful plant of Antilles, scape 3 feet high, fl. yellow. Lindley translated gracile graceful instead of slender! Ep. odorutissimum is probably a $2 \mathrm{~d} \mathrm{sp}$.

805. Sxnadena R. (united gland) diff. Epidendrum, sepalis ineq. 2 later. orbicul. 3 ovatis minor, label. tripart. medio hastato bifido, basi liber. unguic. glandula magna bifida gerens ad apice columna connexa. Acaulis scapo ramoso.-Type S. amabilis (Ep. (lo. L. auct.) fol. Iato lanceol. carnosis. Molucas, figured by Rumph. 6. t. 43. large white fragrant flowers.

806. Xaritonia R. (nympha) diff. Epiden$d \cdot u m$, sepalis 4 ineq. patens, labello bificlo incluso. Parasitica acanlis racemosa-Type X. elegrens Raf. Epid. Apetalum Jaq. am. 142, pict. 216. Vitm ... fol. subul. carinatis, scabropunct. racemo sub-10fl-Jamaica \&c. fl. fragrant varicgated of purple and yellow. The whole G. Epidendrum requires revision as it was quite artificial: this $G$. deviates even from the usual Orchidea having only 5 sepals besides the lip. See other G. below.

807. Taumastos R. (Iris gr.) Cal. 3sepalis carinatis, Cor. petalis 3 unguicul. cord. retusis. stam. 3 basi monadelphis, stig. 3 simpl. cetera 
ut Iris et Sysir.-Quite a peculiar Genus near to my Olsynium new fl. Type T. compressus Raf. Libertia iridea Grah. in bot. mag. 3294. Caule compr. folioso, fol. gladiatis uninervis acutissimis. From Magellania, fl. white. Of nat. family Gạlaxidia while Libertia belongs to Irides by free stamens and Corolla 6parted.

808. Aulica Raf. diff. Amaryllis. Cor. sepalis 6 ineq. ringens, ineq. stam. ineq. declin. glandulis angul. ad basi stam et stylo, stig. 3 acutis,caps 3gona. Scapo tereto 2fl.-Although the G. Amaryllis has been so much reformed by the English botanists, it includes yet many anomalous sp. since Hooker in 1834 gives it 6 variable characters Cor. subeq. vel. ringens! fauce nuda vel squam! stam. declin vel recta! what absurdity! 'The $\mathbf{A m}$. aulica and akin sp. must form a peculiar group, of which I will give 2 types. 1. A. latifolia $\mathbf{R}$. fol. latis ligulatis obt. Brazil, large scarlet flowers. The Amar. aulica of Hook. b. m. 3311-2. A. stri-

- ata Raf. fol. angustis glaucis viridi-striatis. fig. bot. mag. 2983-A. platypetala fig. by Lindl. 1038 is a $3 \mathrm{~d}$ sp.

809. Plectronena R. (spur fil.) cor. basi tubul. limbo 6part. subeq. stam. 6 subeq. fil. curvis basi intus calcaratis, antheris lanceol. stylo declinato, stigma trilobo. Scapis $1 \mathrm{f}$-The G. Zephyranthes taken from Amaryllis must itself form 5 Genera, the real Zephyranthes with smooth stamens. 2. Atamasco Ad. with short tube, equal fl. and stam. 3d. Pogonema with bearded filaments, type $\boldsymbol{Z}$. or $\boldsymbol{P}$. carina$t a$, and 4 th this with spured filaments. Type P. candida (Z. do sims b. m. 2607. Amar. do bot. reg. 724) fol. lin. canal. crassis. Buenos Ayres. The 5 th $G$, is the next. 
810. Mesochloa $\boldsymbol{R}$. diff. Zephiranthes, cor. campanul. 6partita sine tubo, basi connivens, stam. 3 longior subeq. 3 minor, antheris renif. stig. 3 clavata-Type $M$. canaliculata $R$. (Zeph. mesochloa Herb. in bot. reg. 1361.) fol. canalic, acutis, spatha lanc. ad artic. scapo, ovario turbin ... Bucnos Ayres, fl. white, bulb black.-The G. Haylockia of Herbert differs by 3 stamens only on sepals, fi. tubular at base: it thus belong to Irides instead of Amaryllides, type $\boldsymbol{H}$. pusilla of B. Ayres, fol. lin. filif. fl. radicalis ochroleucis.

811. Trisacarpis Raf. diff. Amaryllis, cor. 6 part. ineq. tubo breviss. ad basis intus corona calyptrata supera, stam. declin. ineq. filif. stig. 3 capsula magna triloba ad basis triscata, sem. dupl. series plana alata, scapo 2floro.-Very singular and distinct $\boldsymbol{G}$. so much blended with Amaryllis that a sp. of it was called $A$. aulica! 3 types at least. 1. Tr. Falcata Raf. (A. calyptrata, bot. reg. $164 \& \mathrm{c}$ ) fol. falcatis lanceol. calyptra triangularis. Cape, fl. green.-2 $\boldsymbol{T} r$. rubra R. (A. aulica ker. bot. reg. 444!) fol. lanc. rectis, calyptra cuculata, sepalis rubris lanc. 2 ovatis acum. infero involuto. Brazil-3 Tr.psittacina (A. do. auct.)

812. Evsarcops Raf. diff. Amaryllis, cor. campanul. ineq. basi tubul. stam. adscendens ineq. stigma clavato. caps. baccata 3loc. sem. baccata paucis globosa nigra in arillis rubris involutis. Scapo multifl. umbell-Type' $\boldsymbol{E}$. reticulata $\mathrm{R}$. (Am. do L'her red, 424. Andr. 179. b. m. 65\%. b. reg. 352) fol. cuneatis basi canalic. medio albo vittatis, umb. multifl. bract. obl. fl. nutans roseis reticulatis. Brazil, a striking $G$. by the fruit, yet often figured as an Amaryllis. 
813. Laticona P. diff. Amaryllis, cor. 6 part. unilateralis, stam. declin. remotis filif. stig. 3loba polysperma. Scapofl. umbellatisType L. compressa Raf. (Am. laticoma E. b. reg. 497.) fol. loricatis, scapo compresso scabro, umb. multifl. S. Africa fine sp. with habit of Brunsvigia, fl. incarnate, sepals with a red nerve. This Gr. is akin to the Lycoris of Herbert, the $\boldsymbol{L}$. or Am. radiata has also unilateral petals (only 5 ! undul. in the fig. b. mag. 596.) but the stamens are cuneate \& $\mathbf{c}$, is it also a $\mathbf{N}$. G? Pleurastis Raf.

814. Heroion R. diff. Asphodelus, stam. declin. ineq. filif. incurvis, 3 superis brevior, stylo incurvo declin.-Il. filiformis Raf. (Asph. tenuior bot.' mag. 2626) caule folioso, fol. filiformis, fl. luteis. Akin to A. luteis, but with quite different stamens. Mt. Caucasus.

815. Ifuon R. diff. Asphodelus, Cor. tubular 6fid, not 6parted nor spreading. Type I. nutans R. Asph. liburnicus \$cop. t. 12. Vitm. W. P.\&c. Caule basi folioso, fol. setaceis subtrig. fl. pendulis laxis flavis-In Istria, singular G. akin to Borboya 606. Both Ifuon and Heroion were old grecian names of $\boldsymbol{A}$ sphodelus. 816. Eutereia R. (well divided) diff. Dracontium, spatha cucul. spadix tereto undique florifero, cal. campanul. 8-9partitus, stam. 8-9, anth. biloc. biporosis, bacca 3loc. 3sp.Rad. tuber. scaposa, fol. divisis.-Type E. nigricans R. Drac. polyphylum L. auct. Scapo breviss. genic. tuberc. fol. laceris, foliolis 3 part. pinnatif.-Douth America, with blackish spathas oft. figured. It is strange that Linneus and all authors should have joined this and the next G. to Dracontium, differing by habit and calix, ber- 
ries \&c. The scandent sp. appear the type of Dracontium, which Adanson changed to Honstera perhaps a better name.

817. Spathyema Raf. 1803 des ribed 1808. Ictodes Bigelow 1818, Anier. Authors Dracontium L. 4. pus Sal. 1815, Beck 1833. Spatha ventricosa, spadix pedunc. ovato florifero, cal. 4part. persistens, lobis cucul. crass. spongiosis. stam 4. stylo 4 gono pyram. baccis uniloc. oligosp. in spadix immersis. Radix crussa, acaulis, fol. simpl. spathis radicalis-Very distinct G. shuffled into many, I restore the best and first name given to it in 1803 as soon as observed, meaning winter spatha. There are 3 types at least see my monogr.. N. N. 1. Sp. latifolia R. Drac. fetidum L. oft. fig. fol. subrotundis subcordatis, spatha ovata acum. macul. North America-2. Sp.angusta R. fol. ovatis lanceol. spatha angustata. N. Amer.-3. Sp.lanceolate Raf. Drac. camchatense I. fol. lanceol. spatha lanceol. apice plana. In Sibiria.

818. Strepsantriera Raf. (turned anth.) Raf. diff. Pothos, spatha reflexa plana, spadix tereto elongato, apice masculifero, cal. 4part. crasso truncato ineq. persistens,stam 4 ineq. antheris bilobis reversis (dorso ad pist. opp.) pist. obov. 4 gono trunc. stig, sess. punctis. bacca 2 loc. $2 \mathrm{sp}$. sem. tuberc. Parasilica, acaulis, fol. simpl. -Type Str. macrophyla Raf. fol. cord. obt. undul. lobis divaric. spatha lanceol. undulata South America, often figured and shuffled in 3 genera. Pothos macrophyla Sw. W. bot. m. 2801. P. grandifol. Jaq. ic. 610. Arum acanle Burm. 36. Dracont. cordatum Aubl. Plum. t. 51,63 \&c.

819. Potros I. non Ad . . This Linnean G. 
includes many sp. of different habit, the flowers of which have not been well described, and must be examined again, I have taken $3 \mathbf{G}$. out of it already and more may be blended. Pothos derived from Potha ceylonese name of some,the sp. with cal. 4part. 4equal stam. and 2 seeds will belong here; but Pothos of Adanson meaning desire in Greek, was the $\mathbf{G}$. Polyanthes.

820. Tapanava Ad. Raf. diff. Pothos, spathis ovatis carinatis, spadix globoso florifero, cal. 3-4squamoso, stam. squamis 3-4obovatis intus 2polliniferis, stigma sess. umbilic. Bacca 1loc. 1sp, scandens, fol. simpl. disticha, spathis axillaris.-Several Asiatic sp. blended in Pothos scandens belong here, of which I will give 2 types. 1. T. indica Raf. fol. lanc. striat, petiolis elongatis alatis, spathis pedunc. reflexa. Ceylon and Malabar-2. T. chinensis Raf. (Pothos scandens bot. mag. 1337) fol. lanceol. acum. falcatis, petiolis, brevis obovatis, spathis subsess. acutis brevis. In China, the spadix is yellow and fragrant.

821. Podospadix R. diff. Pothos, spatha reflexa ovata undulata, spadix peduncul. tereto florifero, cal. truncato 4 fido, lac. ineq. coalescens, stam. 2-4eq.. antheris exertis peltatis, bacca 1 sp.? Acaulis, fol. simpl. nervosisType P. reticulata Raf. Pothos crassinervia Jaq. ic. 610. W. P. fol. oblongis cuneatis acum. subtus reticulatis, nervo medio angul, striato, scapo sulcato-South America, Caracas, singular smooth plant, seen dry, in my specimen leaf semipedal on short petiol, scape slender; spatha small undate like a bract, flowers fuscate, on a spadix quite removed and raised on a peduncle. 
822. Amonena Ad. diff. Oruntium, cal. 6part. 3 alt. latior, stig. sess. drupa oliveformis sp. nucleo cordato. Scaposa spica term. fl. distinctis bracteatis.-Type A. japonica R. Oront. do. L. auct. fol. convol. venosis scapo brevior, bracteis membr. subrot. Quite distinct from our O. aquaticum cal. spong. eq. baca spongiosa Isp. sem. cornea in spadix immersa.

823. Trlcusta Raf. cal. (ipart. stam. 6, stig. sess. bacca 3locul, 3-6sp. Habit of AmidenaType 'T' nepalensis $\mathbf{R}$. fol. convol. bract. elongatis fl. multo longior. MIts. of Nipal: indicated by Buchanan and Smith under the native name of Tilcusta, akin to next G. T'upistra.

824. Tupistra Wal. spadix nudus multiff. cal. 6fidus, antheris 6 sessiles cal. inserta. stylo sulcato, stigma peltato umbil. 3lobo, bacca 3loc. 3sp. Rhizoma fol. et scapis ferens, fl. distinctis bracteatis. - Two types T. nutans b. reg. 1223 , b. mag. 3054 , fol. ovatolanc: acum. spadix obl. nutans. cal. camp. Sylhet in India, $f$. fulvous with brown dots.-2. T. squealida b. reg. 704. b. m. 1655. fol. lanceol. acutis, scapo brevi spadix recto, cal. urceolato. Amboyna and Molucas, fl. cineroaus. The G. Aspidistra and Macrogyne very akin in habit, differ from this by lack of spadix, and solitary radical $\mathrm{f}$. but the pistil being free cannot belong to Asarides, they are Monocotyle linking the Acorides and Asparagoides.

825. EMPRo'TIA, tire Arornes. This order of Monocotyles as reformed by me in 1815 contained 2 sub orders and 6 families, which I now will enumerate here with their Genera. The presence of a spadix is the main character of all.

1. Gymadia flowers without perigone. 
First family, EQUISETIA, type Equisetum. 2d Fam. SAURURIDIA, no spatha, types Saururus, Aponogeton $\mathcal{S} \cdot \mathrm{c}$.

3d Fam. ARISARIA, a spatha, G. Arisarum 661, Homaida, 662, Desmesia 663, Megotigea 666, Alocasia 668, Dracunculus 669, Peltandra 670, Colocasia 671, Caladium 6\%2, Arosma 673, Telipodus 676, Kunda 305 , Provenzalia 679, Otosma 801, Spirospatha 802, Pleurospa 803 ... Zostera, Ambrosinia of c.

II S. O. Caliciniá flowers with a perigone or calix.

4th Fam. PO'THIDIA, the Pothides a spatha, G. Seguinum 677, Dracontium, Eutereia 816, Spathyema 817, Strepsanthera 818, Pothos 819, Tapanava 820, Podospadix 821, Houttuyna \&c.

5th Fam. ACORIDIA, the Acorides, no spatha, G. Acorus, Orontium, Amidena 821, Tilcusta 822, Tupistra 823.

6th Fam. TYPHACEA, types Typha and Sparganium.

To which I may add my new family of UNIseMIDEs as a 7th which I united doubtfully to Asparagoides in 1815, and is another link of those orders, see 6 .

826. Nemanpsis R. (fil. curvis) diff. Dracena perig. basi inflato, tubo filif. 6fido, lac. filif. curvis ad faux lac. eq. stylo filif. curvo, stig. capit. Type N. ternifolia R. Drac. surculosa L. auct. b. reg. 1169 , surculis teretis annulatis subaph. fol. ternis ovatobl. acum. racemis term. corymbosis, bract. subulatis brevis. In West Africa, fl. white. Quite distinct from Dracena.

827. Euphyleia $R$ (well leafy) diff. Dracena, Perig. corollato persistens urceol. 6part. stam. 6 subulata, stylo, stig. 3, bacca 3loc, 
polysp. Arbor, fol. imbricatis, 凡. panicul.Type E. odorata Raf. Drac. australis Forst. W. P. bot. m. 2835. Dr. obtecta Grah. fol. confertis imbric. lanc. acutis planis basi dilat. panic. compos. spicata.' In Australia, fine simple tree, quite leafy with habit of Yucca, A. white fragrant, berries white.-How unlike Nemampsis and my Clintonia, both united to Dracena once! Dracena ought to be spelt Drakaina and the type is Dr.draco. The Dr.marginata forming the G. Plyylloma of Ker. bot. mag. 1585 chiefly differ from Euphyleiu by habit and stamens filiform.

828. Chintonia Raf. 1817 not Clintonia of Lindley 1829 which is my Gynampsis 1833. Beautiful G. of mine shuffled into Dracena, Convallaria, Smilacina . . . by the Genera shufflers! see my monograph of it in New Flora $\mathbf{4 2 6}$ to 448 , including $20 \mathrm{sp}$. and var. under 2 subgenera Cuscumia and Onyxula. Quite vistinct from all akin by berry 2loc. and stigma bilobe, from Styroudra by 6 sepals and habit. The G. Convellaria was one of the worst, having no characters at all, and have reformed it into 8 Genera, in 1817 and 1830 , see med: ff. and below till 831 .

829. Sipryalis R. (tube bottle) diff. Convallaria, perig. ovato. basi ventricoso, phialiformis fident. stam. 6. antheris sagitt. inclusis, stylo, stig. 3fido villoso. foi. oppositis, umbellis axillaris.-Iype, Siphe nitida Ral: caule tereto, fol. opp. brevi pet. obl. acum. nitidis, umbellis pedunc. nutans :3-10floris. Mts. of India. Conval. oppositifolia, Walich, Lod. 610, Hook. ex. fl. 125, b. max. 3529.

830. Fudea Richard, Ophiopogon Gawior and engl. bot. Perig. corolif. epart. persistens. 
stam. 6, anth. lin. snbsess. stig. obt. bacca 1 loc. 1sp. Habit of Convalaria-2 types blended as Conval. japonica by Th. L. auct. -1 Fl. angulata Raf. fol. rad. plura linearis 3gonis bipedalis apice planis striatis recurvis, scapo apice 4 gono, fl. racemosis secundis $2-6$ fasciculatis. -2 $\boldsymbol{F l}$. anceps Raf. fol. rad. membr. involutis linearis acutis palmaris incurvis, scapo ancepsboth in Japan with white flowers and blue berries.

831. Globeris Raf. med. fl. 1830, diff. Convallaria, perig. corol. globoso 6 fido, stam. brevis disco insertis, antheris ovatis, pistil. 6striat. capsula 3loc. 6sperma.-Type Gl. autumnalis Raf. Conval. spicata Thunb. auct. fol. rad, lin. striatis, scapo brevior striato, fl. racemosis subspic. agregatis ebracteatis. In Japan, flowers violaceous. If this plant has realy a capsule as stated by Thunberg it is not even of Asparagoides family, but of Asphodelides.

The other genera blended in Convallaria are the real Convalisina of which $\boldsymbol{C}$. majalis is the type-2. Sigimlaria or Axillaria Raf. 1837, Polygonatum of 'Tourn. and Desf. Evallaria of Necker : we may choose either except Polygon-atum same as Polygon-um, Sigillaria from Solomon Seal the vulgar name would be the best, if the fossil Sigillaria is modified into Sigillites.-3 Mayantuenum Pers. Smilacina Desf. (same as Smilax!) 'Tovaria Necker not of Adanson-4 Styrandra Raf. 181\%, types the Conv. bifolia and others with 4 stamens, 4 sepals, 2 cells.

832. Daiswa Raf. diff. Paris and Trillium, cal. 5sepalis, petalis 5, Stam. 10, stylo tereto trifido stig. 3, baca 3loc. Fol. verticill, caule 1f.-Type D. polyphyla R. Paris do Sm. \&c. 
fol. 8-10lanceol. trinervis petiolatis. Nipal, the flowers vary with ternary and quaternary parts, but the style is permanent. It is therefore a link between Trillium and Medeola. If not admitted as a G. Paris cannot neither, that has quaternary parts, but 4 reflexed styles and 4 loc berry, leaves 4 ovate sessile trinerve acute. All belong to my family Trimlidia differing from Asparagides by several styles or stigmas, and habit often whorled.

833. Arapus Ad. Papiria Th. not Lam. diff. Gethylis, perig. limbo 8-18part. stam. 8-18, stig. 3lobo, bacca 3locul.-Types $A$. spiralis Raf. Pap. do. Th. Grethylis afra auct. b. reg. 1016. fol. lin. spiral. glabr. S. Africa. The real Gethylis has only 6 stamens, and berry uniloc. both have habit of Colchicum, and the ovary partly adherent forming with Hemanthus \&c, the family of Gethylides differing from Narcissides as do the Asparagoides from Asphodelides, by having berries.

834. Leucodesnis Raf. diff. Haemanthus, perig. cor. infund. limbo 6part. erecto stam. 6 exertis ineq. filif. stig. 3fido, bacca 3loc. 3sp.Type L. pubescens R. Haem. do Ait. L. b. reg. 382. albiflos Jaq. W. b. mag, 1239. fol. obov. ciliatis puberis, invol. 5 phyl. ineq. ovatis viridis, fl. albis congestis, S. Africa.-The African G. Haemanthus contains several anomalous sp. merely united by the habit: the real has stam. equal erect, cor. tubular.

835. Scadoxus R. (umb. glor.) diff. Haemanthus, cor. limbo patulo, stam. ineq. adscendentibus. Umbellis multifl. pedunc. articulatis-Type Sc. multiflorus R. Hacm. do L. auct. often figured. fol. ellipt. acutis concavis, invol. fl. brevior. West Africa. 
836. Preniema R. (around bloody) dift: Ifremanthes, cor. infund. basi Ggibbosa inflata, stam 6. filif. equalis exertis. stig. obtuso, Capsula 3loc. 3sp. fol.binis, invol. urceol, spart. ineq. colorato, fl. inclusis.-Type P. coarctata R. Haem. do Jaq. W. b. reg. 181. fol. ellipt. obt. scapo crasso punctato, invol. rubro amplo, lac. ut foliis. S. Africa, fl. incarnate, anthers yellow ovate. This G. and the next having capsules instead of berries do not even belong to the same family of Gethylides, but to Crinides.

83\%. Serena R. (Nymph) diff. Perilema, cor. turbinata 6 fida, stam. 6 eq. stig. 3lobo,capsula 3loc. 3sp. fol. binis, invol. spathaceo, umbella brevior.-Types 1. S. carnea R. Haemanthus do Ed. b. reg. 509. fol. subrot. hirsutis, spatha sphacelata reflexa, stam. inclusis, cor. carneis, obl. obt. R.-2 S. lancifolia R. Haem. do. Jaq. sch. 80. W. auct. fol. lanceol. glabris ciliatis, spatha brevis, stam. exertis, corolla patens. Perhaps a sub G. both of S. Africa.

838. Narcissus Raf. non auct. This beautiful $G$. included a crowd of sp. with very variable cup or inner corolla, that must form at least $6 \mathrm{G}$. I restrict the real Narcissus to those with-cup campanul. 6fid. including $N$. odorus, pseudo, minor f.c.-Several monographs of the linnean Narcissus have been given by Bellendeu, Salisbury, Ker, Smith, Lamark \&c, which see for sp.

839. Autogenes R. diff. Narcissus, cup rotate meinbranose, entire or crenate-such as A. pocticus, angustif, biflorus, tenuior \&c.

840. Jonquilla R. difi: Narcissus, cup campanulate plicate crisp or crenulate-sueh as $\boldsymbol{J}$. 
odora (Narc. jonquilla, tazetla, bicolor, mujor, incomparabilis, trilobus, viridiflora of.c.

841. Calathinus R. difl: Narcissus, cup campanul. crenate stam. 6 ineq. 3 brevior-such as 1 C. cermuus (Narc. do Sal. pyrenaicus Pers. triandrus L.) 2 Serolinus, 3 multiflorus, N. calathinus anct..

843. Moskerion R. diff. Narcissus, cup. cylindrical sulcate crenate-Type 1. MI. moschatum Raf. Narc. do auct. 2 hispanicum Narc. do Gouan. Vitm. Erion, Calathinus, Autogenes were Greek names of Narcissus.

844. Comianinum $\mathbf{R}$ (Plinius name) diff. $\mathbf{N}$. cup large funnel form, petals linear, stamens and style declinata.-Very distinct G. types 3 sp. blended in Nurc. bulbocodium by Authors. C. montanus, C. minimus of c, see Salisbury.The G. Barlacenia and my Plearostima 368 are very near to Narcissides, connecting with the section of Pancratides that have stamens monadelphous or united to the cup.

845. Pancratiun Raf. non auct. This Limnean G. was equaly inconsistent as Narcissus and 3 Genera have alrearly been removed from it Abapus, Ismene, Eurycles. I shall divide it into 9, and my real Pancratium has the cup or Nectary or inner corolla campanulate with 18 equal teeth, 6 stamens alt. with 2 tecth rising from those opposite to petals. - Such are $\boldsymbol{P}$. maritimum, carolinianum, zeglanicum, $1 \mathrm{li}$ riosme Raf. fl. lud. 5, amenum Sal. And. rep. 556 which is $\mathbf{P}$. declinatum Jaq. \&c. The linnean Pancratium only differed from Narcissus by the cup bearing the stamens, both form the real family of Narcissides with a double corolla, the akin $G$, with a single corolla form the 
family of Crinides, and those with unequal or irregular stamens the Amarzuides, all of the Natural Order YMNODIA.

846. Nemepiodon R. cup. campanul. 12dentate, 6 alt. teeth bearing the stamens-such as N. mexicanus, caribeum, speciosum Sal. Red. 156 (N. carib. b. m. 826.)

874. Ismene Herbert, diff. Pancratium, cor. with a long tube, cup campanulate 12lobed, crenulate, stamens declinate inserted inside.Type I. amancaes, Narcissus do R. P. 283. Pers. Pancrat. do bot. reg. 600. fine G. of S. Amer. quite distinct.

848. Eurycles Sal. 1812. Proiphis Herb. 1821, Stemonix Raf. 1833. diff. Pancratium cup 6parted, dentate, stam. inserted insideseveral sp. of Polynesia and Australia blended as P. amboinense L. Crinum nervosum Lher. Amaryllis rotundif. Lam . . . Hooker reckons 3 sp. E. australis, sylvatica and Cunningleami figured in b. mag. 3399.

849. Zouchis Raf. diff. Pancratium. cup. with 6 bifid segments, stamens in the sines -Type Z. illyrica Raf. Pancr. do auct. often figured. Zouchi is the illyrian name.

850. 'Tomodon R. (cut teeth) diff. Pancratium, cup rotate or camp. Globed multifid, teeth unequal irregular,style declinate.-Types 1.T. rotatum Raf, Pancr. do b. mag. 827 Sm. \& c 2. 'T. Aloridanum R. rotatum Lec. 3 T. riparium Raf. $P$. mexic. Lec. 4 coronarium Raf. Pancr. do Lec. 5 pratense Raf. P. occid. Lec. see my monograph of this G. in New Flora.

851. Siphotoma R. (tube cut) diff. Pancrativem, cup cylindrical multifid, stamens unequal, 3 alt. shorter.-Type S. calathina Raf. Pancr. do. b. mag'. 1561. Sm. \&c. 
852. Braxireon R. (short Ireon) diff. Pancratium, cup quite cuplike short Gfid as in Narcissus, but bearing the stamens.-Type $\boldsymbol{B r}$. humile R. Pancr. do Cav. 20\%. Pers. sf.c.

853. Troxistemon R. diff. Pancratium, cup rotate 6dentate, teeth bearing the stamens, sinusses emarginate or undulate, petals narrow recurved.-Types Tr. littorale and fragrans.

854. Lirtanus R. (sand lily) diff. Crinum, sepalis unguic, Ovario infero, filam. stam. basi gibbosis, fl. umbel.-Type L. 5florus Raf. Crinum arenarium Hook. b. mag. 2531. fol. striatis, scapo 5 floro. Australia. The G. Crinum had also been widely mistaken, since Agapanthus with free ovary had been united to it, besides this $\mathbf{G}$. and the next.

855. Scadranus R. atl.J. 1833 (blue umbel) diff. Agapanthus, cor. tubo fusif. limbo camp. 6 fid. lacinis canalicul. 3 latior obt. 3 angustior acutis. stam. 6 ineq. incurvis filif. tubo inserta. ov. libero obl. stylo filif. recto, stig. obt.-Type Sc. multiflorus Raf. fol. ligulatis planis acum. scapo tereto, umbella multifl.-Fine plant seen alive in our gardens, native of Florida and Louisiana, it is the blue Crinum of Bartram, $\mathrm{Cr}$. americ. Pursh \&.c not L. the Agapanthus of our Gardeners, quite different from this $\Lambda$ frican genus. Leaves pedal, one inch broad, fl. inodorous uncial azure blue. The Gr. Amunon of Ad. differs only by long tube, he quotes the Crinum 4 L. and figures of Commelin, Breyn, Plukenet which must be verified. Is it the Agapanthus? Of family Alordes (not Crinides) with all the Narcissides that have a free pistil.

856. Scaduakintos R. (umb. hyac) cor. campanul. Gpartita, stam. 6. filam. alt. mem- 
branosis insertis apex segm. cor. ovar. stipitat. stigma ... caps. 3loc. polysp. Facies Alliacen, scaposus umbella multifl. cerulea.-I'ype Sc umbellaris R. Brodiea grandiffora Pursh, Nuttal Eat. \&c, which has been proved by Hooker to be quite different from the $\mathrm{Br}$. grandifl. of Smith, not even of same Genus! but it is not a Milla as surmised by him. Of Family Aloides called the Missouri Hyacinth.

85\%. Brodiea Sm. \&c. Hookeria Sal. Cor. tubulosa 6ida, stam. 3, squamis 3 alt. ad tub. inserta. Ovar. stipit. style filif. stigm. 3 caps. 3loc. sem. centralis. scaposa, bulbosa, $f$. umbellatis.-Type Br. grandiflora Sm. b. reg. 1183. b. mag. 287\%. fol. lin. elong. canalic. acum. umbella 5-8flora. In Origon, fine large blue flowers. Type of a new family with Sowerbea, Xiphidium and Leucoryne 657 \&c akin to Xyrides and Commelines, which may be called XYPHIDIA, it differs from them by Corolla equal, from Aloides by 3 stamens, from Irides by ovary free. Does Wachendorfia belong to it?

858. Conanthes R. (cone fl.) diff. Pitcairnia,flowers conical, cal. 3part. Petalis 3 undul. longis nudis stam. 6 ineq. 3 longior, stylo elongato, stig. 3fido. Parasita, scaposa, racemosa. -Type C. albiflos R. Pitc. do Hook. b. m. 2641. fol. lin. lanc. integris acum. scapo racemoque simplex. Brazil, fl. white.

859. Hexalepis R. (6 scales) diff. Tillandsia, cal. 3part. scariosis colorat. convolutis, cor. tereta 3part. convol. squamis 2 ad bas. petalis alt. ad stam. 6, sed ovario circondans, stigma 3lobo fimbriato. fol. imbric. A. spicatis-Type II. psittacina R. Till. do Hook. b. m. 2841. fol. ligilatis acutis basi inflat, spica rachi flex. 
bract. color. fl. eq. In Brazil. fl. bicolor red end yellow. Nearer Pitcairnia than 'Tillandsia by the scales. All these G. belong to my family Arcumna 1835 with Guzmania, Aechmea, Lachenalia, Eucallis below 86: \&c.

860. Dexmborogon R. (tree beard) Neog. 18\%5. ditf. 'Tillandsia, stam. 3 (non 6) stylo, stig. 2-3lobo, caps. 2-3loc. Parasitica, ramosa, fl. axill.-Type the D. usneoides and probably other sp. In fact the G. 'I'illandsia is in utter disorder, it includes several Genera; those with unilocular capsule must form the $\mathbf{G}$. Karreguata of Adanson.

861. Errostax R. (wooly spike) diff. Bromelia, ovar. 3alato, cal. globoso 3lobo, petalis 3 rigidis linearis, stam. 6 ineq. 3 brevior epipetala, stigma dilat. 3lobo. Fol. imbric. spica clavala, lanata, florib. in lana immersis.Type E. glauca Raf. Bromelia melnnantha E. b. reg. 766. fol. inıbric. lanceol. marg. spinosis, spica albo lanata, cal. lutesceus, petalis atropurp. Antilles. Is the fruit a capsule as in the next $\mathrm{G}$ ?

86:. Eucalduas R. diff. Bromelia, ovar. 3 gibboso, cal. spart. undulato, basi tubuloso glanduloso. cor. 3 part. apice spiralis, squamis 2 ad medio petalis, stam. 6 filif. 3 inter sq. insertis antheris filif. stylo 3 gono, stigma 3fido. caps. 3 loc. polysperma. Purasilica stolonif. $\mathrm{fl}$. spicalis bractentis-Type Euc. versicolor Raf. Bromelia zebrina Hook. b. mag. 2686. fol. lanceol. canalic. obt. dent. spinosis, subtus glaucis, albo transverse zonatis, caule albo nutans, bracteis longis lanceol. roscis. Beautiful plant of Brazil, flowers versicolor, calix white, petals and stamens yellow. Quitc distinct from $\mathrm{Bro}$ 
melia by capsule and scales on petals as it Pitcairnia and Hexalepis. 'T he sp. of I?romelia with unilocular berry form the G. I'scilomelia of Necker.

863. Runziss R. (root turte) restudimaria Burchell b. reg. 921. diff. Bioscorea stylis 3 coalitis, Radix amplissima epigea. Hardly diflerent from Dioscora, generic names derived by aria cannot stand. Very singular huge roots edible like yams called Hottentot Bread. 2 . types. 1. Rh. elephantipes R. Tamns do W. P. fol. renif. planis, racenis axill-2. Rh. montana R. fol. cord. subt. glaucis nervosis. Both from S. Africa.

864. Roscus I. auct. this G. must be carefully revised, as it has many anomalies, the $\mathbf{G}$. Danae of Mosnch and Persoon for the R. recemosus must be adop'ted, differing by habit, corolla and fruit. The real Ruscus bears the flowers on the leaves that are perhaps enlarged expansions or peduncles. The R. androgymis is not a Danac, but includes 2 distinct sp. I. $R$. latifolius Raf. andıog. W. Dill. t. 250. Hook. b. m. 3029. fol. subcord. ovatis ocum. fl. subsess, in capitulis multifl. congestis. Madeira, t). yellow white in a notch of the leaf.-2. $\mathbb{R}$. anthopus Raf. androgynus Sims b. mag 1898. fol. ovatobl. acum. fl. solit. perlicelis fl. eq. Canary Ids.-Both must form the S. G. Gurenias (Diosk) by fi. marginal, corolla 6parted rotate, anthers sessile in a central tube or nectary. The G. I'eliosanthes of R. Brown differs by cor. tubulose, ncctary globose staminiferous.

86j. Siraitos R. (11. jap.) difi. Abalom, fl. hermaphr. petalis $\mathbf{6}$ obl. obt. stan. $\mathbf{6}$ filam. subul. brevis, antheris 4 gonis, ovar. orat. stylis 3 revol. caps. unica 3 locul. $-\mathrm{A}$ Genus blended 
with the American Abalon by 'Tlumberg and others although quite distinct, but habit similar. - Type Sir. aquaticus Raf. Melanthium lutcum Th. fl. jap. who quates Veratrum luteum of I in. caule teres flex. striatus, fol. lanceol. integris, fl. spicatis luteis. In Japan in watcrs.

866. Abalon Ad. 1763. Maf. N. f!. 1830. Chamahirimm Wild. 180!) Gray Sul, G. Ophiostachys Red. 1848? Diclinotrys l:al. neog. 1820 - A very distinct $\mathrm{G}$ shufled into $7 \mathrm{G}$. by the linneists \& c, well described as the first G. of my New Flora N. Am. and again by Gray as Helonias rioica. Adanson's name is the first and hest meaning not in a ball. Ophio-stachys and Chama-lirium are bad compound names of Sinchys and Chama.

IIaving just reccived the Monograph of Gray on the Melanthacea or rather my HesoNivis, of North America, I will revise them in my New Flora. I merely state here that his G. Leaco-crinum must be changed into Leucrinis Raf. that his Leimanthium of Wild. is my Evonyxis 8:3-that his Stenanthium S. (4. of Veratrum is my G. Anepsa 89 -that his $A$ mianthum muscoloxicumi was my Crosperma laeta of 1825 a plant shuffled into 3 Genera till made one of by myself since 1801 see G. I00that his Schano-cullon will be my S\%oinolon Raf.-For his 'Tofieldas, with 5 other names Triantha, Hebelia, Isidrogalvia, Leptilix, of $\mathrm{c}$, see Abama of Adanson 1763 in my New Flora with 4 sp. and 2 akin Gencra of which I shall write a inonograph.

867. Barno R. (1) jap.) diff. Uvularia, petalis 6 obl. stam. ( 6 hypogyna, stylus 1, stig. 3 reflexa. coule artic fol. cirrhosis- Type $B$. cirrlosa Raf. Trularia do 'Th. auct. caule te- 
res artic. fol. sessilib. geminatis linearis cirrhosis, pedic. axill. 1 fl. reflexis. In Japan, f. yellow, stamens white. Baimo is as good as Plantago.

868. Simira R. (n. jap.) diff. Ornithogralum petalis 6 lanceol. eq. stam. 6 subul. 3 latiora alt. stig. obt. capsula ovata villosa 3gona 6striata-Type S. japonica Raf. Orn. do Th. auct. scapo striato longo racemoso, fol. linearis planis. Japan, fl. purplish, perhaps only a subgenus. Simira is a pretty japanese name.

869. Strypandia R. Br. another G. akin to Skilla with filiform stamens diff. by filaments retrocurved and anthers with glands, stem foliose, $\{$. paniculate: thus more different by habit than characters. Several sp. from australia. 1 St. glauca fol. glauc. tortilis, $2 \mathrm{St}$. propinqua Cun. b. mag. 3417. fol. gramineis \& c.

870. Artmroponivi R. Br. diff. Skilla and Anihericum by petals reflexed, stamens villose, style declinate, roots fasciculate-several sp. from Australia. A. panicul. A. minus \&c.

871. Chlorophytum R. Br. diff. Skilla, stam. connivens, capsula 3loba polysp. $4 \mathrm{sp}$. from Australia. G. admitted although less distinct than some of mine.

872. Podonix R. (bearded claw) diff. Tuli$p a$, petalis ineq. 3alt. lanc. minor, 3 ovatis major unguiculis barbatis, stam. ineq. basi barbatis, stigma 3poroso, caps. globosa 3loba. Acaules -Type P. albiflora Raf. Tulipa biflora L. auct. often figured, b. reg. 535. fol. 2 linearib. scapo 1-2floro. Near Caspian Sea, flowers small and white. Near to G. Liriopogon 113 to which I once referred it, but distinct.

873. Phariun Herb. cor. 6part. subeq. stam. 
6 monadelphis ad basis membrana conlitis, itylo fistul. recto, stig. perforatum capit. Fucies alliacea.-N. G. near the Repa $\delta \mathrm{c}$, but stamens united. 'T'ype Ph. fistulosum H. li, reg. 1546. fol. fistul. teretis, scapo umbellato, unib. paucifi. invol. 3 subrot. fl. cernuis, stam exertis Mexico.

871. Prasronon R. diff. Pharium, petalis sulcatis, stam. subulatis basi coalitis, stylo filit. stig. acuto, caps. 6sp.-Type Pr. longifolimm Raf. Allium do Sprengel, b. reg. 1034. Schenoprasum do Kiunth. fol. lin. canalic. longissimis, scapo striato subanceps basi folioso, unbella sub 8il. congestal. Mexico, fl. purple. Forming a sabf. Punarides with the last and Spiranihere 885, by united stamens.

875. Eucrosia Edw. diff. Amamjlis, stam. basi monadelphis. 'Type E. bicolor b. reg. 207. fol. ellipt. glaucis, umb. 4f. 4bracteata. S. America, fl. greenish purple, bracts white.'These Genera with united stamens appear to indicate new fimilies. This will be type of sulff. Eucrosides in Narcissides.

876. Phaionlleps R. (brown veins) diff. Sisyrinehium, cor. infundib. Gfida eq. stam. 3 filam. monad. in tubo elongato. Scapost.Family Galaxidia near G. Golaxia-Types 1. Ph. odoratissima R. Sisyr. do Lind.b. reg. 1283. fol. angustiss. glaucis equante ad scapo teres, spatha univ. eonvol. 4-6fl. pedic. nutans. Patagonia, fl, white with brown reins. - Sisyr. flexuosum is a $2 d \mathrm{sp}$.

877. Poganelpha R. (beard brother) diff. Sisyrinchium, stam 3 basi monadelphis pilosis, stylis 3 basi coalitis, ovario piloso-'Types 1 . P. maculata Raf: caule flex. tereto, fol. amplex. brevis lin. obt. spathis 2 lanc. subeq. acutis $2 \mathrm{ft}$. 
peralis orati obt. flavis basi nucula atropurp. Chili, only deened a var. of the next in b. reg. 1915. var. pumilum.-2. P. graminifolia Raf. - Sisyr do. b. reg. 1067. diff. fol. caule longion sessilib. acutis, spathis ineq. lanceol. petalis obovatis luteis, macula fusca ad medio. Chili.

878. Olsvium Raf. newfl. 1 p. 7\%. diff. Pocrolplyer, cor. petalis 6 campanul. non patulis, stam. 3 elongatis liberis, basi coalitis coaretatis glaluis, stylo elongito, stig. 3 acutis.-Type 1. grom diflorum R. Sisyr. do b. reg. 1634, b. mag. 3599. Caule compresso, fol. brevis acutis, spatha ineq. f. linis nutans purpureis- Origon at falls Dakanagan, large purple flowers-2d sp. O. luleum Raf. Marica californica Ker. b. m. 983, cau!e alato, fol. lin. petalis obov. luteis. California, many $\mathrm{G}$. have lately been blended in Sisyrinchimm, even the next with free. stamens!

879. Orthrosantuss Siweet, diff. Sisyl. petalis 6 ineq. 3 ext. angustior, stam. 3 liberis.: sp. of Australia, nearer to Marica than Sisyrinchium. One of them is the Sisyr. ixioides Forst. or Ferverin do W. or Morea do Th. thus put in 3 Gonera.

880. Evertira R. (well free) diff. Sisyr. petalis 6 cqualis, obovatis, stam. 3 liberis-Type multiflora Raf. Orthrosanthes do Sweet, Sisyrinchium cyaneum Lind b. reg. 1090 ! fol. cespitosis linearib. Iongiss. spathis 2 ovatis suber. petalis ceruleis obov. ol:tusis. Australia-'The G. Mrerira chichly differs from this by petals unequal and stamens alt. to stigmas, the G. Cipura besides by stigma petaliformis. Bobrertia is also an akin Cenus.

881. Puamaniris Raf. (brown fl.) diff. IFtores, petalis iner. : alt. quadruplo major basi 
conc. apex planis, 3 pet. minor planis, stmm. 3 subconnatis, stig. 3 lin. planis bidentatis.- This was nearer to sisyr. ly stam. Lut referred by labit to Morea! 'lype Ph. Lurida Kaf. Morea do Edw. b. reg. 31:. Unillera papillosa, fol. 2-3 linearib. caule rq. petalis acum. fucis. South Africa.-How different fiom Pardanthes chimensis of $\mathrm{Ker}$ or Belanenda Red. which was the type of Morca! same as Miorus!

882. Hanonor. Lab Sm. ovar. inf. petalis 6, stam. 3 antheris sessilis petalis insertis, stylo, stig. capit. caps. 3 loc. (isp.-This G. has been made the type of family Hacmodoracer, very near to Irides; but the next $G$. is not of same family: nor is the G. Anigazanthes differing by cor. tubular unilabiate 6dentate and 6stamens,nor my Pleurostima $\mathbf{3 6 8 .}$

883. Prilemocarya.R. Br. Ovar. inf. petalis 6, stam. $\mathbf{6}$ epipetalis antheris sess. stylo, stigma capit. caps. 1loc. 3sp. ad abortu Nux monosp. coronata-'Yype Plil. cilicte Br. Sin. fol. distichis fimbriatis, fl. panicul. Anstralia. Nitho' the insertion of stamens is similar to the last, their double number and fruit makes this type of a subfamily in the funily of Droscommes.

881. Eestiepuus R. 13r, Petalis 6, internis 3 fimbriatis, stam. 6 liberis, or. lib. stigma trigono, caps. pulposa 3icc. 3valv. valvis septiferis polysp. Frutescers, fol. alt-Type E. rugustifolius $\mathrm{Br}$. Sm fol. lin. lanc. A. fascicul. nutans. This G. has the habit of Emilax, and forms a link between Sinuscra and AsprountLIDLS.

885. Śrrantuma Raf. Miff. Emstrephus, stam. basi monadelphis, antlieris apice spiralis - Sp. otate Raf. Eustr. latifolius Br. Em. fol. ovatis fl. fascic. pedic. nutans. Auntralia. 'The 
minion of the stamens is akin to G. Ruscus, yet this must be referred to the subf. of Pharides in firmily Aspirodemids. See 8\%4.

856. Eulara L. auct. based on the Restio elegia since made $E$. juncea by Linneus; now having several sp. requiring reform, and becoming type of the family ELEGIDEs differing from JunciDEs by 3 stamens instead of 6 . - The real G. Elegia has cal. 6glumis ineq. stam. $\mathbf{3}$, stylis $\mathbf{3}$, Gaertner says caps. 6loc. 6sp. Smith says 3loc. polysp. it is so at least in E. racemosa if not in $\boldsymbol{E}$. thy rsifera once $\boldsymbol{E}$. juncea.

88\%. Tristemon Raf̈. 181\%. diff. Juncus,cal. 6glumis ineq. 3 ext. aristatis, stam. 3, stylo 1 , stig. 3, caps. 3loc. polysp.-This includes all the Juncus with 3 stamens, nearer Elegia and same family, such as Tr. marginatus, odoratus, polycephalus, cunglomeratus \&c see my monograph in New Flora.- The family of JunCIDES with 6 stamens includes only funcus, Luzula, Aptylanthes, Rapatea, Pollia and a few others.

888. Batoskion Raf. (Ball rush) diff. Restio capitulis globosis, fl. fem. 4 valvis, stylis 2 , capsula 2loc. 2sp.-'Type B. dichotomum Raf. R. 4phylus Lab. P. Sm. Br. dichotome, foliose, spicis panicul. Australia. This as well as $\mathbf{C a}$ lorophus Lab. also belong to Elegrnes instead of Restides.

889. Aввотіа Raf. diff. Triglochin, cal. 3-4 glumis deciduis, stam. 3 sessilis. interdum 1-2. pistillis 3 -4coalitis, stigmas sess. glandulosis fissis. capsulis 3-4coalitis monosp. Acaulis, $\boldsymbol{f}$. racem.-This G. containing $3 \mathrm{sp}$. Ab. filiformis, palustris, pumila is described in the monograph of my New Flora I p. 36. It belongs to Elegines, and is the link with the G. Triglo- 
chin.-Thus the ELEGIDES include Elegia, T'ristemon, Calorophus, Balostion, Abbotia and perhaps other Genera; they are very near to Xurmes 17, differing by no real internal corolla.

890. Cinosmopetalon Rotb. diff. Reslio cal. ineq. 3 interuis major cartilagineis-Type. C $h$. tectorism. Restio do L. auct.

8)1. Lenka Raf. (one less) diff. Restio stam. 2. stylis '2. all the sp. with " 2 instead of $\mathbf{3}$ stamens and styles belong here. 'I'he Restrnes or Restiacea fumily differ from Juncides by fruit monosperm, and besides these 3 Genera inclurle others from the Southern IIemisphere.

892. Puy undu Gacetn. Sm. Garciana Lour. Perigono 2phylo corolliforme, stam. 3 ad uno petalo inserta. Ovar. libero, stylo filif. stig. capit. caps. 3loc. 3valv. polysp. rad. fibrosis, fol. ensutis, fl. spicatis.-2 sp. Ph. Ianuginosum and pyomenm. Of family Xurrdia, but type of a subf. Phylidrines.

893. Nenitrs R. (fil. pl.) Apteria Nut. 1834. Ov. inf. cor. tubul. camp. Gidentatis alt. minor, stam 3 in tubo, stylo filit. stig. 3 cmeatis, caps. coronata monoloc. polysp. placentas 3. Rad. fibr.canle symamoso.- Type Nemilis setacea Raf. Apteria do Nut. ic. caule filif. 1-41. squamis remotis brevis sphacelatis. Fiorida, Alabana and Missouri! minute annual plant, fl. white.-Nuttal makes a new fumily of this with Wripterella, it difiers from it as Luzula from Juncus; but Burnammia with 6 stamens cannot belong to it. All the monocotyle with 3 stamens are essentialy different from those with 6. In fact the staminate numbers are of greater importance in Monocotyles than in 
Dicotyles, which some Botanists are not aware of: I had to change the name of Apteria which is a class of insects!

894. Sowerbea Sim. petalis 6. stam, 3 fertilis, anther is bilobis disjunctis, stam. 3 sterilis castratis, caps. 3loc. 3-6ip. Fincies Alliercert'Type s. juucers.m. ic. b. mag. 1104, andr. rep. 81. fol. canalic. linearib. scapo unbelia multitl. pedic. articul. Australia, 11. rosate. family of Xiphidia see Brodica 857.

695. Spanaxis Ker. difi. Iris, cor. tubulosiz 6fda regularis eg. stam. 3 patulis, stig 3 oblongis, spatha ?ulois lacera.-Deveral speciez Sp. anemoniflora, frregraus, tricolor, gran. difl. bulbifere, fimbrialre \&c; but those with irregular corolla must form the next Genus.

893. Anactomon R. (glad. diosk) diff. sparaxis, cor irregularis, segmentis connivens, vel uno patulo, stam. erectis-Type 2 Sp. An. bicolor R. Sp. do Ker.-2 An. unalentum R. Ep. do Ker. Gladiolus do Jaq. W. P. perhaps a subg. by bilabiate corolla, Pasganon Raf: Of family Gradroluna differing fiom Irides as Amaryllides from Cinides by the irregular corolla or stamens.

๑97. Paneguia R. diff. Mlarica and Sisyr. petalis basi coalitis campanul. stylo trifido.-'Types 1. P. striala. R. Sisyr. do Sm. ic. 9, Red.66. S. spicata Cav. Marica striata Kier. b. m. 701. f. fascic. 1 spathis bract. membr. ad pedic corollis mucronatis.-2 P. palmifolia $\mathbf{R}$. Sisyr. do L. Horea do Th. fl. panicul. corymbosis. Perhaps subg..

893. Priopatalon R. (saw pet) diff. Alstroemeria, ovar. globoso 6costato, petalis o patulis serrulatis, 2 ext. eq. dilatatis, 3 int. an- 
gustior 2 sup. minor. Stam. 6 declin. 2 sup. antheris brevior. - T'ype Pr.prillidum R. A. do Grah. b. mag. 3010. ful. sparsis lin. lanc. denticul. subanuplex. fl. umbellat. ¿-jpeíalis acum. cxt. obov. int. ellipt-Chili, fl. incarmate, the figure represents the style clavate entire while the description tells it is 3 gone with 3 stigmas! - Whi!e the $G$. Amarylis has undergone such a revision and division the akin Gr. Alstroemeria has been left untouched, although presenting striking anomalies, I therefore present this and the 2 next reformed Genera out of it, but there are probably more.

899. Lulavia R. (bird lily) diff. Alstroemeria, cor. campan. subeq. petalis 3 internis angustior brevior, stam. 6 subeq. rectis, antheris 3 alt. major ellipt. stigma 3, caps. 3gona.-Type L. psittacina R. Alstr. do. Lehm. Sweet t. 15. b. Inag. 3033. Caule flexunso, fol. cuneat. obovatisque obt. tortis, umb. 4-5ff. involucrata, petrilis cuneat. acum. Mexico, fine red flowers with purple spots, and greenish tips.

90í). Donecasperara R. diff. Alsto. cor. camp. petalis connivens subeq. 3 int. unguicul. stam. inclusis subeq. ovar. semisupero, stylo basi incrassato recto, stiona 3 . caps. plana apice trivalvis, valvis septif, scptis ad utrinque latere 2sp. sem. 12 globosis coccineis.-Type D. acutifolia R. Alstr. do Link t. 29. b. mag. 3059. Volubile, fol. petiol, lanceol. acum. subtus pubescens, fl. umbel. bracteis lanceol. petalis ext. obl. croceis, internis spatulatis flavis. Mexico disc. by Deppe. Certainly quite unlilie the two above in habit and characters. 


\section{CEN'TURIA X MONOCOT.}

ORCHIDEs' or Synarmia. 'This fine Nat. Order of plants shall be continued here; having already given many Genera of it, see 1, 117 to 138,183 to $186,201,211,220$, to 228,318 to 344,372 to 387,382 to 385,804 to 806 . It is now become one of the most interesting and prolific orders, evincing the vast progress of modern Botany. Linneus had only () Genera of it, Adanson only 7 although he had Vumilla omitted by Linneus, Necker in 1790 had 17 Genera, Jussieu, Swartz, Richard, 'Thouars had vastly increased them,(Persoon had 30) and now Lindley has over 200 Genera; but 100 have been or will be added by myself. The single Genus Epidendrum which was a confuse medley has furnished 30 Genera, and even as reformed lately it contains 10 or 15 more as I have shown. Necker had 3 which I could not ascertain as he gave no typical sp. but I give here their characters that they may be ascertained and restored.

901. Pranrosantires Neck. 1474. Petalis 4-5patulis incq: undul. label. basi tubul. quadrato dilatato ad apex, anther. 2. stig. infundib. Scrposa-This must include several I Dendrobiums, and Necker intimated that like Epidendrum, it had many anomalies.

902. Eymisantiena Neck. 1475 petalis 5 lincar. subeq. Label. basi tubul. columna amplectens, anthera cuculata 4 loc. pollinis 8. stylo tubo adnato, stigma infundib. caps. ventricosa contorta. Cuulescens.-Is it the Octomeria? of late Authors.

903. A Buocirs Neck. 14\%0. diff. Orchis, petalis 5 ineq. label. resupinato ad basi galei- 
forne calcarato, antheris 2 cuculatis. Ovar. contortum. Scriposa. Necker only says this includes 10 of the limnean Orchis, diflerent from his Inachlorhiza with fasciculated roots, a sibg. of Orchis. It must include some $\mathbf{H a}$ benaria.

901. Conostrus R. (hollow St.) dif?. Epidemdrum. Petalis 5 eq. patulis, label. ad col. inscrto, 3 part. lateralis ciliatis latis, medialis nuda lincaris. columna vel stylo clavato, apex infundib. labiato bialato, stig. antherisque inter cavitas inclusis. 'Terrestrial, subcuulescens, $f l$. racem. amplis.-.'lwo types blended as lip. linearis L. 1. C'oil. obtusifolia Raf. fol. '2. biculatis obt. connatis, petalis linearib, cuspidatis, columna campanul. dentata, lab. lac. media, elongata. In Antilles, root clavate, flowers triuncial petals greenish, lip and column white.2 Coil. emarginata R. Ep. ciliare b. m. 463 non .L. Ep. cuspirdatum Lod. 10. b. reg. 783. fol. ternis ligulatis emarg. petalis lanceol. Antilles, fl. large yellow, the column white.

905. Okxwe R. diff. Aerides, label. infundi bulif. calcar conico incurvo, col. rostrata, an. thera rostrata baici 2loc. pollen 2 Prarusit. conelese. A. spicalis.-Type (). cormua R. Aerides do IRoxb. b. reg. 1485. fol. linulatis apice obliq. emarg. spicis pendulis racemosisIndia, fl. white incarnate, smell of tuberose. The Aerides or air flower's differ from Lipial. by a spur.

906. Sumprta R. (Nymph) diff. Epid. Labe!lo libero 3lobo, disco callo magno obl. sulcato, col. auric. stigm. cavo cordato, anthera nuda terminalis. Parasit. bulbisterrestris bifolintis, spicatis-Type S. odorata R. Epid. do. W. reg. 1415, Encyclia patens Hock. b. m. 3013. 
Macradenia do looi) bulbis ovatis suicatis, fol. 2. ligulatis carinatis, sp. racem. paucifl. bract. breviss. sepalis obov. subeq. labello brevis. Brazil, green firgunt flowers. Whis like the next lias been shuhicd in various alien Genera.

907. Onunsmu R. (necklace stem) diff. Epid. petalis subeq. ovatis, 3 ext. basi gibbosis coalitis, labello contormi cuculato ovato acuto basi scrobiculato. C'aulib. claratis, articulis tumidis monilif. fol. distichis extr-rxilluris -Types 1. O. purpurea R. (Epid. monilif. I. Dendrobium do Sw. W. b. reg. 1314.) fol. obl. obliq. emarg. obt. petiolis dilat. amplex. China, f. purple-2. O. albiflora. R. Epid. monile Thunb? diff. fol. acutis, fl. albis. Japan.

998. J ENSOA R. (Jap. bot.) diff. Epicl. petalis obl. 2 internis, label. obl. basi cucul. apice deflexo obt. col. curva antice concava, stigm. obt. antheris 2 anticis, caps. teres tortilis. $\mathbf{S c r}$ pusa, genic. fol. ensatis.-Type I. ensuta R. (Epid. do L. Limodorum do 'I'b) fol. gladiatis, scapo terei $\mathrm{i}$ genicul. bract. vagin. lanceol. Japan, f. yellow, spicate?

909. Jinensia R. (Jap. bot.) Petalis ovatis concavis, 2 internis, label. trificlo emarg. basi callis 2 obl. medio concavo, col. filif. incurva, stig. hifd. concavo, antheris 2 dorsalis, capsula clavata. Scopose, fol, gladiatis, fl. spicatisType J, nervesa R. Limodorum striatum Th. f. jap. scapo angulato, fol. rad. glad. nerrosis, fl. cernuis, bracteatis. Japan, f. yellow. 'The G. Limodorum contained many anomalies also, sp. with or without spurs, beards or no beards, many kinds of pillars or styles or clinandres \& $\mathbf{c}$. The shapes of capsules will afford good characters in Orchides too much neglected heretofore. The Japanese, Chinese, Hindu and Arabic bo- 
tanists deserve comemoration like ours.

910. Dinotnon R. (" wats) ditt. Aper.. Lav bello tripartito basi bicalluso, lac. nurdia unguic. ovata acuta, col. clavata, cap-ula filiformis. caule arlic. clavato, bifoliata. scap)is syenrm. imbric. fl. spicntis-Type \$). clectum K. Epid. do 1. \&e. bot. reg. 1840. fol. tern. lanceolatis binispetalis lin. conneatis acutis. Cumana, fl. green, lip white.

911. Dicropinca IR. (bicul. fol.) petalis 3 superis galeatis coalitis basi gibbosis, "z inferis patens, labello oblongo basi bullato didymo adnatoque, longe unguic. canalic. Anthera operculata 2loc. "pollen. Scrposa, riazomula, fi. spicalis-'Type D. clegans $\mathrm{L}$. Gocdyera discolor E. bot. reg. 271. fol, ovatis ellipt. cuspid. subtus margineque rubris, scapo spathaceo. Brazil, fine sp. rhizona violet, fl. white, very long claw to the lip.

912. Inmex $\mathbb{R}$. (Jap. mane) petahis resupinatis unguicul. oratis, 3 cxternis, labcllo supero ovato basi bicalloso apice galeato, col. semiteres, antheris 2 sess. caps. filif. tortilis. Scrpo-

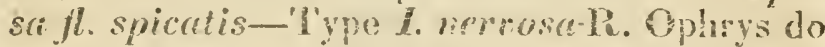
'ih. fl. jap. fol. rad. oratobl. nervosis sulcatis, scapo nudo angulato. Japan, spike of purplish incarnate flowers. Unlike any of the fir. remo. red from Ophrys, habit of Spiranthes and Goodyera.

913. Dotmlopme R. (wa!t crested) diff. Epid. sepalis 3 lane petalis 2 ovatis, labello conforme orato acum. integro concavó, basi callo suicato cristato, ad col. adherens. caulescens $f$. spicatis amplis bracteatis-Туре $D$. purpurec R. Epid. Skinneri Lindl. b. reg. 188!. fol. amplex. lato lanceol. acutis bract. obl. breris. Cruatemala, fine large purple flowers, 
another blended G. the warts or glands of the lip afford excellent generic characters.

914. Psychusis R. (butterfly lip) diff. Epid. petalis eq. labello unguicul. libero, latere bialato, disco incrassato lobato, apex obcord. Bulbis terestris trifoliatis, scapo raginato $f l$. thyprsoideis-Type Ps. amenu Raf. (Lipid. bifidum Aubl. Sw. W. Red. 64, b. reg. 1879) Bulbis obl. artic. striatis, fol. term. 3 ellipt. obt. trinervis fl. laxis, petalis obl, acutis. South $\Lambda$ mer. beautiful and strange flowers, petals green dotted purple, the lip tricolor white red and yellow.

915. Psrcnopsis R (butterfly form) difi. Oncidium, petalis bilabiatis, 3 cxt. eq. lin. erectis, 2 internis recurvis falcatis unclul. labello 3 lobo, disco crista triloba, Col. bialata fimbriata glandul. anthera term 2loc. goleata 4pollin, Balbis terrestris unifol. scupo arlic. piaurifl. - Type P. picta R. Oncidium papilio Lind. b. r.910, H. b. In. 2795. Bulbis violaceis, folia ovata fusca, viride picta, scapo anceps. Trinidad, quite distinct $G$. near the last, large flowers 3 or 4 inches wide, variegated of yellow and orange.

916. Gruzonon $\mathbf{R}$ diff. Oncilium, petalis eq. la hello truncato mucron. sinuato, disco lame!lis sinuato dentatis truncatis, Col. bialata, gynizus bident. Bulbis ter. fol. subtus et supri bulbis, scapis paucifl-Туре G. vusselirmum $\mathbf{R}$. Onc. do Lind. b. reg. 1830. Bulbis ovat. angul. fol. lanceol. petalis ovatis acum. label. obovato. Brazil, large flowers fuscate, lip with purple spots. What different G. from the last!

917. Lophiaris. R (thick crest) Petalis carnosis conformis, labello pandurato, disco crista triloba carnosa, Col. alis carnosis, anthera 
cristata. Acaulis,scapo ramoso-Type L. fragrans Raf. (Epidendrum! lanceanum Lind. b. reg. 1887) tol. lato obl. rervosis carnosis, $\mathrm{fl}$. confertis, petalis ovatis undulatis. Guyana, fl. yellow with purple spots, lip purple, fine smell like Aerides and Pink. Genus nearer to Oncidium and the last than to Epidendrum!

918. Ditulima R. (2. warts stig) diff. Dend.obium, pet. ext. ovat. connatis, 2int. minor, lab. unguicul. cuncato emarg. sine callo, stigma bicalloso inter cavitas, anthera pedicel. pollen 4 didyma libera. Cuule arlicul. fol. distichis, $\mathrm{f}$. axil.-Type D. anceps R. (Dendr. do Sw. W. b. reg. 1239.) caule compresso, articulis cuneat. truncat. fol. lanceol. ensatis, fl. nutans, lab. crenulato. Bengal, Pegu, fl. greenish.

919. Pierardia R. diff: Dendrobium, pet. 3 ext. lanc. 2 int. latior, lab. spatulato, unguic. lato invol. basi saccato calcarato. caule parasit.artic. fol.alt. $l$. spicalis-Type $P$. bicolor R. (Dendrob. pierardi Lod. 750 , Hook. b. m. 2584 ) fol. lanceol. patulis, labello obovato acuto. Bengill. petals white, lip yellowish base purplish. How different from last genus!

920. Passtreris 13. (quite twisted) petalis omnis difformis angul. tortilis, 2int. minor, lab. stipit. basi et apice saccato, medio plicato, col. bicornib. 2 tubul. mellilluis, Bulbis ter. unifol. scapo unif.-Type P. paradoxa Raf. bulbis costatis, folia lanceol. 3nerva, scapo pendulo, bract. tortilis, ovario sulc. non tortilis. Caraccas, flower yellow with purple dots, lip orange, a most singular flower which Hooker could hardly describe and has wrongly united to 2 Genera, heing his Gongora and Coryanthes macrantha, b. misc. 80, b. mag. 3102 . I. ind. b. reg. 1811. 
921. Plectrelmintius R. (spur worm) diff. Angrecum, pet. cq. lanc. acum. lab. obov. rostrato serrato, calcar longissime vermiformis flexuoso apice bilobo, col. basi angul. rostellata, pollinis 2 caucatis. Rad. fasc. teret. fol. imbric, scapis divisis.-Type Pl. bicolor $\mathbf{R}$. Angr. caudatum Lind. b. reg. 1844. fol. canal. emarg. scapis pendulis flexuosis 4fl. West Africa fl. green, lip white, spur 9 or 10 inches longAngr. sesquipedale of Thouars is perhaps a second species.

922. Onkerirus R. (swelled beneath) diff. Maxillavia, cor. basi saccata, petalis subeq. connivens, lab. integro undulato, basi bitubercul. pollinis 4 ineq. binis anticis brevior. parasit. bulb. ter. scapis radic.-'Type O. pallidus R. (Maxill. pallidifl. Hook. b. mag. 2806.) bulbis teretis, fol. lato lanceol. "trinervis, scapo breve, bract. linearib. St. Vincent Antilles, ff. pale yellow. Maxillaria is another heterogenous $G$. of 40 sp. requiring revision, Lindley unites Colax and Xylobium to it wrongly, no constant characters even in pollen! compare Dendrobium and Xylobium squalens with this Genus.

923. Pentulors R. (5 warts like) diff. Maxillaria, sepalis 3 ext. longior liberis, petalis 2 minor basi coalitis, labello cuculato, integro, basi callis 5 paralelis cristeformis. Bulb. Ier. unifol. scapo squamoso parecifl.-Type $P$. discolor R. (Maxil. do Hook. b. m. 1549. Xylobium Iindl.) Bulbis ovatis levis, folia ovata ellipt. acum. multinervis, Jamaica \&c fiowers ochreleucos few on short radical scaly scapes. Quite unlike the last G. except in habit.

924. Tulexis R. (warts outside) petalis patulis tuberculatis, lab). undul, carinato truncato, 
col. erectia antice bident. postice 3dent. ciliata, anthera 8locul. capsula tereta tuberculata. Terrestris, rad. fibr. carn. scapis artic. unifol. unifl.-Type 'T'. bicolor Raf. (Brassavola tuberculata Hook. b. m. 2878) folia crassa graminea acum. supra sulcata, pet. lin. lanceol. Brazil, fl yellow with red spots, lip white'The type of the G. Brassavola was Epidendrum cuculatum b. m. 543 or Cymbidium do Sw. W. with undivided ciliated lip with flat claw and smooth petals, capsule of.c. Cymbidium included many G. the C. cchinocarpon and muricatum must be compared with this.

925. Lysimna R. (Nymph) diff. Brassavola and Tulexis, petalis eq. linearib. levis, lab. cordata acum. integro unque longior, col. integra postice calcarata, capsula clava levis. Epiphyta rad. vermic et bulb, unifoliata scapo squamoso pancifloro.-Type $\boldsymbol{L}$. bicolor $\mathbf{R}$. (Brassav. cordata Lindl. b. reg. 1914. folia ang. lanceol. carinata, scapo sq. remotis paucifl. Brazil fl. green, lip white, small round bulbs mixt with the worm like roots. 'Thus quite different from last $G$.

926. Dilonilis R. (2 brim lip) diff. Octomeria, petalis eq. patulis lin. labello libero ligulato vix trilobo, disco bialato vel cristatis 2 longitud. crassis, anthera opercul. 8loc. 8pol. caulescens, fol. distichis, fl. spicatis-Type D. serrata R. (Octom. do Hook. b. mag. 2823) fol, lin. lanceol. apice dentic, scrratis, spica laxa paucifl. Brazil, fl. whitish. Quite distinct in habit and characters from the real Octomeria with adnate lip, type Dendrob. angustif. W.

927. Enotire ea R. (nymph) diff. Octomeria, petalis subeq. connivens ovatis acum, lab. conforme basi bilobo tuberculis 2 oblongis, col. se- 
miteres, anthera ovata, 2loc. poll. 8 in fasciculis 2 coalitis. Rhizoma repens squam, scapis unifol. Al. axil.-Type E. graminifolia R. scapis setosis, folia lanceol. pedunculis axillaris geminata. Antilles. Wrongly put into 3 G. Epidendrum graminif. L. Dendrobium do W. Octomeria do Hook. b. m. 2764. All these G. with 8 stamens or pollen form the group Octomerides with many Genera.

928. Froscula R. (Nymph) petalis 5 lanerol. supero fornicato, 2 lat. cum columna adnatis, desinens cum labello in calcar conico, lab. cucul. infundıb. dentic. Col. brevis cava biloba, anthera 2loc, opercul. pollen sulcato bipartibilis caps. flexuosa. Caulcscens paucifl.-'Type Fr. hispida R. caulib. erectis hispid is flexuosis, fol. lauceol. obt. apice, oblique cmarg. fl. 1-3 term. sess. bracteis ovatis brevis calcar rigido obt. Mts. of Nipal, fl, white, lip with yellow streaks. Quite a peculiar $G$. although it is Dendrobium longicorne Lindl. b. reg. 1315. More akin to G. Pedilonum of Blume, but the type of it is Dendr. secundum of Sumatra, with petals equal secund like fingers, all coalescent with lip entire into a spur, stem articulate $\mathcal{f}^{\circ} \cdot$.

929. Hecabe R. (nymph) diff. Rletia, petalis 5 patulis, lab. calcarato 3lobo, lobis invol. crenato undul. col. libera semiteres, anthera ad apice immersa, pollen 4 bilobis. Bulbis ter. foliosis, scapis later. artic. spicatis.-Type H. lutea R. (Bl. woodfordi Hook. b. m. 2719) bulbis striatis, caule folioso sterilis, fol. lanc. acutis plicatis macul. scapis radicalis floriferis. Trinidad, fl. yellow. Very distinct G. the Genera Pachyne Sal. or Phaius Lour. Spathoglotis Blume,-Gyas Sal. or my Anthogyas, have 
all been blended in Bletia by Hooker \&c.Also my Hexalectris, see 940.

930. Cochleantues R. (shell fl.) petalis connivens ovat. undul. lab. cochleato bilobo, basi crista cochleata (ut pecton plicata, calcar col. clavata, anth. 2loc. 2labiata, pollinia 4eq. caulescens, fl. axillaris-Type C. fragrans R. fol. lanceol. 5aervis, pedic. 1fl. axillaris. 'Irinidad. fl. ample fiagrant white, lip purple. It is Zygopetalum cochleare Lindl. b. l'eg. 1857, but quite a different G. from Z. makui H. b. m. 2748 , with petals secund, lip flabellate with a spur, anther calciform, 4 uneq. pollen. 'Ihe 'Z. rostratum b. $\mathrm{m} .2319$ is probably another G. Mencudenium Raf. no spur, pillar winged, lunular gland \&c. The Epid. fragrans and emulum appear to form a subg. near these, BuцвoDrctis Raf. by petals free equal, lip cocheate entire bulbs reticulate \&c.

931. Cyphredium I. auct. 'I'his fine Genus has lately been increased by many sp. and some appear the types of peculiar Genera or subg. at least. One of them forms my Gr. Criosanthes established since 1817. I propose the following subgenera or rather Genera: for the N. Aner. sp. see my new flora.

932. Saconon R. (bag toothed) diff. petalo infero bident. lab. saccato orifice dentato, col. obl. incurva, 2 anth. calcaratis ad basis, appendice ovali obt. sulcato Caulescens.-Types 1 C. ventricosum W. Sweet t. l. pet. 2 int. angustis longis, orif. emarg. dentis parvis-2 C. macranthon W. Sw. Hook. b. m. 2938, pet. 2 int. subeq. ovat. basi villosis, lab. reticul. orif. parvo. - Both from Sibiria with red fl. smooth unifl. leaves ovate undulate nervose.

933. Stmegas R. (stig. large) diff. petalis 
ineq. sup. renit. cuculato, $\stackrel{\bullet}{\geq}$ int. ciliatis cuncatis obt. labello panduato hasi cucul apex calciforme, col. apex dilatata in stigma trilobo,anth. $\because$ ad basis lateralis. Scapo amifl. fol. rad. dislichis-Type st. vemslum R. Cypr. do 11 . b. 11. 21:29. Lindl. b. reg. 783. fol. lanceol. obl. acum. canalic, enervis macul. scapo villoso.Fine sp. of Nepal, leaves with purple dots beneatl, fl. varied of purple and green.

934. Cornula R. (nymph) diff. pet. sup. fornicato emarg. 2 lat. ellipt. undul. lab. brevi saccato integro non fisso, col. obcord. sine app. dorsalis, filam. 2 pendulis antheriferis ineq. bilobis. Scapo unifl.-Tyрe C. insignis R. Hook. ex. fl. 34. b. mag. 3412, Lod. 1321 fol. ligulatis, scapo piloso, bractea obl. obt. fl. eq. Also from Nipal, large green f. with purple spots and veins.

935. Menerhora R. (moon bearing) diff. lab. basi canalic. apex auricul. saccata, append. lunato. Scopo unifl-Type M. bicolor Raf. fol. obl. acut. macul. scapo pubescens, pet, sup. ciliato margine revoluto, ceteris obl. undulatis. Bornco and Java, seen alive in gardens, fl. dull purple, but upper petal white broader ovate.

936. Criosantres Raf. 181\%. Arietinun Beck 1833. diff. petalis 5 (non 4,) 4 linearis, 1 supero latior, labello parvo obconico inflato acuto, caulescens-Type Cr. parviflora $\mathbf{R}$. 1817. Arietinum Americanum Beck 1833. Cyprip. arietinum. Pursh and Amer. botfol. lanceol. pet. sup. ovatobl. acuto pet. 2 inf. deflexis, lab. reticulato. Canada and Mts. of Vermont \&c. fl. small, greenish brown, having the aspect of a Ram's head whence my name. This G. even lack the character of $\mathbf{4}$ petals, I 
have it in my herbal. Cordu!n lackis the appendix, yet these 2 characters were the main of the Genus, that must therefore be reformed. 'They are the types of my family of Dipiantulres established 1815 in orler of Oncundes.

937. 'Tumcunina Raf. (Birm. name) diff. $\boldsymbol{E}$ pidendrum, petalis 3 ext. lanceol. obt. binis inferis gibbis coalitis, 2 internis ovalis major, lab. cuculato basi carinato, apice bicorne, col. adnata inter cornib. enata, antheris 2. stig. fovea melliflua desinens in tubo ovario penetrans, capsula longissima teres. Fruticosa scundens, articulata, fol. alt. distichis.-Type Th.moschata Raf. Epid. do, Buchanan ic. in Symes travels. Ramosa sulcata, fol. bifaria lanceol. obt. spicis laxis oppositifol. In the Birman empire, fl. yellow very fragrant. purple spots on lip, bracts striated. I thus add another beautiful G. near Vanilla, same habit, but also akin to Dendrobium, diflererit from all by lip and stigma.

938. Isotria Raf, 1808. pet. ineq. 3 ext. eq. longis lincarib. canalicul. 2 int. ovatolanceol. dimidio brevior, lab. trilobo, lobo medio undul. emarg. disco glandula magna tubereul, cristata. subtus sulcata, col. subquadrata, stigna fossula scabro, anthera term. mobilis operc. pedicelata quadrata, caps. filif. clavata. Tollo fuscic. fol. term. vertic. caule unifl.-Types 1. Is. certirillate Raf. $\Lambda$ rethusa and Pogonia do auct. fol. 5 obl. lanceol. acum. petalis juternis ovatis obtusis. Swamps from New York to Georgia says Beck, 1l. fuscate outside, pale inside, 2 . f. medeoloides. Arethusa do anct. fol. 5 obov. acum. petalis internis acutis, Woods of New Jersey \&c, podal, fl. fuscate purplish outside inner petals yellow, lip dull yellow, gland yellow. 
Under the names of Arethusa medeoloides and vertic. the American Authors have blended 2 or 3 plants, of same habit, very unlike the other Orchides, but hardly of same Genus, which I noticed since 1804, see my new flora for other particulars. All quite distinct from Pogonia ophioglossoides in habit and flowers. Many $\mathbf{G}$. were blended in Arethusa, the $\mathbf{A}$. ciliaris $\mathbf{L}$. else Orchis pectinata W. is the Bartholinia do of Brown akin to Blephariglotis but habit unlike unifoliate.

939. Odonectis Raf. 1808. Pet. ineq. 3 ext. lanceol. acut. 2 int. cuneatis emarg. lab. cuneat. 5 dentato, capsula clavata. Cetera et facies ut Isotria-Type $O$. verticillata $\mathbf{R}$. fol. 5-6 rotatis obl. lanceol. fl. 1-3termin. sessilib. Mts. Alleghany, disc. 1804, pedal. perhaps a subg. of last.

940. Hexanectris Raf. 1825 neog. diff. IBletia, lab. ecalcarat. (icristato, col. clavata, antheris 2 fissura dehiscens. Bulbosa, aphyla, scapo squamoso $\boldsymbol{l}$. racemosis- Type $\boldsymbol{H}$. squamosa Raf. Arethusa spicata Walt. Bletia aphylla Nuttal \&c. Scapo tereto, squamis ovatis imbricatis, labello divaric. trifido venoso. Florida \&c, pedal, fl. brownish purpie. Quite distinct by habit from all the Bletias, also by lack of spur and not 4 anthers.

- 941. Anistylis Raf. 1825 neog. Liparis sp. Lindl. diff, petalis patentib. 3 cxt. lincarib. 2 int. filiformis, lab. obovato integro, hasi melliflua, apice bialata involvens anthera opercul. bilocul. capsula tortilis, bulbosa, bifoliatra. fl. spicaits. - Types the American sp. of Malaxis, blended even with M. lilifolia and loeseli of Europe, although not even of same Genus. 1. A. convalluvisu. 2. A. lutea soc, My sulog. Diphrygllum 
1808 difters by internal petals bifid. The real G. Liparis or rather iny Mesoptera (since there is a $\mathbf{G}$. of fish Liparis) differs by 2 lateral wings to the middle of the pillar and bilobed lip. The G. Listera of Brown differs by wingless column. All have the same habit, see my monograph in New Flora.

942. Drturlis R. (2 wart lip) diff. from Lis. tera and Liparis by 2 warts on the lip, parasite plants. Type D. nepalensis Raf. Liparis bituberculata Lindley.

943. Acroanthes R. 1808 (uncolored fi.) Microstylis Nut. 1818. This G. was once a Malaxis, 1 published it ten years before Nuttal in my remarks on Am. Gen. See my Monograph in New Flora, but Lindley has added many sp. to Microstylis some of which require revision.-I could still increase my $\mathbf{N}$. G. of Orchides by other types, but these surely evince how much remained to be done in revising them. The lips, spurs, crests, pillars (also called clinandre, androphore \&c) glands, capsules \&c, offer good characters often neglected for the anthers and pollens. The uniflore Disa like D. grandiflora are the type of the Genus, $D$. cormuta and other spiked sp. must form my G. Gramaria-Eria and Stenia of Lindley worst of his names are my Exeria and Stenopolen -His Sophronia or Sophronitis, name preocupied is my Lophoglotis of c. His Georchis is inadmissible unless Orchis be abolished, it is my Geobina. His Mon-Acanthus is my Cuculina $\mathcal{f}^{\circ} c$. I now conclude the Orchides and shall end the Monocotyles by a few G. of DriMIRIIIES.

944. Ennoconos R. (in bell) diff. Maranta, 
cor. triplex. ext. 3part, eq. media 3part. ineq. interna 3part. ineq. 2 segm. latior. 1 labellif. cucul. anthera unica 1loc. ferens, stylus filif. basi coalito, stigma infundib. capsula 3loc. 3sp. Scaposa, fl. congestis panicul. in spatha campanulata.-Type E. zebrinum Raf. Maranta do bot. mag. 1926, bot. reg. 385 . fol. longe petiol. ellipt. obt. maculatis, scapis fol. brevior, spathis erosis 2-3fl. Brazil, singular habit akin to Calathea.

945. Zelmira R. (nymph) diff. Calathea,cal. 3sepalus, cor. duplex. infundib. limbi 3part. lacinia 1 major difformis cuculata cornuta, filam. bilob. lobo unico antherifero, stigma fistulosum rostratum, caps. 3loc. 3sp. caulescens, fl. capitatis, spathis concentricis.-Type Z . violacea Raf. cal. do Lindl. b. reg. 961. fol. ovatis petiol. capitulo ovali multifloro. Brazil. The real G. Calathea has corolla regular, 3 petaloid stamens, 2 sterile, style curved, stigma concave, type C. grandifl. C. longibracteata, unless the G. Calla and Thea. become Provenzalia and my Theaphyla 1830, this will be an erroneous compound.

946. Siphotria 12. (tubes 3) diff. Alpinia, cal. duplex utrinque tubulatis bilabiatis vel. ineq. 3lobo, labio 1-2dent. cor. tubulosa labio incluso, stam. sessilis. Caule folioso diviso ad scapo squamoso.-Type S. squamosa Raf. Alp. tubulata Edw. b. reg. 737. fol. lanceol. undul. acutis, scapo radic. brevis squamoso. Guyana, fl. redish. Quite distinct from Alpinia by tubular perigones.

947. Bojeria R. (bot.) diff. Alpinia, cal. 3 fid. ineq. stylo filif. pubesc. basi glar da biloba, stig. capit. fissura transv. caps. 3loc. sem. pyrif. in pulpa.-Type B. magnifica R. Alp. do. Ros- 
coe, Hook. b. mag. 3192 who says it ought to be a Genus. Mauritius Id. If Bojer had a G. already, we may substitute Doxanties meaning glorious flowers. Brown has wrongly united Renealmia of L. to Alpinia, and framed a N. G. Renealmia my Ezcria near my Olsynium differing by unequal petals, but his R. pulchella with 3 inner petals subeq. unguic. and free stamens is probably an Orthrosantes.

948. Ilytuuria R. (nymph) diff. 'Thalia, cal. 3part. eq. acum. cor. tubo trigono, limbus duplex, ext. 3fid. eq. undul. intern. 3fid. ineq. uno segm. cuculato, filam. petaloid. bipart. anthera unica clavata adnata, stylo teres incurvo, stig. concavo, drupa uniloc.-Type 1 l. cannaformis Raf. Thalia do, Forst, Pers. Buch. ic. in Symes travels, dichot. fol. ovat. ellipt. acum. petiolis spathaceis, pedic. artic: furcatis multif. fl. alt. spathis lanc. from Pegu to Polynesia.-The Thalia geniculata of America agrees in nothing but the fruit a drupe stated to be bilocular by L. the corolla has 5 petals only, the 2 internal urceolate, the filament is lanceolate \&c. It is the type of 'Thalia.

949. Spirostylis R. diff. Thalia, cal. 3sepalis subeq. ovatis acutis, cor. duplex. 6petala, pet. 3 ext. obov. eq. 3 int. ineq. filam. petaliform apice concavo, basi ferens anthera loc. longe pedunc. filif. stylo spiralis, stig. magno labiato concavo; fructus obov. levis nux flexilis 1 loc. 1 sperma. Acaulis, fl. panic. spathis 2valvis 2fl-Type $S p$. biflora Raf. Thalia dealbata Rosc. Pursh. El. \&c fol. petiol. ovatis, fl. panic. spathis 2fl. subeq. convol. Florida and Carolina, seen alive, fl. purplish binate small sessile. Nearer to Ilythuria by fruit and corolla, but 
distinct by petals, style, anther. I had called it Malacarya in $181 \%$.

950. Xyphostylis R. diff. Canna, cor. basi tubulosa, segm. 3 int. ineq. undul. filam. ineq. bipart. anthera adnata, stylo ensiformis, stig. lin. involuto-Types 1. X. lutea Raf. Canna flaccida Rosc. and Amer. Authors, fol. ovatobl. fl. luteis-2. $\boldsymbol{X}$. angustif. R. Canna do W. auct. fol. lanceol. fl. rubris, cor. segm. int. rectis emarg. Both from Florida to Louisiana. The real G. Canna (root of Canna-bis) or rather Katubala of Adanson has style clavate, stigma obtuse and other differences.

951, CRATODIA Raf. 1815. This order of mine distinguished from Orchides by a calix and fruit commonly multilocular, was the Nat. family Scrtaminea of Linneus; but this name has been restricted to the G. Musa, Heliconia and akin with berries \&e by Ventenat. The Gingembres of Adanson or Cratophytorum of Necker included also the Bromelides properly separated by Jussieu. But many Nat. families are included in the Cratodes, besides Scitamines, such as

Dryminizes of Ventenat, Types Amomum, Maranta, Alpiria, ofc Curcumides a subfamily with several stamens.

Stratides Raf. 1815, types Stratiotes, Hydrocharis \&c, and perhaps Aspidistra with Macrogyne.

The Valisnermes and Pistides with unilocular fruit, may with the Cratodes that have such a fruit, be formed into a link with Orchides, as a Sub Order of either, or perhaps a peculiar order Synarmes Raf. 1815. To revise all the akin Genera would be a task, at present I shall merely indicate two old Genera of Adan- 
son and Necker that appear to have been forgotten. It has been properly stated that notwithstanding the labors of Roscoe and others these families require as much correction as the Orchides.

952. Psydaranta Necker 1488, diff. Maranta, cal. 3sepala caduca, cor. tubulosa 5 part. 1 lac. bifida, anth. obl. tubo insidens pedic. stylo clavato basi adnato, capsula ovata 3 loc. polysp. Scaposa, spathis bifar. Type Ps.comosa Raf. Maranta do L. auct. which L. intimated was a peculiar Genus, the caulescent types of Maranta have a drupe 3loc. 3sp. stig. trigone, cal. 3part. cor. ringent \&c.

953. Zingiber Ad. diff. Amomum, cal. tubul. 3fid. cor. duplex, ineq. bilab. lab. sup. integro, inf. bipart. cor. interna 3loba of c. This G. Ginger must be restored, it includes several sp. and the Am. zerumbet; but A. zedoaria is perhaps a Genus Zedoaria Raf. The G. Pacoseroca of Adanson was Amomum L. with equal corolla. The Costus echinatus W. P. must form a G. Acinax Raf. diff. from Costus by capsule baccate echinate. The G. Hellenia same as Helenium L. must be changed into Allagas Raf.

954. Melorma R. (honey pits) diff. Fritillaria, petalis 6 campanul. 3 int. obov. latior, omnis basi ext. gibbosis, intus fossula melea, stam. 6 glabris filif. anth. lin. stig. 3. Fol. opp. et vertic. cirrhosis, fl. axill.-Type 1R. cirrhosa R. Frit. do Grah. Imperialis leucantha Fischer. Fol. inf. oppos. ovatis, sup. vertic. lin. lanceol. carinatis cirrhosis, fl. paucis axill. pendulis. Altaic Mts. fl. white. Add this G. to the Lilies near my G. Baimo 867, with same habit, and my IIexorima of 1808 sp. of Streptopus. 


\section{I C O T' Y L E S . (Peripetalic.)}

955. Veronica L. I begin the revision of this fine series of plants by a Genus that I had divided into ten groups before 1815 : few Botanists have seen the necessity of this, and except Hebe hardly any have been attempted ever since, except Leptandra of Nuttal which was my Calistachyre of 1808. Yet this $\mathbf{G}$. contains over $100 \mathrm{sp}$. offering the greatest anomalies of good characters. 1 shall now reform it into 14 Genera and 8 subgenera quite distinct, that form quite a peculiar family my VERONICIA of 1815, with Pederota, Solomonia and all the diandrous G. wrongly united to Pediculares by Jussieu or Scrophularides by others. They are easily known by corolla subregular, 2 stamens, one stigma, capsule bilocular, partition contrary to valves of $\mathrm{c}$. The real $G$. Veronica will be yet very prolific and known by calix 4 partito ineq. 2 minor. corolla subrotata, 4loba subeq. 1 minor, stam. 2 glabris filif. stylo filif. capsula compressa. 2 locul. sub 4valv. oligosperma:-It contains 7 subg. at least.

1. Becabunga R. caps. obcord. compressa, cal. ineq.-Types almost all the sp. Ver. becabunga, anagallis, arvensis, digitata, precox, reniformis, pectinata, and many other sp. but the Authors seldom notice the differential characters.

2. Isonica Raf. capsula obcord. vel. bisculata, compr. cal. 4part. equalis.-'Types V. chamedrys, peduncularis, urticifolia, biloba, verna, pinnata, scutellata, complicata \&c.

3. Fimbrula R. caps. biscutata, cal. ineq. 
ampla fimbriato.-Types V.agrestis, calycina, and akin species.

4. Orbonica R. caps. orbicul. compressa,cal. obov. amplo-'Type $\boldsymbol{V}$. arguta \&c,

5. Dodecula R. cal. 4part. subeq. cuneatis trifidis-Type $V$. glauca Sm. fl. gr. 17. procera, fol. cord. serrat. ped. 1floris.

6. Alponica R. cal. bracteato cil. caps. obov. emarg. stylo persistens, stig. capitato.-Types V. alpina, Wormskioldi, \&c.

7. Endasia R. cal. 4part. corolla 4part: undulata, crenata faux villosa. caps. ovata semi 4 valv.-Types $\boldsymbol{V}$. maritima, spicata, spuria, crenulata, elliptica \&•c.

8. Meionica R. cal, 4 fidus subeq. caps. orbiculata vel truncata-Types $V$. montana, taurica, pedunculata \&c.

956. Odicardis R. (2 teeth heart) diff. Veronica, cal, diphyllo. sepalis magnis cordatis serratis bidentatis. Capsula biscutellata 2 loc. 2sp. marg. dehiscens, sem. concavis pendulis rugosis (in V. plerumque planis levis)-Type Od.cristagalli R. Ver. do Stev. Sn. fol. ovatis serratis sess. fl. solit. axill. Mts. Caucasus.

957. Panoxis R. $1830 \mathrm{~m}$. fl. diff. Veronica, cal. 4 part. eq. cor. tubulosa 4 fida eq. caps. obl. acuta.-'Types V. salicifolia, cataracta, macrocarpa \&.c, compare with 960.

95\%. Derwentia R. diff. Veronica, cal. 4 part. eq. cor. vix rotata bilabiata, lab. sup. integro erecto lanc. acut. lab. inf. trilobo, stylo declinato-Types 1 D. suaveolens $\mathbf{R}$. (Ver. derwentia Andr. rep. t. 531. V. labiata Br. Sm. H. b. m. 3461.) fol. lato lanceol. acum. serratis, racemis axill. elong. Australia fl. white fragrant 2 D. perfoliata R. (V. do Br. b. mag. 1936) 
fol. ovatis connatis 5nervis acutis, racemis gracilis. Australia fl. blue. Probably many other sp. of Oceania.

959. Ponaria R. $1330 \mathrm{~m}$. fl.-calix 5part. subeq. cor. 4 loba subeq.-Types Ver. pona, latifolia, laciniata \&c. The G. Amphianthus 'Torrey differs by cor, tubular infund. stam. included, stig. bifid.

960. Eustaxia .R. 181\%, Calistachia R. 1808 not of Vent. Leptandra Nut. 1818, diff. Veronica, cal. 5part. subeq. cor. tubulosa, apex 4lob. ineq. filam. exertis filif. stylo similis. caps. ovata acum. 2loc. polysp. apex bivalvis, valvis inflexis, placenta contrario. Fol.vertic.oppos. fl. spicatis.-Fine G. first distinguished by myself in 1802, published 1808, hardly of family Veronicia like the two next. Several American and Asiatic Sp. see my monographs of 1820 of Med. fl. 1830, and of New Flora : E. alba, purpurea, villosa, oppositifolia, sibirica, japonica \&c. It belongs to family Lruacines? with Lilac, Nyctanthes, Hebe \&c, the capsular $\mathbf{J}_{\text {AS- }}$ MINIDES.

961. Hebe Com. Jus. diff. Veronica, cal. 4 part. cor. hypocraterif. limbus 4lobus, caps. ovalis biloc. valvis 2 invol. placenta centralis, stipitato. Frutex. fol.opp.racemis, panic.Removed by Jussieu to the Jasminea, akin to Lilac-Type $\boldsymbol{H}$, magellanica Juss. fol. perennantib. ellipt. integris, racemis axill. called Veronica decussata by $W . P$. and nearly all the Authors.

962. Alloplein R. 1830, diff. Veronica, cal. campan. 4-5fid. cor. rotata 4-5fida ineq. stam. 3-4incurvis, stig. truncato, caps. obcordata. Caule artic. fl. axill.-Type A. rotundifolia Raf. Ver. do RP. fol. subrot. 1-3 ad articulis. 
Peru. Although the capsule is quite like Veronica, the extra number of stamens render it a doubtful G. of the family.

All the Genera of capsular peripetal flowers with 2 stamens ought perhaps to form a peculiar order ARINEMIA, and then the families Veronicoides, Justicoinns, Iillacines \&c, should belong to it, as well as Gratromues with unilocular capsules-Cextririma Raf. 1815 with spurs, types Pinguicula and UtriculariaCalceolines, types Calccolaria, Bacola, Jovelana, Pederota, Wulfenia \&c. This would be a very distinct natural order, although some may contend that as in Labiates, 2 or 4 stamens are not essential, why not as much as valves and placentas?

963. Ygranela R. 1833 atl. J. diff. Limo. sella, cal. camp. 4fid. ineq. cor. camp. subeq. 5 loba, 2 erectis, 3 patulis inf. stam. 2 , stylo brevis, stig. bilobo, caps. globosa uniloc. bivalv. polysp. sem, centralis, fol. vadic, scapis unifl. -Type Y. maritima, R. fol. flif. obt. glabris, scapis fol brevior. Sea Shores of N. Amer. in New Jersey, cespitose, uncial, fl. bluish white. - Limosella silesiaca and capensis having two stamens are probably 2 other sp. but if they have the calix 5 fid as in Limosella, they must form a subg. or G. Trutafenic Raf. This with the next $G$. is of fumily Gratrolinss. Limosella with 4 stamens and cal. 5fid, was wrongly put among Lysimrechides by Jussieu, it must belong with Besleria, Maturea and many $G$. akin to Gratiola by uniloc. capsule, to the family of Mesopiones, that lrave 4 unequal stamens.

964. Prutunela R. 1833 (small pelt. stig.) diff. Limoseila, calix 3 dent. cor. 4 partita, stylo incurvo, stigma peltato, stam. 2.-Type P.cun- 
eata R. Limos. diandra L. \&c. fol. lin. cuneatis, scapis equante-India. Quite distinct G. although habit similar, belonging to GratioliNES as well as Hemianthus, Micranthemum \&c.

965. Ilysanties R. 1820 annáls. This includes the American sp. of Lindernia, with cor. tubut. bilab. 2fertile stam. and 2sterile, stigma bilam. caps. uniloc. myriosperma, placenta fiee central. Many sp. see their monograph and that of our Gratiolas in my new flora. Gratiola includes also several Genera, and the subg. Psidiola, without calicule.

966. Ambulia Ad. diff. Gratiola, cor. tubul. vel urceol. limbo 4 lobo subbilab. inf. 3lobo. stam. 4 fertiles in tubo. stigma bilobo.-Type A. alata R. 4 gona Hook. b. m. 3134. glabra, caule 4 gono 4 alato, fol. lanceol. serrat. subauricul. fl. subspic. sess. cor. lobis retusis. cal. 2 bract. B. Ayres, f. blue-2. A. rigida R. (Gr. do fl. tex, 5. Gr. acuminata Walt. El. \&c) glabra rigida, caule 2-4gono, fol. rhomboideis, basi cuneatis, apice serratis obtusis, fl. axill. ped. cal. ebract. cor. lobis subdent. Florida to Texas. Subg. Aotilix by no calicule. Some asiatic sp. belong here. This Gr. by 4 stamens belongs to my family Antrostomia 1815 which has many subfamilies Celsides, Digitalides, Antirhinides besides Herpestides to which it belongs with 4 G. blended in Herpestis, Monniera, Habershamia, Macuillamia, see my Neogenyton 1825 and new flora, where all akin G. are rectified. The G. Bramia of Ad. only differs by upperlip bifid forming a 5lobed corolla.

96\%. Isoloba R. diff. Pinguicula, cal. camp. subeq. 5lobo, cor. camp. equalis 5loba, lobis emarg. calcar subul. stam. 2 connexa, antheris peltatis adnatis, stig. sess. petaloideus bilab. 
caps. globose 1loc. 4valvis?-Types 1. I. lutea R. (Ping. do Walt. Vahl, Mx. L. \&c. campanulata Lam. Pers. \&c.) pubescens, viscidula, fol. ovat. obt. fl. luteis, calcar brevis recto. Carol. Florida $f \cdot c-2$ I. pumila R. (Ping. do $\mathbf{M x}$. P. E. \&c,) pumila, pilis capitatis, fol. ovat obt. f. pallide cerul. cal. et caps. villosa, calcar tubo eq. Carol. Florida-3. 1. recurva R. (Ping. edentula Hook.) pubescens, fl. nutans luteis, calcar recurvo cor. brevior, fol. sess. ovatis acutis.-4. I. elatior R. (P. do Mx. P. E.) fol. spat. ovatis obt. viscidis, scapo basi villoso, $\mathrm{ft}$. purpuro striatis, corolla tubo ventric. calcar compr. obt. Is the $\boldsymbol{P}$. acutifolia $\mathbf{M x}$. a real Pinguicula or same as Is. recurva? Ping. lusitanica appears another sp. of Isoloba, the cor. having 5 lobes eq. emarg. stig. conc. bilab. but the spur is only saccate-The real European G. Pinguicula differs by cor. bilabiate, capsule oblong or obcordal. P. cristallina fl. gr. t. 11. has spur saccate, capsule globose.

968. Justica Necker, Justicia! L. auct. Adatoda Tourn. Ad.\&c. The tropical regions swarm with plants referable to this group, rather than Genus. Linneus had 37 sp. with Dianthera, Persoon had 100 in 8 sections, each real Genera. Vahl and Vitman had $\mathbf{1 1 7}$ and now we know nearly 200. Dianthera was improperly united by some botanists; but Necker had 2 detached G. Geunsia and Roslinia; he properly stated that Justicia like Veritas or Prudentia could not be generic names without modifications. Few Genera offered so many distinct characters for good Genera, as stated by $\boldsymbol{R}$. Brown, 40 such will be afforded when all the sp. will be well revised: meantime Crossandra, Apelandra, and Elytraria have been separated. 
I will now offer 28 typical Genera, two of which were in first part 311,378 . The real JusticorDEs have all 2 stamens, and are a group very near to Veronicordes, while those with 4 stamens are nearer to Ruellia in the family of Acanthides. - 'The real G. Justica will still contain all the sp. with calix simplex, 5part. ineq. cor. tubul. bilab. sup. emarg. vel bident. inf. trilobo, vel 3fido anitheris monoloc. sp. 19 to 42 of Persoon ; but even this affords subgenera, such as.

1. Ecbolia Raf. cor. tubo filif. incurvo, lab. sup. lin. bifido reflex inf. ineq. trifido-Types $\boldsymbol{J}$. ciliata or Ecbolium L. with most of the Justicia 19 to 32 of Persoon.

2. Gandarusa Rumph. cor. tubo recto, $f$. verticillatis, 'Types J. gandarusa, vertic. and others akin.

3. Pulcolia Raf. (n. ind.) cal. parvus, tubo cor. filif. lab. sup. linearis angusto integro? reflexo, inf. eq. sfido. stam. exerta. 'Type J. nasuta I. and akin sp. a link with next Genus.

4. Hirselina Raf. cor. pubescens, filam. hirsutis, capsula compr. marginata. Type J.paniculata $\mathbf{L}$. and akin.

5. Loncotoma Raf. (cut lance) cal. foliaceus ineq. lab. sup. recto lanceol. bifido.-Type $J$. carthaginensis I. \&.c-See till 990 for the revised Genera. 'The G. Elytraria chiefly differs. by habit scapose, cal. 4 parted, cor. subeq. 5lobo The G. Nelsonia of R. Brown, very akin to this differs by no sterile stamens, Australian G. of 2 sp. N. rotundif, campestris. Aphelandra of R. Br. chiefly difiers by habit, 4gone bracteate spikes, lower lip subentire.

969. Adatoda Raf. diff. Justica, cor. ringens, lab. sup. recto concaro integlo.-Types $\boldsymbol{A}$. 
arborca R. (J. adatoda L.) A. betonica, repens? \&c.

970. Stethoma R. (pectoral) diff. Justica, cal. camp. eq. 5part. cor. campanul. lab. sup. brevis integro fornicato, lab. inf. dilatato equalis 3lobo-'Type st. pectoralis R. (Just. do Vahl. Siv. Jaq. 3 t. :, Vitm. W. P. b. reg. 796.) fol. ov. lanc. brevipet. spicis gracilis panicul. Antilles, fl. small red, balsamic: perhaps other sp. akin. Put among the Diantheras by L. and some botanists, but others say anthers uniloc.

971. Geunsia Necker, Hypoestes R. Brown, diff. Justica, cal. duplex ext. 4part. interno tubul. 4fido. Cor. resupinata tubul. lab. sup. trident. infero integro, nectario ad disco campanul. postice fissum, bisetoso, caps. loc. 2spermis -Type G. fustuosa Raf. (Just. do L. auct. panicul. Forsk) frutic. ramis teretib. fol. petiol. ellipt. fl. panic. thyrsoideis. Arabia, India. Certainly a very peculiar G. perhaps the sp. 2 to 8 of first Section in Persoon belong to it,but the flowers must be verified. Hypoestes a posterior name may be applied to J. Forskalei and floribunda as a subgenus.

972. Adelona R. (n. ind.) diff. Justica, cal. duplex vel. calicul. sep. 2. cor. tubul, bilab. utrinque integris, antheris 2loc. divisis ut $\mathrm{Di}$ anthera.-'Types 1. A. serrata R. fol. ovat. serratis. Malabar fl. white, figured by Rheed. 9. t. 43-2 A. iniegra $\mathbb{R}$. fol. ovatolanceol. integris. Meganesia fl. red, fig. Rumph 6. t. 22. Both blended in Just. bivalvis L. \&c, habit similar fl. ped. axil. $6 f l$ bracts ovate.

973. Kuninia R. (n. ind.) diff. Adeloda, calic. 2 ineq. cor, lab. sup. falcato (emarg?) lab. inf, 3lobo.-'Iypes $\boldsymbol{K}$. falcata, malabarica, \&c Just. do auct. 
974. Carima R. (n. ind.) diff. Adeloda, cal. duplex utrinque 4part. cor. villosa, labiis .... A. spic. vertic.-Type C. sulcata R. Just. et Dianth. do auct.

975. Marama R..(n. ind.) diff. Justica, cal. parvus camp. urceolat. eq. 5 dent. cor. ringens, lab. sup. incurv. emarg. inf. patens 3fid eq. faux cor. inflata.-Type M. picta R. Just. do L. beautiful asiatic sp. often figured, bot. reg. 1227. Shrub with red fl. and leaves yellow in the middle, ovatoblong acum.

976. Lustrinia Raf. diff. Justica, cal. 5part. eq. cor. tubulosa tereta recta, labis parvis angustis, sup. integro, inf. 3crenato-'Type L. geniculata R. Just. do b. m. 248\%. fol. ov. lanceol. acum. paniculis laxis cernuis, bract. subul. Antilles, fl. scarlet. Is it a subg. of Adatoda? are the anthers uniloc?

97\%. Dianthera L. diff. Justica, stam. antheris 2locul. loculis remotis sepe ineq.-This contains the sp. 43 to 54 Pers. but has several anomalies yet, affording subg. and $\mathbf{G}$.

1. Eupodanthes R. cal. 5part. subeq. cor. tubo ventric. lab. sup. reflexo emarg. inf. trifido. stigma bilobo, capsulis substipitatis.-The various $\mathbf{N}$. Amer. sp. blended in $\mathbf{D}$. and $\mathbf{J}$, americana, pedunculosa, ensiformis, humilis $\&$ c, see their monograph in New Flora.

2. Oximula R. cal. 5part. subeg. cor. incurva, lab. sup. bident. inf. trident. stig. acut. $f$. involucr.-Type D. eustachiana L.

3. Osmularia R. capsula 4 gona. Type D. odora Forsk, \&c compare with Sarcanthera 982.

4, Uranthera $\mathbf{R}$, antheris caudatis.-Types D. secundifl. ciliata, appendiculata of R.P. and Peru. 
5. Dothicroa R. cal. 5part. ineq. cor. Iab. sup. bifido, inf, 3fido subeq. antheris ineq. Type D. guttata R. Just, do Wal. b. reg. I334. fol. obl. subcren. fl, spic, ochroleucis rubro guttatis. India.

978. Emularia Raf, diff. Adatoda by anthers as in Dianthera. Types sp. 69 to 79 of Persoon. J. secunda Vahl. has galea linear.

979. Flavicoma R, diff, Dianthera, cal. 5 part. subeq. setaceus cor. tubulosa, ringens, galea emarg. lab. 3part. anth. loculis 2 paralelis, stigma clavato bilobo caps. substip. Herb. fl. capit. bract.-Types 1. Fl. capitata R. Just. flavicoma Lind. b. reg. 1027. fol. petiol. lanc. acum. undul. bract, imbricatis subul. Brazil, fl. yellowish._2 $\mathrm{Fl}$. paniculata R. (Just. calytricha Link. Hook. ex. fl. 212, b. mag. 2816) fol. longe pet. obl-lanc. basi subcord. repandis acutis, paniculis congestis. Brazil, fl. yellow. very large 3 inches long.

980. Janasia R. (nymph) diff. Dianthera, cal. 5 part. calic 3 sep. cor. tubo brevi compresso gibboso, limbo ventricoso camp. 5fido subeq. stam. incurvis. Antheris loculis eq. paralelis, stig. clavato.-Dichotoma, fl. spic. bract.Types 1. J. Roseı Raf. (Just. Aangularis Sims. b. mag. 1440, b. reg. 1340.) dich. 4ang. fol. ovato lancéol. acum. subdent. bract. subul. Sylhet Mts. fl. rosate.-2 J. rubra Raf. (Just. 4 angul. Hook. b. m, 2845 non Sims) caule artic. 4ang. fol. petiol. ovatolanc. acutis serratis, bracteis ternis. Madagascar, fl. red. $2 \mathrm{sp}$. in bot. mag. under same name!

981. Éruesia R. (nympli) diff. Dianthera, cal. tubulosus 5fidus, cor. tubul. galea elongata emarg. lab. inf. 3lobo medio brevior. Stam. 
apice uncinatis, antheris reniformis 2loc. equalis-Type Eth. carnea. Raf. (Just. do Lindl. b. reg. 1397) fol. pet. ov. lanc. acum. subcren. spicis obl. densis, bract. lanceol. Brazil, fl. incarnate.

982. Sarcantulera R. (thick anth) diff. Dianthera, cal. parvus camp. eq. 5dent. cor. tubul. ad medio angustior, limbo camp. bilab. labis subeq. bifido et trifido. Antheris carnosis ad basis gerens loculis 2 linearib. eq. capsula 4 gona.-Type S. venusta Raf. (Just. do Wallich, Lind. b. reg. 1380) frut. fol. ovat. acum. pubesc. fl. panic,laxis. Pandua Mts. of Bengal, fl. small dark purple.

983. Meiosperia R. diff: Dianthera, capsula monosperma! thus not even of this family, are the cells monosperm? the cor. is bilabiate but undescribed. Type D. debilis Forsk. Vitm.

984. Panemata R. (all bloody) diff. Dianthera, cor. tubulosa, subtrifida, lac. 2ineq. lanceol. Type $\boldsymbol{P}$. zeylonica R. Just. sanguinolenta Vahl, Vitm. \&c repens fol. obl. obt. petiol. pedunc. axil. 1floris. Ceylon, whole plant bloody color. Compare Adatoda repens.

985. Calasias R. (cal. hairy) diff. Dianthera, cal. duplex, ext. 2sep. internus camp. 5fido villoso. cor. tubul. longissima. lab. sup. latior.C. bracteata R. (D. trisulca Forsk. J. biflora Lam \&.c) frutex brachiato. fol. obl. integris coriaceis, pedunc. axill. 2-3fl. bract. lanc. flavis Arabia, fl. orange color.

986. Crateola R. diff. Justica, cor. hypocraterif. tubo brevis basi inflato, limbo plano 5 lobo vel 5partito equalis. Types the J. vincoides, J. parviflora Ortega non Retz, \&c, pubesc. lanceol. fl. subvertic. bract. subul. Mexico.

987. Oplonia R. (weapons) diff: Justica,cor. 
hypocraterif. tubo tereto, limbus 5lobo, bilabiato, lab. sup. bilobo plano. 'Type Opl.spinos R. Justicia do L. auct. Prickly shrub, and probably all the prickly $\Lambda$ merican sp. J. microphyla, armata, acicularis $f \cdot c$.

988. Crossandra Sal. Harrackia Jaq. diff. Justica, cor. infundib. tubo filif. limbo amplo, lobis 5, infimo major. Stam. 4.-Type $\boldsymbol{C r}$. undulatu Sal. 1, reg. 69. fine plant shuflled into 4 Genera, Just. infundib. L. \&c. Ruellia do Andr, rep. 542. Harrackia speciosa Jaq. ecl. 33.- J. sinuata is probably a 2 nd sp. Not even of Justicides group, but akin to Ruellia in ACANTHIDES.

989. Amathea Raf. Roslinia Neck. not Mench (see 495) cal. 5part. cor. ringens, tubo, compr. faux ampliata, galea erecta bifida, lab. 3fid. media major lanc, deftexa. Stam. 4 subcq. frutic. fl. spic. bract.-Type Am. pulchra Raf. Justicia do Jaq. pict. 259. Lin. \&c, fol. ovat. pet. acum. subt. tom. spicis 4 gonis, bract. cordatis. South Amer. fl. red large. Quite a distinct $\mathrm{G}$. akin to my Dimanisa 310 , that differs by double calix and unequal stamens, besides habit. These $2 \mathrm{G}$. belong to the Acanthides,

990. Rubluia L. auct. 'This G. chiefly different from Justica by 4 stamens suliequal, but had fewer anomalies, the corolla was commonly subequal camp. 5lobed. Persoon had $60 \mathrm{sp}$. Smith refers here the Insticia gangetica $L$. which I suspect to be a Crossandra, or a peculiar subg. Camirama Raf.-IIe says that R. depressa is not cven of same family: while $\mathbf{R}$. blechum and anisophyla with equal calix form the G. Blexum Br. Jus. R. burbata has beard- 
ed stamens and anthers aristate, perhaps not of this G. R. strepens has the filaments connected by membranes, thus a subg. Hemelosia Raf. The Gerardia tuberosa real type of Gerardia has been united to Rucllia by Swartz but wrongly. Gaissomeria differs by cor. tubulose and curved, Lepidagathis besides by regular limb.

991. Intrusaria Raf. diff. Ruellia, cor. lac. infima intrusa fornicata, caps. loculis dispermis. -Type Intr. bicolor Raf. R. intrusa Forsk. Vahl. Vitm. W.P. \&c. Arabia, and perhaps R. macrophila Vahl, is a $2 \mathrm{~d} \mathrm{sp}$. with unequal lobes and same firuit.

992. Antueilema diff. Ruellia, cal. ineq. lac. supera major. cor. tubulosa filif. bilabiata, lab. sup. 2fido, infer 3fido eq.-Type Anth. paniculata IR. Ruellia do L. auct. and Browalia alienata L. put in two Genera! Akin to the G. Phaylopsis, is it the same? R. imbricata Forsk is a $2 \mathrm{~d} \mathrm{sp}$.

993. Hygrophina R. Br. Sm. diff. Butllia, cal. basi tubul. 5fido ineq. caliculatus, cor. tubul. camp. limbo rlngens ineq. 5lobo-Types 1 H. malabarica Raf. (R. ringens L. auct.) 2 balsamica L. 3 angustif. Br. 4 ocymoides Cav. t. 416.-5 H, oblongifolia Raf. R. do $\mathbf{M x}$. It is of this plant that Leconte (the opponent of botanical improvement) has said it was a wonder that the Genera manufacturers had not separated it from Ruellia! The G. to which it realy belongs had been noticed long ago! and since fixed by Robert Brown,no contemptible improver and Genera maker!

994. Lrunalia Raf. (n. ind.) diff. Ruellia, stam. 2 fertiles, 2 steriles.-Type $U p$. pulchella Raf. Ruellia varians Vent. cels. t. 48. P. Eran- 
themum pulchellum Andr. t. 88. 'Thus another tink with Justica.

995. Geirariota Plumier non I. nec auct. diff. Ruellia oal. 5fid, oor. tubul. bilab. galea erecta emarg. labio inf. 3lobo refl. lat. emarg. medio bifido. Caps 2loc. valvis non septiferis \&.c. Not of family Acanturines, but the real Personates of my family AN'THOSTOMIA having partitions paralel to valves. I have proved (following Smith) in my new flora that this is the real original G. Gerardia, and I have given the monograph of $40 \mathrm{~N}$. Aner. sp. blended with it, but belonging to the G. Aureolaria, Panctenis, Agalinis, Tomanthera, Dasistema, Seymeria, Dasanthera, Pagesia, Orostima,Russelia, Macrantliera \&c, see 360 to 408. 'The African Gerardias are the G. Melasma, the Asiatic the Grenus Lophanthera with crested anthers and camp, corols. Meantime the types of Gerardia are $\boldsymbol{G}$. tuberosa, $\boldsymbol{G}$. mupestris (Ruellia do Sw. P.) G. scabrosa (Ruellia do Sw.P.) and probably other blended tuberose sp. of Ruellia \&c.

996. Plevremidis R. (side half 2) diff. Thunbergia, cal. simplex spathaceo fisso univalvis bifido, cor. ineq. 5fida-Type Pl. grandiflora R. 'Thunb. do Roxb. b. reg. 492. fol. opp. pet. cordat. angul. pedic. axill. Ifl. India, large blue flowers.

997. Endonelas R. (inside blací) diff. Thunbergia, cal. simplex, bipart. lac. cordat. trinervis carinatis, cor. tubo curvo, 5lobis rotatis subeq. flabellatis-Type E. alata $\mathrm{R}$. Thunb. do Sims b. mag. 2591. Scandens, fol. cord. deltoideis sinuato dentatis, 5nervis, petiolis alatis, subtus tomentosis, ped. axil. 1floris-ZZanzibar in East Africa, fl. yellowish, tube inside 
blackish.--The real Gr. Thunbergia has a double calix, the inner camp. multident. cor. camp. The Th. repens or Septas repens of Loureiro is probably a peculiar Genus Septilia Raf.

998. Dis'reika Raf. (2 ster.) cal. 5 fidus ineq. ext. bibract. coloratus, cor. ventricosa hians 4 loba, lobo inf. latissimo, stam. 2 fertilia, 2 sterilia, stig. 2 clavatis. Facies Thunbergia.Type D. angulosa Raf. Martynia do Lam. Mart. diandra W. P. often figured, fol. opp. cord. angul. ped. axil. multifl. Mexico.-Of family SESAMIDES Raf. 1815 with capsule 4locular, several G. Seramum, Martynia, Craniolaria, Basonca, Dysosmon \&c. Craniolaria of L. wrongly united to Martynia differs by a double calix, the inner tubular spathaceous. Cran. annua L; but his Cran. fruticosa has been united to Gesneria! while it forms a G. of Gesnerides Petramnia Raf. Basonca Raf. has tubular cor. base of tube gibbose around, type B. longiflora, Martynia do Ait.-M. perennis has become the G. Gloxinia, and thus every sp. of Martynia was the type of a Genus! my Dysosmon f. ludov. differs fiom them and Sesamum, by corolla unilabiate.

999. Ammana Raf. (nymph) cal. tubul. 5fidus, cor. infund. limbo camp. ineq. 5lobo. Stam. 4. didyn. Ovario immerso glandulis 5, stig. capit. umbilic. caps. uniloc. 2 placeatas later. Scaposa -Type Alm. hirsuta Raf. Gloxinia do Lindl. b. reg. 1004, fol. ovat. rugosis hirsutis crenatis, scapis agreg. Ifl. Brazil, fl. blue. Of family Pedalides differing from Gratiolides by seeds not central. T'otaly unlike Gloxinia of family Gesneridis, that has calix superior 5parted, nearer to Cyrtandra that has 2 sterile stamens: while habit and capsule like Ramonda 1068. 
1000. Rotheca R. (11. ind.) cal. 5dent. cor. subbilab. vel 5 lobis ineq. 5 deflexis, infero parvo, cetera ut Clerodendron.-Types $1 \boldsymbol{R}$. bicolor Raf. Clerod. macrophyl. Sims b. m.2536. fol. oppos. latis ovatis acum. sess. serrat. subt. toment. fl. panic. Mizuritius fl. yellow, lower lip. blue.-2 R. ternifolir Raf. Volkameria serrata L. auct. Rheed. 4 t. 29. fol. ternatis ovat. selrat. f. concolor Malabar. See Egena $31 \%$.

CENTURIA XI.

\section{DICOTYLES-PERIPLTALIC.}

1001. Convorvulus. This G. and Ipomea have been so blended and perplexed by botanists that they are still in utter confusion; nearly $250 \mathrm{sp}$. belong thereto, $(\mathbf{1 6 6} \mathrm{sp}$. are in the monograph of Convolv. by Desrousseaux and Smith which are constantly shuffled from one to another there being no distinction between them! although they offer 50 good characters to distinguish 50 Genera out of them.-Calix single or double, equal or unequal, corolla rotate or campanul. or infund. or hypocr. or tubular, entire or dentatc or lobate, stamens quite unequal or subequal, stigmas 1 or 2, cripitate or lobed, capsule 1-2-3-4locular, cells 1 to 4 seeds Sc.-Necker began a reform by proposing the G. Milhania, Apomea, Stevogtia .... others have added Calystegia, Pharbitis, Murucoa \&c. I proposed between 1808 and 1820 Stylisma, Diatrema, Ornithosperma, Rhodoxylon and see my Stomadena 11, Coiladena 12 in this flora. I will now still increase them to 48 Genera with 30 subgenera and thus reform this fine group of Genera, refering 175 typical sp. to them.-The real G. Convolvulu's Raf. has calix simplex 
5part. ineq. cor. campan. subintegra, stam. 5 ineq- filam. filif: antheris linearib. stigm. 2 linearib. vel. 2lamellaris caps. 2locul. 2 valvis 4 sperma-It has many subgenera yet.

1. Taranelis R. (n. ind.) cal. lac. 3ext. sagittatis. Type C. medium, \&c.

2. Ballela R. (n. ind.) cor. sub 10crenata. Type C. repens \& .

3. Lacara R. (many heads) fl. capitate involucrate or bracteate.'Iypes C.capitatus Vahl. 2 subtrilobus, 3 villosus, 4 scabra Raf. (capitatus Raf. fl. lud.) 5 saxatilis, \&.c.

4. Scadiara R. fl. umbellate involucrate. Types C. ruber, C. cneorum, \& c.

5. Pentantuus R. cor. camp. subrotate, edge 5angular, stamens subequal, base bearded, stigmas reflexed. Type C. pentinthus Jaq. W. \&c. Also C. violaceus with reflexed stigmas.

6. Lizeron R. the true Convolv. lacking the above distinctions, as C. arvensis, persicus,verticillatus, chinensis, tricolor \& Sc $^{\circ}$ with many other species perhaps. 'The deeply biparted stigma almost 2 and linear ought to be found in all however.

1002. Stevogtia Neck. 642 diff. Convolv. cal. 5fidus, caps. 2loc. 2sperma, folia compositaTypes not mentioned, but $\mathbb{C}$. platicarpos Cav. with racemose $\mathrm{fl}$. and capsule compressed may be one, and also C. tomentosus?

1003. Sanilum Raf. (n. egypt) diff. Convolv. corolla 5 fida, stigma capitate? - Types I S. copticum, 2 S. Itumile Jaq. 5pentaloides Lin. 3 bracteatum, 4 bicolor \&c, as Convolv. and probably others. But C. priviflorus L. has 2 revolute stigmas, and forms subg. Exostreps Raf. 
1004. Milinania Neck. 645. Calistegia RB. diff. Convolv. cal. duplex. persistens, ext. bipart. major, internus 5part. subeq. stigm. 1 capit. obl. s. glob.-Types C. sepium, 2 riparia Raf. 3 involucrata, 4 bracteata, 5 pentaphyla (stig. bilobo, cor. 5 loba, 8 spithamea, 9, 10 villosa and mutabilis Raf. 1. ludor. 11 Catesbiana, with many others.

1005. Trrtalia R. (n.ind.) diff. Convolv. cal. equalis, vel. subeq. stig. capit. bilobo-Types T. striata 2.angustif. 3 emarginat. 4 maxima, 5 Sibirica \&c all Convolv. of Authors. C. filicaulis with stigma simpl. obtuse is probably a subgenus Aplimia IRaf.-A 3d subg. Priacstis has cal. unequal, such as C. domin. gensis, gemellus, triflorus, hispidus, anceps. triqueter, macrorhiza, Vitifulius, dissectus, 5 lobus, umbellatus with villose seeds \&c, malabaricus with villose corolla \&c, panduratus with costate calix and stigma \&c.

1006. Turpetuun Raf. diff. Convolv. cal. subtriplex, ext. caducus bracteis 2 magnis, internus persist. 5part. ineq. 2 major ext. involvens, 3 int. minor eq. corolla infundib. 5loba emarg. plicis oppositis, stam. 5 subcq. antheris spiralis. stig. capit. caps, didyma 2 loc. 2-4sp.-Types 1. T. indicum Raf. Ipomea et Convolv. do Auct. Herm. t. 178. bot. reg. 2\%9. Villosa, fol. obl. vel deltoideis repandis cuspid. ped. 2fl. Ceylon, Malabar $\mathcal{O} \cdot c$. fl. white.-2 T. australe Raf. Ip. turp. R. Br. fol. cord, acutis integris, ped. multifl. caule angulato. Australia and Tahiti. Probably other sp.

1007. Priaritis Choisy, diff. Convolv. stig. capit. granulato 3-4lobo stellato, caps. 3-4loc. 3-4valv, 6-8sp. - All the Convolv. with bell flowers, capitate stigma and more than 2 cells be- 
long here and they are numerous. C.hederacea, aristolochif.. Miller Sin. Ip. punctuta also and many others.

Scamonia is a subg. of it by capsule 3-4loc. calix dilatate emarg. Pl. scamonia.

1008. Diatrema Raf. 1808, diff. Convolv. cor. 5loba, stigma capit, subintegro, capsula uniloc. basi biloc. 4sperma-Types the Conv. purpureus, and blended sp. with others akin thereto, not C. nil as once stated. 2 types at least, $\boldsymbol{D}$. purpurca, fol. subrot. fl. purp. $2 D$. alba, fol. cordatis fl. albis, both American figured by Dillen. D. muricatus probably a $\mathbf{3 d}$ sp.

1009. Ornitroosperma Raf. 1817 diff. Convolv. cor. subinfundib. stigma elliptico integro, capsula monoloc. 3sperma.-Type O. aritumnalis Raf. Ipomea avicularis fl. lud, 145. Volub. fol. cord. 3lobis, acutis, ped. 1fl-Louisiana banks of rivers, fl. white small. This and the last $\mathbf{G}$. deviate by the monolocular capsules, quite a striking character, type of a new family ApLarNIA R. with Ramondia 1053.

1010. Apopleumon Raf. (n. grec) diff. Convolv. cal. campanul. 5fido ineq. cor. tubulosa infund. limbo 5lobo crispato, stigma bilobo.Type Ap. bignonioides Raf. Ipom. do bot. mag. 2015. tuberosa scandens, fol. cord. trilobis, lobis imbric. angul. repandis subt. pallidis, ped. nutans multifl. South Amer. fl. violaceous white 2 inches long.

1011. Samudra Raf. (n. ind.) diff. Convolv. cal. imbric. subeq. ccr. tubo apex ventricoso, limbo plano 5plicato, disco glanduloso 5lobo, stylo flexuoso, stig. 2lobo.-Type S. speciosa Raf. Conv. do W. Ipomea do Pers. C. nervosus Lam. Burm. t. 20. Rheed 11 t. 61, bot. mag. 2446. fol. cord. acutis subt. argenteis, ped. mul- 
tifl. bract. cordatis. Indiat-Cone. cuneatus $\mathbf{W}$. is probably a $2 \mathrm{~d}$ sp. with swelled tube,leaves cuneate, axillary corymbs.

1012. Isypes Raf. (eq. under) diff. Convolv. cal. camp. 5lid equalis cor. 5loba, lobis crenatis apiculatis, stam. ineq. stig. capit.-Type $\boldsymbol{I}$. ochraceus Rat. Conv. do lind. b. reg. 1056. volub. piloso, fol. cord. acum. integris, ped. 1 fl. West Africa, fl. yellow. Akin to Stevogtia 1001 by 5 fid calix yet equal, but stamens unequal.

1013. Kolofonia Raf. (n. grec) diff. Convolv. cor. infundib. limbo plano 5lobo, lobis obcord. stella plicata ad sinutb. oppos (in Conv. altern) stig. capit.-Type R. albinervia Raf. Conv. do Lind. b. reg. 1116. fruticosa, scandens tuberc. fol. cord. repandis rugosis subtus retic. fl. term. solit. bract. binis lanceol.-S. Africa Algoabay, fl. large white, tube and star strawcolor.

1014. Murucoa Aublet, diff. Convolv. cal. turb. 5 part. eq. cor. infund. limbo patens 5lobo, stam. $5 \mathrm{eq}$. lobis oppositis, disco ovar. cingens, stig. 2lamel. caps. 2-3loc. 2-3sp-Type $M$. violacea Aubl. t. 54. Conv. macrospermus W. \&c, frutex scandens, fol. ovat. acut. rigidis, fl. axill. corymbosis. Guyana, fl. blue.-'This is perhaps not even of family Covrouvulines which ought to have alternate stamens, and will belong to Gentranides! all the Conv. with equal and oppos. stamens must be removed in the natural orders.

1015. Apanboe R. (n. ind.) diff. Convolv. cal. duplex, ext. 2phyl. orbic. cor. canp. Lloba, lobis ineq. uno emarg.-'Type A. bicolor Raf. Ipomea campanulata L. Rheed 11. t. 56. repens piloso, fol. cord. ped. multifl. Malabar, ft. red 
and white. By the irregular corolla hardly of this family, the stamens stigmas and fruit must be examined.

1016. Iponea Raf, diff. Convolv, cor. infundib. tubo longo, limbo camp. stigma capit. caps. 3locul. 6sp.-Types all the sp. having these characters, several subgenera yet-Ip. puncta$t a$ has a 3lobe stigma.

1. Pullis R. tubo cor. 5angul. Type.Ip. hepaticifolia $\mathrm{L}$.

2. Lomalix R. cal. patulus marginatus, type Conv. anotheroides $\mathrm{L}$.

3. Kemopsis $R$. (ivy form) cor. indivisa, stam. exsertis longissimis. Type Ip. hederifolia $\boldsymbol{L}$.

4. Hemilasis R. cal. inequaliss. cor. limbo 5 plicato 5dent. filam. basi barbatis. Type $\mathbf{I} p$. heterophyla Ortega, perhaps a Genus.

5. Sericosperma Raf. cor. tubo longo tereto caps. vel. sem. sericeis, types Conv. sericeus and eriospermus of authors.

1017. Quamoclita Raf. (n. Amer. diff. Conv. cor. hypocraterif. tubo longo, limbo plano stellato 5 dent. stigma capit.. caps. 2loc. 4valvis, 4 sp. -Types bipinnata, cordata, hybrida, lacunosa, rubra, coccinea, solanifolia, denticulata, R. Br. \&c chiefly Ipomeas of Authors, with many akin in 5 subgenera.

1. Apomea Necker, leaves pinnate.

2. Tulotropis Raf. calix carinate, keel warty. Type Ip. verrucosa Ortega.

3. Troxula R. tubo camp. limbo rotato 5lobo, caps. sericea, type Conv. literalis.

4. Melasis Raf. capsule membranose sub Agone, Aseeded. 'Type Ip. tuberosa L.

5. Lomptia R. (edge reversed) cor. limbo 
integrorevoluto, stigma ovatum integrum. 'Type Ip. bracteata Cav. perhaps a Genus.

1018. Gynoisia Raf. (fem. hairy) diff. Convolv. stam. basi vill. ovar. villoso, stigma globoso indiviso, capsula vill. 4loc. 4sp.-Type $G$. carolina Raf. Conv. and Ip. do or trichocarpa L. et auct. see my new flora. Different from Pharbitis by cells monosperm. Conv. quinque folius I. is probably a $2 \mathrm{dl}$ sp. with corolla 5 dentate.

1019. Euryloma R. (broad edge) diff. Conv. cal. 3segm. interuis major, cor. infundib. limbo profunde 5lobo, lobis latis rotundis, stam. exsertis subineq. stigma bilobo-Types $1 \mathrm{E}$. grandiflora Raf. Conv. do L. -2 E. latiflora R. Conv. do Poiret, Ip. do RS. bot. reg. 889. Ip. grandif. Andr. b. rep. 403. fol. cord. acum. glabris, ped. 3fl. Antilles, large white flowers3 E. leucantha? Conv. do D. Sm. Ipoinea do Jaq. Scop. t. 4. Vitm. fol. cord. subangul. acum. glabris subtus rubris, ped. 2f. America,fl. white small, capsule brown.

1020. Doxema R. (glory bloody) diff. Convolv. calix urceolatus, lac. 5ineq. filif. basi membr. coalitis, cor. infund. tubo curvo clavato, limbo inflato 5dent. stam. exserta ineq. arcuata, stylo arcuato, stylo arcuato, stig. capitato-Type $\boldsymbol{D}$. sanguinea R. Ipomea do W. P. bot. reg. 9. Quite unlike the other Genera by curved tube, stamens calix \&c.

1021. Modesta R. (nymph.) diff. Convolv. calix imbric marginato, cor. hypocrat. tubo crasso limbo plano 5 fido, lobis dilat. emarg. stam. basi barbatis, stig. didymo, caps. 2loc. 4valvis, semina lanata - 'Types $M$. paniculata R. (Conv. do L. Ipom. do b. reg. 62. Ip. mauritiana Jaq.) fol. palm. 3-5fidis, lobis ovatis acut. undul. inte- 
gris, pedunc. paniculatis. East Indies \&c-2. M. insignis Raf. Ipomea do Andr. rep. 636. bot. reg. 75. fol. cord. repando lobatis acum. fl. cymosis. E. Indies, fl. rosate.-3 MI. macrorhiza, R. Ip. do Mx. \&c.-This G. has 3 subgenera somewhat different Decaloba, Caulotulis and Gomphipus.

1022. Decaloba Raf. diff. cal. subeq. cor. 10loba vel 10emarg. stig. capit. emarg. granuulato. Types 1 IITod. mutabilis Raf. Ipomea do bot. reg. 39. frutic pub. fol. cord. integris 3lobisve acum. supra villosis scabris, subt. toment. ped. cymosis. South America.-2. $M$. congesta $\mathbf{R}$. Iि. do bot. reg. 333 . caule tuberc. fol. palmatis lac. cuneatis acutis, ped. 1-4fl. Buenos Ayres, A. incarnate.-4 M. coriacea $R$. Conv. hypocraterif. Desr. Lam. Rees 55, frut. fol. cord. coriaceis, ped 11. sepe geminis. E. Indies.

1023. Caulotulis Raf. diff. cal. segm. 3 ext. cordata major, cor. infund. 5fidis, lobis dilat. cuspidatis plicis oppositis.-Types Mr. tubercuIata R. (Ip. do bot. reg. 86. Convolv. digit. Roxb.) frutic. ramis tuberc. fol. pedatis 5-9fidis, lac. obl. ped. 3-4f. East Indies-Ip. dasycarpa Jaq. is perhaps a $2 \mathrm{~d}$ sp. Both perhaps akin to Ipomea, but calix,stamens, stigma, capsule as in Modesta.

1024. Gonphipus R. (club under) diff. exactly like Decaloba except corolla semi 5fid, not 10lobed. Type M. setosa R. Ipom. do bot. reg. 335. setosa hispida, fol. cord. trilob. dent. acum. pedunc, multifl. pedic. clavatis. Brazil, said to be akin to Ip. batatas and platanifolia, are they of same group? I never could meet Ip. batatas in bloom.

1025. Inalia R. (nymph) diff. Convolv. calix 
infundib. 5fido ineq. ciliato, cor. rotata camp. 5 fida, stam. 5 subeq. in tubo, stylo brevi, stig. ¿ linearis. caps globosa 2loc. 2sperma.-Type Id. albiflora Raf. (Convolv. elongatus W. P. bot. reg. 498) fol. subsess. cord. acut. pilosis pedunc, elongatis 1-2fl. bracteis subulatis vestitis. Canary Ids. Near to G. Stevogtia and 'I'urpethum by 2 seeded capsules.

1026. Bonanox R. diff. Convolv. cal. connivens margine membranaceo, cor. hypocrat. tubo longo clavato, limbo rotato integro vel 5 fido, stam. ineq. ad apex tubo, stigma capit. Alobo, capsula 2loc. 4sperma-Types 1 B. riparia Raf. Ip. bonanox L. auct. bot. mag. 752. Caule scabro, prostr. fol. cord. angul. acum. cal. a!istatis. Antilles and Florida, fl, large and white, forming with the 2 next the subg. Mundavalis Raf. by corolla entire. $-2 B$. orbiculata Raf. Ip. do Elliot, canle scalro prostrato, fol. orbic. emarg. venosis glabris, ped. subtrifl. cal. ovat. mucron. Florida, fl. purple-3 $\boldsymbol{B}$, indica Raf. Ip. bonanox L. Rheed 11 t. 50. caule scandens aculeato. fol. cord. acut. integris, ped. tortuosis 2-3fl. India, large violet flowers-2d subg. Gonphula Raf, corolla 5fid. 4 B. muricate R. Convolv. do L. auct. Ip. bonanox bot. reg. 290. caule muricato, fol. cord. acum. pedunc unifl. clavatis, cal. acum. East Indies, f. blue.

1027. Clemenera R. (morning glory) diff. Convolv. cal. subeq. connivens subul. hisp. cor. infund. limbo 5angulis, vel 5dentatis, plicis ad dentis opp. stam. vix ineq. sîig. capit. 2-3lobo, caps. $2-3$ loc. loculis $2 \mathrm{sp}$.- Types the various sp. blended as Conv. and Ip. nil, at least 6-1. $\mathrm{Cl}$. hederacen R. (Ip. do Jaq. col. t. 124, bot. reg. 85. Ip. barbata Roth, Ip. nil Pursh, Conv. nil Mx.) fol. subtrilobis hirsutis, ped. brevis trif. 
North Amer. fl. blue-2 Cl. hirsuta R. Ip. nil Elliot. Dillen f. 92. Hirsuta, fol. cord. acum. nonulis 3lobis, ped. brevis 1-3f. cal. hirsutissimis muricatis longe acum. Carolina, Kentuky sc-3 Cl. cuspidata R. Dillen f. 96.-4 Cl. peruviana R. Ip. do RP. $119-5 \mathrm{Cl}$. guinensis R. Dillen f. 93-6 Cl. indica R. Ip. nil L. bot. mag. 188. Dillen f. 91.

1028. Plesiagopus Raf. (near goat foot) diff: Convolv. cor. infundib. vix 5loba, stam. omnib. ineq. filam. subul. villosis, stig. capit. emarg. caps 2loc. 4valv. 4sperma.-Near to G. Modesta including all the sp. akin to Conv. pescapra, 'Types 1 Pl. sovance Raf. C. pescapra L. \&c, Pheed 11 t. 5\%. Rumph 5.159 \&c, fol. bilobis carnosis lucidis, ped. $1 \mathrm{fl}$. India fl, bicolor, rosate, bottom dark purple-2 $\mathbf{P l}$. maritima $\mathbf{R}$. Ip. do bot. reg. 319. fol. obcord. bilobis basi truncatis. Tropical sea shores. $-3 \mathrm{Pl}$.rotundifolia, R. Ip. marit. R. Br. fol. ovato subrot. emarg. sepe obliquis ped. $1 \mathrm{fl}$. Australia fl. rosate-4 Pl? brasiliensis R. Conv. do L. plum. t. 104. fol. subrot emarg. nitidis, basi bigland. ped. $3 \mathrm{fl}$. Brazil, fl. purple, this is stated to have caps. 3loc. 3sp. and would thus be a Latrienda. 5 Pl. cuneifolia R. Ip. do Lam. fol. cuneat. emarg. ped. $1 \mathrm{fl}$.

1029. Ketiosia R. (nymph) diff. Convolv. cal triplex, ext. bipart. medio bipart. major. eq. internum 3part, ineq. cor. hypocrat. tubo brevis, limbo rotato 5angul. faux coronata intus 10dentatus, stam. subeq. filam. planis alatis membran. antheris inter dentes corona, stig. 2 divaric. capsula uniloc. basi semibiloc. 4sperma.-Type $\boldsymbol{K}$. involucrata R. Convolv, do W. P. b. reg. 318 \& c, fol. cord hast. pub. ped. elongatis sub 3f. Mauritius and Africa, fl. yellow white, 
mouth red. A very singular and distinct $\mathbf{G}$. capsule as in Diatrema, calix near Milhania, stamens and crown quite peculiar, of family Aplarnia, see Ramonda 1053.

1030. Poinaetra R. (foot mealy) diff. Conv. cal. teres erectus, cor. infundib. tubo 5 gono, limbo 5 fido, filam. ad tubo toto adnatis, disco glanduloso, stig. 2oblongis, caps. 4loc. 4sp.-A very distinct G. fruit as in Gynoisia, but 2stigmas and thus nearer to Stylisma, but corolla quite different, yet same family. Type $\boldsymbol{P}$. farinosa $\mathbf{R}$. Corv. do L. auct. fol. cord. acum. repandis rugosis, caule forinoso volub. ped. farin. 3ftoris.

1031. Ampinone R. (nymph) diff. Convolv. cor. tubulosa 5dent. stig. bilobo didymo-Types 1 A. lobata R, Ip. 3loba L. Conv. sub trilob. Sm.-2 A. chenopodia R. Conv. do D. Sm. fol. ov. dent.-3 A. asarifolia R. Conv, do D. Lam. Sm. fol. renif. ped. 1-2fl. Senegal-4 $A$. tiliefolia R. Conv. do Com. Sm. frut. fol. cord. ped. Ifl. S. Africa, fl. triuncial, forming a subg. by calix rotate, cor. with a short tube and long tubular limb-5 A. cymosa, Conv. do D. L. fol. cord. obl. acum ped. cymosis E. Indies-6 $\boldsymbol{A}$. venosa. Conv. do D. Vahl. \&.c

1032. Breweria R. Br. diff. Convolv. cor. infund. 5 plicata, stylus bifidus, stig. 2capit. caps. 2loc. 4 sp.-3 types each with deviations, $1 \mathbf{B r}$. linearis fol. lin. cal. eq. stylo bipart-2 Br. media cal. eq. stylo bifido ineq.-3 $\mathrm{Br}$. pannosa toment. cal. ineq. stylo bipart. ineq. all from Australia, near to Evolvulus chief diff. the corolla only.

1033. Ruodoxylon Raf. 1815, diff. Convolv. cal. subeq. cor. plicata stigma capit? capsula uniloc. monosp. basi dehiscens 10valvis! Fru- 
tescens, fol. angustis, fl. racemosis vel thyrsoideis-A fine G. indicated by Ventenat, long ago proposed by me, it is the Rosewood as the name implies, capsule quite peculiar,type of the family APLARNIA with Ornithosperma, Melascus, Turbina, Diatrema, Kethosia, Ramonda,\&c all with uniloc. capsules-2 sp. Rhod. floridum and scoparium Raf. Conv. do L. auct.

1034. Cleiostroma R. (shut mouth) diff. Conv. cal. ineq. cor. tubulosa tereta, apex 5 fida clausa, antheris sagittatis, stig. capit. caps. 2loc. 2sp. fl. capit. invol. periantho campanul. 12part. ineq. 10-12fl.-Type Cl. villoso, Raf. Conv. agregatus Lour. Mart. Rees. caule scandens villoso, fol. palmat. Flobis ovatis. Anam, large white flowers, quite distinct $\mathrm{G}$. by habit and corolla, near Amphione 1031.

1035. Nemanthera Raf. (filif. anth.) diff. Convolv. antheris filiformis spiralis, capsula 2loc. 2sp.-N. bufalina Raf. Conv. do Lour. Mant. Rees, frutic. fol. cord. sagitt. pedunc. multifl. Anam.

1036. Merascus R. (membre box.) diff. Conv. cal. ineq. 2 internis major, cor. hypocraterif. limbo plano sinuato, stig. 3-4lobo, capsula membranacea unilocul. 3-4sperma turbinata-Type M. latifolius Raf. Conv. do Desr. Lain. Rees, fol. cord. glabris, ped. 3f. South Amer. fl. white ample, 5 inches in diameter: family Aplanna. 103\%. Exocroa R. (out col.) diff. Convolv. cal. eq. 5phyl. scariosus coloratus, cor. infundib. antheris sagitt. stig. cap. striato-'Type $E$, egyptiace R. Conv. cairicus and egypt. L. auct, Ip. palmata Forsk. fol. pinn. palm. serrat. ped. filif. panic. Perhaps $C$. arenarius is a $2 \mathrm{~d}$ sp. having a membranose calix, 
1038. Lobake Raf. diff. Convolv. cal. eq. cor. 5loba, lobis acuminatis, stig. 2 reflexis, capsula villosa-Type $\boldsymbol{L}$. guyanensis R. Conv, do Aubl. t. 52 auct. ciner. toment. fol. ovatobl. $\mathrm{f}$. capitatis. Akin to Exotreps subg. of Sanilum 1003, differs by cal. equal \&c.

1039. Enulina R. diff: Convolv. cor. 5fida, capsula 4locul. 4sp.-Type E. parviflura $\mathbf{R}$. Conv, do I. Lam. not L. solanifol. Rees, fol. cord. obl. ped. multifl. Antilles. Akin to Gynoisia 1018, but diflerent corolla, stamens and probably stigma! L.

1040. Latrienda R. diff. Conv. or rather Pharbitis by stigma capit. capsula 3locular. sepe trigona, 3sperma-Perhaps only a subg. of Pharbitis but the cells are monosperm and constantly 3. Types 1. L. soldanella R. Conv. do L. auct. cal. double, a subg. with the next $2 \mathrm{~L}$. imperati R. Conv. do auct. 3 L. mulliflora R. Conv. do Miller, Martens, Rees.-4 I. palmatus R. Conv. do of same authors.-5 L. brasiliensis R. Conv. do R. auct. sec 1028.--'The C. 5 folius Miller not I. is stated to have 2 seeds in each 3 cells, and thus would be a Pharbitis 5folia Raf, fruticosa, fol. 5part. Mexico, large purple fl. and fruit like an apple! 1041. Turbina R. diff. Convolv. capsula turbinata, uniloc. membranosa, 2-3sp.-Type $\boldsymbol{T}$. corymbosa R. Conv. do L. auct. Genus near Melascus and Ornithosperma, but cor. regular campanul, how is the stigma? family Aplaria. 1042. Canonea R. (nymph) diff. Convolv. cal. equalis, cor. infundib. limbo 5lobis acutis, stig. capit. bilobo.-Type C. bifida Raf. Conv. do Vahl. auct. fol. cord. obl. acum. mollis, ped bifidis multifl. Java \&c. 
1043. Nemostmma R. (fil. stig.) diff', Convolv, cal. equalis, cor, subrotata plana, stig. 2 filiformis, vel stylo bifido. stig. acutis-Types $1 \mathrm{~N}$. canariensis R. Conv. do L. auct. frutic. villosa fol. cord. ped, multifl-2 N. camtabrica R. Conv. do L. auct. Erectus, fol. linearib, ped. multifl. Spain and Maroco-Besides two subg. Trichima R. cor. rather campanulate fl. not involucrate 'Types Conv. hystrix, lineatus, spicafolius, of authors-Periexa R. cor. camp. fl. involucrate, 6phyl, 3 alt. major, cal. linearib. 2bract. stylo bifido. stig. acutis-Type Conv. lanatus L. (cneorum Forsk.)

1044. Calrxnos Raf. (slender cal.) diff. Conv. cal. subeq. setaceus. cor. subinfund. stig. capit. bilobo, fol. oppositis!-Habit quite peculiar,no other Convolv. has opposite leaves, Type $C$. trinervius Raf. Conv, do L, auct. glabra volub. fol, pet. cord. obl. acum. trinervis, fl. axil. subsess. sepe binis. Japan fl. purple: they must offer additional characters, fruit unknown!

1045. Distrmake R. ( 2 ac. stig.) diff. Convolv. cor. infundibul. limbo 5lobo, stigm. 2 longis acutis.-This has the corolla of Ipomea and stigmas of Nemostima, type D. glaber Raf. Conv. do Aubl. t. 53 and authors. Volub. glabra, fol. digit. fl. axil. racemosis.-Guyana,large white fl. thick root, a milky plant.

1046. Evolvulus L. This G. has also been blended with Convolvulus, and forms many distinct $\mathbf{G}$. the main differences are the rotate corolla and split style, yct 20 sp. with those characters are put in Convolvulus even quite lately. I shall describe 7 Genera of them some of which by the equal or opposi-e stamens do not even belong to Convolvulides. I have dry in fruit a doubtful sp. of this G. E. cnneifolius 
Raf. with cal. eq. caps. monoloc 1-2sp. 2 styles, stig. obt.-fol. sess. lin. cuneatis, pedunc. 1fl. pilosis bibract. caps. cal. longior pilosa. New Jersey, probably a N. Gr. Plesilia Raf.

1017. Fraxina Raf. (separ. stig) diff. Convolv. cal. subeq. cor. camp, infundib. limbo integro, filam. ineq. basi barbatis, stigm. 2 globosis divisis, et stylus interdum. caps. 2loc. 2valv. 4sp. -'Types 1 'Tr. sagittifolia, 2 umbellata, 3 quinquefolia, 4 tridentata, 5 mindunensis Raf. Conv. spherostigma Cav. P. 6 Sherardi Raf. Calystegia paradoxa Pursh, 7 ebracteata, C. do Lam. \&c all ranged in Convolvulus, the generic character is chiefly from the first, all must be fully described: this is the first link with Evolvulus.

1048. Exaltosis R. (out different) diff. from Traxima by calix very unequal, sepals all different, 3 cordate, cor. 5 fid. 15lobed. E. biflora Raf. Ip. and Conv. do aact, fol. obl. cord. pubesc. ped, geminis. China.

1049. Symerrus Raf. (flum. sic) diff. Convolv. calix duplex, ext. 2partito amplo, interno 5fido ineq. 3 ext. major, corolla rotata 5 fida, stam 5 ineq. stylo bifido, stig. 2 linearis. caps. glob. 2 loc. 4sp.-Types Sym. siculus Raf. Convolv. do L. auct. b. reg. 445 . prostratus flexuosus, fol. ovatis delt. pedic. 1fl. bract. Ianceol. Sicily, fl. bluish small, seen alive 1809 .

1050. Stylisua Raf. 181\% dif: Conv. and Symethus. cal. subeq. simplex, cor. campanul. subintegra sub 10dent. filam. 5 equalis villosis ad basis cor. antheris sagitt. disco glanduloso arnularis, stylo bipart. stig. 2 globosis. caps. 4 loc. 4sperma-Types 1 St. tenella Raf. Conv. do Jam. W. P. trichosanthes Mx. P. \&c-2 St. aquaticu Raf. Conv. do Walt. El.-3 St. sher- 
ardi Raf. Conv. do P.E. and some other sp. see my new flora, all North American. Certainly as near Evolvulus as Convolvulus! capsule like Gynoisia 1018.

1051. 'T'inyelua R. (nymph) diff. Convolv, and Symethus. cal. simple subeq. cor, campan. 5 dentata, filam 5 ineq. stylo bifido, stig. 2 globosis, caps 4 gona 2loc. 4sp.- Types 1 Th. tamnifolia R. Ipom. do L. Conv. do El. forming a subg. Microle Raf, by cor. 5dent. eq. to calix, fl. capitate involucrate.-2 T\%. obtusiloba Raf. Conv. do Mx. P. E. another subg. Ocripha Raf. by cor. large with a tube, filaments tomentose at base, pod. 1f. 2stipulate-C. lanuginosus and incanus probably belong to this Genus.

1.052. Ditereia R. (twice cut) diff. Conv, and Evolvulus, calix campanul. 5 fido subeq. cor. rotata 5loba, stum. 5 eq. lobis oppositis! stylis 2 bipart. stig. 4 capit.-Type D. parviflora $\mathbf{R}$. Evolv. latif. Iidw. b. reg. 401. suffirut. villos. fol. sess. obl. cord. acum. axillis 3 floris. Brazils, small white flowers. Not of this family, but probably of GENTIANIDEs, are other akin Genera with similar stamens? compare Dichondra, Cuscuta ofe.

1053. Bucinarea Raf. (bot. Arab) diff. Conv. and Evolv. cal. 5part. subeq. imbric. cor. rotata integra, vix 5emarg. plicis ad emarg. opp. stam. 5 subeq. basi pilosis, stylo brevis bifidus, stig. 2 clavatis, caps. 2loc.-Type R. maderensis Raf. Convolv. suffiruticosus Dryander, bot. reg. 133 (non Desf. nec P.) caule levi, fol. obl. subcord. acutis, ped. 1-3fl. Madeira-2 B? atlantica Raf. Conv. suffrut. Desf. t. 48. P. caule villoso, fol. lanccol. ped. Ifl. elongatis Mts. At- 
las, fl. rosate and white. These 2 shruls. appear to form a very natural Genus, near Erolvulus and next. Dedicated to Buchar a great botanist and traveller of Malaga in the 10th century.

1054. Perrerias Raf. (around cup) diff. Conv. and Evolv. cal. basi camp. limbo 5 fido rotato. cor. rotata 5angulata, plicis opp. angulis. stam. 5 equalis! filam. subul. pilosis, antheris incurvis extrorse dehiscens, nectarium cupularis in disco carnoso ovario cingens stylo bifido, stig. 2 clavatis-Type P. pamnifolius. Raf. Convolv. do Salisb. par 20, bot. reg. 222. Volub. pilos. fol. cord. obl. ped. 3-5fl. bract. lanc. remotis. Canary Ids. fl. pale violaceous. Quite a distinct G. by calix and nectary, probably not of this family. Near to Phacelia, Ellisia s.c or rather of Cuscutaria family with Evolvulus, Hydrolca \&c.

1055. ECHIDIA Raf. 1815, pronounce Ekidia. This family of mine is perfectly distinct from the Labiates by $\mathbf{5}$ stamens, and from Borragines or 'T'rachytes by irregular or labiate corolla with 5 unequal stamens, the $\mathbf{G}$. Lycopsis appears by its curved tube to form a link between them and also the Verbenacea. They all belong to the natural order Lobogrvis with lobed ovary and one central style. 'The Gr. belonging to Ecunes are 1 Echum with oblique campanul. corolla, stigma bifid, calix 5parted unequal. 2 Exroxy con Desf. P. Raf. with cal. 4parted and cor. bilabiate, 3 Isorium Raf. see 219 with equal cor. but unequal stamens, besides the 6 next Genera till 1061.

1056. Traxara R. (rough head) diff. Echium cor. infundibulif. limbo campanul. 5 lobo eq. stam. longissimis filif. ineq. stylo declinato, stig- 
ma bifido. Frutic, A. cripil. corymbosis-'T'ype Tr. capilata Raf. E. do L. auct. Hispida, capitulis term. S. Africa.

1057. Isoplesion R. (nearly eq.) diff. Echium cor. infundib. fere regularis stam. ineq. declinata 2 longior-Types, Is. italicum, giganteum, $p y$ renaicum, vibram, \&c all Echium of Authors. 1058. Oplexion R. (armed E.) diff. Echium, cor. hypocraterif. tubo brevi, limbo patulo 5lobo subregularis-Type op. ferox R. Ech. do Andr. t. 39. P. fiuticoso aculeato, fl. spicatis. S. Africa.

1059. Larepines $R$. (much covered) diff. Echium. cor. campanulata subregul. non obliquata, calice inclusa, stam. brevis ineq. inclusis, stylo bifido-Type L. parviflorum R. Ech. do Roth, Mench.P. Poir. \&c. Dichotom. fol. ovaliobl. Africa.

1060. Argyrexias R. (silvery E) diff. Echi$u m$, cal. equalis 5part. cor. subbilab. lab. 2-3lobis eq. stam. exertis declinatis ineq. stig. obtuso.--Type A. condicrens Raf. Ech. do L. auct. bot. reg. 44 . fruticoso, fol. lanc. racemis panicul. secundis. Madera.

1051. Prentuysa R. (5 fimbr.) diff. Ėchium, cal. subbilab. 2-3part. cor. camp. 5loba ineq. basi appendices 5 fimbriatis villosis 5lobis clausa, stam. declin. omnis ineq. stig. bifido.-P. Types $1 \boldsymbol{P}$. strigosa R. Ech. fruticos. L. auct. oft. figured, bot. reg. 36. frut. fol. lanc. strigosis avenis,-2 P. glauca R. Ech. do Jaq. Andr. 325 \&c, frutic. fol. lanc. glabris glancis avenis-3 $\boldsymbol{P}$. levigatı R. Wch. do I. auct. suffirut. levis, fol. lanc. marg. scabris, fl. spicatis, sem. muricatis. All from Africa, the $\mathbb{E}$. glabrum Thunb. is probably a 4 th sp. This $\mathrm{G}$. is very distinct by the nectary. All the Echiums forming thus 9 Gen- 
era had been united together and to Borragines by mere rough aspect!

1062. NOLANIDIA Raf. 1815. another nat. family of mine of same order Lobogr nia, differing from Borragines by the 5lobed ovary and 5 nuts or capsules. Nolana has the calix so different in all the sp. as to indicate several Genera ; I had united Siphonanthus to it, but it appears the type of another family. Nolana prostrata has calix sagittate, $N$. coronata calix cordate, $N$. spathuluta calix spath. $N$. inflata and revoluta have calix ventricose, they will form the subg. Iohypa, Periesta, Spatulina, Gastrina.

1063. Pernloba R. diff. Nolana, cal. carinatis 3 vel 5angul. non sagitt. cor. camp. 5loba, lobis trilobatis, disco 5lobo, caps. 5 monosp.Type P. paradoxa 12. Nol. do Lind. b. reg. 895, b. mag. 2603. prostrata fol. pet. ovatis obt. Chili, fl. blue.

1064. SIPHONANTIITA Raf. this nat. fam. differs from Nolanides and Borragines by cor. 4fid, stam. 4, berries 4 as in Prasium : it is thus nearer Labiates, but the corollas are quite equal. 'The habit of Siphonanthus with ternate leaves and racemose $\mathrm{fl}$. is also like Labiate. Is it a subfamily of them? do any other akin types exist? Falkia and Coldenia difler by sereral styles. But Jussien mentions a Coldenia of Peru with 1 style, corolla 5 fid, 5 stamens which is either a Nolana or a N. G. of Siphonanthia, Monomesia Raf-But Perama hirsuta Aubl. (or Mattnschkea W.P.\&c) is another G. of this family, having same habit, although it has 4 seeds instead of berries, and the flowers agregate as in Globularia. 'The G. Monniera, Ra- 
putia, \&c form another akin family MonIErrnes; differing by irregular corolla and stam. from Nolanidia.

1065. POLIMIA Raf. 1815. This nat. order. of mine next to the Loвogy NIs is easily known by one ovary, several styles or stigmas, fruit without seminiferous valves. I shall give here the 5 families of it as established then, although there may be one or two others, to mention all the $\mathbf{G}$. belonging thereto, would be a task, but several of my N, G. out of Convolvulus belong here.

1. Drcondrania R. 1815. Ovary lobed, lobes 1seeded. Types Dicondra, Coldenia, Falkia.

2. Staticia R. Statice, Armeria \&c, fruit monosperm.

3. Cuscutaria R. fruit capsule multilocular. Types Cuscuta, Kadula R. Kadaras R. Evolvulus, (and all my revised Genera of it) Hydrolea, Eucodon Raf. (Codon Jus.) Sagonea, Ophioxylon, Nama, Sterisia R. \&c.

4. Cressaria R. capsule monolocular Cressa, Aldea R. P. Porana of $\bullet$.

5. llexia R. fruit berry or drupe. Types Cordia, Ehretia, Menais, Varronia, Schrebera, Ilex, Nemopanthes R. and akin Genera.

The next order Ericura with seminiferous valves included the Gentianides and Chironides, with Orobanchides that have irregular corolla; but these last must be removed to the next order Cirasmantuia or Personate, while Epiclia will belong to the class NANTIANDRIA by stamens opposite as in Lysmachides. While the real Convolvulides were removed to order Darynia near to Epacrides by having a regular corolla, although their unequal stamens con- 
nect them with many other families, and they ought now to form with Verbascordes a peculiar order ol suborder Pentanisia with 5 une. qual stamens.

1066. Verbascun, this $G$. is the type of a large family well distinguished from Soi.Anides by a capsule and unequal stamens, corolla irregular ; from Scrophulaidides by 5 stamens; as from Convorvelines, by corolla irregular. Hyosciamus, Blenocoes 716 till Stimenes 722 belong thereto; but Nicotiana and Datura with their reformed Genera with regular corolla, belong to Convorvulines. - Verbascum must also be reformed and includes 4 Genera: the real VerBASCU M has cal. 5part. cor. rotata patens 5loba ineq. stam. 5ineq. filam. inclinatis lanatis antheris conformis, stylo, stig. 1. caps. 2loc. 2valv. polysp. sem. centralis.

1. Thapsus, R. fil. basi lanatis. V. thapsus, thapsoides, montanum, nigrum, pulverulentum, mucronatum, lychnitis, blattaria, sinuatum, floccosum, pheniceum \&c.

2. Lasiake l?. fil. apice villosis, $V$. plicatum sm. fl. gr. 226. - V. lyratum. has calix 5phylous serrate. Subg? Prionula R.-3. 1. FLowosia R. (Diosk) diff. Verbascum, antheris difformis ineq. 3 renif, 20 bl-Types $\mathbf{F l}$. phlomoides, condensata, nemorosa \&c. Verbasc. of Authors, probably a Genus.

1067. Leiosannea R. (smooth st) diff. Verbascum, stam. glabris, antheris ineq. difformis 2 oblongis-Types L. crassifolia and cuspidata Verbasc. do Authors.

1068. RAyonda Richard (not of Mirbel which is Lygodium) diff. Verbascum, cor. 5partita regularis, stam. approx. antheris apice perfora: 
tis, capsula 1 locul. polysp. placentas 2 parietalís. Scaposa-'Type R. pyreanica Rich. Pers. Verbascum myconi L. auct. fol. rad. ovalib. crenatis tomentosis. Mts. pyrenees, fl. blue. Hardly of this family, probably type of a new family with those Solanides and Convolvulides that have a capsule unilocular, such as Diatrema \& c, APLARNIA see 1009; but akin to GratioliDEs differing by 5 stamens.

1069. Cuscuta I. auct. This G. was based upon mere habit with capsule; Cassytha ought to have been united to it by these principles! it includes at least 8 Genera! my real G. Cuscuta has cal. 4-5̃fidus, cor. camp. 4-5fida persistens, appendices nullis, stam. 4-5alt. stylis 2, stig. acutis, caps. pyxidium, 2loc. 2sp. Aphylla, filamentosa, volubilis, fl. subsess. - Type only C. vulgaris or europea of $\mathrm{L}$.

1070. Kadula R. (Diosk) diff. Cuscuta, cor. ovatis 4 fidis, stam. 4, stigm. 2 capitatis. $f$. pedunc corymbosis-Type R. corymbosa $\mathbf{R}$. Cusc. do RP. t. 115. P. Peru.

1071. Antrianema R. (fl. sine fil.) diff. Cuscu$t a$, cal. membr. 5part. cor. urceolata 4-5fida, stam. 4-5, stylis 2 longissimis, stigm. capit. $\boldsymbol{A}$ caulis, parasiica, caulib. evanescens, fl. glomeratis densis-Types 1 A. paradoxa Raf. (s. verticillata) Cusc. do Raf. annals 97, glomerulis amplectens, verticillatis, cor. subtub. 4 fidis. Kentuky-2 A. capitutu Raf. acaulis 98 annals, fl. glomer. capitatis. cor. subcamp. 5fidis. Kent. Illinois.

1072. Pentake R. (5 points) diff. Cuscuta, cal. angulato magno cor. eq. 5 fido, cor. conica כdentis corniculatis, stig. 2 capit. fl. puniculatis-P. chinense R. Cusc. do Lam. W. P. Vitm. 
1073. Kadurias R. (Diosk) difl. Cuscuta, cor. 5part. reftexa, $A$. racemosis-K. reflexa $\mathrm{R}$. Cusc. do Roxb. cor. t. 104. P. India.

1074. Nemepis R. (on filaments) diff. Cuscuta cal. 5part. cor. camp. vel. urceol. 5fida, stam. 5, appendices squamis 5 opp. stam. fimbriatis, stig. capit. Caulib. ramosis, fl. varis disp.-'Types 1 N. odorata R. Cusc. do RP. t. 105. P. fl. congestis sessilib. cor. camp. Peru, fl. with purple dots-2 $N$. americana $R$. Cusc. do $L$. auct. fl. congestis pedunc. cor. urceol. filis croceis, fl. albis. North America-3 N. prolifera R. Cusc. Amer. nonnulis, surinamensis Schill. Vitm. fl. fascicul. proliferis, cor. tubul. lac. patulis, filis flavis, $\mathrm{fl}$. viridescens. Antilles and $\mathrm{S}$. Amer.-4 S. africana R. Cusc. do W. P. \&c. americana. 'Thunb. pedunculis unifl. bracteatic. 1075. Lepimenes R. (scale lunular) diff. Citscuta, cal. 4part. cor. infundib. 4fida, stam. 4, squamis 4 opp. lunulata crenata, stigm. 2 acutis-Type L. epithymum R. Cusc. do Sm. W. P. europea Lam. fl. sessilib. rubellis. North of Europe.

1076. Aplostylis Raf. diff. Cuscuta,cal. 4 fidis cor. ovata 1 dent. stam. 4, append. nullis, stylo unico capit. $f$. racemosis-Type A. lupuliformis R. Cusc. do Krok. Sil. t. 36, monogyna W. P. Sm.-This G. by the single style is not even of the same family and nearer Convolvulines; but should the stam. be opposed to corolla as in Cassytha, it will be a lirk with that $\mathbf{G}$. and of same family.

1077. Cassytura 1. a genus widely mistaken by all botanists, with habit of Cuscuta and Viscum! of doubtful aff. in Jussieu, deemed by him and Necker akin to Laurus! which might be if it is dicotyle, but it is rather monocotyle and 
akin to Olax, Bassia-cal 3part. cor. globosa 6fida, stam. 6 fert. segm. opp. 6 interna sterilia, stylus 1, stig. sub 3fid. caps. glob. 1sp. ad corolla subbacata tecta.-Types C. filiformis L. filif. verrucosa, fl. spicat. India-2 other subg. or Gen. not yet well descr. belong here, all the sp. of Viscum L. without leaves belong here according to Smith and probably form a peculiar akin Genus. Most of the Botanists have given different characters to this Genus! hardly 2 or 3 agree which indicates several sp. or G. most of them unite Volutella to it or ascribe its character to Cassytha. The Cassutha cornea of Rumph, or C. corniculata I. has never been well described, having spines, leaves linear lanceolate and horned flowers, it must probably form a G. perhaps the Collodion of Loureiro?While the Cuscuta 5. t. 184 of Rumph. which is the Cassyta zeylanica Gaertn. $t 27$, \&c ought to form another G. RuMpuTRIs Raf. diff. by stam. 6 ineq. 3 shorter alt. 3 longer biglandulose at base, 1 style, 3 stigmas, nut longer than baccate coroila. Type $\boldsymbol{R}$. fasciculata $R$ af. fol. paucis fascicul. tenuis.

1078. Ozart inis Raf. diff. Cassytha fl . . stylo tereto persistens, caulib. artic. aphylis flowers to be described ...types 10 . opuntioides $\mathbf{R}$. artic. compr. $2 O$. triquetra $R$. artic. 3quetris (V. artic. Burm) -3 O. capense $R$. artic. teretis baccis opp. -40 . paradoxa Raf. (Visc. vertic. L. sine fol.) rámis teretis, baccis confertis: all those united to Viscum by L. \&c O. opuntioides is besides Cactus pendulus! Ait and Cassytha baccifera Sal. thus put in 3 Genera! 1079. Spironema R, Volutelia Forsk. Vitm. (n. mal. ad Voluta) diff. Cassytha, cal. 6phyl. deciduus, cor. ineq. 6part. 3 ext. hasi intus cor- 
moculatis, anth. 9 sessiles, 3 int. steriles glandnlif. stig. capit.-ip. aphyla R. V. do F. V. filif. striata, villosa, f. spicatis. Arabia-If these (i. do not belong to OrAXIA and are monocotyle they may form a peculiar family CASY'IIDES by the corolla persistent and baccate, but they agree with Olax and akin G. by the fruit and corolla anomalous, some sterile stamens \&c. I add the 3 Genera blended in Olax.

1080. Olax L. J. \&c. cal. integro, cor. infund. .3loba, stam. 3 fert. lobis opp. 4 steriles, styl. 1, stig. 1, fruct. lsp. frut. volub. ramis flagelif. fol. alt. avenis-O. scandens Roxb. zeylanica L. fol. pet. ovata, ped. axil. ramosis. E. Ind. and Ceylon, put in Sapotilles by Jussieu, while he puts the next G. in Aurantia! while Smith deems them of same Genus!

1081. Fissilia Com. Jus. diff. Olax. cor. 3part. stam. 3 fertiles gerens, 3 sterilis furcatis, stig. 3lobo, drupa 1sp. arbor.fol.alt.-F. psittacina Jus. Olax fissilis Smo fol. integris laurinis semperv. racemis. axil. African Ids.

1082. Spermoxyron Lab. diff. Olax, cor. 5part. 1-2liberis petalif. 3 coalitis cnm stam. 3 opp. stam. 2 sterilia, capsula 1sp. Aphyla cassythif.-Sp. australis L. Olax do Sm. flagellis strictis aphylis, probably alt. 3 G. monocotyle.

1083. SYNAR'THIA Raf. 1815. 'This family of mine includes the Dipsacea of Jussieu, which Decandole has ascertained to have a free ovary, together with Globularia, Alypum and akin Genera. They are distinguished by corollas irregular, single seeds, stamens commonly 2 or 4, flowers capitate, often with a perianthe. It is a family of nat. order. OtisPermia, where belong also Pyrenaria, Verbenides, Vitexides \&.c. The G. Opercularia, Cryp- 
tospermum and Evea must be added to Dipsacea; but the Valerianides of Dec. are quite a peculiar family. 'I'he G. Scabiosa I. the most numerous in species offered a crowd of anomalies and yet has been left nearly untouched except by Vaillant and Necker ; the linneists blending to this day 16 fine Genera into Scabiosa, which I have distinguished since 1815, and shall now enumerate, having verified most of them alive or dry.

1084. Scabiosa Necker, Raf. non L. nec auct. Periantho multipartito, patens ineq. phorantho piloso, corollis 5fidis ineq. radiatis. stam. 4, akena angulata denticulis coronata-Types Sc. columbaria, eburmea, argentea, atropurpurea, prolifera, alba, grandiflora, ucranica, cretica, graminif, pumila? indurata, maritima \&c.

1085. Astrocelinalus Vail. Ad. Chetastrum Neck. diff. Scabiosa, Phorantho paleaceo, cor. camp. 4fidis, ineq. akena globosa, coronatis setis 5longis patulis-Types Sc. sylvatica, ciliata, rigida, tatarica, uralensis, integrifolia \&oc.

1086. Prerocephalus Vail. Ad. Pteropogon Neck. diff. Scabiosa, Phorantho nudo, cor. camp. 5fidis ineq. akena globosa, corona duplici ext. marginata, int. papposa, pappus plumosusTypes 1 Pt. incanus Raf. Sc. pteroceph L. auct. 2 Pt. plumosus R. Sc. plumosa Sm. Knantia do L. this forms a subg. 'Trichopteris Necker by Per. aphyl. pappus 12 pilose.

108\%. Succisa Neck. diff. Scabiosi, Per. imbricato, phorantho paleaceo, corollis 4 fidis subeq. akena ventricosa obl. 4dentata-Types 1 S. vulgaris R. Scab. succisa L. 2 S. ambrosioides, 3 dichotoma, 4 arvensis, 5 ciliata, 6 scabra \&c. 
1088. Cephalaria Schr. diff. Scabiosa, Per. globoso imbric. cor. 4 fidis subeq. akenis 8 aristatis-Types C. S. Sc. alpina, syriaca, attenuata, \&c.

1089. Euptilia Raf. (well feathered) diff. Scabiosa, per. glob. imbric. cor. 5 fidis ineq. akenis aristatis et papposis-Type E. cretica Raf. Sc. papposa involucrata Sm.

1090. Presiopsora Raf. diff. Scabiosa, per. patens 8-10part. cor. 5lidis subeq. akenis denticulatis-Types $\mathrm{Pl}$. sicula, afvicana, monspeliaca, of $\cdot c$.

1091. 'Teremphas R. (cut cup) diff. Scabiosa. Perianthis urceolatis multifidis.--Type $T$. urceolata R. Sc. do Desf.-A. subg. Pentexis Raf. has per. turbin. 5fido, type Sc. rutefolia Vahl. 1092. Xetola R. (aristulate) diff. Scabiosa, Periantho 5 phyllo, fol. longe aristatis, corollis 4fidis subeq. phorantho paleaceo aristato.Types 1 X. transylvanica R. Sc. do L. $-2 X$. trenta R. Sc. do Haquest.Vitm. Sc. haqueti Lam. wrongly united to Sc. leucantha by Persoon.

1093. Lonelosia R. (edge membr) diff. Scabiosa, Phorantho globoso, akenis corona membranacea plana rotata orbiculata, integra vel. emarg. nervosa vel scabra.-Types L. stellata and Coronopifolia Raf. Scab. do auct. L. palestina will be a $3 \mathrm{~d}$ sp. perhaps a subg. by segments of corolla trifid. L. simplex a 4 th and a subg. by Crown campanulate Codostelma Paf. 1094. Leveopsora R. (white Sc) diff. Scabiosa, per. globoso imbric. cor. 4 fidis subeq. lac. inf. deflexa, akenis trigonis 3dentatis-Type S. leucantha Raf. Scab. do I. Sc. ustulata is perhaps a 2d sp.

1095. Sixalix R. (carrot cal.) diff. Scabiosa, 
per. pinnatifido villoso-Type S. daucoides $\mathbf{R}$. Scab. do Desf. $\mathcal{f} \cdot \mathrm{c}$.

1096. Gonokeros R. (angle horned) diff. Scabiosa, per. imbricato, cor. 4 fidis subeq. akena angulis in cornibus desinentib-Type G. hungaricus R. Scab. corniculata Waldst. Pers. 1097. Pentena R. (5 or 1 ) diff. Scabiosa, per: patens multif. phorantho ovato, akenis aristis 1 vel 5 instructis-Types P. ochroleuca Scab. do L. aristis 5-2 P. uniseta R. Scab. do Savi t. 2 , Vitm. arista unica.

1098. 'I'remastelma R. (perf. croïn) diff. Scabiosa, pet. 6phylus, akenis corona ext. dilatata 10perforata, 10nervosa, corona int. papposa.- Types 1. Tr. brachiata Raf. Scab. do Sm. Knautia palestina $L_{\text {. }}$ auct. fol. integris $-2 \boldsymbol{T r}$. sibthorpi R. Scab. do Sm. fol. pinnatif. Cyprus. 1099. Tulasidia R. (compr. 2) diff. Scabiosa cor. 4fid. ineq. radiatis, akenis compressis bidentatis.-Type Thl. bidens $\mathbf{R}$. Scab, do Sm.

1100. Diototheca Raf. fl. lud. not of Vaillant which is Morina 'T, L. diff. Scabiosa, per imbric. scariosus, phorantho glob. paleaceo, paleis scariosis, corollis 1fidis irreg. lac. sup. brevis emarg. inf. longior, stam. 2 brevis in tubo cor. akenis ovatis, faux bifida-G. akin to the last, differing by the 2 stamens as in Morina. Type D. repens Raf. repens pubescens 4gona, fol. opp. petiolatis ovat. dent. scabris, fl. axill. Louisiana.

In all these $G$. the fruit is a single seed free within a hollow calix perforated at the end, somewhat as in the CAR ExIDEs, and all have a single style with simple stigma: such fruit ought to be called an utricule rather than akena which is a kind of nut. 


\section{CENTURIA XII.}

1101. A "istolocma, no botanist had thought to revise this Genus, till I partly did in my med. fl. 18:25: yet it ollers perigone unilabiate or bilabiate or regular, 5 or 6 or 12 stamens, 1 to (i stigmas ame many limels of capsules! therefore forms a group of plants including 12 Genera-my Asistorochis Raf. has perigono adherens, tubulato, sepe incurvo, unilabiato basi ventricoso, apex lingulato integro, antheris 6 sess. epigynis stigma stellato Gipart, capsula 6 gona, 6locular, polysperma-4 subgenera.

1. Chenatops, labio oblongo vel ovato, Types A. anguicisla, maurorum, betice, indica, clematitis, rutunda, longh, pistolochia, sempervirens? liempferi, punciula, barbata, pallida, hirla \&c.

2. Eirtoglossa. labio dilatato spatulato retuso vel cordato. 'I'ypes A. surinamensis, reniformis, odoratissima, glanca, altissima \&c.

3. Glossura, labio caudato vel appendiculato 'Types A. canduta, A. pelteta, A. maxima, \&c.

4. Mestrenipius, tubo curvo coarctato apice angulato, labio dentato. 'I'ypes $\boldsymbol{A}$. grandifiora, A. fetens \&c.

1102. Hexaplectris R. (6 spurs) diff. Aristol. Perig incurvo (icostato, basi 6 calcaratis, labio dilatato vel cuculato longe candato, capsula clavata-Types $\mathbf{I} \boldsymbol{H}$. bicolor R. Arist. do L. b. reg. 139!, fol. cord. 3lobis, longe pet. stip. acuta fl. axill. sess. S. Am. fl. yellow, lip fuscate cuculate, tail pedal-2 $\boldsymbol{H}$. fuscuta R. Arist. candata Buoth, b. reg, 1453 (non Jaq. I) fol. renif. 3lobis brevi pet. nicro macul. stipula obl. labio renif. Brazil, fl. brown, tail pedal.-Per- 
haps some sp. of Glossura belong here, if they have spurs.

1103. Plagistra R. (obliqual) diff. Aristol. Perig. tubuloso incurvo, non labiato, apex integro oblique truncato-Types $P l$. cretica Raf. Arist. do Lam. P.- the hirln of L. included this sp.

110 . Tropexa R. ( 6 keels) diff. Aristol. perig. infundibulif. tubo curvo Ggono, labio spatulato, capsula globosa 6carinata-Type Tr. bilobr. R. Arist. do L. caule scand. fol bilobis.

1105. Einoneia R. (1 less) diff. Aristol. stam. 5 caps. 5locul.-Type $\mathbf{L}$. bracteata Raf. Arist. pentandra L. \&c.

1106. Anbexa R. (n. Arab) diff. Aristol. perigono sacato inflato, unilab. lab. producto contorto dilatato bilobo.- Type $A m b$. labiosa $\mathbf{R}$. Arist. do b. reg. 68!), b. mag. 2545. ringens Link. t. 13 non Vahl. Volub. angul. fol. subrenif. Brazil.

110\%. Pistolochia R. diff. Aristol. perig. bilabiato, incurvo, subtrifido, stigma sess. unico magno capit. lobato-lypes the various sp. blended in Arist. serpentaria see my med. fl. and new fl. 1. P. serpentaria med. fl. t. 10-2 latifolin-3 angustifolia of c.

1108 . Diglossezis R. diff. $\Lambda$ ristol. perig. bilabiato, labis ineq, tubo bilobo, caps. obl. striata, stigma caps. 1 locul-Types 1 D. trinervis $\mathbf{R}$. Arist. bilabiata L. auct. fol. ovatobl. oht. 3nervis.-2 D. latifolia R. Arist. ringens Vahl. \&c fol. subrot. cord. lab. sup. spatul. inf. lanceol3 D. cymbifera Raf. Arist. do Mart. t. 49. b. reg. 1543. fol. renif. lab. sup. ovato acuto, inf. pandulato emarg. Brazil: the A. cordifl gigartea \& $\mathrm{c}$ perhaps belong to this $\mathrm{G}$.

1109. ENDoneca R. 1828 (12 inside) diff. 
A ristolochia, stam. 12, labio plerumque ovato'Types E. semprevirens R. $\Lambda \mathrm{r}$. do anct.-2 $\boldsymbol{E}$. hastata R. Arist. do Nut. \&c.

1110. Psopniza R. (false snake root) diff. Arist. perig. bilabiato, stam. 12. stigma unico sess. lohato convoluto.-'Type P's. undulata Raf. Arist. serpent. Big. med. fl. ic. fol. obl. subcord. acutis undulat. trinervis, Florida \&c. fl. red. one of the sp. blended among our snakeroots.

1111. Ptrenipus R. (winged tube)diff. Aristol. perig. tubulosis tripteris trilobis, stam. 6-8, stig. subsessile trilobo, caps 3 loc? - Type Pt. tripteris R. Arist. do Raf. fl. lud. 65 sarmentosus,fol. cord. fl. spic. bracteatis. Louisiana, fl. sinall white, see my remarks in new fl. on possible mistake of Robin.

1112. Sirminsa R. 1828, diff. Aristol. perig. tubuloso, apex trilobo subeq. non alato, stam. epistylis, stylo crasso, stig. 3-6-Types 1. Siph. glabra Raf. Arist. sipho Lher \&c-2 S. tomentosa R. Ar. do Sims \&c both scen alive.

1113. Hixastyus R. 1825 neog. 24. diff. Aristol. and Asarum, cal. tubular trifid, stam. 12. ovar. semilib. tereto, apex concavo, stylis 6 lateralis erectis, stig. 6 obliquis truncatis bicornis. caps. 6loc. oliyosp-'Type $\boldsymbol{H}$. arirolia R. A sarum do Mx. El. \&c. fol. cord. sagitt. Ken. tucky to Carolina. Seen alive nearer to Siphisia than Asarum!

1114. Bragantia Lom. non Vand. perig. tubul. ineq. 3fido, patens, corona brevi cyathif. stam. 6. caps. siliquef. 4 gona 4 loc. 4ralv.-'Types 1 Br. racemosa Lour. fol. lanceol-2 Br. latifolia Wal. fol. obl. subcord. caulib. 1-2phylis. Birman.-The Genera Munnickia and Ceramium (bad name employed for a Fucus) tomen- 
tosum of Blıme. Br. blumi Lind. must be verined, near Siphisia.

1I15. Trineriza Lind. perig. 5part. stam. 9 in 3 phalanges radiatis, stig. Gdent. caps. ut Bragantia-'Iype 'Tr. piporitu, caule flex. genic. fol, ovatolanc. costatis, subt. reticul. pubesc. racemis pancifl. axil. Ceylon.

1116. Stembxa R. (6 ster.) Trichopus Gaertn. fil. Lind. (non 'Trichopus Muller (im.) Perig. tubul. 6fido, stam. Gfertilis, 6sterilis in foveis stylus 1, stig. 3 bipart. caps. 3quetra 3locul. ipu a, loculis 1-2sp. indehiscens-3 types from Cev on. 1 St. cordata R. fol. cord. triang. obt. retusis-2 $s t$. media, fol. ovatolanc. acum.-3 St. angustif. R. fol. lin. lanc. acum. caps. 1 spermis, an Genus? - Lindly asserts that all these G. and Aristolochia, have a peculiar anatomical structure of stems different from the Dicotyle plants and shrubs. Perhaps like Cuscuta, Cassytha, Cactus \&

111\%. Mezorea Aubl. Jus. Vit. Tephranthus Necker. Rhopium Schr. W. P. Perig, 6part. foveis 6. stan. 3 stylis adnatis. ovar. libero. stylis 3, caps 3gona 6loc, Gvalv. 6sp. Frutex, fol. alt. stipulatis. $f$. corymb-'Type $M$. gujanensis vel. Rh. citrifolium. fol. sulsess. ovat. acut. lucida integra-Very singular G. of doubtful affinities, introduced here to show that it links with $\mathbb{H e x a s t y l i s} \mathbf{1 1 1 3}$, and Steirexa. If all these $G$. are to be l'emoved by anatomical structure, the ovary free, half fiee or adherent will be less essential, forming however 3 families Asarides, Meborides, (jutinines with a berry, that stand in the nat. method at the very end of the Dicotyles, as a transition to the first order of Monocotyles including the Epigyne families, Hydrocharides, Valisnerides, Orchides \&c. 
Perhaps they are even Monocotyles and Endogenous, as the ternary numbers of organs appear to indicate-The G. Salacia and Strunifia form another akin fanily sisucunes having petals besides a divided catix and epigyne stamens-Necker puts this G. Meborea near to Passiflora, and perhaps the stamens are monadelphous as in Passiflora and Sisyrinehi $t m$ once deemed gynandrous by Linnens.

1118. Nepentues L. auct. Bandura Burm. Ad. a wonderful $G$. of doubtful affinities, which Lindley reduces to Aristolochia! an egregious absurdity since it is dioical. with a free pistil ... It is the type of my family Nepentinues, near to Sarazinides differing by dioical monadelphous stamens, akin also to the order Eprmesia or Euphorbides, and the families of it with united stamens lilie Ricinines $\mathbb{E} c$ chief difference a single large stigma. The G. Aspidistra and Macrogyne are so near to Nepenthes that all must be monocotyle (nay perhaps all the Asarides?) if so they form a peculiar series and link with Sarazinia on one side and Paris on the other by the quaternary number of parts. Many sp. have been blended in N. distilatoria, Jussieu noticed 3, Wildenow had 3 others, there are 7 at least $1 \mathrm{~N}$. distilatoria $\mathrm{L}$. oft. fig. bot. mag. 26:29. 2798, Lod. 1017 \&c caule ramoso, fol. sparsis avenis, ft. racemosis geminatis. India2 N. madagascuriensis, W. P. -3 N. phylamphora W. P. Genus in Lour simplex fol. app. fl. spicatis-4 $N$. zeylenica Raf. Bandura Burm. zeyl. t. 17-5 N. indica Lan. fol. reticulatis-6 N. cantharifera Jus. Rumph. Aubl. 5. t. 59-7 N. scyphus Jus. Malaca-Perhaps several subr. or Gien. are blended here. 
1119. NEPLNTHIDTS Raf. (monoc? endog?) Perigone and anthers with 4nary parts, a very large peltate stigma, capsule 4loc. habit of Aroides and Ilethonica. :3 ( $\mathrm{r}$. at least.

1 Nepenthes Dioic. cal. Apart. pers. stam. 1218 connatis, caps, polysp. coulescens, fol. appendiculratis.

2. Aspidistra Herm. cal. tubul 8fido, antheris 4-6liberis, caps.4sp. type A. punctata E. b. reg. 9\%\%.

3. Harergyne Link. Merm. cal. camp. 8fido. antheris 8 liberis, stigma 8 gona, caps. 4sp-type M. convalarifolia L. Asp. lurida E. b. reg. 628, b. $\operatorname{mag} .2499$.

1120. PASSINLOREA Raf. 1815. This family of mine was established long ago, and I had reduced $1^{\circ} \mathrm{G}$. to it, mostly blended in Passiffora by the Linneists, Necker and Jussieu had already 4 or 5 . 'They are but slightly akin to Cucurbitacea as deemed by Jussieu, by the fruit and habit. They are nearer the Strigilidis and Malpighinia by the perigone and united stamens, several styles \& $c$, thus put in my natural order Aneripunia along with them. See till 1134.

1121. Passifiora Raf. non L. This G. was badly understood by the Linneists, but reformed by Jussien. Mine has Perigono colorato basi urecol. limbo 10 pratito colorato, 5 alt. petaliformis. Corona multipartita. interua nectarif. basi mecol. gymophoro stam. 5 gerens basi comnatis, stylis 33 , fruct. bacca vel. pepo (cysto Neck) uniloc. polysp. placentas 3, sem. arillatis-Many sp. in 3 subgenera-1 Grana.dilla Ad. no involucre-2 Maracoa Raf. fl. involucrate. 
1122. Trupsinna Raf, diff. Passiflora, fl. involucratis bipimnatif. petalis cal. eq. nect. filif. brevis, capsula inflata, placentas 3 internis seminiferis-'Iype 'l'? fetide R. Pas. do L. b. m. 2619 , seen alive. All the capular sp. must be divided of course from the baccate kinds.

1123. Xerogoxa Raf. dift. P'assift. petalis brevior, corona brevi, nect. int. duplice cupula. Ggona plac. 3 int. seminif-'Type Y. biloba Raf. Pas. capsularis 1. anct. Dec. b. mag. 2868. 3 var. fol. bilobis, fol, bifidis, fol. maculatis.

1124. Moneca Rheed. Jaq. diff. Passifl. dioica, cal. tubul. 5fido. petalis 5, nect. sq. 5-10, stam. 5 liberis, ov. vix stipit. capsula Bvalvis polysperma-Several sp. 6 in Jaq. deviating by the free stamens and valvular capsule. Blended in India with the Convolv. called also Modeca, compare with the Euphorbides.

1125. W acropiora Raf. diff: Passifl. cal. tubo brevi, gynophoro longissimo, nect. dupl. series, stam. dilat. planis basi coalitis, antheris magnis -Type N. sanguiner Raf. Pass. do Sm. \&c fol. 3lobis serratis alcutis egland. cal. et ovar. toment. Antilles.

1126. Meropiris lRaf. diff. Passifl. cal. 5part. petalis nullis, corona duplex, nect. sepe simplex planum-Types M. peltrta, saberose, minima pallidre, multiflora, "mgustif. premiosa, hederacea foc, all Passifloras.

1127. Munucuia T. Jus. Pers. Erndelia Necker, diff. Passifl. cal. basi urceolatus, nect. tubuloso truncato-Type MI. ocelleta Pers. 'Pas. murucuia I. auct.-Persoon had $3 \mathrm{sp}$. in this $\mathbf{G}$. each being types of Gencra.

1128. Bepmistema R. (cil. crown) difi. Passifl. cal. tubuloso, petalis brevis, nect. tubul. lobato ciliato-'Гype Bl. aurentia Raf. Muru- 
cuia do P. passifl. do Forst. W. Sm. Andr. rep. t. 295. fol. glabris 3-5lobis obt. New Caledon.

1129. Perzinis Raf." (around half) dıf. Passiff. no petals, calix globular-Type $\mathbf{I}^{\prime}$. orbiculata R. Murucuia P. Passifl. Cav.

1130. Synactn.a Raf. (un. rays) diff. Passi . no petals, rays of nectary united at the base in a cyl. crown inserted on the tubular 5 parted calix.-C'Iype S. viridiflora R. Pass do Cav. W.

1131. 'Tacsonia Jus. diff. Passifl. cal. tubul. 10lidus, coloratus, corona glandulis, gynophoro elongato-Miany sp. in 4 subgenera. 1 Perimna Raf. invol. nuliis-2 Tristegia Raf. invol. 3phylo-3. Siphostegia Raf. invol. tubuloso4 Bralostis R. Cal. globoso, type P. glauca auct. is it a Genus? how is the crown?

1132. Onostecma Raf. (toothed crown) diff. Tacsonia, corona dentata non glandulosa, stam. filam. planis-Types $O$. pedunculata and adulterina Raf. 'Tacsonia do auct.

1133. Erndelia Raf. non Necker, diff. 'Tacsonia cal. et cor, apex reflexa, corona duplice concentrica-Type E. reflexiflora Raf. Pas. do Cav. Tacs. do Pers.

1134. Periconia R. diff. Pass f. cal. crmpanul. lac. subul. petalis dilat. nect. plicato-Type P. perfoliata Raf. Passifl. do L.

1135. Sananunda Clus. Ad. I'asserina-L. ad Passer! 'This G. includes 6 blended: the real hardly differs from Daphne except by fruit. a nut instead of berry. All the sp. with 2 stamens only are the G. Pimelea. Necker applied Sanamunda to the Daphnes with tubular flowers, not funnel shaped. All have 8 fertile stamens in 2 rows on the tube of coroliform calix.

1136. Belvala Ad. diff. cal. longe tubur. 4 fido, extus caliculo diphylo, intus 8dent. vel 
stam, steriles 8 , fertiles 4 eq. capsula membran. Isp. utriculus. fol. oppos. fl. axil. spicatis.Type 1 B. spicata Raf.Passerina dodecandra $\mathbf{L}$. omitted by many botanists.

1137. Trinemandra Raf. diff. Sanam. cal. 5 fidus, stam. 5 eq. unica series-Type Tr. spicata Raf. Pass. pentandra 'Th. W. P. auct. fol. ovat. hirsutis, spicis ovatis terminalis. $\mathbf{S}$. Africa.

1138. Stenoctis R. (8 ster.) diff. Sanam. Stam. 16, 8 steriles glanduliformis superis, 8 fertiles inferis in tubo-'Types St. capituta and uniflora Raf. Passerina do auct.

1139. Pausia R. (olea lat.) diff. S. cal. tubo campanul. ut olea, utriculo baccato-nearer to Daphne by fruit, type $\boldsymbol{P}$. orientalis Raf, Pass. do L. auct. fol. lanceol. obt. fl. axil.

1140. Balendasia R. (ball inside hairy) diff. S. cal. globoso 4dent. intus hirsuto, utriculo baccato-Type B. ericoides Raf. Pass. do L. auct. fol. lin. imbric, glabris. S. Africa.

1141. Gastrilia R. diff. Daphne, dioica, cal. basi ventricoso limbo 4fido-Types 1 G. geminata Raf. Daphne dioica I. Vitm. Gouan t. 17. fol. imbric. lin. lanceol. fl. axil. geminis. S. Europe, fl. ochroleucos, blended with Passerina hirsuta by some botanists-2 G. umbellata Raf. Daphne occid. Siv. P. fol. alt. lanceol. glabris, f. term. subumbelatis. Artilles.

1142. 'I'umelaia R. (n. grec.) Sanamunda Necker non Ad. diff. Daplne, cal. tubuloso, apex 4 fido erecto non infundib.-All the sp. of Daphnes with tubular flowers.

1143. Octoplis R. diff. Daphne, cal. hirsuto, intus nect. $8 \mathrm{vel}$ petalis 8 minimis, fol. sparsis fl capit. bracteatis-Type O. polistachya Raf. 
Gnidia do Berg, Vitm. \&c. fol. linear. capitulis panicul. bract. ovatis, obtusis. S. Africa, near to G. Steiroctis 1138, but petaliform nectaries.

1144. Nemoctis R. (8 fil.) diff. Daphne cal. villoso, limbo 4part. stam. 8 exertis, filam. capillaris, petalis 4 ut in Gnidia, fol. sparsis $f l$. capit. involucr.-Types 1 N. buxifolia Raf. Gnidia filamentosa L. auct. Lachnea buxif. Lam. P. fol. sessil. ovalibus-2 N. angustifolire Raf. var. prec. auct. fol. sess. lanceol. both S. Africa. Lachnea differs by unequal cal. stam. and drupaceous nut.

1.145. Dessenia Raf. diff. Ginidia, cal. filif, limbo 5part. petalis 5, stam. 10, 5 exerta, fol. sparsis, fl. capit. involucr.-Genus medial between Gnidia and Dais. Dessenia was Adanson's name for Gnidia. Types 1 D. lanceolata Raf. Gn. capit. L. auct. fol. lanc. fl. term. invol. 8phylo. S. Africa.-2 D. daphnefolia R. Gn. do L. $\& \cdot c$ fol. obl. cuneatis glabris, capit. ped. invol. 5ph. Madagascar-3 D. hirsuta Raf. var L. fol. obl. spatul. hirsutis canis, invol. $5 \mathrm{ph}$. Madagascar-Subg. Xaiasue Raf. diff. by no petals, seeds rostrate not baccate $f l$. umbel-Type $\boldsymbol{X}$. bicolor R. Stellera chamajasme L. auct. fol. lanc. fl. albo purpureis. Sibiria. Stellera is octandrous.

1146. Ścopolia L. supl. not Forster (Griselinia) nor Smith (Todalia) diff. Daphne, antheris connatis in duplice columna alteram posita, fol. alt. fl. axil. capitatis, involucris diphylis. -Type Sc. pendula Raf. composita L. Vitm. Daphne penclula $\mathbf{S m}$. W. P. Arborea, fol. pet. obl. acum. pedunc. pendulis axil, 1 floris. Java -Wrongly pat in Gynandria octandria by $L$.

1147. Nestronia R. new fl. 503, Dioica, cal. camp. 1fido, stam. 1 fil. brevis lin. drupo 1sper- 
mo. fol. oppos, pedunc, multifl.-See my long account of this new $G$. in my new flora. 'Types $1 \mathrm{~N}$. umbelluta R. 504. fol. ovat. rhombeis integris planis glabris, ped. axil. umbellatis 3-5fl$2 N$ ? undulata R. 505. fol. lato lanceol. undulatis, drupis ped. solit-Both frem Florida and Georgia, small shrubs. Of family Dapunidia like all the preceding since 11:35.

1148. CLY'THRELIA Raf. 1815. LentiBUlaria Rich. Lind. This family based on Utricularia and Pinguicula, will now contain 20 genera. It is a very distinct group, which was very improperly annexed to Prinulacea by Jussieu because the fruit is somewhat alike, but there is not a single affinity besides. I put it next to Gratiolines; but have now my doubts about it, and have been led to consider this family as rather belonging to Monocotyles and Lndogenous, when it would widely change place and come near the Commelines with irregular corollas. Nay these aquatic plants like many others partake somewhat of the Cellular structure, very evident in the vesicular organization of Utricularia. We have no positive evidence of the seeds being dicotyle and even if they are, they may like Juncus be exogenous notwithstanding. Yet here the calix alone is persistent, while the corolla is very deciduous, not at all marcescent.

1149. Utricularia L. Lentibularia Ad. This aquatic G. of only 9sp. in Linneus has been increased to nearly 80 lately, the tropical climates abound with unnoticed sp. Brown found 24 new ones in Australia, in North America nearly 20 have been detected, see my monograph, Leconte had already 13, Vahl and Poiret described 36sp. the fine monograph of Smith in Rees 
contains 62 sp.-But out of so many, disparities occur and several Genera are indicated thereby, which I have endeavored to distinguish. The real original genus has calix bivalvis eq. persistens, cor. ringens calcarata, bilabiata, labis clausis integris, faux gibbosa, stam. 2 inclusis in tubo, St.stig. 1, cups. globosa vix valvata uniloc. polysp. sem. centralis. Facies aphỳla, vel. fol. squam. scaposis, radicib. sepe fluitans utriculatis.-The types are U. alpina, vulgaris, obtusa, furcata, cerulea? and $\mathbf{4 0}$ more; but the flowers of each must be examined again.

1150. Lentibularia Raf. (or Xananthes,open $f$. if prefered) diff. Utric. corollu hians pervia, faux non gibbosa, calcar obsoleto carinato decurens-Type $\boldsymbol{L}$. minor Raf. Utr. do L. auct. Lentibularia of Gesner was applied to the whole G. by Adanson and since to the whole family by Richard, whence my 2 names.

1151. Trixapias Raf. diff. Utric. calcar globoso, capsula subulata-Type Tr. capillacea $\mathbf{R}$. Utric. do V. Sin.

1152. Askofake R. (box lent) diff. Utric. capsula lenticularis bivalvis non globosa-Type $\boldsymbol{A}$. recurva $R$. Utr. do Lour. Sm. This and the last deviate so much in the capsular shape quite globular in all others, that this indicates other characters to be sought for.

1153. Stomorsia R. (hairy mouth) diff. Utric. cal. ineq. ovat. lab. inf. dilat. deftexo trilobo, palato villoso-Type St. cornuta R. Utric. do Mx. \&c, seen alive see my flora.

1154. Meionula R. (minute) diff. Utric. cal. inequalissimo, fol. inf. magna concava carinata, infructo inflata-Type $M$. parviflora Raf. Utric. 
minutissima V. Sm. scapo capillaris 1-4f. squamis acutis, calcar subul. Malaca, ft. minute blue.

1155. Hanulia R. (n. Arab.) diff. Utric. cor. lab. sup. concavo emarg. infero orbicul. integro calcar obt. curvo, capsula stylosa membranacea subplurivalvis, ad basi cal. inflato coalita.Types 1. H. alba Raf. Utr. inflexa Forsk. Sm. fl. albis-2 H. flava Raf. Utr. stellaris Sm. fl, flavis-very dift. from that of $\mathbf{L}$. of next $G$.

1156. Lepinctis R. (sq. stel.) diff. Utric. corolla sine calcar, lab. inf. saccato, caule squamis vertic rad. sine utriculis-Type Lep. stellaris R. Utric. do L. W. P. caule filif. apice ramoso, squamis 4-6nis oratis ciliatis. India, very distinct if realy without spur.

1157. Meloneura Raf. (membr. nervose) diff. Utric. cal. membr. ineq. fol. sup. magna orbiculata nervosa emarg. cor. lab. inf. bifido, caps. membr.-Type $M$. purpurea Raf. Utric. striatula Sm. fol. petiol. conc. scapo 3-4f. West Africa, fl. purple.

1158. Nelipus R. (n. ind.) diff. Utric. cor. lab. inf. bifido vel bilobo, ad 1157 cal. equalisTypes N. bifida, limosa, spivalis, biloba Raf. all Utric of Sm. in monograph.

1159. Vesiculina Raf. diff. Utric. cor. labio inf. trilobo, lobo medio sepe emarg.-Types V: saccata, setacea, purpurea, gibba, albiflora, compressa, graminifol. tenclla, pygmea \&c. all Utricul. of authors see Sm. and Lec.

1160. ENskide R. (one split) diff. Utric. cor. lab. sup. bifido, lab. inf. 3-5lobo. palato sepe barbato.-Types $1 \boldsymbol{E}$. Garbala $\mathbf{R}$. scapo paucifl. calc. subul-2 E. flava R. fl. sparsis congestis $3 \boldsymbol{E}$. chrysantha R. fl. 3hracteatis, lab. inf. 4lobo, all from Australia and Utricul. of $\mathbf{R}$. Brown, Sm. 
1161. Cosmuza R. (fine split) diff. Utric. cor. lab. sup. trifido lac. subul. labio inf. 3lobo, lobis omnis emarg.-Type C. coccinea Raf. Utric. multifida Sm. fol. spatulat. scapo bifloro, calcar obt. compr. Australia, scarlet flowers.

1162. Megozipa R. (large branched under) diff. Utric. cor. lab. sup. trilobo, inf. integro.'Types MI. macrorhiza, fornicata, longirostris, integra Raf. \&c all Utric. Lec. \&c.

1163. 'Trilobulina Raf. diff. Utric. cor. lab. sup et infero subeq. et ambi trilobatis vel crenatis.-Types $\boldsymbol{T r}$. fibrosa, striata, crenata Raf. all Utric. of Walter, Lec. Sm.

1164. Personula Raf. diff. Utric. cor. lab. sup. emarg. inf. integro, palato magno eminens bilobo. $l$. racemosis-Type $P$. grandiflora Raf. Utr. personata Lec. E.

1165. Plesisa R. (near eq.) diff. Utric. cal. ineq. sub 3part. vel lobo inf. bipart. cor. lab. subeq. subintegris-Type $\mathbf{P l}$. bipartita Raf. Utric. do Elliot.

1166. Plectoma R. (cut spur.) diff. Utricul. cal. subeq. conc. nerv. inf. emarg. cor. lab. inf. trilobata ineq. calcar bifido, stam. 2 submonadelphis, stigma bilab. ineq. capsula ovata bivalvis. fluitans fol. vertic. artic. ramosis inflatis $f$. racemosis flavis-Types $P l$.inflata Ràf. $2 \mathrm{Pl}$, stellata Raf.-both blended as Utric. inflata or ceratophyla by authors, a very distinct Genus, see my new flora.

Thus it appears that Utricularia as it stood, included sp. with calix equal or unequal, cor. with lips, entire or with 2,3 or more lobes, different stigmas and spurs, capsule with one, or 2 or more valves, glob ${ }^{-a}$ or lenticular or ovate or subulate !.. such is zongruity of botanical 
genera! The sp. are yet in utter confusion by their simplicity and will never be properly known till refered to my Genera; the stamens and stigmas must also be noticed, and the color of the flowers is very essential in this series of plants.

1167. EUPHORBIDES Raf. is a family of the order EPIMESIA or the 'Tricoca (Euphorbia of some) based chiefly on the G. Euphorbia of L. or 'Tithymalus Ad. which was a jumble of nearly $\mathbf{3 0}$ good Genera! easily known by having a perianthe with many male flowers around a female and commonly without calix, which L. had mistaken for dodecandria! The other families of this extensive order are easily distinguished-Cyrtosides by lack of perianthe, fruit dicocus, type Mercurialis-Tragides by flowers separate, type Tragia-Phalarsides by stamens monadelphous and determinate,type Sapium-Rrcrsides by stamens united and many, type Ricinus \&c. I mean here chiefiy to revise in part the G. Euphorbia, left uncorrected by all except Necker who made 5 G. out of it, and Persoon who divided his $156 \mathrm{sp}$. in two subg. Tithymalus and Esula. A few akin G. have lately been admitted, but the $200 \mathrm{sp}$. now known afford a crowd of good characters, while the old greek names of these plants afford many good names for them.

1168. Eupiorbia Necker, periantho duplex, ambi globosis truncatis integris, fl. masc. paucis sepe $\mathbf{5}$, monandris, gynophoro pistilo ferens, stylis 3 , stig. 6 caps. tricoca, trisperma. Caulib. perennis carnosis, aphylis, spinosis, fl: sparsis-Type $\boldsymbol{E}$, antiquorum, mamillaris, officinarum \&c and akin sp. but the flowers of all must be verified. The smooth sp. will form the 
subg. Alskebra arabic name, the spinose the subg. SADIDA.

1169. A thymalus Neck. diff. Euph. periantho ext. turbinato cavo lobato, interior 5sepalis, cuculatis furcatis alternans. fl. masc - plura 15-20. -I do not know which of the leafless Euphorbias belong here as Necker omits the types; but observers will easily ascertian.

1170. Torfasadis R. (n. afric.) diff. Euph. periantho clauso dentib. 5, externe 5sepalis carnosis obtusis-T'ype T. cańariensis Raf. E. do L. and probably several other sp.

1171. Dactylantines Haworth diff. Euph. per. simplex 4-5sepalis tubulatis bilabiatis, lab. sup. brevi trilobo, inf. longior palmato tridactyloTypes D. anacantha, tuberculata, medusa and akin sp. many blended in E. medusa, also D. globosa H. Euph. do bot. mag. 2624. Perhaps 2 subg. Anacantha and Medusita, this with sepals oft. 4parted not tubular.

1172. Tirucalia R. (n. ind) diff. Euph. per. simplex ventricoso, 4-5lobo lobis planis rotatis integris coloratis fl. masc. pluris ... caulescens, fruticosa, foliosa, $f$. non umbel-This answers to the Tithymaloides of Tournefort and includes the T. indica. Enph. tirucali 1. with many akin sp. but the flowers must be well described in all, as several form peculiar genera.

1173. Adenorna Raf. (gland. pit) diff. Euph. periantho tubuloso, apex 4dent. glandulis 4 magnis umbilicatis alt. fl.masc. Insertis ad per. latere, bract. subul. antheris ineq. bilobis, stylo trifido, stig. acutis. frutic. foicosa, fl. umbrl. bracteatis.-Type Ad. punicea Raf. E. do Sw. W, P. bot. reg. 190. fol. lanc, cuneat. subt. glaucis, umbellis 5 fidis, invol. lanceol. pedic $1 \mathrm{fl}$. bracteis 
2 obov. coccineis ad floribus. Antilles. 1134. Plturinena Raf. 1833 atl. journ. Poinsetia Grah. 1836. diff. Luph. periantho urceol. carnoso apice sub 8lobo, ad latere glandula magna tiansversa unica concava melliflua, phorantho villoso alveolato, fl. masc. paucis inclusis, antheris planis 2loc. stylis 3 bifidis. frutic, foliosa, $f$ l. umb. involucratis-'Type Pl. coccinea Raf. atl. j. p. 182. Euph. poinsetiana et pulcherima Hortis. Poinsetia pulcherima Gr, b. mag. 3493. fol. ovat. subang. acutis, umbella depressa corymbosa, invol. bracteif. obl. coccineis. Mexico, akin to the last G. described and named by me 3 years before Graham. Poinset was no botanist, he merely sent the seeds to our gardens where seen alive.

1175. Desuonema R. 1833 (fasc. fil.) diff. Euph. periantho duplex, ext. tubul. 5dent. vel 5part. connivens, per. int. 5 petaloideis membranosis cuneatis emarg. stam. vel fl. masc. fasciculatis ad gynophoro insertis, plurimis flif. vix artic. interdum castratis. Gynophoro trigono elongato, ovar. 3lobo, stylis 3 simplices. Herbacea foliosa, fl. umbel. invol.-Type D. hirta Raf. atl. j. 178. fl. tex. 19. canle simpl. striato scabro, apice hirto, fol. opp. petiol. ovat. obt. hirtis dentatis, imis alt. ovatolanc. acum. invol. triphylo lanceol. sess. From Texas to West Kentucky very rare, pedal, fl. green, petals white not glued to the perianth as in the others. G. akin to 'I'ragia, a link with the 'Tragides.

1176. Lepanena R. (scaly gland) diff. Euph. periantho simplex urceol. limbus 8fidıs, 4 alt. brevis barbatis, 4 alt. major subrot. coloratis, ad basis ferens squanis magnis carnosis glanduliformis concavis dilatatis, phorantho aristato vel fl. masc. castratis mixtis, gynophoro tereto, stylis 
3 trifidis, stig. 9. herbacea, foliosa umbel. invol. - Type L. leucoloma Raf. Euph. do Raf. ff. tex. 11. atl. j. fol. sparsis obov. integris acutis, umb. trifida, invol. fol. sinilis albo marginatis. Arkanzas and Missouri, seen alive in our gardens, where it has some varieties, simplex, elatior, cuneifolin \&c. When much handled it produces a kind of numbness.

1177. Kanopikon R. (n. grec.) diff. Euph.per. duplex, ext. bilobo plano, interno urceol. ad margine 4 glanduloso, fl. masc. 4 omnis bifilis diandris, gynophoro brevis, stylis 3 bifidis, caps. globosa. Frutic. inermis umbel.invul.-Type $\boldsymbol{R}$. atropurpureum liaf. Euph. do W. b. mag. 3321. fol. imbric. reflex. glaucis cuneatis, umbellis sessilis 4 fidis, invol. 4 obov. Flowers dark purple, glands yelíow.

1178. Aklema R. (n. grec.) diff. Euph. per. simplex, lobis subrot, crenatis, stylis connatis, stig. 3. Frutic. inermis, fl. cymosis nudis.-Type A. nudiflora R. Euph. do Jaq. rar. t. 499. P. \& c. Subtrichot. fol. ovat. integris undulatis.

1179. Tumalis R. (n. grec.) diff. Euphorbia or rather my G. Lacanthis 356 by-Per. cupularis, 5 dent. glandul. 5 alt. fimbriatis, $f$. et fruct. pedunc. cum gynophoro.-Type T. bojeri Raf. Luph. do Hook. b. mag. 352 \% fol. cuneat. coriaceis retusis, ped. axil. cymosis dichotomis, bract. 2. semiorb. coccin. basi coalitis. Madagascar. Habit of Lacanthis, less spinose; the bracts alınost an outer perianth. These with Kanopikon, are remarkable by the androphores realy diandrous.

1180. Vallakis R. (nom. lat.) diff. Euph. per. lobis 10, 5 alt. major scutellatis crassis planis. phorantio setoso, androphoris vel fl. masc. in 5 
phalangis 4-6andris. Herbacea, fol. oppos. $f$. varis, sepe dichotomis.-'Types all the Euphorbias, with fimbriate calix not already mentioned, and they are numerous, such as $\boldsymbol{V}$. ipecacuana, portulacoides, uniflora, missurica, fimbriata foc $\mathrm{c}$, and many others.

1181. Xamesike R. (n. grec.) diff. Euphorbia per. simplex campanulatus 4 lobo, lobis parvis interris vel crenatis. Herbaced dichotoma sepe diffusa, fol. oppos. fl. axil. fasc.vel dichot.A very extensive $G$. easily known by habit, fi. not very diflerent from other genera, but several must be separated and better distinguished; for many new Sp. of $\mathbf{N}$. Amer. see my new flora. Probably several subg. must be established. Xamesile vulgaris (Euph. chamesyce L.) has crenate lobes, $\boldsymbol{X}$. scordifolia serrate lobes, $\boldsymbol{X} \boldsymbol{a}$ mobala has entire lobes.

-1182. Tithymalis Ad. Necker, Raf. diff. Euph. periantho simplex, ventricoso, sepe 5lobo lobis scutellatis equalis planis crassis subrot. integris, dentib. interjectis alt. obsoletis, fl. masc. sepe 12 ineq. frutic. et herb. fol. sparsis, umbellis compositis, involucratis.-The most numerous in sp. divided into 5 subgenera.

1. Paralias R. capsulis glabris. Such as T. or E. maritima (paralias L.) gerardi, dendroides, linearis, rosea, helioscopia, paniculata. and many more.

2. Tulocarpa $R$. capsulis verrucosis, such as T. or E. spinosa, carniolica, palustris, platiphylos, hiberna, micrantha, literata, angulata, \& $c$.

3. 'Tuloisra R. caps. verrucosis pilosisque. T. or E. verrucosa, pilosa \& $c$.

4. Xarakias R. caps. villosis vel lanatis. T or E. characias vel serotina Raf. gramulata. coralloides caps. lanatis. 
5. Pytuiusa R. caps. echinatis vel setosis. T or E. dulcis, pythiusa vel fuscata, epithymoides, obtusata \&c.

1183. A llobia R. (diff. lobes) diff. Tithymalis, perianthis monoicis, masculis lobis integris, femineis lobis lunatis, capsulis angulis inuricatis -Type A. portlandica Raf. Eaph. do L. auct. 1184. Keraserma Necker. diff.' T'ithym. perianthis lobis lunatis vel bicornis, capsulis sepe glabris.-Many sp. in 3 subgenera.

1. Esula R. lobis sepe 5 emarg. vel bicornis: such as L. or E. falcata, esula, lucida, diversifolia, sylvatica, retusa, \&c.

2. Latiy ris R. lobis 5 lunatis. K. or E. lathyris, peplus vel oleracen, genistoides, provincialis, segetalis, cyparisias. squamosa, pallida, leplophyla, corifolia \&.c.

8. Karuites R. lobis 4 lunatis. $K$. or $\mathbf{E}$. exigua, rubra, seguieri \&c.

1185. Murterias R. (n. grec.) diff. Tithym. periantho tubuloso ore serrato multifido, lobis scutellis 4 lunulatis-Type M. myrsinites Raf. Euph. do L. auct.

1186. Nisonenes R. (uneq. lun) diff. Tithym. per. lobis 4 lunatis ineq. 2 oppositis duplo major capsula glabra-Type N. diffusa Raf. Euph. do L. auct.

1187. Lophobios R. (crest cobios) diff. Tithym. pet. lobis cristatis vel lobatis crenatis, caps glabra.-Types L. cristata, terracina R. Euph. do auct. Kobios was a grecian Tithymalus.

1188. Agaloma R. (pretty border) diff. Tithym. perianthis sepe dioicis, cupularis vel campanul. lobis 5 eq. membranaceis rotatis integris dilatatis corollatis, ad basis glandulis $\mathbf{5}$ oppositis planis, 'fl. masc. filam. fascicul. clavatis, antheris 2locul. capsulis glabris, fl. umbellatis vel dichot. 
-A very pretty genus based on the $\boldsymbol{E}$. corolla$\boldsymbol{t a}$ and blended or akin sp. such as E. anguslif. graminif. Sc. See my monograph.

1189. Pedilanthus Necker 1790, Kunth, Edwards, Crepidaria Haworth, difl. ad Euphorbia, Periantho caliculato vel bracteis binis ad basis, irregularis calceiformis angul. gibbosis hians, lobis 4 fornicatis ineq. stam $12-16$ exsertis. Frutic. fl. corymbosis \& c.-Very striking G. lately adopted by many; types the $\boldsymbol{E}$. myrtifolia, $t$ thymaloides \&c of Authors, also F'ed. canaliculata Raf. Crep. do Haw. Euph. do L.od. 727. E. carinata Don b. mag. 2514. fol. ellipt. subt. carinatis, fl. term. corymb. fascic. nutans coccineis. Trinidad.

1190. Cyathophora R. diff. Euphorbia, periantho tubul. tereto apex multifido vel laciniato dentato, dentis 5 major linearibus. phorantho papilloso, fl. masc. sepe 10. Herbacea, fol. sparsis, fl. termin. fascicul.-Types 1. C. picta Raf. E. cyathophora Scop. del. t. 3 Vitm. Auct. fol, lyratis et panduratis oblongis integris, apice coccinea et alba picta, sepe lanceol. ped. fascic. sepe 3 floris. Florida $\& \cdot c$, seen alive in gardens-2. C. ciliat $\mathbf{R a f}$. E. heterophyla Jaq. W. E. cyathoph. bot. reg. 735. petiolis cilıatis, bl. ovatis serratis acum. fl. subcorymb. bract. basi coccineis-3 C. heteropliyla Raf. E. do L. auct. fol. petiol. difformis, ovatis lanceol. panduratis serratis glaucis, fi. pedunc. term. S. Amer.

1191. Synexemia Raf. neog. 1825, Mascalanthus Nut. 1835. Monoica, cal. Gpartito eq. cor. o, stam. 6 basi coalitis, fl. fem. stylis 3 bifidis, stig, 6 capsula 6valvis 6sperma. Herba, fol. alt. distichis, fl. axil, sepe geminatis.-The Phylanthus carolinianus of North America and sp. blended therein, are the types of this $\mathbf{G}$. 
which Nuttal has published 10 years after myself. under another name. It contains 4 or 5 sp. see iny monograph. The $G$. Phylanthus, Croton, Tragia \&c, and others akin to Euphorbias contain many neglected $G$. partly given in my Neogenyton and New Flora..i

1192. STaenerrena Raf. this linnean G. was merely distinguished from Serratula by the bisetose anthers as in Inula: while Serratula has long ago been reformed, and Vernonia, Liatris \&c separated, this had been left untouched, except by Necker, although offering many more irregularities: which I will endeavor to correct. My Staneluna has P'eriuntho imbricato, inermis sepe ovnto multifloro, flosculis eq. phorantho paleis multifidis, antheris basi appendiculatis, sem. gabris obov. pappus plumosus -Frutic. fol. sparsis, fl. term.-Even 3 subg. must be distinguished that might be as many genera: I have them dry.

1. Cinmepeuce R. (pr. Xamepeuke) diff. sq. phoranth. multi-aristatis, antheris biaristatis, pappus non digitatus. - I'ype St. chamepeuce L. auct. or Ch. imbricala Raf. fol. imbric. longiss. lin. revol. fl. term. 2-3. Creta, Grecia.

2. Anaxeton Raf. (gnaph. diosk.) diff. sq. per. apice scariosis reflexis, sq. phor. multif. stylo bifidus, pappus basi connato palmato vel polyadelpho, antheris basi biplumosis.-Type $\boldsymbol{S t}$. squar.. rosa R. Leysera do Th. P. Steh. gnaphaloides I. auct. (non Leyseria do) fol. filif. toment. obt. bași subampl. fl. paucis toment. S. Africa.Leyseria has radiate flowers!

3. Staeifelina (veris) diff. flosc. nonnulis neutris, sq. phor. multif. pappus ramoso plumoso (Lin.) scabro palmato (Sm.)-Type \$t. arborescens L. auct. fol. pet. oval. subt. arg. fl. 
corymb. puniceis. Cieta, Grecia. st. fruticosia and spinose probably belong here, altho' flowers not well described, spinosa has hairy seeds.

1193. Roccardia Necker, diff: Staehelina, Periantho tereto scarioso,- 8-10flosculosis. antheris bibarbatis ad hasis, sem. 4gona, propus simplex basi connatus palmatus.-Type $\boldsymbol{R}$. purpurea Raf. Staehel. dubia L. auct. fol. lin. dentic. fl. 2-3glomer. sq. per. lanceol. Italy, spain. Isinneus deemed it medial between 3 G. Staehel. Serratula and Gnaphalium! the down not plumose is the main generic feature. how is the phoranthe?

1194. Aplina R. (nymph and simplicity) diff. Staehel. Periantho tereto scarioso, flosculo unico gerens! antheris basi biplumosis barbatis, sq. phor. simpl. pappo digitato plumoso.-Type Apl. fuscuta Raf. Staehel. unifloscula Sm. fl. greca 846 . fol. ovat. acut. dent. subt. canis, per. fuscato. Mt. Parnassus. G. akin to the last, very singular by the florets reduced to unity!

1195. Alnibias R. (n. arab.) diff. Stachel. per. tereto turbin. squ. carinatis, phorantho subnudo, sem. villosus, pappus subsinpl. apice dentato.Type A. hastatia R. Staeh. do Vahl, Pers Sm. Chrysocoma spatulata Forsk. Vitm. probably the other Arabic fruticose chrysocoma belong here, Chr. mucronata, ovate of fiorsk. 'The real Chrysoc. have the per. hemisph. phoranthe naked, down quite simple.

1196. Tuloclinia R. (warty bed) diff. Staehel. per. turbin. phorantho mulo verrucoso, pappo subplumosus.-I'ype T. imbricata R. Stach. do $\mathbf{L}$. auct. fol. subul. subt. toment. fl. binatis $\mathbf{S}$. Africa. How are the appendages?

116\%. Plectreca R. (10 spurs.) diff. Staehel. 
per. hemisph. paucifl. phorantho nudo celluloso, antheris basi bicalcar. pappus paucisetis scabro non plumoso-'Type P'l. corymbosa R. Staeh. do L. auct. fol. cuneat. dent. toment. fl. coryınb. panicul. S. Africa.

1198. Ocneron R. (n. grec.) diff. Staehel. per. hemisph. lanato, sq. phor, rigidis 2-3dent. antheris biaristatis, pappo scabro non plumoso. frutic. fol. petiol. opp.et. sp. fl. panicul. luteis Types 2 sp. blended in St. ilicifolia L. disting. by Smith 1. Ocn. cordatum R. fol. oppos. cord. dent. obt. supra lucidis subt. toment. S. Amer.2 Ocn. aquifolium R. St. do Sm. plum. ic. 123. fol. sparsis subrot. dent. spinosis, subt. lanatis. Antilles. Thus quite an American Genus. Ocneron was a Greek name for Ilex or Ruscus.

1199. Lachnospermum Wild. diff. Staehel. periantho tereto, phorantho villoso, sem. villis involutis.-Type L. ericifolium Wild. Staehel. fasciculata 'Th. Pers. \&c, fol. fascic. ter. subul. tomentosis, ramis divaricatis rigidis.-It thus appears that $\mathbf{L}$. and others threw into this $\mathbf{G}$. all the shrubs akin to Serratula! even that G. requires further revision, and I shall conclude here by another $\mathbf{G}$. out of it.

1200. 'Tulakenia R. (warty seed) diff. Serratula \&c, periantho squarroso arachnoides, sq. setaceis planis, phorantho aristato, sem. 4gona obverse pyramid. tubercul. pappo rigido ineq. scabro. Unifloris-Types 1. T, mollis Raf. Carduus do Jaq. W. Cirsium do Scop. Serratula simplex Poir. Dec. Sims b. mag. 2482.-2 T. blanda $\mathbf{R}$. Serrat. do auct.-3 T. cyanoides $\mathbf{R}$. Serrat. do auct. \&c. 


\section{ADDI'TIONS AND CORRECTIONS.}

1201. My Onovora 351 has since been called Curxsers by Lindley, who has thus acknowledged that Esch-scholtzia was a wrong name! but my name is previous and better, Chryseis being nearly the same as Chrysis a genus of flies.

1202. For Hexastruis 706 substitute StyLExi Raf. having had another G. Hexastylis since 1825, see 1113.

1203. Rupifraga cuscuteformis Raf. Saxifraga do Lod. Cab. 186, bot. mag. 2631, a second sp. of this G. see 243, differing from $\boldsymbol{R}$. sarmentosa by small size, leaves smaller, flowers larger less unequal, shoots filiform. scapes biflore. China.

1204. Piaropus undulatus Raf. Pontederia azurea Luman. hort. Jam. non alis. Another fine sp. of this $G$. see 301, proving my assertion that many sp. exist-fol. subrot. acum. undulatis. Lunan describes the flowers with upper lobe of corolla larger ovate, anthers hastate, ovary trigone, style filiform, stigma clavate. Jamaica \&e.

1205. Einadxa R. (1 or 2) diff. Chenopodium, cal. 5fido baccato, stam 1 vel 2, stylis 2.-Type $\boldsymbol{E}$. linifolia Raf. Rhagodia do R. Br. Sm. frutescens decumbens, fol. lin. lanc. integris. Australia.-The Chenop. baccatum of Labillardiere was the type of the G. Rhagodia of $\mathbf{R}$. Brown, who has added many sp. to this G. billardieri, crassifol. hastata, nutans, spinescens \&c, which have all 5 stamens, instead of 1 or 2 . Both G. belong to my family Baseldides 571 .

1206. For my Lunania 7 substitute TriexasTrma (3-6 stig.) Raf, since I perceive a previous G. Lunania in Decandole, although mine was 16 
established since 1830, what is the date of the other?

1207. Streptyuis Raf. (twisted style) diff: Commelina. cal, et cor. eq. stam. 3 villosis ad basis, sterilis 3 nectarif. cilict. antheris glandula, stylo et stig. spiralis persistens-Type Str. bracteolata Raf. Com. do Lam. Com. spirata L. are 2 sp. blended here? 'This is another G. to be removed from Commelina and is very distinct.

1208. Add to Sarcoperis 23, bacca triloba carnosa ad cor. baccans, intus capsula 3loc. apice 3valvis, sem, 5, uno locolo monospermo.

1209. Add to Heminema 31, stylus brevis crasso, stig. 3 villosis, capsula 3lo $\therefore$. 3sp.-It is also the Tradescantia multifl. of Lunan, quite different from that of Jaquin my Tripogandra 28.

1210. Allosperma R. (different seeds) diff. Commelina, capsula 3locul. bivalvis! valva sup. 2loc. 4sp. sem. rotundis rugosis, loc. et valva inf. monosp, semen elliptico lenticularis.-A very strange structure of fruit! 'Type $\boldsymbol{A}$. tuberosa $\mathbf{R}$. Com. do $\mathbf{L}$. auct. fol. ovatolanc, ciliatis subtus viliosis, pedunc. multifl. Mexico. pedal fl. blue.

1211. Add to Dirtea 689. Commelina polygama probably belongs here, and all the sp. blended in C. communis said to grow in Asia, Africa and America, but 5 or 6 sp. are blended probably! the African sp. is quite distinct $\boldsymbol{D}$. africana $\mathbf{R}$. fol. nervosis basi ciliatis fl. 2-3 axil. caps. 2loc. 2valv. 4sperma. Probably a subg. Diclisa Raf. very different from Com. africana of Persoon. The Com. erecta includes also several blended sp. having commonly the capsule trigone trilocular 3sperme: thus another subg. or G. perhaps same as my G. Ananthopus 690.

1212. Dilasia R. (2 villose) diff. Dirtea, cal. 3 
lanc. pet. 3 eq. ovatis, stam. 2 fertilis villosis, 4 sterilis nectarif. gla ris, stylo recurvo-Type $\boldsymbol{D}$. vaginata Raf. Commel. do auct. fol. linearib. $\mathrm{fl}$. term. involucro convoluto vaginato. India.

1213. Queltia Salisi. diff. Narcissus, cor, tubulosa, limbo patulo, corona tubul. urceolata erosa, stam. 6 eq. inclusis connivens, stigma trilobo.-Thus very near to my Moskerion 843, 'Type Q. montana Raf, poculigera Salisb. Narc. montanus Pyr. Edw. b. reg. 123. fol. planis obt. fl. 1-2 cernuis albis, \&c.

1214. Add to 'Troxistemon 853, the Pancratium littorale and its varieties driandri, distichum, mexicanum, which are as many sp. werè framed into the G. Hymenocalis by Sims; but his characters of nect. erosum s. dent. filam. liberis flaccidis, was very loose and inacurate. It may be a subgenus.

1215. Nesynstylis Raf. (not un. St.) diff. Strumaria, stam. liberis non monadelphis erectis stylis adnatis.-The Gr. Strumaria was put in 3 linnean classes Monadelphia, Gynandria and Hexandria! the monadelphous sp. belong to Narcissides, the free sp. to Hypoxides, they are $N$. filifolia and zindulata R. Strum. do auct. the first was pui in Crinum and Leucojum by L. and others, thus in 3 Genera! figured in bot. reg. 440, habit Alliaceous, fol, rad. filif. scapo teres, umbella 10-12ff. bract. 2ineq. lin. petalis albis obl. acutis.

1216. Agapanthus umbellatus Lher. W. P. bot. mag. 500, bot. reg. 699. Crinum africanum L. Mauhlia Th. Tulbaghia Heist. fol. vittatis angustis bifaris acutis, fl. umbel. nudis ceruleis, cor. infund. 6fidis, 3 alt, apex incrass. uncinatis,stam. declin. stigma trifido.-I have added this to shew its contrast with my G. Scadianus $\mathbf{8 5 5}$.

121\%. Vagnera of Adanson is the G. out of 
Convallaria, since named Smilacina, Mayanthemum, Tovara \&c: being among the additions of Adanson it had escaped my notice ; but being the first and best must be restored, see 831 .

1218. Add to Podospadix 821, many sp. have been blended in Pothos crassinervia, that probably all belong to this $\mathbf{G}$. I will add $\mathbf{5}$ other types -2 Pod. teres R. Pothos do auct. P. crassinervia bot. mag. 2987, fol. petiol. cuneat. obtusiusc. nervosis, scapo teres, spatha lanceol. spadix triuncialis obl. tereto. Demerara, the sp. of Hamboldt is different by a pedal spadix. Pod. Inımboldi R-3 Pod. angustif. R. fol. lanceol. acum. scapo angulato, spadix gracile: this is probably the sp. of Jaquin.-4 Pod. microphyla R. Pothos do Hook. b. mag. 2953, fol. ovat. acutis costatis, petiolis apex incrassatis, spatha lanceol. revoluta, spadix obl. brevis. Brazil.-S. Pod. harisi R. Pothos do Hook. ex. fl. 211 fol. lanc. magnis, spadix tereto, cetera ut 4.

1219. 'The G. Lunovia Pers. and PhilodenDron Lindl. just published in bot. reg. 1958 must be added to the Aroides, this last has the habit of Tapanava 820 but is quite distinct by multilocular berries ofc: this proves how many fine G. of Aroides were yet concealed in Pothos and Arum,

1220. Tremasperia R. (perf. seeds) diff. Bonanox 1026. cor. integra, capsula globosa baccata uniloc. cortex coriacea, pulpa fungosa alba 3-4sperma, sem. umbilic. perforatis.-Type $\boldsymbol{T} \boldsymbol{r}$. bonanox Ráf. Ipomea do Lunan. fol. cord. acum ped. 1-2fl. Jamaica, quite peculiar fruit and of family Aplarinia.

1221. Tereietra Raf. (cut in 4) diff. Ornithosperma 1009 cor. campan. integra, capsula uni- 
loc. Avalvis 4sperma-'iype ' $\mathbf{L}$ '. violacea $\mathbf{R}$. Ipomea do Luman, Jamaica.

1222. Neortrosis R. (not strait) near Doxema 10:0, cal. 5 fidus, cor. tubo curvo, limbo plicato stam. exerta, stig. 4 sulcat. caps. 4 loc. 4 sp. sem. angul.-Types N. cocciner and tigrina Raf. Ipomea do of Lunan Jam. perhaps not of others. G. quite peculiar by curved corolla like Doxema perhaps a subg. of it.

1223. Dactruepis Raf. another G. near Cuscuta and Nemepis.-Type $N$. brownei Raf. Cuscuta do Lunan, ramósissima. fl. aggregatis, cal. 5dent. cor. 5fidis, stam. 5 sq. nect. 5 palmatis 5 fidis. caps. 4 spermis. Júmaica.

1224. Eronema R. (love threads) diff. Cuscuta and Nemepis, cal. colorato 5 part. lanc. corolla cup:slaris, 5́dent. stam. 5 antheris didymis, sq. nect. fimbriatis, caps. 4loc. 4sp.-Type $\boldsymbol{E}$. robirsoni R. Cuscuta do Lunan, fl. pedunculatis. Jamaica, called love bush, medical, diuretic, speritive.

1225. For Bragantia 1114 adopt Munnickia, since there was a genus Bragantia of Vandelli see 534 previous (?) to that of Loureiro.

N. B. Add to Lepadena 1176, it is the Euphorbia marginata of Pursh and North America, not of Kunth and Mexico, which is perhaps a $2 \mathrm{~d}$ sp. of the Genus. 
126

INDEX OF GENERA \&c.

IN Centuries 9, 10, 11, 12.

Natural Orders and Families are in Capitals, Synonyms in Italics.

Abalon 865, 6

Abama 866

Abapus 833

Abbotia 889)

Abrochis 903

Abumon 855

Acinax 953

Acoridia 825

Acroanthes 943

Adamboe 1015.

Adatoda 969.

Adeloda 972

Adenorima 1173

Aechmidia 859

Agaloma 1188

Agapanthus 1216

Aklema 1178

Alkibias 1195

Allagas 953

Allium 874

Allobia $\mathbf{1 1 8 3}$

Allopleia 972

Allosperma 1210

Almana 999

Aloides 855

Alpinia 946, 7

Alponica 955

Alskebra 1168

Alstromeria 898 to 900

Amaryllis 805 to 813

Amathea 989

Ambulia 966

Ambuya 1106

Amianthum 866.

Amidena 822

Amomum 953

Amphianthus 959

Amphione 1031

Anacantha 1171

Anactorion 896

Anaxeton 1192

Anigozanthes 882

Anistylis 941

Anthanema 1071

Antheilema 992

Anthostonia 966.995.

Aplarnia 1033, 68

Aplina 1194

Aplostylis 1076

Apomea 1014

Apopleumon 1010

Apteria 893

Arethusa 938 to 40

Argyrexias 1060

Arietinum 936

Arinemia 962

Aristolochia 1101 to

1112

Arthropodium 870

Aromes and ArisaRIA 825

Arum 802, 3, 818

Asarum 1113

Askofake 1152 
nnodelus 814,15 Aspidistra 824, 1119 Astrocephalus 1085 Atamasco 809 Athymalus 1169 Aulica 808

Autogenes 8.39 Axillaria 831

Baimo 867

Balendasia 1140 Ballela 1001 Baloskion 888 Balostis 1131 Bartholinia 938 Basonca 998 Becabunga 955 13elvala 1136 Blephistelma 1128 Bletia 929, 940 Blexum 990 Bonanox 1026 Bojeria 947 Bragantia 1114, 1225 Bramia 966 Brassavola 924, 5 Braxireon 852 Brewera 1032 Brodiea 856, 7 Bromelia 861, 2 Bulbodictis 960

Calathea 945

Calathinus 841 Calasias 985 Calla 801, 2 Calistachia 960 Calistegia 1004 Calixnos 1044
Camonea 1042

Canirama 990

Canna 950

Carima 974

Cassytha 1077

Caulotulis 1023

Cephalaria 1088

Chamalivium 866

Chamepeuce 1192

Chondropetalon 890

Chlorophytum 871

Chryseis 1201

Cipura 880

Cleicmera 1027

Cleiostoma 1034

Clematops 1100

Clerodendron 1000

Clintonia 828

Ciytirrelia 1148

Cochleanthes 930

Codiaminum 844

Coilostylis 904

Commelina 1207 to 1 :

Conanthes 858

Convallaria 828 to 831 , 1217

Convolvulus 1001 to 1054,1221 to 23

Cordula 934

Coryanthes 920

Cosmiza 1161

Costus 953

Craniolaria 998

Crateola 986

Cratodia 951"

Crepidaria 1189

Ciressaria 1065

Crinides 845 
Crinum 854, 5, 1216 Criosanthes 9:36 Crossandra 988

Cuculina 943

Cuscuta 1069 to 76 , 1223,4

Cuscutaria 1065

Cyathophora 1190

Cymbidium 924

Cypripedium 931 to 36 Cyitosites $116 \%$

Dactylanthes 1171

Dactylepis 12:23

Dactylorhiza 903

Daiswa 832

Daphne 1141 to 44

Decaloba 1022

Dendrobium 918,9,926 to $\mathbf{9 2 8}$

Dendropogon 860

Derwentia 958

Desmonema 1175

Dessenia 1145

Dianthera 977

Diatrema 1008

Diclinotris 866

Dicondrania 1065

Didothion 910

Diglosselis 1108

Dilasia 1212

Dilomelis 926

Diototheca 1100

Diplanthera 936

Diphrylum 941

Dirtea 1211

Disa 943

Distimake 1045
Distcira 998

Ditereia 105:

Dituilis 942

Ditulima 918

Dodecasperma 900

Dodecula 955

Doxanthes 945

Doxema 1020

Doxosma 804

Dothicroa 97\%

Dothilophis 913

Dracena 826 to 28

Dracontium 816 to 818

Drymirhizes 951

Ecbolia 968

Ecrinida 1055

Echium 1055 to 1061

Einadia 1205

Einomeia 1105

Elegia 886

Elegides 886 to 889

Euprotia 825

Emularia 978

Emulina 1039

Encyclia 906

Endasia 955

Endocodon 944

Endodeca 1109

Endomelas 997

Enothrea 927

Enskido 1160

Ericlia 1085

Epidendrum 804-6,907= $14,917,924,937$

Eprimesia $116 \%$

Equisetia 825

Eranthemum 991 
Eronema 1224

Eryostax 861

Eindelia 1133

Esula 1184

Ethesia 981

Eucallias 862

Eucrosia 875

Eucrosines 875

Euphorbia 1168 to 1190

Euphorbides 1167

Euphylleia 827

Eupodanthes 978

Euptilia 1089

Eurycles 848

Euryloma 1019

Eusarcops 812

Eustaxia 960

Eustrephus 884

Eutereia 816

Evallaria 831

Eveltra 880

Evolvulus 1046 to 1054

Exallosis 1048

Exeria (Eria) 943

Exioxylon 1055

Exocroa 1037

Eydisanthema 902

Ferraria 879

Fimbrula 955

Fissilia 1081

Flavicoma 979

Flugea 830

Fraxima 1047

Fritilaria 954

Froscula 928
Gamaria 943

Gandarusa 968

Gastrilia 1141

Geobina (georchis) 945

Gerardia 995

Gethylis 833

Geunsia 971

Globeris 831

Glossura 1100

Gloxinia 998, 9

Gongora 920

Gonokeros 1096

Gomphipus 1024

Goodyera 911

Granadilla 1121

Gratiola 965, 6

Gurenias 864

Gynampsis 828

Gynizodon 916

Gynoisia 1018

Haemanthus 834 to 37

Haemodora 882

Hamulia 1155

Harrackia 988

Haylockia 810

Hebe 961

Hecabe 929

Hellenia 953

Hemelosia 960

Hemilasis 1016

Heminema 1209

Heroion 814

Hexalepis 859

Hexalectris 940

Hexaplectris 1102 
Hexastylis 1113, 1202

Hirselina 968

Hookeria 857

Hygrophila 993

Hypoestes 971

\section{Ictodes 817}

Idalia 1025

Iebine 912

Ifuon 815

ILEXIA 1065

llysanthes 965

Ilythuria 948

Intrusaria 961

Ipomea 1016 \&c

Ismene 847

Isoloba $\mathbf{9 6 7}$

Isonica 955

Isoplesion $\mathbf{1 0 5 7}$

Isotria 938

Isypus 1012

Janasia 980

Jensoa 908

Jimensia 909

Jonquilla 840

Juncus sit 7

Justica 968 to 989

Justicoides 968

Kadula 1070

Kadurias 1073

Kanopikon 1178

Karaguata 860

Karuites 1184

Katubala 950

Kemopsis 1016

Keraselma 1184
Kethosia 1029

Kolofonia 1013

Kuniria 973

Lacara 1001

Lachnospermum 1199

Larephes 1059

Lasiake 1066.

Lathyris $\mathbf{1 1 8 4}$

Laticoma 813

1.atrienda 1040

Leiena 891

Leiosandra 1067

Lentibularia 1150

Lepadena 1176

Lepiactis 1056

Lepimenes 1075

Leplandra 960

Leucrinis 866

Leucodermis 834

Leucopsora 1094

Libertia 807

Lilavia 899

Limodorum 908,9

Limosella 963, 4

Liparis 941

Liriamus 854

Lizeron 1001

Lobake 1038

Lomalix 1016

Lomelosia 1093

Lomiptia 1014

Loncotoma 968

Lophiaris 917

Lophobios 1187

Lophoglotis 943

Lunania 1208 
L.ustrinia 976

Macradenia 906

Macrogyne 1119

Macrophora 1125

Malaxis 941 to 43

Maracoa 11:21

Marama 975

Maranta 914, 952

Martynia 998

Mascalanthus 1191

Mattushlien 1061

Maxillaria 923, 3

Mayanthemum 831, $121 \%$.

Meborea 1117

Medusita 1171

Megozipa 1162

Meionica 955

Meionula 1154

Meioperis $\mathbf{1 1 2 6}$

Meiosperma $\mathbf{9 8 1}$

Melanthium 865, 6

Melascus 1036

Melasis 1014

Meloneura 1117

Melorima 954

Menephora 936

Mesodoa 810

Mesophor es 963

Mesteniphus 1101

Microla 105 I

Microstylis 943

Milhania 1004

Modeca 1124

Modesta 1021

Monnieridia 1064
Monomesia 1064

Monstera 831

Morea 879, 831

Moskerion 813

Munnicliia 1225

Murtekias 1185

Murucoa 1014

Murucuia 1127

Mutafinia 963

Narcissides 838 to 853

Narcissus 838 to 844 , 1212

Nemampsis 826

Nemanthera 1035

Nemapiodon 846

Nemepisia 1074,1223

Nemitis 893

Nemoctis 1014

Nemostima I043

Neorthosis 1222

Nepenthes ～ 1118

Nepentuines $\} \mathbf{1 1 1 9}$

Nesipus 1158

Nestronia 1145

Nesynstylis 1215

Nisomenes 1186

Nolana $\quad\{1032$

Nolanidia $\left\{\begin{array}{l}1063 \\ \text { Nata }\end{array}\right.$

Ocneron 1198

Ocripha 105I

Octomeria 926, 7

Octoplis 1143

Odicardis 956

Odonectis 939

Odostelma 1131 
Olax 1080

Olsynium 878

Omonoia 1201

Oncidium 915, 16

Onkeripus 922

Ophiopogon 830

Ophiostachys 866

Ophrys 912

Oplonia 987

Drbonica 955

Orcindes 901 to 943

Ormostema 907

Ornithogralum 868

Ornithospermum 1009

Grontium 822, 3

Orthosanthes 879

Orxera 905

Osmularia 977

Otosma 801

Oximula 978

Dzarthris $10 \% 8$

Pancratium 815 to 853

Paneguia 897

Panemata 984

Panoxis 957

Panstrepis 920

Papiria 833

Paralias 1182

'Taris 832

Pasganon 896

Passerina 1135 to 1140

Passiflorea, Passiflora 1120 to 1134

Paussia 1139

Pedilanthus 1189

Pedilonum 928
Peliosanthes 864

Peltimela 964

Pentake 1072

Pentanthus 1001

Pentanisia 1065

Pentena 1097

Penthysa 1061

Pentulops 923

Perama 1064

Peremis 1129

Perihema 836

Periexa 1043

Pericodia 1134

Periloba 1063

Periphas 1054

Personula 1164

Phaianthus 881

Phaiobleps 876

Phadrosanthus 901

Phalarsides 1167

Pharides, Pharium

873,4

Pharbitis 1007

Philodendron 1219

Phlebocarya 883

Phylidrum 892

Phylloma 827

Piaropus 1204

Pierardia 919

Pinguicula 967

Pistides 961

Pistolochia 1107

Pitcairnia 868

Plagistra 1103

Plectoma 1166

Plectreca 1197

Plectronema 809 
Plectrelminthus 921

Plesiagopus 1028

Pleuradena $\mathbf{1 1 7 4}$

Pleurastis $\mathbf{8 1 3}$

Pleuremidis 966

Pleurospa 803

Plexisa 1165

Podaletra 1030

Podonix 872

Podospadix 821, 1218

Pogadelpha 877

Pogonema 809

Pogonia 938

Poinsetia 1174

Polinisa 1065

Pontederia 1204

Potiridia 825

Pothos 817 to 821 , 1218

Praskoinon 874 Priopetalon 898

Pseudomelia 862

Psophiza 1110

Psydarantha 952

Psychilis 914

Psychopsis 915

Pteriphis 1111

Pterocephalus 1086

Pulcolia 968

Pullis 1016

Pythiusa 1182

Quamoclita 1014

Queltia 1213

Ramonda 1068

Restio 886 to 891
Rhagodia 1205

Rhizemis 863

Rhodoxylon 1033

Rhopium 1117

Ricinides 1167

Roslinia 989

Rotheca 1000

Ruellia 990 to 95

Rumputris 1077

Rupifraga 1203

Ruscus 864

Sacodon 932

Salacides 1117

Samudra 1011

Sanamunda 1135

Sanilum 1003

Sarcanthera 982

Sarcoperis 1208

Saurulidia 825

Scabiosa 1084 to 1099

Scadianus 85̃

Scadiara 1001

Scadoxus 835

Scaduakintos 853

Scamonea 1007

Schenoprasum 874

Scopolia 1146

Septilia 997

Serena 837

Sericosperma 1016

Serratula 1200

Sesamides 998

Sigillaria 831

Simira 868

Siphisia 1112

Siphonanthus 1064 
Siphotoma 851

Siphostegia 1131

Siphyalis 829

Siraitos 865

Sisyrinchim 876 to 880,897

Sixalis 1095

Skoinolon 866

Smilacina 831, 1217

Sowerbea 894

Sparaxis 895

Spathyema 817

Spermoxyron 1082

Spiranthera 885

Spironema 1079

Spirospatha 802

Spirostylis 949

Staehelina 1192 to 99

Statricıdes 1065

Steirexa 1116

Steiroct is $\mathbf{1 1 3 8}$

Stenanthum 866

Stenopolen (Stenia)

943

Stethoma $\mathbf{9 7 0}$

Stevogtia 1002

Stimegas 933

Stomoisia 1153

Stratides 951

Strepsanthera 818

Streptylis 1207

Strumaria 1215

Stylexia 1202

Stypandra 869

Styrandra 831

Stylisma 1050

Succisa 1087
Sulpitia 906

Symethus 1019

Symplocarpus 817

Synactila 1130

Synadena 805

Synarmia 901, 951

Synarthia 1083

Synexemia 1191

Tacsonia 1131

Talanelis 1001

Tamus 863

Tapanava 820

Taumastos 807

Tephranthus 1117

Tereietra 1221

T'ereiphas 1091

Testudiuaria 863

Thalia 948, 9

Thapsus 1066

Thicuania $\mathbf{9 3 7}$

Thlasidia 1099

Thunbergia 996, 7

Thyella 1051

Tilcusta 82:3

Tillandsia 859, 60

Tirucalia 1172

Tirtalia 1005

Tomodon 850

Torfasadis $\mathbf{1 1 7 0}$

Tragides 1167

T'raxara 1056

Tremasperma $\mathbf{1 2 2 0}$

Tremastelma 1098

Trichima 1043

Trichopus 1116

Triexastima 1206 
Triglochin 889

Tikillidia 832

Trilobulina 1163

Trimeiandra 1137

'Trimeriza 1115

Trisacarpis 811

Tristegia 1131

Tristemon 887.

Trixapias 1161

Troxistemon 853,1214

Troxula 1014

Tulakenia 1200

Tulexis 924

Tulipa 8\%2

Tulocarpa 1182

Tuloclinia 1196

'T'uloisia 1182

Tulotropis 1014

Tumelaia 1142

Tumalis 1179

Tupistra 824

Turbina 1041

Turpethum 1006

Trimacea 825

Upudalia 994

Uranthera 977

Utricularia 1149 to 1066

Uvularia 867
Vagnera 1217

Vallaris 1180

VALLISNERIDES 951

Verbascum 1066, 68

Veronicides 955

Veronica 95.5 to 62

Vesiculina 1159

$\mathrm{V}$ iscum $10 \% 8$

Volkameria 1000

Volutella 1079

Xaiasme 1145

Xamesike $\{1181$

Xamobala 1181

Xarakias 1182.

Xaritonia 806

Xerogona 1123

Xetola 1092

Xylobium 923

Xyphimia 855, 894

Xyphostylis 950

Ygramela 963

Y MnODIa 845

Zelmira 945

Zephyranthes 809, 10

Zingiber 953

Zygopetalon 930 




\section{NOTICES.}

Late works published by Prof. Rafinesqne.

History of the American Nations, before and after Columbus - 2 volumes published $-\$ 5$ for 6 volumes to subscribers.

Life, travels and researches of Prof. Rafinesque, in both Hemispheres-one vol. $12 \mathrm{mo}$. 75 Cents.

The philosophy of Instability-one volume 8 vo. 1,50 .

New Flora of North America-One volume 8 vo. - \$5.

Herbarium Rafinesquianum-\$1.

Atlantic Journal, with 200 tracts on Sciences, one vol. 8vo. complete-\$2.

A few copies of former works for sale-Analysis of nature-Principles of Somiology-Outlines of discoveries-New Genera of Animals and Plants of Sicily-Ditto of North America - Fishes and Shells of the River Ohio-MIedical flora of the United States, \&c. Unique Copy of Autikon Botanikon or 2500 Self figures of new and rare plants, folio $\$ 500$

Icones plant. rariorum N. Amer. folio, 600 figures-\$ 300 . 

S

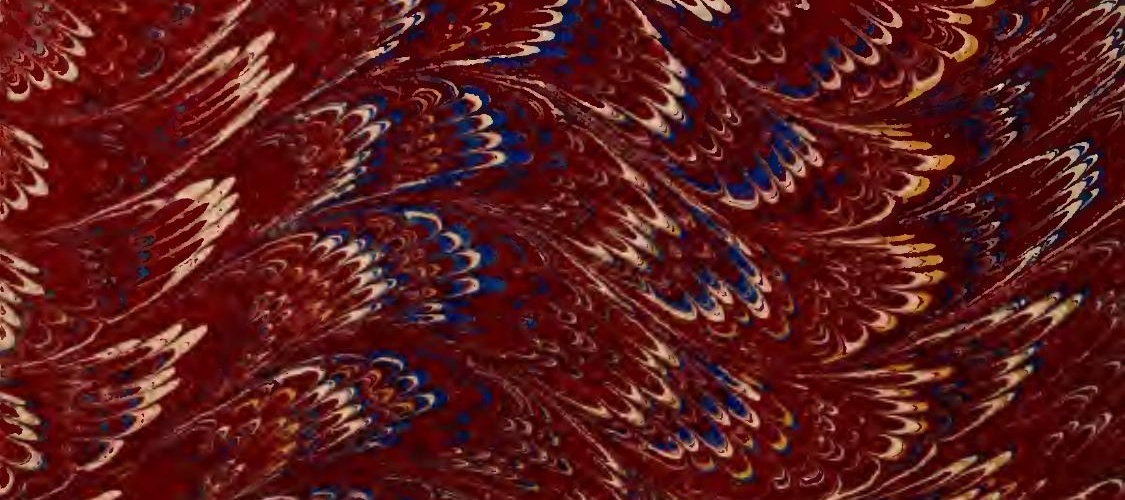
J

3

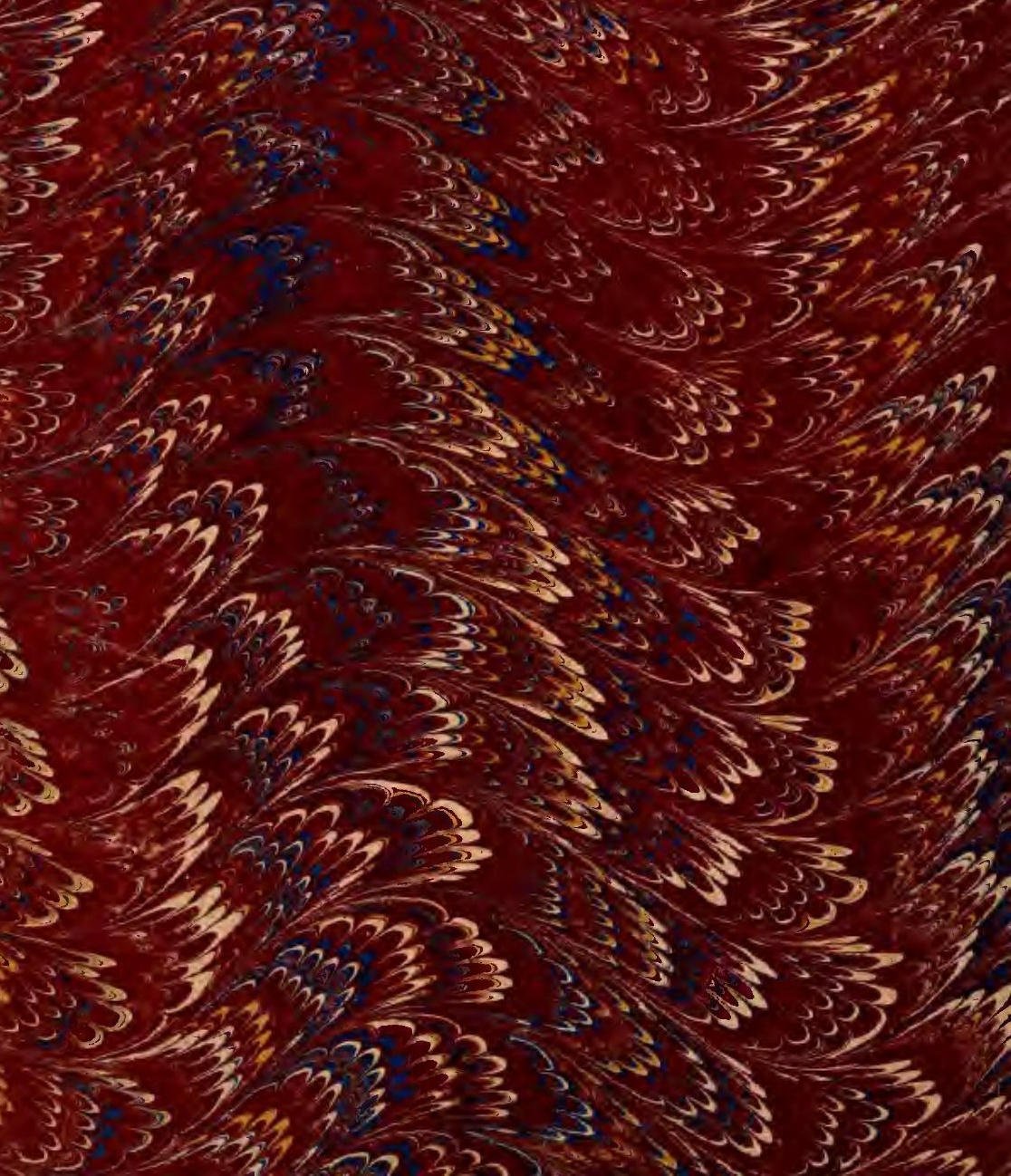




\section{$5>^{13}$}

- 5

3.5
3

QK94.R3

Rafinesque, Constantine/Flora Telluriana

gen

s.

3.

32.

s.

.

(3)

(3)

3,3
3
3
3

(2)

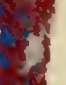

,

s

sis

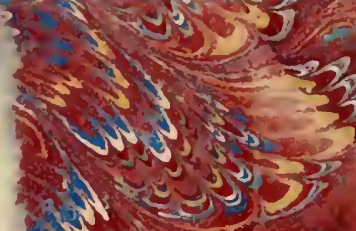

2.

$5 \rightarrow$ is

ex

).

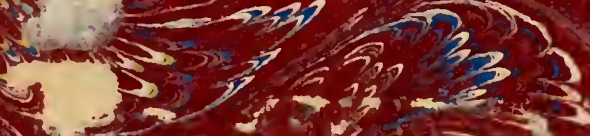

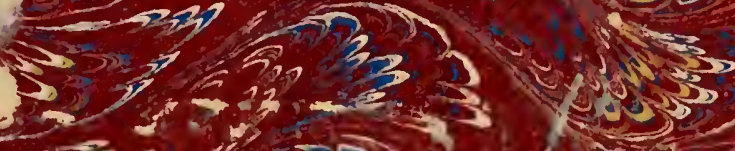

(1)

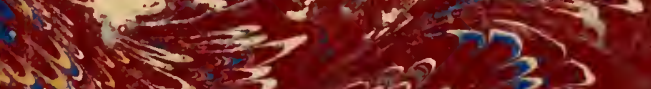

-

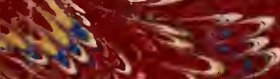

, 3

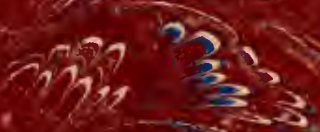

N

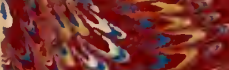

$23^{3}, 2,3$

$x_{x \rightarrow 3} \rightarrow 3$

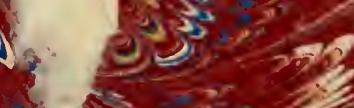
翣

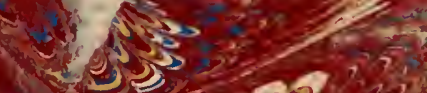

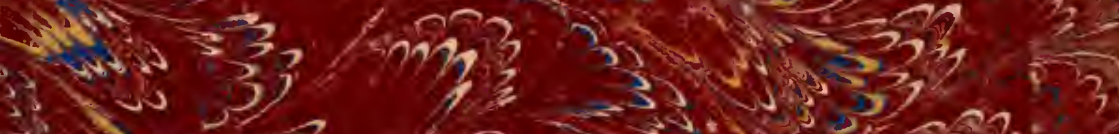

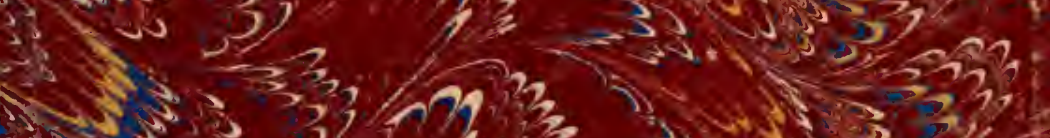

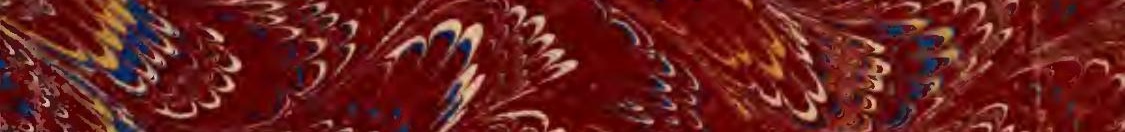

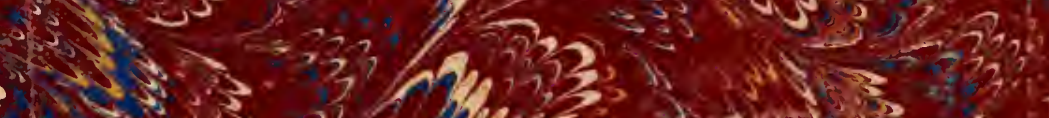

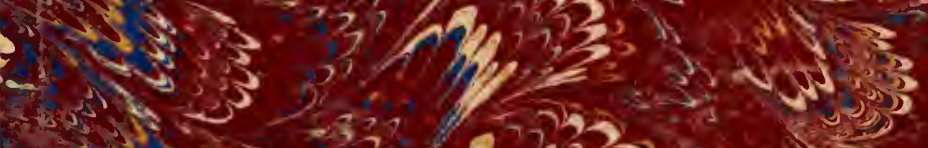
N N

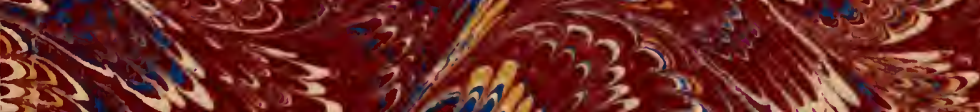




\section{$28 x=53$}

(5) 35

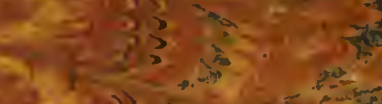

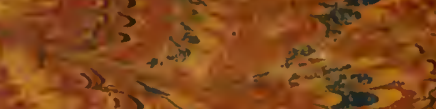

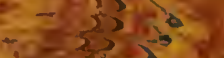

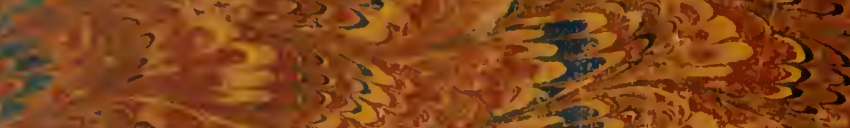

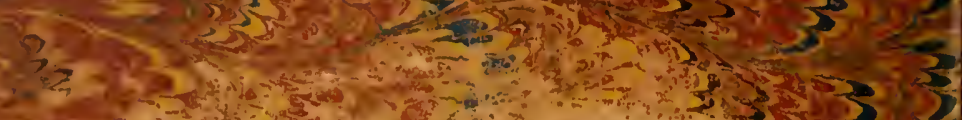

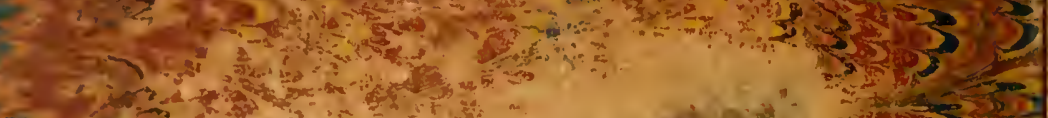

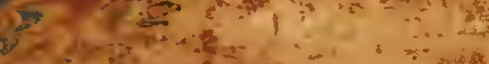

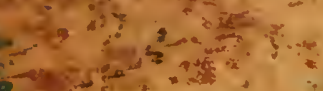

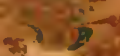

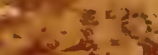

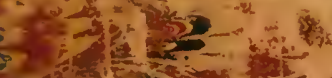

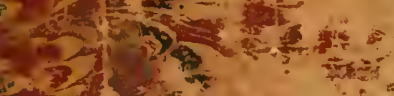

3)

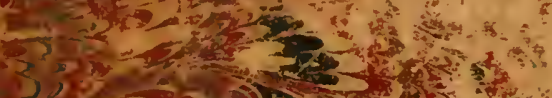

$3,23, y)=3$

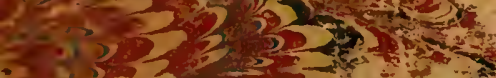

3)

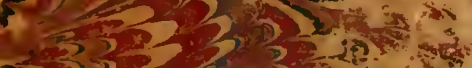

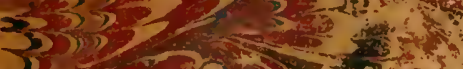

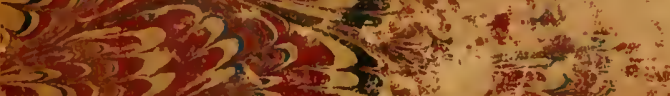

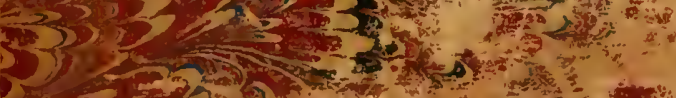

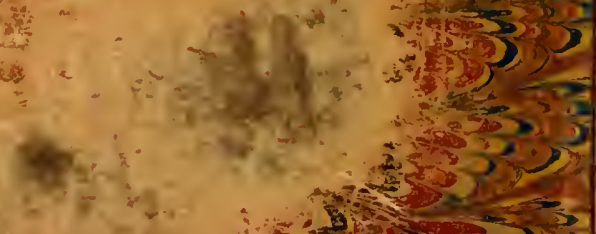

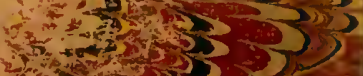
$2 * 3+230$

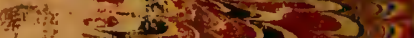

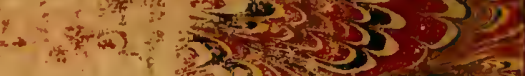

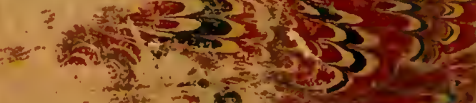

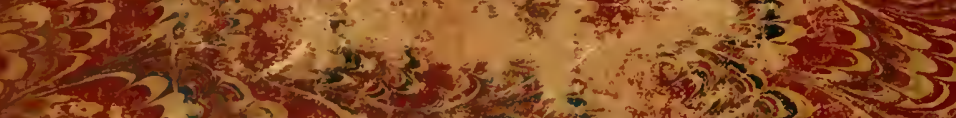

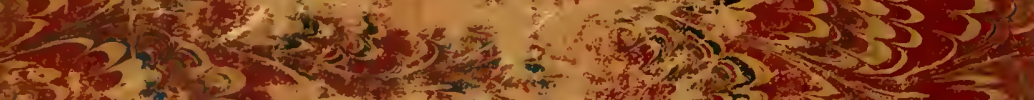

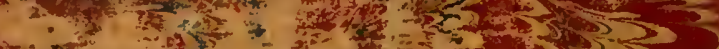

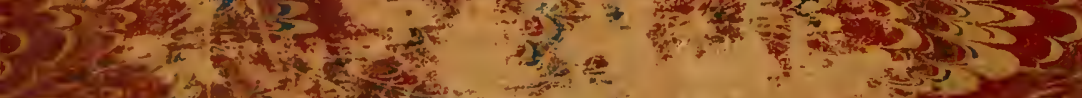

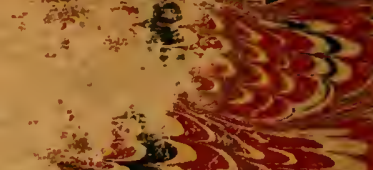

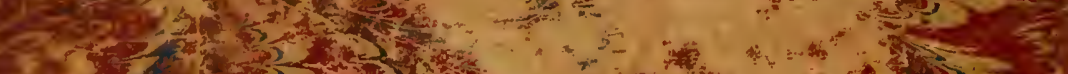

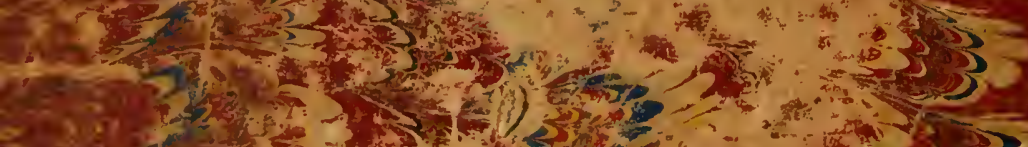

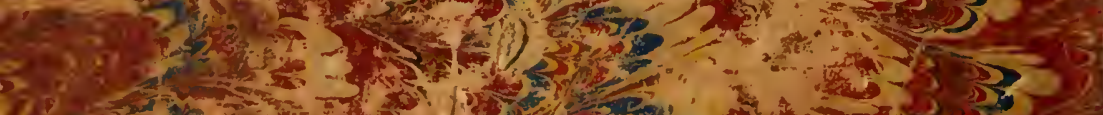

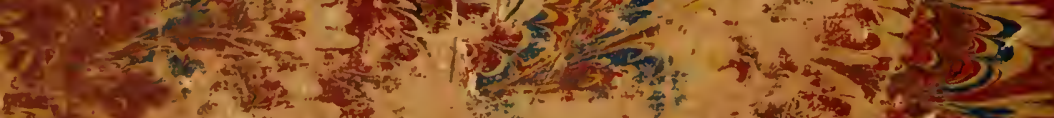

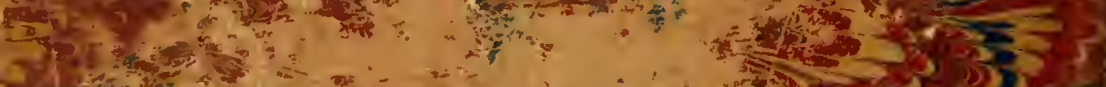

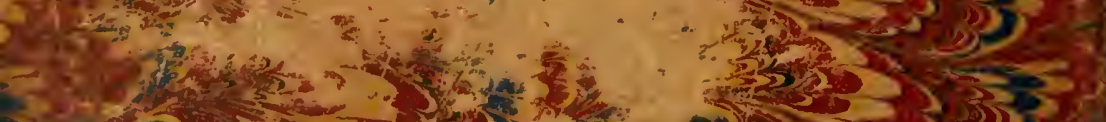

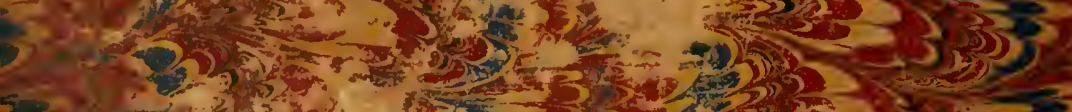

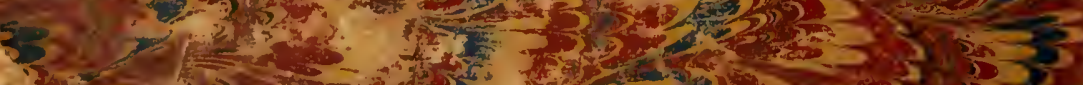

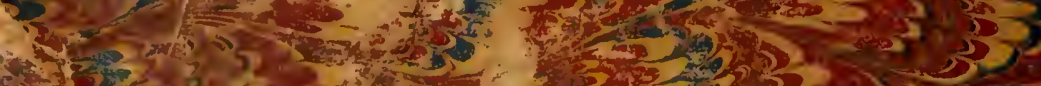

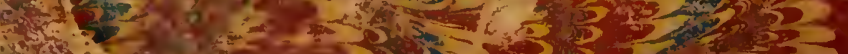

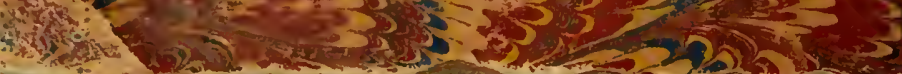
is 35 